\title{
Sealing Performance of Bentonite and Bentonite/Crushed Rock Borehole Plugs
}

Received by DSTI

AUG 121992

\section{Prepared by}

S. Ouyang, J. J. K. Daemen

Department of Mining and Geological Engineering University of Arizona

Prepared for

U.S. Nuclear Regulatory Commission 


\section{AVAILABILITY NOTICE}

\section{Availability of Reference Materials Cited in NRC Publications}

Most documents cited in NRC publications will be avallable from one of the following sources:

1. The NRC Public Document Room, 2120 L Street, NW., Lower Level, Washington, DC 20555

2. The Superintendent of Documents, U.S'. Government Printing Office, P.O. Box 37082, Washington, DC 20013-7082

3. The National Technical Information Service, Springfield, VA 22161

Although the Ilsting that follows represents the majority of documents clted in NRC publications, it is not Intended to be exhaustive.

Referenced documents avallable for inspection and copying for a fee from the NRC Public Document Room Include NRC correspondence and internal NRC memoranda; NRC bulletins, circulars, information notices, Inspection and investigation notices; licensee event reports; vendor reports and correspondence; Commission papers; and applicant and licensee documents and correspondence.

The following documents in the NUREG series are available for purchase from the GPO Sales Program: formal NRC staff and contractor reports. NRC-sponsored conference proceedings, international agreement reports, grant publications, and NRC booklets and brochures. Also available are regulatory guides, NRC regulations in the Code of Federal Regulations, and Nuclear Regulatory Commission Issuances.

Documents avaliable from the National Technical Information Service include NUREG-series reports and technical reports prepared by other Federal agencies and reports prepared by the Atomic Energy Commisslon, forerunner agency to the Nuclear Regulatory Commission.

Documents avallable from public and special technical libraries include all open literature items, such as books, Journal articles, and transactions. Federal Register notices, Federal and State legislation, and congressional reports can usually be obtained from these libraries.

Documents such as theses, dissertations, foreign reports and translations, and non-NRC conference proceedings are available for purchase from the organization sponsoring the publication cited.

Single copies of NRC draft reports are available free, to the extent of supply, upon written request to the Office of Administration, Distribution and Mail Services Section, U.S. Nuclear Regulatory Commission, Washington, DC 20555.

Coples of industry codes and standards used in a substantive manner in the NRC regulatory process are maintained at the NRC Library, 7920 Norfolk Avenue, Bethesda, Maryland, for use by the public. Codes and standards are usually copyrighted and may be purchased from the originating organization or, if they are American National Standards, from the American National Standards Institute, 1430 Broadway, New York, NY 10018.

\section{DISCLAIMER NOTICE}

This report was prepared as an account of work sponsored by an agency of the United States Government. Neither the United States Government nor any agency thereof, or any of their employees, makes any warranty, expressed or implied, or assumes any legal liability of responsibility for any third party's use, or the results of such use, of any information, apparatus, product or process disclosed in this report, or represents that its use by such third party would not infringe privately owned rights. 


\section{DISCLAIMER}

Portions of this document may be illegible in electronic image products. Images are produced from the best available original document. 
NUREG/CR-5685

$\mathrm{CH}, \mathrm{RW}, \mathrm{WA}, \mathrm{WD}, \mathrm{WN}, \mathrm{XC}$

\section{Sealing Performance of Bentonite and Bentonite/Crushed Rock Borehole Plugs}

Prepared by

S. Ouyang, J. J. K. Daemen

Department of Mining and Geological Engineering University of Arizona

Tucson, AZ 85721

Prepared for

Division of Regulatory Applications

Office of Nuclear Regulatory Research

U.S. Nuclear Regulatory Commission

Washington, DC 20555

NRC FIN L1476 



\section{ABSTRACT}

This study includes a systematic investigation of the sealing performance of bentonite and bentonite/crushed rock plugs. American Colloid C/S granular bentonite and crushed Apache Leap tuff have been mixed to prepare samples for laboratory flow testing. Bentonite weight percent and crushed tuff gradation are the major variables studied. The sealing performance assessments include high injection pressure flow tests, polyaxial flow tests, high temperature flow tests, and piping tests. The results indicate that a composition to yield a permeability lower than $5 \mathrm{x} \cdot 10^{-8} \mathrm{~cm} / \mathrm{s}$ would have at least 258 bentonite by weight mixed with well-graded crushed rock. Hydraulic properties of the mixture plugs may be highly anisotropic if significant particle segregation occurs during sample installation and compaction.

Temperature has no significant effect on the sealing performance within the test range from room temperature to $60^{\circ} \mathrm{C}$. Piping damage to the sealing performance is small if the maximum hydraulic gradient does not exceed 120 and 280 for samples with a bentonite content of 25 and 358 , respectively. The hydraulic gradients above which flow of bentonite may take place are deemed critical.

Analytical work includes the introduction of bentonite occupancy percentage and water content at saturation as two major parameters for plug design. A permeability model is developed for the prediction of permeability in clays, especially in view of the difficulties in obtaining this property experimentally. A piping model is derived based on plastic flow theory. This piping model permits the estimation of critical hydraulic gradients at which flow of bentonite takes place. The model can also be used to define the maximum allowable pore diameter of a protective filter layer. 
ABSTRACT ....................... . . . . . . . . . . . . .

LIST OF FIGURES . . . . . . . . . . . . . . . . . . . ix

LIST OF TABLES ....................... . . . $x v$

ACKNOWLEDGMENTS . . . . . . . . . . . . . . . . . xix

LIST OF SYMBOLS . . . . . . . . . . . . . . . . . . $x x i$

EXECUTIVE SUMMARY . . . . . . . . . . . . . . . . . 1

1. INTRODUCTION . . . . . . . . . . . . . . . . . . 5

1.1 Introduction . . . . . . . . . . . . . . 5

1.2 objectives .................... . . . . 6

1.3 Scope and Limitations . . . . . . . . . . . . . 6

1.4 Organization .. . . . . . . . . . . . . . . 7

1.5 Methods of Investigation . . . . . . . . . . . . . . 8

1.5 Source Location of Test Materials . . . . . . . . 8

2. MATERIAL GHARACTERIZATION AND PROPERTIES RELEVANT TO SEALING 10

2.1 Bentonite .................... 10

2.1.1 Engineering Properties ............ . 10

2.1.2 Cation Exchange Capacity . . . . . . . . . 16

2.1.3 Diffuse Double Layer . . . . . . . . . . . . 19

2.1.4 Sealing Engineering Significance of Cation

Exchange and Double Layer . . . . . . . . 22

2.1.5 Microstructure . . . . . . . . . . . . . . 24

2.2 Apache Leap Tuff . . . . . . . . . . . . . . . . . 27

2.2.1 Physical and Mechanical Properties . . . . . . 27

2.2.2 Hydrological Properties . . . . . . . . . 27

2.3 Bentonite/Crushed Tuff .... . . . . . . . . . . 31

2.3.1 Bentonite Content .............. . 31

2.3.2 Crushed Tuff Gradation . . . . . . . . . . 33

2.4 Permeant.................... 35

3. FLOW TEST RESULTS AND ANALYSIS . . . . . . . . . . . . 36

3.1 Sample Preparation and Installation . . . . . . . . 36

3.1.1 Bentonite Plugs . . . . . . . . . . . . . . . . . 36

3.1.2 Bentonite/Crushed Tuff Plugs . . . . . . . . 36

3.1.3 Sample Designation . . . . . . . . . . . . . 37

3.2 Flow Test Methods . . . . . . . . . . . . . . . . . 37

3.3 Results of Bentonite Permeability Testing . . . . . . . 39

3.3.1 Influence of Permeant . . . . . . . . . . . . . 41

3.3.2 Influence of Sample Size .. . . . . . . . 41 
3.3.2.1 Results of First Flow Test Sequence . . 48

3.3.2.2 Results of Second Flow Test Sequence . . 52

3.3.2.3 Water Content Distribution of Sample B-C-4-B . . . . . . . . . . 52

3.3.2.4 Cracking Patterns of Bentonite Disks . . 55

3.3.3 Results of High Injection Pressure Flow Tests . . 55

3.3.4 Permeability of Bentonite to Air . . . . . . . . 62

3.4 Permeability of Bentonite/Crushed Tuff . . . . . . . . 65

3.4.1 Longitudinal Flow Tests........ . . . . 65

3.4.1.1 Mixed Samples with 15\% Bentonite by

Weight............... . 65

3.4.1.2 Samples with 25 or $35 \%$ Bentonite

by Weight - First Test

Sequence (Downward Permeation). . . . 65

3.4.1.3 Samples with 25 or $35 \%$ Bentonite

by Weight - Second Test

Sequence (Downward Permeation) . . . . . 73

3.4.1.4 Samples with 25 or $35 \%$ Bentonite

by Weight - Third Test

Sequence (Upward Permeation) . . . . . . 77

3.4.1.5 Samples with 25 or $35 \%$ Bentonite

by Weight - Fourth Test

Sequence (Downward Permeation) . . . . . 91

3.4.1.6 Samples B/AL-C-12-25/A and

B/AL-C-12-35/A ............. 91

3.4.1.7 Effects of Piping . . . . . . . . . . . 100

3.4.1.8 Mixed Samples in Stainless Steel

Permeameters ............. 102

3.4.1.8.1 Double-Pipette Falling Head

Flow Testing ....... 102

$\begin{array}{lll}\text { 3.4.1.8.2 } & \begin{array}{l}\text { Effect of the Upward Seepage } \\ \text { Force on Sealing Performance }\end{array} \\ & 108\end{array}$

3.4.1.8.3 High Injection Pressure Flow

Testing on Samples B/AL-C-

8-25/A-S and B/AL-C-8-35/A-S 108

3.4.1.9 Flow Testing of Samples Containing

Crushed Tuff with Ideal Gradation . . . 118

3.4.1.9.1 Tests in PVC Permeameters . . 118

3.4.1.9.2 Tests in Stainless Steel

Permeameters ...... . 125

3.4.2 Polyaxial Flow Tests . . . . . . . . . . . 125

3.4.3 High Temperature Flow Tests... . . . . . . . 136

3.4.4 Piping Tests . . . . . . . . . . . . . 139

3.4.4.1 Sample B/AL-C-4-25/A-P-B . . . . . . 139

3.4.4.2 Sample B/AL-C-4-25/A-P-A . . . . . . . 142

3.4.4.3 Samples B/AL-C-4-35/A-P-A and
B/AL-C-4-35/A-P-B ....... . . 142

3.4.5 Flow Tests of Bentonite/Crushed Tuff Seals in
Tuff Cylinders . . . . . . . . . . . . 144 
TABLE OF CONTENTS-- Continued

Page

3.4.5.1 Mixed Sample in RC-1 . . . . . . . . 144

3.4.5.2 Mixed Sample in RC-2 . . . . . . . . . 146

3.4.5.3 Mixed Sample in RC-3 . . . . . . . . 148

3.4.5.4 Mixed Sample in RG-4 . . . . . . . . . 151

3.5 Analysis . . . . . . . . . . . . . . . . . . . . . 151

3.5.1 Bentonite Permeability as a Function of Void

Ratio . . . . . . . . . . . . . . . 151

3.5.2 Sealing Performance of Bentonite/Crushed Tuff

Plugs . . . . . . . . . . . . . . . 151

3.5.3 Influence of Gradation of Crushed Tuff . . . . . 158

3.5.3.1 Bulk Porosity of Systems of Crushed

Tuff Particles............ . 158

3.5.3.2 Permeability of Systems of Crushed

Tuff Particles ........... . . 160

3.5.3.3 Bulk Porosity of Systems of Crushed

Tuff Particles in the Presence of

Bentonite . . . . . . . . . 165

3.5.3.4 Discussion .... . . . . . . . . 165

3.5.4 Bentonite Occupancy Percentage and Water
Content at Saturation . . . . . . . . 168

3.5.5 Mechanical Factors Affecting the Deterioration
of Sealing Performance . . . . . . . . 170

4. PIPING, EROSION AND FLOW OF BENTONITE . . . . . . . . . . 172

4.1 Introduction . . . . . . . . . . . . . . . . 172

4.2 Effect of Dispersion Erosion . . . . . . . . . . . 173

4.3 Zaslavsky-Kassiff Mechanical Model of Piping . . . . . 175

4.4 Model of Bentonite Flow . . . . . . . . . . . . . . . . 177

4.4.1 Theory of Flow through Capillaries . . . . . . . 177

4.4.2 Factors Influencing the Yield Stress of Bentonite 179

4.4.3 Determination of Yield Stress of Bentonite

Mixed with Distilled Water . . . . . . . . 180

4.4.4 Relation Between Water Content and Yield

Stress of Bentonite . . . . . . . . . . . 183

4.4.5 Reliability of Prediction of Bentonite Yield
Stress . . . . . . . . . . . . . . . 184

4.4.6 Prediction of Critical Gradient Using the
Relation Between Yield Stress and Water Content . 184

4.4.7 Filter Design ... . . . . . . . . . . 190

5. PREDICTION OF PERMEABILITY AND SWELLING PRESSURE OF BENTONITE 193

5.1 Prediction of Permeability . . . . . . . . . . . 193

5.1.1 Introduction ..... . . . . . . . . . 193

5.1.2 Kozeny-Carman Equation . . . . . . . . . . 194

5.1.3 Refined Kozeny Carman Equation for Clays . . . . 195

5.1.4 Validation of the Refined Kozeny-Garman Equation 200

5.1.5 Discussion . . . . . . . . . . . . . 203 
TABLE OF CONTENTS-- -

Page

5.2 Prediction of Swelling Pressure . . . . . . . . . 203

5.2.1 Introduction . . . . . . . . . . . . 203

5.2.2 Bolt's Model .. . . . . . . . . . . . . 207

5.2.3 Yong and Warkentin's Model . . . . . . . . 208

5.2.4 Sridharan-Jayadeva's Model . . . . . . . . . 210

5.2.5 Validation ................ 210

5.2 .6 Discussion . . . . . . . . . . . . . 212

6. SUMMARY, CONCLUSIONS, AND RECOMMENDATIONS . . . . . . . . . . 214

6.1 Summary of Results . . . . . . . . . . . . . . . 214

6.1.1 Bentonite Plugs................. 214

6.1.2 Bentonite/Crushed Tuff Plugs . . . . . . . . 215

6.2 Conclusions .. . . . . . . . . . . . . . . 217

6.3 Recommendations . . . . . . . . . . . . . 219

REFERENCES . . . . . . . . . . . . . . . . . . . . 223

APPENDIX A: SAMPLE PREPARATION AND INSTALLATION FOR

PERMEABILITY TESTING ON BENTONITE AND BENTONITE/

CRUSHED TUFF PLUGS TEST PROCEDURE . . . . . . 239

APPENDIX B: PERMEABILITY TESTING OF BENTONITE AND BENTONITE/

CRUSHED TUFF PLUGS TEST PROCEDURE . . . . . . . 242

APPENDIX C: FLOW TEST RESULTS OF COMPACTED BENTONITE PLUGS • • 248

APPENDIX D: FLOW TEST RESULTS OF THE 10.16 CM DIAMETER

MIXTURE SAMPLES INSTALLED IN PVC PERMEAMETERS . . . 261

APPENDIX E: FLOW TEST RESULTS OF THE 10.16 CM DIAMETER

MIXTURE SAMPLES INSTALLED IN PVC PERMEAMETERS

(SECOND AND THIRD TEST SEQUENCES) . . . . . . . 268

APPENDIX F: EXPERIMENTAL RECORDS OF PIPING TESTS . . . . . . . . 296

APPENDIX G: DERIVATION OF THE BUCKINGHAM EQUATION FOR PIASTIC

FLOW THROUGH PIPES . . . . . . . . . . . . 307

APPENDIX H: PROCEDURE FOR THE DETERMINATION OF YIELD STRESS

OF BENTONITE FLOWING IN GLASS TUBES . . . . . . . . 311 


\section{LIST OF FIGURES}

Figure

Page

1.1 Flow chart of the investigation for sealing performance assessments of bentonite and bentonite/crushed tuff plugs .............

2.1 Moisture-density relations of American Colloid G/S granular bentonite (Test 1) . . . . . . . . . .

2.2 Moisture-density relations of American Colloid C/S granular bentonite (Test 2) . . . . . . . . . . .

2.3 Push-out test results for compacted bentonite plugs . . . 17

2.4 Diagrammatic sketch of the montmorillonite structure . . 18

2.5 Effect of electrolyte concentration on ion distribution in the double layer................. 21

2.6 Effect of cation valence on double layer concentrations . 23

2.7 Modes of particle association in clay suspensions . . . . 25

2.8 Scanning electron microscope view of sedimented montmorillonite . . . . . . . . . . . . . . 26

2.9 Schematic microstructure of soft $\mathrm{Na}$ bentonite saturated and matured at room temperature . . . . . . . . . . . 28

2.10 Grain size distributions of crushed tuff . . . . . . . 34

3.1 Bentonite and bentonite/crushed tuff sample number designation system ................ 38

3.2 Example of discrepancies between permeabilities obtained from a steady flow test and hydraulic and mechanical transient flow tests .............. . 40

3.3 Flow test results of four $25-\mathrm{cm}$ (1-inch) diameter borehole plugs with different permeants . . . . . . .

3.4 Flow test results of Sample B-S-1-A when permeated with $2 \%$ sodium pyrophosphate solution . . . . . . . . . . 44

3.5 Flow test results of Sample B-S-1-B when permeated with distilled water . . . . . . . . . . . . . . 44 44

3.6 Flow test results of Sample B-S-1-C when permeated with $4 \%$ sodium pyrophosphate solution . . . . . . . . . . . 


\section{LIST OF FIGURES-- -}

Figure

Page

3.7 Flow test results of Sample B-S-1-D when permeated with $2 \%$ sodium pyrophosphate solution . . . . . . . . . .

3.8 Permeability results of bentonite Samples B-C-1-A and $B-C-1-B$, using double-pipette falling head method . . . .

3.9 Permeability results of bentonite Samples B-C-2-A and B-C-2-B, using double-pipette falling head method . . .

3.10 Permeability results of bentonite Samples B-C-4-A and B-C-4-B, using double-pipette falling head method and standard falling head method . . . . . . . . . . . .

3.11- Permeability results for bentonite Samples B-C-1-A, 82- 53-

3.15 B-C-1-B, B-C-2-A, B-C-2-B, and B-C-4-A . . . . . . 54

3.16- Bentonite disks $1,2,3$ and 4 sliced from Sample B-C-4-B 56-

3.18 (at 1,24 , and 48 hours after slicing, respectively) 57

3.19 Preliminary permeability results of compacted bentonite samples installed in stainless steel permeameters . . . . 60

3.20- Permeability vs. hydraulic gradient for Samples 92- 61-

3.23 B-C-1-A-S, B-C-1 3/8-A-S, B-C-2 3/8-A-S, and B-C-4-A-S . 63

3.24 Permeability to air of compacted bentonite samples installed in plexiglass permeameters of 25.4 in diameter

3.25 Permeability to air vs. dry density of compacted bentonite samples installed in plexiglass permeameters of 25.4 in diameter . . . . . . . . . . . . . . . . 64

3.26 Permeability results of Sample B/AL-C-4-15/A . . . . . 66

3.27 Permeability results of Sample B/AL-C-4-15/B, using the double-pipette falling head and standard falling head method... . . . . . . . . . . . . . . . .

3.28 Permeability results of Sample B/AL-C-4-15/C, using standard falling head method

3.29 Erosion channels in B/AL-C-4-15/A and B/AL-C-4-15/C ...

3.30 Permeability results of Sample B/AL-C-4-25/A, using constant head method

3.31- Permeability results of Sample B/AL-C-4-35/A, using 


\section{LIST OF FIGURES-- Continued}

Figure

Page

3.33- Permeability results of Sample B/AL-C-4-25/A at

3.35 various injection pressures . . . . . . . . . . .

3.36- Permeability results of Sample B/AL-C-4-25/B at

3.38 various injection pressures . . . . . . . . . . . .

3.39- Permeability results of Sample B/AL-C-4-35/A at

3.41 various injection pressures . . . . . . . . . . 76

3.42- Cumulative inflow and outflow vs. time for Samples 78-

3.44- B/AL-C-4-25/A, B/AL-C-4-25/B and B/AL-C-4-35/A . . . . 79

3.45- Flow rate vs. hydraulic gradient for Samples

3.50 B/AL-C-4-25/A, B/AL-C-4-25/B, B/AL-C-4-25/C, 114- 80-

$B / A L-C-4-35 / A, B / A L-C-4-35 / B$, and $B / A L-C-4-35 / C$. . . 82

3.51- Comparison between first-run and second-run permeability

3.56 results of Samples B/AL-C-4-25/A, B/AL-C-4-25/B,

$B / A L-C-4-25 / C, B / A L-C-4-35 / A, B / A L-C-4-35 / B$, and $117-$

$83-$

$B / A L-C-4-35 / C$ at injection pressures of 207 and $345 \mathrm{kPa}$

3.57- Permeability results of the mixture samples containing

$3.5825 \%$ and $35 \%$ bentonite by weight (measured in the 89 upward permeation) ................. 90

3.59- Upward and downward permeability of Sample B/AL-C-4-25/A 92-

3.60 under various injection pressures . . . . . . . . . . 93

3.61- Upward and downward permeability of Sample B/AL-C-4-35/A 94-

3.62 under various injection pressures . . . . . . . . 95

3.63- Permeability results of Samples B/AL-C-4-25/A, 96-

$3.66 \mathrm{~B} / \mathrm{AL}-\mathrm{C}-4-35 / \mathrm{A}, \mathrm{B} / \mathrm{AL}-\mathrm{C}-4-35 / \mathrm{B}$, and $\mathrm{B} / \mathrm{AL}-\mathrm{C}-4-35 / \mathrm{C}$.... 99

3.67- Permeability results of Samples B/AL-C-12-25/A and

3.68 B/AL-C-12-35/A . . . . . . . . . . . . 101

3.69 Permeability results of the mixture samples installed in stainless steel permeameters (25\% bentonite by weight) . 106

3.70 Permeability results of the mixture samples installed in stainless steel permeameters ( $35 \%$ bentonite by weight).

3.71 Cumulative inflow and outflow vs. time for Samples

$\mathrm{B} / \mathrm{Al}-\mathrm{C}-4-25 / \mathrm{A}-\mathrm{S}$ and $\mathrm{B} / \mathrm{AL}-\mathrm{C}-8-25 / \mathrm{A}-\mathrm{S} . . . . . .$.

3.72 Cumulative inflow and outflow vs. time for Samples B/AL-C-4-35/A-S and B/AL-C-8-35/A-S . . . . . . . . 
LIST OF FIGURES-- - Continued

Figure

Page

3.73 Upward and downward permeability of Sample

B/AL-C-4-25/A-S . . . . . . . . . . . . . . .

3.74 Upward and downward permeability of Samples

B/AL-C-8-25/A-S and B/AL-C-8-35/A-S ..........

3.75- Permeability vs. hydraulic gradient and flow rate vs.

3.76 hydraulic gradient for Sample B/AL-C-8-25/A-S . . . . .

3.77- Permeability vs. hydraulic gradient and flow rate vs.

3.78 hydraulic gradient for Sample B/AL-C-8-35/A-S . . . . . 116

3.79 Sample B/AL-C-8-25/A-S after flow testing . . . . . . 117

3.80 Sample B/AL-C-8-35/A-S after flow testing . . . . . . 119

3.81 Permeability results of Samples B/AL-C-4-15/FA-A, $B / A L-C-4-15 / F A-B, B / A L-C-4-25 / F A-A$, and B/AL-C-4-25/FA-B

3.82 Cumulative inflow and outflow vs. time for Samples

B/AL-C-4-15/FA-A and B/AL-C-4-15/FA-B . . . . . . .

3.83- Room temperature effect on the permeability measurement 122-

3.84 of Samples B/AL-C-4-15/FA-A and B/AL-C-4-15/FA-B . . . 123

3.85- Permeability vs. hydraulic gradient and flow rate vs.

3.86 hydraulic gradient for Sample B/AL-C-4-25/FA-A

(downward flow testing)

3.87- Permeability vs. hydraulic gradient and flow rate vs.

3.88 hydraulic gradient for Sample B/AL-C-4-25/FA-A

(upward flow testing) . . . . . . . . . . .

3.89- Permeability vs. hydraulic gradient and flow rate vs.

3.90 hydraulic gradient for Sample B/AL-C-4-25/FA-B

(downward flow testing)

3.91- Permeability vs. hydraulic gradient and flow rate vs.

3.92 hydraulic gradient for Sample B/AL-C-4-25/FA-B

(upward flow testing) . . . . . . . . . . . .

3.93 Vertical and horizontal permeabilities of a mixture containing $25 \%$ bentonite and $75 \%$ type A crushed tuff

3.94 Vertical and horizontal permeabilities of a mixture containing $35 \%$ bentonite and $65 \%$ type A crushed tuff

3.95 Permeabilities of Sample B/AL-C-4-25/A-S at room and at elevated temperatures .. . . . . . . . . . . . 


\section{LIST OF FIGURES-- Continued}

Figure

Page

3.96 Permeabilities of Sample B/AL-C-4-35/A-S at room and at elevated temperatures . . . . . . . . . . . . . . 138

3.97 Vertical permeabilities of Sample B/AL-C-4-25/A-P-B before and after piping . . . . . . . . . . . . . . . . 140

3.98 Failure of Sample B/AL-C-4-25/A-P-B; flow channels impregnated with cast resin (white) . . . . . . . . . .

3.99 Variations of vertical permeability of Sample B/AL-C4-25/A-P-A as a function of hydraulic gradient during a piping test with the bottom and side outlets open . . . 143

3.100 Bentonite/crushed tuff cylinder pushed out of rock cylinder RC-1 . . . . . . . . . . . . . . . .

3.101 Piping channels that developed along the rock-plug interface of tuff cylinder RC-2 . . . . . . . . . . . .

3.102 Shrinkage and desiccation cracks of the bentonite/ crushed tuff plug in rock cylinder RC-2 after the top of the sample had been exposed to the atmosphere for about 14 days . . . . . . . . . . . . . . . .

3.103 The healing of shrinkage and desiccation cracks of the bentonite/crushed tuff seal in rock cylinder RC-2 upon rehydration ....................

3.104 Bentonite/crushed tuff cylinder pushed out of rock cylinder RC-2 . . . . . . . . . . . . . . . . .

3.105 Permeability of the C/S granular bentonite as a function of void ratio

3.106 Permeability of Wyoming sodium bentonite as a function of void ratio......................

3.107 Permeability of bentonite/crushed tuff mixtures as a function of bentonite content ..............

3.108 Appearance of particle segregation highlighted by desiccation cracks

3.109 Appearance of crushed tuff aggregates emplaced by scooping and by pouring

4.1 Yield stress of bentonite pastes vs bentonite weight percent . . . . . . . . . . . . . . 


\section{LIST OF FIGURES - - - Continued}

Figure

Page

4.2 Relations between the critical hydraulic gradient and pore radius for various water contents of bentonite . . . 185

4.3 Relations between the critical hydraulic gradient and bentonite water content for different pore radii . . . . 192

5.1 Discrepancies between measured flow rates and flow rates predicted from the Kozeny-Carman equation . . 196

5.2 Cluster model of clays . . . . . . . . . . . . 197

5.3 Possible discrepancies for systems of clusters . . . . . 198

5.4 Discrepancies between flow rates predicted from the Kozeny-Carman equation and from the refined equation . . 202

5.5 Linear relationship between the permeability of bentonite and the porosity function $n^{4} /(1-n)^{3}$. . . . 206 


\section{LIST OF TABLES}

Table

Page

2.1 Chemical Analysis of C/S Granular Bentonite . . . . . . . 11

2.2 Grain Size Distribution of American Colloid C/S

Granular Bentonite (Air-Dried) . . . . . . . . . . 12

2.3 Moisture-Density Relations of American Colloid C/S

Granular Bentonite . . . . . . . . . . . . . 13

2.4 Capacity of Metallic Bases of C/S Granular Bentonite . . 20

2.5 Chemical Composition of Tuff . . . . . . . . . . . 29

2.6 Mechanical and Physical Properties of Apache Leap and

Topopah Spring Tuff Specimens . . . . . . . . . 30

2.7 Summary of Water Hydraulic Conductivities of Tuffs . . . 32

3.1 Sample Descriptions of Sedimented Bentonite Plugs . . . . 42

3.2 Sample Descriptions of Compacted Bentonite Plugs . . . . 46

3.3 Changes in Sample Length of Compacted Bentonite Plugs . . 47

3.4 Thickness, Relative Position and Use of Disks

3.5 Water Content Distribution (Sample B-C-4-B) . . . . . 58

3.6 Sample Characteristics of Compacted Plugs Installed
in Stainless Steel Permeameters . . . . . . . . 59

3.7 Inflow-Outflow Record for Bentonite Plugs . . . . . . . 103

3.8 Measurements' of Solids Carried in the Outflows of Samples B/AL-C-4-25/A and B/AL-C-4-25/B........ 104

3.9 Flow Rates and Solids Carried in the Outflows of Mixed Samples Installed with $25 \%$ and $35 \%$ Bentonite by Weight..................... 105

3.10 Sample Dimensions and Bulk Density of Mixed Samples Installed in Stainless Steel Permeameters . . . . . . . . 111 
LIST OF TABLES-- -

Table

Page

3.11 Flow Test Results of Samples B/AL-C-4-25/FA-A, B/AL-C-4-25/FA-B and B/AL-C-4-25/A . . . . . . . . 124

3.12 Summary of Flow Test Results of Samples B/AL-C-8-25/FC-S and $B / A L=C-8-35 / F C-S$. . . . . . . . . . . . . 130

3.13 Summary of Polyaxial Flow Test Results . . . . . . . 135

3.14 Results of Flow Tests at Elevated Temperatures . . . . 135

3.15 Characteristics of Crushed Tuff/Bentonite Plugs Installed in Tuff Cylinders . . . . . . . . . . . . . . 145

3.16 Flow Test Results for the Bentonite/Crushed Tuff Plugs in Tuff Cylinders RC-3 and RC-4 . . . . . . . . . . . . 145

3.17 Measured Permeabilities of Bentonites . . . . . . . . 152

3.18 Particle Size Distribution of Nine Grushed Tuff Gradations . . . . . . . . . . . . . . 159

3.19 Bulk Porosities of Packings of Crushed Tuff Particles . . 161

3.20 Flow Test Results of Crushed Tuff Aggregates . . . . . . 164

3.21 Calculated Bentonite Occupancy Percentage and Its

Water Content at Saturation . . . . . . . . . . . . 166

4.1 Dispersive Erosion Rates of Mixed Samples Installed with $25 \%$ and $35 \%$ Bentonite by Weight . . . . . . . . . 174

4.2 Results of Bentonite Flow Tests through a $3.6 \mathrm{~mm}$ Glass Capillary . . . . . . . . . . . . . . . 181

4.3 Correlation Coefficients of Yield Stress-Water Content . 186

4.4 Comparison of Predicted Yield Stresses and the Experimental Axial Shear Strengths for Different Bentonite Products . . . . . . . . . . . . . .

4.5 Predicted and Experimental Critical Pressure Gradients for Bentonite Flow

5.1 Differences Between Predicted Permeabilities from the Original and Refined Kozeny-Carman Equations . . . 201

5.2 Measured and Predicted Permeabilities of Bentonites . . . 204

5.3 Measured and Predicted Permeabilities of Bentonite MX-80 205 
LIST OF TABLES--Gontinued

Table

Page

5.4 Summary of Swelling Test Results . . . . . . . . . . . 211

5.5 Measured and Predicted Swelling Pressures of Bentonite • 213 



\section{ACKNOWLEDGMENTS}

The research reported on has been performed by the University of Arizona, Department of Mining and Geological Engineering, for the U.S. Nuclear Regulatory Commission (NRC). Project monitor for the NRC is Mr. Jacob Philip. The project is under Mr. M. Silberberg, Chief, Waste Management Branch, Division of Engineering, Office of Nuclear Regulatory Research.

Dr. Jaak J.K. Daemen, Adjunct Associate Professor, Department of Mining and Geological Engineering, University of Arizona, is the Principal Investigator. Dr. James G. McCray, Acting Director, Nuclear Fuel Cycle Research Program, Department of Nuclear and Energy Engineering, University of Arizona, has provided project management assistance and nuclear waste technical assistance. S. Ouyang has performed the theoretical and experimental work as part of his Ph.D. degree requirements. Experimental assistance has been provided by Colin Sharpe, Jinshan Tang, David Smith and Chongwei Ran. Mike Porter has typed, assembled, and produced the report.

Mr. Doug Excell of American Colloid, Arlington Heights, IL, has provided $\mathrm{C} / \mathrm{S}$ granular bentonite. We gratefully acknowledge the cooperation of Magma Copper Co., and in particular of Mr. Frank Florez, for allowing us to collect tuff blocks. 



\section{LIST OF SYMBOLS}

\section{Chapters 1 and 2}

$1 / \mathrm{k}$ : thickness of the diffusive double layer

$\mathrm{C}_{0}$ : cation concentration of pore fluid.

D : dielectric constant

k : Boltzmann constant ( $\left.1.38 \times 10^{16} \mathrm{erg} /{ }^{\circ} \mathrm{K}\right)$

T : absolute temperature $\left({ }^{\circ} \mathrm{K}\right)$, or time

e : unit electronic charge (16 $\times 10^{-20}$ coulomb, or void ratio

$\mathrm{v} \quad$ : valence of cation

$n_{0} \quad$ : ion concentration of electrolyte at great distance from clay particle surface

cec : cation exchange capacity

$\mathrm{p}_{w} \quad$ : weight percent passing sieve aperture $d$

d : sieve aperture or half-distance between two clay plates

$\mathrm{D}_{\max }$ : maximum particle size

$\mathrm{n}$ : porosity or exponent of the Fuller-Thompson grading equation

cu : coefficient of uniformity $\left(d_{60} / d_{10}\right)$

$d_{60}$ : sieve aperture at which $60 \%$ by weight of particles pass ( similarly, for $d_{50}, d_{15}, d_{10}, d_{5}$ ).

ppm : parts per million

$\mathrm{K}$ : coefficient of permeability (or hydraulic conductivity) when expressed in $\mathrm{L} / \mathrm{T}$; intrinsic (or specific) permeability when expressed in $L^{2}$

Q : discharge $\left(L^{3}\right)$ or flow rate $\left(L^{3} / T\right)$

L : sample length

A : cross-sectional area of permeameters

a : cross-sectional area of the inflow and outflow reservoirs, or a geometric coefficient

$t$ : elapsed time of discharge

h : head difference across the specimen

$h^{1}$ : head difference across specimen at time $t^{1}$

$h^{2} \quad:$ head difference across specimen at time $t^{2}$

$F_{\text {in }} \quad$ : amount of inflow

$F_{\text {out }} \quad$ : amount of outflow 


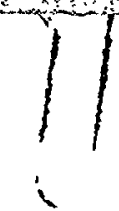

Chapter 3

$P_{w 1} \quad$ : bentonite weight percent based on dry weight

$n_{t} \quad$ : bulk porosity of crushed-tuff phase

$\gamma_{t}:$ dry unit weight of tuff particle

$G_{g b}:$ specific gravity of bentonite

$\gamma_{\mathrm{w}} \quad$ : unit weight of water

$w_{b s}$ : water content of bentonite at saturation

$p_{w z}$ : bentonite weight percent based on air-dried weight

$w_{x t}$ : water content of air-dried crushed tuff

$w_{r b}$ : water content of air-dried bentonite

$p_{w 3}$ : bentonite weight percent required to fill the voids between crushed tuff particles and obtain the maximum dry density of bentonite (ASTM D698) in a mixture

$W_{b}$ : water content of bentonite

$\gamma_{b}$ : unit weight of bentonite at water content of $w_{b}$

$W_{I b}:$ air-dried weight of bentonite

$v_{v} \quad$ : volume of voids between crushed tuff particles

$W_{I t}$ : air -dried weight of crushed tuff

\section{Chapter 4}

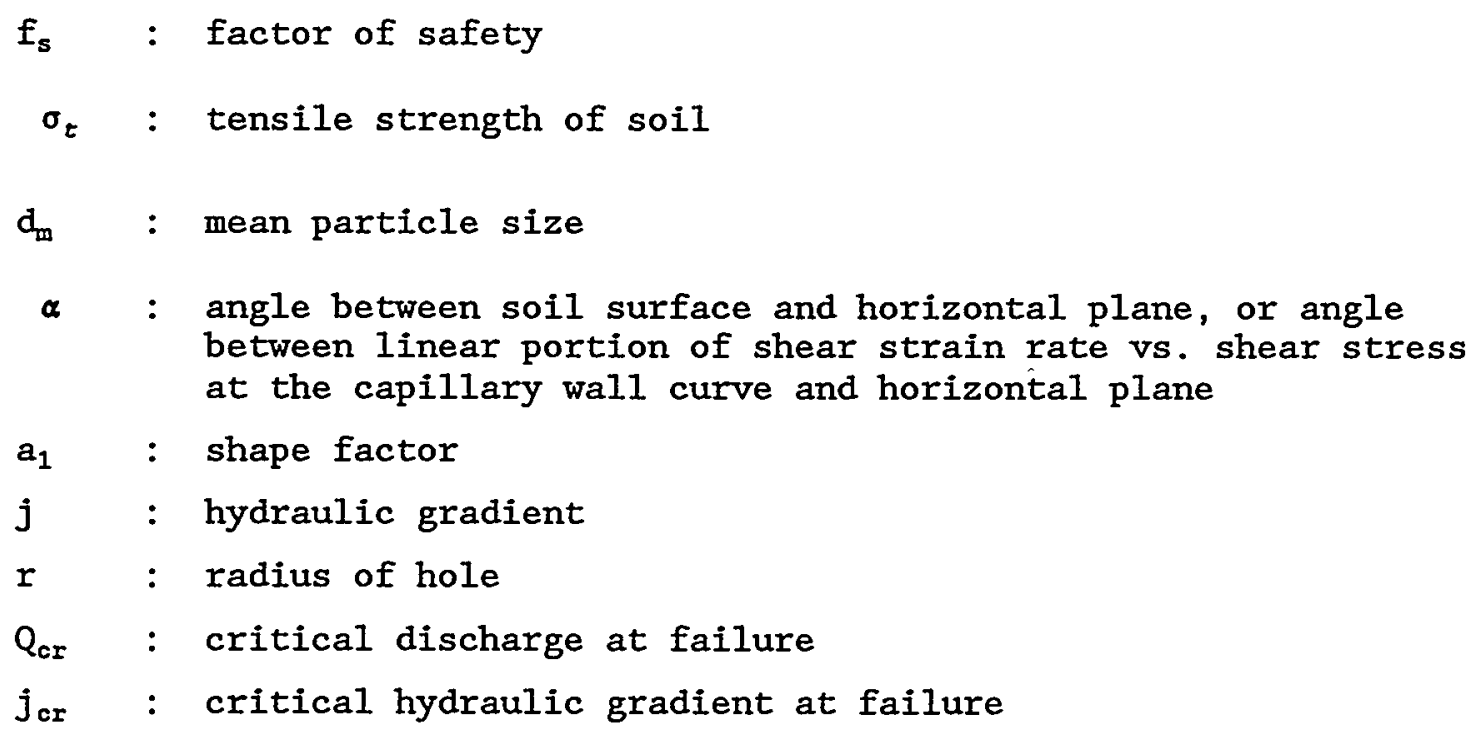


$P_{o}, \Delta P$ : pressure difference between length $\Delta L$

$\Delta L$ : length difference between two points or levels

$\mathrm{R}$ : radius of capillary, or gas constant

$\eta \quad$ : fluid viscosity

$\eta_{p 1}:$ plastic viscosity

$\tau \quad:$ shear stress

$\tau_{f} \quad:$ shear stress at failure, or yield stress

$e_{B} \quad$ : shear strain

dt : time differential

$\mathrm{V}$ : volume of discharge

$u \quad:$ mobility of material $=1 / \eta_{p 1}$

f : yield stress of material

p : $21 f / R$

$\mathrm{V}_{\mathrm{r}} \quad$ : slip velocity at wall of capillary

$\tau_{R} \quad$ : shear stress at wall of capillary

w : water content

$\mathrm{x}$ : bentonite content

$\mathrm{R}^{2} \quad$ : correlation coefficient

$i_{c, p} \quad$ : critical pressure gradient

$\mathrm{R}_{m} \quad$ : representative pore radius of crushed rock skeleton

$i_{c, 1}$ : critical pressure gradient obtained experimentally

Chapter 5 ,

m : shape factor of conducting pores

t : tortuosity

so : specific surface $\left[\right.$ in $\left(L^{2} / L^{3}\right)$ or $\left.\left(L^{2} / M\right)\right]$

$\eta_{1}$ : effective porosity

$\mathrm{N} \quad$ : number of clay particles per cluster

$e_{c} \quad$ : intra-cluster void ratio

$e_{p} \quad$ : inter-cluster void ratio 


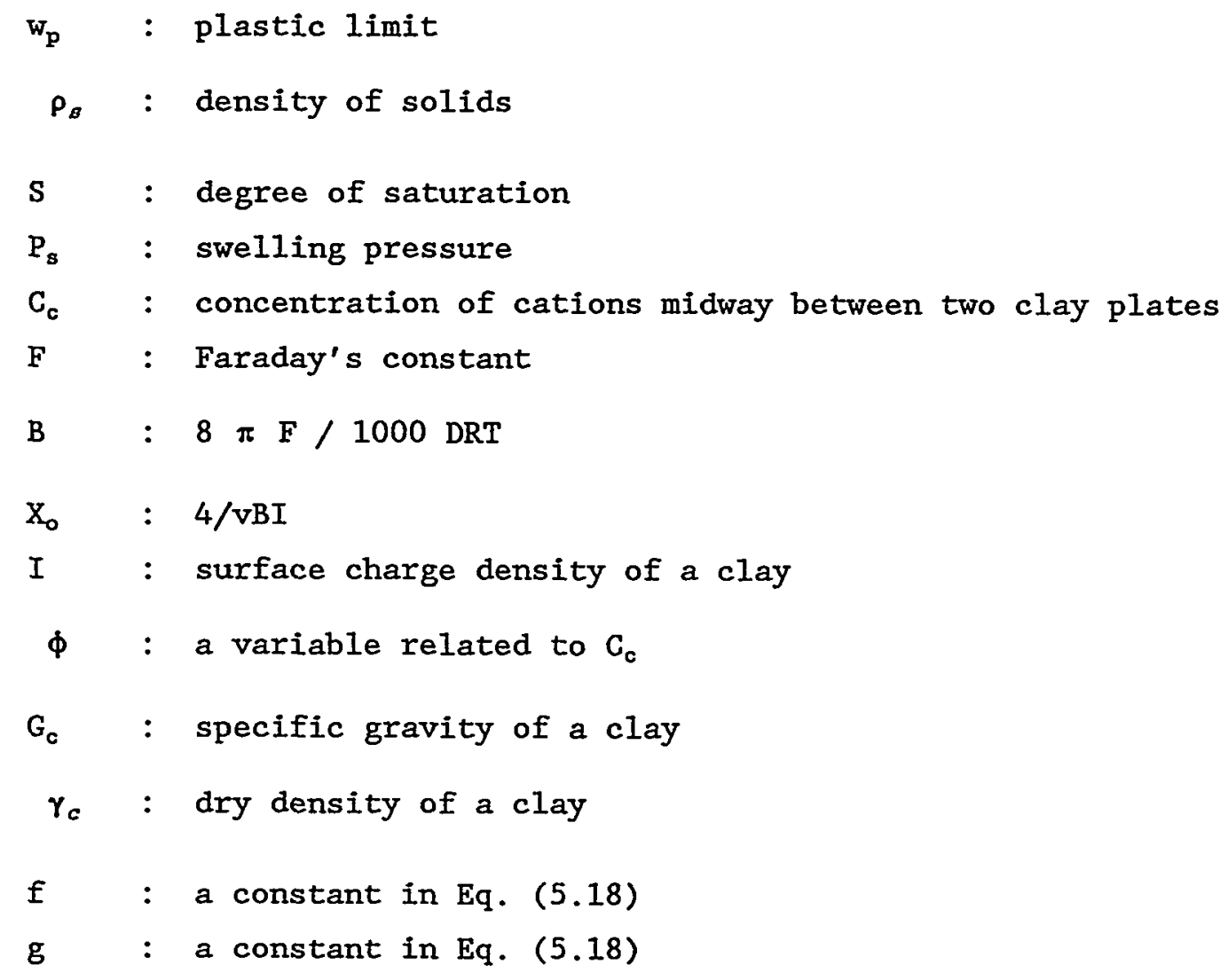




\section{SEALING PERFORMANCE OF BENTONITE AND BENTONITE/CROSHED ROCK BOREHOLE PLUGS}

\section{EXECUTIVE SUMMARY}

Bentonite is an excellent sealant material due to its swelling and selfhealing characteristics, low permeability, sorptive qualities, and longevity in nature. The use of bentonite in constructing hydraulic barriers for waste containment has greatly increased in recent years. Bentonite and bentonite/crushed tuff as well as portland cement-based concrete are being considered as sealing materials for high-level radioactive waste repositories. It may be desirable to use bentonite and bentonite/crushed tuff to minimize deterioration of seal properties and adverse effects on ground water chemistry in the repository environment. Sealing performance evaluation of such plugs is needed under diverse conditions to allow for overall repository performance assessments.

American Colloid C/S granular bentonite and crushed densely welded Apache Leap tuff* have been used to prepare samples for flow testing. The material properties are described in Chapter 2. Bentonite content and crushed tuff gradation are the major variables in sample preparation. The sealing performance assessments include high injection pressure flow tests, polyaxial flow tests, high temperature flow tests, and piping tests. Chapter 3 gives detailed experimental results. Analytical work includes the introduction of bentonite occupancy percentage and water content at saturation as two primary parameters for plug design. A piping model is developed to evaluate the susceptibility of the seals to piping as a result of bentonite flow (Chapter 4). The proposed permeability model in Chapter 5 allows making predictions of permeability in bentonite.

Flow test results of sedimented bentonite plugs indicate the dependence of permeability on the molding water and permeant. The bentonite sample deposited in and permeated with the synthetic groundwater gives a permeability $\left(7 \times 10^{-8} \mathrm{~cm} / \mathrm{s}\right)$ very similar to that of the sample prepared and tested with deaired distilled water. The permeability of bentonite can be reduced by molding or percolating with a dispersing solution (e.g. 28 sodium pyrophosphate solution). During flow testing, the water content tends to become highly nonuniform, as confirmed by multiple observations. Therefore, the permeability calculated assuming the material is uniform should be treated as some equivalent measure of a sample's ability to transmit water.

\footnotetext{
*Unless explicitly noted otherwise, in this report all references to Apache leap tuff refer to the densely welded brown unit of the Apache Leap tuff formation.
} 
Mixtures of bentonite and crushed densely welded Apache Leap tuff can be engineered to yield a low permeability, close to that of bentonite itself. An appropriate composition to yield a permeability lower than 5 $x 10^{-8} \mathrm{~cm} / \mathrm{s}$ would contain at least 258 bentonite by weight mixed with well-graded crushed rock. A mixture containing 258 bentonite and $75 \%$ crushed tuff of type A (maximum particle size of $9.42 \mathrm{~mm}$ ) gradation is a promising seal material. Crushed tuff of FA or FC gradations (FullerThompson gradations, $\mathrm{n}=0.5$ and $\mathrm{Dmax}=9.42 \mathrm{~mm}$ and $19.05 \mathrm{~mm}$, respectively) may also be good candidates for mixing with bentonite. The sealing performance of mixture plugs is enhanced by increasing the amount of bentonite to 35\%. The increase in bentonite content improves the bentonite occupancy percentage and reduces the water content of bentonite at saturation, giving better resistance to piping, erosion and flow. Similar effects have been observed if crushed rock constituting a Fuller-Thompson grading curve (e.g. type FA) is used.

Compaction and bentonite content are decisive factors in producing good mixture seals. The effectiveness of compaction in reducing bulk porosity is hindered by the soft bentonite buffer. To reduce the porosities of the plugs containing 258 or more bentonite, a compaction energy higher than that of the standard Proctor compaction is necessary. For a loosely or ineffectively compacted mixture containing 258 bentonite or less, the sealing performance can be damaged by dynamic disturbances. The influence of such disturbances is greatly reduced when more bentonite is added.

Bentonite/crushed tuff mixtures tested in this study exhibit heterogeneity and anisotropy. A difference of up to one or two orders in magnitude can be expected between the vertical and horizontal permeabilities. The high horizontal permeability results from the uneven bentonite distribution in the pores between crushed rock particles due to particle segregation occurring during sample installation and compaction. Moreover, the contact between adjacent compacted layers may serve as a preferential flow path. Increasing the bentonite content from 25 to $35 \%$ reduces the vertical permeability by nearly an order of magnitude, but results in little change in the horizontal permeability. Adequate sealing ability of the mixture plugs in the transverse direction may be necessary to minimize the lateral flow of groundwater or gases into and out of fractures in the host rock. Compromising the sealing ability in the transverse direction may jeopardize the entire sealing performance if piping occurs. Temperature has no significant effect on the sealing performance of bentonite/crushed tuff plugs within the test range from room temperature to $60^{\circ} \mathrm{C}$. The specific permeability reaches a maximum at $35^{\circ} \mathrm{C}$ and decreases with increasing temperature, indicating the effect of temperature on the structure of the samples. The decreases in the specific permeability are likely due to the thermal expansion of crushed tuff particles and the expansion of the diffuse double layer of bentonite. The structural change is reversible within the temperature range tested. 
The possibility for piping to occur in passageways created by the radial expansion of pores due to an increasing injection pressure is small except for the mixture plugs containing a low bentonite content (e.g. 158 by weight). The effect of pore expansion is believed to be counteracted by pore clogging resulting from the migration of fine particles. The fine particle migration is evidenced by bentonite flow between crushed tuff aggregates. The migration also may be the cause of the breakdown of the linear relation between flow rate and hydraulic gradient observed in all high injection pressure flow tests. The breakdown is believed to indicate the onset of bentonite flow. For mixtures consisting of type A crushed tuff and $25 \%$ or 358 bentonite, the piping damage is small if the maximum hydraulic gradient does not exceed approximately 120 and 280 , respectively.

Piping can occur if bentonite is lost externally. A piping model is developed which combines the yield stress characteristics of bentonite and the flow of bentonite through capillaries. The model provides an analytical means to determine the critical pressure gradient at which bentonite of a given water content starts to flow. The concept of yield stress and critical pressure gradient has been extended to filter design. For bentonite water contents from 50 to 3008 and hydraulic gradients of no more than 1000 , filters with an effective pore diameter of no more than $0.5 \mathrm{~mm}$ are necessary to prevent piping, erosion and flow of bentonite. This may suggest that for seals in contact with open joints or fractures, discontinuities having apertures larger than $0.5 \mathrm{~mm}$ should be grouted to minimize any loss of bentonite. This generalization from pore voids to (approximately) rectangular slots needs further validation.

The Kozeny-Carman equation has been modified to improve the predictability of the saturated permeability in clays. The modification includes a correction factor to account for the microstructural changes which develop in clays in response to changes in water content. Permeability measurements of eleven bentonite samples obtained in this study, along with five measurements reported in the literature, are used to examine the validity of the model proposed. The ratio between the predicted and measured permeabilities varies from 0.8 to 3.75 . The prediction is within 348 of the measured permeability for eleven out of sixteen samples.

Swelling pressures of bentonite can be predicted using the modified Yong and Warkentin model as well as the Sridharan-Jayadeva model (for bentonites with low salt concentrations). The former model gives predictions close to the measured swelling pressures, with differences ranging from 0 to 25.88. Swelling pressures calculated from the Sridharan-Jayadeva model are approximately 8 times higher than the experimental measurements. Assuming a microstructure of three clay sheets per cluster and correspondingly reducing the specific surface of montmorillonite $(800$ $\mathrm{m}^{2} / \mathrm{g}$ ) by a factor of three, the Sridharan-Jayadeva model yields predictions comparable to the experimental results. The difference between predicted and measured swelling pressures varies from 0 to 25.68 .

Several recommendations for future studies can be drawn from this investigation: 
(1) High injection pressure flow tests and piping tests in the transverse direction are recommended to evaluate the consequences of the permeability anisotropy.

(2) If more homogeneous and isotropic bentonite/crushed tuff plugs are deemed desirable, methods are needed to minimize the particle segregation and to assure a uniform distribution of bentonite. The permeability anisotropy may be reduced by emplacing a layer of bentonite on top of each compacted layer. The crushed rock, during subsequent compaction, should carve into the bentonite layers above and below to tie together adjacent lifts. This method deserves further investigation.

(3) Precautions have been taken to reduce the particle segregation during the sample installation, e.g. by emplacing thoroughly mixed materials by scooping. The differences observed between the vertical and horizontal permeabilities therefore may only be minimal, i.e. smaller than in field installations. Particle segregation and uneven distribution of bentonite may be more severe when the mixtures are emplaced in small-diameter long boreholes. The sealing performance evaluation of small-diameter and long bentonite/crushed tuff plugs is warranted.

(4) The proposed permeability model adequately predicts permeabilities of Wyoming sodium bentonite mixed and permeated with distilled water. The model is believed to be adjustable for other fine-grained clays and for different pore water chemistries. Different material type and pore water chemistry likely require changes in parameters of the specific surface and plastic limit. Further studies are suggested.

(5) Changes in the pore water chemistry influence the structure and hence the yield stress of bentonite. Studies of the influence of pore water chemistry on the yield stress of bentonite are recommended.

(6) The effect of bentonite loss into fractures on the sealing performance deserves further investigation. This effect may be evaluated by conducting flow tests on seals installed in rock containing fractures.

(7) Dynamic effects on the sealing performance of bentonite-based plugs should be studied, in view of the possible disturbances caused by earthquakes.

(8) The bond strength of seals in boreholes in rock needs to be determined in order to allow seal design for anticipated loads. 
CHAPTER ONE

INTRODUCTION

\subsection{Introduction}

Bentonite is considered as a promising repository sealant material because of its desirable swelling and self-healing characteristics, low permeability, sorptive qualities, and longevity in nature (e.g. Meyer and Howard, 1983; Pusch, 1983).

The use of bentonite in constructing compacted clay liners or admixed liners has greatly increased in recent years. Boyes (1986) recommends the use of bentonite for liners, rather than local clays, where longterm storage of liquids is needed. Bentonite/crushed rock mixtures have been used to form cap layers to reduce infiltration for uranium mill tailings ${ }^{1}$ (Southwestern Pay Dirt, March 1990, p. 7A). The use of bentonite/crushed rock mixtures may be extended further if the disposal of hazardous wastes in salt mines (Schoenberger, 1988; Stegmann, 1988), abandoned open pits (Libicki, 1989), or abandoned underground mined openings can be demonstrated to be practical and acceptable.

The Nuclear Waste Policy Amendments Act of 1987 designated Yucca Mountain, Nevada, as the candidate repository site for the underground disposal of high-level radioactive waste in welded tuff (U.S. Dept. of Energy, 1988). Al1 penetrations of a high-level waste repository must be properly sealed to retard any radionuclide migration to an acceptable level. Bentonite and bentonite/crushed tuff as well as portland cement and concrete have been proposed as sealing materials for a nuclear waste repository in tuff formations. The current preference, however, is to propose bentonite and bentonite/crushed tuff for as many applications as possible to minimize potential degradation of physical properties and potential adverse effects on ground water chemistry in the repository environment (Fernandez et al., 1987, p. 7-16). One of the strong arguments in favor of using bentonite for sealing a repository at Yucca Mountain is the pervasive natural occurrence of bentonitic materials throughout and below the repository horizon (e.g. Bish et al., 1984). While the compatibility between host tuff and bentonite deserves further investigation, it is reasonable to expect that these two materials should be highly compatible, i.e. not conducive to detrimental interactions.

An immediate gain from adding crushed rock to bentonite is to reduce the amount of waste rock to be disposed of (Smith et. al, 1980). According to Dixon et al. (1985), several advantages are gained by adding sand to

${ }^{1}$ Reith, Jacobs Engineering, and Weslee, Morrison-Knudsen Environmental Services, personal communications, Feb. 1990. 
the clay. The addition of up to 508 of sand: (1) increases the achievable compacted density, (2) does not change (or decrease) the swelling pressure developed by the clay (Taylor et al., 1980, p. 151, on the other hand, show a nearly steady decrease of swelling pressure over a sand content range from 25 to 908), (3) decreases the shrinkage potential, (4) increases thermal conductivity, and (5) increases the bearing capacity of the backfill, minimizing creep or settlement. It is possible that the addition of crushed rock to bentonite may result in similar advantages.

The sealing performance, in particular permeability, of bentonite and bentonite/crushed tuff plugs under diverse conditions needs to be studied to allow for overall repository performance assessments (Binnall et al., 1987; Thompson, 1988). The seal components may be required to retain adequate sealing performance over a long period of time

(Fernandez et al., 1987). The effect of piping and erosion, as learned from the failures of earth dams, embankments, and natural slopes (e.g. Rosewe11, 1977; Sherard et a1., 1972, 1977; Goodman and Sundaram, 1980) is important for the long-term sealing performance of the sealant materials, especially if the hydraulic barriers are installed in locations which are intercepted by joints and/or fractures.

\subsection{Objectives}

The main objective of this study is to evaluate the sealing performance of bentonite and bentonite/crushed tuff sealants under diverse conditions, such as various hydraulic pressure gradients, temperatures, and flow directions. Bentonite weight percent and crushed tuff gradation are the major variables in sample construction. Seals of different diameters are tested to allow an evaluation of size effects, and possibly for some extrapolation from laboratory to field scale.

A second objective is to assess the effect of piping on the deterioration of sealing performance. The results may bear important implications as to the long-term sealing performance of seals that contain bentonite in contact with open joints and/or fractures. A piping model of bentonite, based on plastic flow theory, is proposed. A third objective is to investigate the predictability of permeability and swelling pressure of bentonite.

\subsection{Scope and Limitations}

This study includes a laboratory investigation of the sealing performance of bentonite and of bentonite/crushed tuff plugs. American Colloid C/S granular bentonite and crushed Apache Leap tuff (from the densely welded brown unit, Superior, Arizona) have been used to prepare samples. The sealing performance assessments include high injection pressure flow tests, polyaxial flow tests, high temperature flow tests (up to $60^{\circ}$ ), and piping tests.

The sample diameter varies from 2.54 to $10.16 \mathrm{~cm}$ for bentonite plugs, and from 10.16 to $30.15 \mathrm{~cm}$ for bentonite/crushed tuff plugs. Fixedwalled permeameters (PVC or stainless steel) are used for flow testing. The majority of the plugs are compacted inside the permeameters, and 
receive a compactive effort equivalent to that of the standard Proctor compaction. All the flow tests are performed using either the constant head or the falling head method. High injection pressures are achieved by applying compressed gas (helium) pressures to the inflow reservoir of a constant head flow system. A rectangular Plexiglass permeameter, $12.5 \times 11.4 \times 11.4 \mathrm{~cm}$ in size, is used for polyaxial flow testing. The walls are detachable. Thin porous plates can be installed at inflow and outflow ends. Deaired distilled water is the primary permeant used in this study. Some flow tests have been conducted with different water chemistries, notably including either a dispersing agent or a flocculent.

The flow tests are aimed at evaluating the sealing performance of bentonite and of bentonite/crushed tuff plugs under diverse conditions. They are also aimed at identifying problems and possible improvements in plug design. Theoretical analyses are performed to develop permeability and piping models for bentonite.

\subsection{Organization}

The first section of this first chapter introduces the subject of repository sealing, and identifies other sealing applications of bentonite. The second section describes the objectives of the work reported on. The third section identifies scope and limitations. This fourth section gives a chapter-by-chapter overview of this report. The fifth section introduces the methods of investigation, including a flow chart summarizing the study. Section 1.6 summarizes the general characteristics of the materials tested and identifies the sources from which they have been obtained.

Chapter Two describes material properties and characteristics relevant to sealing.

Sample preparation and installation, experimental procedures and results are summarized in Chapter Three. Details are included in Appendices A through E. Section 3.5 gives analyses and interpretations of the experimental results. Influences on permeability of bentonite content and of crushed tuff gradation are discussed. Also given in this chapter are the methods to calculate the occupancy percentage and consequently the saturated water content of bentonite, which are useful for the design of bentonite/crushed rock seals.

Chapter Four evaluates the effects of piping, erosion, and flow of bentonite on the sealing performance of bentonite and of bentonite/ crushed tuff plugs. A piping model, based on the theory of plastic flow, is proposed.

Chapter Five discusses the inadequacy of the Kozeny-Carman equation in predicting permeability of clays. A refinement of the equation is suggested to account for the changes of microstructures in clays. An experimental validation of the proposed permeability model is included. The latter part of Chapter Five reviews existing models for predicting swelling pressures of clays. Two models are recommended for calculating swelling pressure of bentonite for conditions of diluted salt concentra- 
tions. The predicted swelling pressures are compared with experimental results.

Chapter Six gives a summary, conclusions and recommendations for further research.

\subsection{Methods of Investigation}

Figure 1.1 gives a flow chart of the investigation, which includes an experimental part and an analytical part. Results of the flow tests are used to evaluate the influence of key parameters (bentonite content, crushed rock gradation, injection pressure (or hydraulic gradient), temperature, and sample size on the sealing performance of the plugs. The analytical work includes the development of a permeability model and of a plastic flow piping model for bentonite. Results of longitudinal and high injection pressure flow tests are used to evaluate the two models. Permeability measurements of bentonite reported in the literature are also analyzed to test the validity of the proposed permeability model. Results of bentonite swelling tests are used to assess the predictive capability of some published swelling pressure models.

\subsection{Source Location of Test Materials.}

The bentonite used in this study is American Colloid $\mathrm{C} / \mathrm{S}$ granular Volclay from Upton, Wyoming. The bentonite, a highly colloidal, expansive clay, is an alteration product of volcanic ash (Mitchel1, 1976, p. 39). The volcanic ash was first deposited in a salten sea near the Black Hills region. Later uplift of the region subjected the ash particles to weathering and altered them from their fragile, glassy state to a swelling, paste-forming Na-rich bentonite (Jepsen, 1984). The powdered form of Volclay $(\mathrm{MX}-80)$ has been selected by researchers in Sweden (e.g. Pusch, 1978, 1983; Pusch et al., 1987, 1989) as a potential buffer and borehole sealing material for underground nuclear waste storage.

The crushed tuff is produced from the densely welded brown unit of the Apache Leap tuff. The rock samples are collected along old highway 60, approximately 1 mile east of Superior, Arizona. Tuff is a volcanic ash which has been compressed under its own weight and sometimes is welded due to its high temperature during deposition. Welded tuff, because of its low permeability and high sorptive capacity, is considered a primary candidate for disposal of high-level nuclear waste (Organization for Economic Cooperation and Development, 1982, p. 15). 


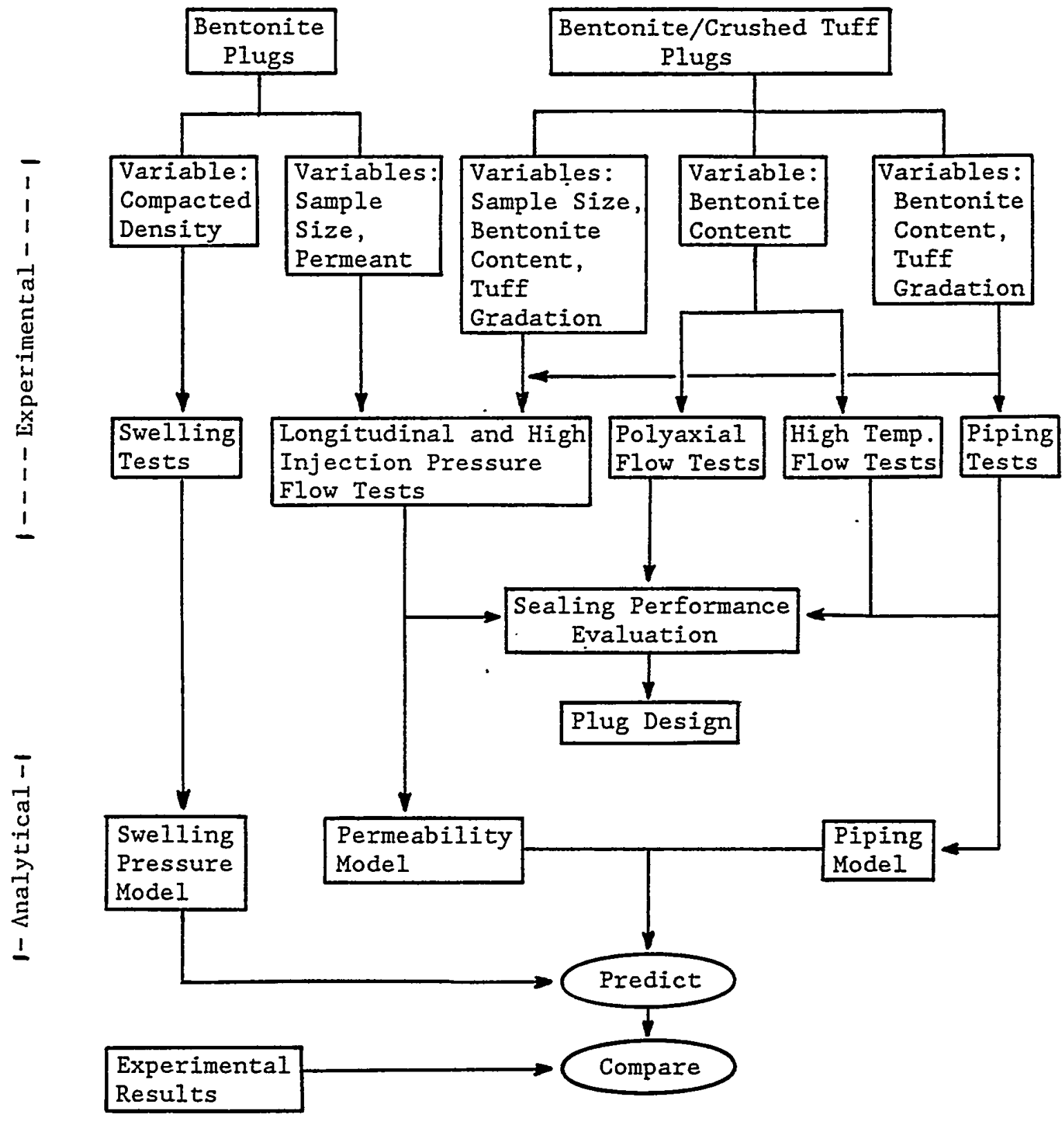

Figure 1.1 Flow chart showing the path of investigation for sealing performance assessments of bentonite and bentonite/crushed tuff plugs. 
CHAPTER TWO

\section{MATERIAL CHARACTERIZATION AND PROPERTIES RELEVANT TO SEALING}

\subsection{Bentonite}

American Colloid C/S granular bentonite from Upton, Wyoming, is used in constructing bentonite and bentonite/crushed rock plugs for flow testing. This sodium bentonite, a colloidal, expansive clay, is an alteration product of volcanic ash (Mitchell, 1976, p. 39). The C/S granular bentonite consists primarily of the clay mineral montmorillonite, with traces of quartz, feldspar and biotite. Chemical analysis of the bentonite has been performed by American Colloid Company (Data No. 202) and by Sawyer and Daemen (1987). The results are shown in Table 2.1. This highly plastic clay has the ability to adsorb nearly 5 times its weight in water and to swell to 10 to 13 times its dry size upon complete hydration (Jepsen and Place, 1985).

Bentonites have a wide variety of industrial uses including drilling muds, ceramics, foundry molding sands, and various geotechnical applications. Of particular interest within the present context is that bentonite finds widespread use for many "sealing" applications, e.g. waste and water pond liners, slurry trench walls, dam cores and aprons, borehole sealing slurries, piezometer sealing, etc. (e.g. Mitchell, 1976 , p. 39).

\subsubsection{Engineering Properties}

The $\mathrm{C} / \mathrm{S}$ granular bentonite has an average specific gravity of 2.92 , liquid limit of 4338, plastic limit of 508, and plasticity index of 3838. Particles smaller than $4.75 \mathrm{~mm}$ (sieve \#4) are used for the determination of specific gravity following ASTM Standard D854-83. Determination of the plastic and liquid limits and plasticity index is performed in accordance with ASTM D4318-84, Sections 16, 17 and 19, respectively. A mechanical sieving, as opposed to the washing operation (D4318-84, 10.1.2.2) is performed to obtain 150 to $200 \mathrm{~g}$ of material passing No. 40 sieve for the testing.

The $\mathrm{C} / \mathrm{S}$ granular bentonite has an average initial (air-dried) moisture content of 9.568 over a range from 9.41 to 9.698 . Table 2.2 shows the grain size distribution of the air-dried bentonite. Moisture-density relations of the bentonite are determined following the compaction method A described in ASTM Standard D698-78. Summarized in Table 2.3 are two moisture-density relations determined by two separate compaction tests on samples from different batches. The results are illustrated in Figures 2.1 and 2.2 .

As shown in Table 2.3, the optimal moisture content varies by up to 7.58 between the two results (i.e. 318 vs. 23.58 ), while the maximum dry 
Table 2.1 Chemical Analysis of C/S Granular Bentonite

\begin{tabular}{lccc} 
& $\begin{array}{c}\text { Sawyer } \\
\text { Batch \#2 }\end{array}$ & $\begin{array}{c}\text { Daemen } \\
\text { Batch \#3 }\end{array}$ & $\begin{array}{c}\text { American } \\
\text { Colloid } \\
\text { Company }\end{array}$ \\
$\mathrm{SiO}_{2}$ & 61.6 & 59.8 & 64.33 \\
$\mathrm{Al}_{2} \mathrm{O}_{3}$ & 20.4 & 20.1 & 20.74 \\
$\mathrm{CaO}$ & 3.4 & 4.9 & 0.52 \\
$\mathrm{MgO}^{*}$ & 3.56 & 3.78 & 2.30 \\
$\mathrm{~K}_{2} \mathrm{O}$ & 1.42 & 1.72 & 0.39 \\
$\mathrm{Na}_{2} \mathrm{O}$ & 3.40 & 3.32 & 2.59 \\
$\mathrm{Fe}_{2} \mathrm{O}_{3}$ & 5.00 & 5.43 & 3.03 \\
$\mathrm{TiO}_{2}$ & 0.16 & 0.17 & 0.14 \\
$\mathrm{P}_{2} \mathrm{O}_{5}$ & 0.10 & 0.09 & 0.01 \\
$\mathrm{H}_{2} \mathrm{O}$ & 0.3 & 0.4 & 5.14 \\
Other & 0.66 & 0.29 & 0.82 \\
\hline
\end{tabular}

*From Sawyer and Daemen (1987, Table 4.1, p. 48).

** From American Colloid Company Data No. 202.

Reproduced with permission from American Golloid Company, IC-352 (Data No. 202, p. 1), Arlington Heights, IL. 
Table 2.2 Grain Size Distribution of American Colloid C/S Granular Bentonite (Air-Dried)

\begin{tabular}{|c|c|c|c|c|c|}
\hline \multirow[t]{2}{*}{$\begin{array}{ll} & \text { U.S. } \\
\text { Mesh Number }\end{array}$} & $\begin{array}{l}\text { Sieve Aperture } \\
\text { (in/mm) }\end{array}$ & \multicolumn{4}{|c|}{ Weight Percent Passing } \\
\hline & & $\# 1$ & $\# 2$ & $\# 3$ & Ave. \\
\hline 10 & $0.0787 / 2.0$ & 100 & 100 & 100 & 100 \\
\hline 20 & $0.0331 / 0.841$ & 94.0 & 92.2 & 91.4 & 92.5 \\
\hline 40 & $0.0165 / 0.419$ & 8.2 & 14.3 & 12.1 & 11.5 \\
\hline 60 & $0.0098 / 0.249$ & 2.8 & 7.2 & 5.9 & 5.3 \\
\hline 100 & $0.0059 / 0.150$ & 0.8 & 2.7 & 1.8 & 1.8 \\
\hline 200 & $0.0029 / 0.074$ & 0.4 & 0.5 & 0.4 & 0.4 \\
\hline
\end{tabular}


Table 2.3 Moisture-Density Relations of American Colloid C/S Granular Bentonite

\begin{tabular}{|c|c|c|c|c|c|}
\hline \multirow{2}{*}{\multicolumn{3}{|c|}{ Moisture Content $(8)$}} & \multirow[t]{2}{*}{ Average } & \multirow{2}{*}{$\begin{array}{l}\text { Moisture Content } \\
(8)\end{array}$} & \multirow{2}{*}{$\begin{array}{l}\text { Dry Density } \\
\left(\mathrm{kN} / \mathrm{m}^{3}\right)\end{array}$} \\
\hline & & Bottom & & & \\
\hline
\end{tabular}

(Compaction Test 1: $G_{S}=2.89$, temperature $=26^{\circ} \mathrm{C}$, sample curing time $=24 \mathrm{hrs}$ )

$\begin{array}{lllll}14.07 & 14.09 & 13.91 & 14.02 & 11.12 \\ 18.07 & 18.85 & 18.01 & 18.31 & 11.35 \\ 20.52 & 21.64 & 23.33 & 21.83 & 11.82 \\ 24.68 & 25.33 & 25.10 & 25.04 & 12.03 \\ 28.89 & 27.06 & 27.46 & 27.80 & 12.10 \\ 30.34 & 36.18 & 26.25 & 30.92 * & 12.13 * * \\ 33.24 & 34.50 & 33.66 & 33.80 & 11.94 \\ 38.18 & 37.86 & 35.82 & 37.29 & 11.85 \\ 41.40 & 40.19 & 39.22 & 40.3 & 11.74\end{array}$

(Compaction Test 2: $G_{S}=2.95$, temperature $=23.5^{\circ} \mathrm{C}$, sample curing time = 72 hrs)

\begin{tabular}{lllll}
17.67 & - & 17.89 & 17.78 & 11.69 \\
18.23 & - & 20.05 & 19.14 & 11.88 \\
21.58 & - & 21.79 & 21.69 & 12.01 \\
23.50 & - & 23.60 & $23.55 *$ & $12.21 * *$ \\
25.54 & - & 25.68 & 25.61 & 12.19 \\
\hline
\end{tabular}

*Optimal moisture content **Maximum dry density

$$
G_{B}=\text { specific gravity }
$$




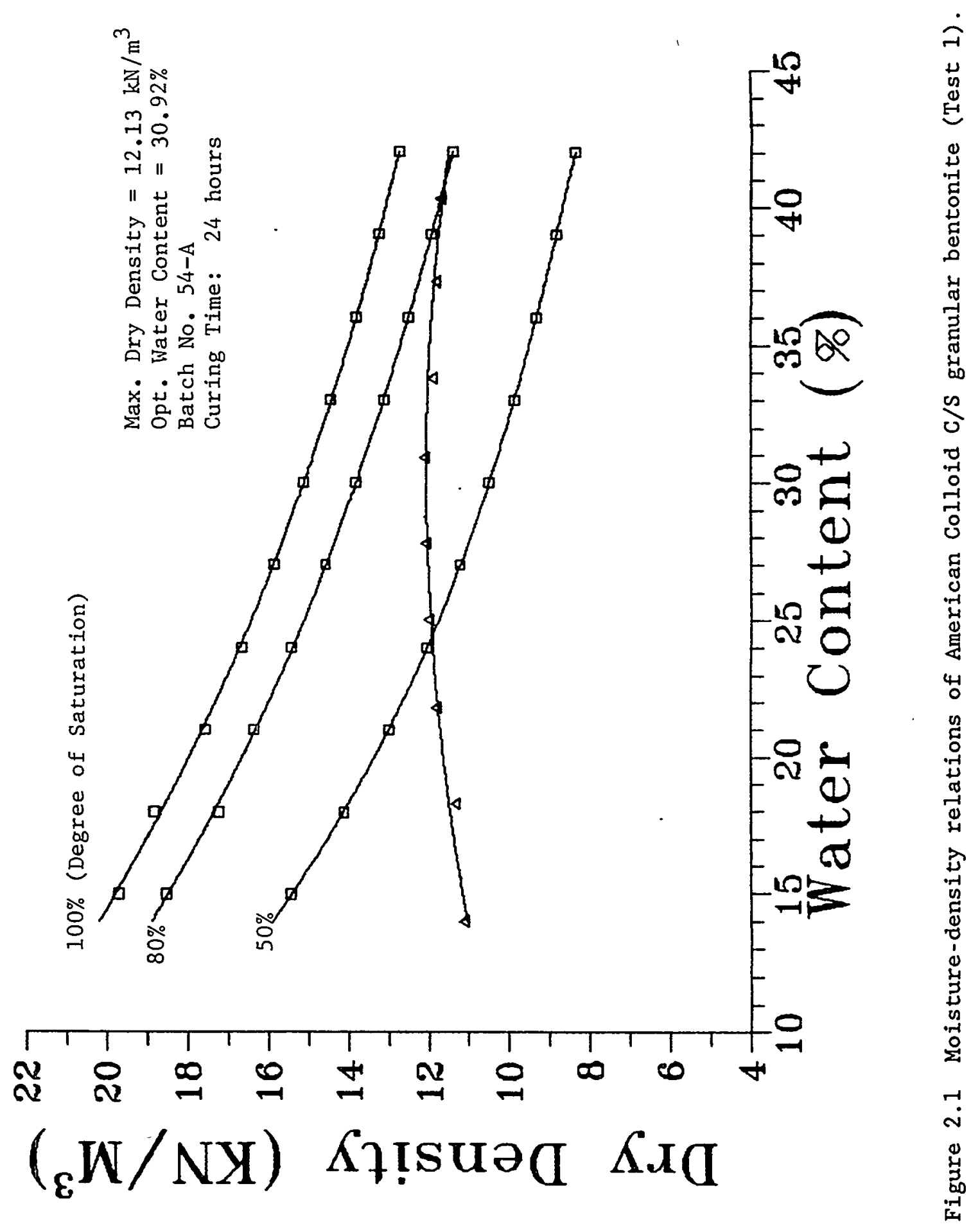




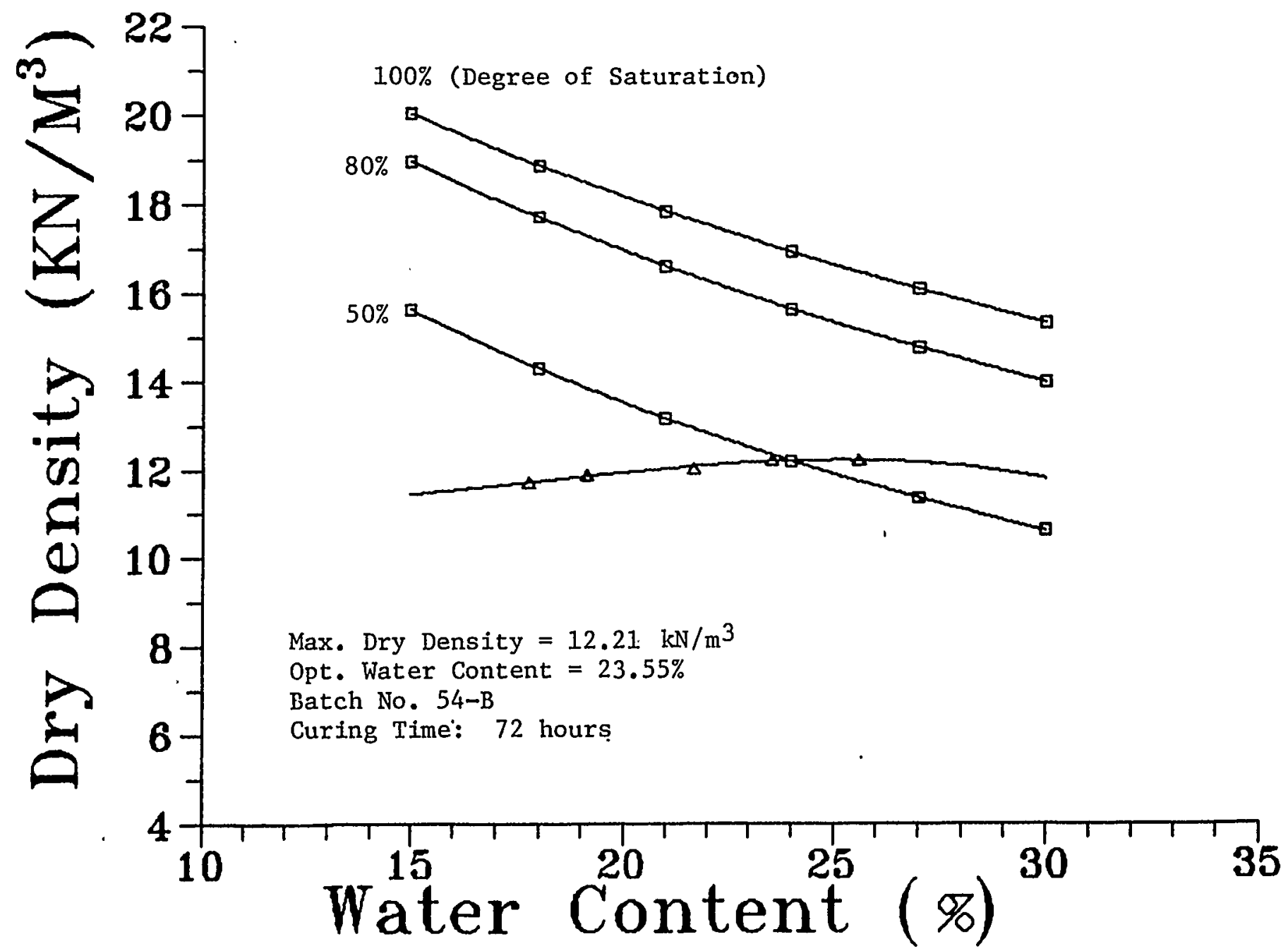

Figure 2.2 Moisture-density relations of American Colloid C/S granular bentonite (Test 2). 
density differs only slightly (12.13 vs. $\left.12.21 \mathrm{kN} / \mathrm{m}^{3}\right)$. This discrepancy may have largely resulted from the difference in sample curing time. It is evident by visual inspection that the moisture is more uniformly distributed for bentonite samples cured for 72 hours than for samples cured for 24 hours. Figure 2.2 is therefore considered to represent the moisture-density relations of the American Colloid C/S granular bentonite used in this study.

Push-out tests were performed on the five compacted bentonite samples from the second compaction test series after specimens were collected from the top and bottom for the determination of moisture content. The compaction mold was placed on a rigid hollow cylinder so that, upon application of an axial force from the top, the sample could move downward without touching the inner surface of the cylinder. The axial force and the sample displacement were monitored during the push-out test. The results are shown in Figure 2.3 in the form of average shear stress vs. displacement.

\subsubsection{Cation Exchange Capacity}

Approximately 908 of the C/S granular bentonite is montmorillonite (American Colloid Company, Data No. 202). Structurally, the montmorillonite clay mineral is classified in the expansive $2: 1$ clay mineral group. The 2:1 designation indicates a clay composed of an octahedral sheet sandwiched between two silicate tetrahedral sheets. The tetrahedral and octahedral sheets combine so that the oxygens forming the tips of the tetrahedron are common to the octahedral layer (Grim, 1953, p. 55). The theoretical composition of $2: 1$ clays is $(\mathrm{OH})_{4} \mathrm{Si}_{8} \mathrm{Al}_{4} \mathrm{O}_{20} \mathrm{n}$ (interlayer) $\mathrm{H}_{2} \mathrm{O}$ (Mitchell, 1976, p. 37). (Figure 2.4)

The theoretical composition of 2:1 clays is almost never found due to isomorphous substitution in the crystal lattice. Isomorphous substitution is defined by Mitchel1 (1976, p. 22) as "substitution of ions of one kind by ions of another type, with the same or different valence, but with retention of the same crystal structure." In montmorillonite, isomorphous substitution occurs predominately at the aluminum sites in the octahedral sheets, where magnesium, $\mathrm{Mg}^{2+}$, replaces every sixth $\mathrm{Al}^{3+}$ cation. The isomorphous substitution results in a net charge deficiency of 0.66 per unit cell. The net charge deficiency may be balanced by exchangeable cations adsorbed between successive unit layers and around the edges of the unit cell (Grim, 1953, p. 59; Mitchell, 1976, p. 38). The structural formula for montmorillonite suggested by Ross and Hendricks (1945, p. 48) can be written in the form: $(\mathrm{OH})_{4} \mathrm{Si}_{8}\left(\mathrm{Al}_{3.34}\right.$ $\left.\mathrm{Mg}_{0.66}\right) \mathrm{O}_{20}$. An approximate chemical formula for the Wyoming bentonite (American Colloid Company, Data No. 202) is ( $\left.\mathrm{Al}, \mathrm{Fe}_{1.67} \mathrm{Mg}_{0.33}\right) \mathrm{Si}_{4} \mathrm{O}_{10}$ $(\mathrm{OH})_{2} \mathrm{Na}, \mathrm{Ca}_{0.33}$, indicating a significant isomorphous substitution of $\mathrm{Al}^{3+}$ by $\mathrm{Fe}^{2+}$ and $\mathrm{a}$ predominance of $\mathrm{Na}$ ions adsorbed. Bonding between successive montmorilionite layers is by van der Waals force (due to instantaneously fluctuating dipoles) and by cations that are present to balance the net charge deficiency of the unit structure. These bonds are relatively weak. Unit layers may be separated easily by adsorption of water or other polar molecules as well as by cleavage (Mitche11, 1976, pp. 36-37). 


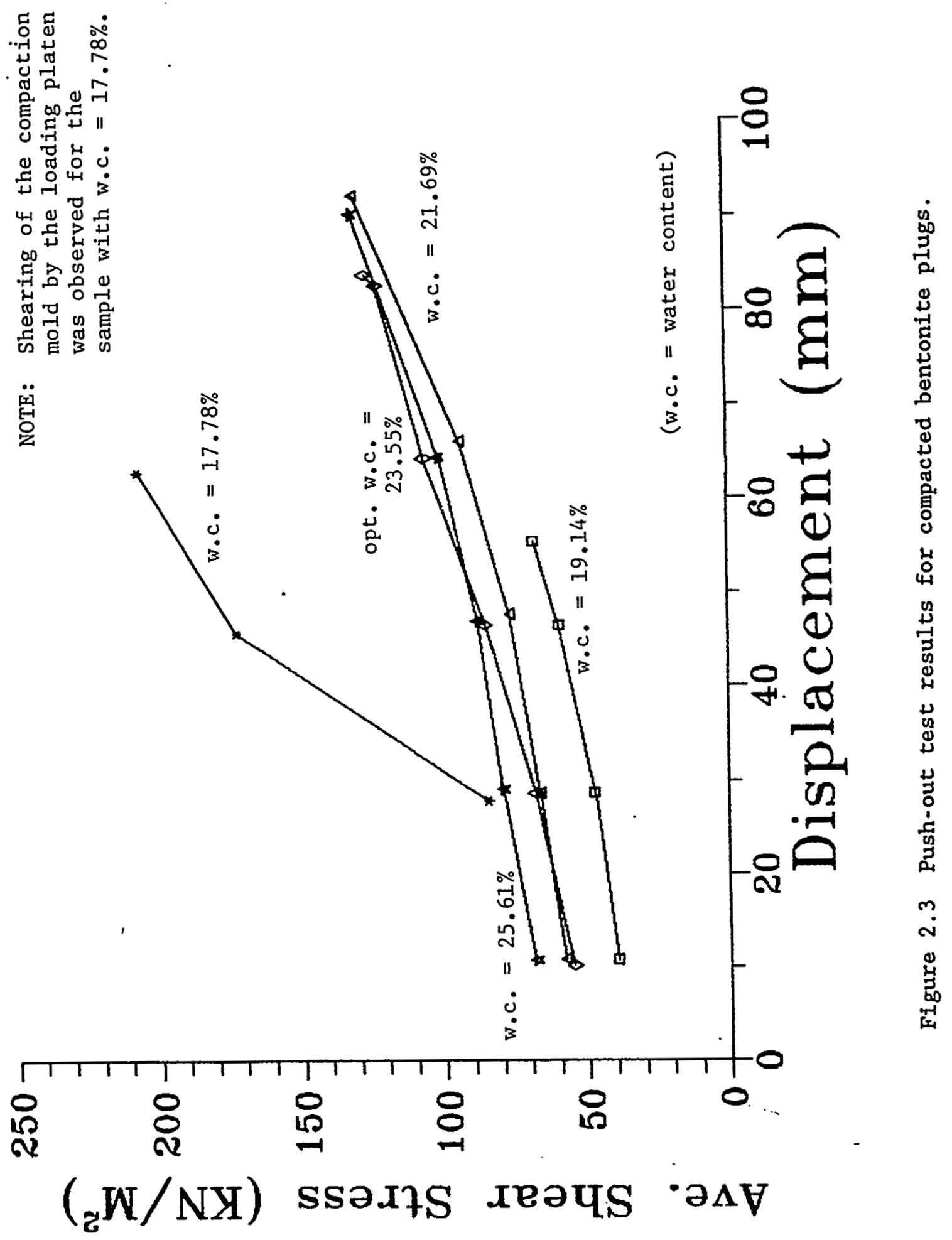



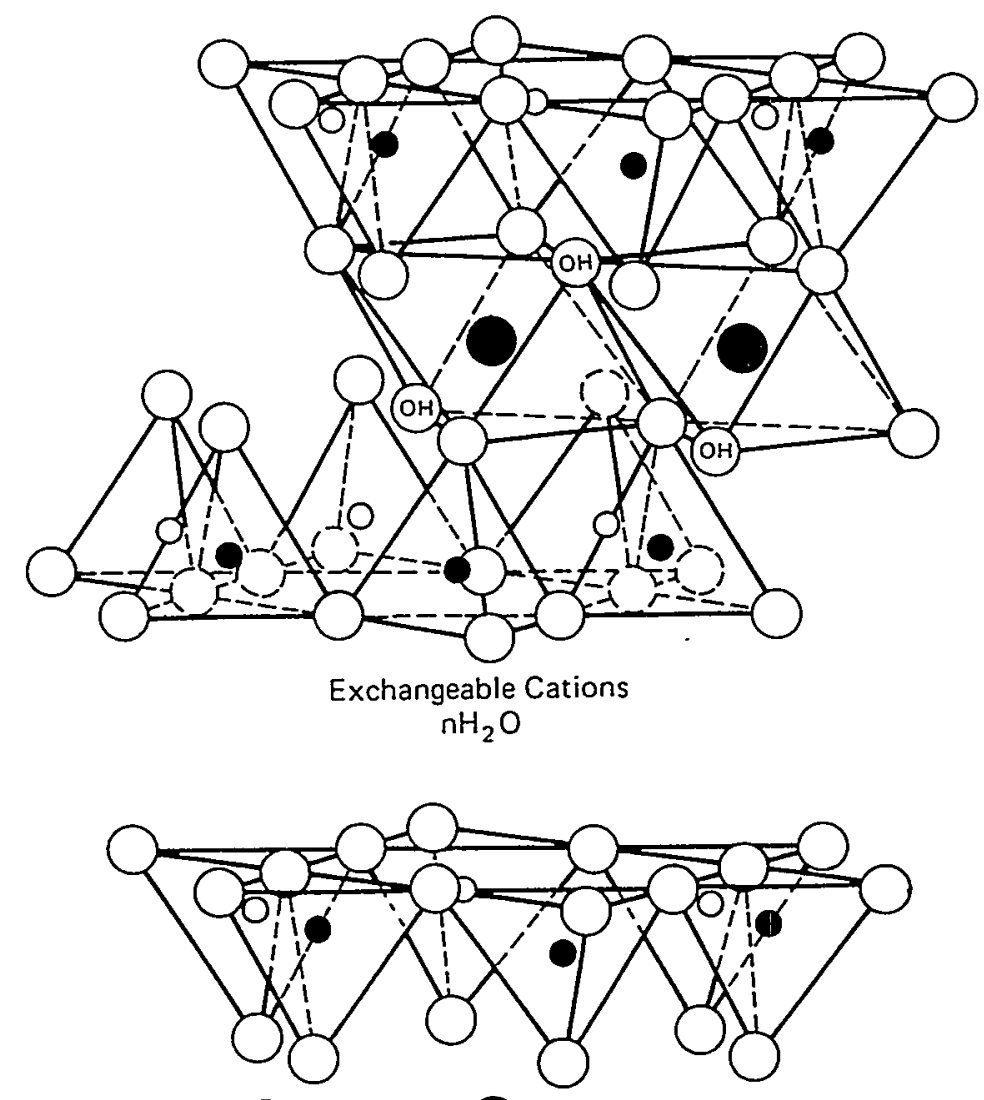

Oxygens (OH) Hydroxyls

Aluminum, Iron, Magnesium

$O$ and Silicon, Occasionally Aluminum

Figure 2.4 Diagrammatic sketch of the montmorillonite structure.

Reproduced with permission from J.K. Mitchell, Fundamentals of Soil Behavior, Figure 3.14, p. 37. Copyright 1976 by John Wiley and Sons, Inc. 
In an aqueous environment, the adsorbed ions are exchangeable with other ions. The capacity for such ion exchanges is termed the cation exchange capacity. The cation exchange capacity is measured in milliequivalent per 100 grams of dry clay. The equivalent weight of an element is its atomic weight (in grams) divided by its valence. The smectite clay group, which includes montmorillonite, typically has a cation exchange

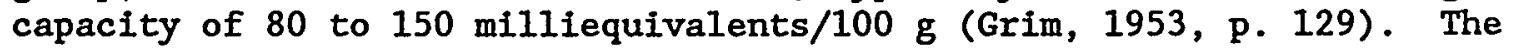
exchangeable metallic bases of the Wyoming bentonite, determined quantitatively by leaching with ammonium acetate (American Colloid Company, Data No. 202), are summarized in Table 2.4.

No universal replaceability series of cations exists (Grim, 1968, p. 212). The series for a particular clay mineral depends upon the ions involved, and upon the test or field conditions. Mitchell (1976, p. 131) lists a typical replaceability series:

$$
\begin{aligned}
& \mathrm{Na}^{+}<\mathrm{Li}^{+}<\mathrm{K}^{+}<\mathrm{Rb}^{+}<\mathrm{Cs}^{+}<\mathrm{Mg}^{2+}<\mathrm{Ca}^{2+}<\mathrm{Ba}^{2+}<\mathrm{Cu}^{2+} \\
& <\mathrm{Al}^{3+}<\mathrm{Fe}^{3+}<\mathrm{Th}^{4+} .
\end{aligned}
$$

This series indicates that if a montmorillonite with adsorbed $\mathrm{Na}^{+}$ions comes in contact with an aqueous solution containing $\mathrm{Ca}^{2+}$, the $\mathrm{Ca}^{2+}$ ions will replace the $\mathrm{Na}^{+}$ions on the clay surfaces. It is possible to replace cations of high replacing power with cations of low replacing power by mass action. In this case, a concentrated solution with cations of low replacing power is necessary.

\subsubsection{Diffuse Double Layer}

A net negative charge of a montmorillonite particle is due primarily to isomorphous substitution. It is also due to a combination of broken chemical bonds and dissociation of hydrogen from hydroxyl molecules (Grim, 1968, pp. 193-195; Mitchell, 1976, p. 130). The negatively charged montmorillonite clay particle creates an electric field. Exchangeable cations are consequently adsorbed on the clay surface to balance this field. In the dry state, the cations are held tight on the clay surface. Any cations in excess of those necessary for charge balancing form a soluble salt precipitate (with its associated anions) in and around the clay particles (Sawyer and Daemen, 1987). In the presence of free pore water, the salts go into solution. This process leads to the formation of a higher molar concentration near the clay surfaces, and the cations tend to diffuse into the surrounding lowerconcentration pore water to create a homogeneous ion concentration throughout the clay-water system. The cation diffusion is opposed by the negative electric field of the clay particle. The negatively charged clay sheet and the positively charged cation distribution in the pore water are called the "diffuse double layer" or simply the "double layer" (Mitchel1, 1976, p. 113; Wu, 1976, p. 398). (Figure 2.5)

The behavior of the diffuse double layer is often described by the GouyChapman theory (Gouy, 1910; Chapman, 1913). According to Mitchell (1976, p. 113), the theory was developed based on the following idealizations: 
Table 2.4 Capacity of Metallic Bases of C/S Granular Bentonite

\begin{tabular}{lc} 
Metallic Base & $\begin{array}{c}\text { Exchange Capacity } \\
\text { (meq/100 grams) }\end{array}$ \\
Sodium & $60-65$ \\
Potassium & $1-5$ \\
Calcium & $15-20$ \\
Magnesium & $15-20$ \\
& \\
Sum (corrected for sulphates) & $85-90$ \\
\hline
\end{tabular}

${ }^{*}$ Reproduced with permission from American Colloid Company, IC-352 (Data No. 202, p. 1), Arlington Heights, IL. 


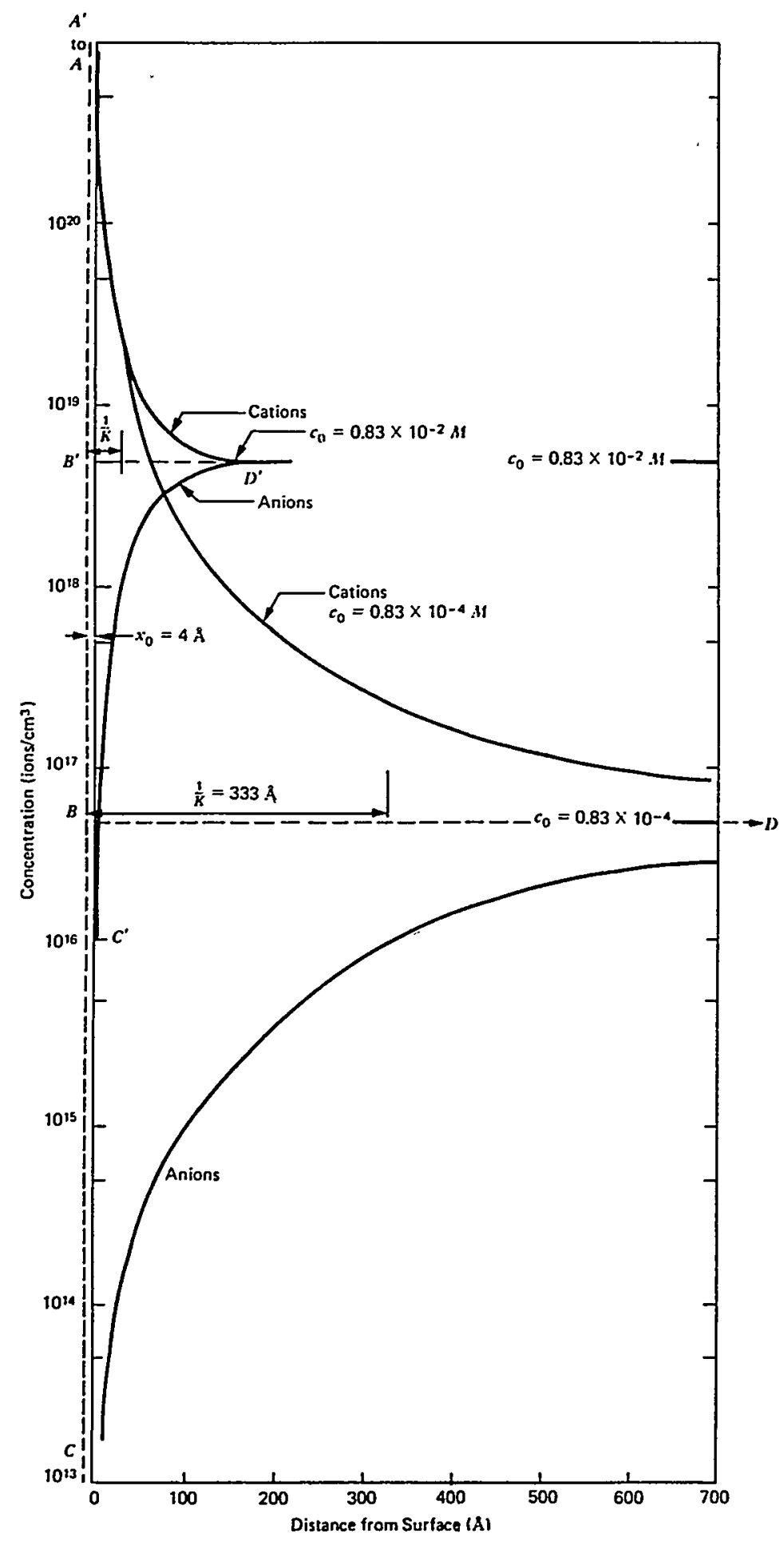

Figure 2.5 Effect of electrolyte concentration on ion distribution in the double layer. 1/K: double layer thickness. $c_{0}$ : cation concentration.

Reproduced with permission from J.K. Mitche11, Fundamentals of Soil Behavior, Figure 7.8, p. 121.

Copyright 1976 by John Wiley and Sons, Inc. 
1) Ions in the double layer are considered as point charges with no interactions between them.

2) Charges on the clay surface are uniformly distributed.

3) The particle surface is a plate whose plane dimensions are large relative to the thickness of the double layer.

4) The dielectric constant of the medium (usually water) is independent of position.

This theory has been derived for colloidal suspensions of clay particles, and considers a single diffuse double layer only. A measure of the extent of the diffuse double layer is the distance from the clay particle surface to the centroid of the diffuse cation charge (Mitche11, 1976, p. 115). This distance is called the "thickness" of the double layer, denoted as $1 / \mathrm{K}$. Theoretical work on ionic and potential distributions near the charged clay particle surface shows that the thickness of the double layer is a function of surface charge potential, electrolyte concentration, cation valence (Figure 2.6), dielectride constant of the medium (usually water) and temperature (Mitchel1, 1976, p. 118). The double layer thickness is given by:

$$
\frac{1}{\mathrm{~K}}-\left[\frac{D k T}{8 \pi n_{0} e^{2} v^{2}}\right]^{1 / 2}
$$

where $1 / K$ - thickness of the diffuse double layer,

D = dielectric constant of the medium,

$\mathrm{k}=$ Boltzmann Constant $\left(1.38 \times 10^{16} \mathrm{erg} /{ }^{\circ} \mathrm{K}\right)$,

$\mathrm{T}$ - absolute temperature $\left({ }^{\circ} \mathrm{K}\right)$,

$n_{0}=$ concentration of ions in the electrolyte (ions $/ \mathrm{cm}^{3}$ ) at great distance from the clay particle surface,

e unit electronic charge (16.0 $\times 10^{-20}$ coulomb), and

$v=$ valence of cation.

Factors such as ion size, $\mathrm{pH}$ and anion adsorption also influence the extent of the diffuse double layer (Mitchel1, 1976, p. 126-127). 2.1.4 Sealing Engineering Significance of Cation Exchange and Double
Layer

The development of the diffuse double layer and cation exchange can account for many observed changes in the engineering behavior of clays. Grim (1953, p. 127) points out the great sensitivity of the physical properties of clays to the type of exchangeable ions carried. Grim and Guven (1978, p. 238) state that various properties of a clay may change due to a change in cation and/or cation concentration. Singh (1982) indicates that the liquid, plastic and shrinkage limits, as well as the permeabilities of various montmorillonites, are a function of the adsorbed cation, either $\mathrm{Na}^{+}$or $\mathrm{Ca}^{2+}$. Results from Endell et al. (1938), reported by Grim and Guven (1978, pp. 242-3), indicate that mixtures of $\mathrm{Ca}$ montmorillonites with sand have permeabilities two orders of magnitude higher than similar mixtures of $\mathrm{Na}$ montmorillonites with sand. 


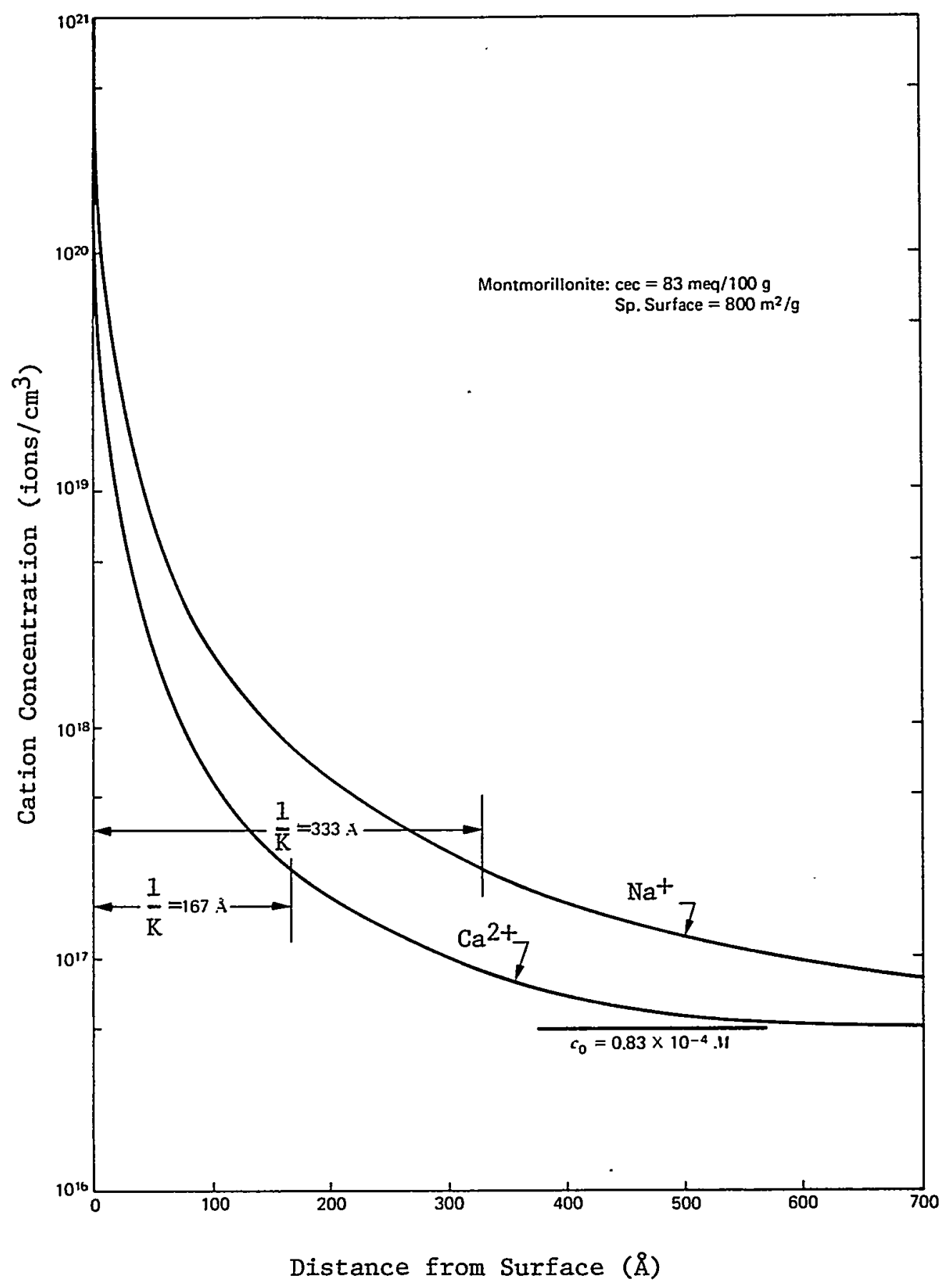

Figure 2.6 Effect of cation valence on double layer concentrations. cec: cation exchange capacity. 1/K: double layer thickness.

Reproduced with permission from J.K. Mitchell, Fundamentals of Soil Behavior, Figure 7.11, p. 124.

Copyright 1976 by John Wiley and Sons, Inc. 
Similar permeability ratios for the two bentonite types are also reported by Mesri and 01son (1971). The discrepancy may be explained by the double layer thickness and cation exchange. As shown in Eq. (2.1), double layer thickness is inversely proportional to the cation valence. As valence increases from $\mathrm{Na}^{+}$to $\mathrm{Ca}^{2+}$ by cation exchange, the double layer collapses and the clay particles may be expected to flocculate (in a clay suspension) or "consolidate" (in a compacted clay plug). Flocculation or consolidation would increase the void ratio and subsequently provide more cross-sectional area for water flow through the bentonite plug (Pusch et al., 1982).

The plastic and liquid limits of montmorillonites vary, depending upon the adsorbed cation (Grim and Guven, 1978, pp. 238-9; Mitche11, 1976, p. 127). Na montmorillonites have extremely high liquid limits. Their double layer is thicker than that in $\mathrm{Ca}$ montmorillonites. The difference in shear strength between sodium and calcium Wyoming bentonites is quoted by Grim and Guven (1978, p. 241) from Samuels (1950). The same authors also show substantial differences between the two materials for compressibility and for consolidation rates.

Dispersive behavior and resulting cracking and piping failures depend on the cation type adsorbed by montmorillonite clays (e.g. Jones, 1981). A laboratory study by Statton and Mitchell (1977) identified the strong difference in influence on montmorillonite shale dispersivity between fluids loaded with sodium or with calcium.

\subsubsection{Microstructure}

The structure of a clay determines its properties. The term "structure" was initially limited to the arrangement of soil particles (Lambe, 1958). For fine grained soils, it may be more appropriate to term "structure" as the arrangement of soil particles and the electrical forces acting between adjacent particles (Lambe, 1958), or as the combined effects of fabric, composition, and interparticle forces (Mitchell, 1976, p. 135).

According to van Olphen (1963), particle associations in clay suspensions can be described as follows:

1. Dispersed. No face-to-face association of clay particles.

2. Aggregated. Face-to-face association of several clay particles.

3. Flocculated. Edge-to-edge or edge-to-face association of aggregates.

4. Deflocculated. No association between aggregates.

Different modes of particle association in clay suspensions are shown in Figure 2.7.

In most soils and sediments, individual particle associations are quite rare. Aggregates of several clay plates are the more common structural forming units (Mitche11, 1976, p. 137). Figure 2.8 shows a scanning electron microscopic photograph of sedimented montmorillonite. The 


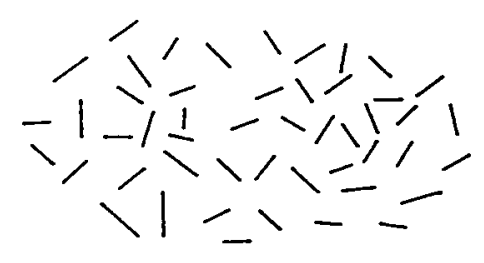

(a)

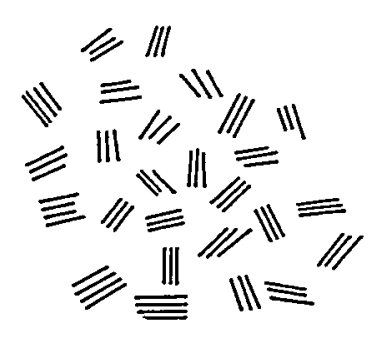

(b)

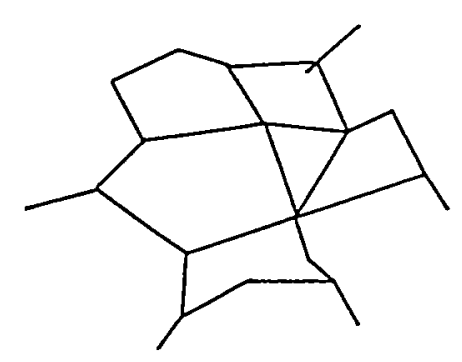

(d)

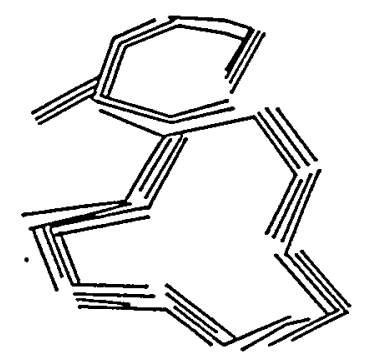

(f)

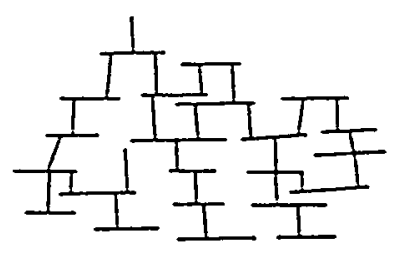

(c)

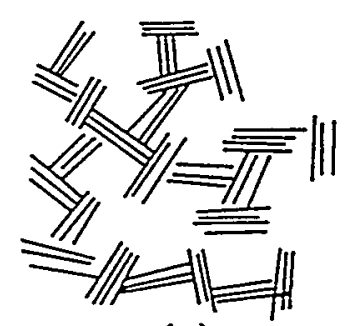

(e)

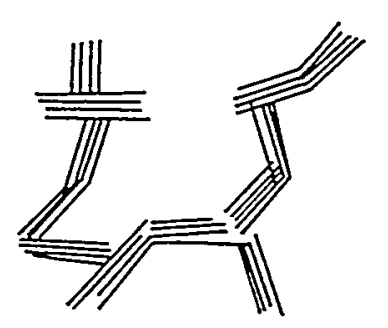

(g)

Figure 2.7 Modes of particle association in clay suspensions. (a) Dispersed and deflocculated. (b) Aggregated but deflocculated. (c) Edge-to-face flocculated but dispersed. (d) Edge-to-edge flocculated but dispersed. (e) Edge-to-face flocculated and aggregated. (g) Edge-to-face and edge-toedge flocculated and aggregated.

Reproduced with permission from J.K. Mitche11, Fundamentals of Soil Behavior, Figure 8.1, p. 136.

Copyright 1976 by John Wiley and Sons, Inc. 


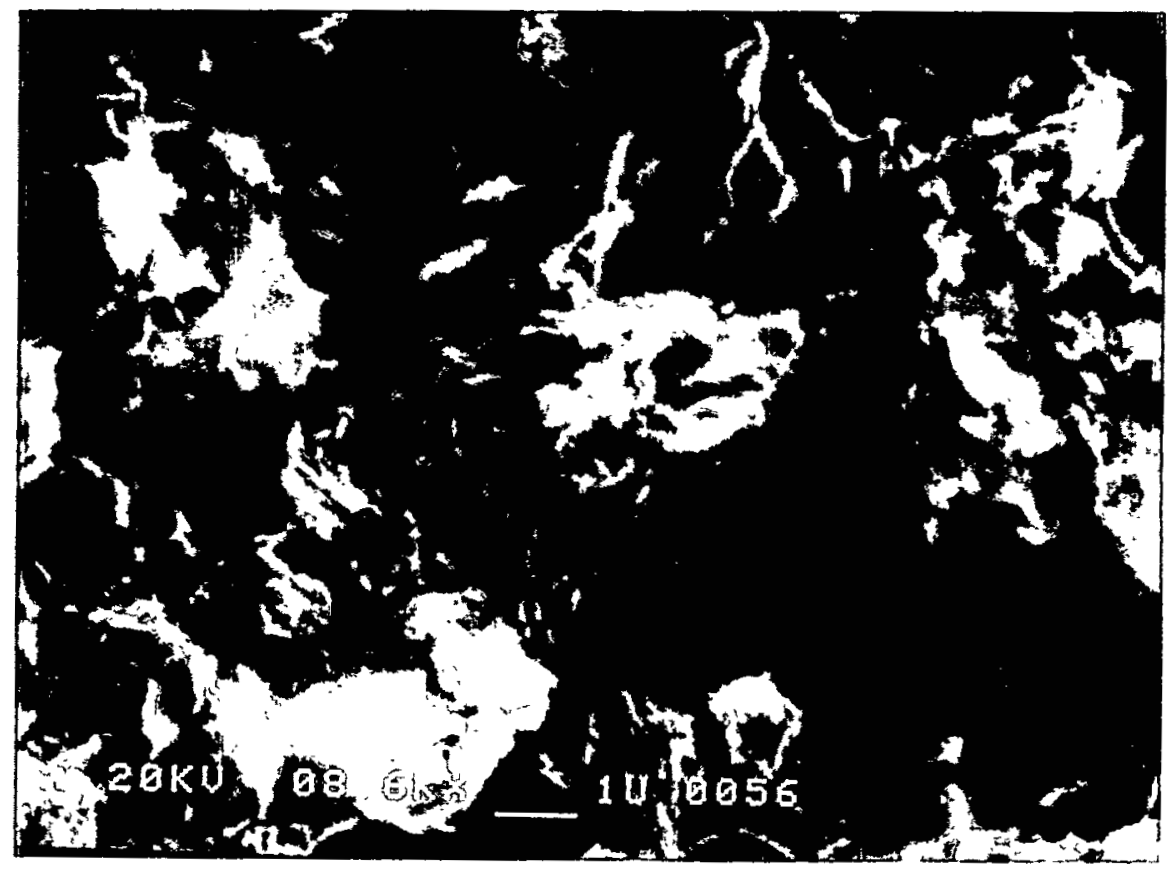

Figure 2.8 Scanning electron microscopic view of sedimented montmorillonite. 
schematic microstructure of soft $\mathrm{Na}$ bentonite $(\mathrm{Mx}-80)$ saturated and matured at room temperature (Pusch et al., 1989) is shown in Figure 2.9. Both figures demonstrate an aggregated structure and a distribution of unequal pore sizes.

According to Pusch et al. (1989), large parts of many grains shown in Figure 2.9 are not expanded. This may suggest that the external surfaces of clay aggregates are primarily involved in the hydration process. The hydration, however, may not necessarily be matured (possibly is only in a pseudo-equilibrium state) and may continue further through diffusion at a much slower rate until the adsorbing capacity and/or the water supply are exhausted. The aggregated structure also suggests that the water permeation will occur predominantly in pores between clay aggregates. The inadequacy of the Kozeny-Carman equation in predicting permeabilities in clays has been attributed to the distribution of unequal pore sizes (01sen, 1962).

\subsection{Apache Leap Tuff}

The rock used to produce crushed tuff is from the densely welded brown unit of the Apache Leap tuff. This tuff has a high porosity and low permeability and resembles Topopah Spring tuff, in which a proposed nuclear waste repository may be located. The chemical composition of Apache Leap tuff reported by Fuenkajorn and Daemen (1991) is compared with that of Topopah Spring tuff in Table 2.5. Apache Leap tuff contains more iron oxide and less silica than does Topopah Spring tuff. The percentages of calcium, magnesium and aluminum oxides of Apache Leap tuff are slightly higher than those of Topopah Spring tuff.

\subsubsection{Physical and Mechanical Properties}

Physical and mechanical properties of Apache Leap tuff have been determined by Fuenkajorn and Daemen (1991) and are shown in Table 2.6. Also included in this table are the properties of Topopah Spring tuff reported by Scully (1984), Price and Bauer (1985), Zimmerman et al. (1985), and Anderson (1981).

The elastic modulus, dry bulk density, and porosity of Apache Leap tuff are comparable to those of Topopah Spring tuff. The latter tuff has higher compressive and tensile strengths than does the former one.

\subsubsection{Hydrological Properties}

Of particular interest is the permeability of the Apache Leap tuff. Constant head radial flow testing has been performed on two hollow rock cylinders to determine the permeability of the tuff. The two hollow cylinders have an outer diameter of $7.55 \mathrm{~cm}$, and inner diameters of 2.575 and $2.582 \mathrm{~cm}$, respectively.

The first cylinder contains an oblique $4-\mathrm{cm}$ long natural fracture. This cylinder was sealed at both ends with mechanical drainage packers. The space between the packers was filled with distilled water. Under an initial $0.4 \mathrm{~m}$ water head, no leakage was detected. Water pressure was then increased to $69 \mathrm{kPa}$ (a water head of $7 \mathrm{~m}$ ) in an attempt to saturate 


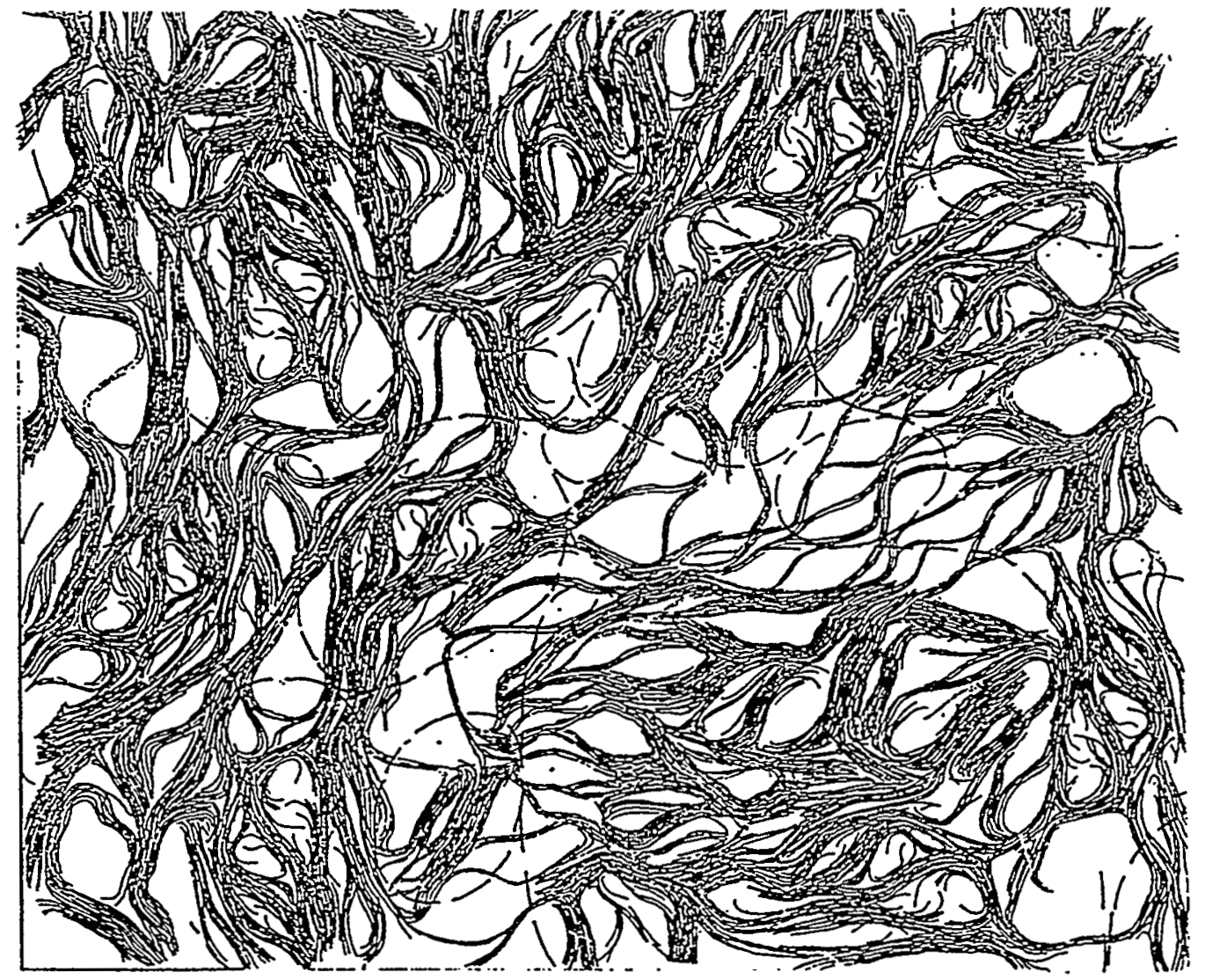

Figure 2.9 Schematic microstructure of soft $\mathrm{Na}$ bentonite saturated and matured at room temperature. Large parts of many grains are not expanded.

Reproduced with permission from Pusch, R., O. Karnland, and A. Muurinen, "Transport and Microstructural Phenomena in Bentonite Clay with Respect to the Behavior and Influence of $\mathrm{Na}, \mathrm{Cu}$ and $\mathrm{U}, "$ SKB Technical Report 89-34, Figure 53, p. 82. Copyright 1989, Swedish Nuclear Fuel and Waste Management Co., Stockholm. 
Table 2.5 Chemical Composition of Tuff

$\mathrm{SiO}_{2}$

$\mathrm{Na}_{2} \mathrm{O}$

$\mathrm{K}_{2} \mathrm{O}$

$\mathrm{CaO}$

$\mathrm{MgO}$

$\mathrm{Al}_{2} \mathrm{O}_{3}$

$\mathrm{Fe}_{2} \mathrm{O}_{3}$

$\mathrm{FeO}$

\begin{tabular}{c} 
Apache Leap \\
Tuff \\
$(8)$ \\
\hline
\end{tabular}

71.7

3.8

2.5

0.4

0.21

14.8

3.3

0.10
Topopah Spring Tuff

$(8)$

76.8

4.1

3.8

0.3

0.11

12.4

1.0

0.05 
Table 2.6 Mechanical and Physical Properties of Apache Leap and Topopah Spring Tuff Specimens

\begin{tabular}{|c|c|c|}
\hline & Apache Leap Tuff* & Topopah Spring Tuff ${ }^{* * *}$ \\
\hline \multicolumn{3}{|l|}{ Uniaxial Compressive } \\
\hline Strength (MPa) & $73.2 \pm 16.5$ & $95.9 \pm 35.5$ \\
\hline Elastic Modulus (GPa) & $22.6 \pm 5.7$ & $26.7 \pm 7.7$ \\
\hline Poisson's Ratio & $0.20 \pm 0.03$ & $0.14 \pm 0.05$ \\
\hline \multicolumn{3}{|l|}{ Brazilian Tensile } \\
\hline P-Wave Velocity $(\mathrm{km} / \mathrm{s})$ & $6.4 \pm 1.5$ & $4.1-4.6$ \\
\hline $\begin{array}{c}\text { Internal Friction } \\
\text { Angle (degrees) }\end{array}$ & 43 & 26 \\
\hline Dry Density $(g / c c)$ & $2.37 \pm 0.42$ & $\begin{array}{l}1.32 \text { (non-welded) } \\
2.0-2.3 \text { (welded) }\end{array}$ \\
\hline Porosity $(8)$ & $7-10$ & $6-20$ \\
\hline${ }^{*}$ From Fuenkajorn and & Daemen (1991) & \\
\hline
\end{tabular}


the sample. Approximately 90 minutes after the pressure increase, the fracture was found wet. About 2 hours later, water started dripping from the fracture. Subsequent measurements indicate an average flow rate of $2.3 \times 10^{-4} \mathrm{cc} / \mathrm{s}$. A slightly higher flow rate of $2.6 \times 10^{-4} \mathrm{cc} / \mathrm{s}$ was obtained under an injection pressure of $103.4 \mathrm{kPa}$.

The second sample contains no visible fractures. The average flow rate measured is almost one order of magnitude lower than that of the first sample. The sample was then subjected to injection pressures of 69 and $103.4 \mathrm{kPa}$, for 9 and 24 days, respectively. The permeability values determined at this stage vary from 6.3 to $2.3 \times 10^{-10} \mathrm{~cm} / \mathrm{s}$, compared to the range of $<10^{-10}$ to $10^{-8} \mathrm{~cm} / \mathrm{s}$ for Topopah Spring tuff (Anderson, 1981; Zimmerman et al., 1985). Fuenkajorn and Daemen (1991) present permeability measurements of the welded Apache Leap tuff under various conditions. Table 2.7 summarizes the permeability results for the two types of tuff.

\subsection{Bentonite/Crushed Tuff}

A mixture of bentonite and ballast material (e.g. quartz sand and crushed rock) is being considered as backfill and sealant for nuclear waste repositories (Pusch et al., 1980; Nilsson, 1985; Holopainen, 1985; Yong et al., 1986; Williams and Daemen, 1987). Primary advantages for adding ballast material to bentonite are to increase thermal conductivity (Pusch et al., 1980; Dixon et al., 1985), to decrease shrinkage potential, and to increase the bearing capacity of the sealant, minimizing creep or settlement (Dixon et al., 1985). According to Nilsson (1985), a suitable composition for such sealant must fulfill the following requirements: (1) low permeability, (2) low compressibility, (3) small average pore size to prevent bentonite migration, and (4) some swelling potential. Immediate gains from adding crushed host rock to bentonite are to alter the natural geochemical properties as 1ittle as possible (Holopainen, 1985) and to reduce the amount of waste rock to be disposed of (Smith et al., 1980).

Bentonite content and the gradation of ballast material are two decisive factors in the design of mixture plugs. If the grading is not proper or the mixture not thoroughly homogenized, or if the amount of bentonite is not sufficient to fill the ballast pores, soft parts of the bentonite gel can be displaced and torn-off fragments transported through channels that are formed at a relatively low water overpressure (Pusch et al., 1987). In this study, bentonite weight percent and crushed tuff gradation are varied.

\subsubsection{Bentonite Content}

A literature survey has been conducted to obtain appropriate bentonite contents used in this investigation for the construction of bentonite/crushed tuff plugs. Pusch et al. (1980) report that the permeability of water-saturated bentonite/quartz mixtures with a weight ratio varying from $1: 10$ to $1: 5$ (i.e. 9 and $16.7 \mathrm{wt}$. percent of sodium bentonite, respectively) ranges between $10^{-7}$ to $10^{-11} \mathrm{~cm} / \mathrm{s}$. Holopainen (1985) indicates permeabilities of $5 \times 10^{-7}$ to $10^{-8} \mathrm{~cm} / \mathrm{s}$ for bentonite/crushed rock mixtures with 158 sodium bentonite. Bentonite/sand percentages of 
Table 2.7 Summary of Water Hydraulic Conductivities of Tuffs

\begin{tabular}{|c|c|c|c|c|}
\hline $\begin{array}{l}\text { Rock } \\
\text { Type }\end{array}$ & $\begin{array}{l}\text { Hydraulic } \\
\text { Conductivity } \\
(\mathrm{cm} / \mathrm{s})\end{array}$ & Test Method & Conditions & Source \\
\hline $\begin{array}{l}\text { Apache Leap } \\
\text { Tuff, Brown } \\
\text { Unit (densely } \\
\text { welded) }\end{array}$ & $10^{-10}-10^{-9}$ & $\begin{array}{l}\text { Radial permeameter } \\
\text { test }\end{array}$ & Unconfined & $\begin{array}{l}\text { Fuenkajorn and } \\
\text { Daemen (1991)* }\end{array}$ \\
\hline$"$ & $10^{-12}-10^{-10}$ & $\begin{array}{l}\text { Radial permeameter } \\
\text { test }\end{array}$ & $\begin{array}{l}12 \mathrm{MPa} \text { confining } \\
\text { stress }\end{array}$ & $"$ \\
\hline$"$ & $\sim 10-12$ & $\begin{array}{l}\text { Radial permeameter } \\
\text { test }\end{array}$ & $\begin{array}{l}7 \mathrm{MPa} \text { confining } \\
\text { stress }\end{array}$ & $"$ \\
\hline$"$ & $<6 \times 10^{-11}$ & $\begin{array}{l}\text { In-situ borehole } \\
\text { flow test }\end{array}$ & $4.3 \mathrm{~m}$ deep & $"$ \\
\hline$"$ & $10^{-9}-10^{-8}$ & $\begin{array}{c}\text { Back-calculated from } \\
\text { porosity }\end{array}$ & - & $"$ \\
\hline$"$ & $<10^{-9}$ to $10^{-8}$ & Falling head test & Unconfined & $"$ \\
\hline$"$ & $<3 \times 10^{-9}$ & Falling head test & $\begin{array}{l}10 \mathrm{MPa} \text { uniaxial } \\
\text { stress }\end{array}$ & $"$ \\
\hline$"$ & $6.3-2.3 \times 10^{-10}$ & $\begin{array}{c}\text { Constant head flow } \\
\text { test }\end{array}$ & Unconfined & This study. \\
\hline $\begin{array}{l}\text { Topopah Spring } \\
\text { Tuff }\end{array}$ & $<10^{-10}$ to $10^{-8}$ & - & - & $\begin{array}{l}\text { Zimmerman et al. } \\
\text { (1985) and Ander- } \\
\text { son (1981) }\end{array}$ \\
\hline
\end{tabular}

*Fuenkajorn, K. and J.J.K. Daemen, 1991, "Mechanical Characterization of Densely Welded Apache Leap Tuff," Technical Report NUREG/CR-5688, U.S. Nuclear Regulatory Commission. (In print) 
$10 / 90$ and $20 / 80$ \& have been used by Nilsson (1985). Bentonite contents of $5,15,25$, and 35 weight percent are selected by Williams and Daemen (1987) in preparing bentonite/crushed basalt plugs. Their flow test results indicate massive failure for the mixtures with 58 of bentonite. In an extensive study of clay/crushed granite mixtures for backfilling a nuclear fuel waste disposal vault, Yong et al. (1986) conclude that the candidate backfill should contain between 20 and 308 clay. Based on the literature research, three bentonite weight percentages $(15,25$, and 35\%) are chosen for the preparation of bentonite/crushed tuff plugs in this study.

\subsubsection{Crushed Tuff Gradation}

Five different crushed tuff gradations have been used to construct mixture samples. Three gradations (Types A, B, and C) are derived from the "coarse" type by first eliminating all particles finer than $0.074 \mathrm{~mm}$ (U.S. mesh \#200) and then successively removing crushed tuff particles larger than $9.42,6.68$ and $4.75 \mathrm{~mm}$. The "coarse" gradation is obtained from crushing Apache Leap tuff chunks of approximately $15 \times 15 \times 20 \mathrm{~cm}$ ( $6 \times 6 \times 8$ in), using a jaw crusher and an adjustable roller crusher in sequence. The other two gradation types (FA and FC) are obtained using $\mathrm{n}=0.5$ and $\mathrm{D}_{\max }=9.42$ and $19.05 \mathrm{~mm}$, respectively, in the FullerThompson grading equation (Eq. 2.2).

$$
P_{W}=100\left(\frac{d}{D_{\max }}\right)^{n}
$$

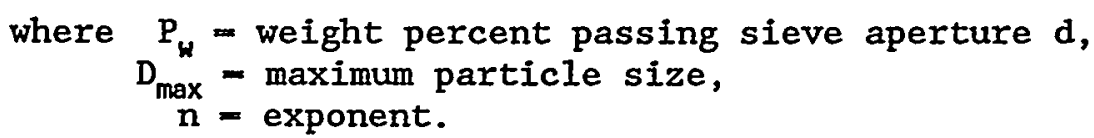

The Fuller-Thompson grading curve is considered to be an ideal grading which may result in the densest possible state of packing (Winterkorn, 1975; Head, 1980, p. 150). The U.S. Bureau of Public Roads (USBPR, 1962 ; as cited by Winterkorn, 1975) recommends 0.45 for $n$ as the best overall value. Head $(1980, p .150)$ suggests an $n$ value of 0.5 . The Fuller-Thompson equation has been used in formulating backfill material for a nuclear waste disposal vault (Pusch and Alstermark, 1985, in which the value of $n$ is not reported; Yong et al., 1986, $n=0.25$ ).

Figure 2.10 shows the grain size distributions of the five gradation types along with the "coarse" one. Also shown in the figure are the coefficients of uniformity $\left(d_{60} / d_{10}\right)$. The uniformity coefficients for Types FA and FC are theoretical values computed from Eq. (2.1). The grain size analysis of crushed tuff aggregates smaller than $0.074 \mathrm{~mm}$ has not been performed. Gradation types FA and FC are quite different from the "coarse" gradation. To obtain crushed tuff of the FA and FC gradations requires sieving and blending. Crushed tuff of $A, B$, and $C$ gradations is obtained easily from the "coarse" aggregates as discussed earlier. 


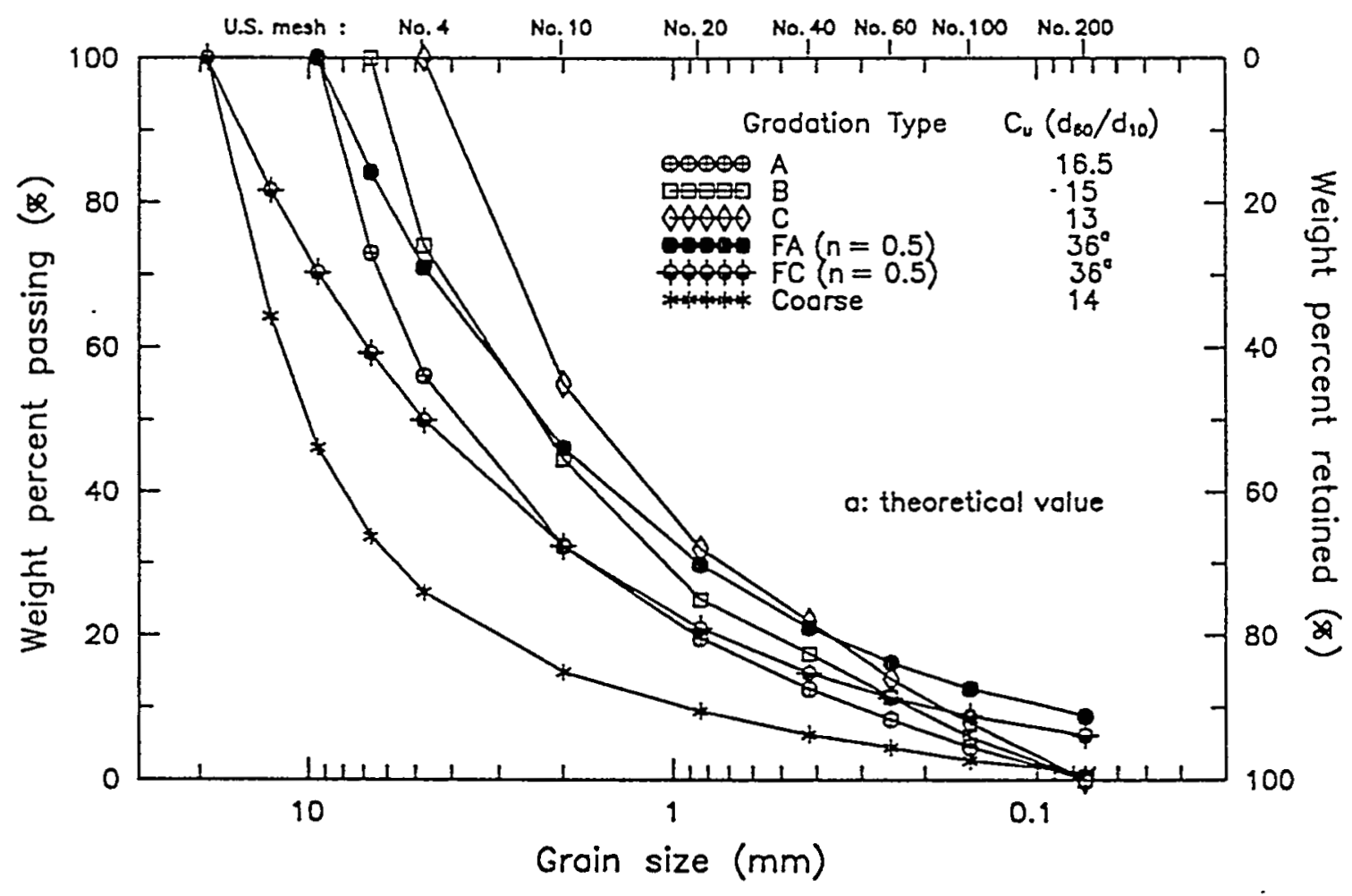

Figure 2.10 Grain size distributions of crushed tuff. 


\section{$\underline{2.4}$ Permeant}

Permeability testing on bentonite and bentonite/crushed tuff plugs with the local (site-specific) ground water is desirable to incorporate any possible physical-chemical interactions (Gaudette and Daemen, 1988; Neuzil, 1986). However, the site-representative ground water is not available and emphasis has been placed on the use of deaired distilled water as the primary permeant.

Air dissolved in distilled water can be removed by boiling. According to Lambe (1951, p. 57), boiling can reduce the dissolved air in water to about $0.75 \mathrm{ppm}$ of oxygen. Water which has been deaired is slow in regaining its air. An experiment conducted at M.I.T. indicates that the deaired water is only 608 saturated after exposure to air for 13 days (Lambe, 1951, p. 58). The distilled water used in this study has been boiled in a stainless steel vessel for at least 30 minutes. The vessel is covered to prevent the collection of foreign matter from the atmosphere. The water is allowed to cool to room temperature. The deaired distilled water is then ready to be used. The excess water is stored in an air-tight plastic bottle. The water in the plastic bottle is no longer used when the storage time exceeds 72 hours.

The deaired distilled water has a $\mathrm{pH}$ value of 6.4 , which increases to 7.3 when measured at room temperature after the water has been boiled in the presence of crushed Apache Leap tuff. Crushed tuff particles used in the boiling range from 12.7 to $19.05 \mathrm{~mm}$ in size. The $\mathrm{pH}$ of bentonite suspensions varies from 8.5 to 9.5 (American Colloid Company, Data No. 202). 
CHAPTER THREE

FLOW TEST RESULTS AND ANALYSIS

\subsection{Sample Preparation and Installation}

\subsubsection{Bentonite Plugs}

Four $25.4 \mathrm{~mm}$ diameter bentonite plugs, for flow testing with different permeants, have been constructed using the sedimentation method by gradually dropping $16.45 \mathrm{~g}$ of air-dried bentonite powder into a fluid column. The samples have not been compacted and have been allowed to hydrate for three weeks to a month prior to permeability testing. Saturation of the samples has been aided by intermittent vacuuming from the top. The applied vacuum was less than $34.5 \mathrm{kPa}$ to avoid upward movement of the samples.

Bentonite of the optimal water content (23.58) has been used to prepare the compacted samples. To achieve a uniform water content, a plant sprayer is used to evenly distribute the (distilled) water across the clay while mixing. The bentonite is then cured for 72 hours before installation. For sample diameters greater than $50.8 \mathrm{~mm}$ (2 in), a manual rammer as specified by ASTM D698-78 (Standard Proctor Method), D3.2.1, is used to compact the samples. A hammer compactor with hammer weight of $0.53 \mathrm{~kg}(1.16 \mathrm{lb}$ ) and circular specimen contact of $25.4 \mathrm{~mm}$ (1 in) in diameter, is used for compacting samples of smaller diameters. For either case, the drop height and the number of drops per layer are adjusted to provide the same energy input as for the Standard Proctor Test $\left(593 \mathrm{~kJ} / \mathrm{m}^{3} ; 12375 \mathrm{ft}-1 \mathrm{~b} / \mathrm{ft}^{3}\right)$.

The compacted bentonite samples installed in PVC permeameters have no immediate confinement on top, and therefore swell vertically upon further hydration. They are saturated under a water pressure of 24.5 $\mathrm{kPa}$ ( $2.5 \mathrm{~m}$ water column) from the bottom port for about 75 days. A vacuum pressure of $34.5 \mathrm{kPa}$ ( $5 \mathrm{psi}$ ) is applied continuously to the top port to help saturation. For the compacted samples totally confined in stainless steel permeameters, saturating has been attempted by an injected water pressure of $345 \mathrm{kPa}$ (50 psi) and an intermittent vacuum pressure of $103.5 \mathrm{kPa}$ (15 psi) for approximately 35 days. Such samples are later subjected to a low water pressure of $69 \mathrm{kPa}$ (10 psi) for about 45 days and then to a pressure of $24.5 \mathrm{kPa}$ for another two weeks, prior to flow testing.

Procedures for sample preparation and installation are given in Appendix A.

\subsubsection{Bentonite/Crushed Tuff Plugs}

The mixture plugs are prepared by thoroughly mixing crushed tuff (of a selected gradation) with a predetermined amount of bentonite having a 
23.58 water content. A mixed sample is stored and cured in an air-tight plastic jar for 72 hours. The sample is then transferred into a permeameter using a small plastic shovel. This emplacement method, termed herein the scooping method, is selected to minimize particle segregation. All the mixture samples receive a compaction energy equivalent to that of the Proctor compaction method, except for the three samples mixed with crushed tuff of the FC gradation, which received only 258 of the Proctor compaction energy.

The procedure for sample saturation follows ASTM D2434-68, Section 6.6.4. To minimize changes in sample structure, the samples are saturated under a $2.5 \mathrm{~m}$ water column $(24.5 \mathrm{kPa})$ from the bottom for approximately 2 months, frequently aided with a vacuum of $103.5 \mathrm{kPa}$ (15 psi) at the top.

Procedures for sample preparation and installation are given in Appendix A.

\subsubsection{Sample Designation}

The sample identification system is given in Figure 3.1. Crushed tuff grain size distribution curves used in preparing the mixture type of plugs are shown in Figure 2.10 along with the "coarse" gradation.

\subsection{Flow Test Methods}

Constant head, standard falling head (Lambe, 1951), and modified falling head (double-pipette falling head) (Williams and Daemen, 1987) methods are used to determine permeabilities of the sealant plugs. They are characterized as steady and quasi-steady (e.g. falling head) flow test methods. Test procedures are described in Appendix B. Equations (3.1), (3.2), and (3.3) are used to compute permeability for the constant head, standard falling head, and modified falling head methods, respectively.

$$
K=\frac{Q L}{A t h}
$$

where $K$ - coefficient of permeability $(\mathrm{cm} / \mathrm{s})$

$Q$ - quantity of water discharged over time $t$ (cc)

$\mathrm{L}$ - sample length (cm)

A - cross-sectional area of the permeameter $\left(\mathrm{cm}^{2}\right)$

$t$ - elapsed time of discharge ( $s$ )

$h=$ head difference across the specimen (cm).

$$
K=\frac{a L}{A\left(t_{2}-t_{1}\right)} \ln \frac{h_{1}}{h_{2}}
$$


(I) Bentonite plugs

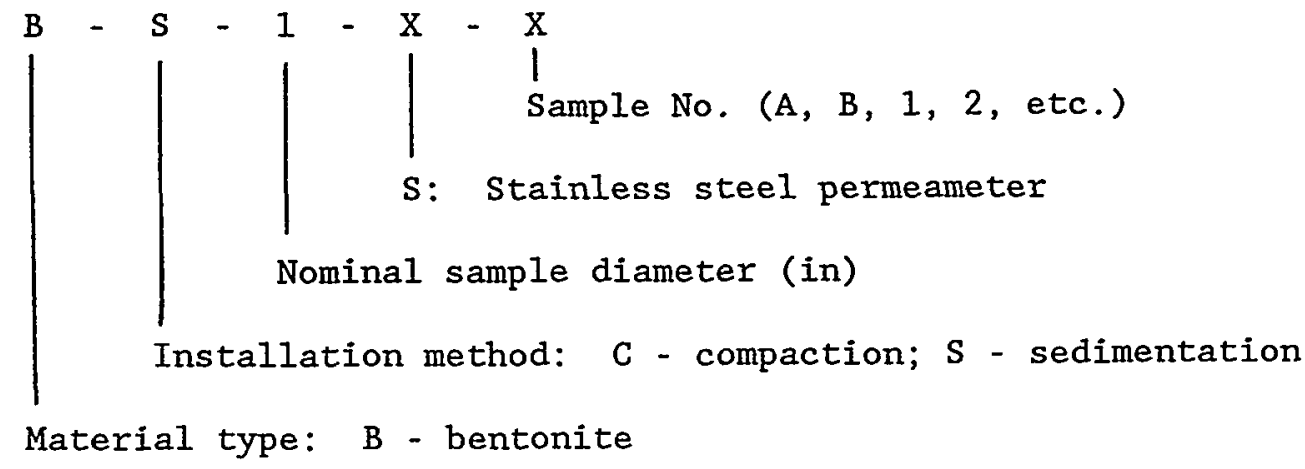

(II) Bentonite/crushed tuff plugs

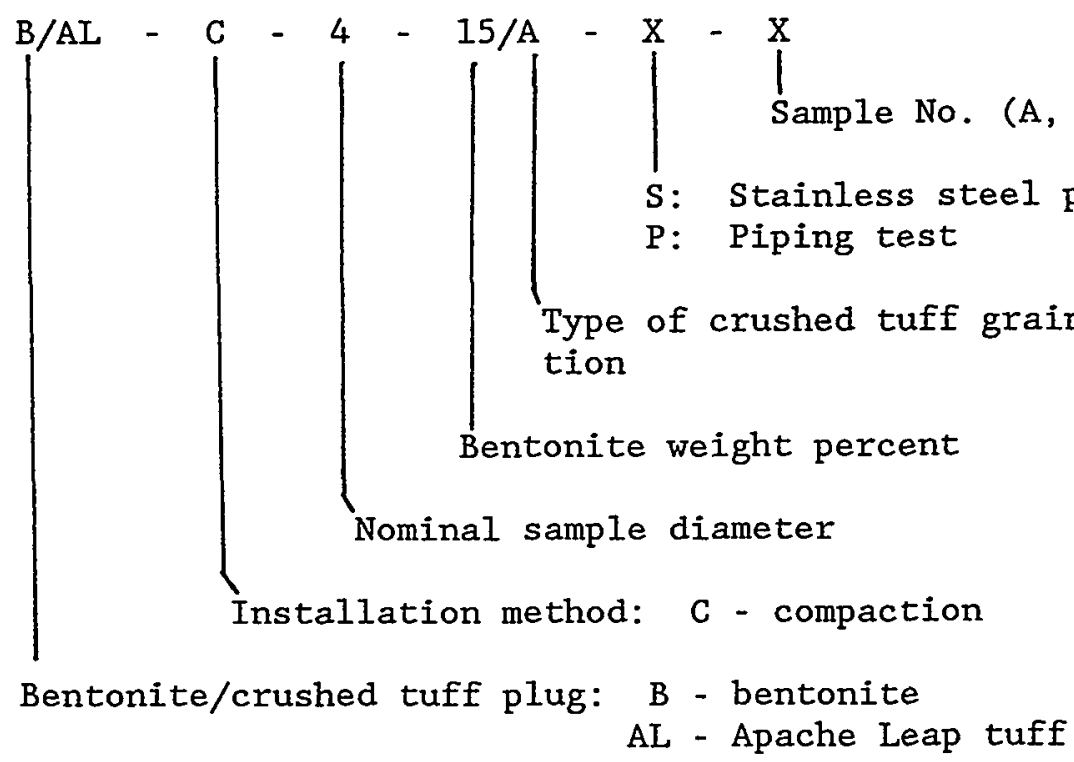

Figure 3.1 Bentonite and bentonite/crushed tuff sample number designation system. 


$$
K=\frac{a L}{2 A\left(t_{2}-t_{1}\right)} \ln \frac{h_{1}}{h_{2}}
$$

where $a=$ cross-sectional area of the inflow and outflow pipettes $\left(\mathrm{cm}^{2}\right)$

$t_{1}=$ time when water level difference is $h_{1}$

$t_{2}=$ time when water level difference is $h_{2}$

$h_{1}=$ height difference between water levels in the pipettes at $t_{1}$

$h_{2}=$ height difference between water levels in the pipettes at $t_{2}$

and other parameters are already defined.

other flow test methods are available, such as the constant flow rate method (Olsen, 1966, Olsen et al., 1985), hydraulic transient flow methods (Brace et a1., 1968; Hsieh et al., 1981; Neuzil et a1., 1981), and mechanical transient flow methods (step load consolidation test, Terzaghi, 1927; Lambe, 1951). The constant flow rate method is not chosen for this study, mainly because this method is not suitable for high injection pressure flow tests, high temperature flow tests, and piping tests, because pressure/gradient is the critical independent variable. The transient flow methods are excluded from this investigation as they tend to yield lower permeability values than those obtained from the steady flow methods (Seaber and Vecchioli, 1966; 01son and Daniel, 1981; Tavenas et al., 1983). Permeability discrepancies between a steady flow test and transient flow tests of the Pierre Shale are reported by Neuzil (1986).

Precise measurement of permeability in highly deformable media (e.g. the bentonite studied here) is difficult with any technique (Gibson et al., 1967; Smiles and Rosenthal, 1968; Smiles, 1968; Kharaka and Smalley, 1976; 01son and Daniel, 1981). In this investigation, low hydraulic gradients are used for the determination of permeability to minimize the deformation of the samples during flow testing. The flow tests with large hydraulic gradients are aimed at studying the sealing performance under high injection pressures. Considerable efforts are devoted to obtaining the mass balance of inflow and outflow.

\subsection{Results of Bentonite Permeability Testing}

Eighteen bentonite samples have been constructed for flow testing: (1) four $2.5-\mathrm{cm}(1-$ inch) diameter plugs for flow testing with different water chemistries, (2) six compacted samples of $2.5,5$, and $10 \mathrm{~cm}(1,2$, and 4 in.) diameter (two for each size) for studying the size effect on permeability, (3) four compacted plugs having diameters of 2.654, 3.475, 6.01 , and $10.246 \mathrm{~cm}$, respectively, for the high injection pressure flow testing, and (4) four $2.5-\mathrm{cm}$ diameter samples for determining the permeability to air. 


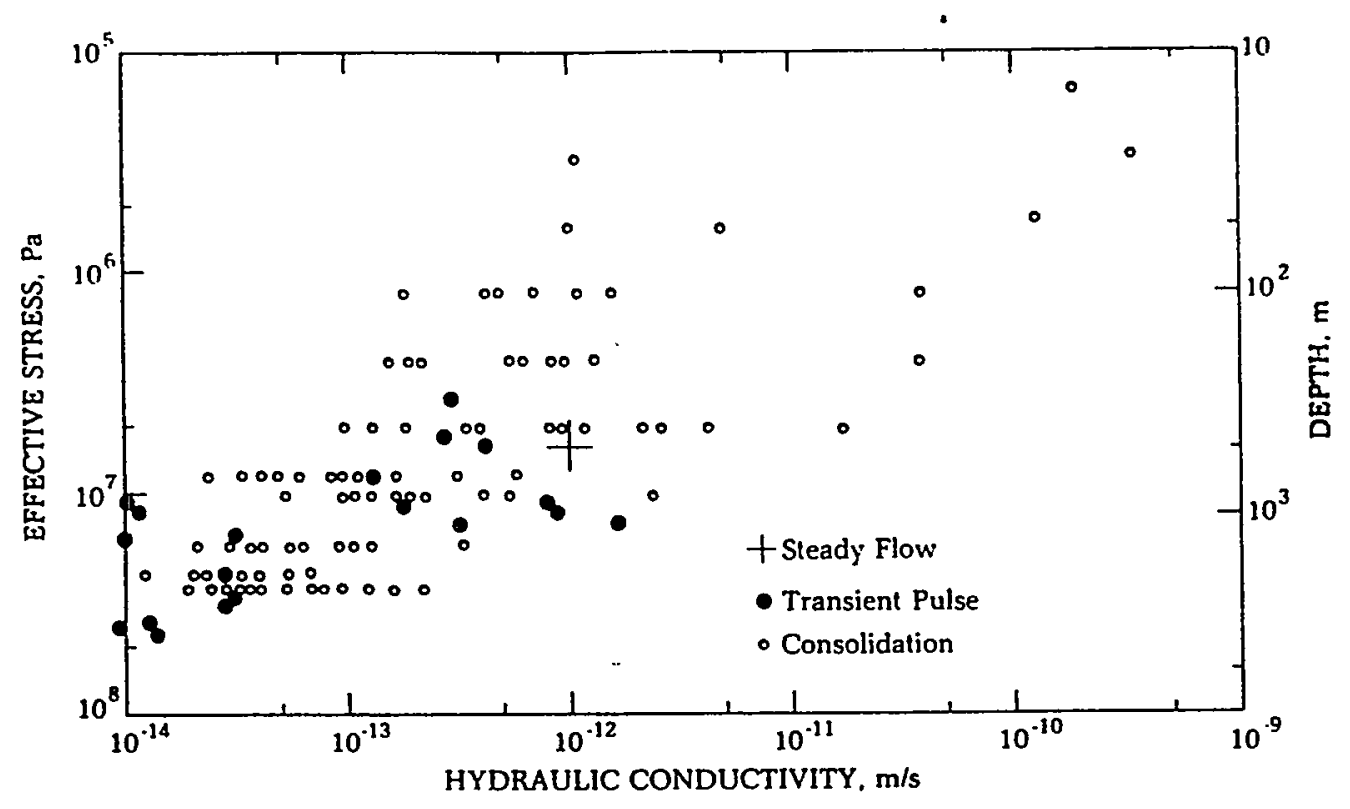

Figure 3.2 Examples of discrepancies between permeabilities obtained from steady flow tests and from hydraulic and mechanical transient flow tests.

Reproduced with permission from Neuzil, C.E., "Groundwater Flow in Low-Permeability Environments", Water Resources Research, Vol. 22, No. 8, pp. 1163-1195, Aug. 1986, American Geophysical Union, Washington, DC. 


\subsubsection{Influence of Water Chemistry}

Samples B-S-1-A, B-S-1-B, B-S-1-C, and B-S-1-D are constructed by gradually dropping $16.45 \mathrm{~g}$ of air-dried bentonite into a water column. The samples are allowed to deposit by sedimentation for about three weeks prior to testing. Sample descriptions and test conditions are given in Table 3.1 .

Sample B-S-1-A, deposited in and tested with distilled water, shows a permeability as low as $6.9 \times 10^{-8} \mathrm{~cm} / \mathrm{s}$ at the end of a testing period of 21 days (Figure 3.3). To replace distilled water with 28 sodium pyrophosphate solution (a dispersing agent), the sample has been flushed with the solution for about 37 days before the flow testing resumed. Two months later, the permeability has dropped to 2 to $3 \times 10^{-8} \mathrm{~cm} / \mathrm{s}$ (Figure 3.4). Sample B-S-1-B has originally been deposited in and permeated with 28 sodium pyrophosphate solution. As shown in Figure 3.3 , the sample has a permeability of $1.4 \times 10^{-8} \mathrm{~cm} / \mathrm{s}$ about 25 days after the test started. The sample has then been flushed with distilled water for 30 days and subsequently tested with the same water for 83 days. A permeability of $1.6 \times 10^{-8} \mathrm{~cm} / \mathrm{s}$ has been measured at the end of the test (Figure 3.5).

Relatively high permeability values, around $10^{-6} \mathrm{~cm} / \mathrm{s}$, are measured for Sample B-S-1-C during a test period of 8 days. This sample has been deposited in a 28 calcium hydroxide suspension (a flocculent agent) and later tested with distilled water. Subsequently, the sample has been flushed with 48 sodium pyrophosphate solution for 48 days. The permeability testing has then been resumed and continued for 72 days. A reduced permeability of $3.4 \times 10^{-7} \mathrm{~cm} / \mathrm{s}$ has been measured at the conclusion of the test (Figure 3.6). Sample B-S-1-D, sedimented in the synthetic water, has a permeability of $7.7 \times 10^{-8} \mathrm{~cm} / \mathrm{s}$ when tested with the synthetic water (Figure 3.3) and a permeability of $2 \times 10^{-8} \mathrm{~cm} / \mathrm{s}$ with the 28 sodium pyrophosphate solution (Figure 3.7 ).

\subsubsection{Influence of Sample Size}

Six compacted bentonite plugs of $2.5,5$ and $10 \mathrm{~cm}(1,2$ and $4 \mathrm{in})$ diameter (two of each size) have been constructed for flow testing in PVC permeameters. The samples are compacted at the optimal moisture content of $23.55 \%$, with compaction energy equal to that of the standard Proctor method (ASTM D698-78). The sample descriptions can be found in Table 3.2. During the saturation stage, the samples are allowed to adsorb water and swell vertically. This hydration-swelling process has continued throughout the subsequent flow testing period. Changes in sample length as a result of the hydration-swelling process are summarized in Table 3.3. The sample length at the end of flow testing is the maximum possible expansion, given the restraint imposed by the end caps of the permeameter. Considering that the bentonite can swell to 10 to 13 times its dry size upon complete hydration (Jepsen and Place, 1985), the final sample lengths in Table 3.3 suggest that the samples are still capable of swelling further. Due to the geometric restraint, a certain amount of swelling pressure must have been generated. This is confirmed by the fact that the bottom cap plate of the PVC permeameter housing Sample B-C-4-B has been detached from the sample chamber (originally 
Table 3.1 Sample Descriptions of Sedimented Bentonite Plugs

\begin{tabular}{|c|c|c|c|c|}
\hline $\begin{array}{l}\text { Sample } \\
\text { Number }\end{array}$ & $\begin{array}{l}\text { Type of Fluid* } \\
\text { for sedimentation }\end{array}$ & $\begin{array}{c}\text { Initial } \\
\text { Sample } \\
\text { Length } \\
\text { (cm) }\end{array}$ & Permeant \#I & Permeant \#2 \\
\hline$B-S-1-A$ & $\begin{array}{l}\text { Distilled water } \\
(\mathrm{pH}=6)\end{array}$ & 15 & Distilled water & $\begin{array}{l}2 \% \text { Sodium } \\
\text { pyrophosphate }\end{array}$ \\
\hline B-S-1-B & $\begin{array}{l}2 \% \text { Sodium } \\
\text { pyrophosphate } \\
(\mathrm{pH}=9)\end{array}$ & 9 & $\begin{array}{l}2 \% \text { Sodium } \\
\text { pyrophosphate }\end{array}$ & Distilled water \\
\hline$B-S-1-C$ & $\begin{array}{l}2 \% \text { Calcium } \\
\text { hydroxide } \\
(\mathrm{pH}=12)\end{array}$ & 14.7 & Distilled water & $\begin{array}{l}4 \% \text { Sodium } \\
\text { pyrophosphate }\end{array}$ \\
\hline$B-S-1-D$ & $\begin{array}{l}\text { Synthetic water }{ }^{*} \\
(\mathrm{pH}=6)\end{array}$ & 19.7 & Synthetic water & $\begin{array}{l}2 \% \text { Sodium } \\
\text { pyrophosphate }\end{array}$ \\
\hline
\end{tabular}

*Percentages refer to the ratio between weight of chemical added and weight of distilled water.

* Synthetic water was produced by boiling distilled water in the presence of A-Mountain crushed tuff 


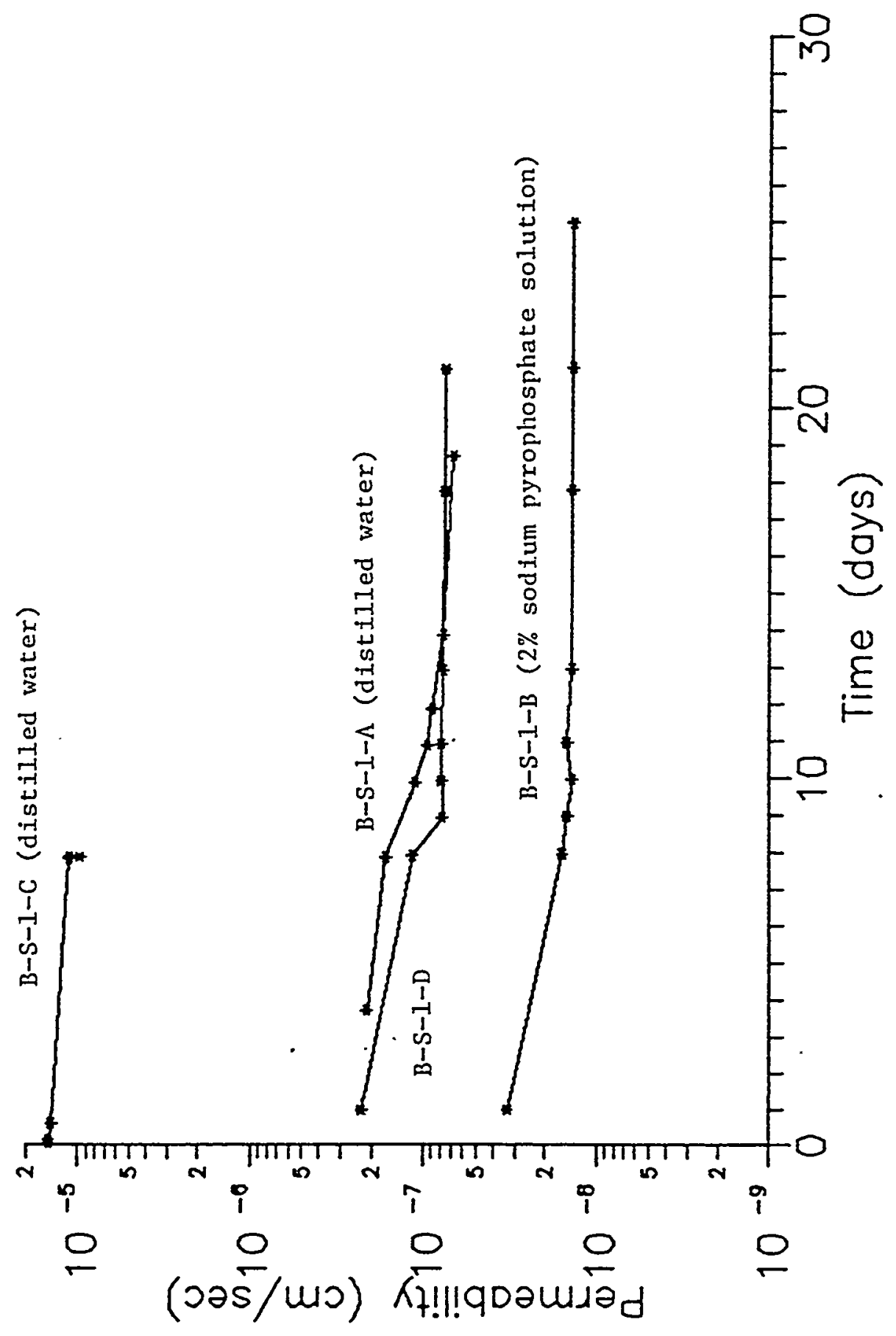

出

岕 


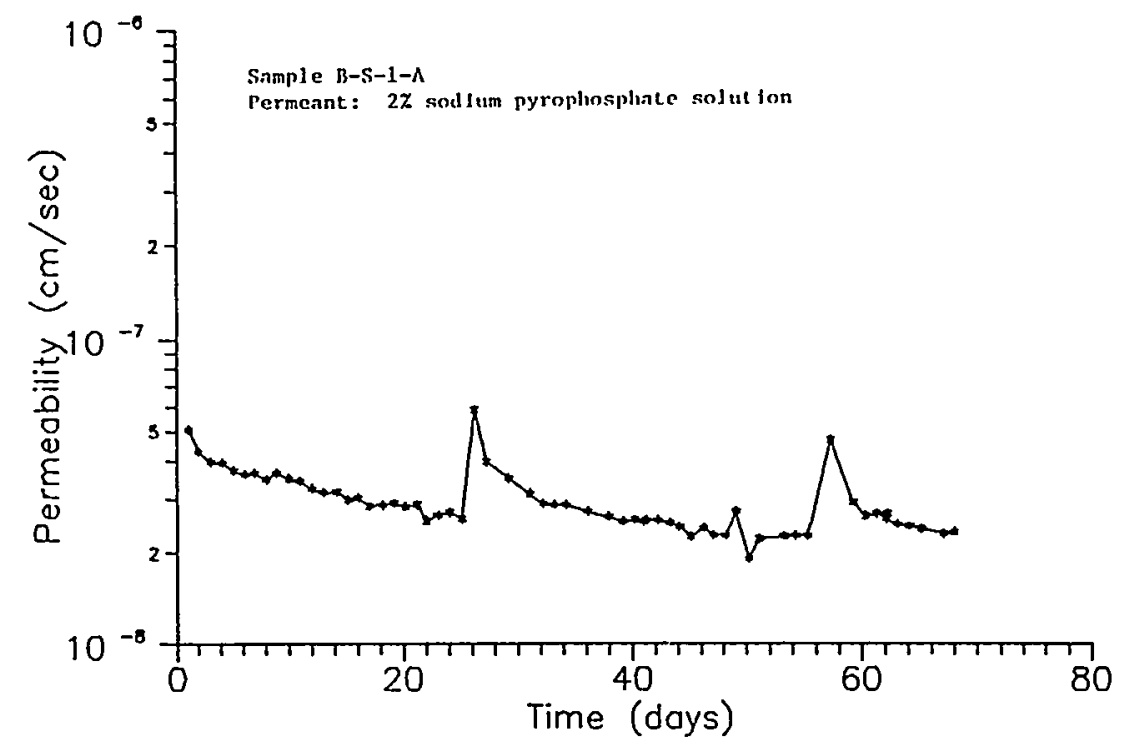

Figure 3.4 Flow test results of Sample B-S-1-A when permeated with 28 sodium pyrophosphate solution.

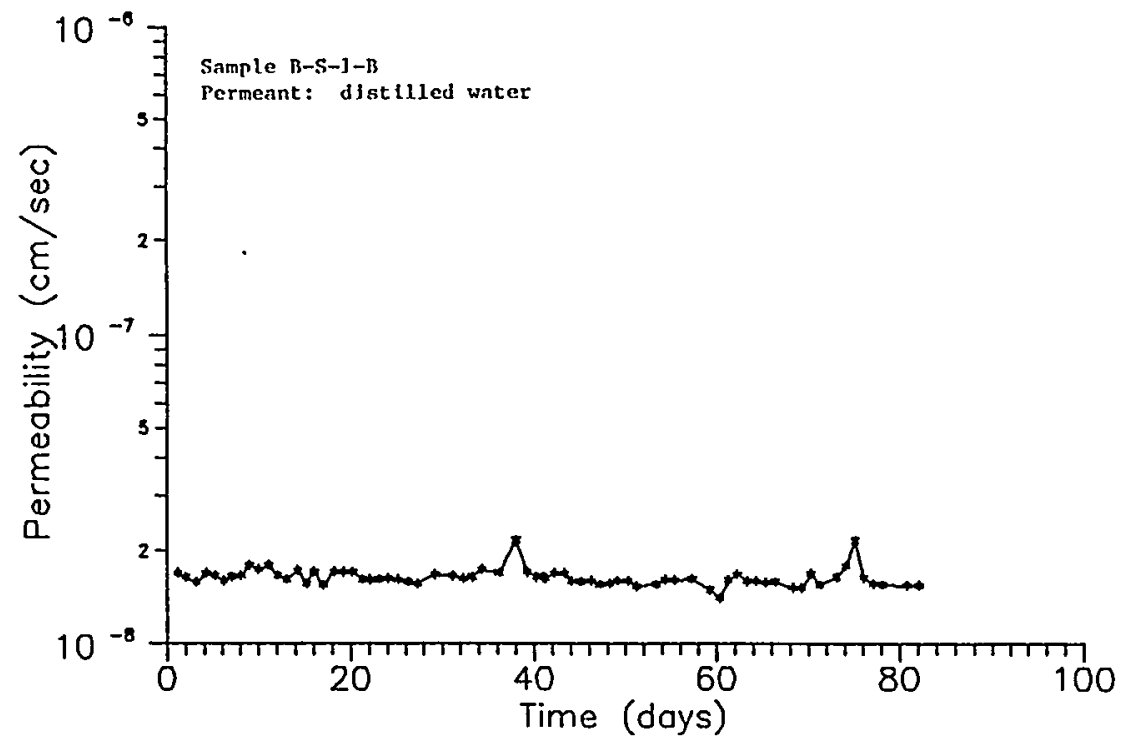

Figure 3.5 Flow test results of Sample B-S-1-B when permeated with distilled water. 


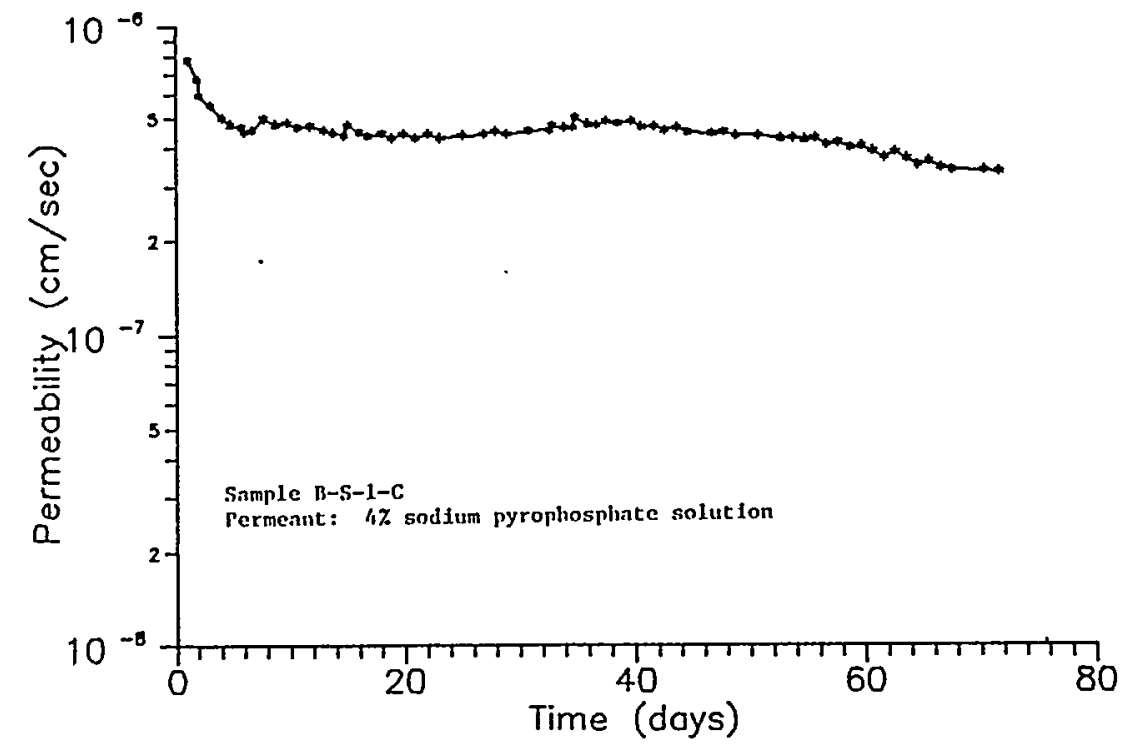

Figure 3.6 Flow test results of Sample B-S-1-C when permeated with 48 sodium pyrophosphate solution.

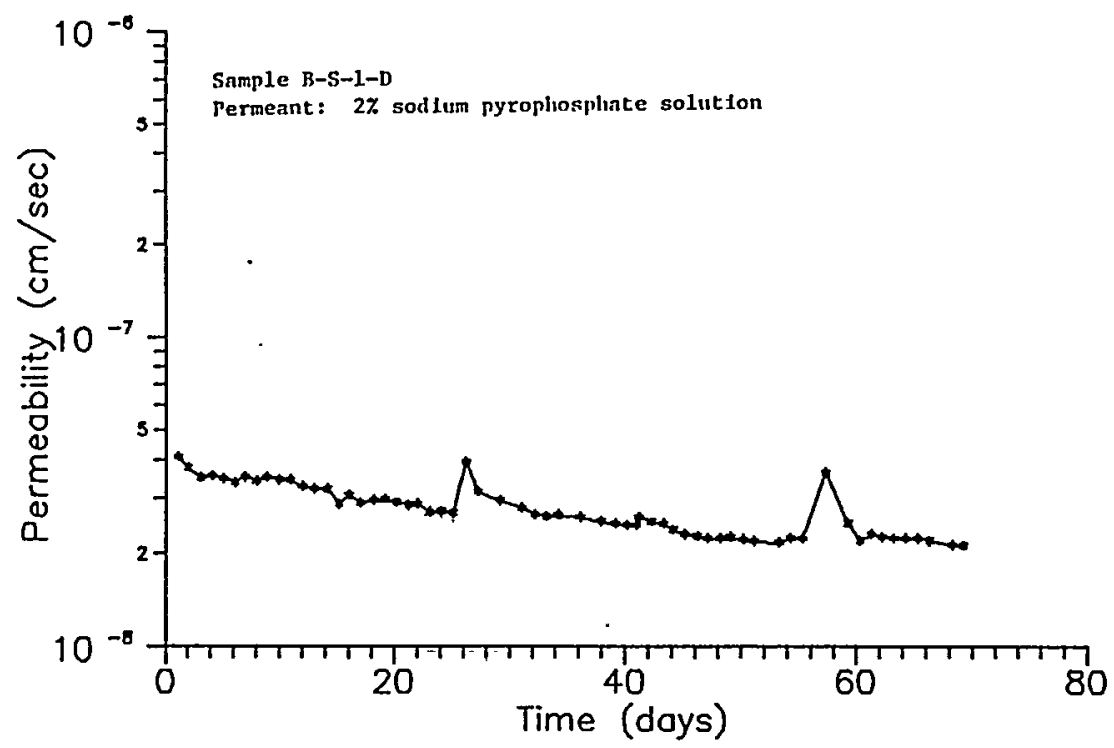

Figure 3.7 Flow test results of Sample B-S-1-D when permeated with 28 sodium pyrophosphate solution. 
Table 3.2 Sample Descriptions of Compacted Bentonite Plugs

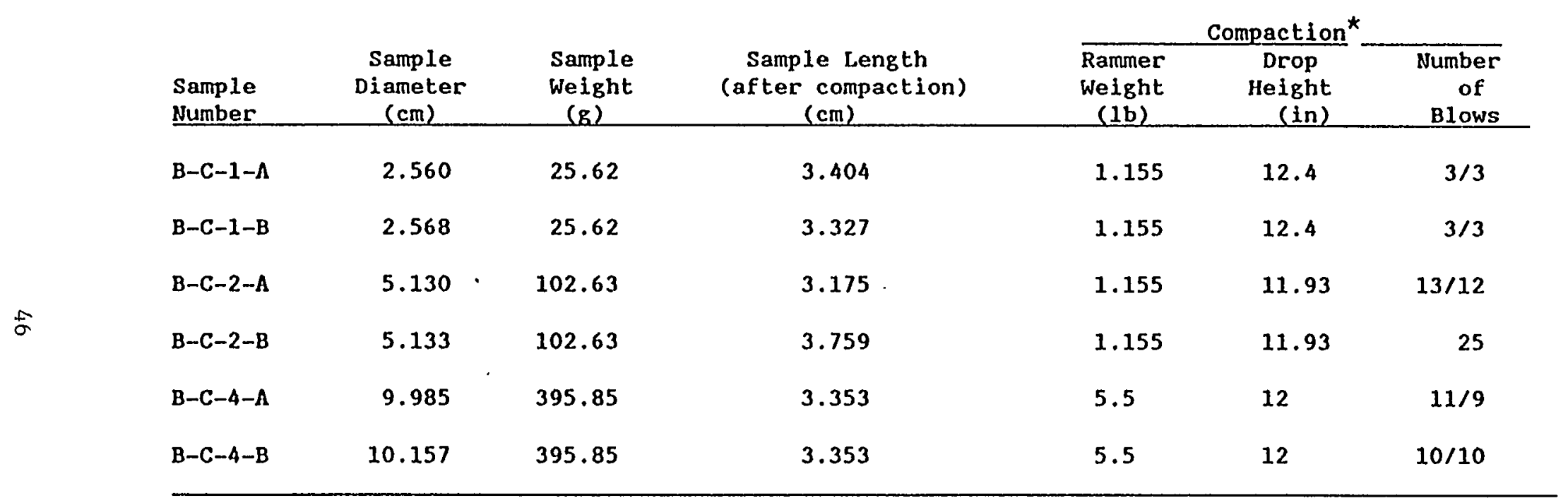

* Compaction was performed in a sequence of 2-1ayer installation except for sample B-C-2-B. Combination of rammer weight, drop height, number of blows results in the standard proctor compaction energy $\left(12375 \mathrm{ft}-1 \mathrm{~b} / \mathrm{ft}^{3}, 593 \mathrm{~kJ} / \mathrm{m}^{3}\right)$. 
Table 3.3 Changes in Sample Length of Compacted Bentonite Plugs

\begin{tabular}{lccc} 
& \multicolumn{3}{c}{ Sample Length (cm) } \\
Sample & $\begin{array}{c}\text { After } \\
\text { Number }\end{array}$ & $\begin{array}{c}\text { Before } \\
\text { Compaction }\end{array}$ & $\begin{array}{c}\text { After } \\
\text { Flow Testing }\end{array}$ \\
\hline B-C-1-A & 3.40 & 11.5 & 12.8 \\
B-C-1-B & 3.33 & 11.35 & 12.8 \\
B-C-2-A & 3.18 & 9 & 9.1 \\
B-C-2-B & 3.76 & 9.2 & 9.4 \\
B-C-4-A & 3.35 & 10.1 & 10.2 \\
B-C-4-B & 3.35 & 9.7 & 10.0 \\
\hline
\end{tabular}


glued on with epoxy). The detachment has been observed for the first time approximately 47 days after the flow testing was started.

\subsubsection{Results of First Flow Test Sequence}

Permeabilities of the six compacted bentonite plugs have been measured continuously for approximately 85 days, using the double-pipette falling head method. For Sample B-C-4-B, the testing method has been changed to the standard falling head method after the detachment of the bottom cap has been noticed. The permeability results are shown in Figures 3.8 through 3.10. Experimental results, including inflow, outflow, elapsed time, as well as calculated permeability, are tabulated in Appendix $C$. The inflow and outflow are out of balance throughout the flow testing. This imbalance most likely is due to the continuing hydration and expansion of bentonite. The permeability values shown in Appendix $C$ (except for the latter part of flow testing on Sample B-C-4-B) have been calculated using Eq. (3.3).

In order to use Eq. (3.3), the measured amounts of inflow and outflow are divided by the cross-sectional area of the measuring pipettes and are transformed into equivalent lengths. The height difference $h_{2}$ is then obtained by subtracting the equivalent lengths from $h_{1}$. For unequal inflow and outflow, the use of corrected equivalent lengths, each corresponding to $1 / 2$ (inflow + outflow), may seem desirable in calculating $h_{2}$ such that the continuity assumption for Eq. (3.1) is maintained. As long as $h_{2}$ is less than $h_{1}$, the correction is automatically included in the computation.

Permeabilities of all six compacted bentonite plugs are of the order of $10^{-9} \mathrm{~cm} / \mathrm{s}$. Recognizing that the samples are not yet completely hydrated, these permeabilities appear quite reasonable when compared with the value of $6.9 \times 10^{-8} \mathrm{~cm} / \mathrm{s}$ (for Sample B-S-1-A, Fig. 3.3) at which complete hydration can be assumed.

The standard falling head method (Appendix B, Method B) has been adopted for the latter part of flow testing on Sample B-C-4-B, after the detachment of the bottom cap plate from the permeameter chamber had been observed. Eq. 3.2 is used to calculate permeability. Due to the breakage of the permeameter, the outflow has been difficult to measure, and therefore has been assumed equal to the inflow. The permeabilities thus calculated are, as expected, higher than the values obtained using the double-pipette falling head equation.

For samples of the same diameter, permeability varies only slightly, and not systematically, e.g. as a function of diameter. Differences typically amount to two to three times the permeability obtained. The small variations suggest that similar test results can be obtained if the same procedures for sample preparation, installation, and flow testing are repeated. Permeabilities of the bentonite plugs appear invariant with sample diameters used in this study. 


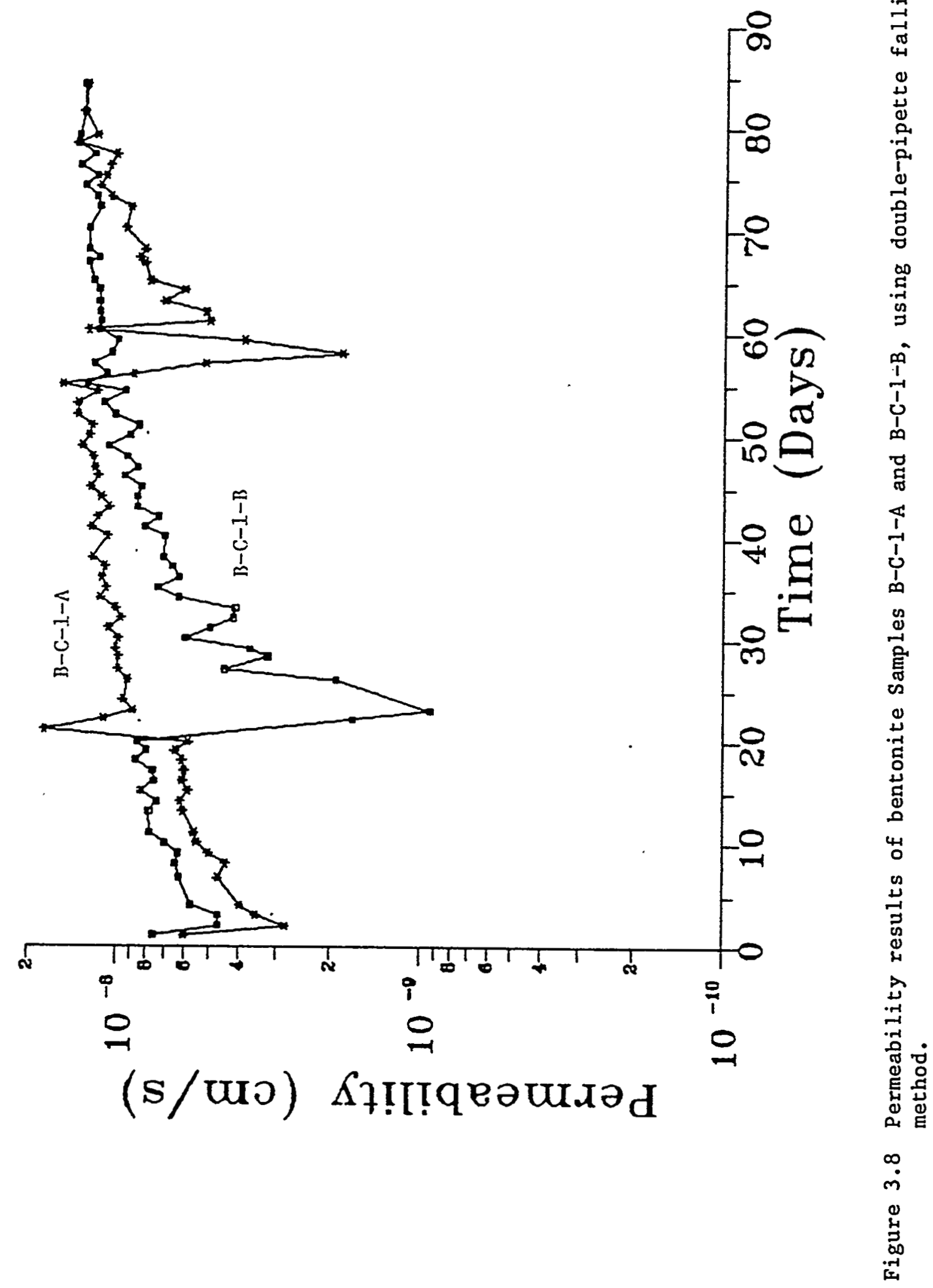




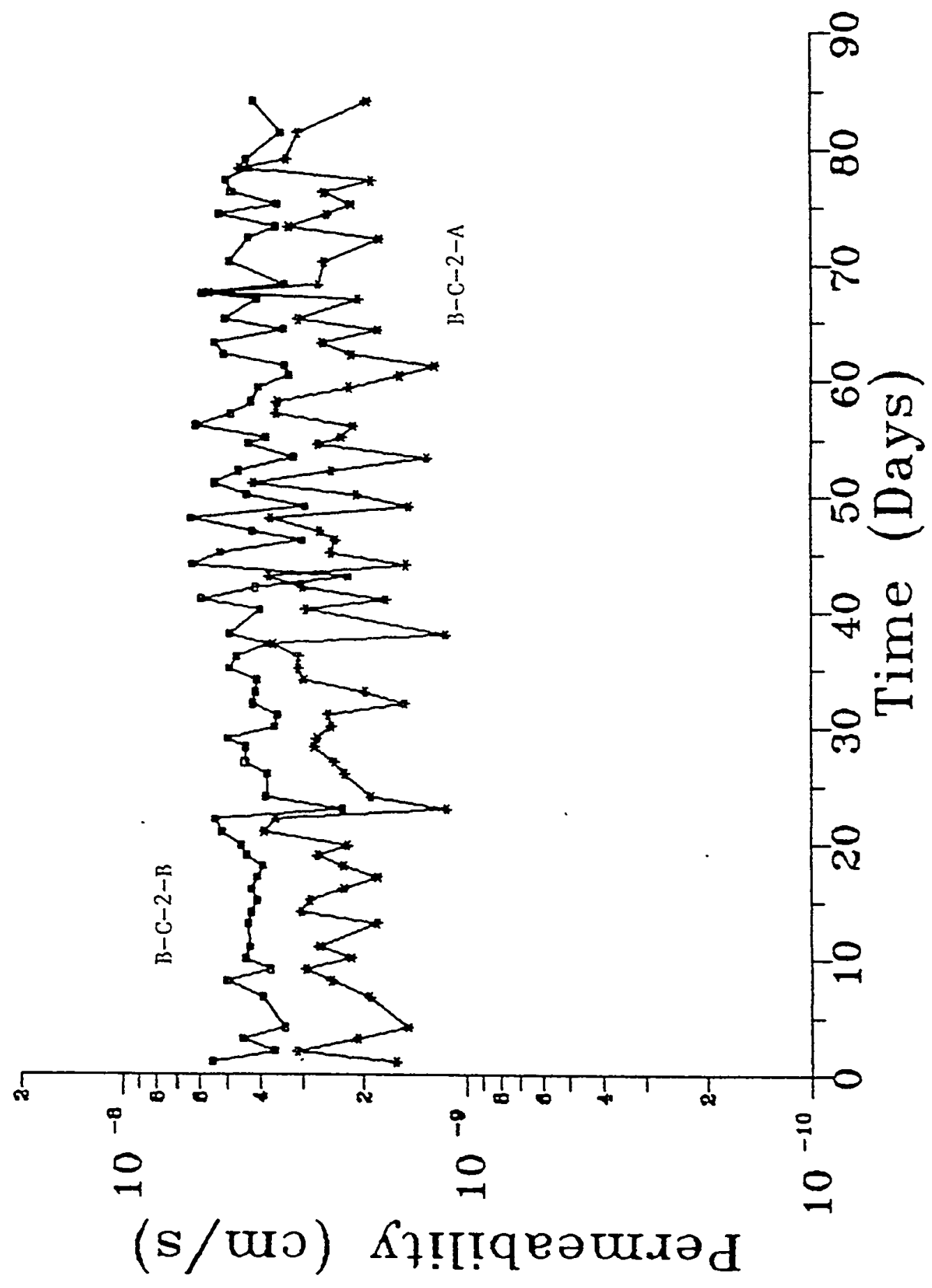

$\underset{\mathbb{Z}}{\mathbb{Z}}$

告

४ै

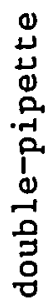

告

$n$
1
01
0
1
0
0
0

告



感

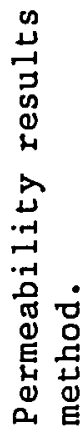

$\underset{\substack{a \\ \infty \\ \infty}}{0}$ 


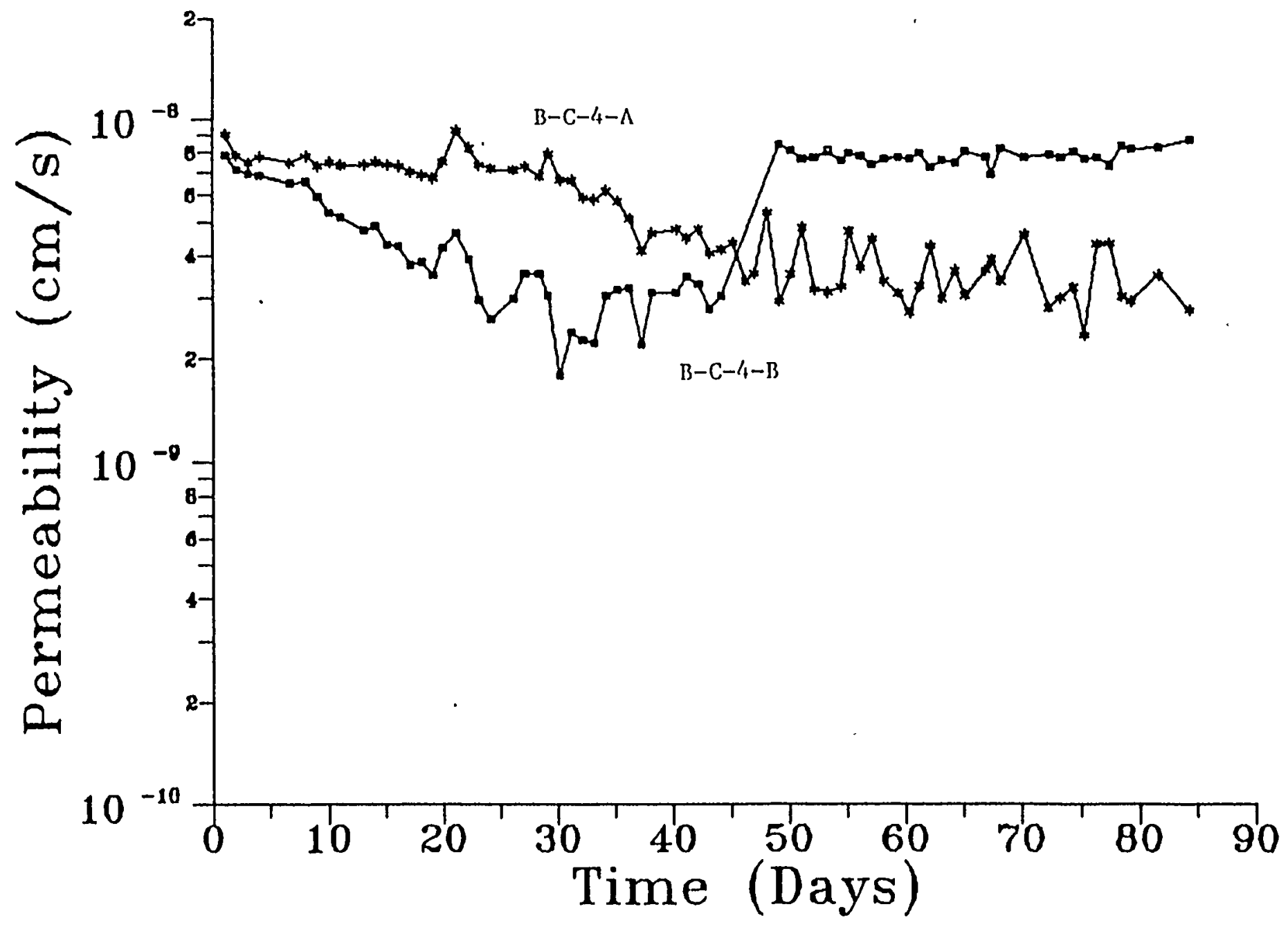

Figure 3.10 Permeability results of bentonite Samples B-C-4-A and B-C-4-B, using double-pipette falling head method and standard falling head method (for the last ten measurements of $B-C-4-B$ ). 


\subsubsection{Results of Second Flow Test Sequence}

In an attempt to improve the mass balance and to examine changes of permeability with continued hydration, five of the six compacted bentonite plugs have been subjected to flow testing for an additional 43 days. Flow testing has not been continued for Sample B-C-4-B because the bottom cap detached from the permeameter chamber. Upon refilling the inflow pipette, this sample responded to the increased hydraulic pressure by slipping. The interface strength is estimated as 2.33 to $2.48 \mathrm{kPa}$ ( 0.34 to $0.36 \mathrm{psi})$, based on the weight of static water column sustained by the sample when the slippage stopped. Sample B-C-4-B has been used for the determination of water content at various locations.

The permeability results show a relatively small variation over the additional test period of approximately 43 days (Figures 3.11 to 3.15 ). Test duration, inflow and outflow, as well as the calculated permeability, can be found in Appendix $C$. The inflow and outflow remain out of balance throughout the testing period. This imbalance may, again, be due to the continuing hydration and expansion of the bentonite.

During the second flow test sequence, Samples B-C-1-A and B-C-I-B yield an average permeability of $2 \times 10^{-8} \mathrm{~cm} / \mathrm{s}$, about 2 times higher than the value obtained from the later measurements of the first test sequence. Samples B-C-2-A, B-C-2-B, and B-C-4-A show essentially the same permeabilities as measured before, in a range from 2 to $4 \times 10^{-9} \mathrm{~cm} / \mathrm{s}$. As shown in Table 3.3, the sample lengths of the latter three plugs vary only slightly, indicating similar hydration and swelling states. No evidence can be discerned for the size effect on permeability.

\subsubsection{Water Content Distribution of Sample B-C-4-B}

Sample B-G-4-B was pushed out of the PVC permeameter and sliced into eight disks for the study of water content distribution and of crack pattern (upon desiccation). The disks are numbered 1, a, 2, b, 3, c, 4 and $d$, in ascending order from the outflow side (bottom) to the inflow side (top). Disks $a, b, c$ and $d$ were used for the determination of water content distribution. Disks $1,2,3$ and 4 remained exposed to air in a room at 408 relative humidity for the study of cracking patterns. Disks $a, b, c$ and $d$ were each divided into three parts, the outer ring ( $1.27 \mathrm{~cm}$ wide), middle ring ( $1.27 \mathrm{~cm}$ annulus width), and inner disk ( $5.08 \mathrm{~cm}$ in diameter). The water content of each part was measured following ASTM Standard D2216-80. Table 3.4 summarizes disk thickness, relative position, and use. The results of the water content determinations are shown in Table 3.5.

The nonuniform water content distribution of Sample B-C-4-B manifests the effect of the continuing hydration and expansion during the flow testing. A uniform water content distribution would be expected if the sample were allowed to reach an equilibiium state within the space provided by the permeameter. It would take a long time for such an equilibrium state to be established. The sample was permitted to absorb water and swell (expand) vertically. The non-uniform distribution of the water content causes problems in interpreting the permeability results (Gaudette and Daemen, 1988, Ch. 6). Rather than related to a 
Sample B-C-1-A

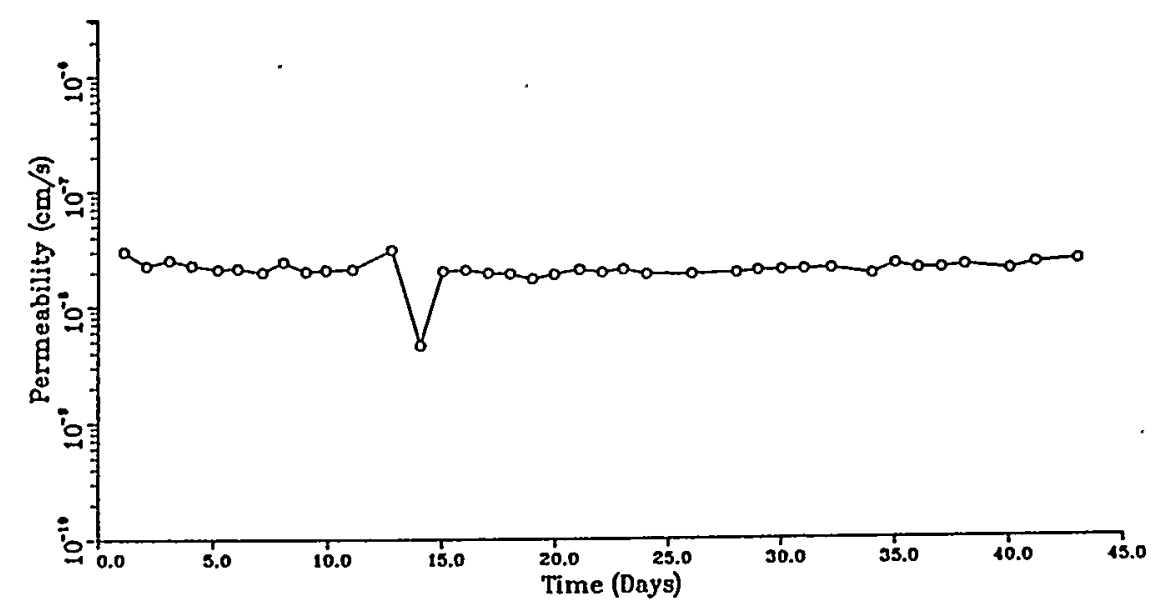

Figure 3.11 Permeability results for bentonite Sample B-C-1-A.

Sample B-C $=-1-B$

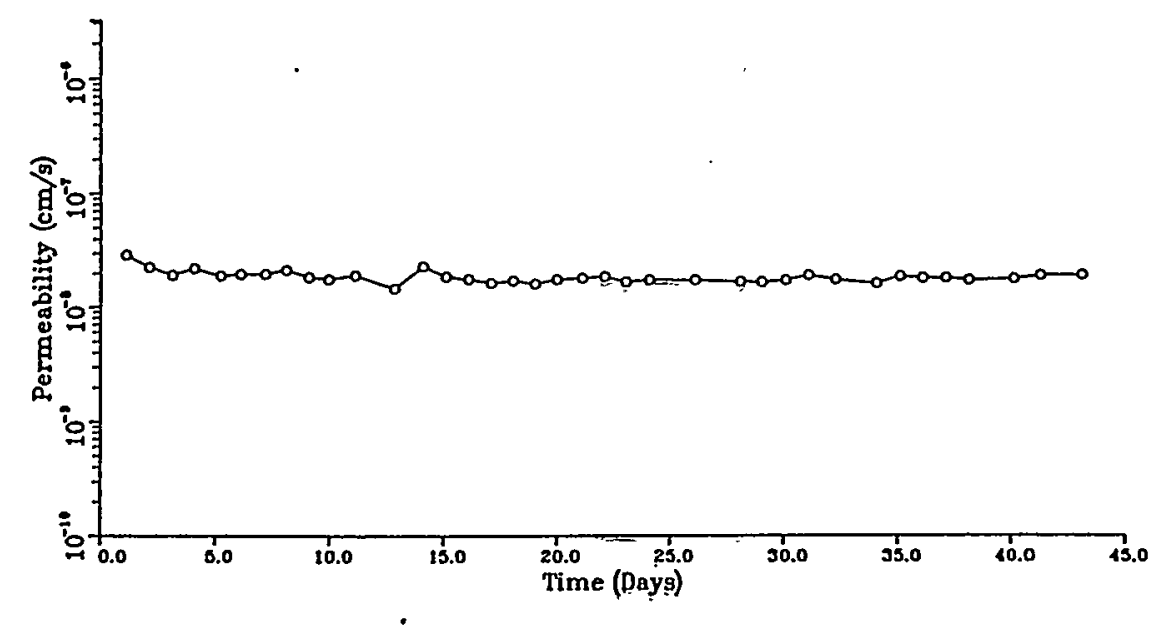

Figure 3.12 Permeability results for bentonite Sample B-C-1-B. 


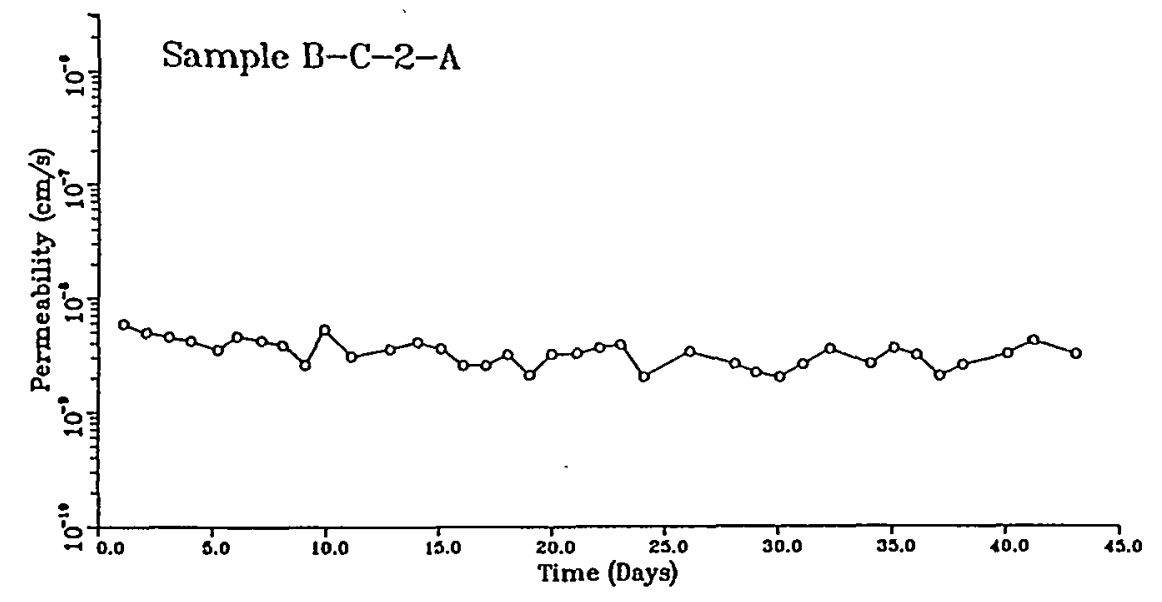

Figure 3.13 Permeability results for bentonite Sample B-C-2-A.

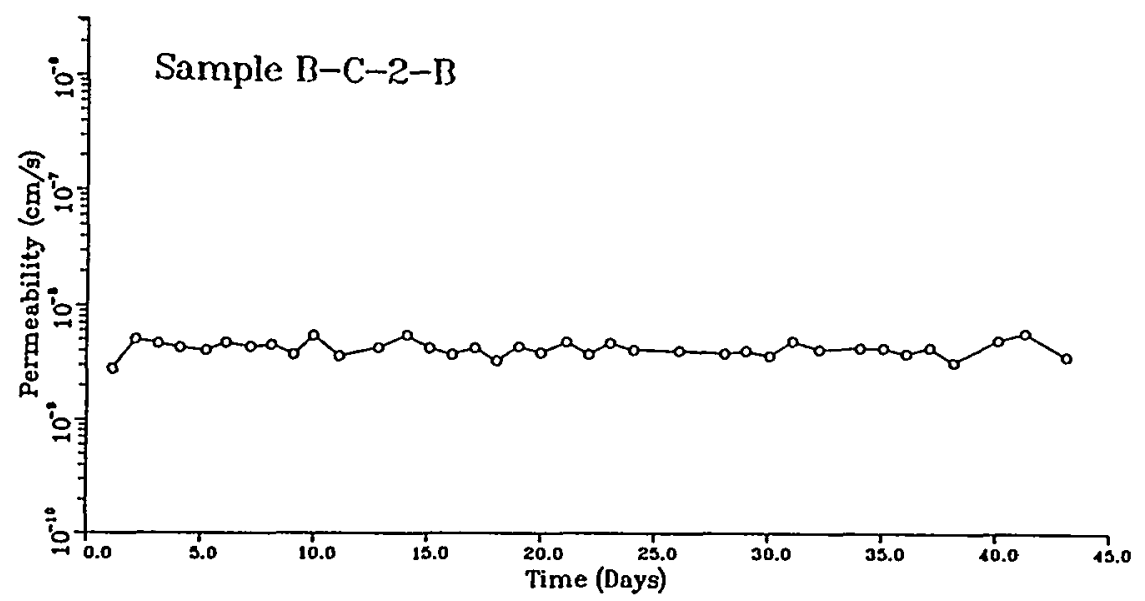

Figure 3.14 Permeability results for bentonite Sample B-C-2-B.

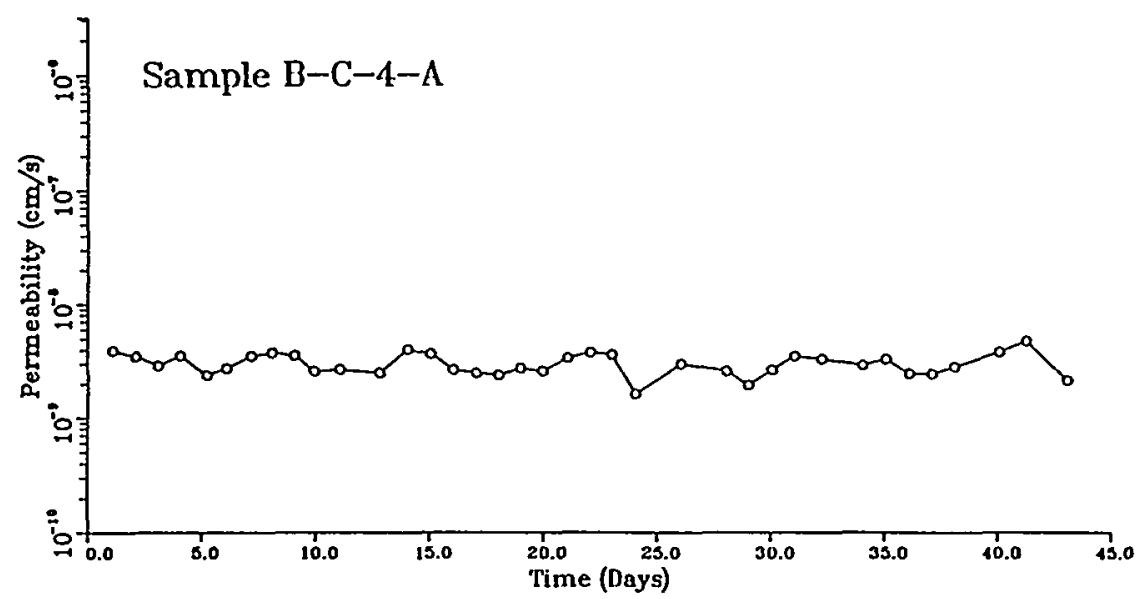

Figure 3.15 Permeability results for bentonite Sample B-C-4-A. 
specific water content and thereby a specific dry density, the calculated permeability should be treated as some equivalent measure (for the system of layers) of the sample's ability to transmit water for the range of water content or dry density of the sample. A similar non-uniform water content distribution may have developed for other bentonite samples installed in PVC permeameters, i.e. in permeameters in which vertical expansion is not restrained.

\subsubsection{Cracking Patterns of Bentonite Disks.}

Disks $1,2,3$, and 4 exhibit different cracking patterns upon drying (Figures 3.16 through 3.18 ). The water contents of the disks are inferred from the water contents measured for the adjacent disks (column 6 in Table 3.5). The disks, lying on plexiglass plates, are exposed to air in a room at $40 \%$ relative humidity. The desiccation cracks first develop along the periphery of the disks, approximately three hours after being exposed to air. Disks 1 and 2, whose water contents are presumably less than 200\%, appear to have more desiccation cracks than disks 3 and 4, with water contents greater than 2208 (Figure 3.17). For the latter two disks, the cracks, with much wider openings, penetrate deeper toward the centers. The volume reduction increases with water content (Figure 3.18).

\subsubsection{Results of High Injection Pressure Flow Tests}

This experimental series includes flow tests of Samples B-C-1-A-S, B-C-1 3/8-A-S, B-C-2 3/8-A-S, and $\mathrm{B}-\mathrm{C}-4-\mathrm{A}-\mathrm{S}$. The numeric in the sample number designation represents the nominal plug diameter in inches. The $S$ stands for stainless steel permeameter. Sample dimensions, initial water content, bulk density, and porosity are given in Table 3.6.

Before flow testing, the samples are subjected to an injection water pressure of $345 \mathrm{kPa}$ (50 psi) for about two months, and intermittently to vacuuming at the top, The double-pipette falling head method is then used to determine permeabilities. With approximately $1.2 \mathrm{~m}$ of water head difference across the samples, the flow testing has continued for more than "a month. No positive outflows have been discerned. The samples have again been subjected to vacuum and the test set up has been replaced with the constant-head method driven by a compressed helium pressurization system. The outflow is monitored by observing the movement of an air bubble in a horizontal pipette. The inflow is calculated from the drop of the water column in a $2.54 \mathrm{~cm}$ diameter PVC water reservoir. Initial permeability results (the upward permeation) obtained under an injection pressure of $34.5 \mathrm{kPa}$ ( 5 psi) are shown in Figure 3.19. No outflow has been detected for Sample B-C-1 3/8-A-S.

Figures 3.20 through 3.23 show the flow test results under injection pressures from 68.9 to $620.5 \mathrm{kPa}$ (10 to $90 \mathrm{psi}$ ). The hydraulic gradients range from 47 to 720 . The permeability values primarily fall between $2 \times 10^{-9}$ to $5 \times 10^{-11} \mathrm{~cm} / \mathrm{s}$, and appear to decrease with increasing hydraulic gradient. Table 3.7 gives the inflow-outflow balance for each sample. 
- (8utotis xә7je xnoy auo Ktə]ewțxoxdde)

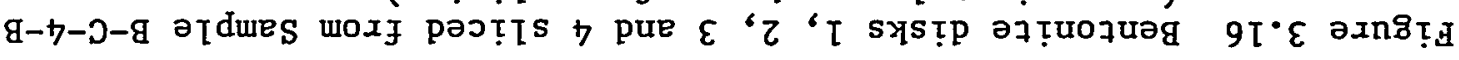

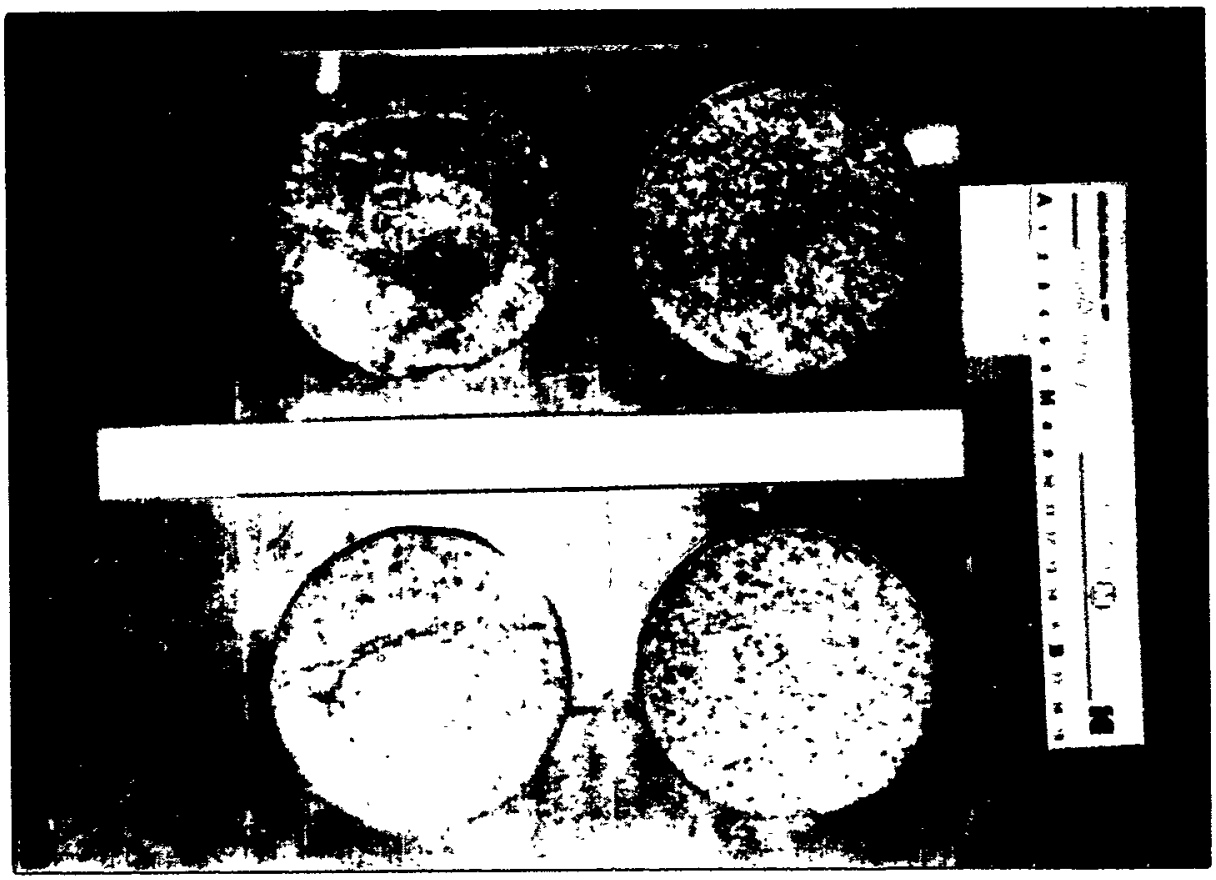




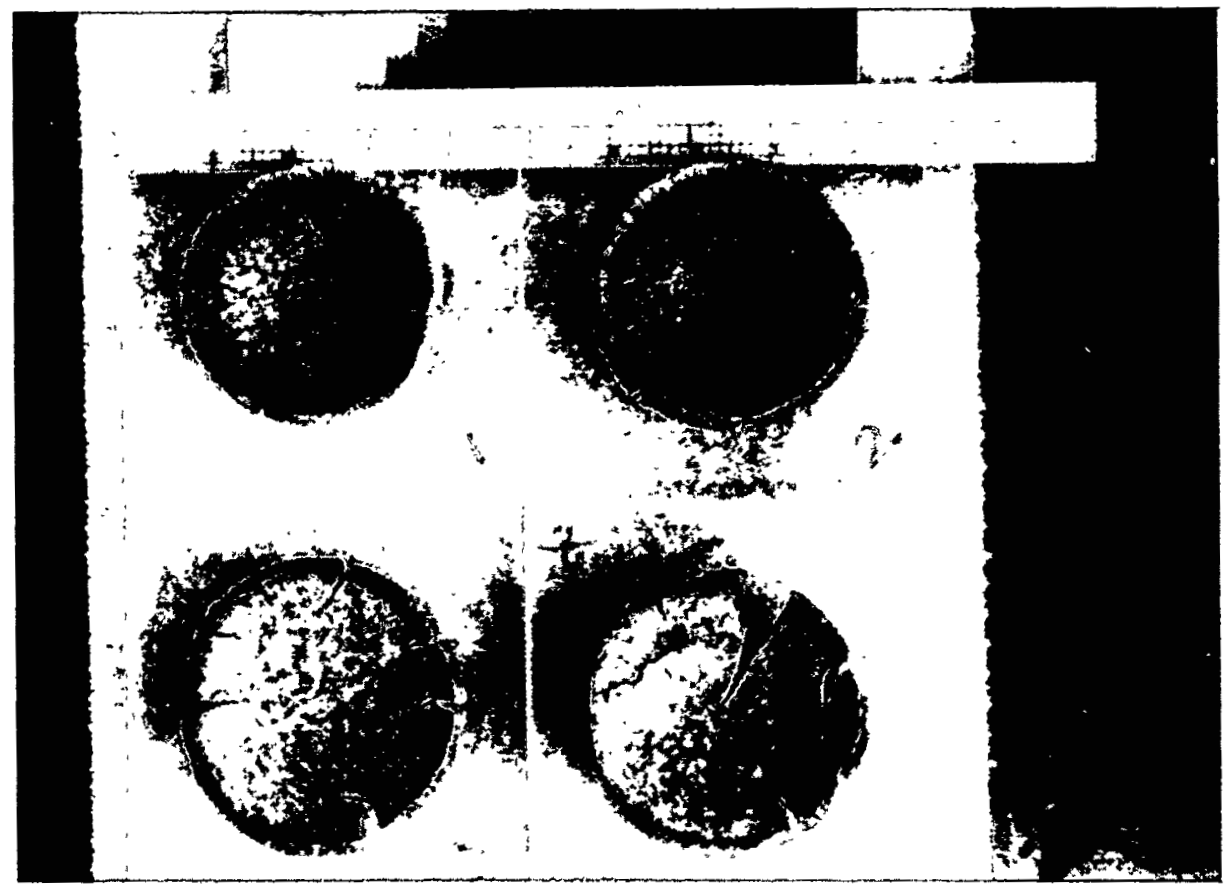

Figure 3.17 Bentonite disks 1, 2, 3 and 4 sliced from Sample B-C-4-B and exposed to relatively dry room environment (approximately 24 hours after slicing).

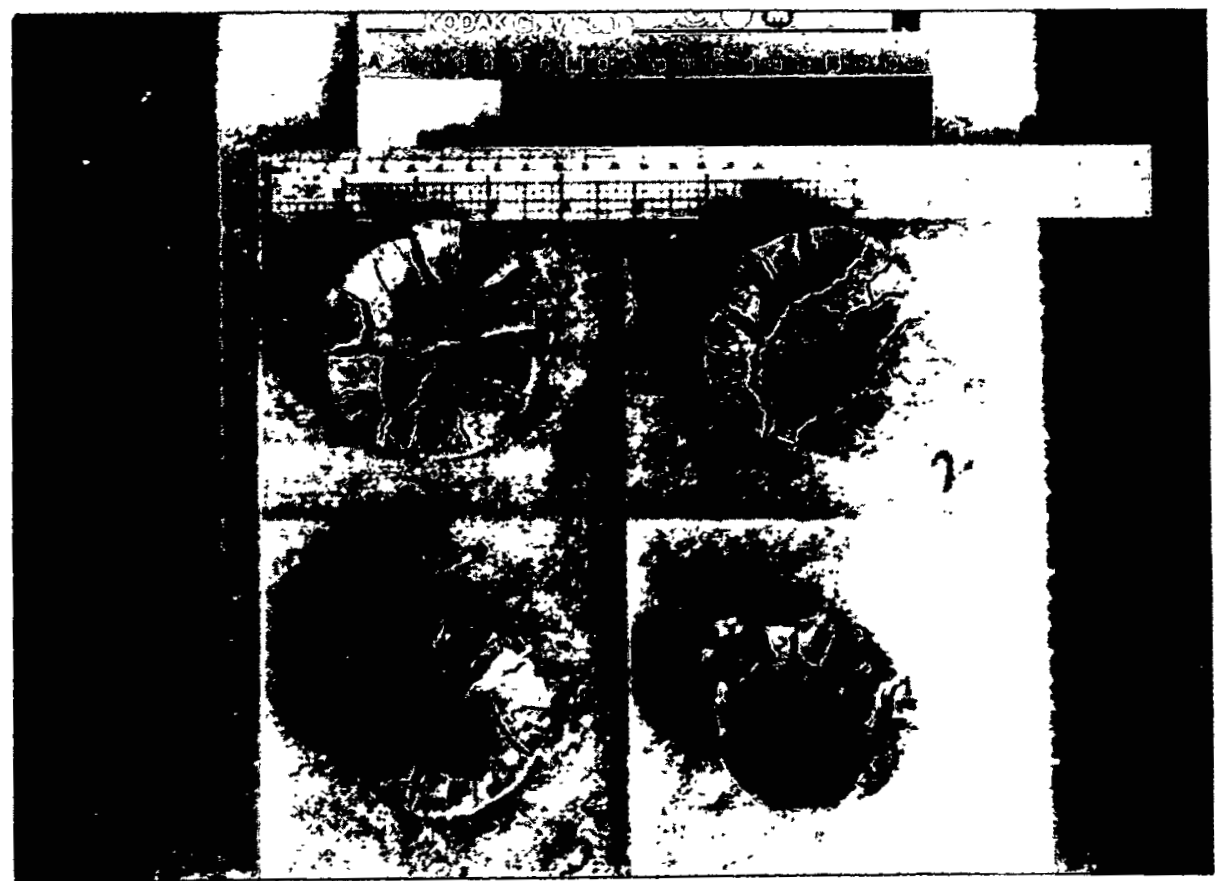

Figure 3.18 Bentonite disks 1, 2, 3 and 4 sliced from Sample B-C-4-B and exposed to relatively dry room environment (approximately 48 hours after slicing). 
Table 3.4 Thickness, Relative Position and Use of Disks Sliced from Sample B-C-4-B

\begin{tabular}{|c|c|c|c|c|}
\hline $\begin{array}{c}\text { Disk } \\
\text { Number }\end{array}$ & $\begin{array}{c}\text { Thickness } \\
(\mathrm{cm})\end{array}$ & $\begin{array}{c}\text { Depth of } \\
\text { Disk Center } \\
\text { (cm) }\end{array}$ & $\begin{array}{c}\text { Water Content } \\
\text { study }\end{array}$ & $\begin{array}{l}\text { Cracking } \\
\text { Pattern } \\
\text { Study }\end{array}$ \\
\hline$d$ & 1.2 & 0.6 & $x$ & \\
\hline 4 & 1.2 & 1.8 & & $x$ \\
\hline c & 1.2 & 3.0 & $\bar{X}$ & \\
\hline 3 & 1.2 & 4.2 & & $x$ \\
\hline b & 1.2 & 5.4 & $x$ & \\
\hline 2 & 1.2 & 6.6 & & $x$ \\
\hline a & 1.2 & 7.8 & $x$ & \\
\hline 1 & 2.2 & 9.5 & & $x$ \\
\hline
\end{tabular}

Table 3.5 Water Content Distribution (Sample B-C-4-B)

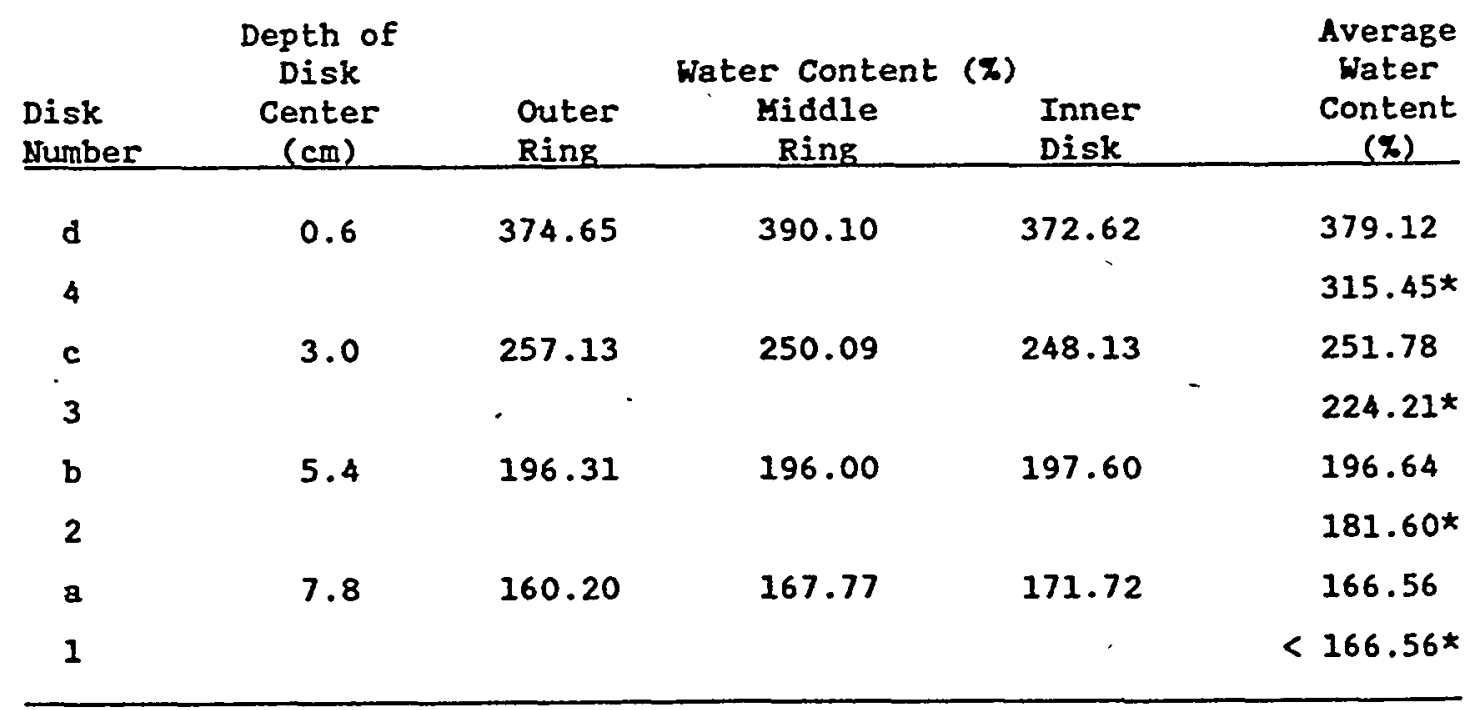

* Water content inferred from water contents of neighboring disks. 
Table 3.6 Sample Characteristics of Compacted Plugs Installed in Stainless Steel Permeameters

\begin{tabular}{|l|c|c|c|c|}
\hline Sample Number & B-C-1-A-S & B-C-1 3/8-A-S & B-C-2 3/8-A-S & B-C-4-S \\
\hline Sample Length (cm) & 8.89 & 9.623 & 14.95 & 13.125 \\
\hline Sample Diameter (cm) & 2.654 & 3.475 & 6.01 & 10.246 \\
\hline $\begin{array}{l}\text { Initial Water Content } \\
(8)\end{array}$ & 23.55 & 32 & 23.55 & 23.55 \\
\hline $\begin{array}{l}\text { Initial Bulk Density } \\
\left(\mathrm{g} / \mathrm{cm}^{3} \text { ) }\right.\end{array}$ & 1.431 & 1.482 & 1.384 & 1.358 \\
\hline $\begin{array}{l}\text { Saturated Water Con- } \\
\text { tent (8) }\end{array}$ & 57.13 & 88.32 & 60.26 & 62.05 \\
\hline $\begin{array}{l}\text { Saturated Bulk Density } \\
\text { (g/cm } 3 \text { ) }\end{array}$ & 1.720 & 1.536 & 1.603 & 1.683 \\
\hline Porosity & 0.625 & 0.721 & 0.638 & 0.644 \\
\hline
\end{tabular}




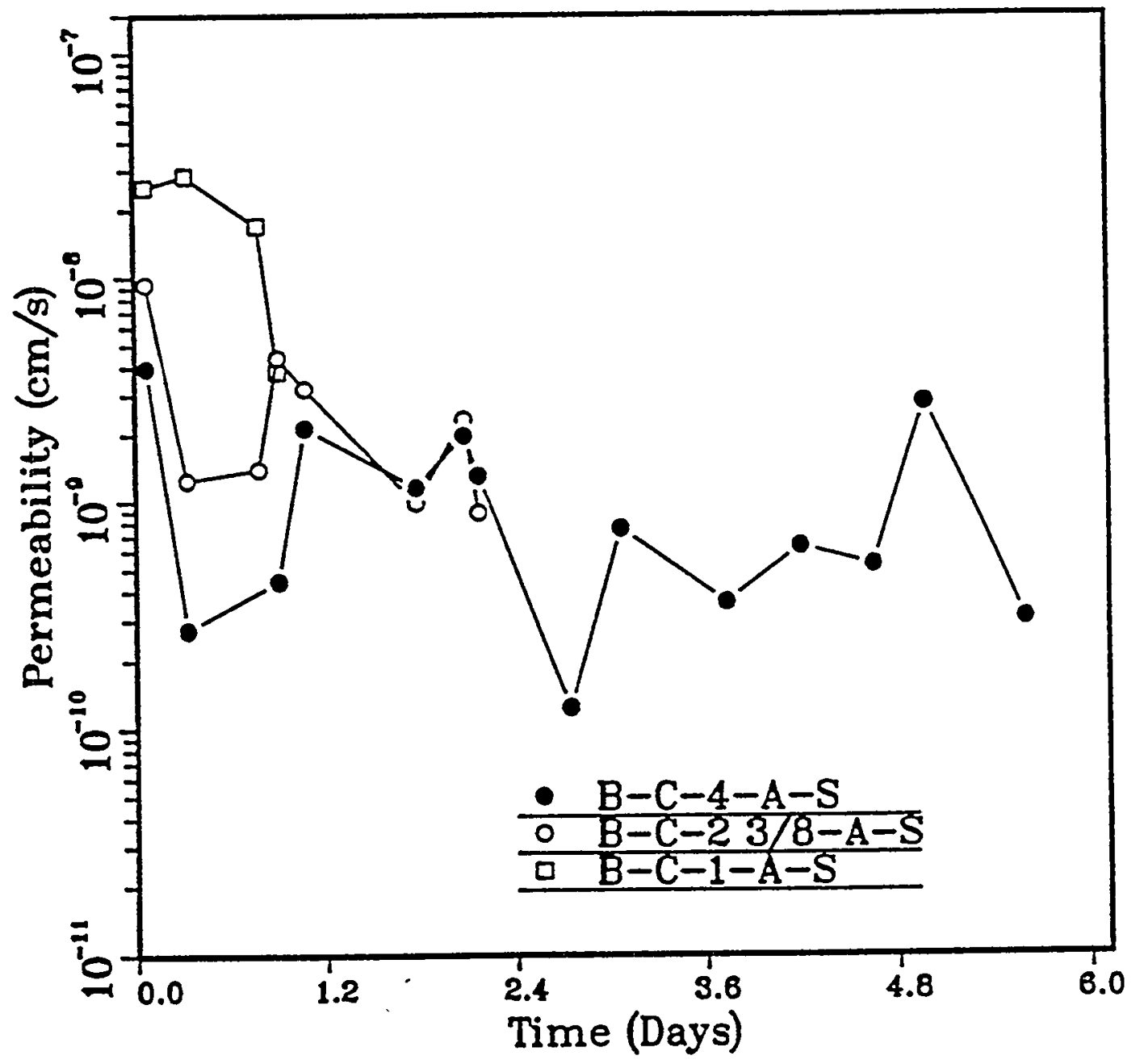

Figure 3.19 Preliminary permeability results of compacted bentonite samples installed in stainless steel permeameters. Hydraulic gradient: $43-45$ for B-C-1-A-S, 25.5-26.7 for B-C-2 3/8-A-S, and $29-31.3$ for $B-C-4-A-S$. 


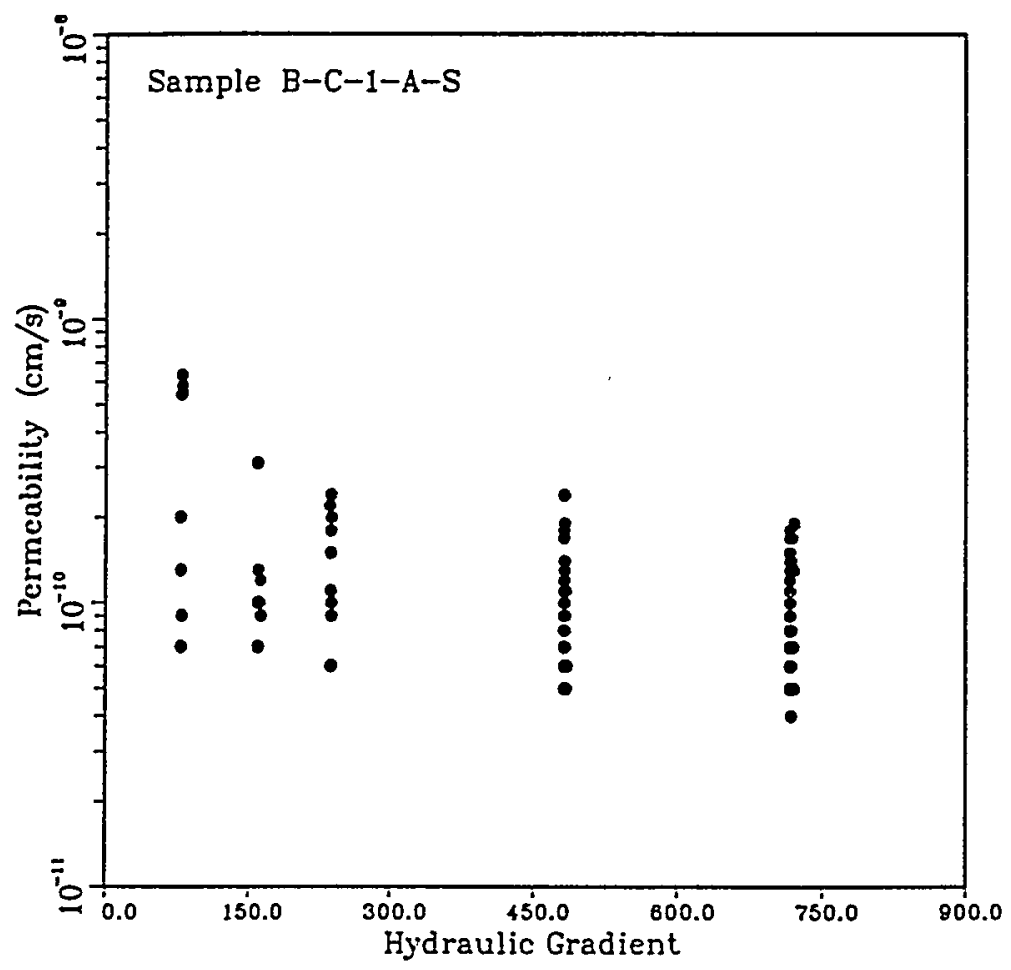

Figure 3.20 Permeability vs, hydraulic gradient for Sample B-C-1-A-S.

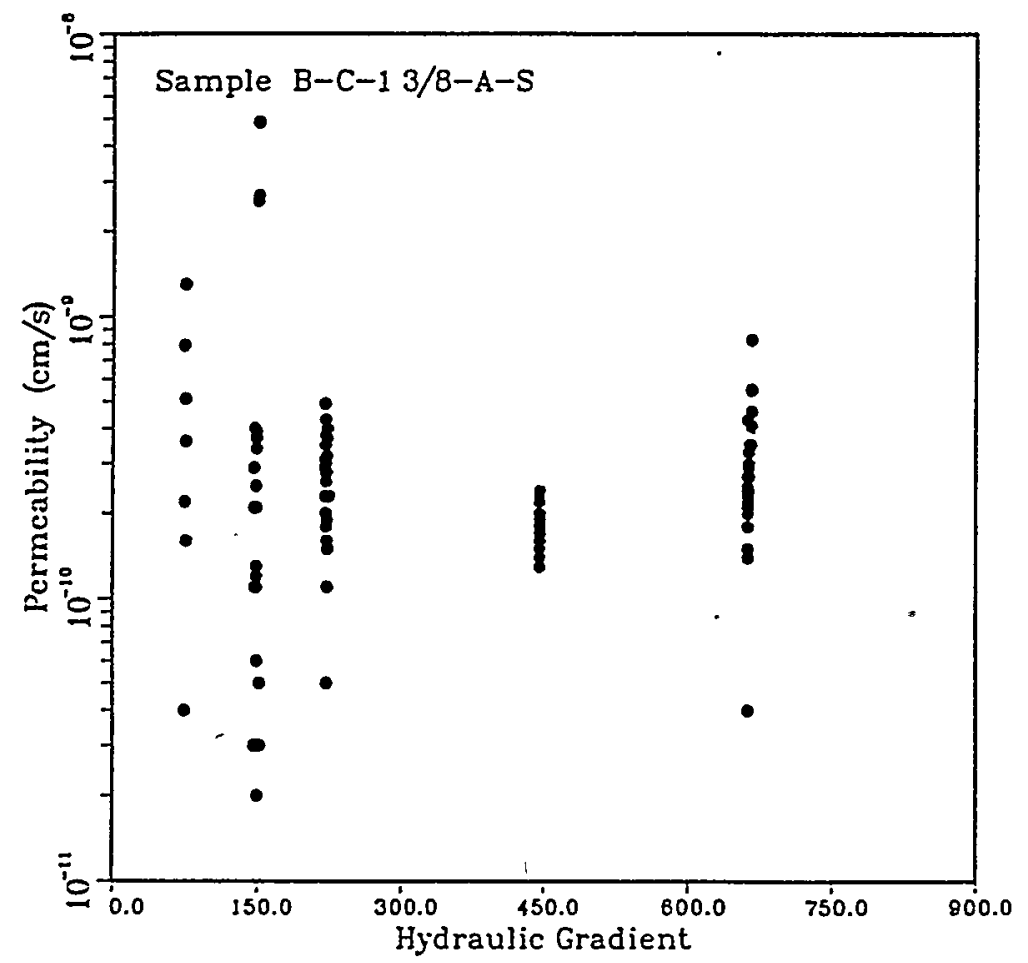

Figure 3.21 Permeability vs. hydraulic gradient for Sample B-C-1 3/8-A-S . 
The sample length, porosity, saturated water content, and bulk density of Sample B-C-4-A-S listed in Table 3.6 may differ somewhat from the actual values. A $10.16 \mathrm{~cm}$ diameter porous plate of sintered bronze is placed on top of this sample. The vertical movement of the sample is prevented by inserting a $3.8 \mathrm{~cm}$ diameter stainless steel rod between the porous plate and the top cap plate of the permeameter. The difference in the diameters of the porous plate $(10.16 \mathrm{~cm})$ and the permeameter chamber $(10.246 \mathrm{~cm})$ allows some bentonite to flow through the annulus into the upper portion of the chamber. Moreover, chemical reactions appear to occur (e.g. cation exchange) between bentonite and the bronze plate, which may be evidenced by the frequent emergence of air bubbles in the then discolored Tygon tubing which connects the exit port and the horizontal pipette. The chemical reactions and bentonite flow may explain why the permeability of this sample, unlike that of the other samples, does not decrease further when hydraulic gradients higher than 300 are imposed.

\subsubsection{Permeability of Bentonite to Air}

The seals to be placed in a nuclear waste repository are required to prevent significant amounts of water from reaching waste. They may be required additionally to prevent significant amounts of gaseous radionuclides from escaping through shafts, ramps, and boreholes (Gupta et al., 1989). Four bentonite samples of $25.4 \mathrm{~mm}$ ( $1 \mathrm{in}$ ) diameter installed in plexiglass permeameters have been tested to determine the permeability to air. The test procedure follows ASTM Standard D4525.

Nine tests have been performed on the four samples. Each sample contains a different initial water content. Various dry densities are achieved by changing the number of layers compacted. The rammer weight and drop height used for compaction are $0.053 \mathrm{~kg}(1.151 \mathrm{bs})$ and $0.27 \mathrm{~m}$ (10.62 in). The permeability results (expressed in $\mathrm{m}^{2}, 1 \mathrm{~m}^{2}=10^{12}$ Darcy) are plotted against the reciprocal of the mean pressure in Figure 3.24. The legend in this figure indicates, in order, the water content, the number of layers compacted, and the number of blows per layer. Figure 3.25 shows the air permeability as a function of dry density.

The permeability to air of the compacted samples ranges from $10^{-11}$ to $10^{-17} \mathrm{~m}^{2}$. It appears that the permeability to air first decreases and then starts to increase. The decrease may be explained by pore clogging due to particle migration. The increase in permeability that occurs later can be, at least in part, accounted for by pore enlarging resulting from loss of moisture. For the samples of high water contents (e.g. 28 and 41.38), the loss of moisture to the percolating air changes the color of the sample from dark grey to a distinctive light gray. To effectively minimize the migration of gaseous radionuclides, highly compacted bentonite plugs at low water content are suggested. 


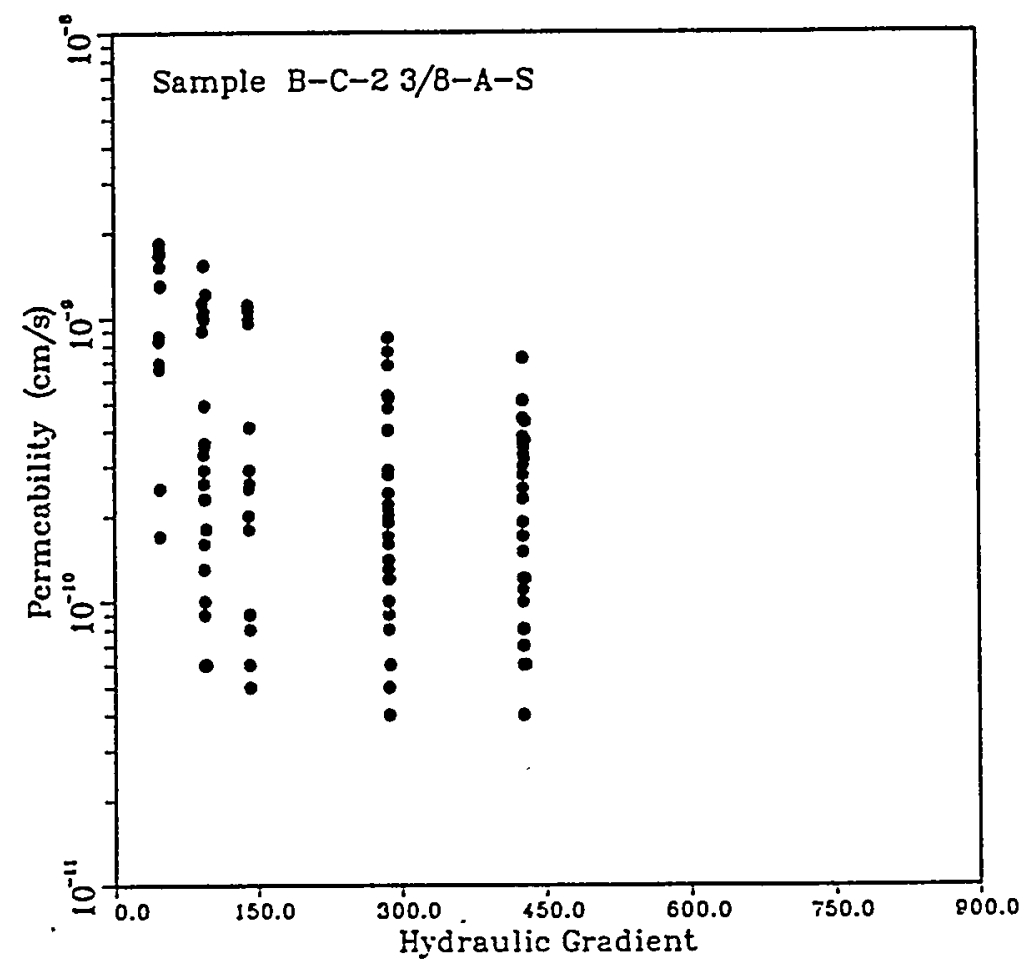

Figure 3.22 Permeability vs. hydraulic gradient for Sample B-C-2 3/8-A-S.

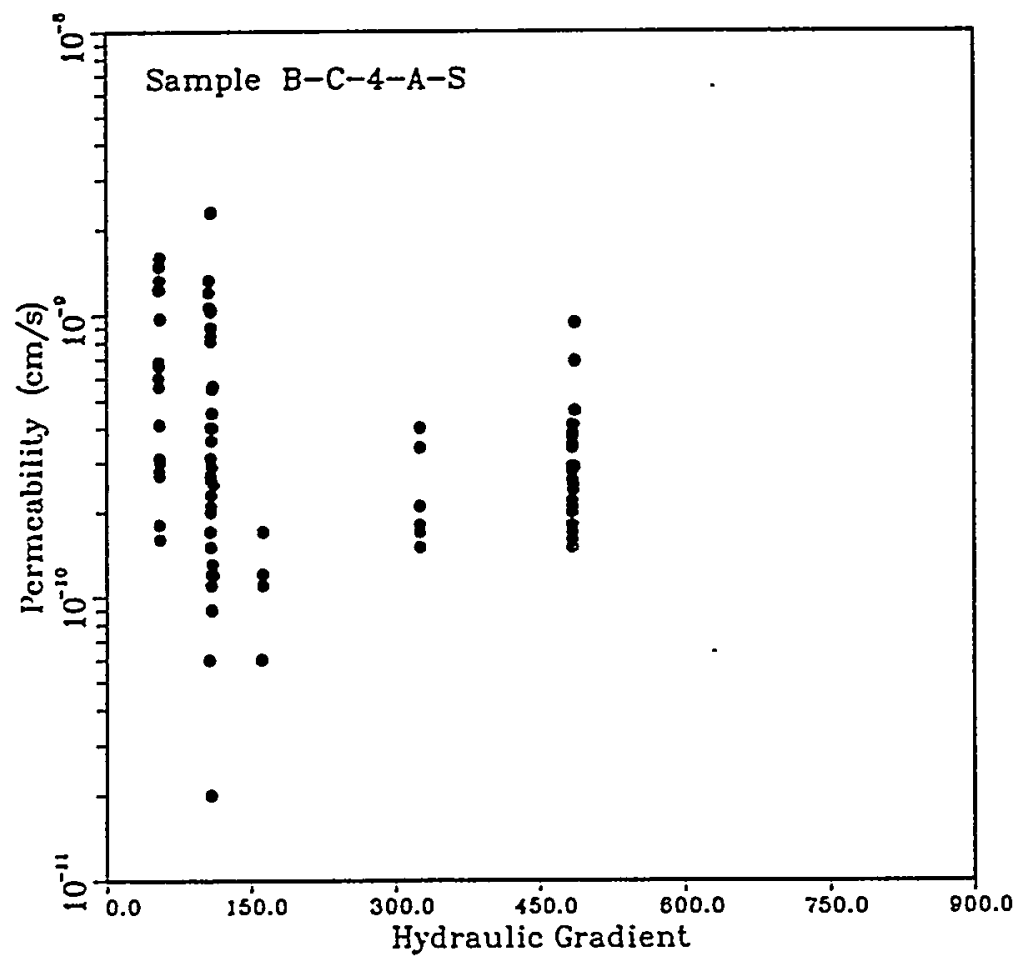

Figure 3.23 Permeability vs. hydraulic gradient for Sample B-C-4-A-S. 


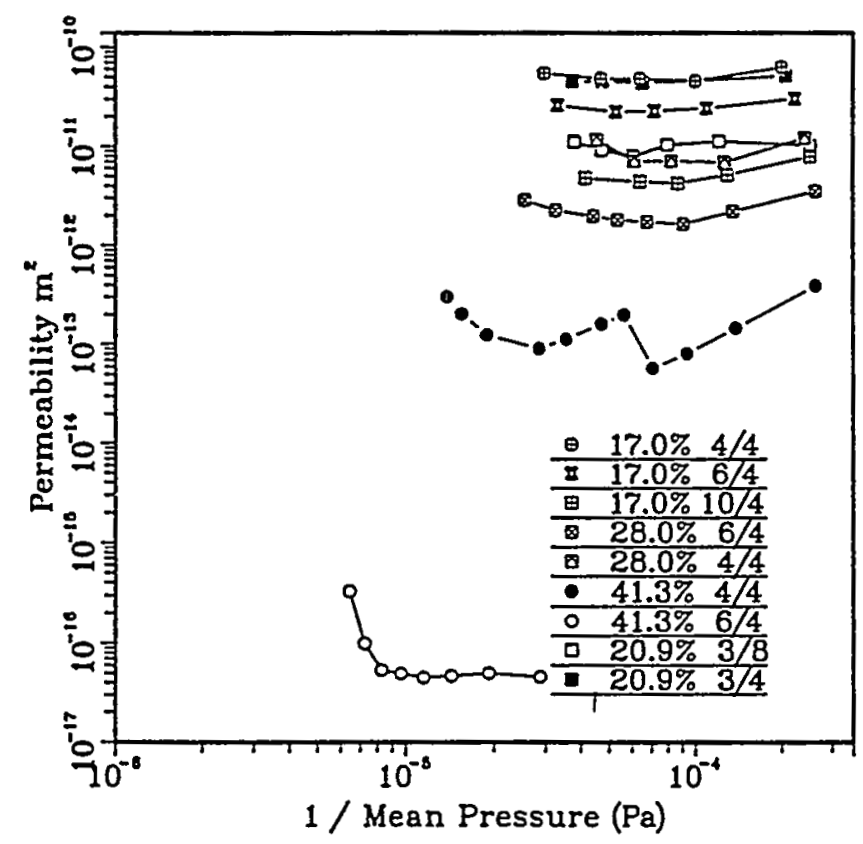

Figure 3.24 Permeability to air of compacted bentonite samples installed in plexiglass permeameters of $25.4 \mathrm{~mm}$ diameter.

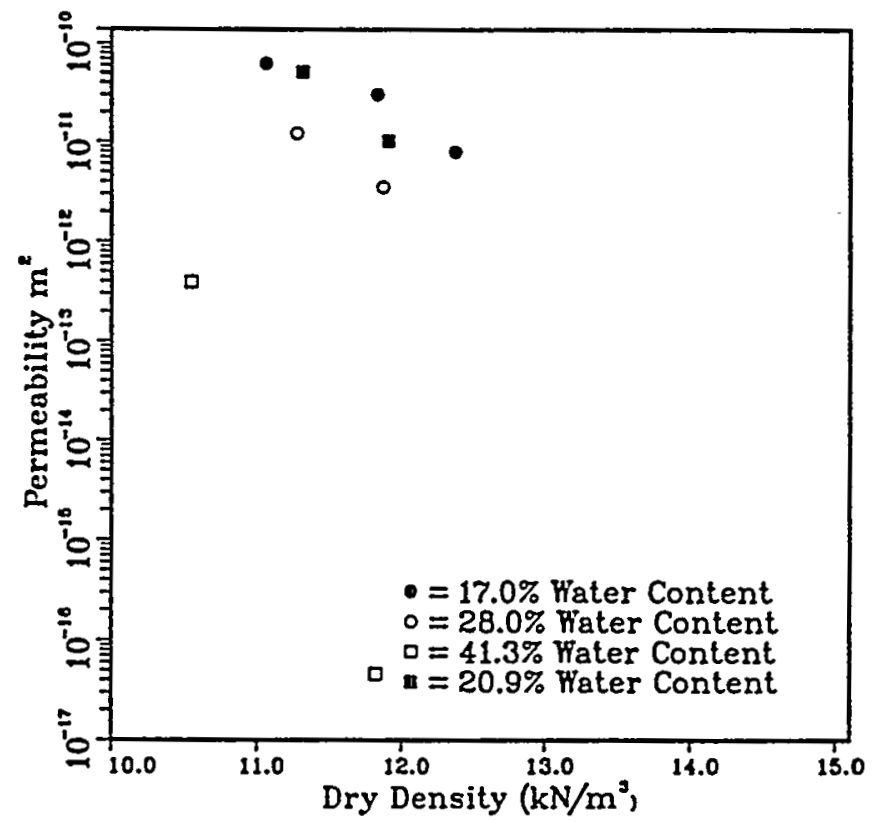

Figure 3.25 Permeability to air vs. dry density for the compacted bentonite samples installed in plexiglass permeameters of $25.4 \mathrm{~mm}$ diameter. 


\subsection{Permeability of Bentonite/Crushed Tuff}

\subsubsection{Longitudinal Flow Tests}

\subsubsection{Mixed Samples with 158 Bentonite by Weight}

Figures 3.26 through 3.28 show the permeability results for Samples $B / A L-C-4-15 / A, B / A L-C-4-15 / B$, and B/AL-C-4-15/C, respectively. These three samples contain $15 \%$ bentonite by weight with different crushed tuff gradations. The first five permeability values in Figure 3.26 have been measured using the double-pipette falling head method. Subsequently, the flow testing has been changed to the standard falling head system due to high flow rates. Permeabilities of Samples B/AL-C-4-15/B and $B / A L-C-4-15 / C$ have been determined by the double-pipette and standard faliing head methods for the former and by the standard falling head method for the latter. Erosion has been observed for Samples B/AL-C-4-15/A and B/AL-C-4-15/C, as evidenced by cloudy outflow. The resultant piping or channelling can be seen in Figure 3.29. Permeability as high as $8 \times 10^{-4} \mathrm{~cm} / \mathrm{s}$ has been registered for $\mathrm{B} / \mathrm{AL}-\mathrm{C}-4-15 / \mathrm{A}$, and 4 $\times 10^{-5} \mathrm{~cm} / \mathrm{s}$ for $B / A L-C-4-15 / C$. No erosion or piping effects have been detected for Sample B/AL-C-4-15/B. The last.ten flow measurements of B/AL-C-4-15/B, using the standard falling head method, yield permeabilities near $4 \times 10^{-8} \mathrm{~cm} / \mathrm{s}$, slightly lower than the values obtained from the double-pipette falling head method. The flow tests on these three samples have not been performed continuously.

\subsection{1:2 Samples with 25 or 358 Bentonite by Weight - First Test Sequence (Downward Permeation)}

The samples have been tested under injection pressures of $2,4,8,15$, 20,30 , and $40 \mathrm{psi}(13.8,27.6,55.2,103.4,138,206.9$ and $275.9 \mathrm{kPa}$, respectively). The injection pressures can be expressed in terms of water head as follows: $1.41,2.82,5.64,10.58,14.1,21.15$ and $28.2 \mathrm{~m}$ at a room temperature of $23.5^{\circ} \mathrm{C}$. Constant injection pressures are maintained by exerting a desired gas pressure, regulated from a compressed helium tank, on top of the water in the inflow reservoirs. The solubility of helium in water is $0.94 \mathrm{cc}$ per $100 \mathrm{cc}$ of water at $0^{\circ} \mathrm{C}$, and $1.05 \mathrm{cc}$ per $100 \mathrm{cc}$ of water at $50^{\circ} \mathrm{C}$ (CRC Handbook of Chemistry and Physics, 1982, p. B104).

Figure 3.30 shows the permeability of Sample B/AL-C-4-25/A. The results for $B / A L-C-4-25 / B$ and $B / A L-C-4-25 / C$ are given in Figures $D .1$ and $D .2$ (Appendix D). Permeability decreases as injection pressure increases. Under an injection pressure of 40 psi (275.9 kPa, $28.2 \mathrm{~m}$ water height), permeabilities of the three samples fall in the upper range of $10^{-9}$ $\mathrm{cm} / \mathrm{s}$. Although the permeabilities of the samples continue to decrease, the outflows appear slightly cloudy after the injection pressure is raised to $30 \mathrm{psi}(206.9 \mathrm{kPa})$.

Figures 3.31 and 3.32 show the flow test results for Sample B/AL-C-435/A. Similar plots for Samples B/AL-C-4-35/B, and B/AL-C-4-35/C can be found in Figures D.3 through D.6 (Appendix D). Significant variations can be seen in the permeabilities measured under injection pressures of 2,4 and 8 psi $(13.8,27.6$ and $55.2 \mathrm{kPa}$, respectively). The variations 


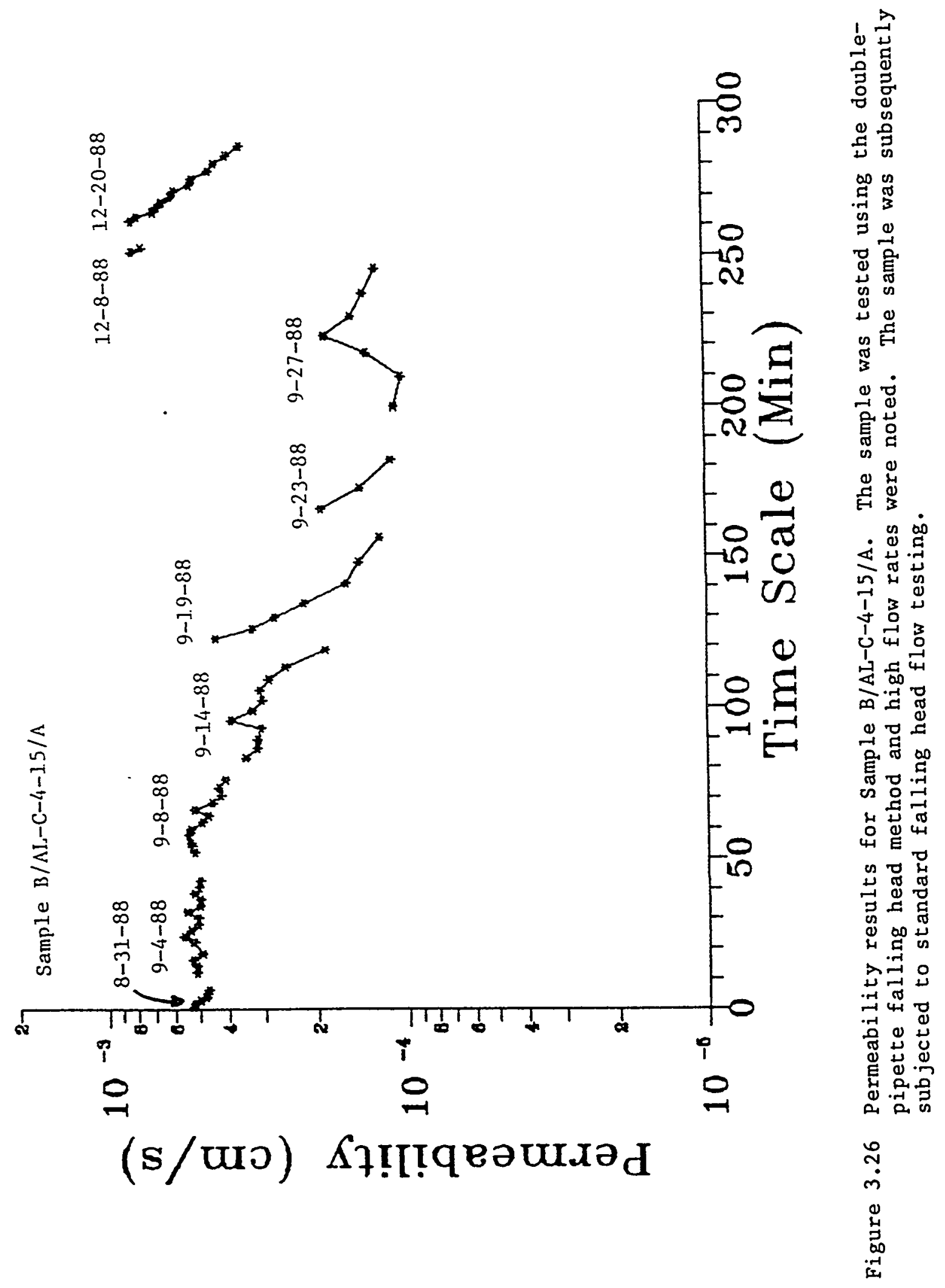




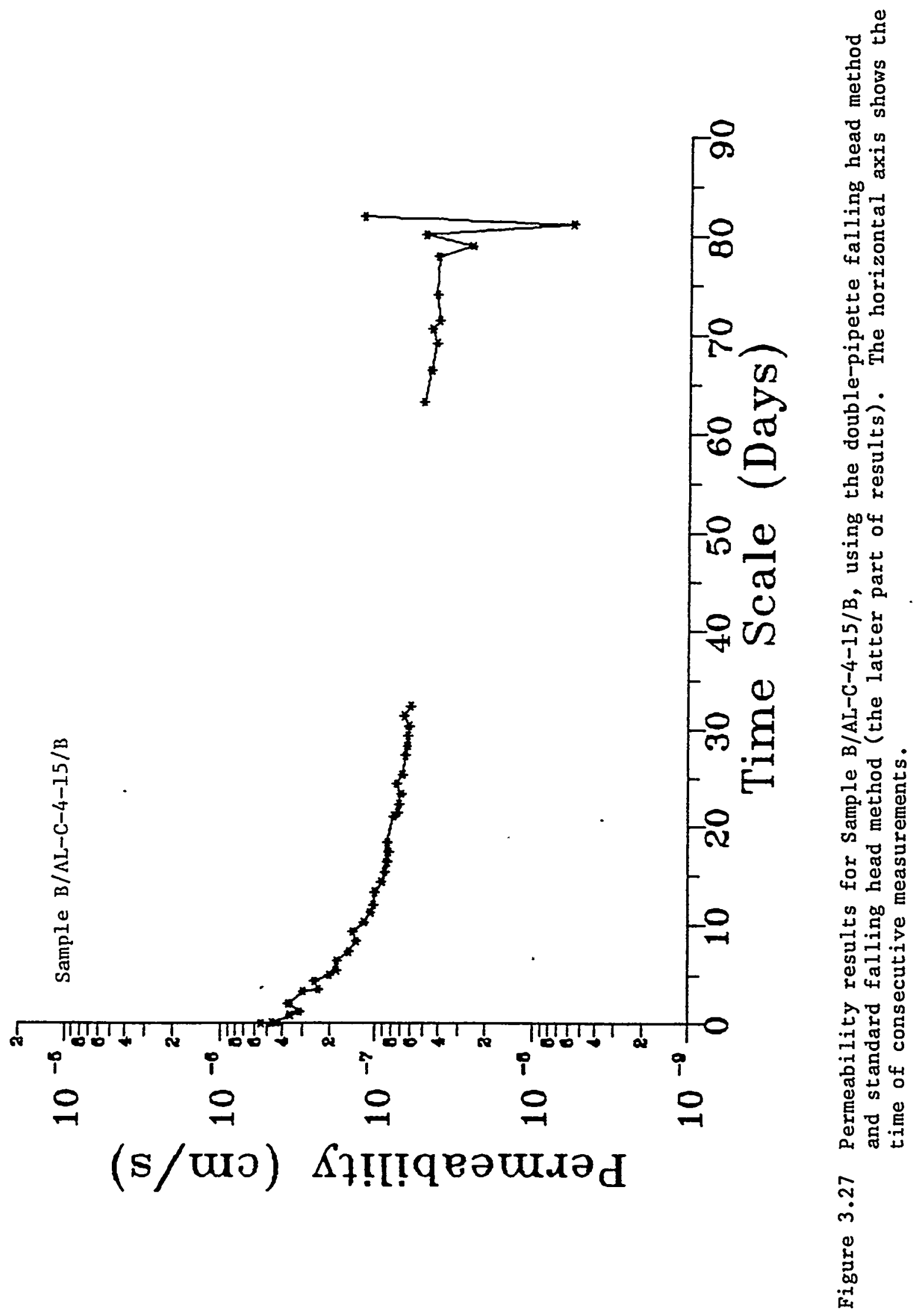




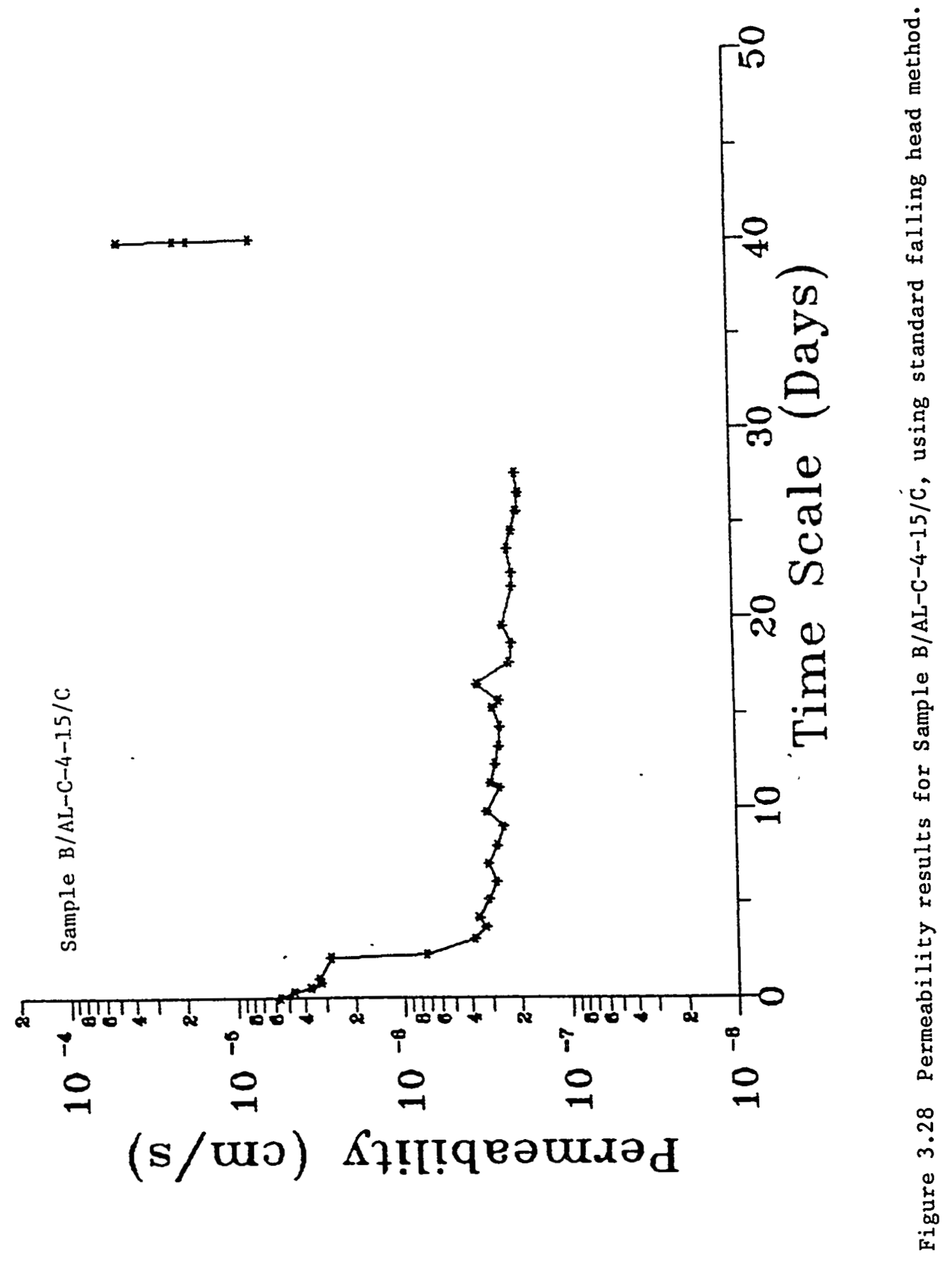




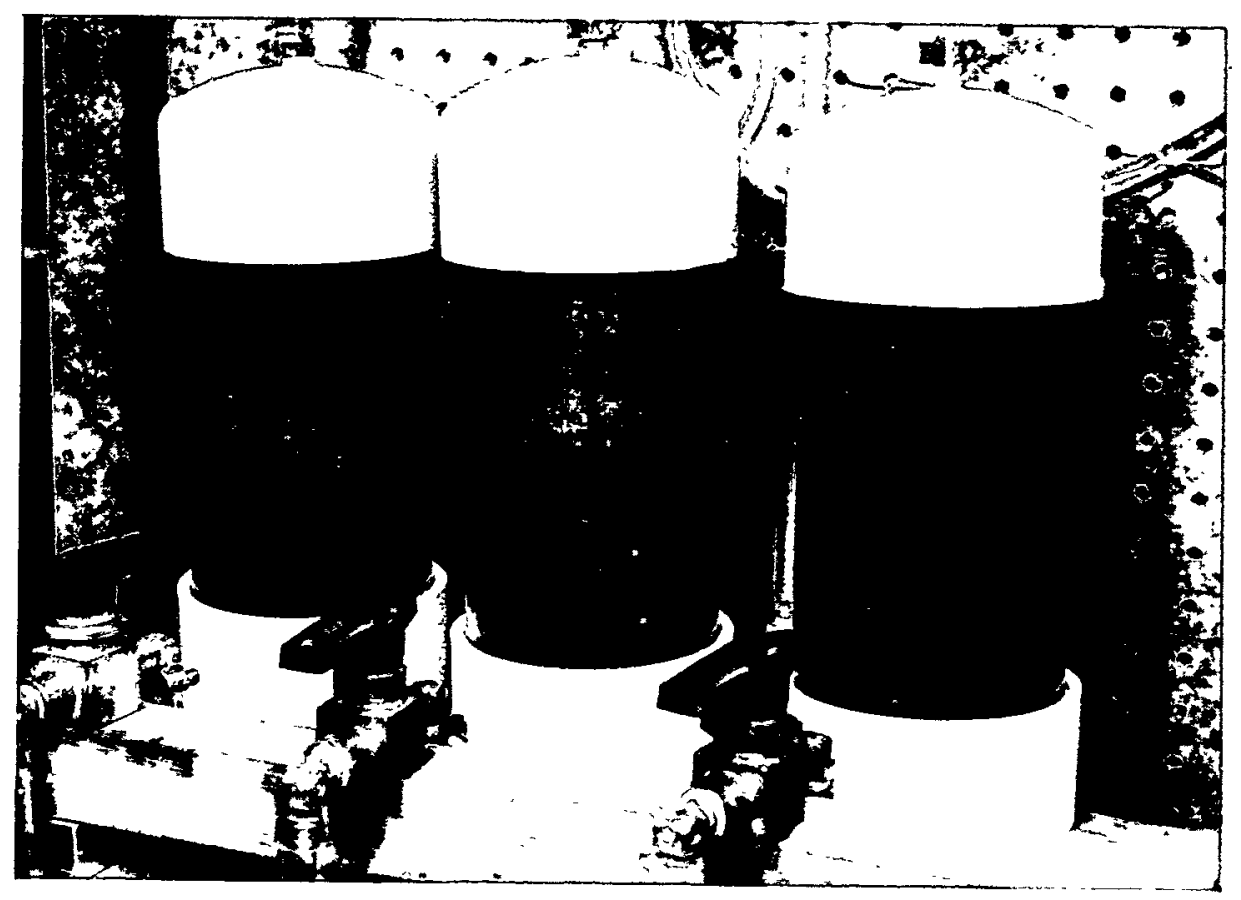

Figure 3.29 Erosion channels in $B / A L-C-4-15 / A$ (left) and $B / A L-C-4-15 / C$ (right). 


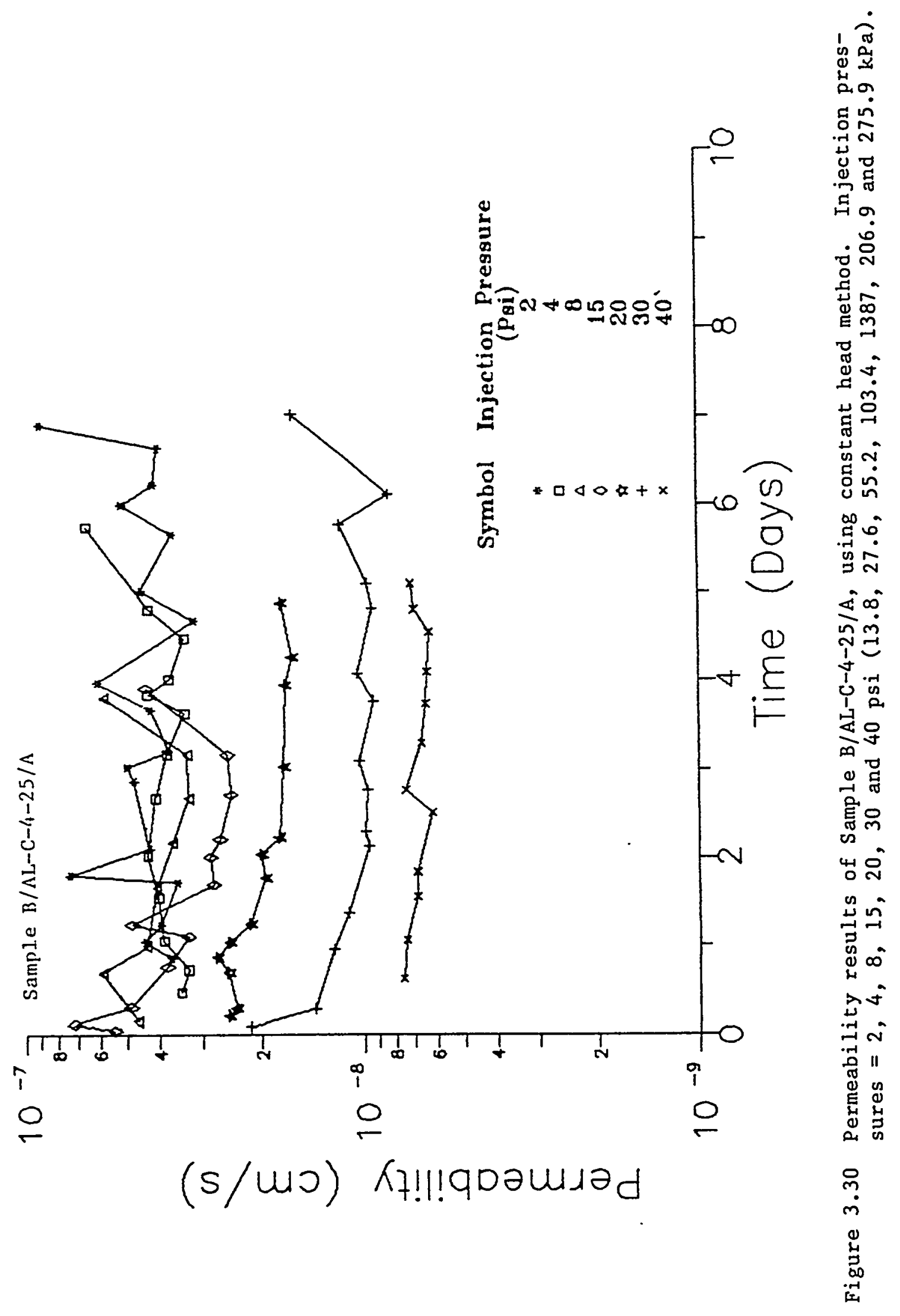




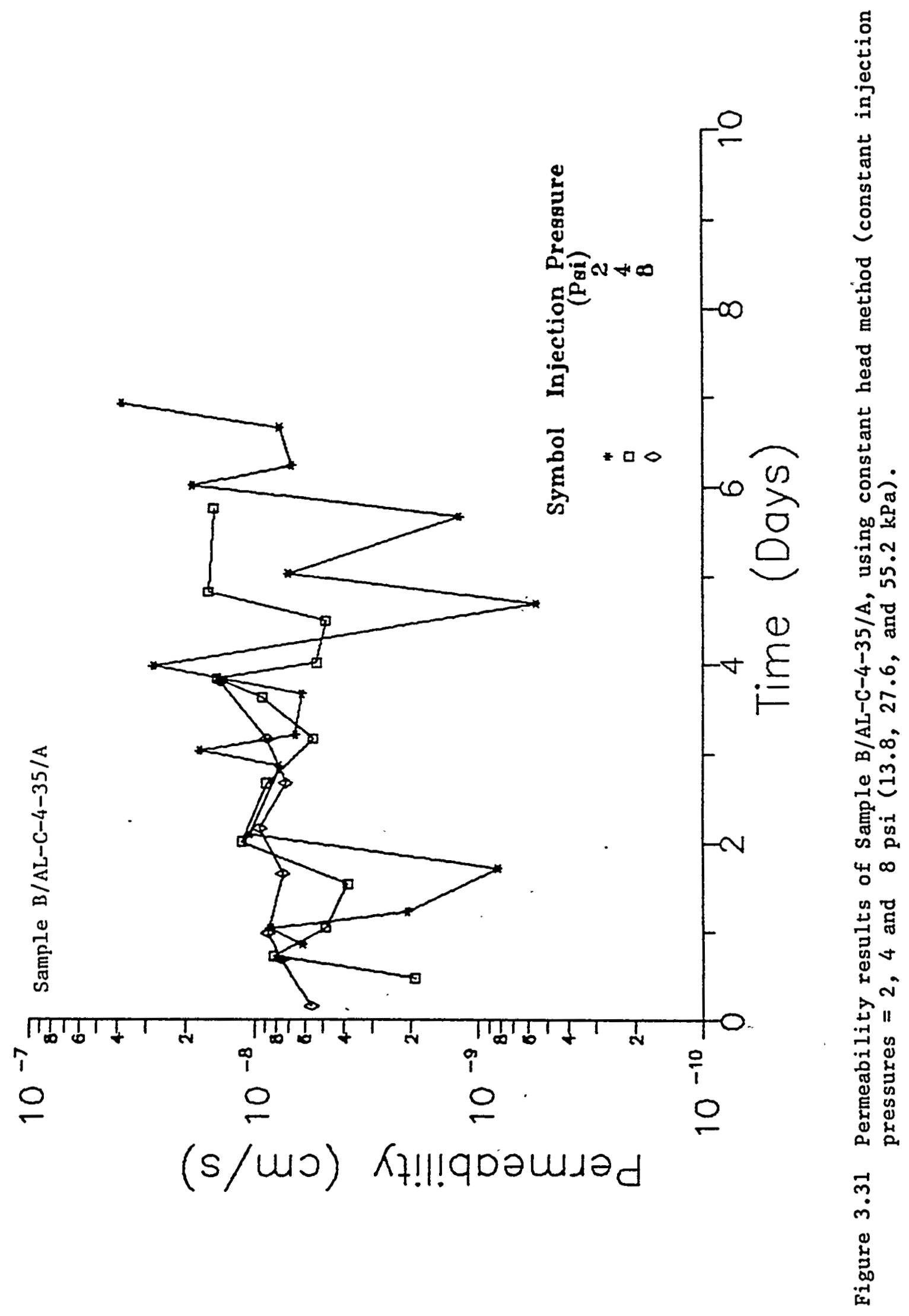




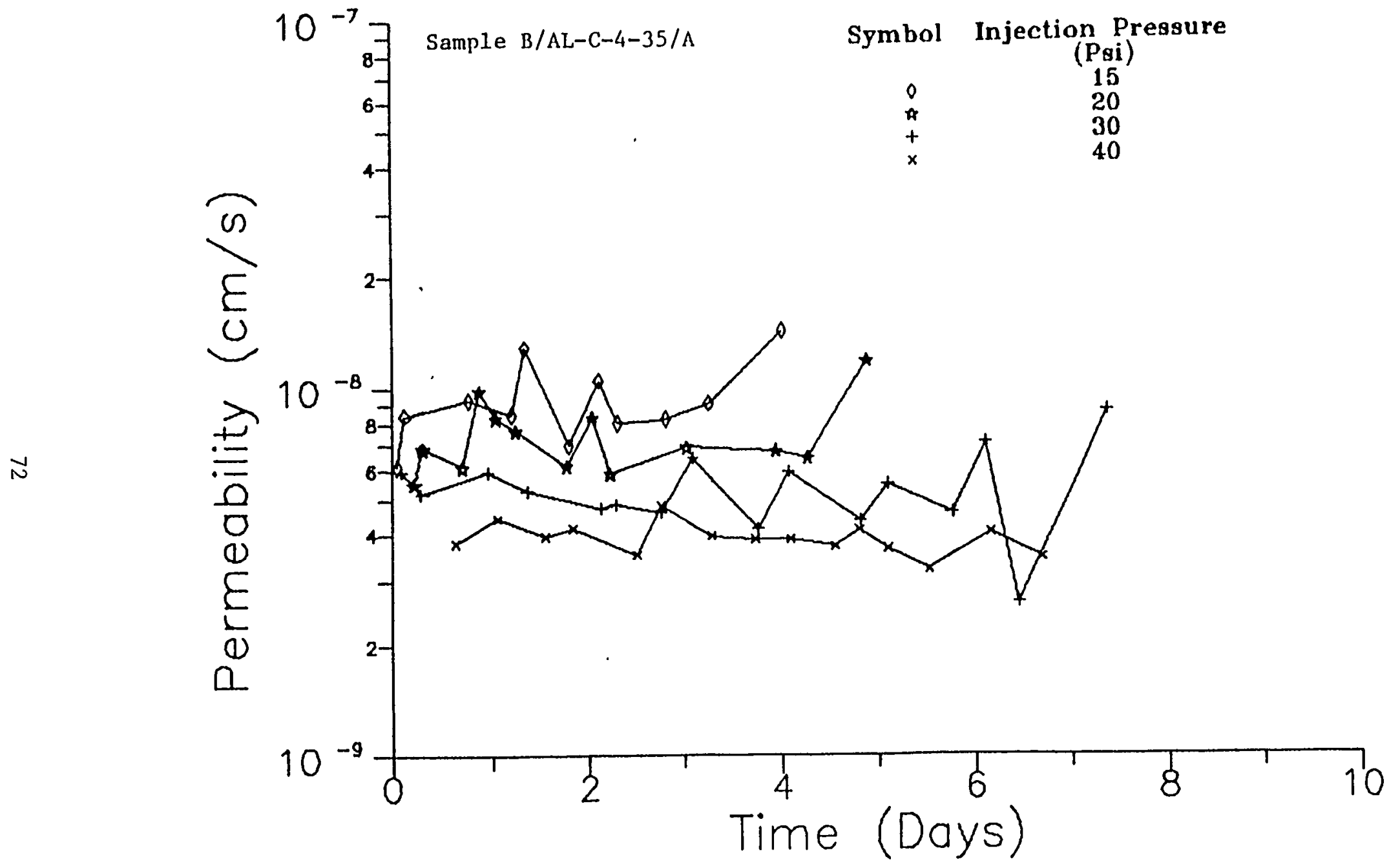

Figure 3.32 Permeability results of Sample B/AL-C-4-35/A, using constant head method (constant injection pressures $=15,20,30$ and 40 psi $(103.5,138,206.9$ and $275.9 \mathrm{kPa})$. 
are most conspicuous for permeability measurements obtained under 2 psi (14 $\mathrm{kPa}$ ) injection pressure and appear to decrease as injection pressure increases. The fluctuations in permeability may indicate that the samples are not saturated and that water adsorption and percolation occur simultaneously. Permeabilities.measured at higher injection pressures become more uniform (Figures 3.34 and 3.36). Under injection pressures of 30 and $40 \mathrm{psi}$. (206.9 and $275.9 \mathrm{kPa}$ ), the samples exhibit permeabilities in the upper and middie ranges of $10^{-9} \mathrm{~cm} / \mathrm{s}$.

Similar to the samples with $25 \%$ bentonite, cloudy outflows are observed first at an injection pressure of $30 \mathrm{psi}$. Visual examinations reveal no evidence of channelling in the samples.

\subsubsection{Samples with 25 or 358 Bentonite by Weight - Second Test Sequence (Downward Permeation)}

Reported in this section are the constant head flow test results of six previously-tested compacted bentonite/crushed tuff plugs subjected to injection pressures from 103.5 to $690 \mathrm{kPa}$ (15 to $100 \mathrm{psi}$ ). The permeability results (except for Sample B/AL-C-4-25/C) are illustrated in three separate plots according to the following injection pressure groups: (1) 138,345 and $552 \mathrm{kPa}(20,50$ and $80 \mathrm{psi})$; (2) 207, 414 and $621 \mathrm{kPa}(30,60$ and $90 \mathrm{psi})$; and (3) $103.5,276,483$ and $690 \mathrm{kPa}$ (15, 40,70 and 100 psi). The results for Samples B/AL-C-4-25/A, B/AL-C-4$25 / B$, and $B / A L-C-4-35 / A$ are shown in Figures 3.33 through 3.41 . The results for the other three samples are shown in Figures E.1 through E.9 (Appendix E).

The outflow agrees well with the inflow, as shown in Figures 3.42 through 3.44. Plots of the inflow-outflow balance for the other three samples are included in Figures E.10 through E.12 (Appendix E). The inflow is calculated from the change of the water level in a translucent inflow reservoir (a PVC pipe of $2.54 \mathrm{~cm}$ diameter). With a few exceptions, the difference between the total inflow and outflow at any injection pressure is less than 108 of the outflow. Under low injection pressures, the outflow appears to be less than the inflow. The trend is reversed as the injection pressure increases. This behavior is common to all six samples. Similar inflow-outflow behavior is observed in flow testing cement plugs emplaced in tuff and in granite cylinders (South and Daemen, 1986, Tables 4:12, 4.13).

The permeabilities measured for the samples with $25 \%$ bentonite lie primarily in the upper to middle range of $10^{-9} \mathrm{~cm} / \mathrm{s}$. Those for the samples with $35 \%$ bentonite fall in the middle to lower range of the same order of magnitude. The results for the six samples reported on earlier (Section 3.4.1.2) include measurements under injection pressures from 13.8 to $275.9 \mathrm{kPa}$ ( 2 to $40 \mathrm{psi}$ ). Tests at the injection pressures of $103.5,138,206.9$ and $275.9 \mathrm{kPa}(15,20,30$ and $40 \mathrm{psi})$ have been repeated during this extended test series. Given the same sample, injection pressure, and testing temperature (room temperature), the permeabilities measured in the second series are noticeably lower than those obtained earlier. The discrepancy suggests that the permeation of water may have caused structural changes in the samples. Moreover, the permeabilities tend to decrease with increasing injection pressure when 


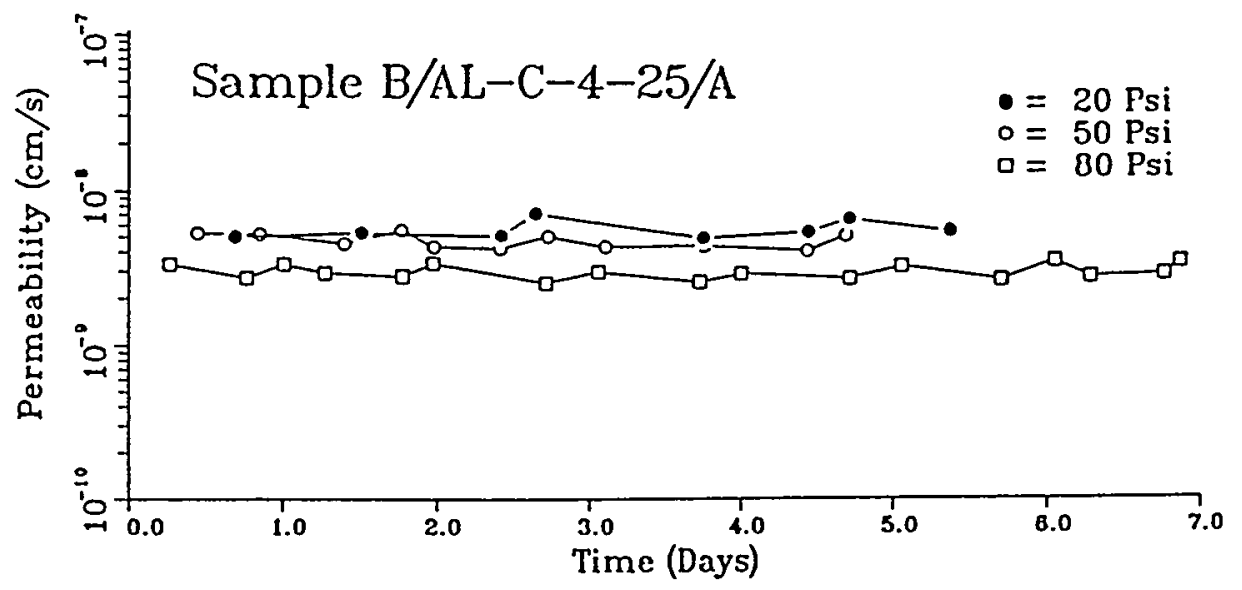

Figure 3.33 Permeability results of Sample B/AL-C-4-25/A at injection pressures $=138,345$ and $552 \mathrm{kPa}(20,50$ and $80 \mathrm{psi})$.

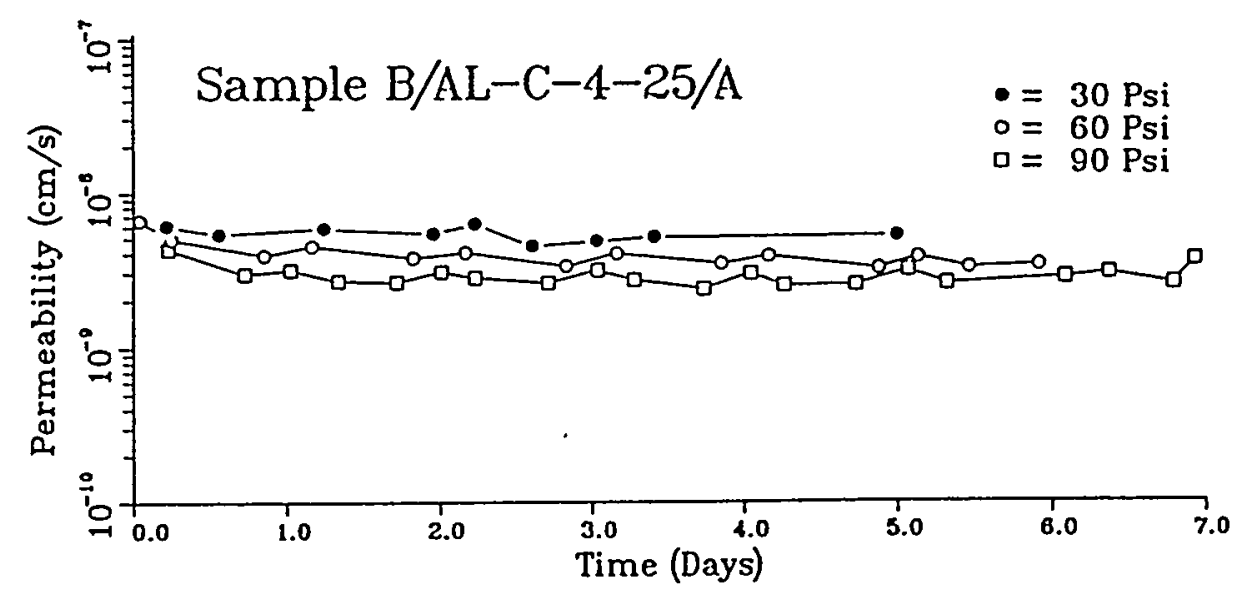

Figure 3.34 Permeability results of Sample B/AL-C-4-25/A at injection pressures $=207,414$ and $621 \mathrm{kPa}(30,60$ and $90 \mathrm{psi})$.

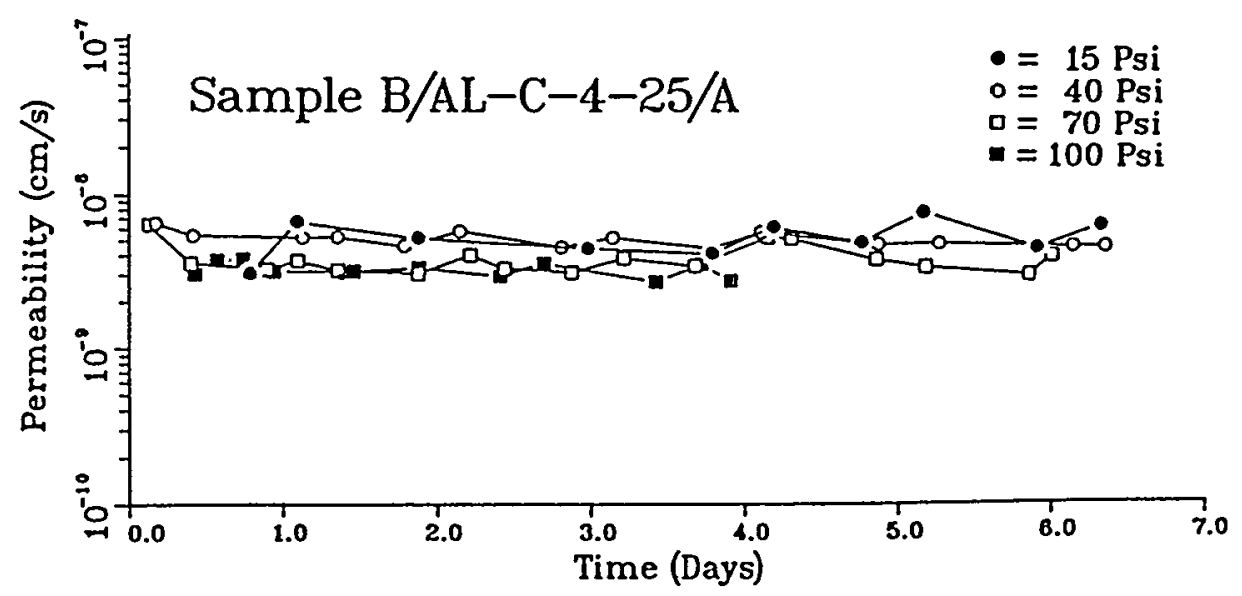

Figure 3.35 Permeability results of Sample B/AL-C-4-25/A at injection pressures $=104,276,483$ and $690 \mathrm{kPa}(15,40,70$ and 100 psi). 


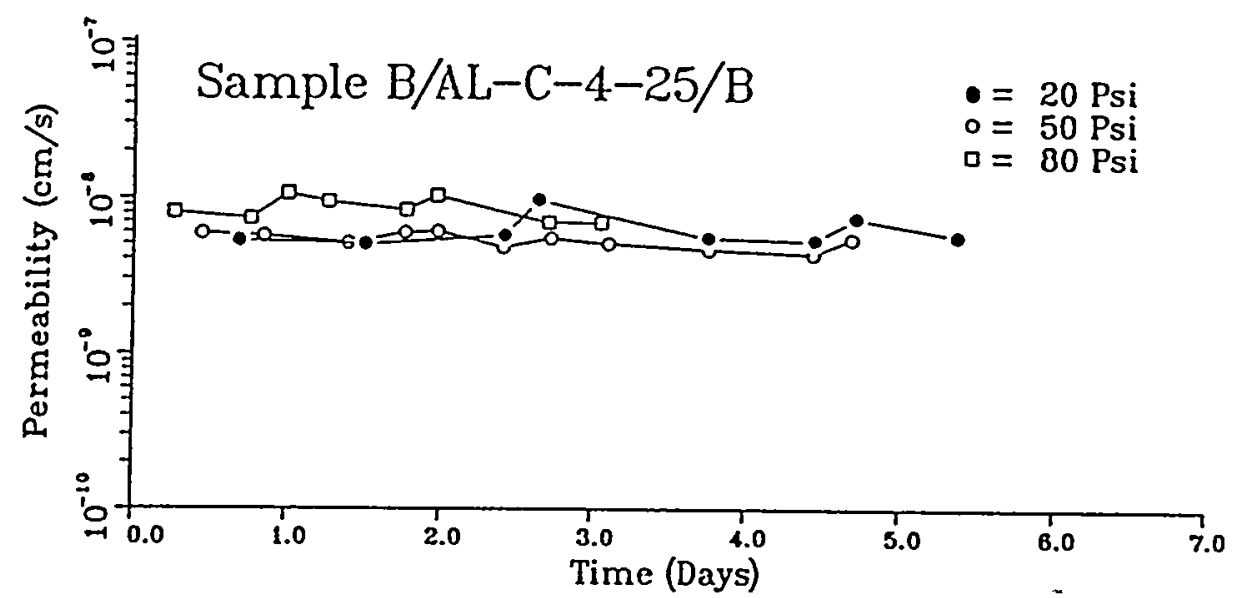

Figure 3.36 Permeability results of Sample B/AL-C-4-25/B at injection pressures $=138,345$ and $552 \mathrm{kPa}(20,50$ and $80 \mathrm{psi})$.

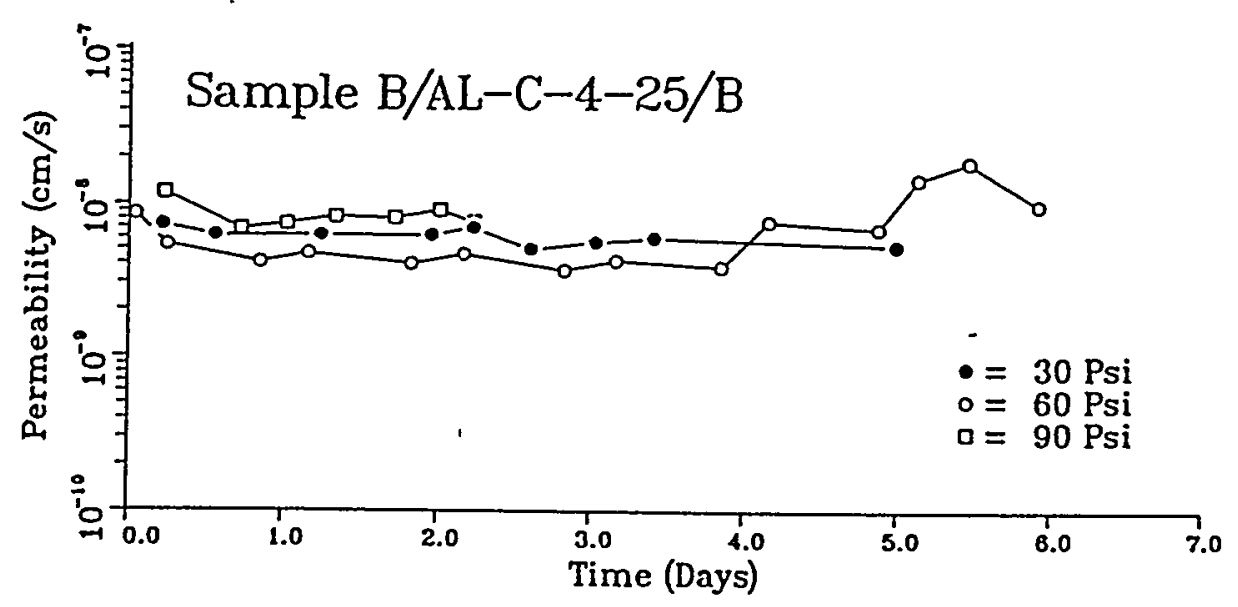

Figure 3.37 Permeability results of Sample B/AL-C-4-25/B at injection pressures $=207,414$ and $621 \mathrm{kPa}(30,60$ and $90 \mathrm{psi})$.

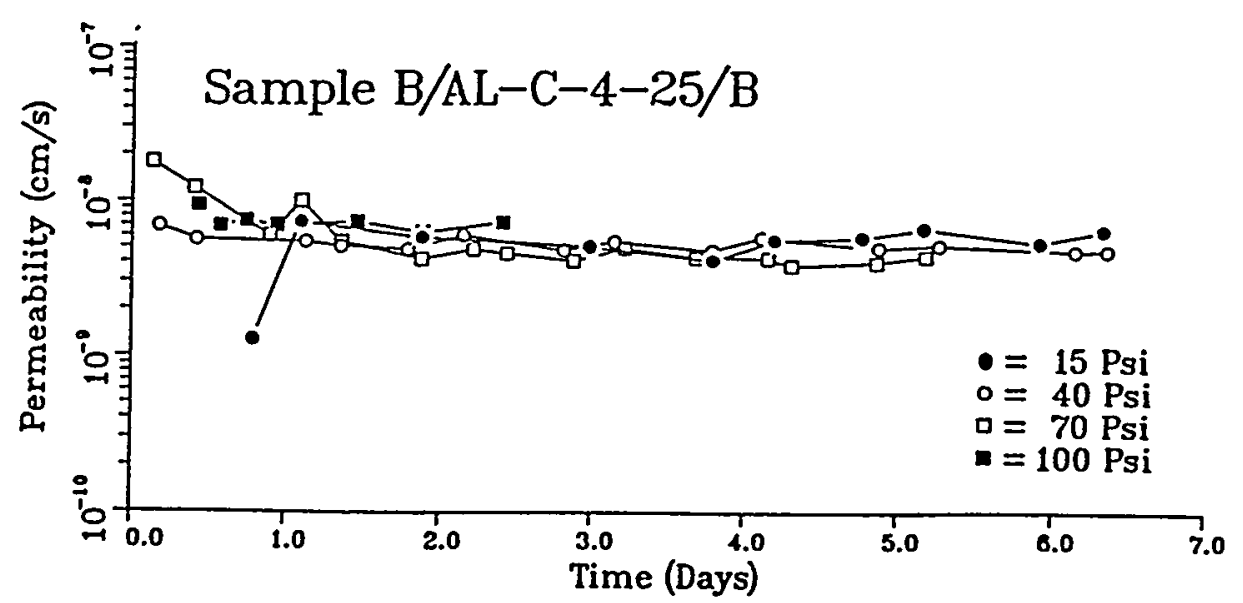

Figure 3.38 Permeability results of Sample B/AL-C-4-25/B at injection pressures $=104,276,483$ and $690 \mathrm{kPa}(15,40,70$ and 100 psi). 


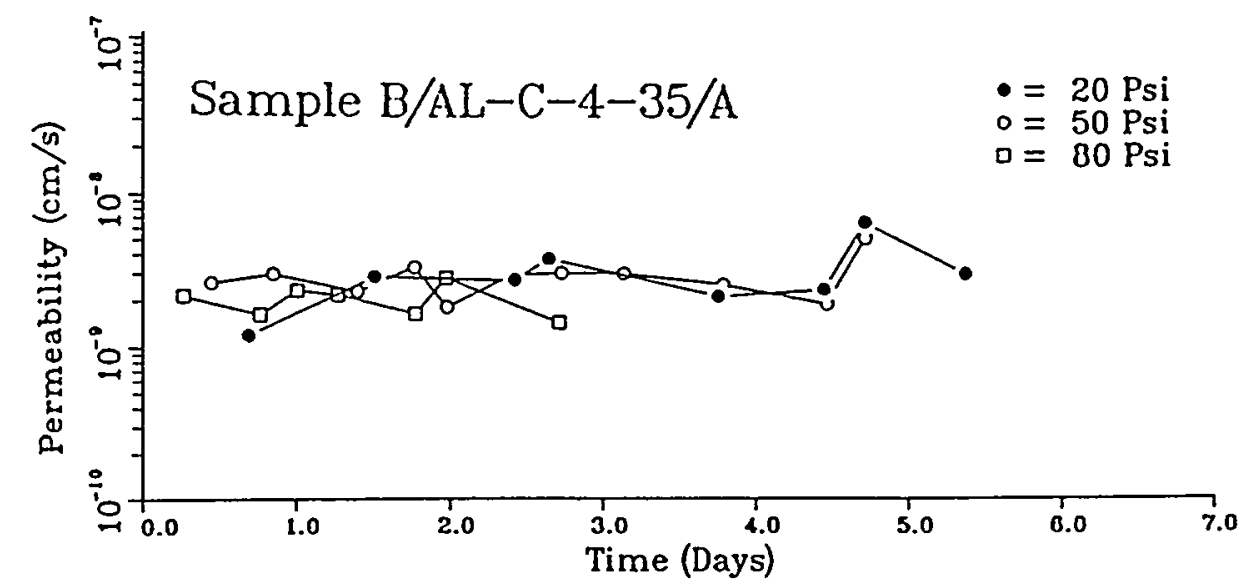

Figure 3.39 Permeability results of Sample B/AL-C-4-35/A at injection pressures $=138,345$ and $552 \mathrm{kPa}(20,50$ and $80 \mathrm{psi})$.

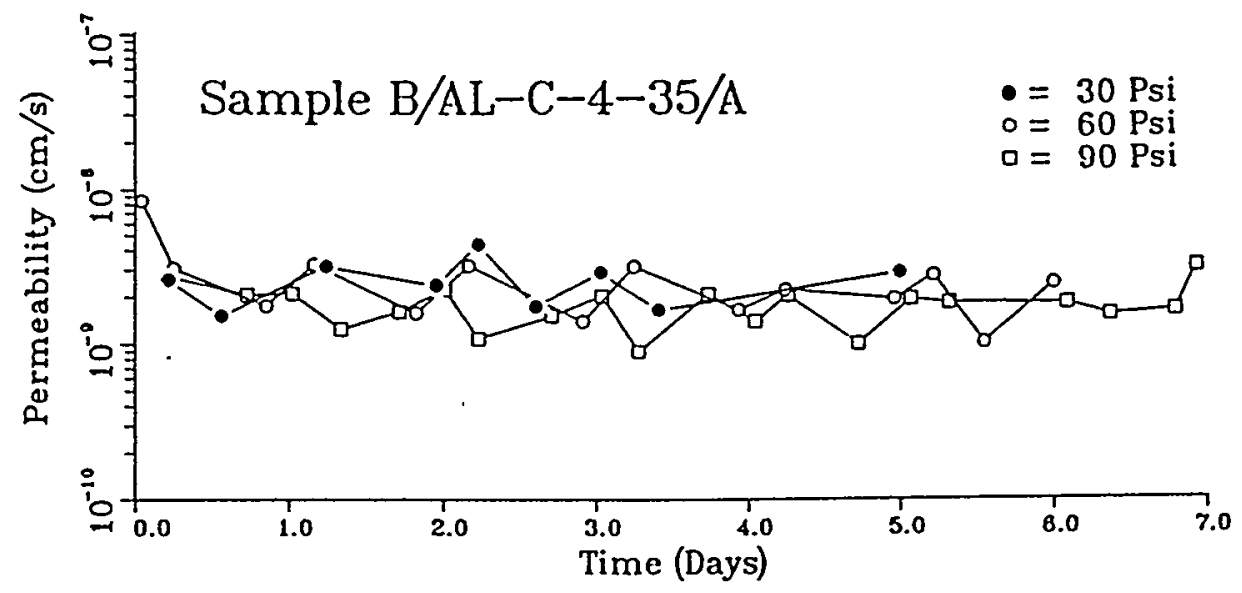

Figure 3.40 Permeability results of Sample B/AL-C-4-35/A at injection pressures $=207,414$ and $621 \mathrm{kPa}(30,60$ and $90 \mathrm{psi})$.

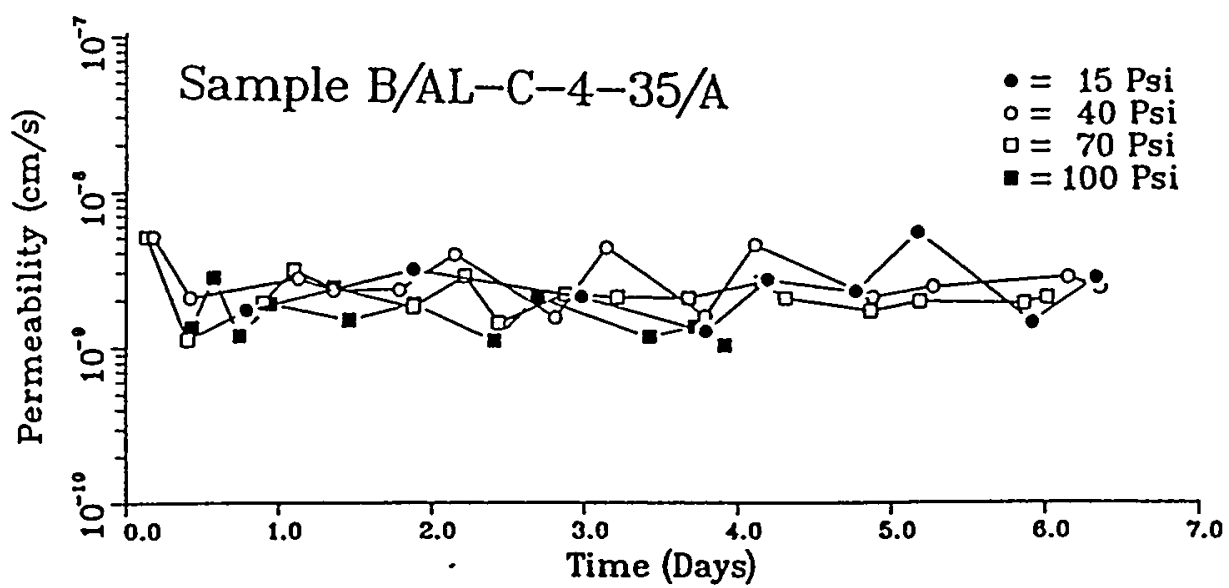

Figure 3.41 Permeability results of Sample B/AL-C-4-35/A at injection pressures $=104,276,483$ and $690 \mathrm{kPa}(15,40,70$ and 100 psi). 
the pressure is raised to $345 \mathrm{kPa}$ ( $50 \mathrm{psi}$ ) and higher, except for Sample B/AL-C-4-25/B as shown in Figures 3.36 to 3.38 . The decrease in permeability is likely due to cloging as a result of particle migration (Mitchell and Younger, 1967). The tendency can also be.seen in the plots of the outflow rate vs. hydraulic gradient (Figures 3.45 through 3.50). For hydraulic gradients up to 150 to 200 for the samples containing 258 bentonite, and up to 300 for the samples containing 358 bentonite, the flow rate increases linearly with hydraulic gradient, an expected relation predicted by Darcy's law. The linear relation is lost, however, when higher hydraulic gradients are imposed. Higher gradients also appear to be accompanied by wider fluctuations of the results. The steep increase in flow rate of Sample B/AL-C-4-25/B (Figure 3.46$)^{\circ}$ at hydraulic gradients of 400 and higher [i.e., injection pressure of $414 \mathrm{kPa}$ ( $60 \mathrm{psi}$ ) and higher] is due to piping.

After having been subjected to injection pressures up to $690 \mathrm{kPa}$ (100 psi), the samples are tested again at injection pressures of 207 and 345 $\mathrm{kPa}$ ( 30 and $50 \mathrm{psi}$ ). The permeability results and the cumulative inflow and outflow for each sample are given in. Figures E.13 through E.21 (Appendix E). The results are compared with those obtained earlier under the same injection pressures in Figures 3.51 through 3.56 . Except for Sample B/AL-C-4-25/B, the second run permeabilities appear to be consistently 2 to 4 times lower than the first run results. This suggests that irreversible changes in sample structure may have occurred. The contrasting behavior of B/AL-C-4-25/B (Figure 3.52) is believed to indicate sealing performance deterioration resulting from piping. The outflow collected from this sample has remained cloudy throughout the testing.

\subsubsection{Samples with 25 or 358 Bentonite by Weight - Third Test Sequence (Upward Permeation)}

In the subsequent flow testing, the flow direction has been reversed (to the upward direction) to study the sealing performance under the action of an upward seepage force. Sample B/AL-C-4-25/B has been omitted from this test series because of the rapid depletion of its inflow reservoir. Limited results (for injection pressures up to $69 \mathrm{kPa}$ ) are shown in Figures 3.57 and 3.58 for the samples with 25 and 35 bentonite weight percent, respectively. Upon incrementing the pressure from $35 \mathrm{kPa}$ (5 psi) to. $69 \mathrm{kpa}$ (10 psi), cracks are seen to develop around the coarse portions (which contain less bentonite) close to the bottom of Sample B/AL-C-4-25/C. This may be due to'insufficient confinement during installation of the sample, or due to particle migration or rearrangement. Sample B/AL-C-4-25/C has been eliminated from this upward flow testing after a leak of its permeameter had been detected at an injection pressure of $276 \mathrm{kPa}(40 \mathrm{psi})$. The other samples have been permeated upward for injection pressures up to $621 \mathrm{kPa}(90 \mathrm{psi})$. The permeabilities obtained from the upward permeation fall in the middle to lower range of $10^{-9} \mathrm{~cm} / \mathrm{s}$ and appear to decrease as the injection pressure increases.

The upward flow test results of Samples B/AL-C-4-25/A and B/AL-C-4-35/A are compared with the downward flow test results obtained earlier under the same injection pressures. Figures 3.59 through 3.62 show the 


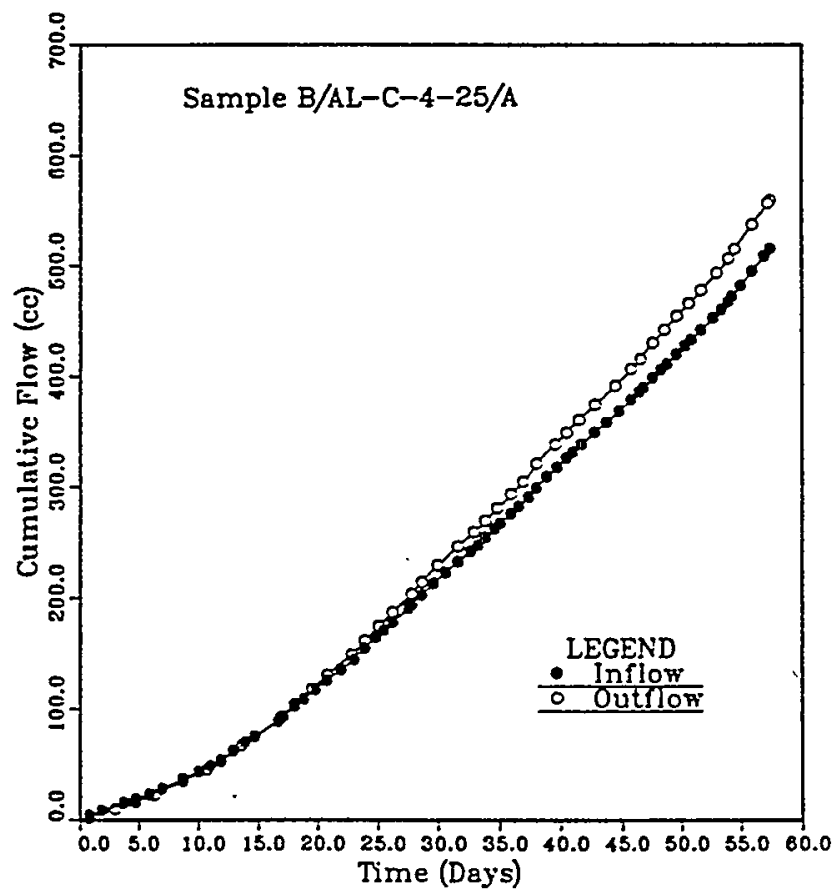

Figure 3.42 Cumulative inflow and outflow vs. time for Sample B/AL-C-4-25/A.

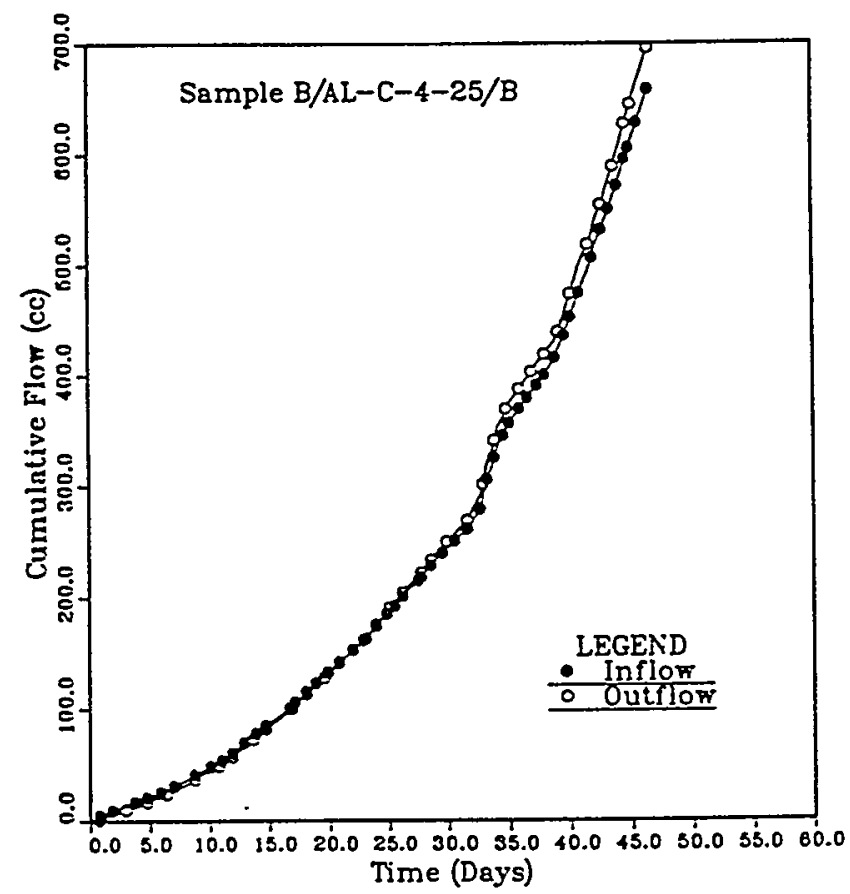

Figure 3.43 Cumulative inflow and outflow vs. time for Sample B/AL-C $-4-25 / B$. 


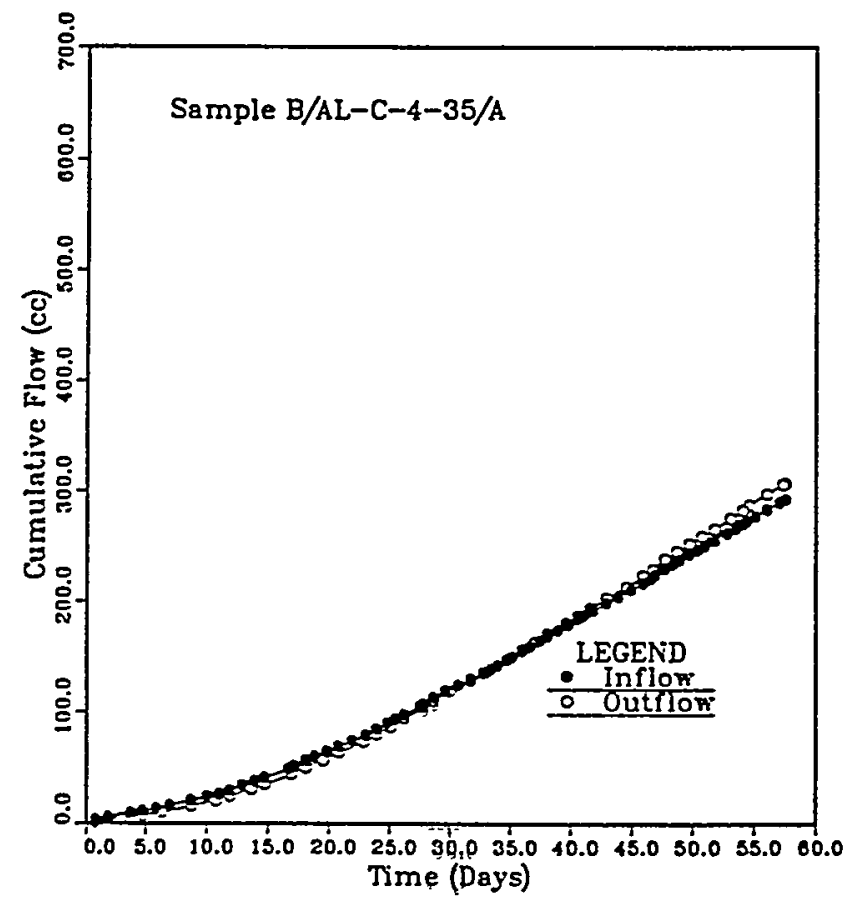

Figure 3.44 Cumulative inflow and outflow vs. time for Sample B/AL-C-4-35/A. 


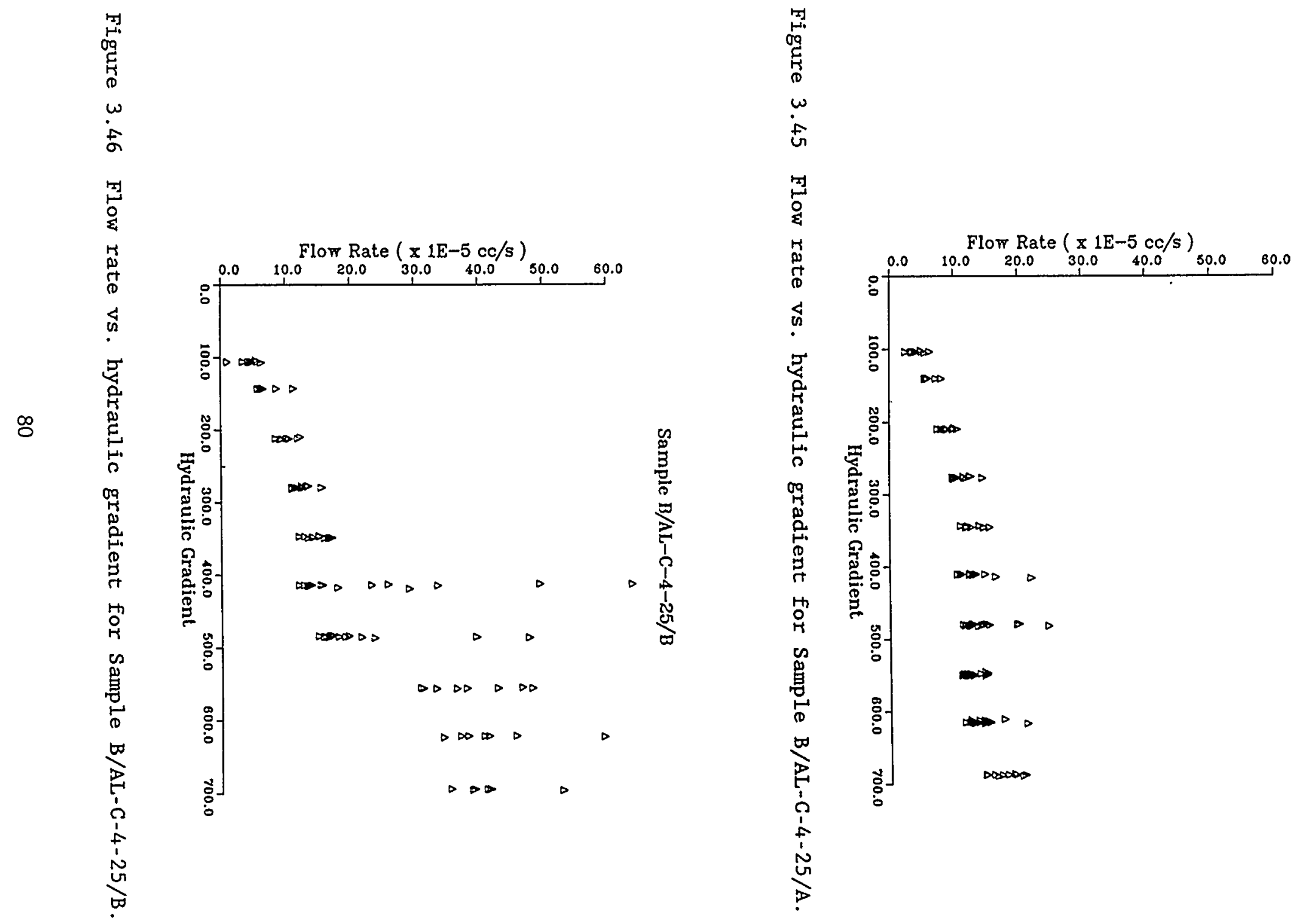


Sample B/AL-C-4-25/C

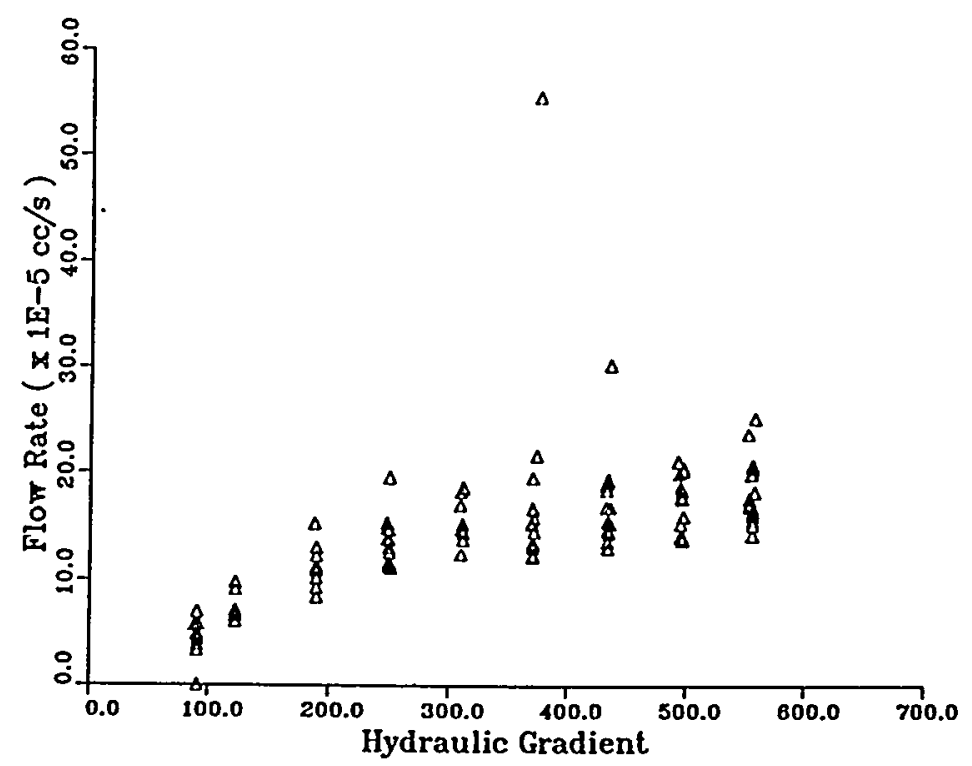

Figure 3.47 Flow rate vs. hydraulic gradient for Sample B/AL-C-4-25/C.

Sample B/AL-C-4-35/A

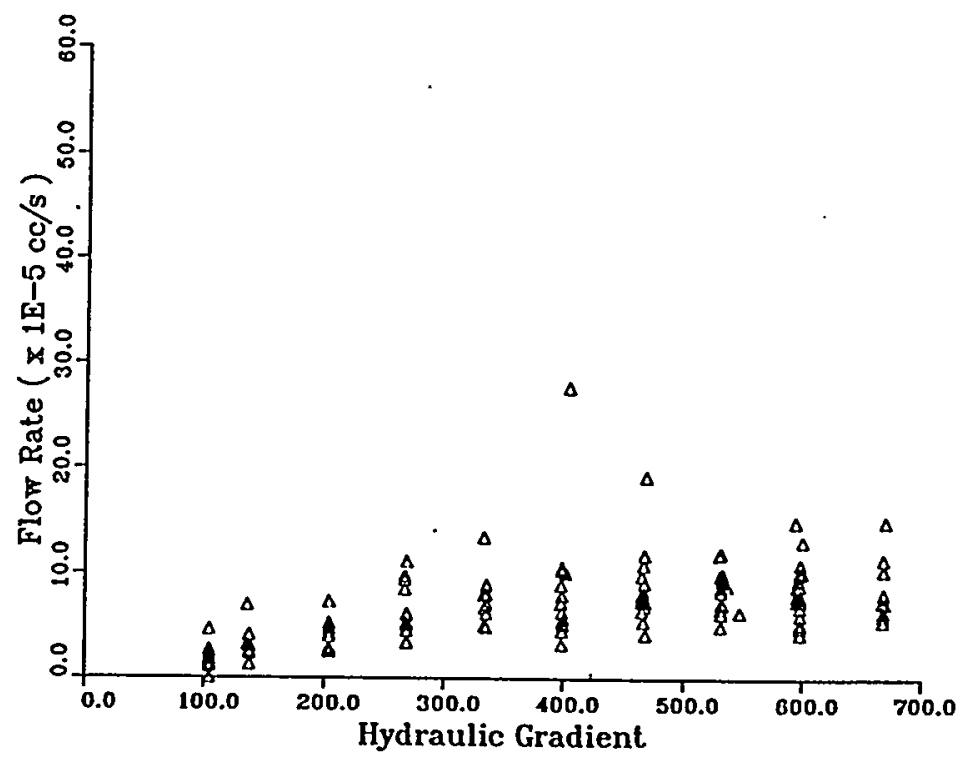

Figure 3.48 Flow rate vs. hydraulic gradient for Sample B/AL-G-4-35/A. 
Sample B/AL-C-4-35/B

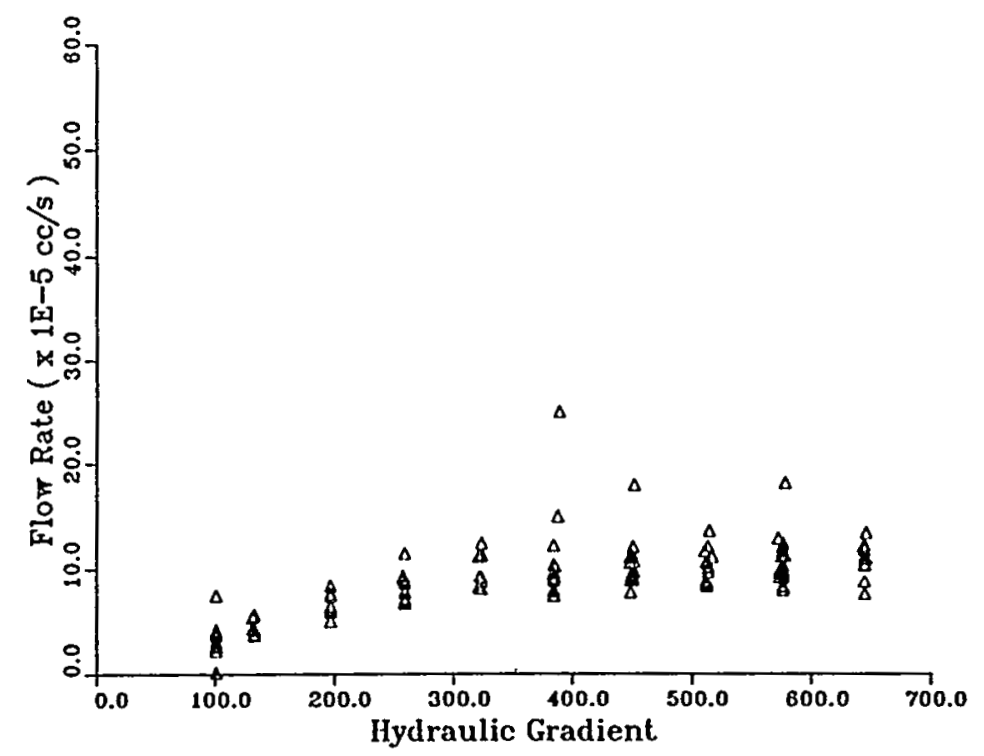

Figure 3.49 Flow rate vs. hydraulic gradient for Sample B/AL-C-4-35/B.

Sample B/AL-C-4-35/C

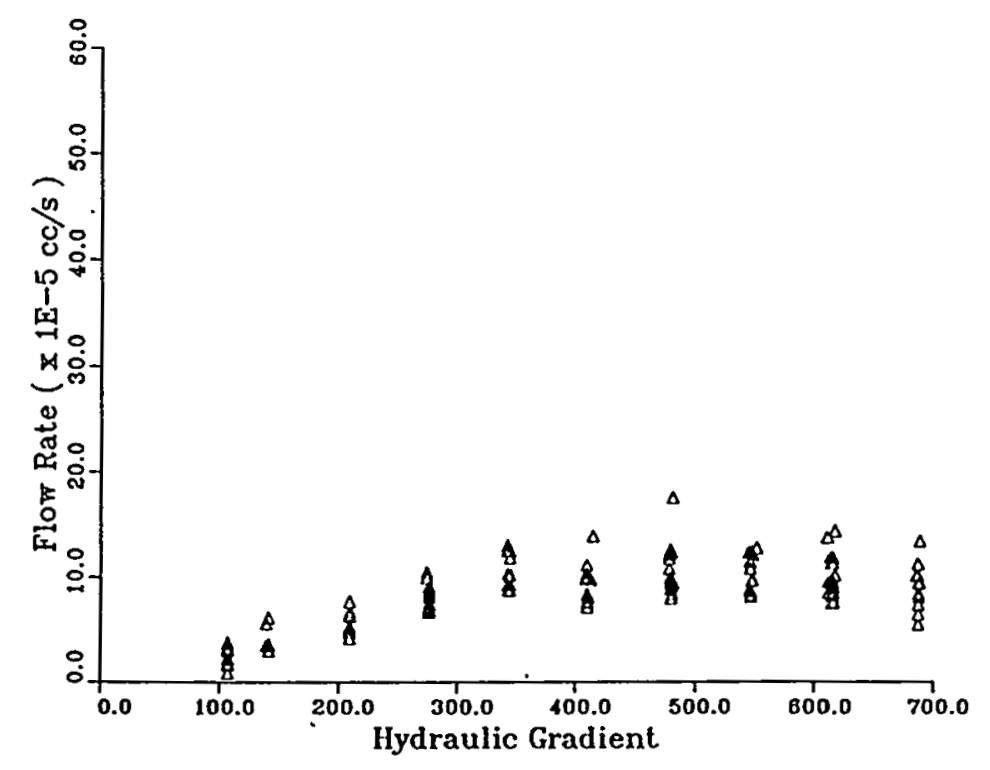

Figure 3.50 Flow rate vs, hyäraulic gradient for Sample B/AL-C-4-35/C. 

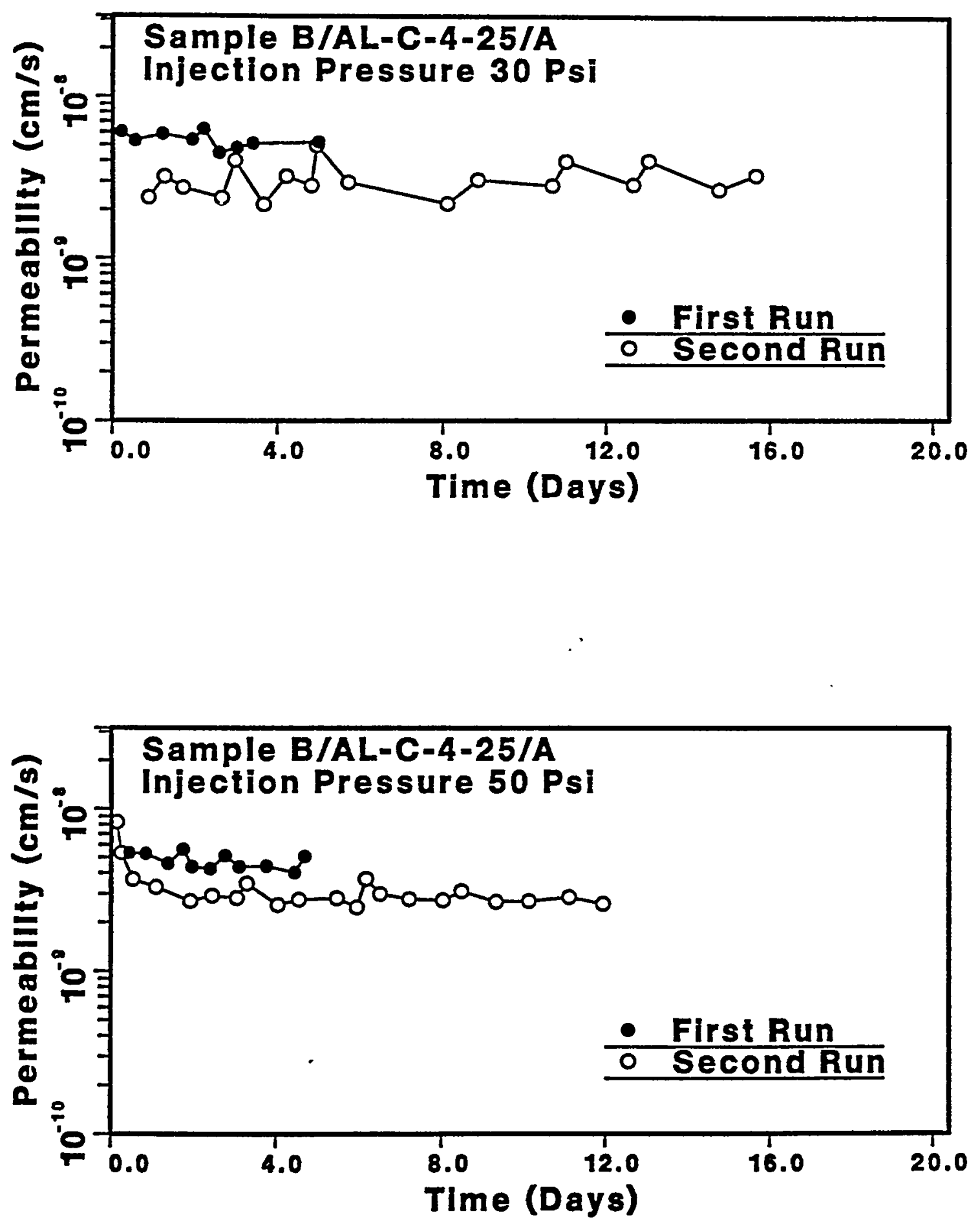

Figure 3.51 Comparison between first-run and second-run permeability results of Sample B/AL-C-4-25/A at injection pressures of 207 and $345 \mathrm{kPa}$ ( 30 and $50 \mathrm{psi}$ ). Second run was after injection pressure of $690 \mathrm{kPa}$ (100 psi) had been applied. 

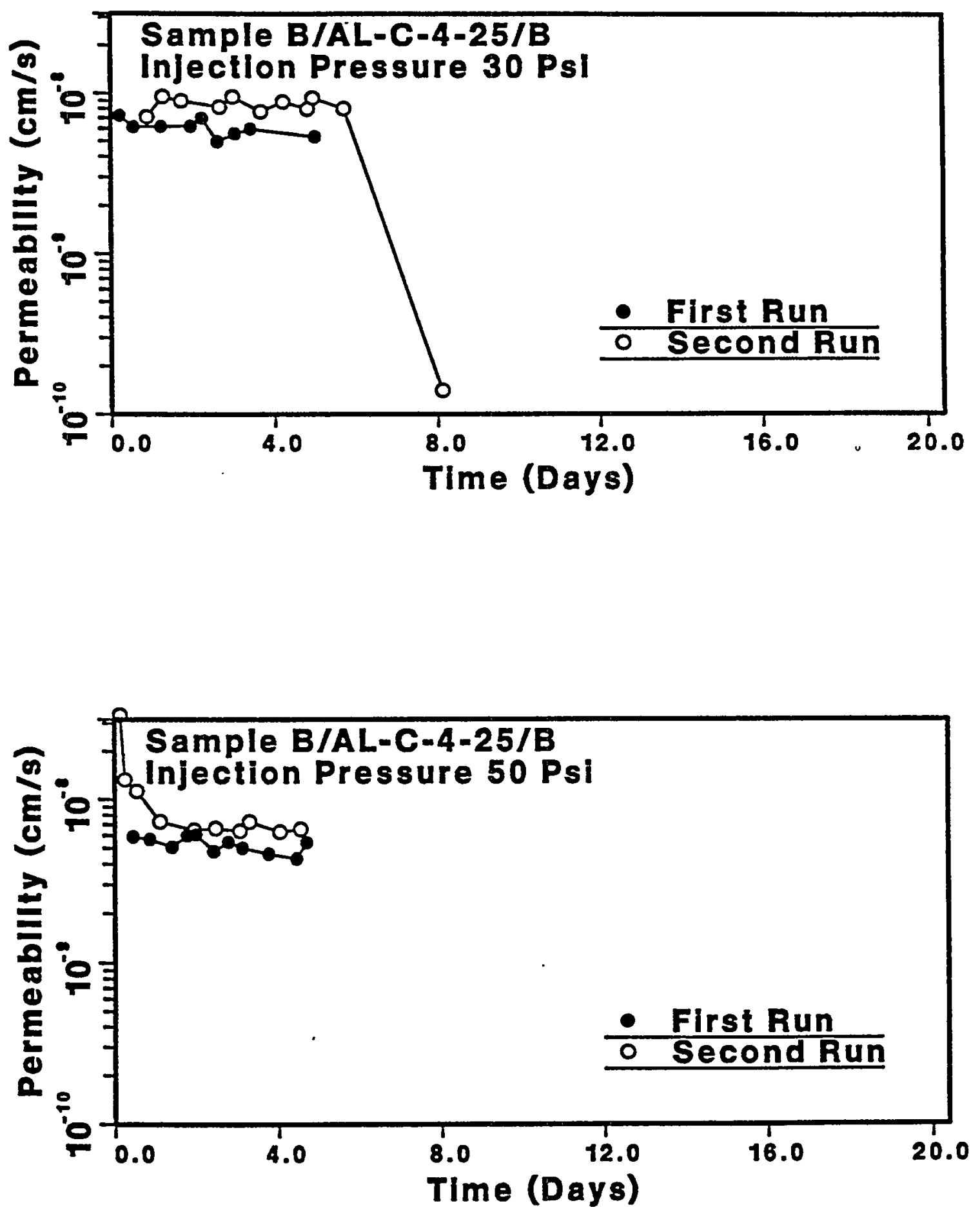

Figure 3.52 Comparison between first-run and second-run permeability results of Sample B/AL-C-4-25/B at injection pressures of 207 and $345 \mathrm{kPa}$ ( 30 and $50 \mathrm{psi}$ ). Second run was after injection pressure of $690 \mathrm{kPa}$ (100 psi) had been applied. 

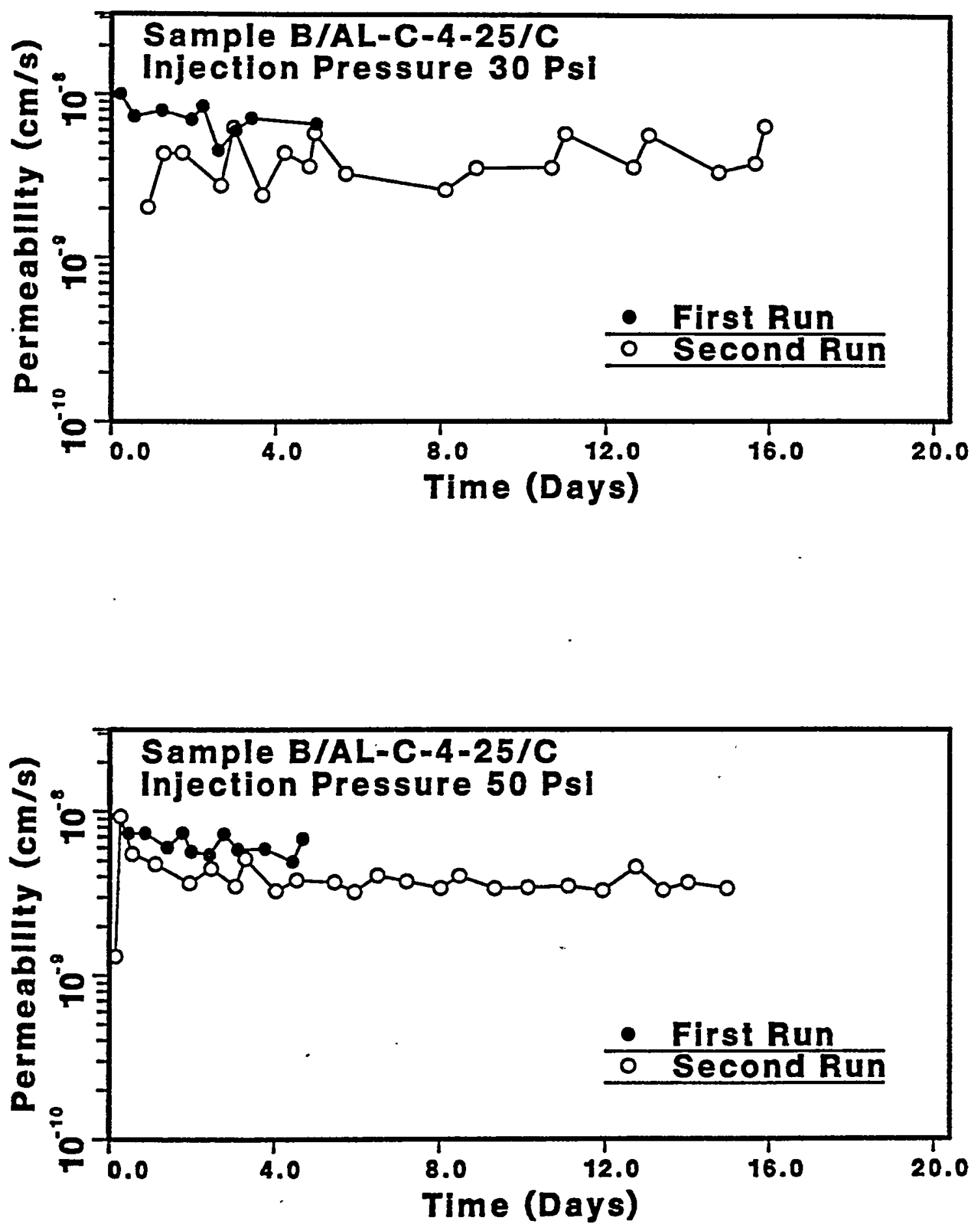

Figure 3.53 Comparison between first-run and second-run permeability results of Sample B/AL-C-4-25/C at injection pressures of 207 and $345 \mathrm{kPa}$ ( 30 and $50 \mathrm{psi}$ ). Second run was after injection pressure of $690 \mathrm{kPa}$ (100 psi) had been applied. 

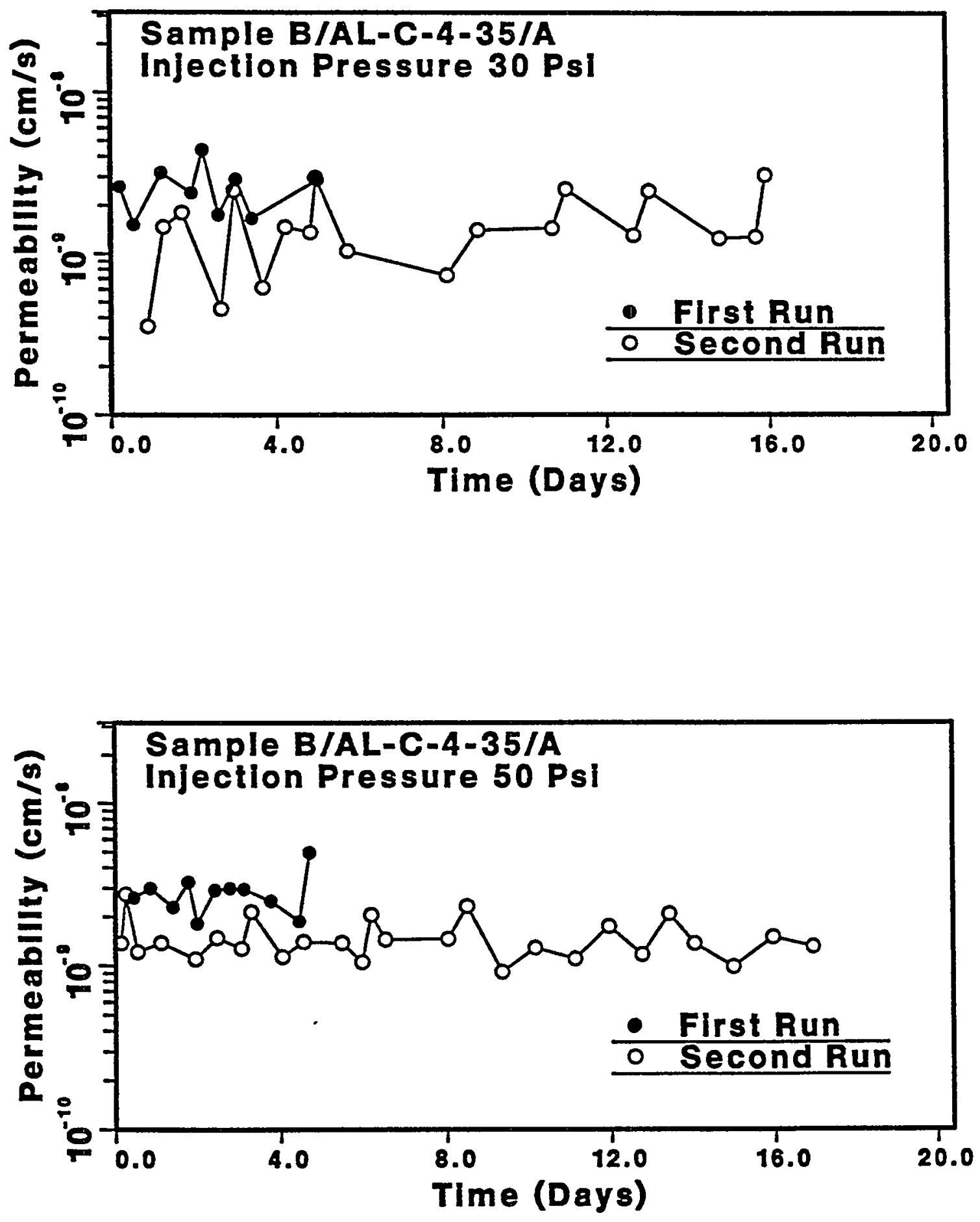

Figure 3.5.4 Comparison between first-run and second-run permeability results of Sample B/AL-C-4-35/A at injection pressures of 207 and $345 \mathrm{kPa}$ ( 30 and $50 \mathrm{psi}$ ). Second run was after injection pressure of $690 \mathrm{kPa}$ (100 psi) had been applied. 

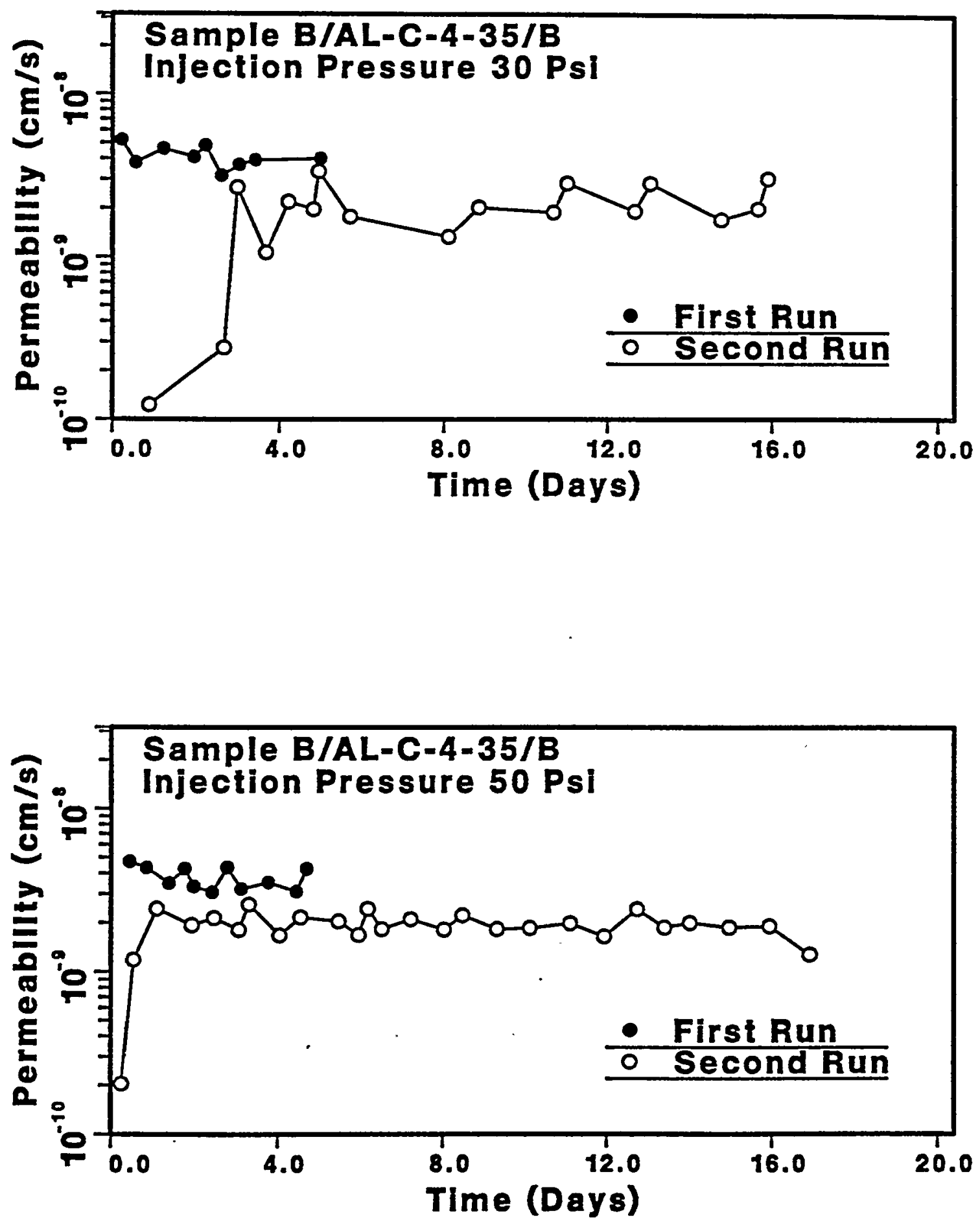

Figure 3.55 Comparison between first-run and second-run permeability results of Sample B/AL-C-4-35/B at injection pressures of 207 and $345 \mathrm{kPa}$ ( 30 and $50 \mathrm{psi}$ ). Second run was after injection pressure of $690 \mathrm{kPa}$ (100 psi) had been applied. 

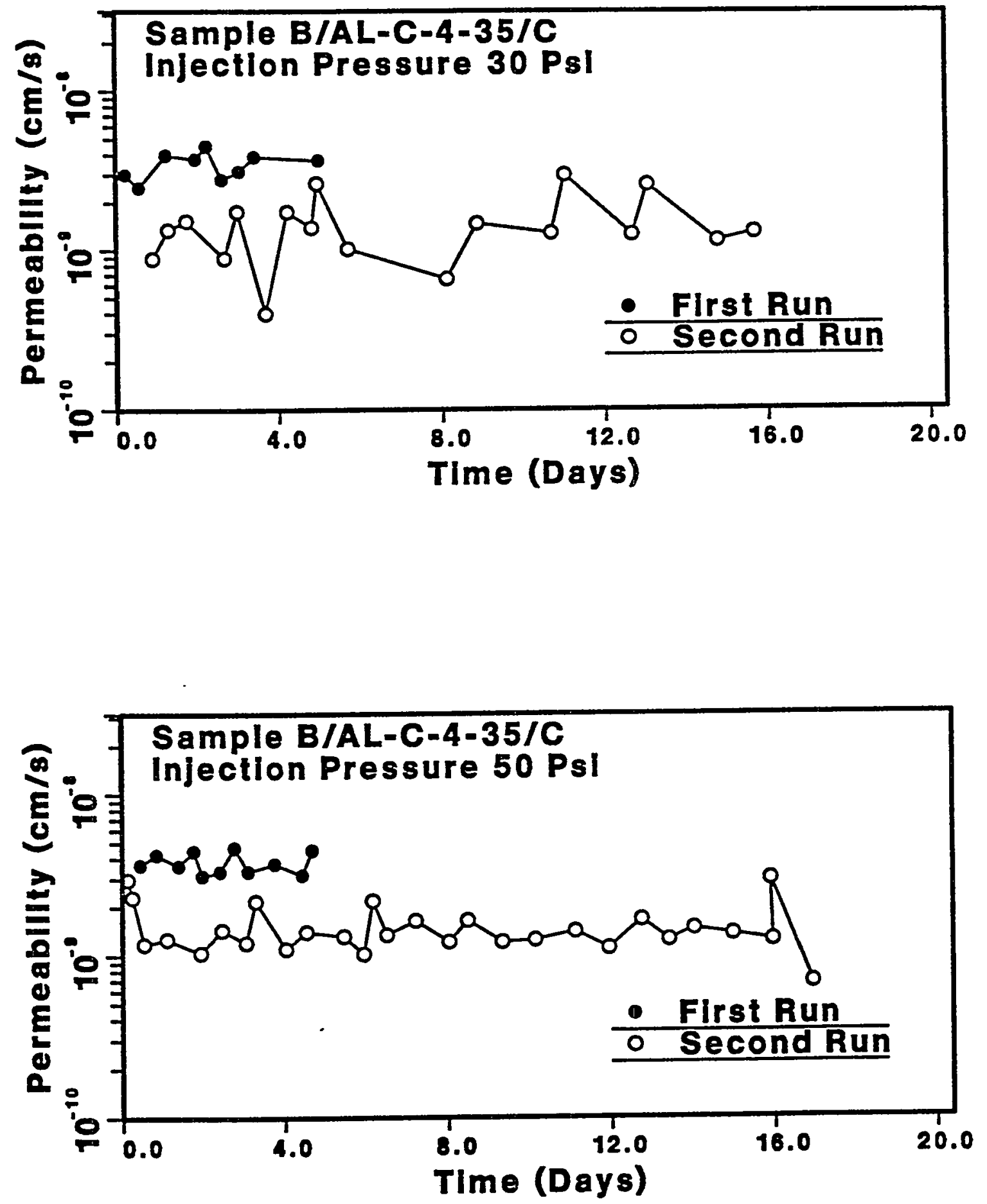

Figure 3.56 Comparison between first-run and second-run permeability results of Sample B/AL-C-4-35/C at injection pressures of 207 and $345 \mathrm{kPa}$ ( 30 and $50 \mathrm{psi}$ ). Second run was after injection pressure of $690 \mathrm{kPa}$ (100 psi) had been applied. 


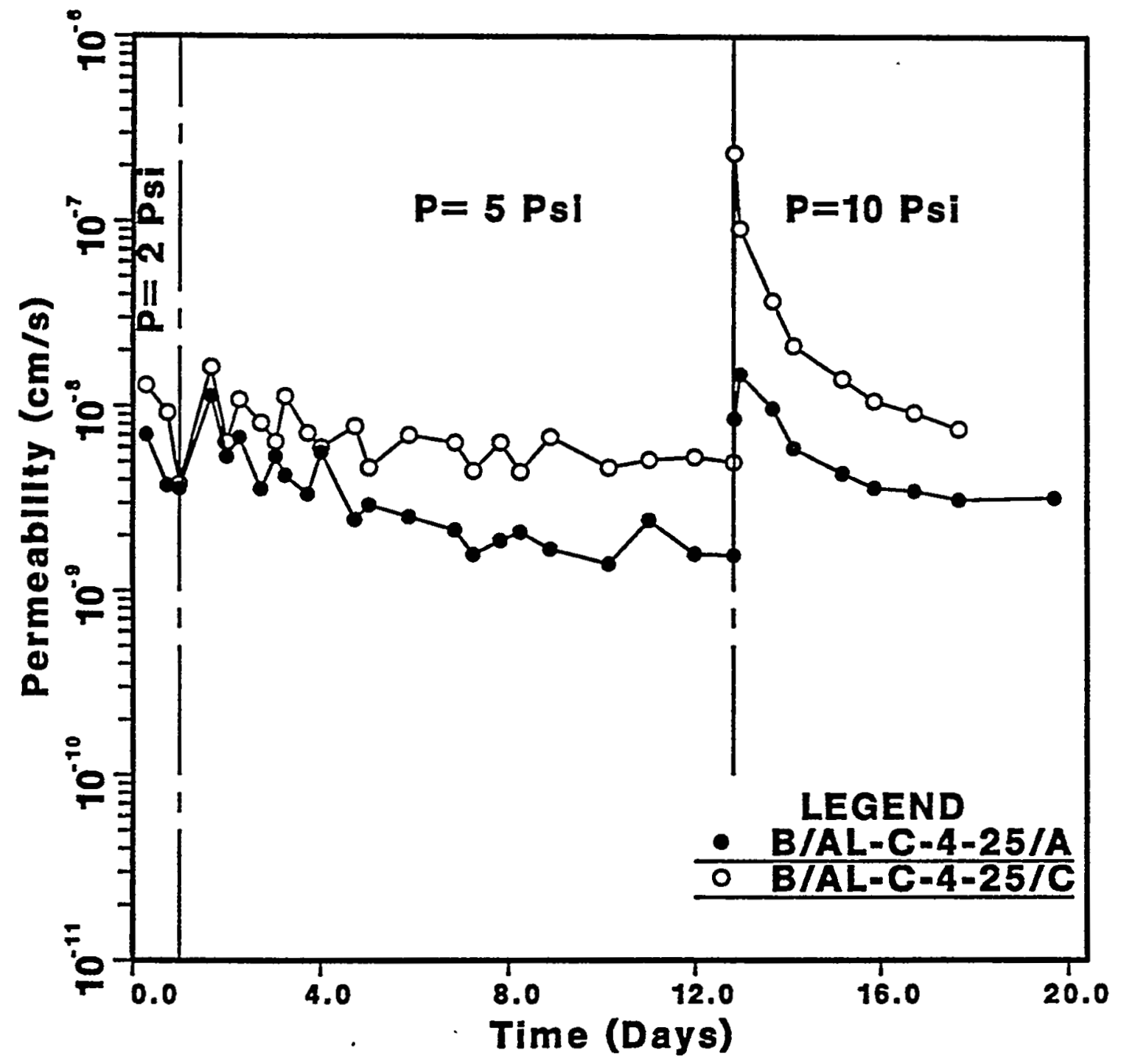

Figure 3.57 Permeability results of the mixture samples containing 258 bentonite by weight (measured in the upward permeation). Injection pressure $\mathrm{p}=13.8,34.5$ and $69 \mathrm{kPa}(2,5$ and 10 psi). Hydraulic gradient: $12-16,30-35$ and $63-70$, respectively. 


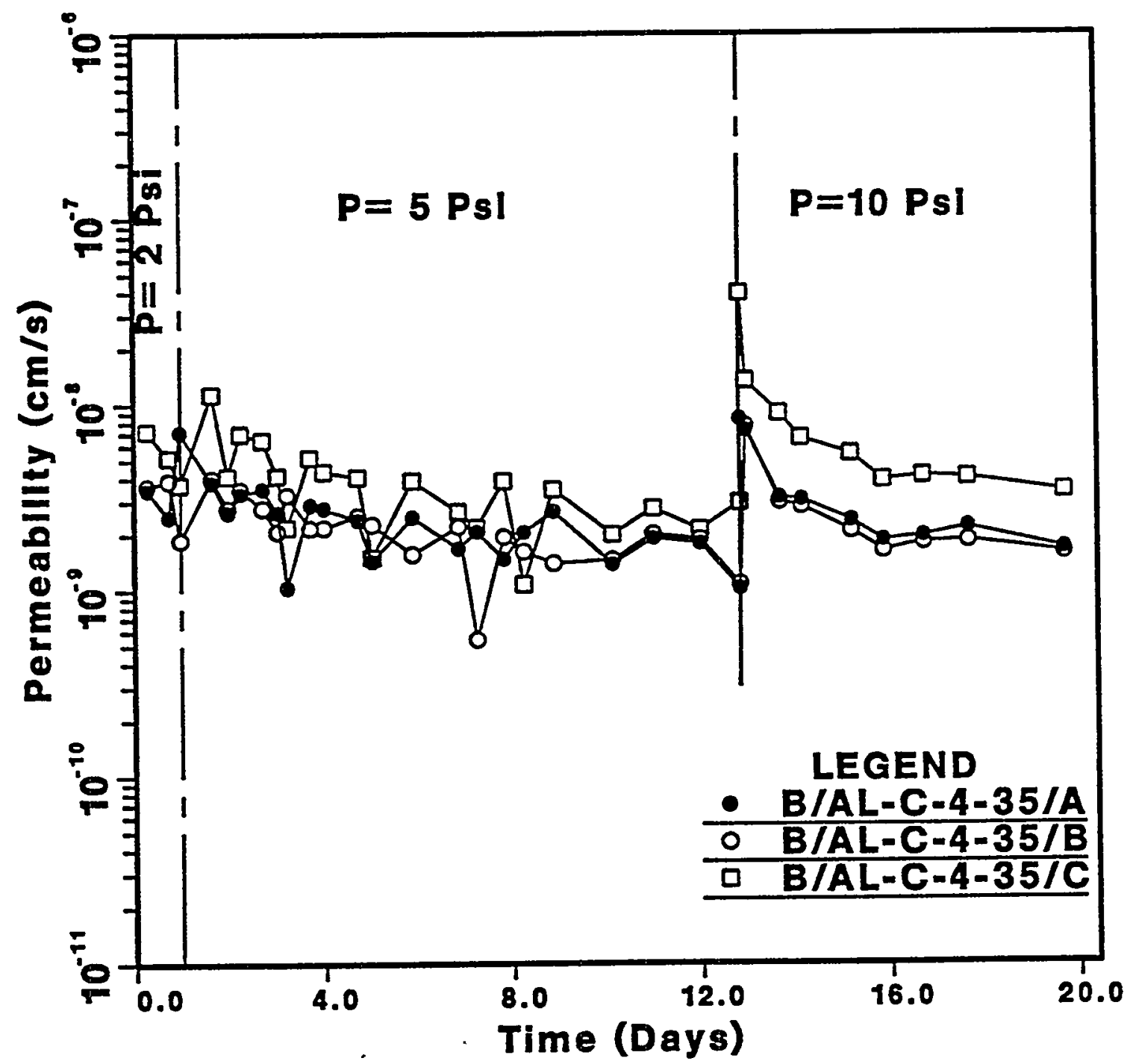

Figure 3.58 Permeability results of the mixture samples containing 358 bentonite by weight (measured in the upward permeation). Injection pressure $\mathrm{p}=13.8,34.5$ and $69 \mathrm{kPa}(2,5$ and 10 psi). Hydraulic gradient: $12-16,31-35$ and $65-71$, respectively. 
permeabilities at injection pressures of 103,138 , and $621 \mathrm{kPa}(15,20$, and $90 \mathrm{psi}$, respectively). The comparisons at other pressures are given in Figures E.22 through E.27 (Appendix E). The permeability measured in the upward flow direction is lower than that measured in the downward direction. The upward seepage forces seem to have no major damaging effect on the sealing performance. It should be recognized that the samples had been subjected previously to injection pressures up to 690 $\mathrm{kPa}$ (100 psi) in the downward flow tests. The lower permeability in the upward permeation may have resulted from particle migration induced at the inflow end and/or consolidation at the outflow end. The effect of upward seepage force on the deterioration of sealing performance is observed from the downward-upward flow testing performed on three fresh mixture samples installed in stainless steel permeameters, described in Section 3.4.1.6.

\subsubsection{Samples with 25 or 358 Bentonite by Weight - Fourth Test Sequence (Downward Permeation)}

Samples B/AL-C-4-25/A, B/AL-C-4-35/A, B/AL-C-4-35/B, and B/AL-C-4-35/C had been tested previously in the downward and upward flow directions. Figures 3.63 through 3.66 present the permeabilities measured in an additional downward flow test sequence for which injection pressures are raised up to $1 \mathrm{MPa}$ ( $145 \mathrm{psi}$ ), equivalent to a $102 \mathrm{~m}$ water column. The maximum hydraulic gradients are between 900 and 1000 . The permeability varies from the lower $10^{-8}$ to below the $10^{-9} \mathrm{~cm} / \mathrm{s}$ range. Variations in permeability are noted and may be attributed, in part, to the fluctuation of ambient temperature (Section 3.4.1.9.1). Temporary and intermittent pore clogging by displaced bentonite gel or individual particle aggregates have also been proposed to explain time-dependent variations in flow rate and permeability (Pusch et al., 1987). Sealing performance of the four samples has been evaluated under various injection pressures and flow directions for more than 9 months. Although depositions of dispersed or eroded bentonite have been observed in the outflow tubing, no deterioration of sealing ability can be detected.

It is recognized that the preservation of low permeability has been obtained in a rather restricted condition which precludes any external lateral flow. The laterally confining boundary provided by a fixed-wall permeameter by no means simulates a possible field situation of open joints or fractures intersecting sealants installed in boreholes or shafts. Fixed-wall permeameters have been modified by drilling circular holes into the wall for flow testing of mixture samples. Results of flow tests in such permeameters are discussed in section 3.4.4.

\subsubsection{Samples B/AL-C-12-25/A and B/AL-C-12-35/A}

The results presented in this section have been obtained from the flow testing of two large bentonite/crushed tuff plugs, $30.15 \mathrm{~cm}$ (12 in) in diameter and 10.5 and $12.3 \mathrm{~cm}$ (4.13 and $4.84 \mathrm{in})$ long. Crushed tuff of Type A gradation $\left(D_{\max }=9.42 \mathrm{~mm}\right.$ ) has been mixed with 25 and 35 weight percent of bentonite. It is important to test large samples so that the performance can be evaluated on a relatively large scale (Olson and Daniel, 1981); especially in light of the variation between field and 

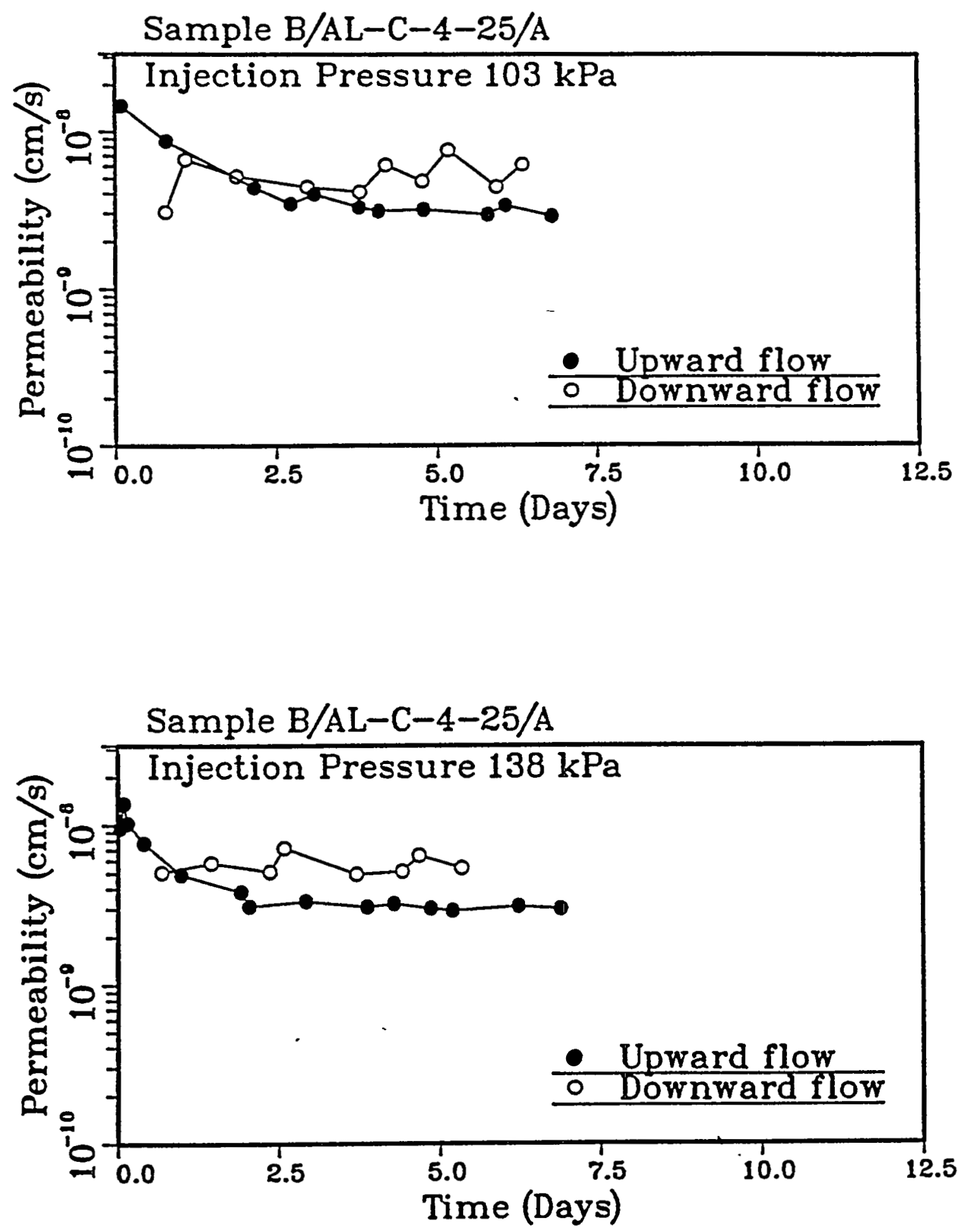

Figure 3.59 Upward and downward permeability of Sample B/AL-C-4-25/A under injection pressures of $103 \mathrm{kPa}$ (15 psi) and $138 \mathrm{kPa}$ (20 psi). Hydraulic gradient: 101.3-108.4 (top), 139.6143.3 (bottom). 


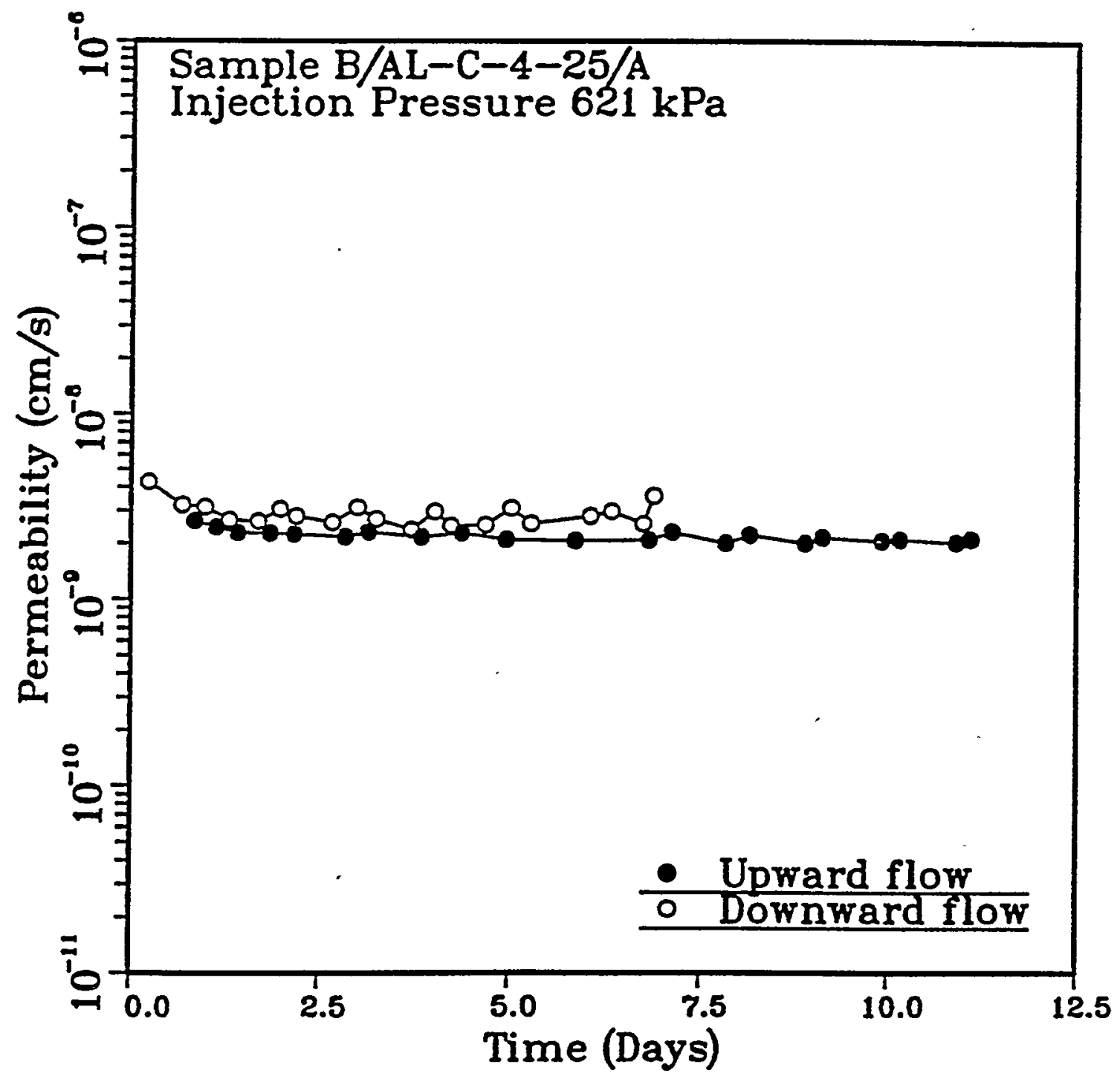

Figure 3.60 Upward and downward permeability of Sample B/AL-C-4-25/A under injection pressure of $621 \mathrm{kPa}$ (90 psi). Hydraulic gradient: $611.1-619.9$. 

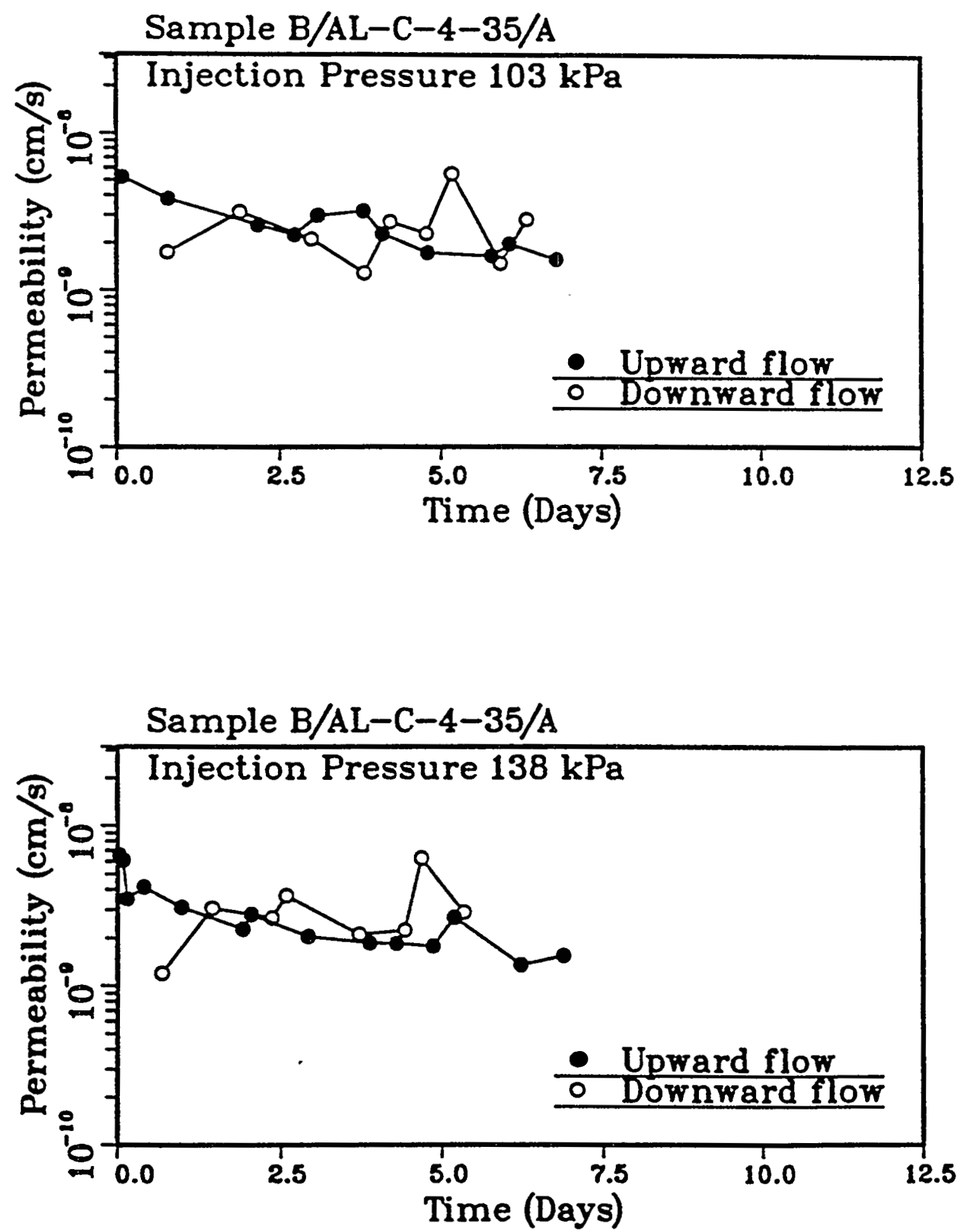

Figure 3.61 Upward and downward permeability of Sample B/AL-C-4-35/A under injection pressures of $103 \mathrm{kPa}$ (15 psi) and $138 \mathrm{kPa}$ (20 psi). Hydraulic gradient: 104.5-105.9 (top), 136.1139.4 (bottom). 


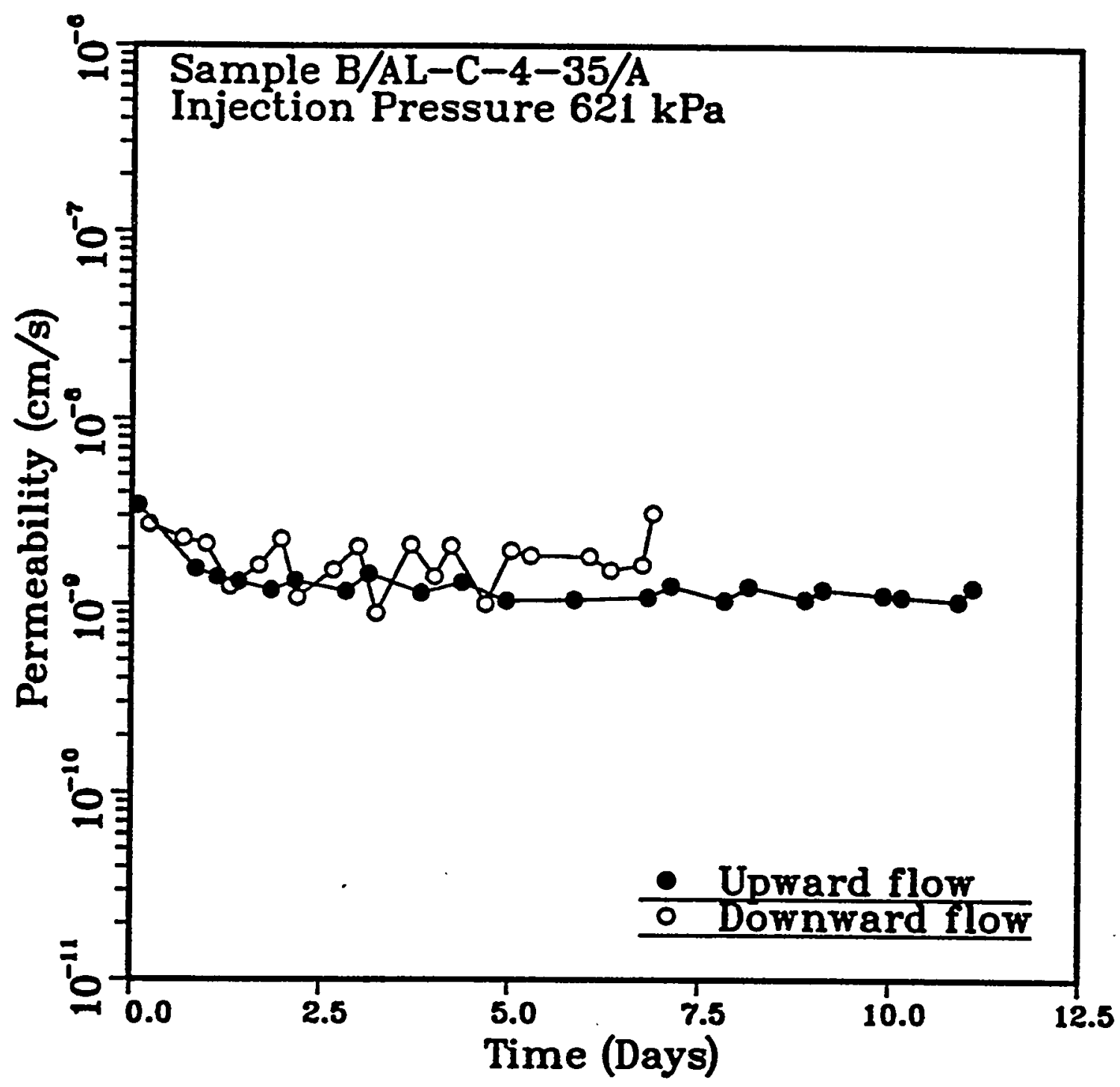

Figure 3.62 Upward and downward permeability of Sample B/AL-C-4-35/A under injection pressure of $621 \mathrm{kPa}$ ( $90 \mathrm{psi}$ ). Hydraulic gradient: 539.9-602.2. 


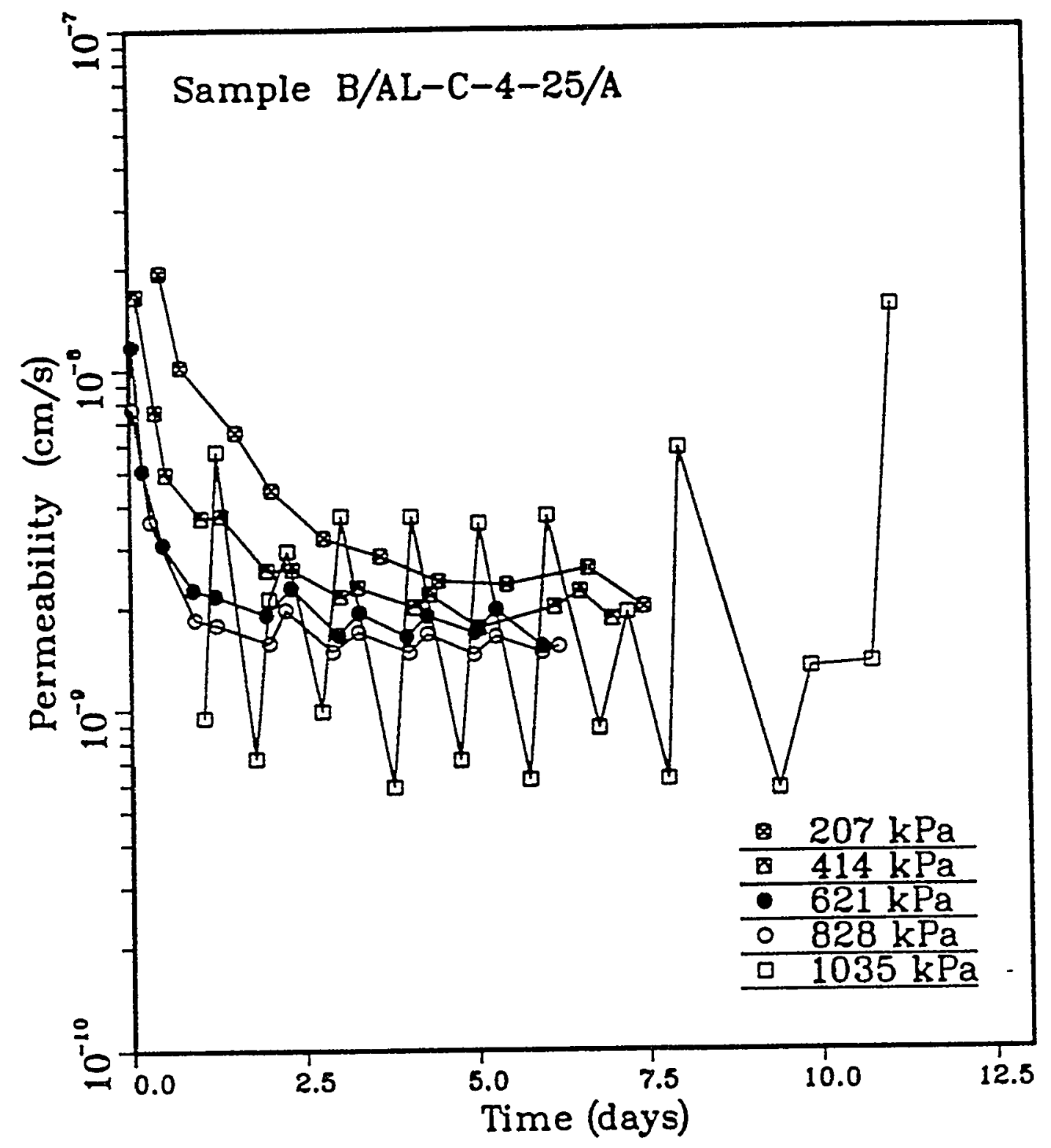

Figure 3.63 Permeability of Sample B/AL-C-4-25/A at five injection pressures as a function of flow test time. 


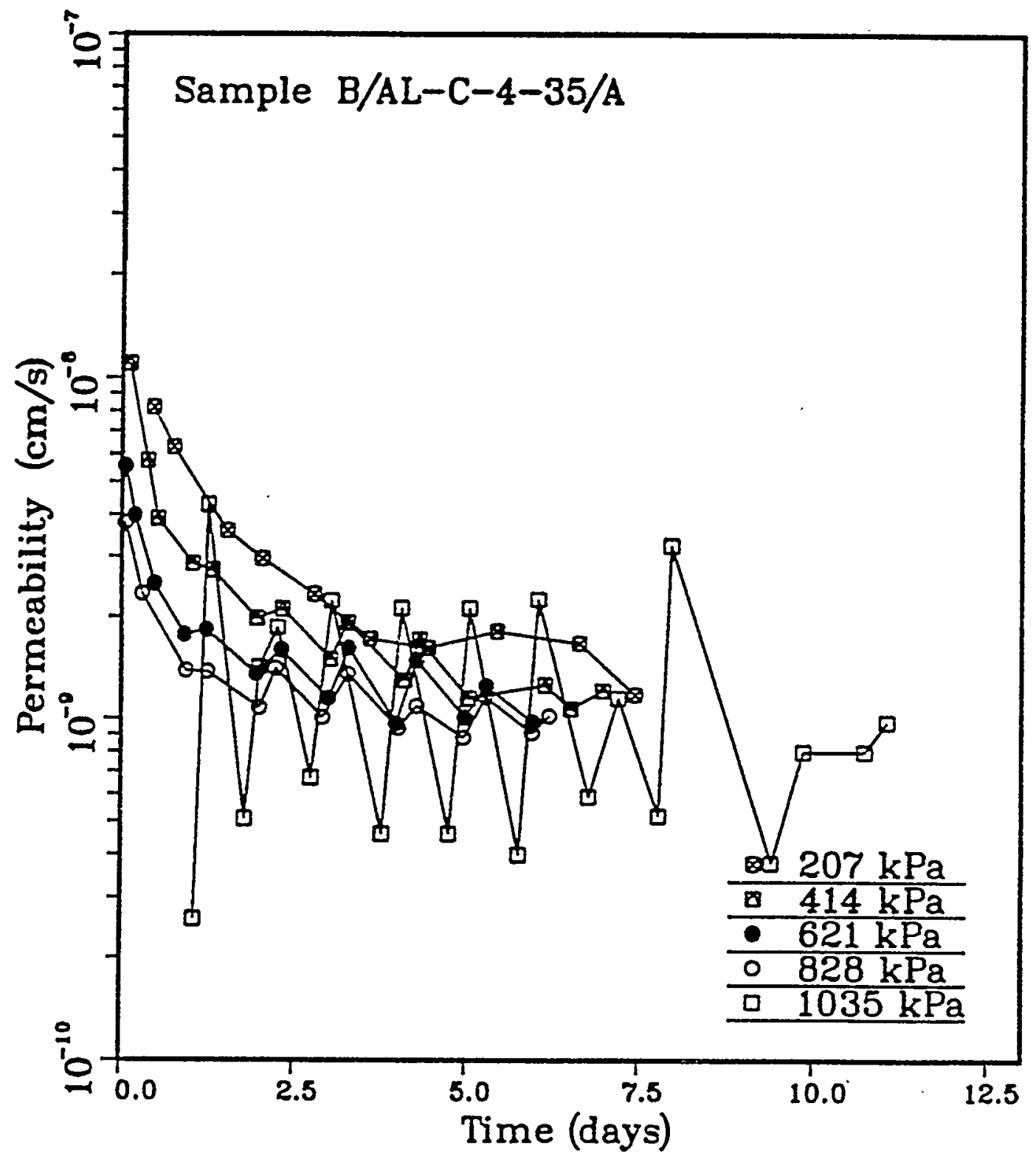

Figure 3.64 Permeability of Sample B/AL-C-4-35/A at five injection pressures as a function of flow test time. 


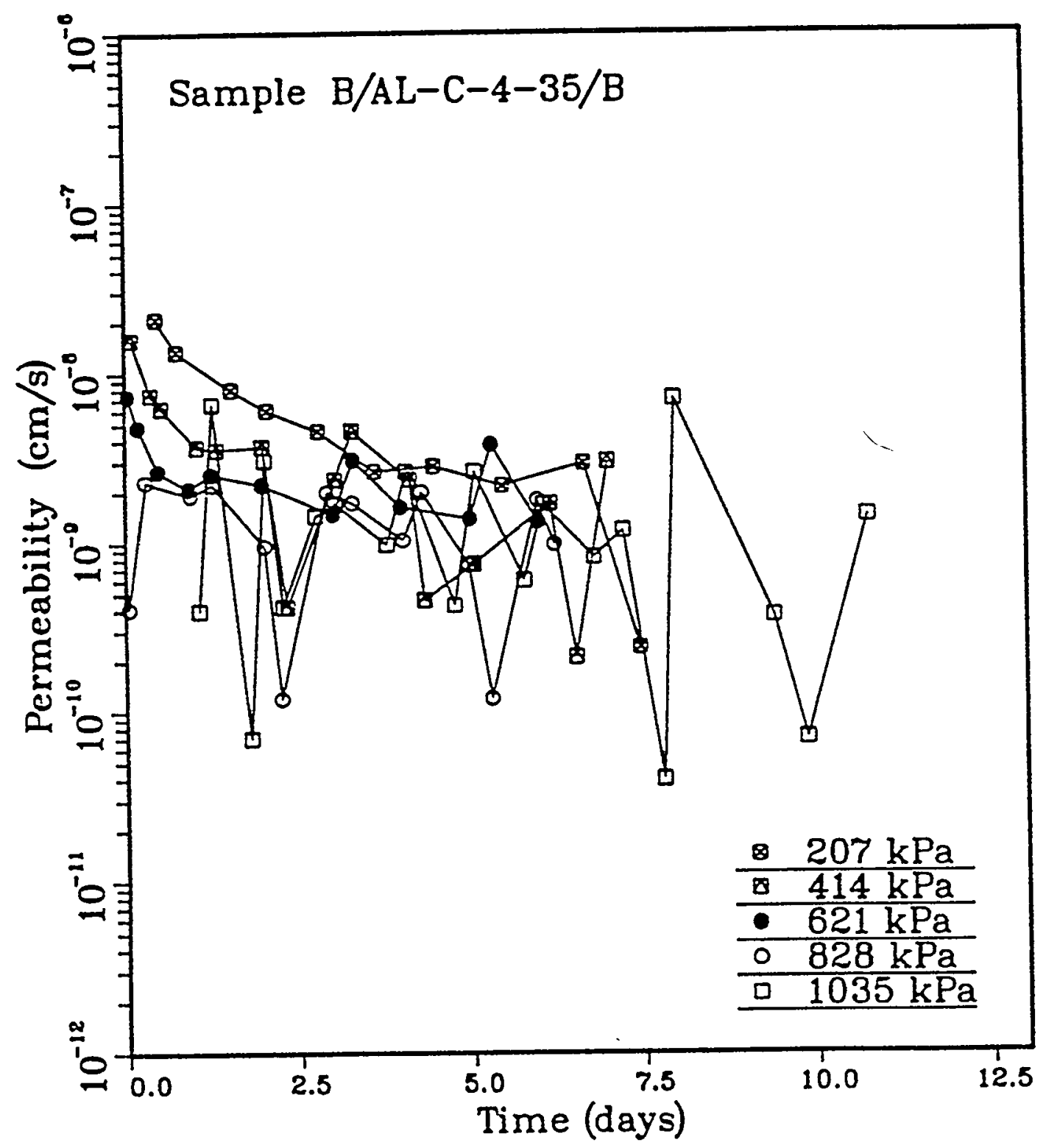

Figure 3.65 Permeability of Sample B/AL-C-4-35/B at five injection pressures as a function of flow test time. 


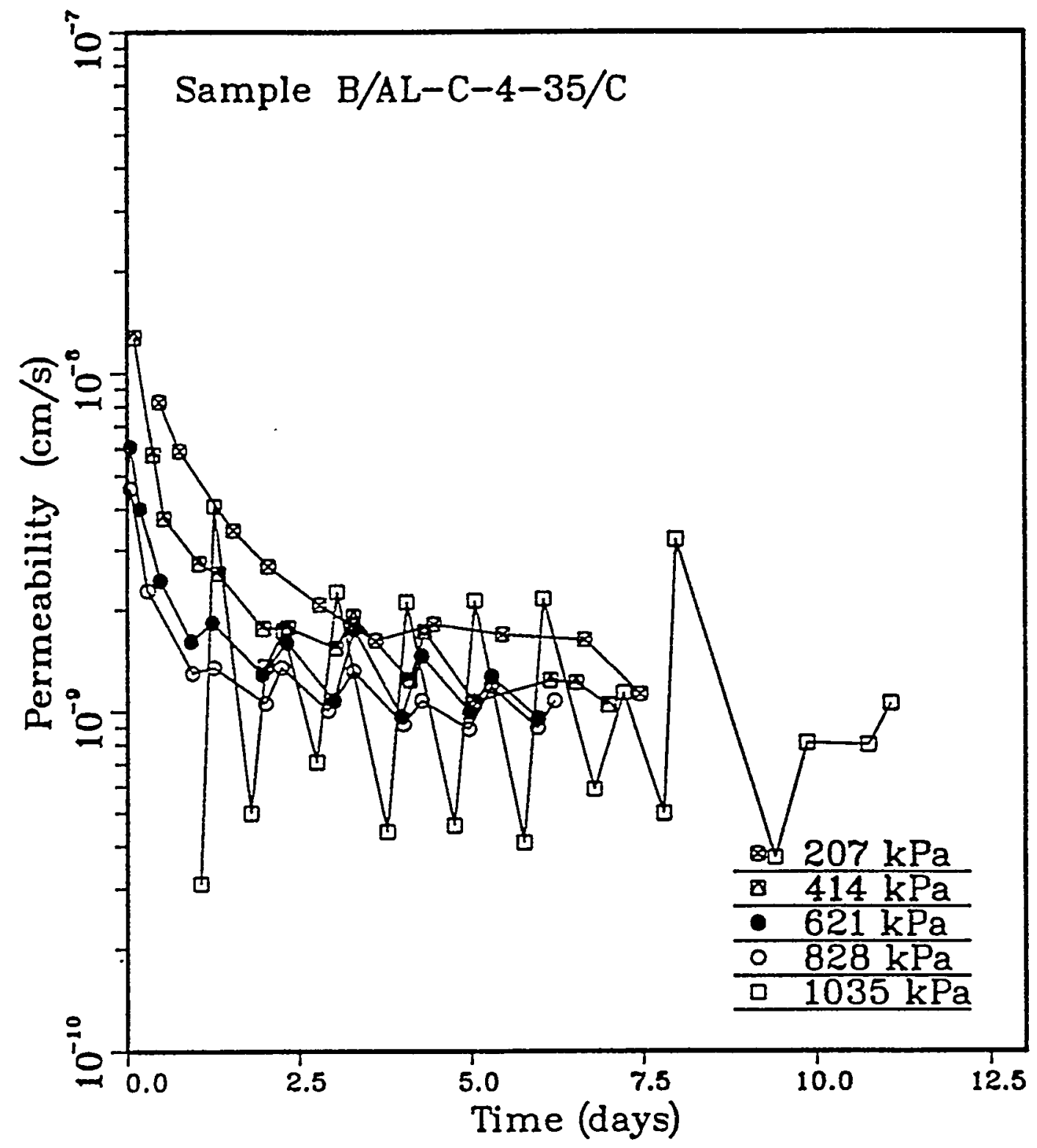

Figure 3.66 Permeability of Sample $B / A L-C-4-35 / C$ at five injection pressures as a function of flow test time. 
laboratory measured permeabilities reported for clay liners (Dunn, 1986; Day and Daniel, 1985; Daniel, 1984).

The Standard Proctor method was used to compact the samples in the permeameters. The samples received 225 blows per layer for 3 layers to adjust the compaction effort for the large sample diameter. To keep the ratio of impact area to sample cross-sectional area close to that in the Proctor compaction (1:4), a $15 \mathrm{~cm}$ diameter aluminum plate was placed on top of the sample and was moved around during compaction. Each blow was aimed at the center of the aluminum plate.

The flow testing with an injection pressure of $34.5 \mathrm{kPa}$ ( $5 \mathrm{psi}$ ) continued for about 18 days. The resulting hydraulic gradients are approximately 31 and 36 for $B / A L-C-12-25 / A$ and $B / A L-C-12 / 35 / A$, respectively. The corresponding total inflows and outflows for 18 days are 338,364 cc, and 73, $57 \mathrm{cc}$. Only limited flow data have been obtained for an injection pressure of $69 \mathrm{kPa}$ (10 psi) due to leaks in the permeameters. Figures 3.67 and 3.68 show the flow test results. In spite of differences in the stiffnesses of the permeameters, in compaction and in the ratio of grain size to permeameter diameter, the low permeability exhibited by these $30.15 \mathrm{~cm}$ diameter samples conforms with those yielded by the smaller samples (e.g. Figures 3.69 and 3.70 for the 10.16 and $20.65 \mathrm{~cm}$ diameter samples). The similarity in the permeability measurements suggests that differences between laboratory and field measurements may be due more to differences in installation procedures than to true size effects. Bentonite/crushed tuff seals of even larger diameters may retain similarly low permeabilities if the proper procedures of mixing, installation and compaction are carefully followed.

\subsubsection{Effects of Piping}

Basic designs for backfilling boreholes, shafts and other seal components in the proposed nuclear waste repository at Yucca Mountain may require the seals to retain adequate sealing performance over a long period of time (Fernandez et al., 1987). The effect of piping, as learned from the failures of earth dams, embankments, and natural slopes (Rosewe11, 1977; Sherard et al., 1972, 1977), may be important for the long-term performance of the backfilling materials. The concern is substantiated by piping observed in tests reported on here, which have caused significant increases in permeability during the flow testing of $\mathrm{B} / \mathrm{AL}-\mathrm{C}-4-15 / \mathrm{A}, \mathrm{B} / \mathrm{AL}-\mathrm{C}-4-15 / \mathrm{C}$, and $\mathrm{B} / \mathrm{AL}-\mathrm{C}-4-25 / \mathrm{B}$.

Various definitions of piping are reviewed by Jones (1981, pp. 7-15). Piping is defined here as "any progressive internal erosion of the soil or rock mass by the flow of water along preferred seepage paths". This definition is modified from the one given by Perry (1975, as cited by Jones, 1981). For flow testing of bentonite plugs with either the standard falling-head or double-pipette falling head method, only minimal piping, or possibly dispersion of bentonite, has been observed. Except for a few gel-like flocks in the tubes close to the permeameter ends, the inflow and outflow has remained clear. For bentonite/crushed tuff plugs with 158 bentonite by weight, piping has been observed in $B / A L-C-4-15 / A$ and $B / A L-C-4-15 / C$, and has caused their permeabilities to 


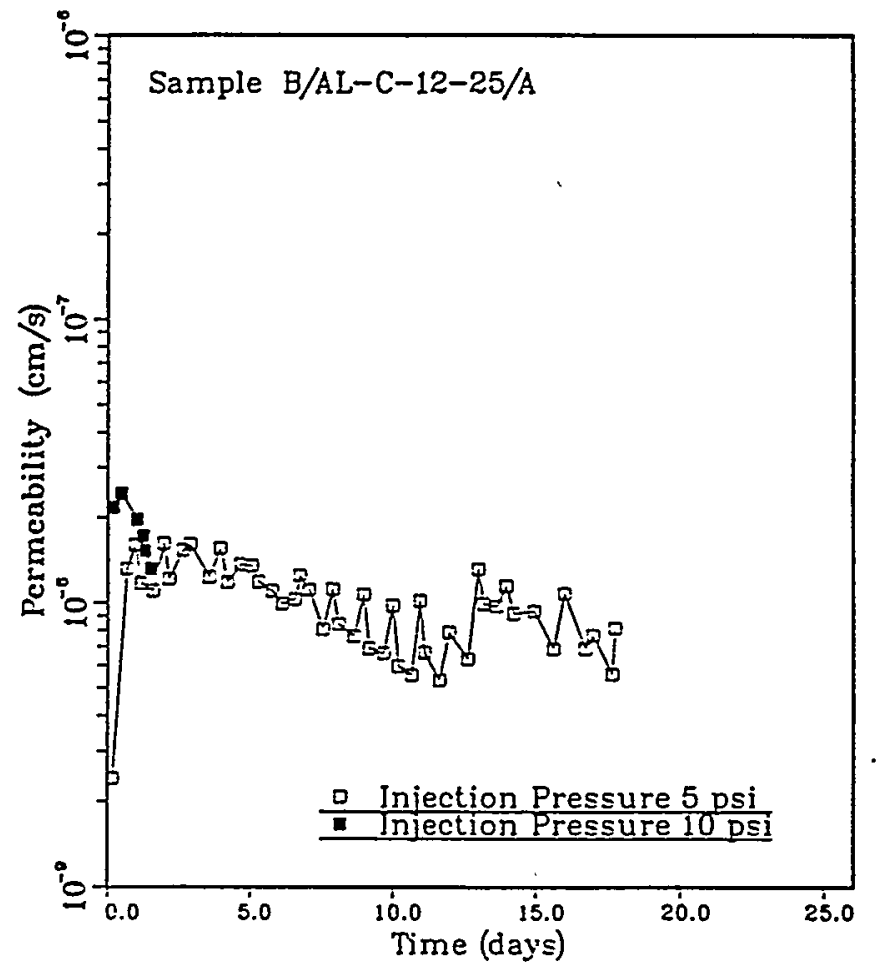

Figure 3.67 Permeability results of Sample B/AL-C-12-25/A.

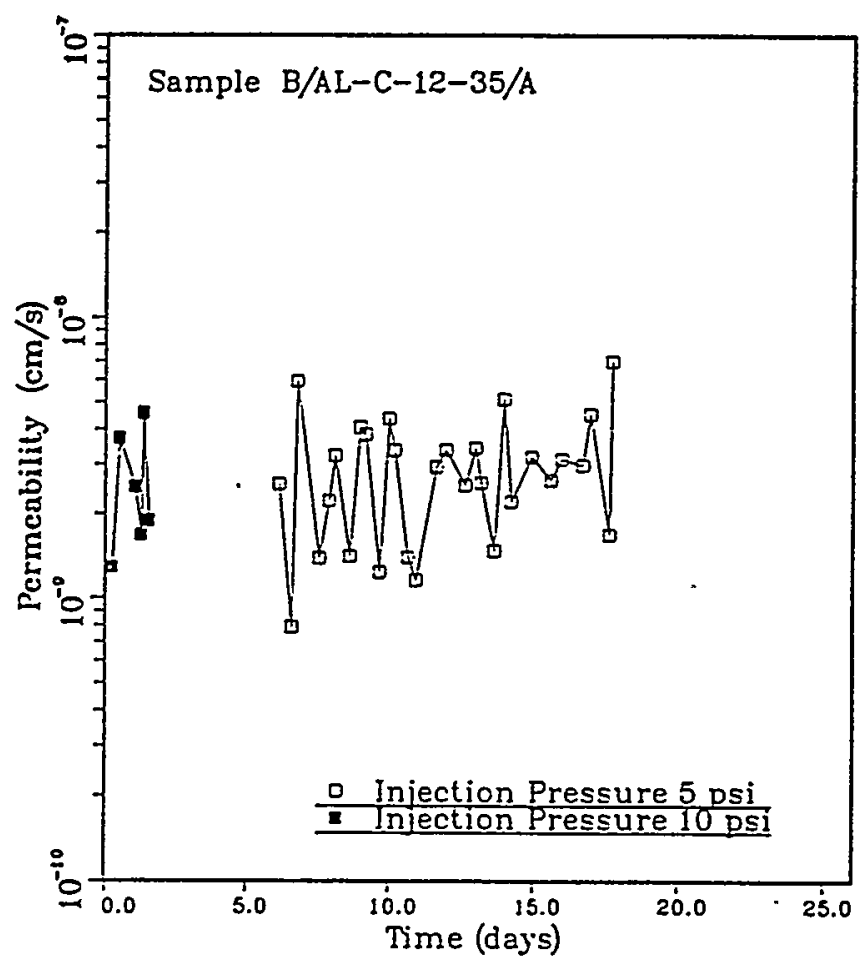

Figure 3.68 Permeability results of Sample B/AL-C-12-35/A. 
increase by approximately one order of magnitude. Erosion channels have developed as a result of piping (Figure 3.29).

Cloudy outflows have been observed when six mixture plugs containing $25 \%$ and 358 bentonite were first subjected to an injection pressure of 206.9 $\mathrm{kPa}$ (30 psi). The outflows appeared cloudy for less than 24 hours and returned to clear under the same injection pressure. The clearing may be accounted for by healing as a result of the migration of fine particles during water percolation. In the second series of flow tests (Section 3.4.1.3), muddy outflow emerged from Sample B/AL-C-4-25/B, while it was subjected to an injection pressure of $414 \mathrm{kPa}$ (60 psi). The permeability increased correspondingly and then decreased to a lesser degree toward the end, before incrementing the injection pressure (Figure 3.37). The outflow collected from this sample remained cloudy at higher injection pressures. The effluent of Sample B/AL-C-4-25/A became slightly foggy about 3 days after the injection pressure was raised to $690 \mathrm{kPa}$ (100 psi). The amounts of suspension and solute carried in the outflows of Samples B/AL-C-4-25/A and B/AL-C-4-25/B have been determined by oven-drying the collected effluents for a number of cases. The results are given in Table 3.8 .

After having been subjected to injection pressures up to $690 \mathrm{kPa}$ (100 psi), the six samples have been tested again at injection pressures of 207 and $345 \mathrm{kPa}$ ( 30 and $50 \mathrm{psi}$ ). The amount of solids carried in the outflows has been measured. The results are summarized in Table 3.9. Also included in the table are the $\mathrm{pH}$ values of the outflows measured at the end of the flow testing at $345 \mathrm{kPa}$ injection pressure. The $\mathrm{pH}$ values of the outflows collected from Samples B/AL-C-4-25/B, $B / A L-C-4-25 / C$ and $B / A L-C-4-35 / B$ appear to be lower than those for the other samples. The lower $\mathrm{pH}$ values seem to be associated with higher flow rates. The de-aired distilled water yields a $\mathrm{pH}$ value of 6.36 , which increases to 7.31 when measured at room temperature after the water has been boiled in the presence of crushed tuff. Crushed tuff particles used in the boiling range from 12.7 to $19.05 \mathrm{~mm}$ in size. The boiling was maintained for 30 minutes, at room pressure.

\subsubsection{Mixed Samples in Stainless Steel Permeameters}

Crushed tuff of gradation type A and with 25 and 35 bentonite weight percent was selected to prepare four samples in stainless steel permeameters. Compactive efforts equivalent to the Standard Proctor method were applied to the samples, i.e. 25 blows per layer for the $101.6 \mathrm{~mm}$ ( 4 in) samples and 100 blows per layer for the $203.2 \mathrm{~mm}$ ( $8 \mathrm{in}$ ) samples. After inserting piston and capping plate, the samples were subjected to a $2.5 \mathrm{~m}$ water head from the bottom port for saturation. The saturation process was aided intermittently by applying vacuum from the top port, at a vacuum of $103.5 \mathrm{kPa}$ ( $15 \mathrm{psi}$ ) for 30 to 45 minutes. During the saturation, the pistons gradually moved upward, responding to the swelling of samples. Such movements were minimized by filling the remaining space between piston and top cap plate with water.

3.4.1.8.1 Double-Pipette Falling Head Flow Testing. The results are presented in Figures 3.69 and 3.70. The samples are tested at room temperature using the double-pipette falling head method. The inflow 
Table 3.7 Inflow-Outflow Record for Bentonite Plugs

\begin{tabular}{|c|c|c|c|c|c|c|c|c|}
\hline \multirow{2}{*}{$\begin{array}{c}\text { Sample Number: } \\
\text { Injection Pressure } \\
(\mathrm{kPa} / \mathrm{ps} i)\end{array}$} & \multicolumn{2}{|c|}{$B-C-1-A-S$} & \multicolumn{2}{|c|}{$\mathrm{B}-\mathrm{C}-1 \quad 3 / 8-\mathrm{A}-\mathrm{S}$} & \multicolumn{2}{|c|}{$\mathrm{B}-\mathrm{C}-2$ 3/8-A-S } & \multicolumn{2}{|c|}{$B-C-4-A-S$} \\
\hline & $\begin{array}{l}F_{\text {in }} \\
(c c)\end{array}$ & $\begin{array}{l}\text { Fout } \\
\text { (cc) }\end{array}$ & $\begin{array}{l}F_{\text {in }} \\
(\mathrm{cc})\end{array}$ & $\begin{array}{l}F_{\text {out }} \\
\text { (cc) }\end{array}$ & $\begin{array}{l}F_{\text {in }} \\
(c c)\end{array}$ & $\begin{array}{l}\text { Fout } \\
\text { (cc) }\end{array}$ & $\begin{array}{l}F_{\text {in }} \\
(c c)\end{array}$ & $\begin{array}{l}\text { Fout } \\
\text { (cc) }\end{array}$ \\
\hline $68.9 / 10$ & $\therefore$ & - & - & - & - & - & - & - \\
\hline \multirow[t]{2}{*}{$137.9 / 20$} & - & - & - & - & - & - & 5.32 & 8.02 \\
\hline & & & & & & & \multicolumn{2}{|c|}{ ( 31.8 days) } \\
\hline \multirow[t]{2}{*}{$206.8 / 30$} & 0.51 & 0.19 & 1.39 & 2.55 & 3.01 & 3.23 & 6.84 & 0.80 \\
\hline & \multicolumn{2}{|c|}{ (67.9 days) } & \multicolumn{2}{|c|}{ (51.0 days) } & \multicolumn{2}{|c|}{ ( 60.5 days) } & \multicolumn{2}{|c|}{ (29.2 days) } \\
\hline $413.7 / 60$ & 1.14 & 1.19 & 2.66 & 1.99 & 4.56 & 3.39 & 1.39 & 1.60 \\
\hline & \multicolumn{2}{|c|}{ (52.0 days) } & \multicolumn{2}{|c|}{ (32.1 days) } & \multicolumn{2}{|c|}{ (32.0 days) } & \multicolumn{2}{|c|}{ (3.3 days) } \\
\hline \multirow[t]{2}{*}{$620.5 / 90$} & 0.76 & 0.48 & 1.52 & 1.52 & 3.04 & 1.37 & 15.34 & 15.74 \\
\hline & \multicolumn{2}{|c|}{ (19.1 days) } & \multicolumn{2}{|c|}{ (14.5 days) } & \multicolumn{2}{|c|}{ (18.4 days) } & \multicolumn{2}{|c|}{ (18.0 days) } \\
\hline TOTAL: & $2 . .41$ & 1.86 & 5.57 & 6.06 & 10.61 & 7.99 & 28.89 & 26.16 \\
\hline
\end{tabular}

NOTES :

1. The actual values of the saturated water content, saturated bulk density, porosity, and sample length for B-C-4-A-S may be somewhat different from the values indicated in the table. This sample is not fully confined vertically on the top. Upon saturation, bentonite may expand through the annulus between a sintered bronze porous plate $(10.16 \mathrm{~cm}$ in diameter) and the permeameter wall into the upper portion of the permeameter chamber. The upward movement of the porous plate is prevented by inserting a $3.8 \mathrm{~cm}$ diameter stainless steel solid rod against the top cap plate.

2. The first numbers in the inflow and outflow columns give the total inflow and outflow from the preceding flow tests under lower injection pressures.

3. The number in parentheses indicates test period in days.

4. Fin: cumulative inflow; Fout: cumulative outflow. 
Table 3.8 Measurements of Solids Carried in the Outflows of Samples B/AL-C-4-25/A and B/AL-C-4-25/B (Second Test Sequence)

\begin{tabular}{lcccc}
$\begin{array}{l}\text { Sample } \\
\text { Number }\end{array}$ & $\begin{array}{c}\text { Injection } \\
\text { Pressure } \\
(\mathrm{kPz} / \mathrm{ps})\end{array}$ & $\begin{array}{c}\text { Outflow } \\
\text { Volume } \\
(\mathrm{cc})\end{array}$ & $\begin{array}{c}\text { Weight of } \\
\text { Solids } \\
(\mathrm{g})\end{array}$ & $\begin{array}{c}\text { Concentration } \\
\text { (8 per 100 cc) }\end{array}$ \\
\hline \multirow{3}{*}{ B/AL-C-4-25/A } & $690 / 100$ & 21.8 & 0.05 & 0.23 \\
& & 25.2 & 0.05 & 0.20 \\
B/AL-C-4-25/B & $552 / 80$ & 25.9 & 0.12 & 0.46 \\
& & 26.5 & 0.13 & 0.50 \\
& $690 / 100$ & 13.0 & 0.10 & 0.77 \\
& & 18.75 & 0.14 & 0.75 \\
& & 19.2 & 0.12 & 0.625 \\
& & 31.2 & 0.14 & 0.45 \\
\hline
\end{tabular}


Table 3.9 Flow Rates and Solids Carried in the Outflows of Mixed Samples with 258 and 358 Bentonite by Weight. (Second test sequence, after being subjected to injection pressures up to $690 \mathrm{kPa}$ )

\begin{tabular}{|c|c|c|c|c|}
\hline Sample Number & $\begin{array}{c}\text { Hydraulic } \\
\text { Gradient }\end{array}$ & $\begin{array}{c}\text { Flow Rate } \\
\left(x 10^{-4} \mathrm{cc} / \mathrm{s}\right) \\
\text { Mean } \pm \text { S.D. }\end{array}$ & $\begin{array}{c}\text { Solids in } \\
\text { Outflow } \\
\text { (g per } 100 \mathrm{cc})\end{array}$ & $\mathrm{pH}$ \\
\hline$B / A L-C-4-25 / A$ & $343-347$ & $0.818 \neq 0.102$ & $\begin{array}{l}0.09 \\
0.08 \\
0.075 \\
0.036\end{array}$ & 9.23 \\
\hline$B / A L-C-4-25 / B$ & $\begin{array}{l}209-211 \\
349-351\end{array}$ & $\begin{array}{l}1.445 \pm 0.142 \\
2.245 \pm 0.723\end{array}$ & $\begin{array}{l}0.26 \\
0.30 \\
0.12 \\
0.24 \\
0.24 \\
0.17 \\
0.18 \\
0.15 \\
0.08 \\
0.082 \\
0.075 \\
0.088 \\
0.12\end{array}$ & $\begin{array}{l}8 \\
8.89\end{array}$ \\
\hline $\mathrm{B} / \mathrm{AL} / \mathrm{C}-4-25 / \mathrm{C}$ & $310-314$ & $0.977 \pm 0.167$ & $\begin{array}{l}0.076 \\
0.083 \\
0.083 \\
0.081\end{array}$ & 9.06 \\
\hline$B / A L-C-4-35 / A$ & $333-337$ & $0.401 \pm 0.122$ & $\begin{array}{l}0.087 \\
0.08\end{array}$ & 9.25 \\
\hline$B / A L-C-4-35 / B$ & $321-325$ & $0.508 \pm 0.087$ & $\begin{array}{l}0.091 \\
0.082\end{array}$ & 9.03 \\
\hline $\mathrm{B} / \mathrm{AL}-\mathrm{C}-4-35 / \mathrm{C}$ & $342-347$ & $0.404 \pm 0.133$ & $\begin{array}{l}0.086 \\
0.081\end{array}$ & 9.15 \\
\hline
\end{tabular}

${ }^{*}$ S.D. = Standard Deviation

1) The first three samples consist of $312.5 \mathrm{~g}$ (air-dried) bentonite and crushed tuff. The latter three samples consist of $437.5 \mathrm{~g}$ bentonite and $812.5 \mathrm{~g}$ crushed tuff. The last letter shown in the sample number designation indicates the type of crushed tuff gradation used for mixing.

2) The air-dried bentonite has a moisture content of $9.5 \%$ 


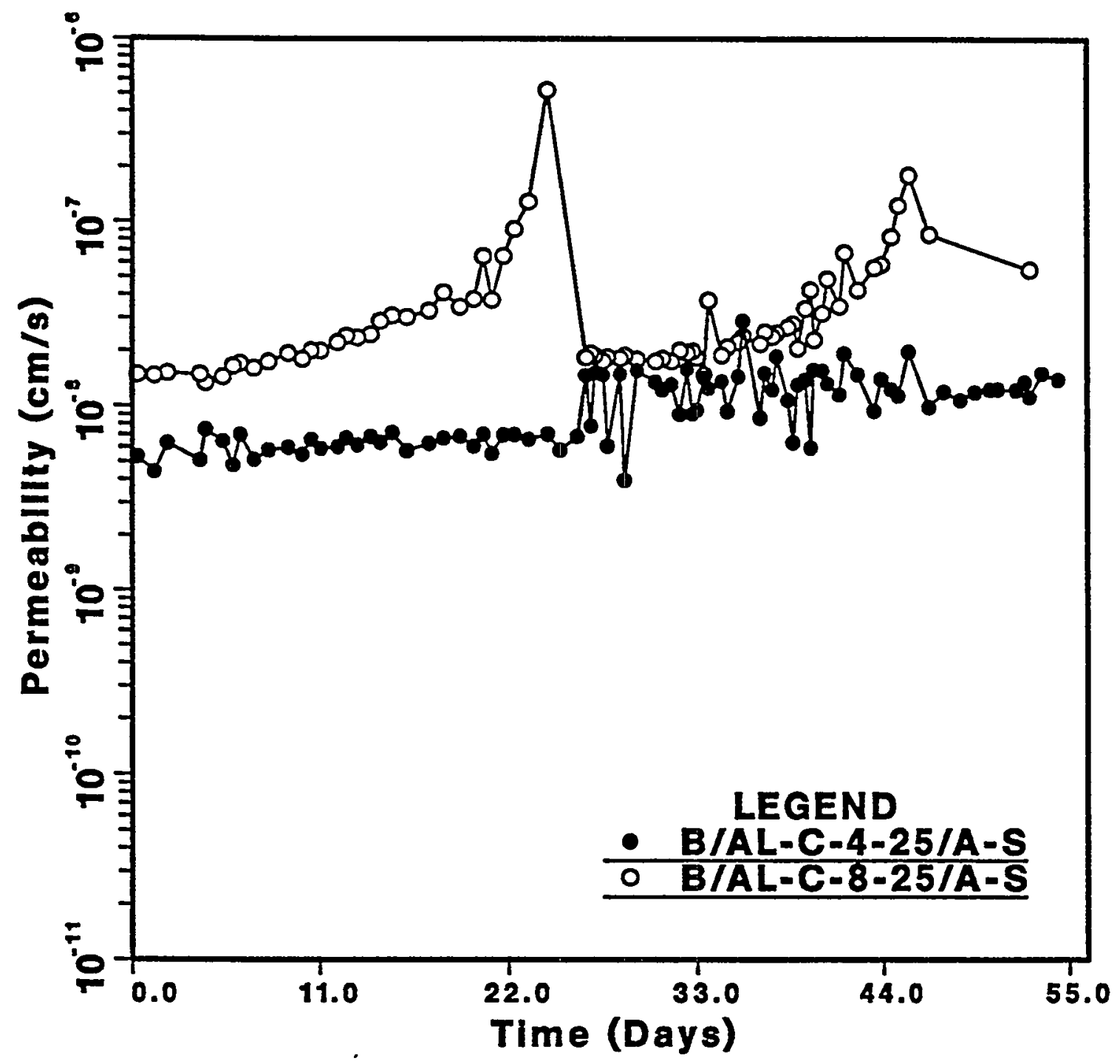

Figure 3.69 Permeability results of the mixture samples installed in stainless steel permeameters ( 258 bentonite by weight). Hydraulic gradient: 6-9.75 for B/AL-C-4-25/A-S and 0.1-6.5 for $B / A L-C-8-25 / A-S$. 


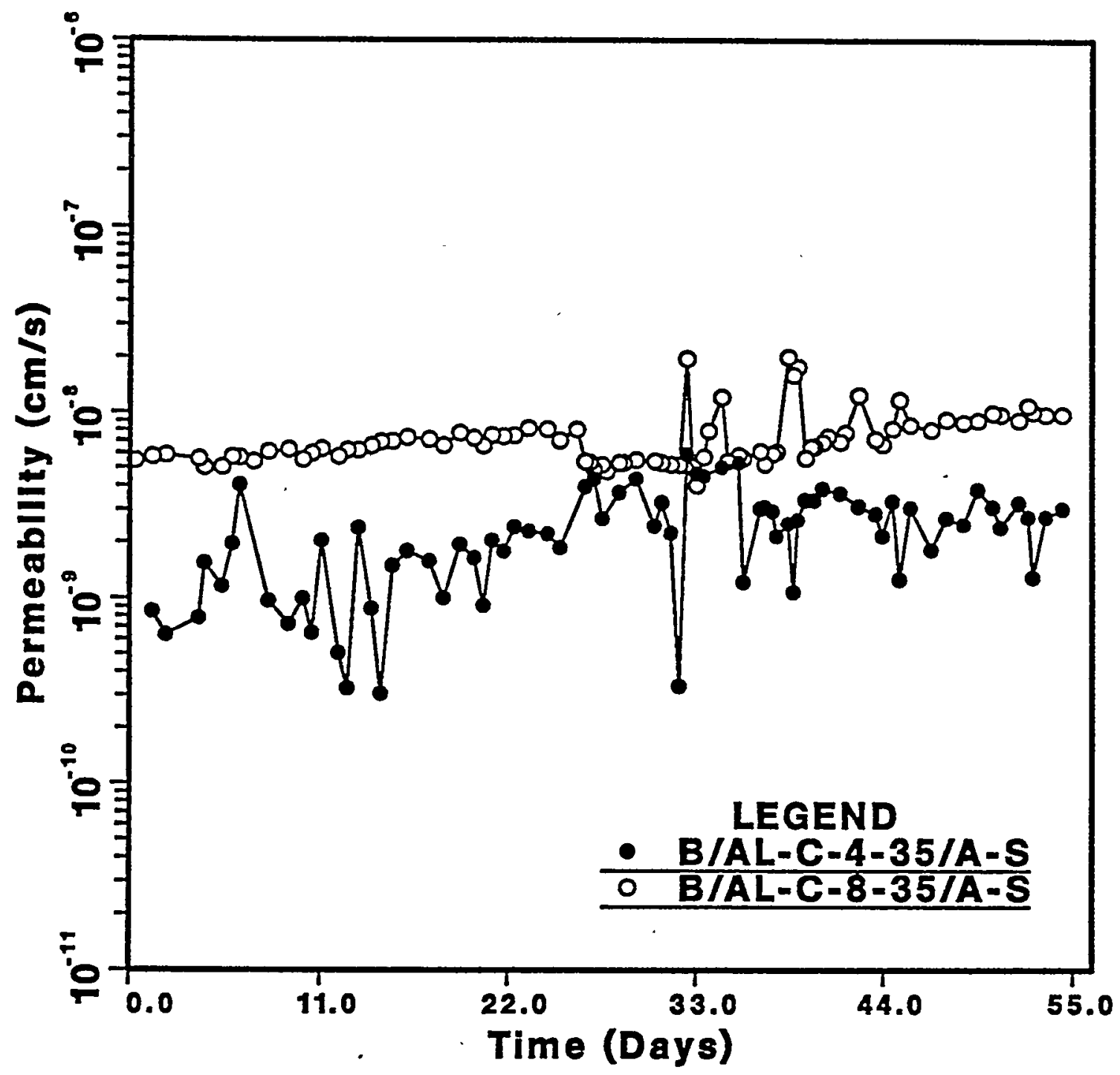

Figure 3.70 Permeability results of mixture samples installed in stainless steel permeameters ( $35 \%$ bentonite by weight). Hydraulic gradient: 9.5-10.6 for $\mathrm{B} / \mathrm{AL}-\mathrm{C}-4-35 / \mathrm{A}-\mathrm{S}$ and $1.5-6$ for $\mathrm{B} / \mathrm{AL}-\mathrm{C}-8-35 / \mathrm{A}-\mathrm{S}$. 
and outflow measurements are shown in Figures 3.71 and 3.72. Sample dimensions and bulk densities before and after saturation are summarized in Table 3.10 .

B/AL-C-8-25/A-S has the lowest bulk density $\left(1.582 \mathrm{~g} / \mathrm{cm}^{3}\right)$ among the four samples and exhibits comparatively high permeability, on the order of $10^{-8} \mathrm{~cm} / \mathrm{s}$. It was noted that, while compacting this sample $(203.2 \mathrm{~mm}$ in diameter), the material in the vicinity of the contact zone heaved with each impact. Lateral movements of particles also were associated with the heaving. The compactor has a rammer of $50.8 \mathrm{~mm}$ ( $2 \mathrm{in}$ ) in diameter and is in accordance with the specifications of ASTM standard D698-78. For samples of $101.6 \mathrm{~mm}$ ( $4 \mathrm{in}$ ) in diameter, each impact of the rammer covers $1 / 4$ of the cross-sectional area. The same rammer covers only $1 / 16$ of the sample area when compacting $203.2 \mathrm{~mm}$ ( 8 in) plugs. The same compaction procedure has been applied to the other $203.2 \mathrm{~mm}$ sample which contains 35 bentonite weight percent (Sample B/AL-C-8-35A-S). In this case, the heaving and the lateral movements of particles seemed to be minimal and the coarse material appeared to be anchored in the fine particles during the compaction. After compaction this sample yielded a bulk density of $1.74 \mathrm{~g} / \mathrm{cm}^{3}$.

For the same bentonite content, the $203.2 \mathrm{~mm}$ ( $8 \mathrm{in}$ ) samples have higher permeability than the $101.6 \mathrm{~mm}$ (4 in) samples. The difference may be due to the variations in compaction (e.g. number of blows) and in the ratio of grain size to permeameter diameter. It may also suggest a possible size effect. Additional tests are needed to confirm the statistical validity of a size effect. If such size effect observations are confirmed, flow tests on larger diameter samples would be warranted.

The break in the curves in Figures 3.71 and 3.72 corresponds to the refilling of the inflow pipette preceded by vacuuming the sample at a vacuum of $103.5 \mathrm{kPa}$ for 30 to 45 minutes. After the vacuuming, the permeability of Sample B/AL-C-4-25/A-S appears to increase, while that of Sample B/AL-C-8-35/A-S seems to decrease. The corresponding changes in permeability can be seen in Figures 3.71 and 3.72 .

3.4.1.8.2 Effect of the Upward Seepage Force on Sealing Performance. Samples B/AL-C-4-25/A-S, B/AL-C-8-25/A-S, and B/AL-C-8-35/A-S have been subjected to the downward-upward flow testing to further investigate the effect of the upward seepage force. The permeabilities have been determined using the double pipette falling head method. The hydraulic gradients are kept low (less than 10) to minimize changes in sample structure. The upward permeability and the downward permeability are compared in Figures 3.73 and 3.74. For all three samples, the upward permeability appears to be roughly three times higher than the downward permeability. The negative effect of the upward seepage force on the sealing ability of the sealants is apparent. Such a sealing performance evaluation is necessary and of importance since an upward flow situation is likely to occur for a nuclear waste repository (Bonne et al., 1985).

3.4.1.8.3 High Injection Pressure Flow Testing on Samples B/AL-C-825/A-S and B/AL-C-8-35/A-S. The flow testing of Samples B/AL-C-8-25/A-S and $B / A L-C-8-35 / A-S$ has been extended to evaluate the sealing performance under high injection water pressures. The maximum injection 

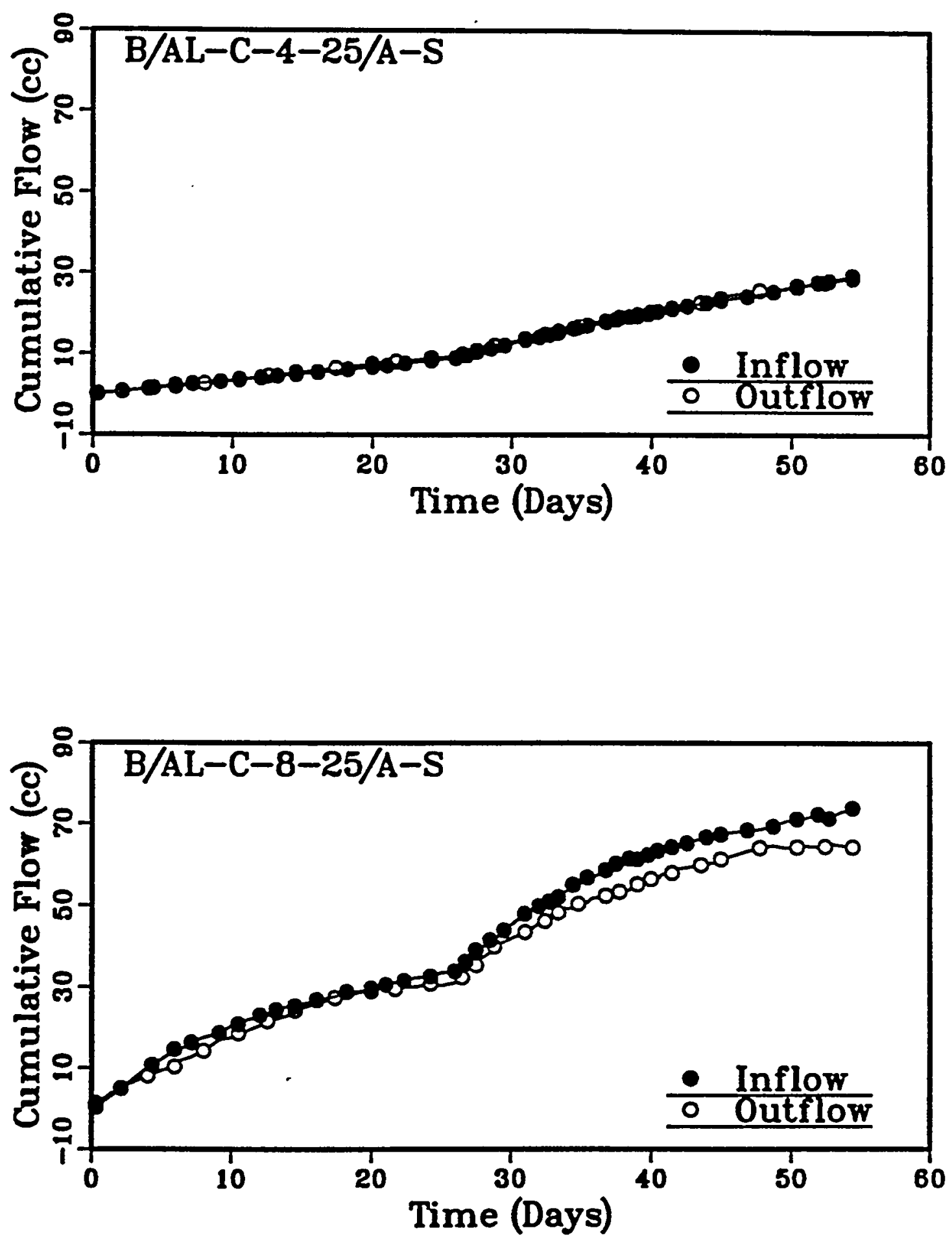

Figure 3.71 Cumulative inflow and outflow vs. time for Samples B/AL-C4-25/A-S and B/AL-C-8-25/A-S. The break in the curves corresponds to the refilling of the inflow pipette preceded by vacuuming samples at a vacuum of $103.5 \mathrm{kPa}$ for 30 to 45 minutes. 

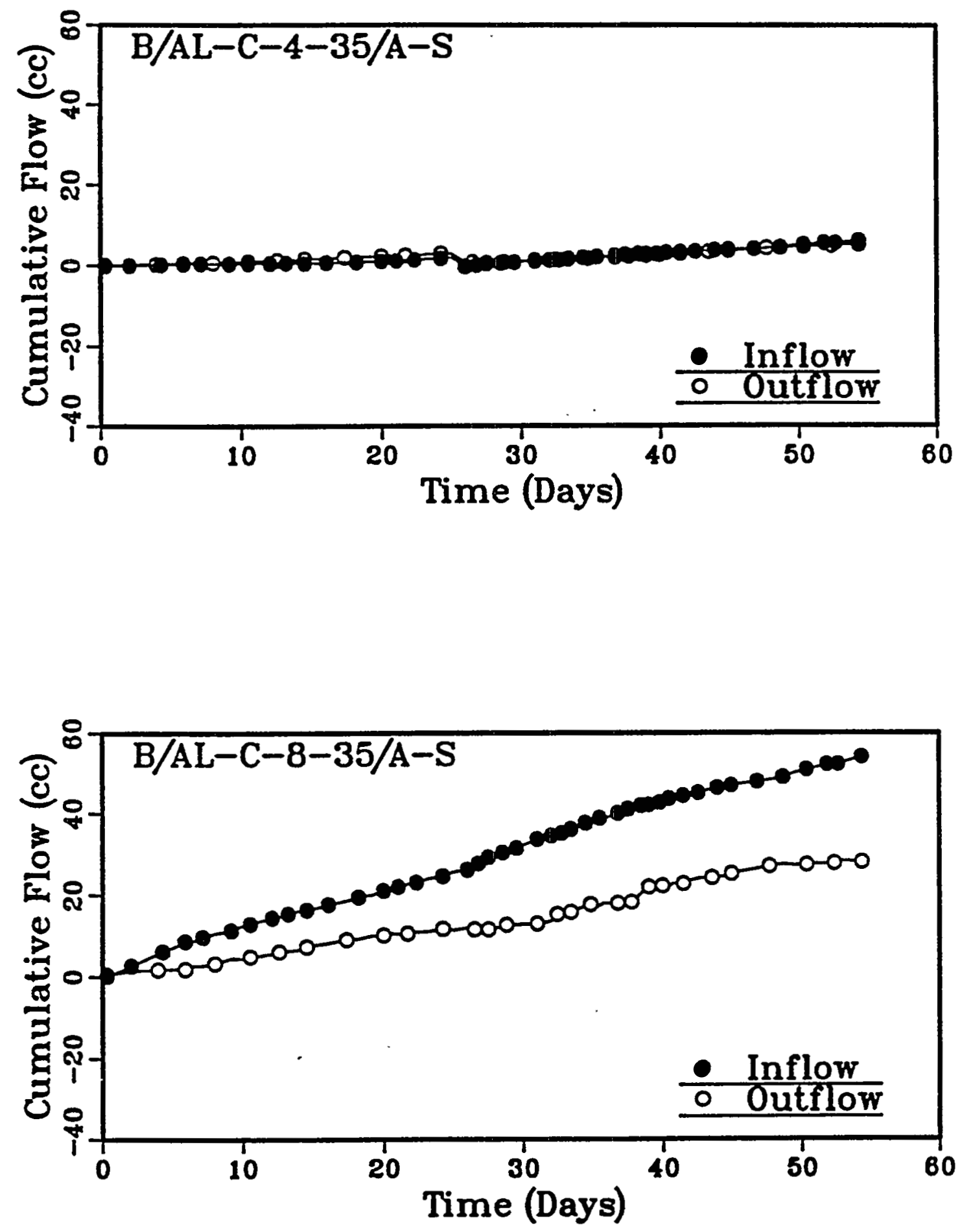

Figure 3.72 Cumulative inflow and outflow vs. time for Samples B/AL-C4-35/A-S and $B / A L-C-8-35 / A-S$. The break in the curves corresponds to the refilling of the inflow pipette preceded by vacuuming samples at a vacuum of $103.5 \mathrm{kPa}$ for 30 to 45 minutes. 
Table 3.10 Sample Dimensions and Bulk Density of Mixed Samples Installed in Stainless Steel Permeameters

\begin{tabular}{|c|c|c|c|c|c|}
\hline \multirow[b]{2}{*}{$\begin{array}{l}\text { Sample } \\
\text { Number }\end{array}$} & \multirow{2}{*}{$\begin{array}{c}\text { Sample } \\
\text { Diameter } \\
\text { (cm) }\end{array}$} & \multicolumn{2}{|c|}{ Sample Length $(\mathrm{cm})$} & \multicolumn{2}{|c|}{ Bulk Density $\left(g / \mathrm{cm}^{3}\right)$} \\
\hline & & $\begin{array}{c}\text { Before } \\
\text { Saturation }\end{array}$ & $\begin{array}{c}\text { After } \\
\text { Saturation }\end{array}$ & $\begin{array}{c}\text { Before } \\
\text { Saturation }\end{array}$ & $\begin{array}{l}\text { After } \\
\text { Saturation }\end{array}$ \\
\hline $\begin{array}{c}B / A L-C-4 \\
-25 / A-S\end{array}$ & 10.24 & 10.8 & 11.05 & 1.633 & 1.596 \\
\hline $\begin{array}{c}B / A L-C-8 \\
-25 / A-S\end{array}$ & 20.65 & 11.0 & 10.90 & 1.582 & 1.597 \\
\hline $\begin{array}{l}B / A L-C-4 \\
-35 / A-S\end{array}$ & 10.25 & 10.8 & 11.25 & 1.644 & 1.579 \\
\hline $\begin{array}{l}B / A L-C-8 \\
-35 / A-S\end{array}$ & 20.65 & 10.05 & 10.65 & 1.740 & 1.642 \\
\hline
\end{tabular}




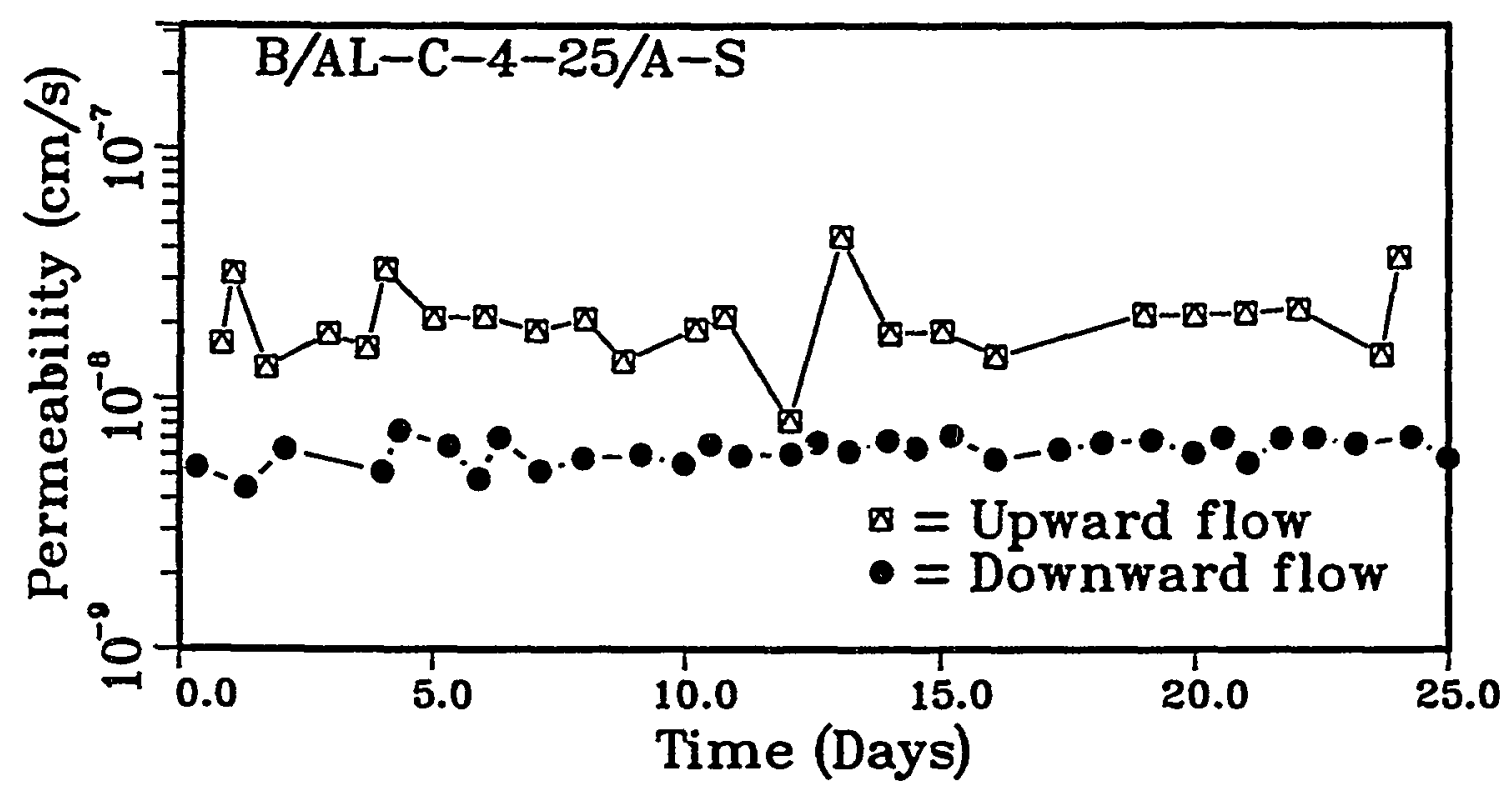

Figure 3.73 Upward and downward permeability of Sample B/AL-C-4-25/A-S. Hydraulic gradient: $1.78-5$ (upward), 6-9.75 (downward). 

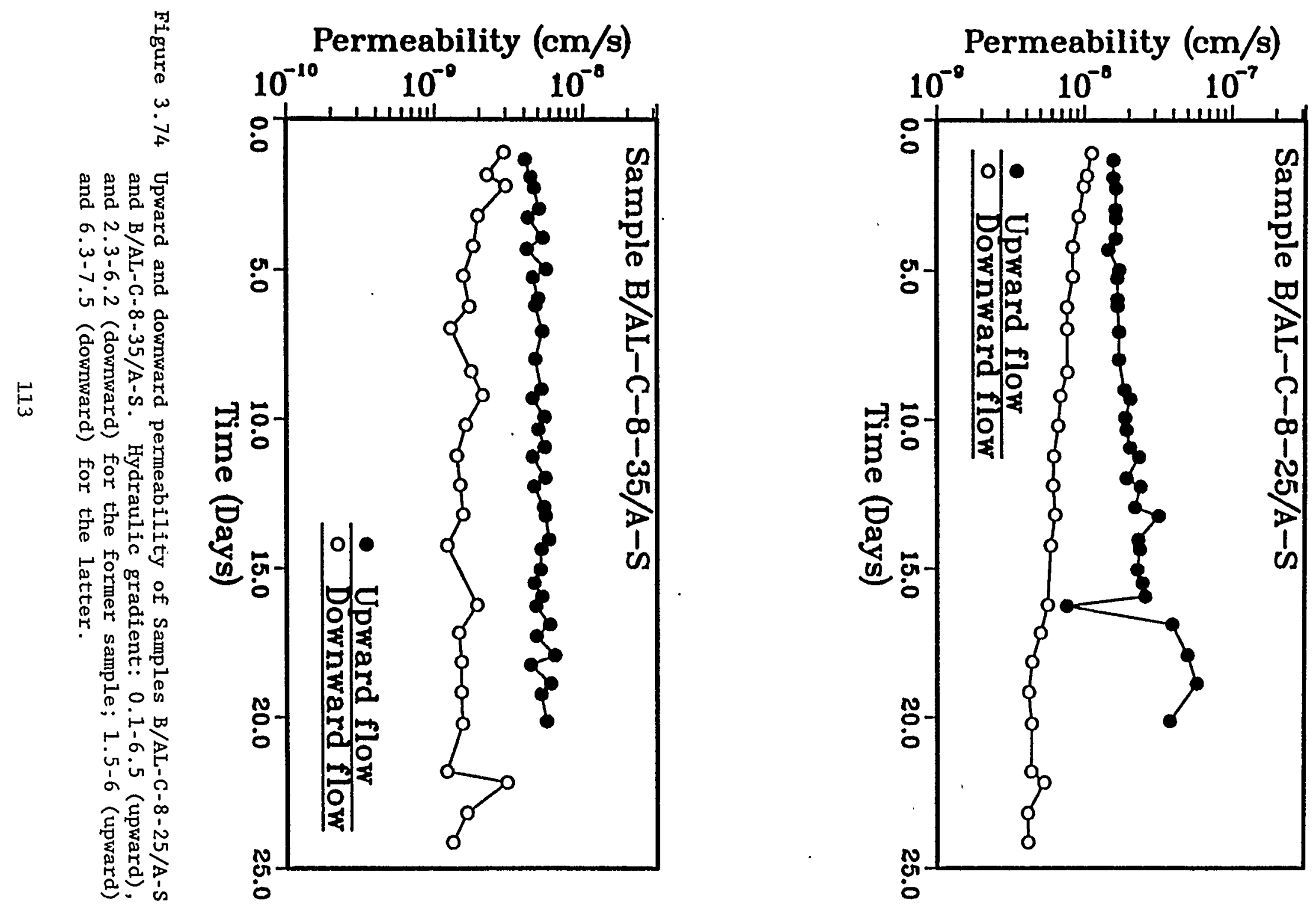
pressures applied are $0.83 \mathrm{MPa}$ (120 psi) for the first sample and 1.86 $\mathrm{MPa}$ (270 psi) for the second, equivalent to 84.5 and $190.2 \mathrm{~m}$ water columns (at $22^{\circ} \mathrm{C}$ ). The corresponding hydraulic gradients amount to 800 and 1800 . Permeability values and flow rates are plotted against hydraulic gradient in Figures 3.75 through 3.78. Permeability appears to be constant for relatively low hydraulic gradients (up to 150 for B/AL-C-8-25/A-S and 280 for B/AL-C-8-35/A-S), and decreases when higher gradients are imposed incrementally. The jumps in permeability shown in Figure 3.77 may result from the radial expansion of pores and hydraulic fracturing due to the quick large increases in injection pressure at which the jumps occur. The increases occur at $389.6 \mathrm{kPa}$ ( $56.5 \mathrm{psi}$ ) for the first permeability jump and $761.9 \mathrm{kPa}$ (110.5 psi) for the second one. No permanent damage in permeability can be detected, which may be indicative of the excellent healing capability of bentonite. The constant permeability implies, based on Darcy's law, a linear variation of flow rate with hydraulic gradient. Such a linear relation can be seen in Figure 3.78 for gradients up to about 250 . The constant permeability may also suggest that the lower hydraulic gradients induce no significant changes in sample structure. The higher hydraulic gradients introduced later start to alter the structure. The permeability reduction may be attributed to bentonite compression and consolidation. These mechanisms do not fully explain the relatively constant permeability measured under low hydraulic gradients. Bentonite slurries exhibit non-Newtonian flow behavior (Jones, 1963; Marsland and Loudon, 1963), which requires a driving pressure exceeding the yield stress for bentonite flow to start. The permeability decreases observed in Figures 3.75 and 3.77 may be explained if bentonite in the mixtures behaves as a non-Newtonian fluid. The assumption is confirmed when the samples are examined after the flow testing. Bentonite has flowed downward, leaving the top quarter of Sample B/AL-C-8-25/A-S comprised primarily of crushed tuff (Figure 3.79, left). This phenomenon is less developed in sample $B / A L-C-8-35 / A-S$, which consists of $35 \%$ bentonite, and can only be discerned by examining the subtle difference in texture between the sample's top and bottom (Figure 3.80).

The yield stress of a bentonite slurry depends on the bentonite concentration in the carrying fluids (Marsland and Loudon, 1963). Bentonites of different water content, therefore, have different yield stresses; the higher the water content, the lower the yield stress. Water contents of saturated bentonite in Samples B/AL-C-8-25/A-S and B/AL-C-8$35 / \mathrm{A}-\mathrm{S}$ are 1158 and 89.58 , respectively. The hydraulic gradient required for bentonite flow in the former sample is thus expected to be lower than for the latter. This reasoning is substantiated when comparing Figures 3.76 and 3.78 . The change of flow rate with hydraulic gradient behaves differently. The flow rate increases continuously up to the highest hydraulic gradient for B/AL-C-8-25/A-S. The flow rate of B/AL-C-8-35/A-S becomes relatively constant for hydraulic gradients from 400 to 800 . The different behaviors suggest that comparatively more permeable channels may exist in Sample B/AL-C-8-25/A-S, which contains only $25 \%$ bentonite. These permeable channels may have been spared from clogging by fine particles and bentonite filling as the pore pressure in the channels would be higher than in the surrounding material because of the smaller head loss. The channels might have maintained a relatively constant permeability throughout the range of hydraulic gradients, so 


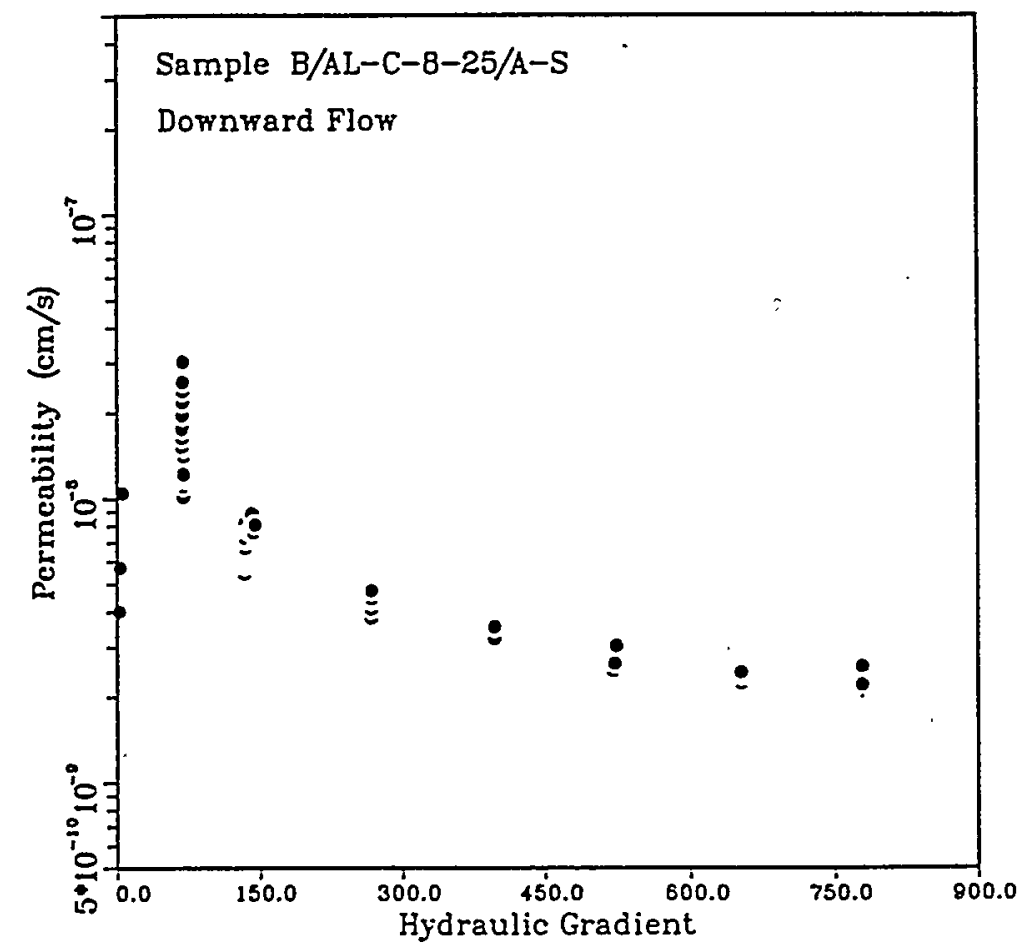

Figure 3.75 Permeability vs. hydraulic gradient for Sample $\mathrm{B} / \mathrm{AL}-\mathrm{C}-8-25 / \mathrm{A}-\mathrm{S}$.

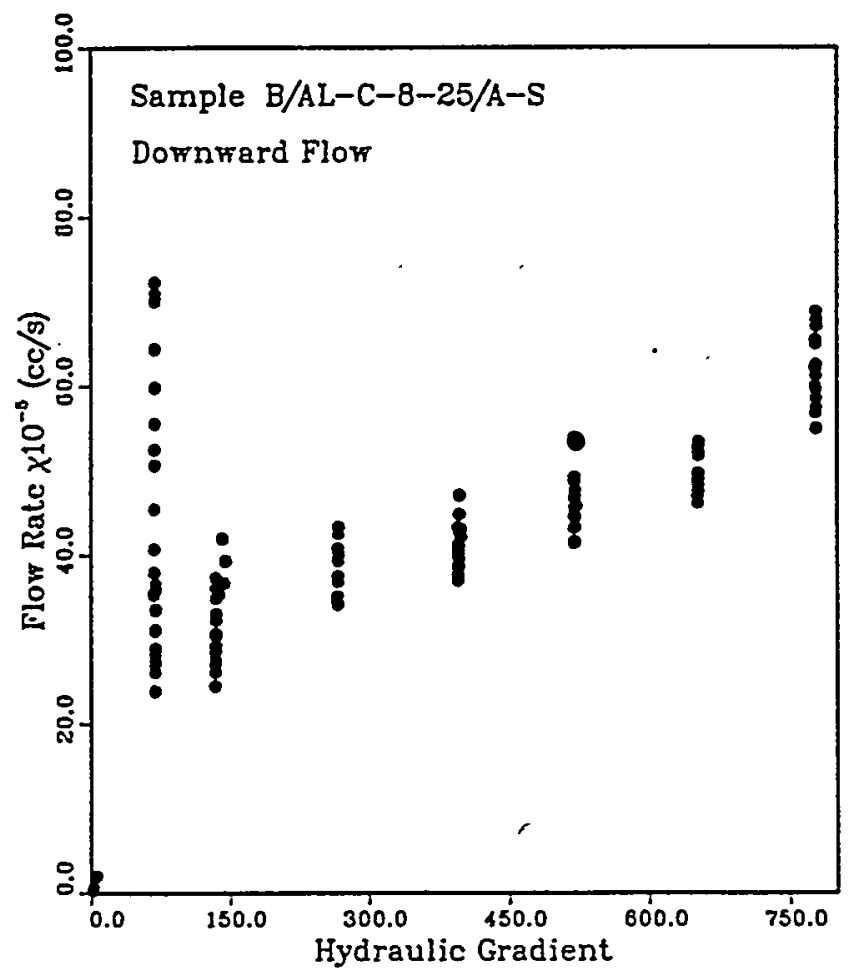

Figure 3.76 Flow rate vs. hydraulic gradient for Sample B/AL-C-8-25/A-S . 


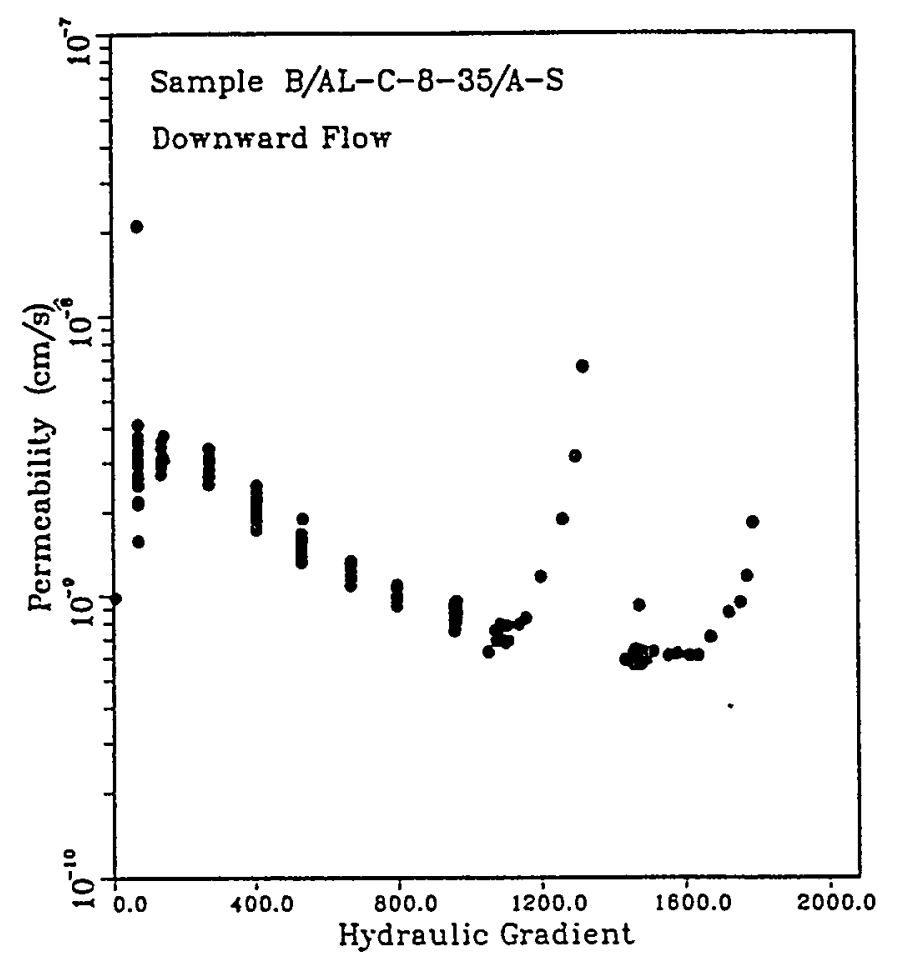

Figure 3.77 Permeability vs. hydraulic gradient for Sample B/AL-C-8-35/A-S .

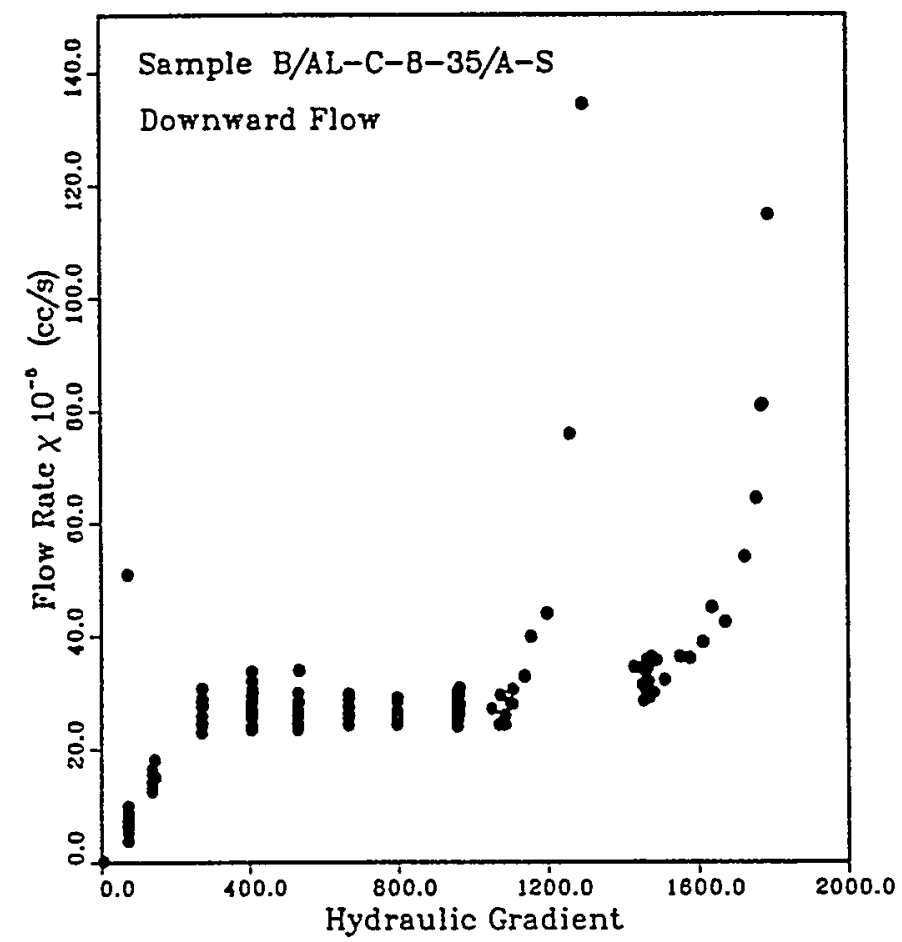

Figure 3.78 Flow rate vs. hydraulic gradient for Sample B/AL-C-8-35/A-S. 


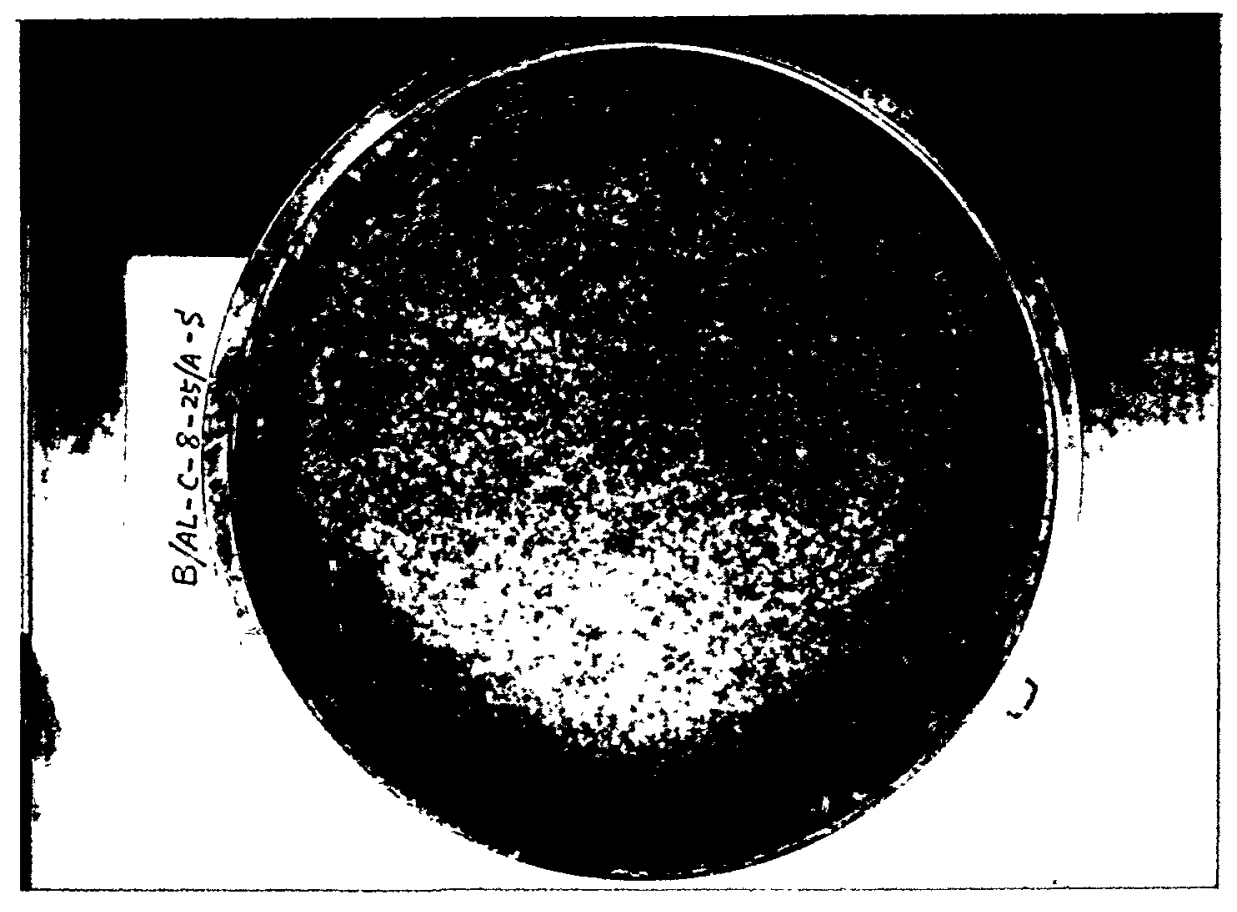

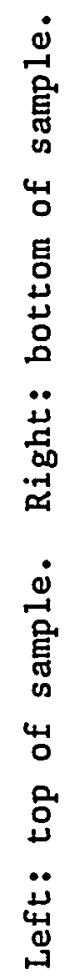

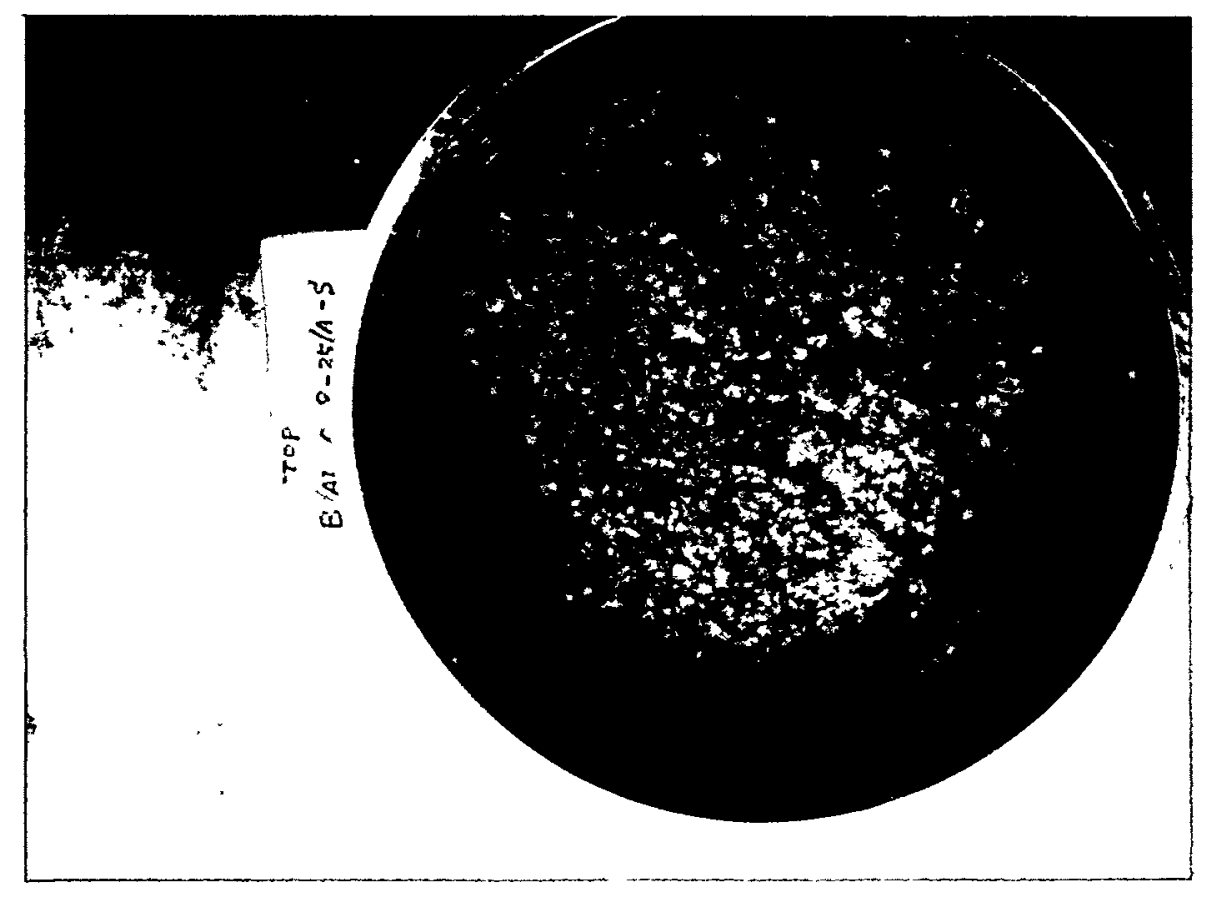

年 
that the flow rate continuously increases as hydraulic gradient increases. The permeable channels appear to be greatly reduced by increasing the bentonite content in the mixture, as demonstrated by the flow rate behavior of B/AL-C-8-35/A-S (Figure 3.78). The bentonite content in the mixtures accounts for 66.38 of the amount. required to fill the void space in between the crushed tuff particles for B/AL-C-8-25/A-S (258 bentonite weight) and 81.48 for B/AL-C-8-35/A-S ( 358 bentonite by weight).. The number and volume of unfilled voids is certainly higher in the former sample than in the latter.

\subsubsection{Flow Testing of Samples Containing Crushed Tuff with Ideal Gradation}

Flow tests have been conducted on an additional six samples to explore further the effect of grain size gradation on the sealing performance. The Fuller-Thompson equation (Eq. 2.2) has been adopted to prepare the crushed tuff portion. The Fuller-Thompson grading curve is considered to be an ideal grading which may result in the densest possible state of packing (Winterkorn, 1975; Head, 1980, p. 150). The Fuller-Thompson curve has been used in formulating backfill material for a nuclear waste disposal vault (Pusch and Alstermark, 1985; Yong et al., 1986).

3.4.1.9.1 Tests in PVC Permeameters. The crushed tuff used in the preparation of four samples follows a grading curve obtained using D $9.42 \mathrm{~mm}$ and $\mathrm{n}=0.5$. They include two each of 15 and of 25 bentonite weight percent. The flow tests are performed downward. The results are shown in Figure 3.81. The cumulative inflow-outflow balances of Samples $B / A L-C-4-15 / F A-A$ and $B / A L-C-4-15 / F A-B$ are given in Figure 3.82. These two samples have been tested ising the double-pipette falling head method. The constant head method with a compressed.gas (helium) pressurization system (Lambe, 1951, p. 58) has been. employed to test Samples B/AL-C-4-25/FA-A and B/AL-C-4-25/FA-B, which contain 258 bentonite by weight.

As shown in Figure 3.81, the permeability.values obtained for each pair of samples are consistent. The conformity may indicate the quality control of the sample preparation, installation and test procedures. The sharp jumps in permeability observed for B/AL-C-4-15/FA-A and B/AL-C-4-15/FA-B correspond to the refilling of the inflow pipette. A similar phenomenon has been reported in Section 3.4.1.8.3. Considering the good inflow-outflow balance (Figure 3.82), the sudden increase in permeability after the refilling is most likely due to hydraulic fracturing. The local fluctuations of permeability in Figure 3.81 correlate with room temperature variations, as illustrated in Figures 3.83 and 3.84. Using the same data, the permeabilities have been recalculated on a time-interval basis such that the temperatures at two ends of each interval are equal or approximately equal. Such a correction reduces the major permeability variations. The corrected permeabilities are represented by open circles in Figures 3.83 and 3.84 .

Samples B/AL-C-4-25/FA-A and B/AL-C-4-25/FA-B were further subjected to higher injection pressures up to $550 \mathrm{kPa}(80 \cdot \mathrm{psi})$. The permeabilities measured at higher gradients are summarized in Table 3.11. Also included in the table are the permeability values of Sample B/AL-C-425/A 


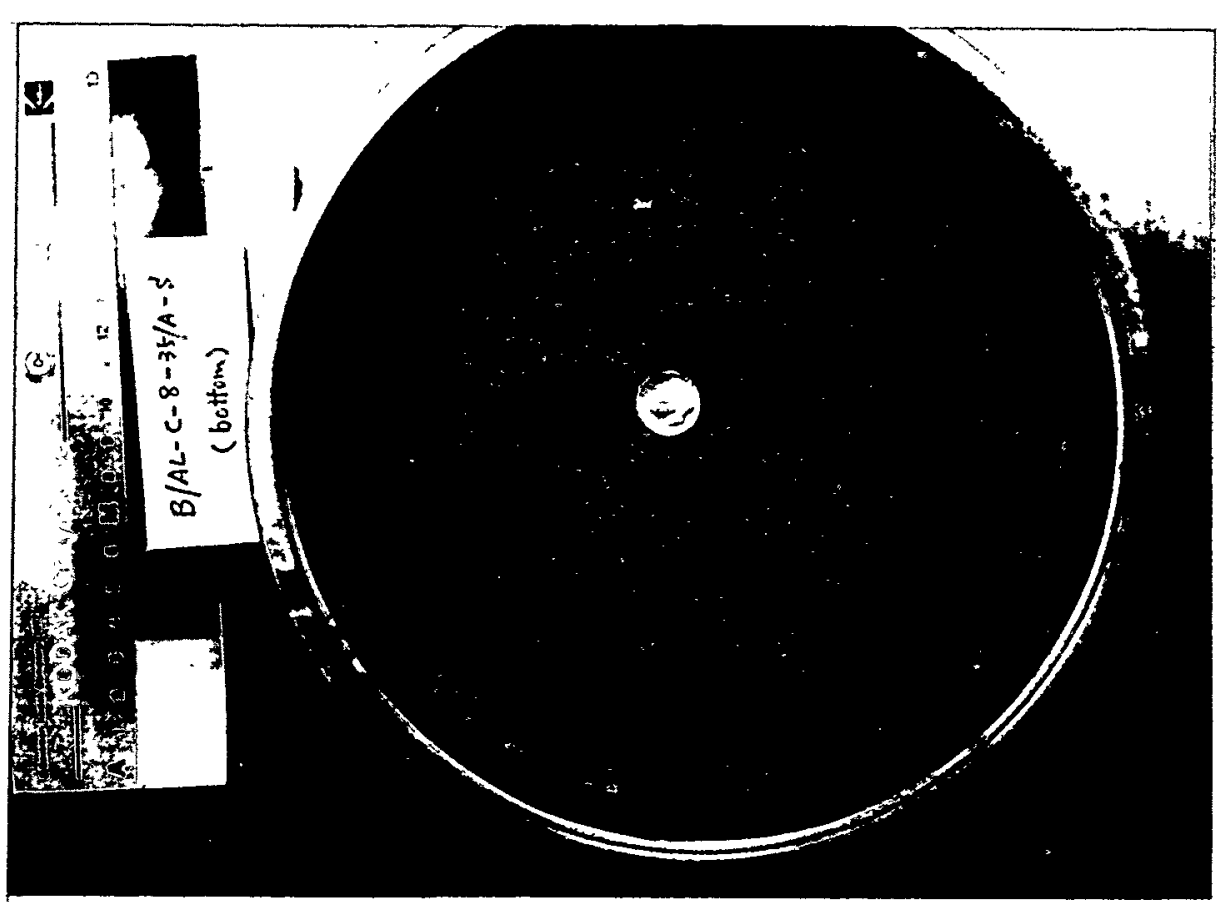

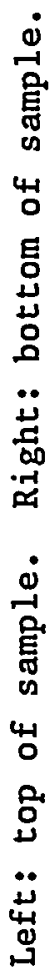

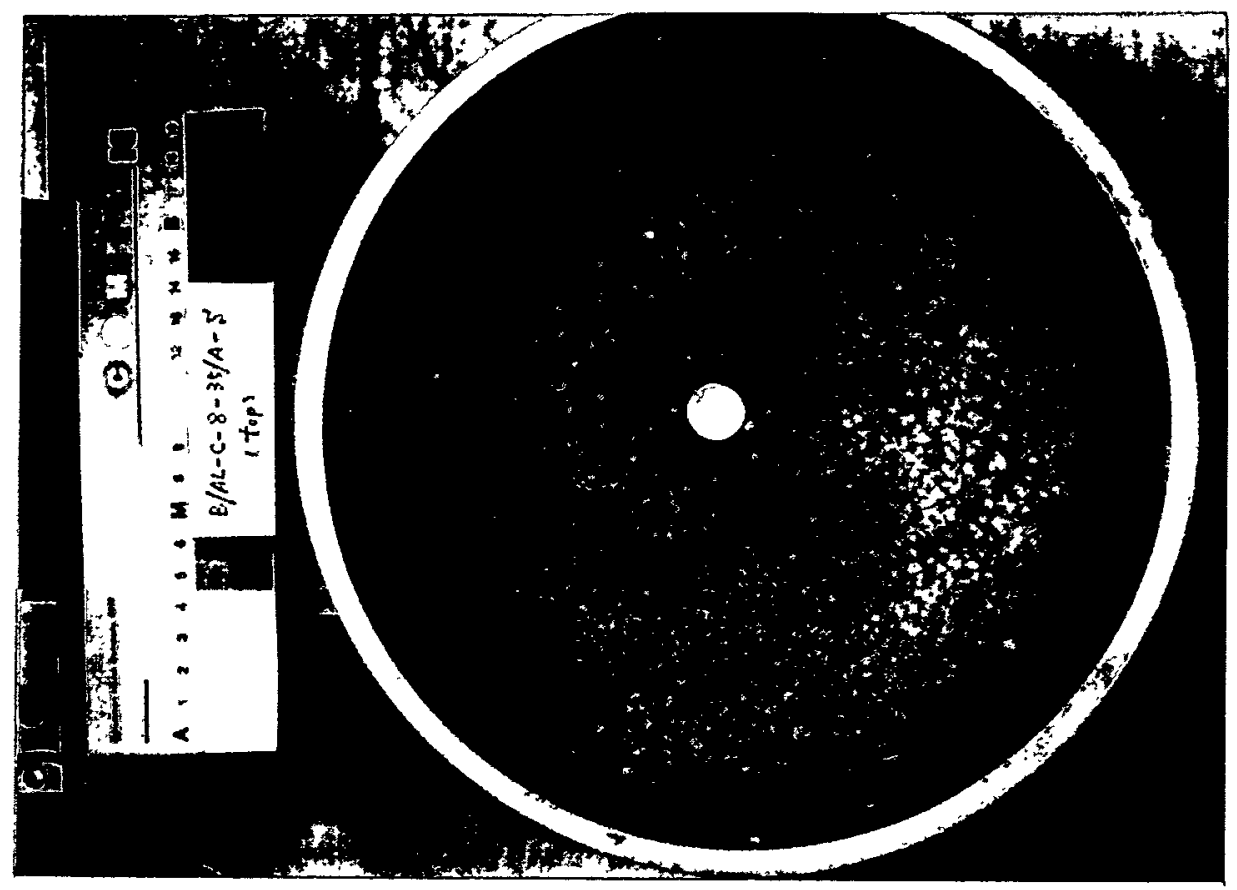

递

总

崩

.



啰 


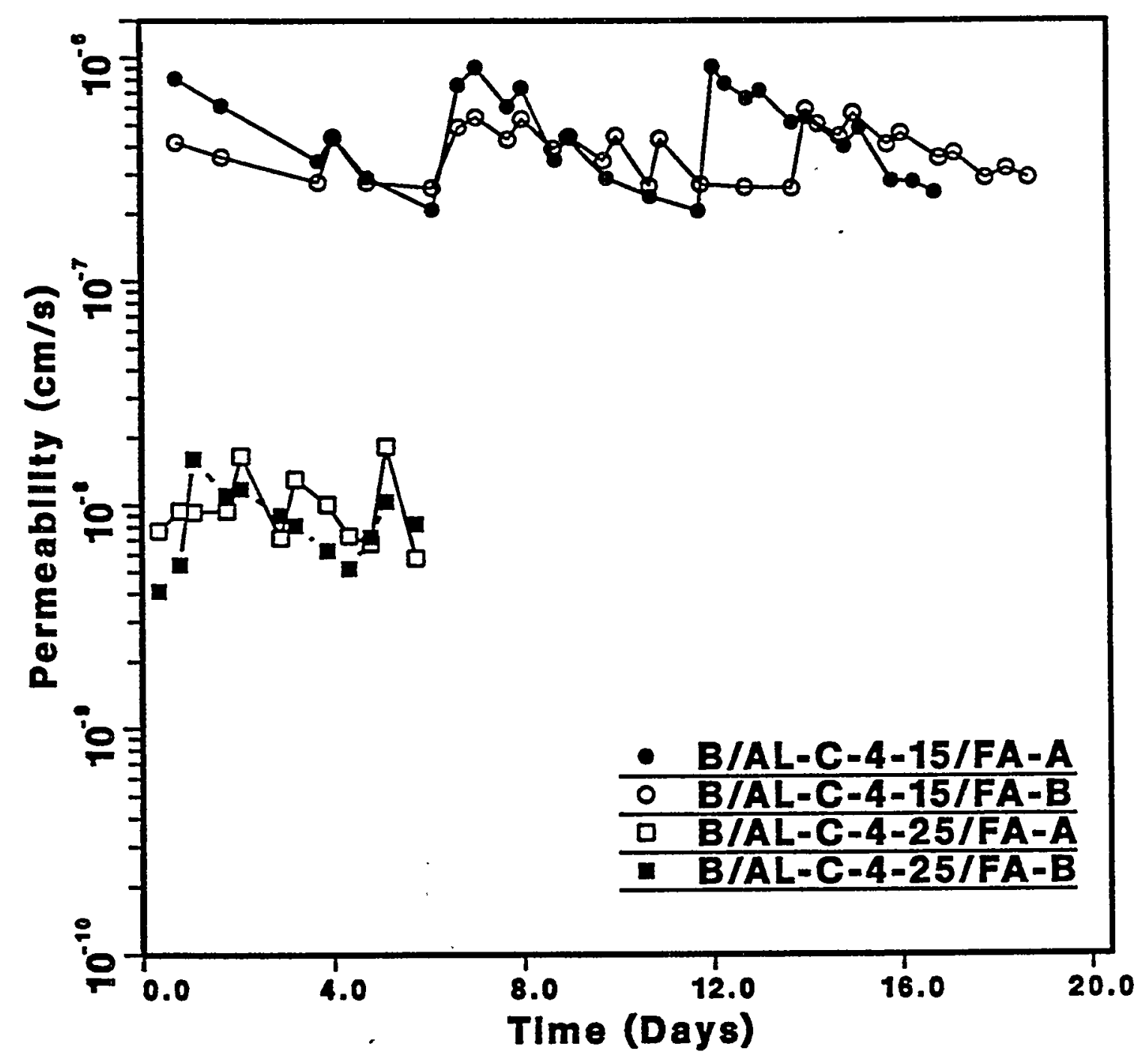

Figure 3.81 Permeability results of Samples B/AL-C-4-15/FA-A, B/AL-C-415/FA-B, B/AL-C-4-25/FA-A and B/AL-C-4-25/FA-B. The grading of the crushed tuff follows the Fuller-Thompson curve. Hydraulic gradient: $1-7,3-7,18-22$ and 17-21, respectively. 

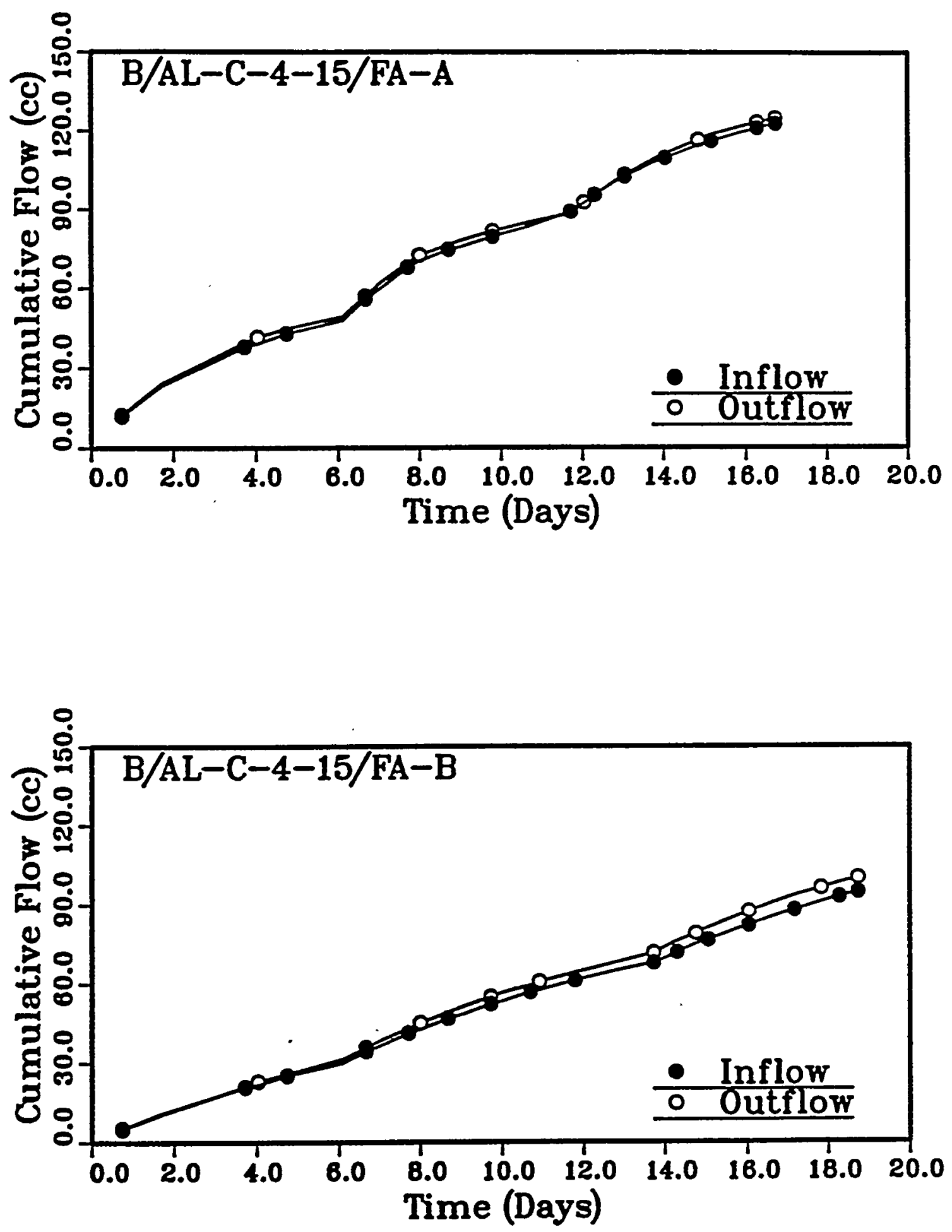

Figure 3.82 Cumulative inflow and outflow vs. time for Samples B/AL-C-415/FA-A and B/AL-C-4-15/FA-B. 


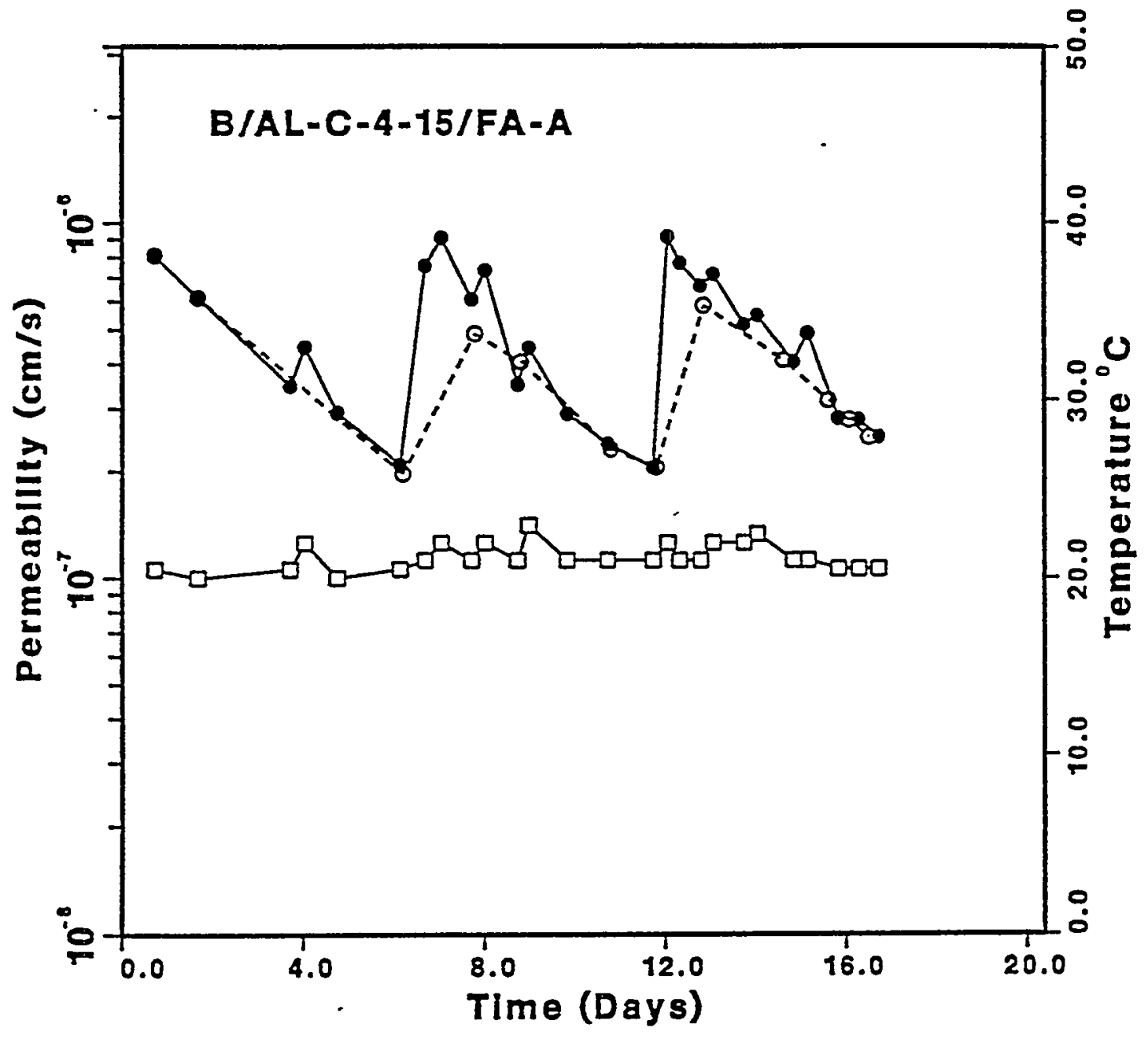

Figure 3.83 Room temperature effect on the permeability measurement of Sample B/AL-C-4-15/FA-A. Solid circles: original data points; open circles: temperature-corrected data points; open square: temperature. 


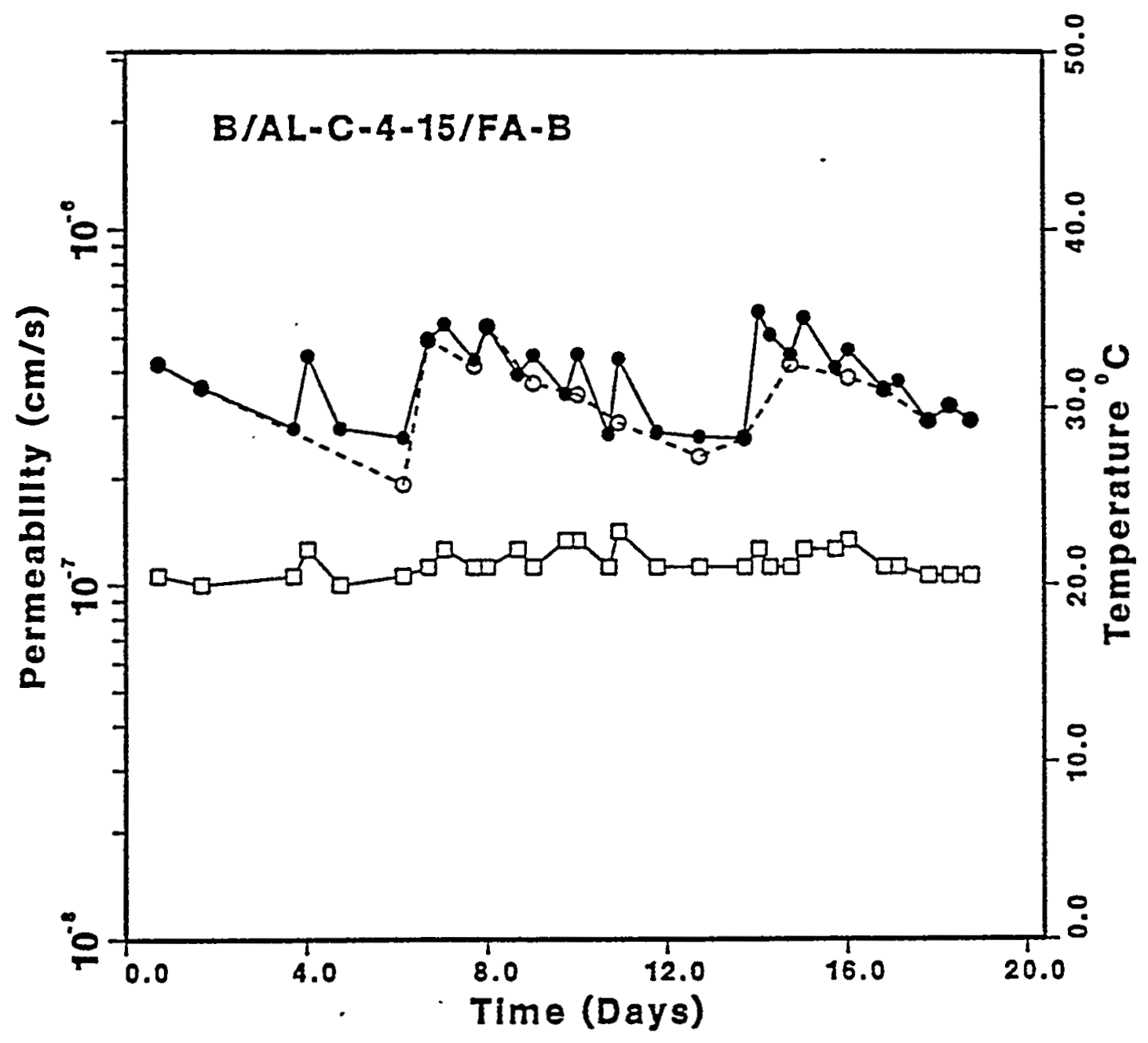

Figure 3.84 Room temperature effect on the permeability measurement of Sample B/AL-C-4-15/FA-B. Solid circle: original data point; open circle: corrected data point; open square: temperature. 
Table 3.11 Flow Test Results of Samples B/AL-C-4-25/FA-A, B/AL-C-4-25/FA-B and B/AL-C-4-25/A

\begin{tabular}{|c|c|c|c|c|c|c|}
\hline \multirow{2}{*}{$\begin{array}{l}\text { Injection } \\
\text { Pressure } \\
\text { (kPa/psi) }\end{array}$} & \multicolumn{2}{|c|}{ B $/ A L-C-4-25 / \mathrm{FA}-\mathrm{A}$} & \multicolumn{2}{|c|}{$\mathrm{B} / \mathrm{AL}-\mathrm{C}-4-25 / \mathrm{FA}-\mathrm{B}$} & \multicolumn{2}{|c|}{$B / A L-C-4-25 / A$} \\
\hline & $\begin{array}{l}\text { Hydraulic } \\
\text { Gradient }\end{array}$ & $\begin{array}{l}\text { Permeability } \\
\left(x 10^{-9} \mathrm{~cm} / \mathrm{s}\right)\end{array}$ & $\begin{array}{l}\text { Hydraulic } \\
\text { Gradient }\end{array}$ & $\begin{array}{l}\text { Permeability } \\
\left(x 10^{-9} \mathrm{~cm} / \mathrm{s}\right)\end{array}$ & $\begin{array}{l}\text { Hydraulic } \\
\text { Gradient }\end{array}$ & $\begin{array}{l}\text { Permeability } \\
\left(x 10^{-9} \mathrm{~cm} / \mathrm{s}\right)\end{array}$ \\
\hline $34.5 / 5$ & $43.7-39.9$ & $18-16$ & $40.1-36.7$ & $21-12$ & - & - \\
\hline $69 / 10$ & $80.3-77.7$ & $29-15$ & $74.3-71.4$ & $31-16$ & - & - \\
\hline $138 / 20$ & $151.2-148.5$ & $19-9.8$ & $138.9-136.4$ & $22-12$ & $140.3-139.6$ & $7.2-5.1$ \\
\hline $276 / 40$ & $294.2-290.5$ & $21-8$ & $270.2-266.8$ & $31-5$ & $278.6-276.8$ & $6.5-4.4$ \\
\hline $414 / 60$ & $441-435.9$ & $10-5.5$ & $405.2-400.5$ & $9-6$ & $415.4-409.6$ & $6.6-3.2$ \\
\hline
\end{tabular}

NOTE: The crushed tuff of Samples B/AL-C-4-25/FA-A and B/AL-C4-25/FA-B follows a Fuller-Thompson curve obtained using $D=9.42 \mathrm{~mm}$ and $\mathrm{n}-0.5$. The crushed tuff of Sample B/AL-C-4-25/A constitutes the type A gradation, which has $D_{\max }=9.42 \mathrm{~mm}$ and $D_{\min }=0.075 \mathrm{~mm}$. All three samples contain 258 bentonite by weight (air-dried) and were installed in $101.6 \mathrm{~mm}$ (4 in) dlameter PVC permeameters. 
for the same injection pressures. The two samples having ideal crushed tuff gradation do not necessarily seal better than the sample with type A crushed tuff gradation. It should be noted that the permeability results in Table 3.11 have been measured only in the downward permeation.

Upward flow tests also have been performed on the samples for the same injection pressures. Downward and upward flow test results are shown in Figures 3.85 through 3.92. The permeabilities measured in the downward direction range from $3 \times 10^{-8}$ to $6 \times 10^{-9} \mathrm{~cm} / \mathrm{s}$, and tend to decrease with increasing hydraulic gradient. The permeability reduction, however, is not as great as that exhibited by Sample B/AL-C-8-25/A-S (Figure 3.75). The flow rate behaviors depicted in Figures 3.86 (for B/AL-C-4-25/FA-A) and 3.90 (for B/AL-C-4-25/FA-B) are similar to that shown in Figure 3.76 for Sample B/AL-C-8-25/A-S. This can be expected as all three samples contain $25 \%$ bentonite by weight. The upward flow testing exhibits relatively constant and significantly lower (about one order of magnitude) permeability. The flow rate increases linearly over the range of hydraulic gradients imposed (Figures 3.88 and 3.92). Samples B/AL-C-425/FA and B/AL-C-4-25/FA-B had become overconsolidated prior to the upward flow testing previously, while being subjected to a $550 \mathrm{kPa}$ injection pressure in the downward flow testing. Similar results have been reported by Mitchell and Younger (1967) for the flow testing of loosely and densely compacted silty clay.

3,4,1,9,2 Tests in Stainless Steel Permeameters. The crushed tuff in Samples B/AL-C-8-25/FC-S (258 bentonite) and B/AL-C-8-35/FC-S (358 bentonite) follows a gradation curve obtained from Eq. (2.2) with $\mathrm{D}_{\max }=19.05 \mathrm{~mm}$ and $\mathrm{n}=0.5$. It is desirable to test such samples as larger particles are likely to be used in the field. The two samples are installed in $203.2 \mathrm{~mm}$ ( $8 \mathrm{in}$ ) diameter stainless steel permeameters so that the ratio of sample diameter to maximum particle size is kept greater than 10, minimizing the possibility of arching (Ouyang and Daemen, 1989, p. 32). The two samples received a compaction effort equivalent to one quarter of the standard Proctor compaction. The samples are sandwiched between two porous stones and two layers of clean sand such that the permeameter chambers are filled. After the initial downward flow testing, the samples are shaken and pounded to try to remove some entrapped air. Shaking and pounding has been pursued after an attempt to remove the air by applying a $69 \mathrm{kPa}$ (10 psi) vacuum failed. In subsequent upward flow testing, Sample B/AL-C-8-25/FC-S exhibits a larger permeability ( 3 to 5 orders of magnitude) while Sample B/AL-C-8-35/FC-S maintains a permeability in the lower $10^{-8} \mathrm{~cm} / \mathrm{s}$ range. The flow test results are shown in Table 3.12 .

\subsubsection{Polyaxial Flow Tests}

The upward and downward flow tests conducted on bentonite/crushed tuff sealants provide information about sealing performance in the longitudinal direction. Adequate sealing in the transverse direction may also be necessary to minimize lateral flow of groundwater or gases into any connected fracture system. Moreover, compromising of the sealing ability in the transverse direction ultimately may jeopardize the entire sealing performance if piping occurs radially. This consideration can 


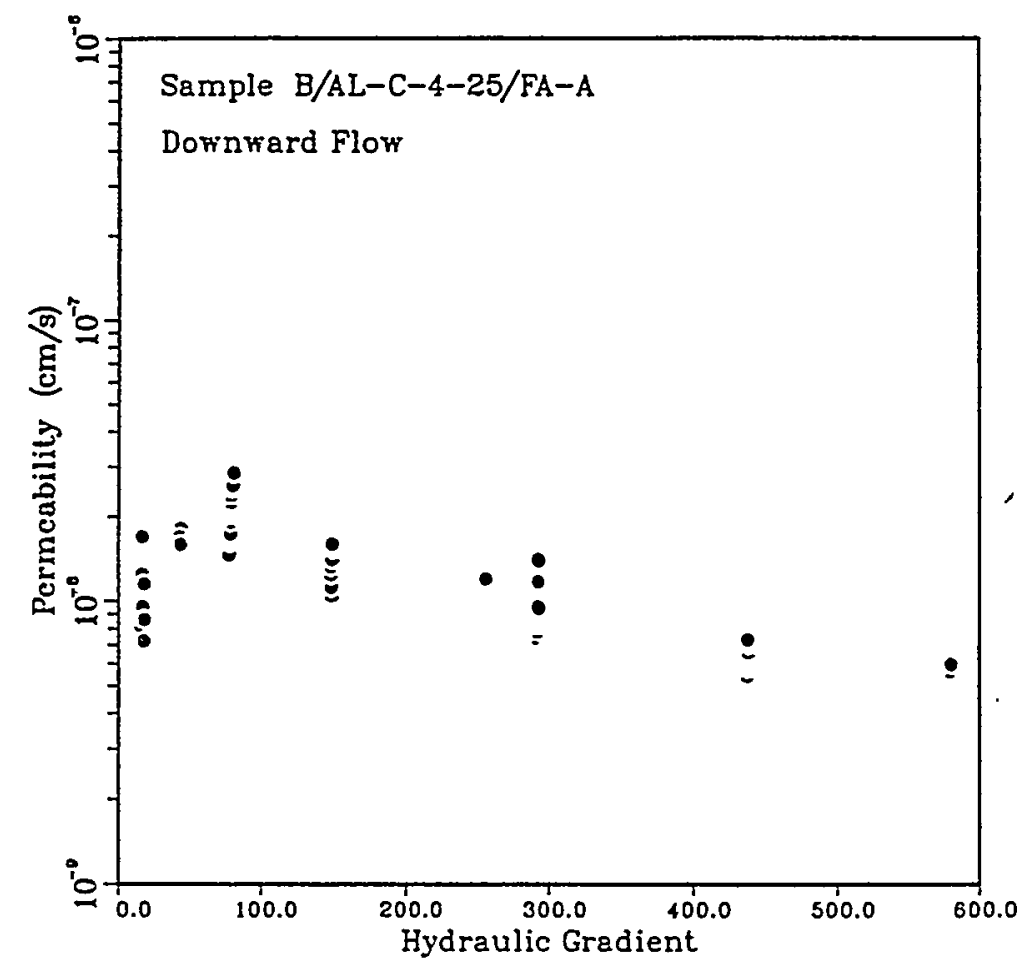

Figure 3.85 Permeability vs. hydraulic gradient for Sample B/AL-C-4-25/FA-A (downward flow testing).

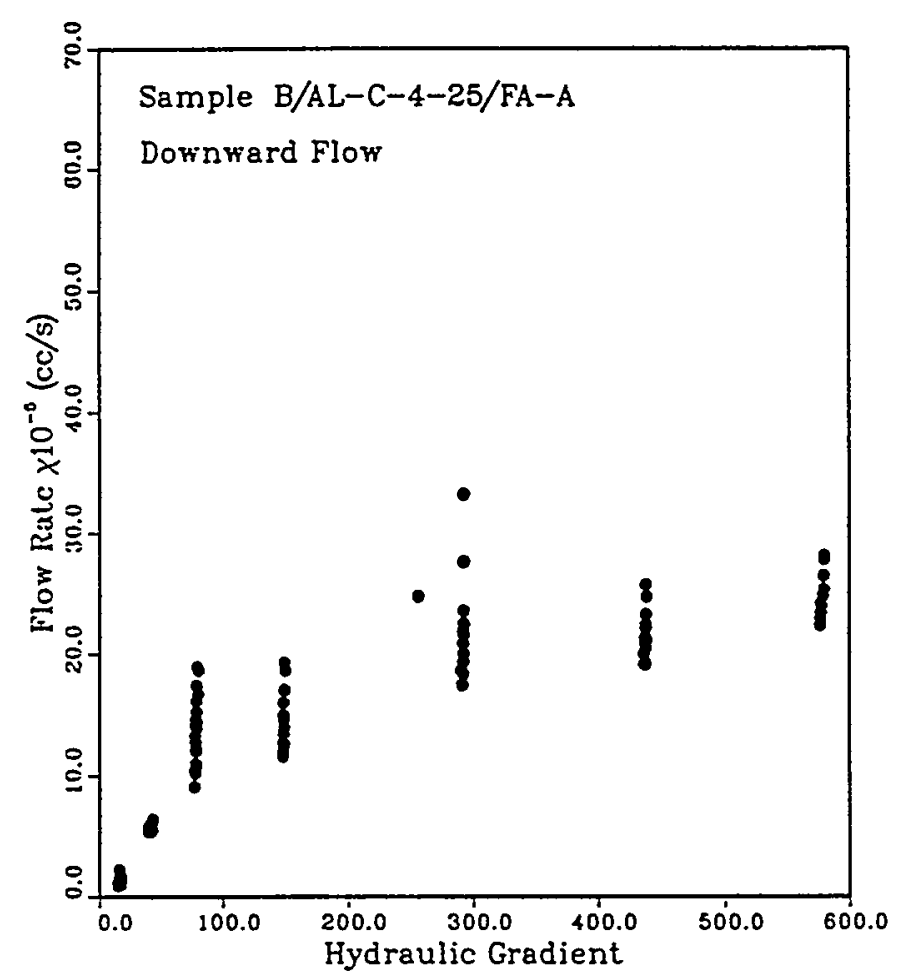

Figure 3.86 Flow rate vs. hydraulic gradient for Sample B/AL-C-4-25/FA-A (downward flow testing). 


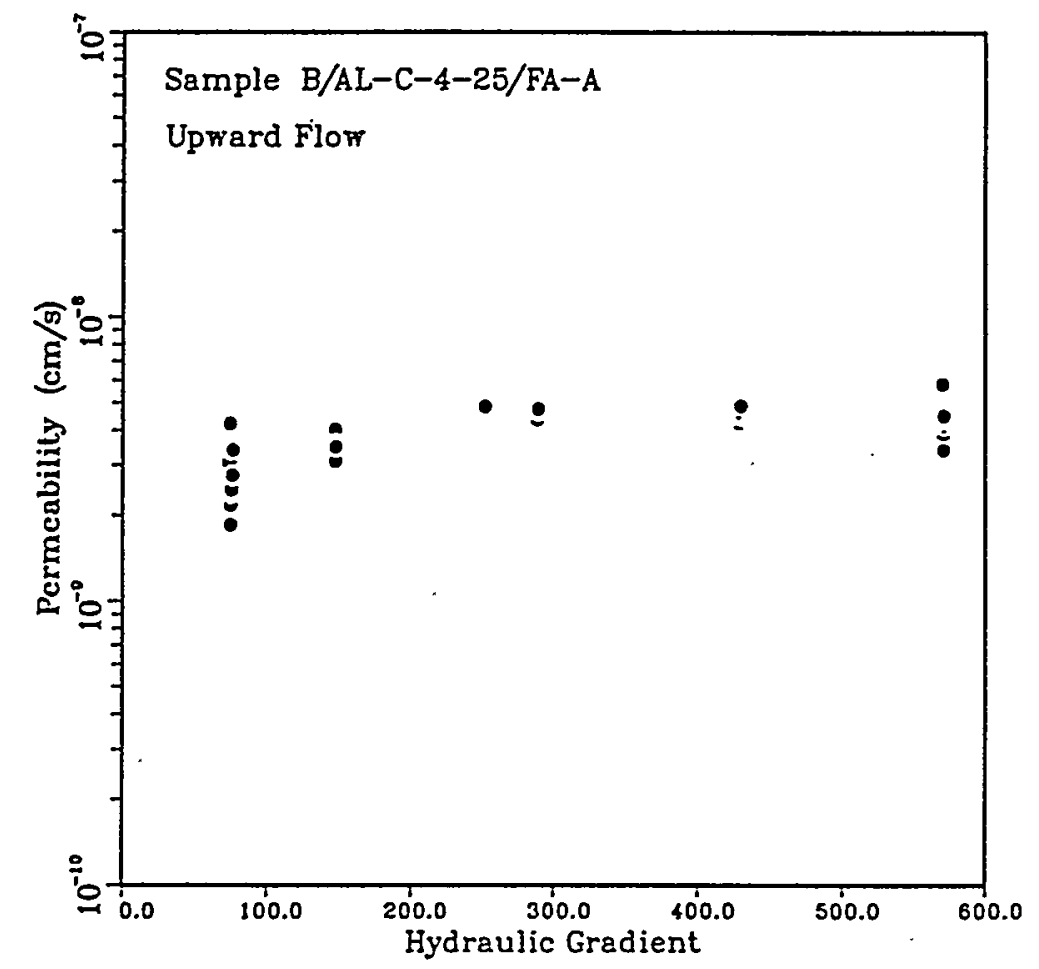

Figure 3.87 Permeability vs. hydraulic gradient for Sample B/AL-C-4-25/FA-A (upward flow testing).

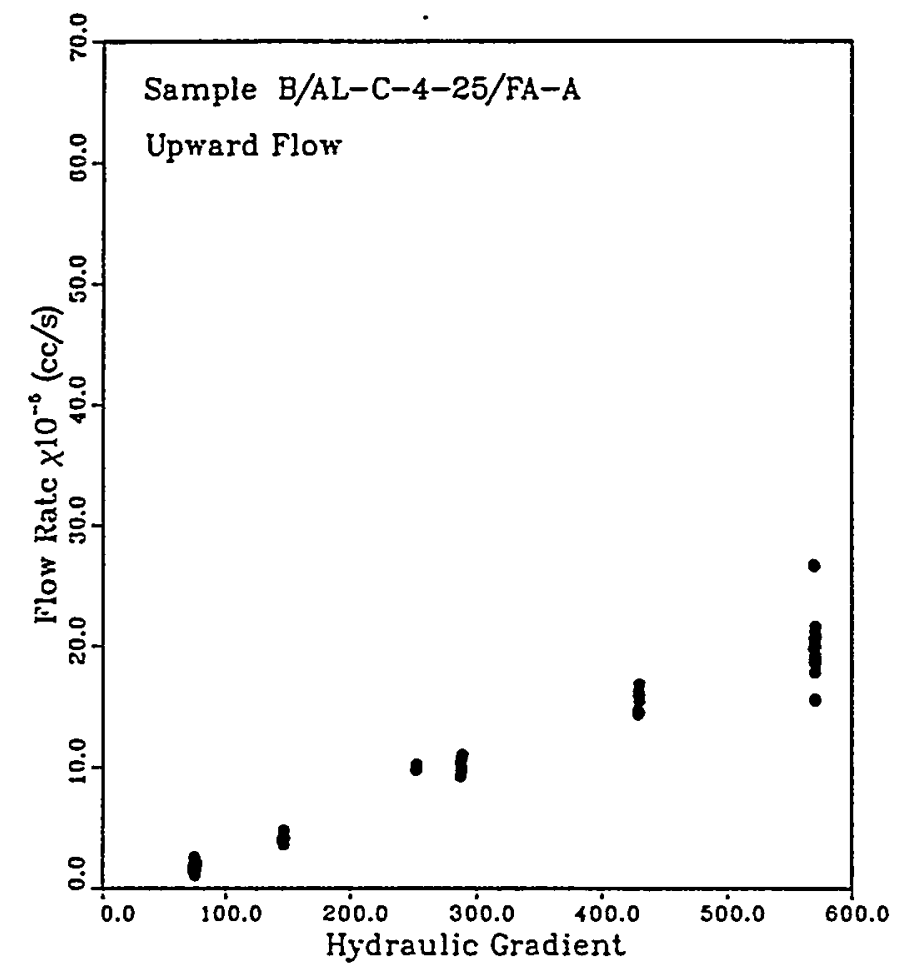

Figure 3.88 Flow rate vs. hydraulic gradient for Sample B/AL-C-4-25/FA-A (upward flow testing). 


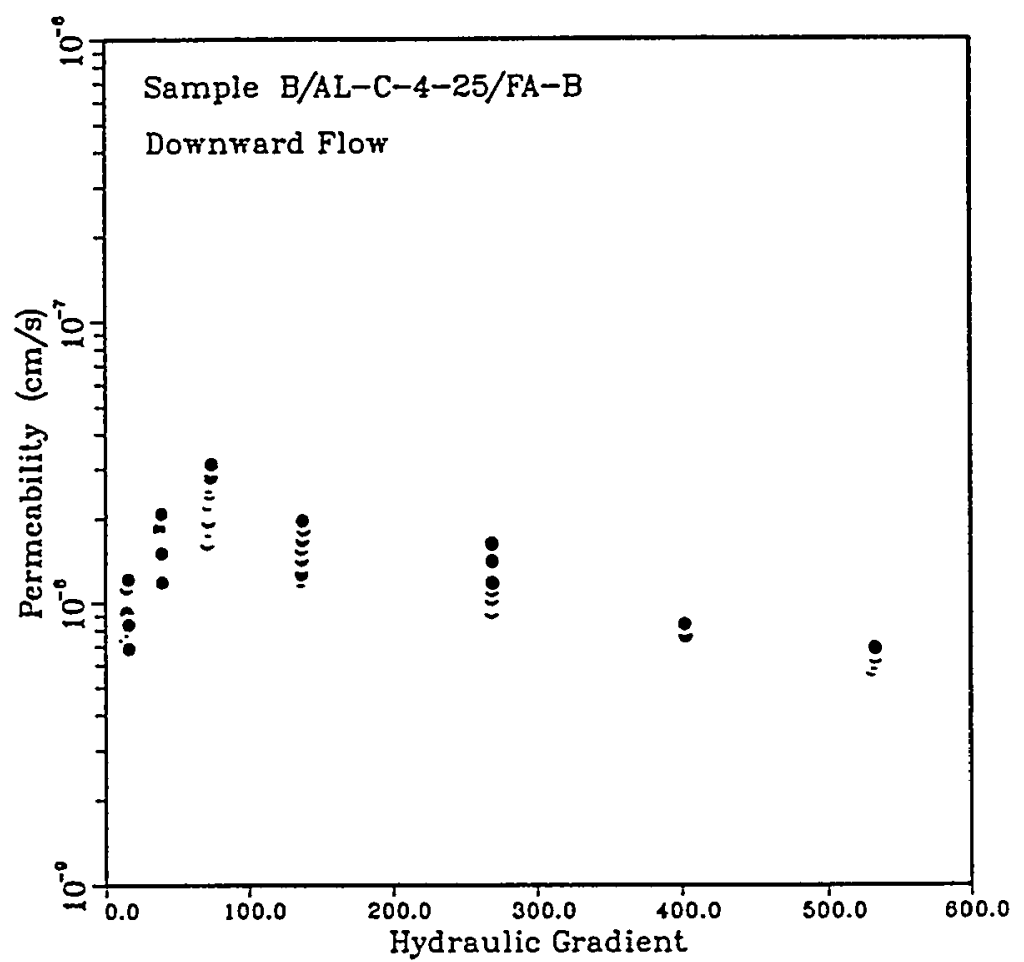

Figure 3.89 Permeability vs. hydraulic gradient for Sample B/AL-C-4-25/FA-B (downward flow testing).

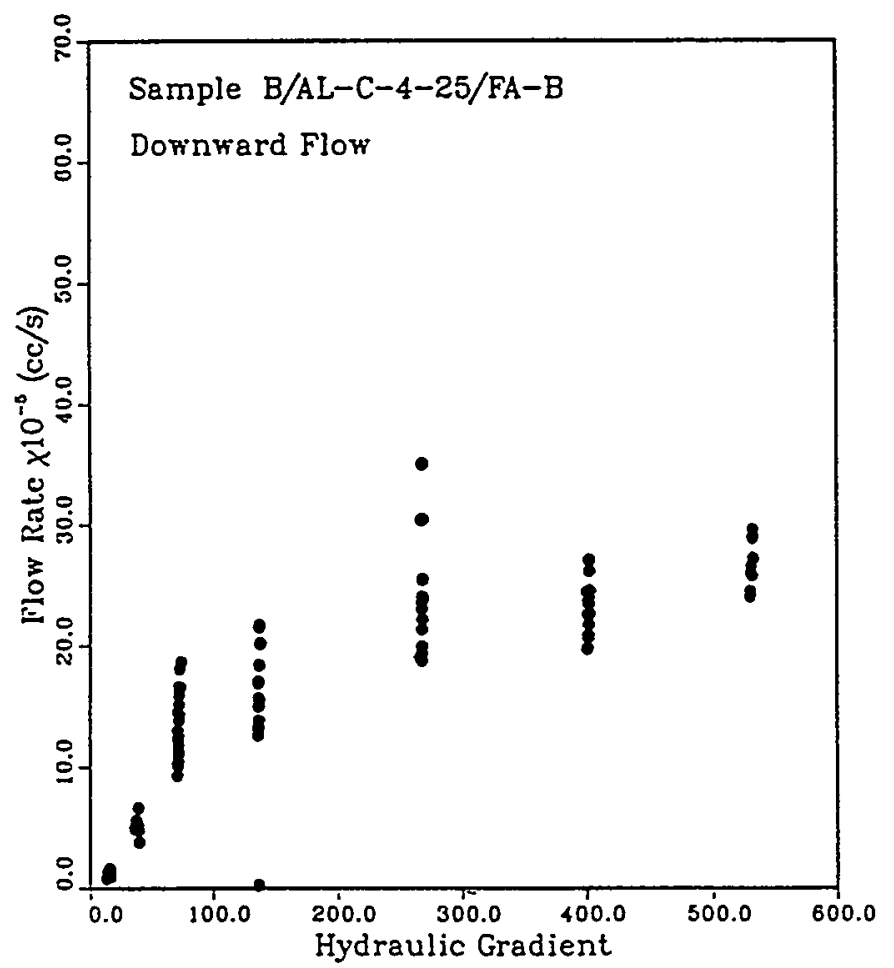

Figure 3.90 Flow rate vs. hydraulic gradient for Sample B/AL-C-4-25/FA-B (downward flow testing). 


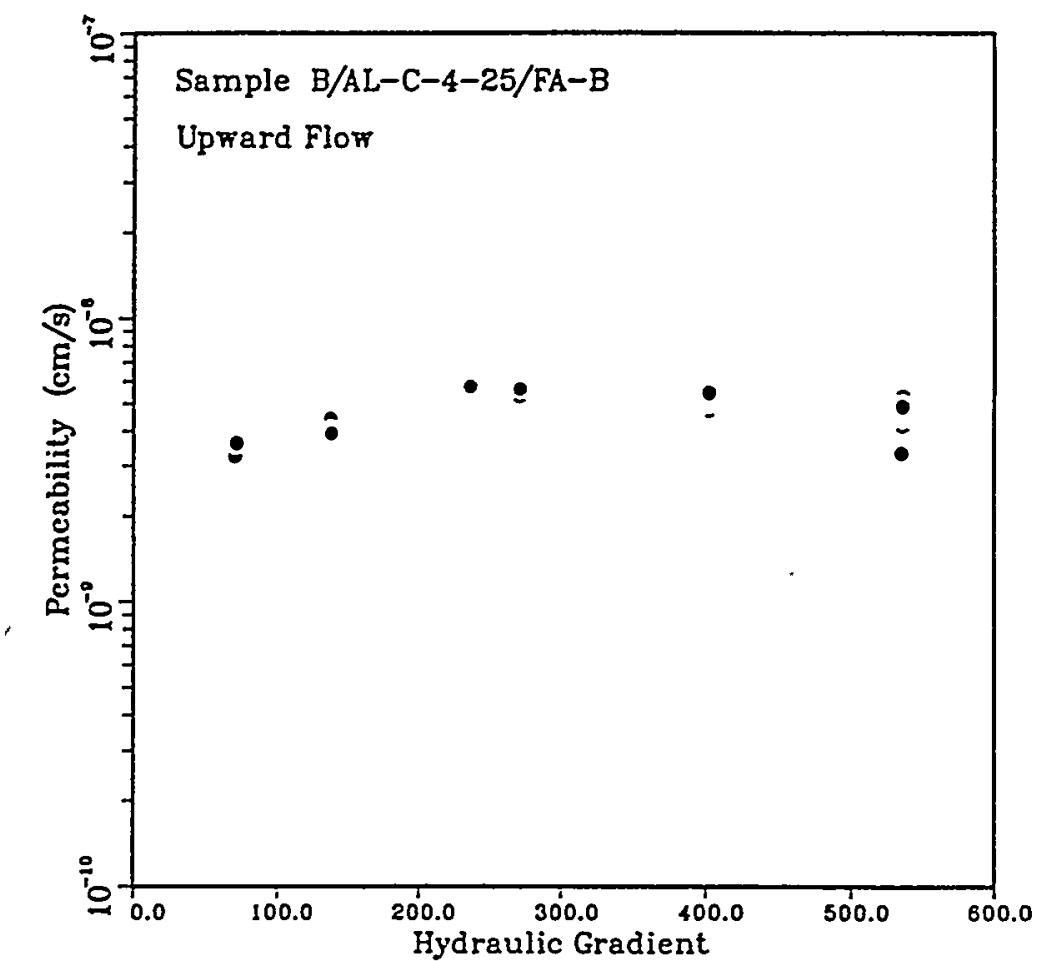

Figure 3.91 Permeability vs. hydraulic gradient for Sample B/AL-C-4-25/FA-B (upward flow testing).

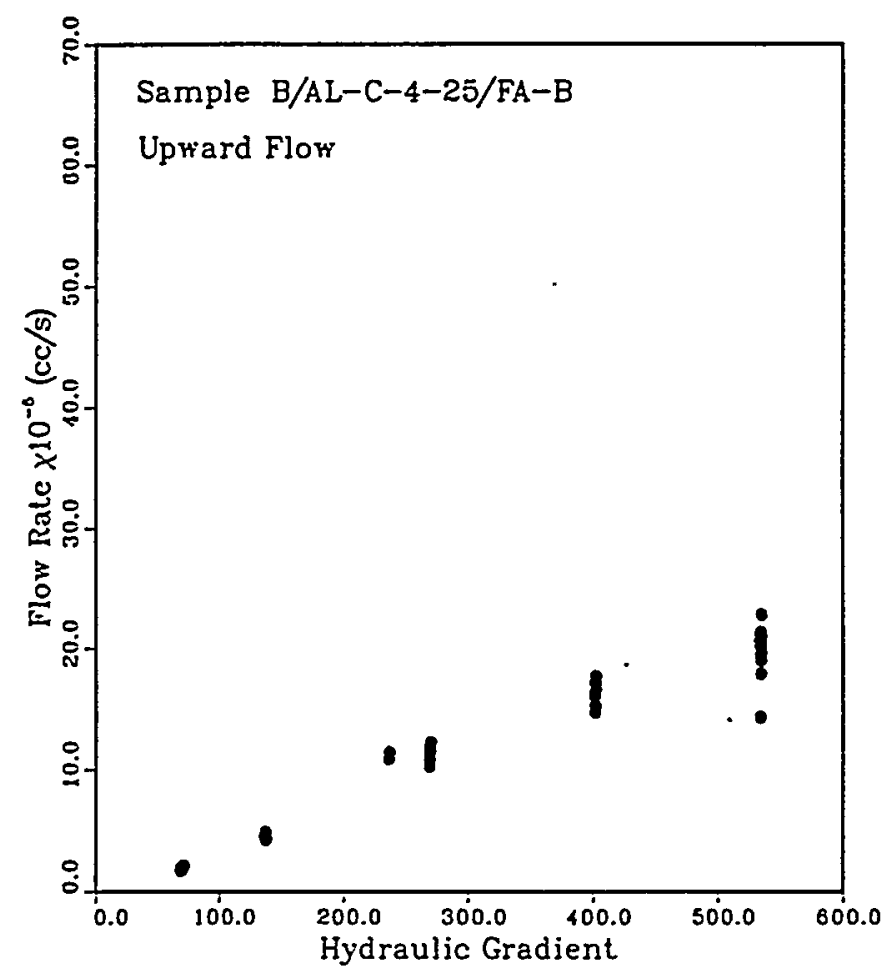

Figure 3.92 Flow rate vs. hydraulic gradient for Sample B/AL-C-4-25/FA-B (upward flow testing). 
Table 3.12 Summary of Flow Test Results of Samples B/AL-C-8-25/FC-S and B/AL-C-8-35/FC-S

\begin{tabular}{|c|c|c|c|c|c|c|}
\hline $\begin{array}{l}\text { Sample } \\
\text { Number }\end{array}$ & $\begin{array}{l}\text { Test } \\
\text { Duration }\end{array}$ & $\begin{array}{l}\text { Inflow } \\
\text { (cc) }\end{array}$ & $\begin{array}{l}\text { Outflow } \\
\text { (cc) }\end{array}$ & $\begin{array}{l}\text { Permeability } \\
(\mathrm{cm} / \mathrm{s})\end{array}$ & $\begin{array}{l}\text { Flow* } \\
\text { Direction }\end{array}$ & Remarks \\
\hline $\begin{array}{l}\text { B/AL-C- } 8 \\
-25 / F C-S\end{array}$ & $\begin{array}{l}1453 \text { min } \\
1355 \\
2774 \\
1614 \\
1317 \\
2949 \\
\end{array}$ & $\begin{array}{l}1.55 \\
1.25 \\
1.45 \\
1.875 \\
0.825 \\
1.275 \\
\end{array}$ & $\begin{array}{l}2.125 \\
0.825 \\
1.55 \\
2.475 \\
0.7 \\
2.5 \\
\end{array}$ & $\begin{array}{l}2.1 \times 10^{-8} \\
1.4 \times 10^{-8} \\
1.4 \times 10^{-8} \\
2.1 \times 10^{-8} \\
1.3 \times 10^{-8} \\
1.6 \times 10^{-8} \\
\end{array}$ & Downward & $\begin{array}{l}\text { Double-pipette } \\
\text { falling head } \\
\text { method. } \\
\text { Hydraulic gradients: } \\
3.4-1.8\end{array}$ \\
\hline & $\begin{array}{l}34 \text { min } \\
15 \\
31 \\
40 \\
27 \\
28 \\
34\end{array}$ & $\begin{array}{l}10.5 \\
0.925 \\
0.475 \\
21.225 \\
18.3 \\
17.175 \\
4.025\end{array}$ & $\begin{array}{l}8.3 \\
0.925 \\
0.9 \\
15.9 \\
13.325 \\
12.825 \\
2.9\end{array}$ & $\begin{array}{l}1.4 \times 10^{-5} \\
1.3 \times 10^{-5} \\
1.6 \times 10^{-5} \\
1.7 \times 10^{-5} \\
1.0 \times 10^{-5} \\
1.3 \times 10^{-5} \\
9.4 \times 10^{-6}\end{array}$ & Upward & $\begin{array}{l}\text { Double-pipette } \\
\text { falling head } \\
\text { method. Hydraulic } \\
\text { gradients: } 3.85-0.02 \\
\text { The sample had been } \\
\text { subjected to shaking } \\
\text { and pounding before the } \\
\text { upward permeation } \\
\text { started. }\end{array}$ \\
\hline & $\begin{array}{l}47.86 \mathrm{~s} \\
45.13 \\
42.31 \\
40.51 \\
36.78 \\
32.98 \\
25.89 \\
23.09 \\
22.24\end{array}$ & $\begin{array}{l}33 \\
33 \\
33 \\
33 \\
33 \\
33 \\
33 \\
33.2 \\
35\end{array}$ & $\begin{array}{l}- \\
- \\
- \\
- \\
- \\
-\end{array}$ & $\begin{array}{l}5.9 \times 10^{-4} \\
6.2 \times 10^{-4} \\
6.7 \times 10^{-4} \\
6.9 \times 10^{-4} \\
7.6 \times 10^{-4} \\
8.5 \times 10^{-4} \\
1.1 \times 10^{-3} \\
1.2 \times 10^{-3} \\
1.5 \times 10^{-3}\end{array}$ & Upward & $\begin{array}{l}\text { Standard falling } \\
\text { head. Hydraulic } \\
\text { gradients: } 5.4-1.74 \\
\text { The sample had not } \\
\text { been tested for } 25 \\
\text { days before this } \\
\text { test sequence. }\end{array}$ \\
\hline
\end{tabular}


Table 3.12 Summary of Flow Test Results of Samples B/AL-C-8-25/FC-S and B/AL-C-8-35/FC-S --Gontinued

\begin{tabular}{|c|c|c|c|c|c|c|}
\hline & $\begin{array}{l}43.62 \\
42.97 \\
42.26 \\
42.58 \\
39.06 \\
37.78 \\
38.44 \\
\end{array}$ & $\begin{array}{l}33 \\
33 \\
33 \\
33 \\
33 \\
33 \\
33 \\
\end{array}$ & $\begin{array}{l}- \\
- \\
- \\
-\end{array}$ & $\begin{array}{l}3.5 \times 10^{-4} \\
3.6 \times 10^{-4} \\
3.6 \times 10^{-4} \\
3.6 \times 10^{-4} \\
3.9 \times 10^{-4} \\
4.0 \times 10^{-4} \\
4.0 \times 10^{-4}\end{array}$ & Downward & $\begin{array}{l}\text { Standard falling } \\
\text { head method. } \\
\text { Hydraulic grandients: } \\
8.26-5.1\end{array}$ \\
\hline $\begin{array}{l}\text { B/AL-C-8 } \\
-35 / F C-S\end{array}$ & $\begin{array}{l}1453 \mathrm{~min} \\
1355 \\
2774 \\
1614 \\
1317 \\
2949\end{array}$ & $\begin{array}{l}2.0 \\
1.85 \\
3.025 \\
2.325 \\
1.45 \\
2.9 \\
\end{array}$ & $\begin{array}{l}2.475 \\
0.875 \\
2.7 \\
2.2 \\
0.825 \\
3.325 \\
\end{array}$ & $\begin{array}{l}2.3 \times 10^{-8} \\
1.6 \times 10^{-8} \\
2.4 \times 10^{-8} \\
1.9 \times 10^{-8} \\
1.6 \times 10^{-8} \\
2.3 \times 10^{-8}\end{array}$ & Downward & $\begin{array}{l}\text { Double pipette } \\
\text { falling head method. } \\
\text { Hydraulic gradients: } \\
3.9-1.8\end{array}$ \\
\hline & $\begin{array}{l}1469 \\
4745 \\
5701 \\
4193 \\
7219 \\
1473\end{array}$ & $\begin{array}{l}2.05 \\
3.825 \\
3.075 \\
1.85 \\
2.475 \\
0.35\end{array}$ & $\begin{array}{l}1.7 \\
3.1 \\
3.6 \\
1.825 \\
3.55 \\
0.725\end{array}$ & $\begin{array}{l}1.7 \times 10^{-8} \\
1.4 \times 10^{-8} \\
1.5 \times 10^{-8} \\
1.6 \times 10^{-8} \\
2.4 \times 10^{-8} \\
3.2 \times 10^{-8}\end{array}$ & Upward & $\begin{array}{l}\text { Double plpette } \\
\text { falling head method. } \\
\text { Hydraulic gradlents: } \\
3.8-0.5 \text {. The sample } \\
\text { had been subjected to } \\
\text { shaking and pounding } \\
\text { before the upward per- } \\
\text { meation started. }\end{array}$ \\
\hline
\end{tabular}


be significant if seals are installed at locations intercepted by joints and/or fractures. It is anticipated that the horizontal permeability will be higher than the vertical permeability for the mixture sealants. Such a permeability variation is common to most geological formations (Freeze and Cherry, 1979, p. 31) and natural soils, as well as compacted fills (Lambe and Whitman, 1979, p. 275).

The polyaxial permeameter, developed independently, is similar to the three-dimensional consolidation permeability cell devised by $B$ udhu ${ }^{1}$ and to the one used by Hsieh (1988). Polyaxial flow testing has been performed on two rectangular samples. One sample consists of $75 \%$ crushed tuff and $25 \%$ bentonite, the other one of 658 crushed tuff and 358 bentonite. The crushed tuff constitutes the type A gradation. The samples are compacted in the vertical direction ( $z$ direction). The energy input is equivalent to the standard Proctor method. The final dimensions of the samples are $11.038 \times 11.032 \times 12.544 \mathrm{~cm}(\mathrm{x}, \mathrm{y}, \mathrm{z})$. The standard falling head method has been employed to measure the permeability in each direction. The $z$-direction coincides with the downward direction. Two porous end plates are emplaced normal to the flow direction before each flow test. The permeabilities measured in the three directions are shown in Figures 3.93 and 3.94 , and are summarized in Table 3.13 .

The flow test results clearly indicate hydraulic anisotropy. For the sample containing 258 bentonite, the permeability in one horizontal direction $\left(K_{x}\right)$ is about 4 times higher than that in the vertical direction $\left(K_{z}\right)$. The permeability in the second horizontal direction $\left(K_{y}\right)$ is approximately 3 times lower. The horizontal permeabilities differ by nearly an order of magnitude. The large decrease in permeability observed along the $\mathrm{x}$ direction is most probably due to migration of particles and clogging of pores (Mitchell and Younger, 1967; Dunn, 1985). For the sample having 358 bentonite by weight, the vertical and one horizontal permeability are about the same, both in the upper $10^{-9}$ $\mathrm{cm} / \mathrm{s}$. The permeability in the other horizontal direction is similar to that of the sample containing 258 bentonite, and differs from the vertical permeability by nearly two orders of magnitude.

Considering the low hydraulic gradients (6.25 to 1.46 for the first sample, 5.52 to 4.69 for the second one) at which the tests have been performed, and the likely occurrence of particle migration, sealants similar to these two plugs may not survive long if piping develops. In particular, it may be difficult to impede particle migration in the lateral direction, unless any open joints or fractures are effectively grouted.

Many variables affect the heterogeneity and the anisotropy in permeability of a crushed tuff/bentonite mixture, including the size and gradation of the rock particles, method of mixing and installation, water content, and the amount of bentonite added. Particle segregation

\footnotetext{
'Personal communication, Prof. M. Budhu, Dept. of Civil Engineering,
} University of Arizona, Tucson, 1989. 


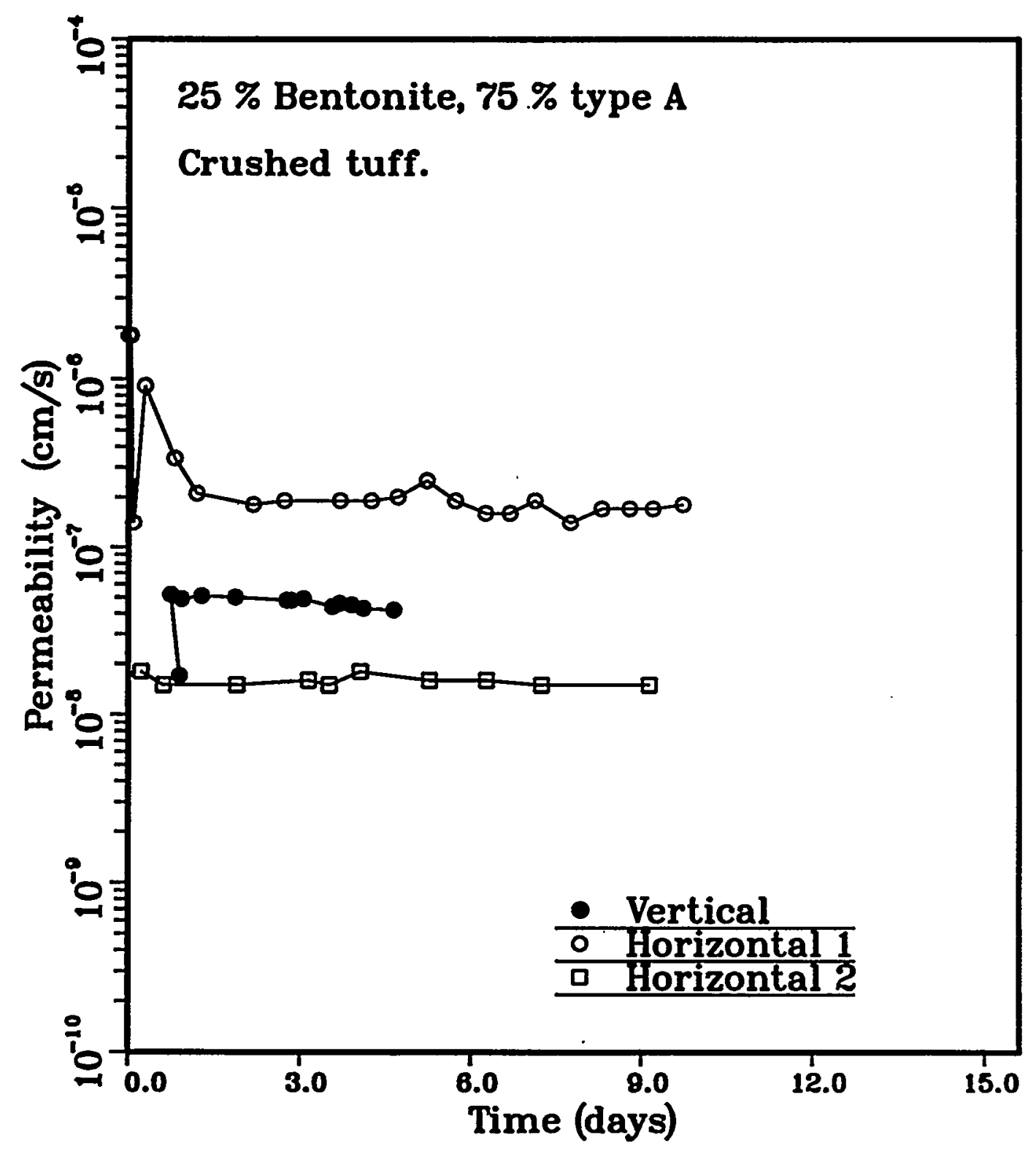

Figure 3.93 Vertical and horizontal permeabilities of a mixture containing $25 \%$ bentonite and $75 \%$ type A crushed tuff. 


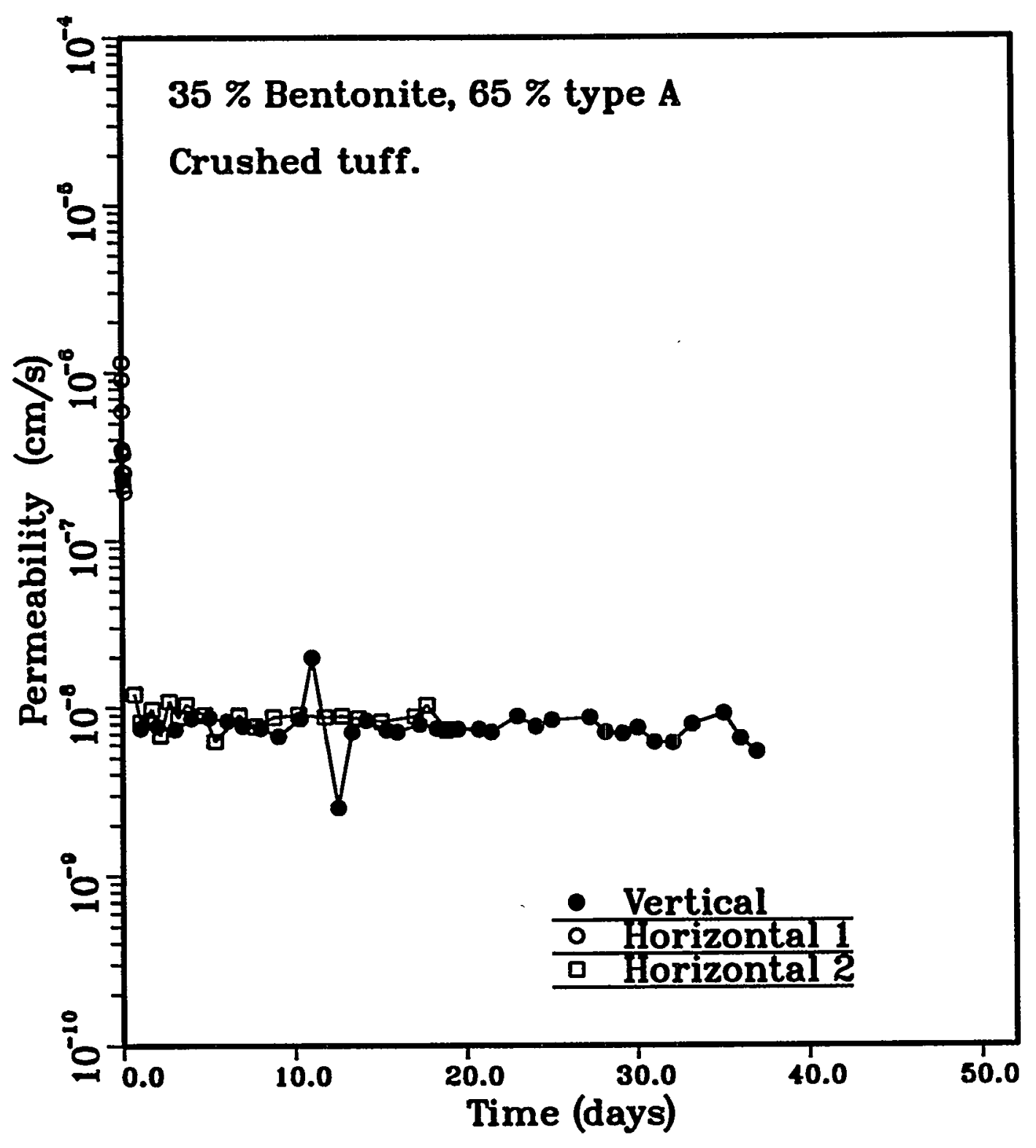

Figure 3.94 Vertical and horizontal permeabilities of a mixture containing 358 bentonite and 658 type A crushed tuff. 
Table 3.13 Summary of Polyaxial Flow Test Results

\begin{tabular}{lccc}
\hline $\begin{array}{l}\text { Wt. Percent } \\
\text { Clay/Tuff }\end{array}$ & \multicolumn{3}{c}{ Permeability (cm/s) } \\
& Vertical & Horizontal(1) & Horizonta1(2) \\
$25 / 75$ & $5.2-1.7 * 10-8$ & $1.8-1.4 * 10-7$ & $1.8-1.5 * 10-8$ \\
$35 / 65$ & $9.2-2.5 * 10-9$ & $11-1.9 * 10-7$ & $12-6.3 * 10-9$ \\
\hline
\end{tabular}

NOTE: The crushed tuff constitutes the type A gradation.

Table 3.14 Results of Flow Tests at Elevated Temperature

\begin{tabular}{ccccccc}
\hline & Test & Total & Total & Permeability & \\
Temp. & $\begin{array}{c}\text { Duration } \\
\text { (days) }\end{array}$ & $\begin{array}{c}\text { Inflow } \\
\text { ( } \mathrm{c} \mathrm{c})\end{array}$ & $\begin{array}{c}\text { Outflow } \\
(\mathrm{cc})\end{array}$ & $\begin{array}{c}\text { Measured } \\
(\mathrm{cm} / \mathrm{s})\end{array}$ & $(\mathrm{cm} / \mathrm{s})$ & $\begin{array}{c}\text { Specific } \\
\left(\mathrm{m}^{2}\right)\end{array}$ \\
\hline
\end{tabular}

Sample 1: $25 \%$ bentonite, $75 \%$ type A crushed tuff

$\begin{array}{rrccccr}21 & 28 & 29.25 & 28.6 & 1.28 \pm 0.40 & 1.28 & \left(* 10^{-17}\right) \\ 35 & 23 & 23.25 & 22.6 & 2.32 \pm 0.15 & 1.72 & 1.28 \\ 45 & 21 & 21.5 & 21.8 & 2.35 \pm 0.12 & 1.62 & 1.45 \\ 60 & 6 & 8.05 & 8.6 & 2.39 \pm 0.31 & 2.05 & 1.16 \\ 35 & 17 & 17.07 & 15.65 & 2.26 \pm 0.10 & 1.72 & 1.68\end{array}$

Sample 2: $35 \%$ bentonite, $65 \%$ type A crushed tuff

\begin{tabular}{rrlllrr}
21 & 28 & 8.1 & 7.25 & $\left(* 10^{-9}\right)$ & $\left(* 10^{-9}\right)$ & $\left(* 10^{-18}\right)$ \\
35 & 23 & 7.25 & 6.15 & $5.02 \pm 1.16$ & 3.06 & 3.05 \\
45 & 21 & 6.45 & 6.1 & $5.26 \pm 0.98$ & 4.13 & 3.72 \\
60 & 4 & 0.8 & 1.2 & $4.26 \pm 1.34$ & 4.88 & 3.24 \\
35 & 17 & 3.65 & 3.67 & $4.27 \pm 1.22$ & 4.13 & 1.58 \\
\hline
\end{tabular}

NOTE: Hydraulic gradients applied are less than 10. 
observed during sample installation and compaction is likely responsible for the occurrences of high horizontal permeability.

\subsubsection{High Temperature Flow Tests}

The heat generated by radioactive decay of high-level nuclear wastes will result in an elevated temperature field around a waste repository (International Atomic Energy Agency, 1984). The temperature changes could affect the integrity or effectiveness of sealants. Temperatures experienced by borehole or shaft seals for the proposed Yucca Mountain repository, however, are expected not to exceed $100^{\circ} \mathrm{C}$ (Fernandez et al., 1987, Table 7.1).

Samples B/AL-C-4-25/A-S and B/AL-C-4-35/A-S installed in stainless steel permeameters are immersed in a constant temperature water bath for flow testing up to $60^{\circ} \mathrm{C}$. The samples are constructed by mixing crushed tuff of type A gradation with 258 and 358 bentonite. Figures 3.95 and 3.96 show the permeability results vs. time. Table 3.14 summarizes the results of high temperature flow testing.

The "measured" permeabilities shown in Table 3.14 are computed directly using the experimental measurements. Assuming the major temperature effects are the changes in viscosity and density of the permeant (i.e. water), permeabilities of the plugs at other temperatures can be predicted based on the average permeability measured at room temperature $\left(21^{\circ} \mathrm{C}\right)$ provided that the permeant properties are adjusted. Results of such calculations are shown in the sixth column of Table 3.14. The specific permeabilities calculated are given in the last column of Table 3.14 .

The measured permeability increases with temperature, except for $B / A L-C$ $4-25 / \mathrm{A}-\mathrm{S}$ at $60^{\circ} \mathrm{C}$. The permeabilities measured at $35^{\circ} \mathrm{C}$ are about 2 times higher than those at $21^{\circ} \mathrm{C}$. A large portion of the permeability increase can be accounted for by the viscosity decrease of the permeant corresponding to the higher temperature. From $21^{\circ} \mathrm{C}$ to $35^{\circ} \mathrm{C}$, this viscosity effect amounts to 748 and 828 of the permeability increase for B/AL-C4-25/A-S and B/AL-C-4-35/A-S, respectively. The percentages indicated above are calculated using the viscosities of pure water at $21^{\circ} \mathrm{C}$ and at $35^{\circ} \mathrm{C}$ (Rouse, 1946 , p. 364; Streeter and Wylie, 1979, p. 534). The actual viscosity of the percolating water may be different from that for pure water; the ratio of the viscosities at two temperatures, however, is sufficiently accurate for practical purposes (Marsland and Loudon, 1963).

The specific permeability of the two samples also changes with temperature, implying an alteration of sample structure. The specific permeability reaches a maximum at $35^{\circ} \mathrm{C}$. The specific permeability at $60^{\circ} \mathrm{C}$ is reduced by 108 for the sample containing 25 percent bentonite, and by 508 for the sample containing 35 percent bentonite, when compared to the specific permeability at $21^{\circ} \mathrm{C}$. 
Sample B/AL-C-4-25/A-S

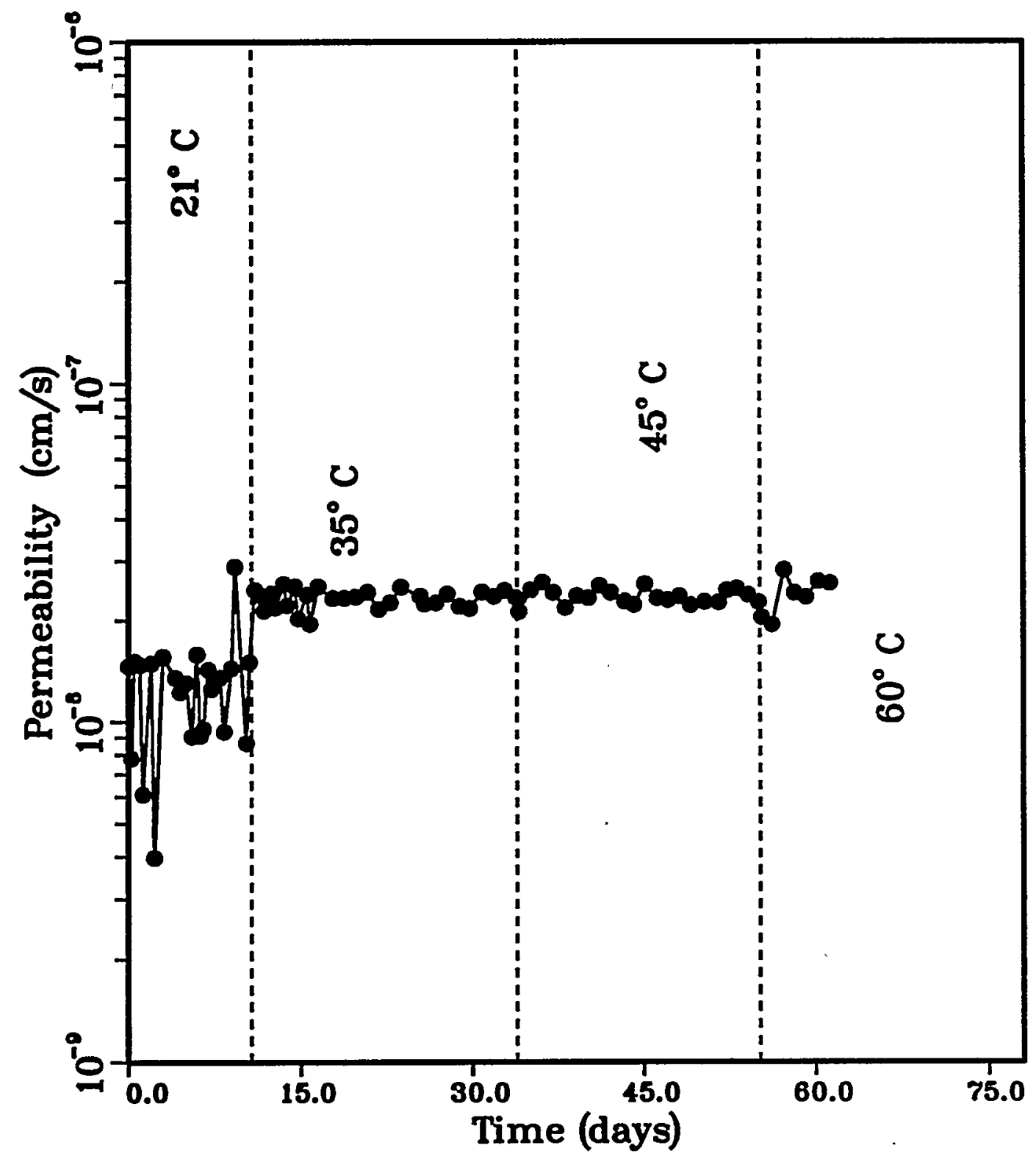

Figure 3.95 Permeabilities of Sample B/AL-C-4-25/A-S at room and elevated temperatures. 


\section{Sample B/AL-C-4-35/A-S}

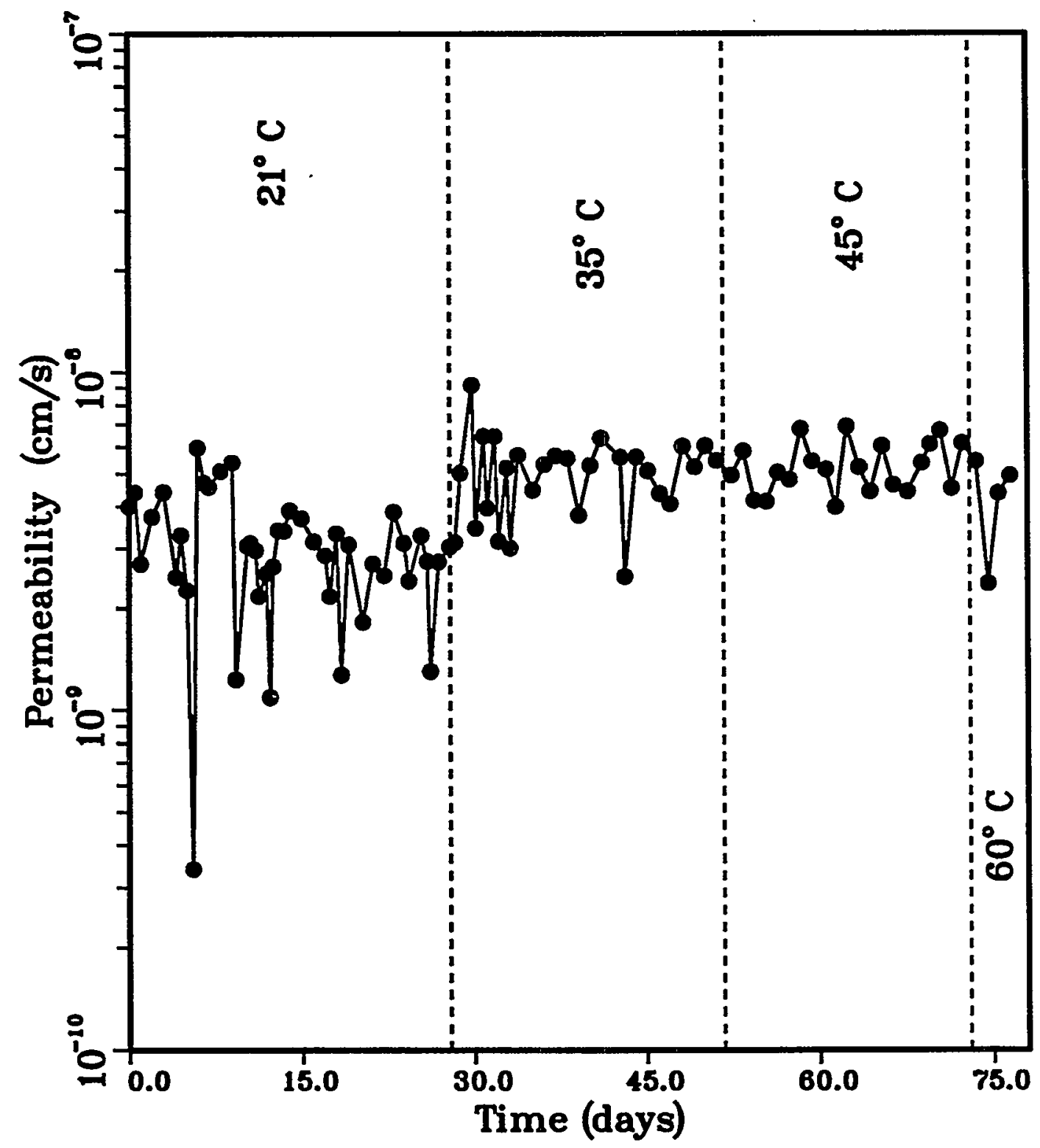

Figure 3.96 Permeabilities of Sample B/AL-C-4-35/A-S at room and elevated temperatures. 


\subsubsection{Piping Tests}

Piping tests have been performed on Samples B/AL-C-4-25/A-P-A, B/AL-C-425/A-P-B, B/AL-C-4-35/A-P-A, 7 and B/AL-C-4-35/A-P-B installed in perforated $10.16 \mathrm{~cm}$ diameter PVC permeameters. A $2.25 \mathrm{~mm}$ diameter hole drilled through the walls of the PVC permeameters housing the first three samples simulates an opening in the walls of boreholes or shafts where seals. have been emplaced. The bottom of the three samples is approximately $1 \mathrm{~cm}$ below. the center of the holes. A $20 \mathrm{~mm}$ diameter hole has been cut in the wall of the fourth permeameter. The bottom of this sample is about $2.4 \mathrm{~cm}$ below the center of the hole. The four samples have been subjected to a series of injection pressures to study the conditions under which bentonite may be lost through the openings, and the possible development of piping channels.

\subsubsection{Sample B/AL-C-4-25/A-P-B}

The initial vertical permeability of B/AL-C-4-25/A-P-B is in the lower $10^{-8} \mathrm{~cm} / \mathrm{s}$ range when all side holes are plugged. Upon completion of these flow tests, the bottom outlet of the permeameter was capped and a side hole of $.2 .25 \mathrm{~mm}$ diameter unplugged. At an injection pressure of $32.75 \mathrm{kPa}$ ( $4.75 \mathrm{psi}$ ), bentonite and fine tuff particles along with a small amount of water move through the open hole into the connecting tubing. Upon re-opening the bottom outlet, the vertical permeability had increased by two orders of magnitude, into the lower $10^{-6} \mathrm{~cm} / \mathrm{s}$ range (Figure 3.97). The detailed results are shown in Appendix F.

In the subsequent.flow testing, the bottom outlet was closed again and the injection pressure increased. The amount of water flowing out of the side hole appeared to increase with injection pressure. Approximately 12 hours after the injection pressure had been raised to $113 \mathrm{kPa}$ (16.4 psi), a spill of wate was found in the laboratory (Figure 3.98, left). The inflow reservoir had been drained completely: The material in the side hole and in the eonnecting tubing was partially flushed out and collected in the graduated glass cylinder. The gross hydraulic gradient induced by the injection pressure $(113 \mathrm{kPa})$ is 116 . The failure of $B / A L-C-4-25 / A-P-B$ is believed to have resulted from piping, channeling, or erosion. The sample was later impregnated with resin so that the permeable flow channels ean be observed (Figure 3.98, right).

The test geometry in which Sample B/AL-C-4-25/A-P-B failed represents an all-around impermeable boundary, except for a small side hole. Under these conditions, the flow net.is expected to be quite different from a configuration in which the injected water is allowed to exit through the side hole and the bottom outlet simultaneously. The former simulation may be warranted when the material underneath the plug forms a practically impermeable boundary. The results of flow testing on Sample B/AL-C-4-25/A-P-B therefore deserve consideration in the multiplecomponent seal design for boreholes and shafts.

For the flow testing on $B / A L=C-4=25 / A-P-A, B / A L-C-4-35 / A-P-A$, and $B / A L-C-4-35 / A-P-B$, the on/off state of the bottom outlet of the permeameters is controlled by a valve such that the sealing performance under either condition can be evaluated. 


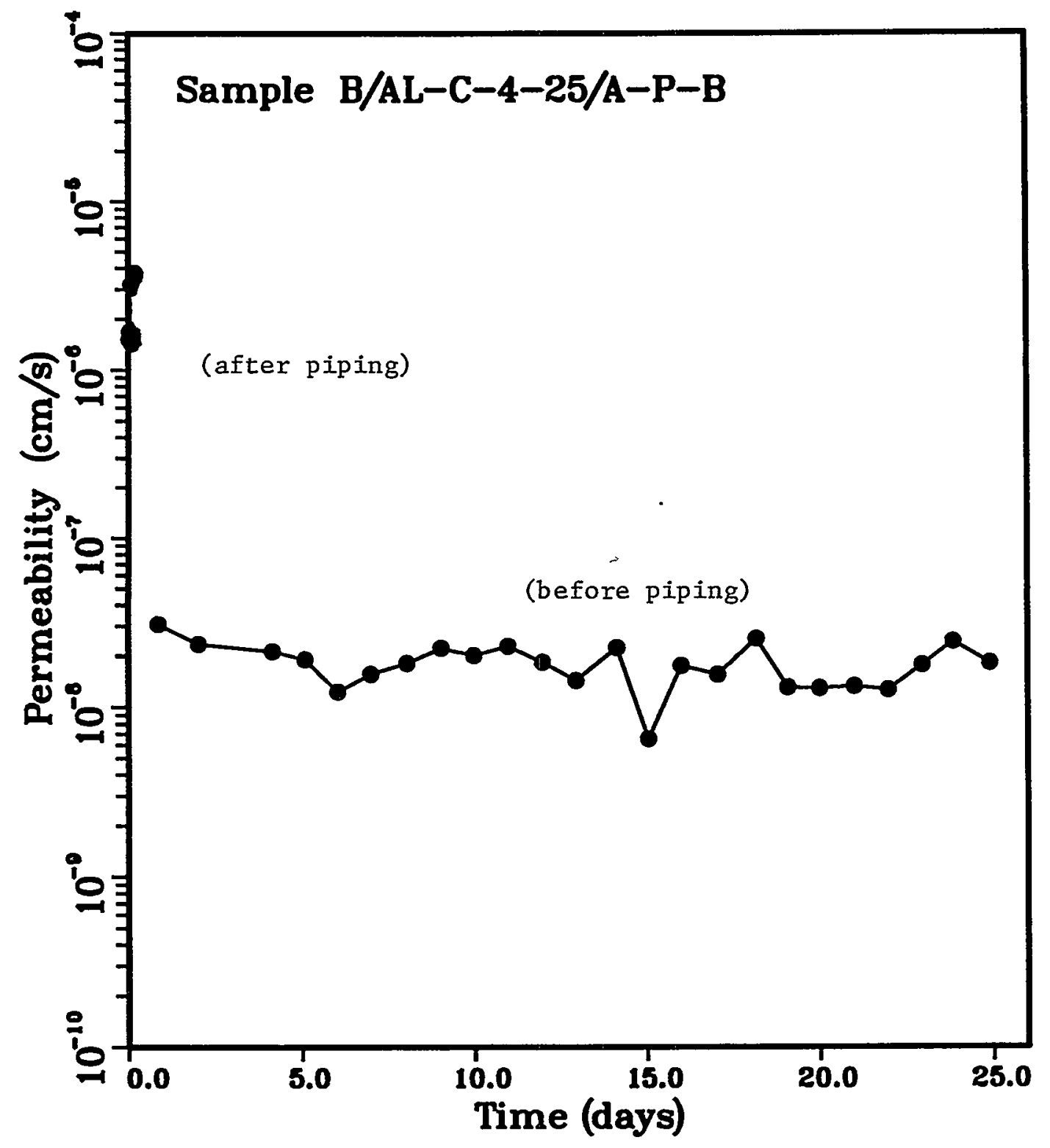

Figure 3.97 Vertical permeabilities of Sample B/AL-C-4-25/A-P-B before and after piping. 


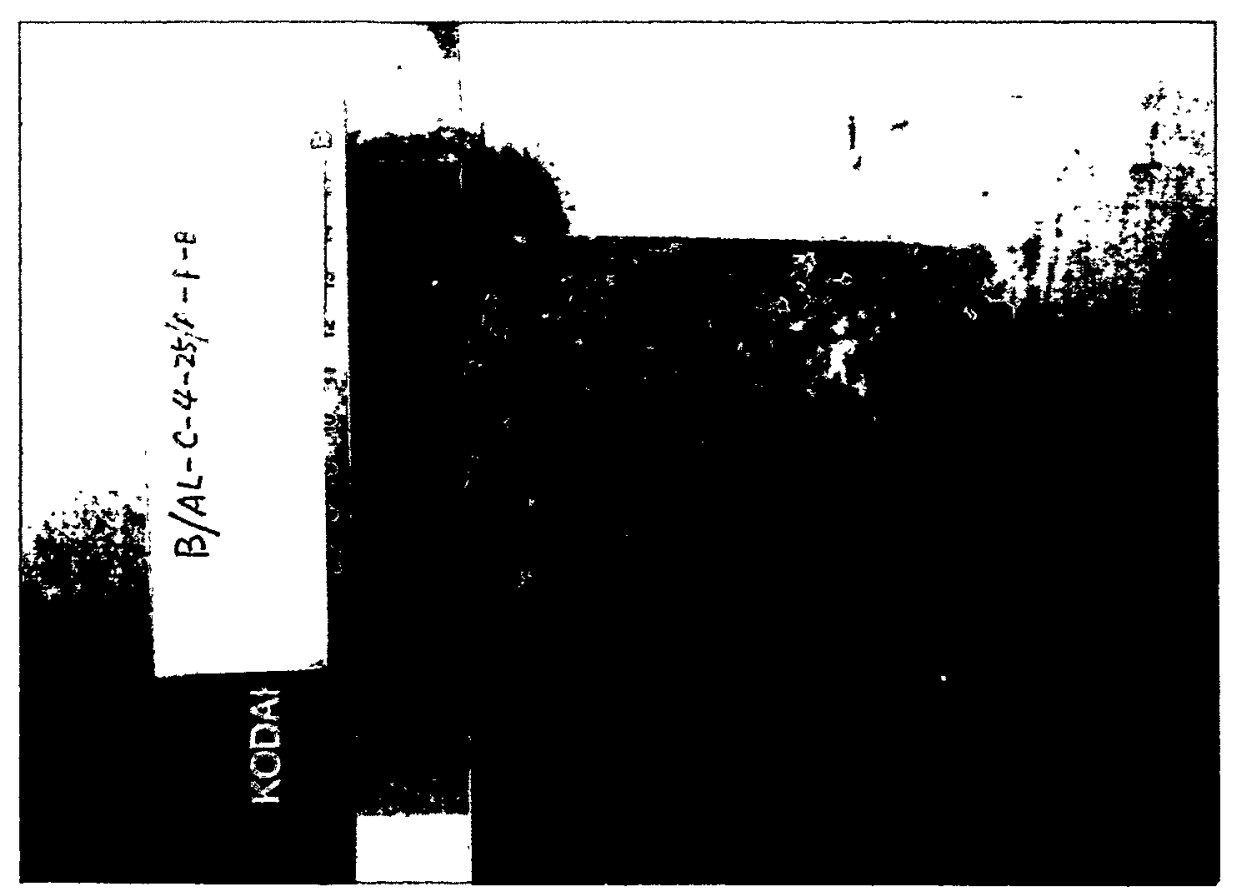

年

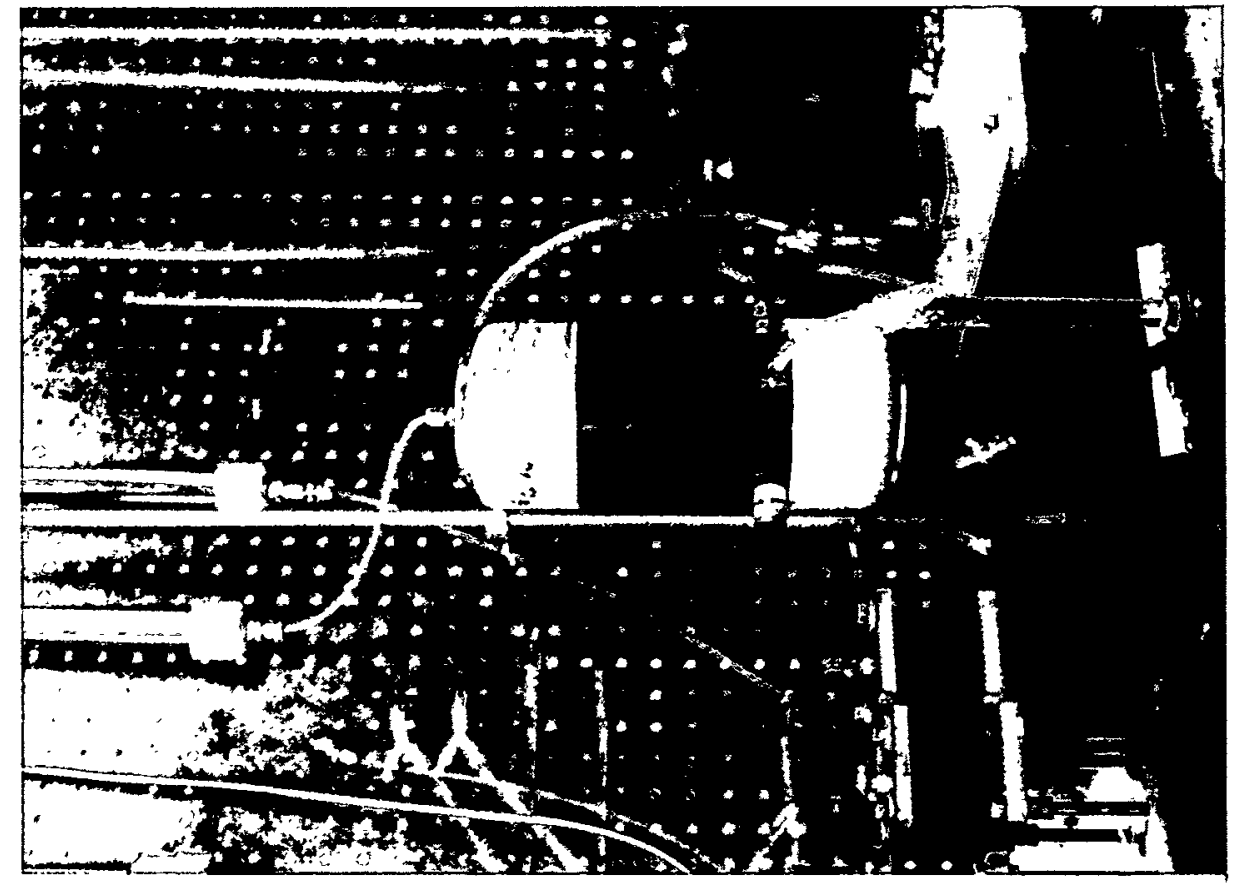

年 


\subsubsection{Sample B/AL-C-4-25/A-P-A}

During flow testing of this sample, the bottom outlet was left open while the injection pressure was increased. Bentonite and fine tuff particles appeared in the tubing connected to the side hole at an injection pressure of $27 \mathrm{kPa}$ ( $4 \mathrm{psi}$ ). No additional signs of piping failure were detected for injection pressures up to $376 \mathrm{kPa}$ ( $54.5 \mathrm{psi}$ ). The amount of water flowing out of the side hole is less than 28 of the amount flowing out of the bottom outlet. The latter outflow was used in calculating the vertical permeability (Figure 3.99). This sample maintained a relatively constant permeability, in the 10 w $10^{-8} \mathrm{~cm} / \mathrm{s}$ range, under injection pressures up to $145 \mathrm{kPa}$ (21 psi, gradient 160). The permeability decreased as the injection pressure increased above 145 $\mathrm{kPa}$. This behavior parallels what has been observed in the high injection pressure flow testing described in Section 3.4.1.8.3.

\subsubsection{Samples B/AL-C-4-35/A-P-A and B/AL-C-4-35/A-P-B}

The PVC permeameter housing Sample B/AL-C-4-35/A-P-A has a side hole of $2.25 \mathrm{~mm}$ diameter. The permeameter for Sample B/AL-C-4-35/A-P-B has a circular sidewall opening of $20 \mathrm{~mm}$ diameter. The two samples were subjected to injection pressures from 20.7 ( $3 \mathrm{psi}$ ) to $207 \mathrm{kPa}$ (30 psi) for about 44 days. The induced hydraulic gradients vary from 30 to 230 . During this stage of flow testing, the bottom and side outlets remained open. The vertical permeabilities measured under a hydraulic gradient of approximately 30 are 1.1 and $2.1 \times 10^{-9} \mathrm{~cm} / \mathrm{s}$, respectively. About two days after the injection pressure has been raised to $207 \mathrm{kPa}$ (30 psi), bentonite in Sample B/AL-C-4-35/A-P-A emerges in the tubing connected to the side outlet. No emergence of bentonite has been observed for Sample B/AL-C-4-35/A-P-B.

After closing the bottom outlets, the two samples were subjected to injection pressures up to $275.8 \mathrm{kPa}$ (40 psi, hydraulic gradient of approximately 305) for 18 days. Measurements of the vertical permeability under injection pressures of 68.95 (10 psi) and $207 \mathrm{kPa}$ (30 psi) were attempted later by opening the bottom outlets. During the flow testing under the reduced injection pressure of $68.95 \mathrm{kPa}$, the inflow and outflow quantities recorded are 5.65 and $17.2 \mathrm{cc}$ for B/AL-C-4-35/A$P-A$, and 13.95 and $13.15 \mathrm{cc}$ for $B / A L-C-4-35 / A-P-B$. The magnitude of the flow mass imbalance suggests that the dissipation of excess pore pressure was faster for the latter sample than for the former one. The difference in pore pressure dissipation appears to be related to the diameter of the side outlets, the only obvious difference between the two samples. The measured permeabilities (based on the outflow) are slightly lower but very close to the ones obtained earlier for the same injection pressures. The detailed experimental record is given in Tables F. 3 and F.4 (Appendix F).

In the subsequent flow testing, the bottom outlets were closed again and the injection pressure was increased to 310.3 , then $345,413.7$, and eventually $482.6 \mathrm{kPa}(45,50,60$, and $70 \mathrm{psi})$. The injection pressures were maintained for $24,9,15$, and 33 days, respectively. Approximately 19 days after the pressure had been raised to $310.3 \mathrm{kPa}$ (45 psi), a burst of cloudy outflow $(14.65 \mathrm{cc}$ ) was noticed (1452 minutes $(24.2 \mathrm{~h}$ ) 


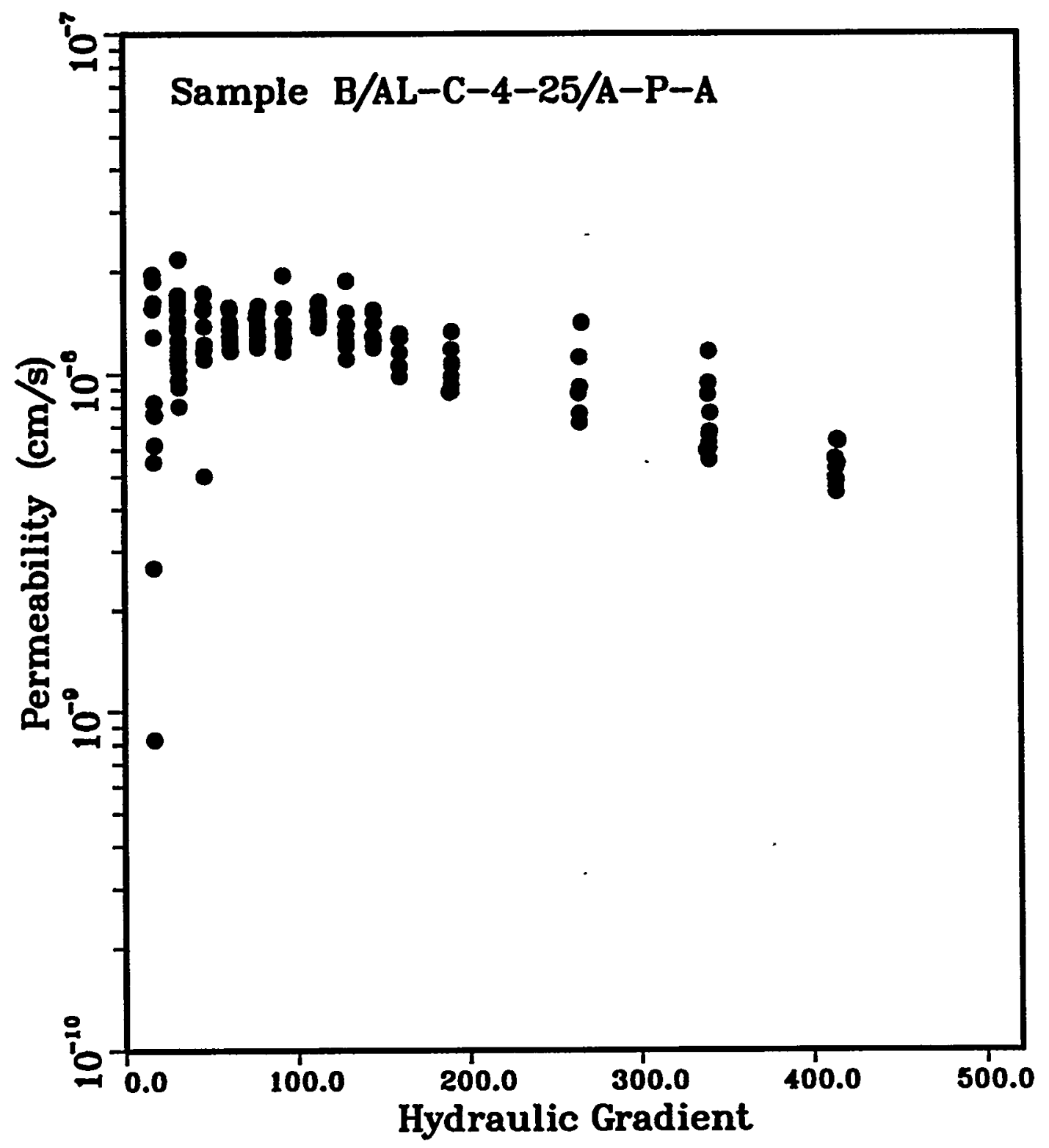

Figure 3.99 Variations of vertical permeability of Sample B/AL-C-4-25/A$\mathrm{P}-\mathrm{A}$ as a function of hydraulic gradient during a piping test with the bottom and side outlets open. 
after the previous measurement), compared with the normal daily amount of 0.4 to $0.45 \mathrm{cc}$. The inflow over the 1452 minutes was $2.05 \mathrm{cc}$.

Unlike Sample B/AL-C-4-25/A-P-B (Section 3.4.4.1) which contains 258 bentonite by weight, the outflow of B/AL-C-4-35/A-P-A (358 bentonite) returned to the normal rate. No further evidence of piping has been detected thereafter.

For Sample B/AL-C-4-35/A-P-B, a slow migration of mud flow through the side outlet was observed at the injection pressure of $482.6 \mathrm{kPa}$ (70 psi, hydraulic gradient: approximately 528). The injection pressure at which the flow of bentonite took place is uncertain because the view was blocked partially by silicon gels applied to glue tubing to the opening. The bentonite flow appeared to be smooth. No outflow bursts were seen.

\subsubsection{Flow Tests of Bentonite/Crushed Tuff Seals in Tuff Cylinders}

Mixtures of crushed tuff and bentonite have been installed in four hollow rock cylinders designated $\mathrm{RC}-1,-2-,-3$, and -4 . The cylinders are prepared from densely welded Apache Leap tuff blocks, except for RC-1, which was obtained from a Topopah Spring welded tuff block from the Nevada Test Site (NTS). According to South and Daemen (1986, p. 66), the NTS tuff block was collected loose on the surface, not broken from an outcrop. The mixtures are prepared by mixing type $A$ crushed Apache Leap tuff with 25 or $35 \%$ bentonite by weight. Sample characteristics are shown in Table 3.15. The mixtures are compacted inside the centered holes in three layers for the two $10-\mathrm{cm}$ diameter samples, and in two layers for the two $5-\mathrm{cm}$ samples. Each impact of the compaction rammer covers $25 \%$ of the cross-sectional area for the $10-\mathrm{cm}$ diameter samples, and close to 1008 for the $5-\mathrm{cm}$ samples. The compaction energy rendered is 368 higher than that of the standard Proctor compaction method (ASTM D698-78). The sealants are confined by a thin layer of sand, followed by a mechanical drain packer at each end. The mixtures are then subjected to $1.2 \mathrm{~m}$ water head from the bottom for over a month, in an attempt to saturate the samples.

\subsubsection{Mixed Sample in RG-1}

During the saturation process, a cone-shaped wetting front has been observed only in the NTS tuff cylinder, indicating that this particular rock sample is more permeable than the Apache Leap tuff cylinders. The NTS rock cylinder was later immersed in a water bath, in order to accelerate the saturation of the mixture inside the cylinder. Graduated pipettes have been attached to the top and:bottom of this plug and the flow rates have been monitored. The average flow rate is $0.15 \mathrm{cc} / \mathrm{mm}$ for the top pipette within 144.4 to $105.5 \mathrm{~cm}$ of water head, and $0.04 \mathrm{cc} / \mathrm{min}$ for the bottom pipette within 161.6 to $152.1 \mathrm{~cm}$ of water head. Most of the water travels through the rock and consequently raises the water level in the bath. The water level has been lowered so that the top surface of the NTS rock cylinder slightly emerges. During the subsequent saturation process, a wet zone with water droplets developed around the periphery of the centered hole. The far end of this ringshaped zone was about $2.5 \mathrm{~cm}$ from the edge of the hole, approximately 258 of the hole diameter. Such a phenomenon may be a result of gravity 
Table 3.15 Characteristics of Crushed Tuff/Bentonite Plugs Installed in Tuff Cylinders

\begin{tabular}{|c|c|c|c|c|c|c|}
\hline $\begin{array}{l}\text { Sample } \\
\text { Number }\end{array}$ & $\begin{array}{l}\text { Bentonite } \\
\text { Weight } \\
\text { Percent }\end{array}$ & $\begin{array}{l}\text { Air-drie } \\
\text { Bentonite }\end{array}$ & $\begin{array}{l}\text { Wt__ }(g) \\
\text { Crushed } \\
\text { Tuff }\end{array}$ & $\begin{array}{c}\text { Sample } \\
\text { Length } \\
\text { (cm) }\end{array}$ & $\begin{array}{c}\text { Sample } \\
\text { Diameter } \\
\text { (cm) }\end{array}$ & $\begin{array}{l}\text { Type } \\
\text { of } \\
\text { Tuff }\end{array}$ \\
\hline 1 & 35 & 317 & 589 & 8.9 & 9.95 & NTS \\
\hline 2 & 25 & 296 & 890 & 8.3 & 10.3 & $\begin{array}{c}\text { Apache } \\
\text { Leap }\end{array}$ \\
\hline 3 & 25 & 37 & 111 & 4.4 & 5.14 & $\begin{array}{c}\text { Apache } \\
\text { Leap }\end{array}$ \\
\hline 4 & 35 & 61 & 114 & 5.4 & 5.14 & $\begin{array}{c}\text { Apache } \\
\text { Leap }\end{array}$ \\
\hline
\end{tabular}

Table 3.16 Flow Test Results for the Bentonite/Crushed Tuff Plugs in Tuff Cylinders RC-3 and RC-4

\begin{tabular}{|c|c|c|c|c|c|c|}
\hline $\begin{array}{c}\text { Test } \\
\text { Dura- } \\
\text { tion } \\
\text { (days) }\end{array}$ & $\begin{array}{l}\text { Nominal } \\
\text { Injection } \\
\text { Pressure } \\
(\mathrm{kPa})\end{array}$ & $\begin{array}{l}\text { Toteal } \\
\text { Inflew } \\
\text { (cc) }\end{array}$ & $\begin{array}{l}\text { Total } \\
\text { Outflow } \\
\text { (cc) }\end{array}$ & $\begin{array}{l}\text { Hydrau- } \\
\text { Iic } \\
\text { Gradient } \\
\text { Range }\end{array}$ & \multicolumn{2}{|c|}{$\begin{array}{c}\text { Permeability } \\
\left(\mathrm{cm} / \mathrm{s} \times 10^{-9}\right) \\
\text { Stand. } \\
\text { Mean Dev. }\end{array}$} \\
\hline \multicolumn{7}{|c|}{ Sample in $\mathrm{RC}-3$ : } \\
\hline 17 & 34.5 & 18.6 & 1.8 & $\begin{array}{c}75.2- \\
66.6\end{array}$ & 1.98 & 1.94 \\
\hline 10.5 & 69 & 17.7 & 6.4 & $\begin{array}{c}135.8- \\
128.2\end{array}$ & 2.96 & 1.02 \\
\hline 10 & 103.5 & 22.8 & 11.8 & $\begin{array}{c}201.8- \\
197.7\end{array}$ & 3.56 & 0.68 \\
\hline 5.3 & 138 & 16.1 & 9.35 & $\begin{array}{c}266.9- \\
263.4\end{array}$ & 3.79 & 0.54 \\
\hline 7.6 & 207 & 30.0 & 20,2 & $\begin{array}{c}408.0- \\
388.2\end{array}$ & 3.71 & 0.22 \\
\hline \multicolumn{7}{|c|}{ Sample in $\mathrm{RC}-4:$} \\
\hline 24 & 345 & 60.4 & 10.8 & $\begin{array}{c}667.8- \\
657.7\end{array}$ & 0.383 & 0.142 \\
\hline
\end{tabular}


pull on the flow upon the water injection from the top, and/or a result of drilling damage.

No outflow through this plug has been observed for a total test period of 12 days. The plug remained in the rock cylinder, which was then exposed to the atmosphere at room temperature of $24 \pm 1^{\circ} \mathrm{C}$ for about 45 days. During this drying period, the top and bottom surfaces of the plug were open to the atmosphere through the drained inflow and outflow tubings passing through the confining packers. After removing the end packers, a gap of approximately $0.2 \mathrm{~mm}$ wide between the plug and the rock wall was found along 758 of the circumference of the center hole. The bond force provided by the remaining interface area, however, was enough to hold the plug in place. It required an additional $50 \mathrm{~N}$ to free the sample. The bond strength is estimated to be $8.8 \mathrm{kPa}$. Figure 3.100 shows the plug taken out of the rock cylinder. The radial permeability of the NTS cylinder itself was determined to range from 3.08 to $2.26 \times 10^{-6} \mathrm{~cm} / \mathrm{s}$, using Eq. 3.4 (Hsieh, 1983, p. 14).

$$
K=\frac{Q}{2 \pi L \Delta h_{w}} \ln \left(\frac{L}{\gamma_{w}}\right) \text { when } L>2 \gamma_{w}
$$

where $Q=$ volumetric injection rate

$L=$ length of injection zone

$\gamma_{w}=$ wellbore or center hole radius

$\Delta h_{\mathrm{w}}=$ hydraulic differential across the effective radius of influence, assumed to be the cylinder wall thickness.

\subsubsection{Mixed Sample in RG-2}

The mixture sample in tuff cylinder RC-2 consists of 258 bentonite by weight blended with 758 type A crushed tuff. This rock cylinder contains an oblique, natural hairline fracture $5 \mathrm{~cm}$ long, forming a $60^{\circ}$ angle with the horizontal and located about $1.5 \mathrm{~cm}$ from the bottom.

After the sample had been saturated under a $1.2 \mathrm{~m}$ water head from the bottom for over a month, a double-pipette flow system was attached to the plug-rock assembly in an attempt to measure the permeability of the plug. The double-pipette flow testing continued for 50 days with no outflow recorded. A constant-head flow system, aided by compressed air, was then employed to impose a higher hydraulic gradient across the plug. Approximately 8 days after the injection pressure had been raised to $34.5 \mathrm{kPa}$ ( $3.5 \mathrm{~m}$ water head), the sample failed as a result of piping. The piping channels developed along the rock-plug interface (Figure $3.101)$, as evidenced by the resurgence of water in the refilled inflow reservoir. The width of the channeling is no greater than $0.5 \mathrm{~cm}$; the channel location is indicated by the arrow in Figure 3.101.

Leaving the top end open to the atmosphere for about two weeks in a room with temperature of $24.5 \pm 0.5^{\circ} \mathrm{C}$ and relative humidity from 55 to 658 


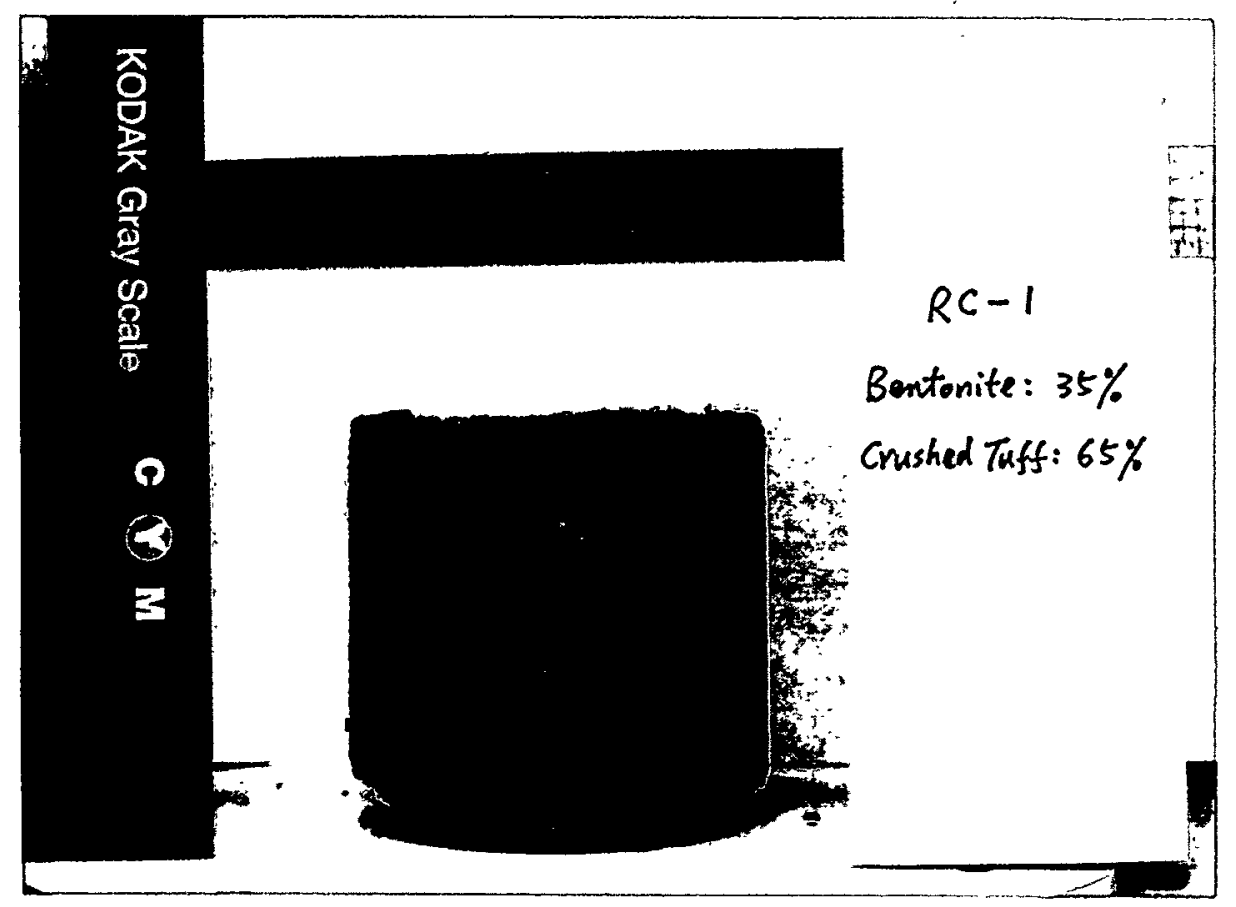

Figure 3.100 Bentonite/crushed tuff cylinder pushed out of rock cylinder RC-1.

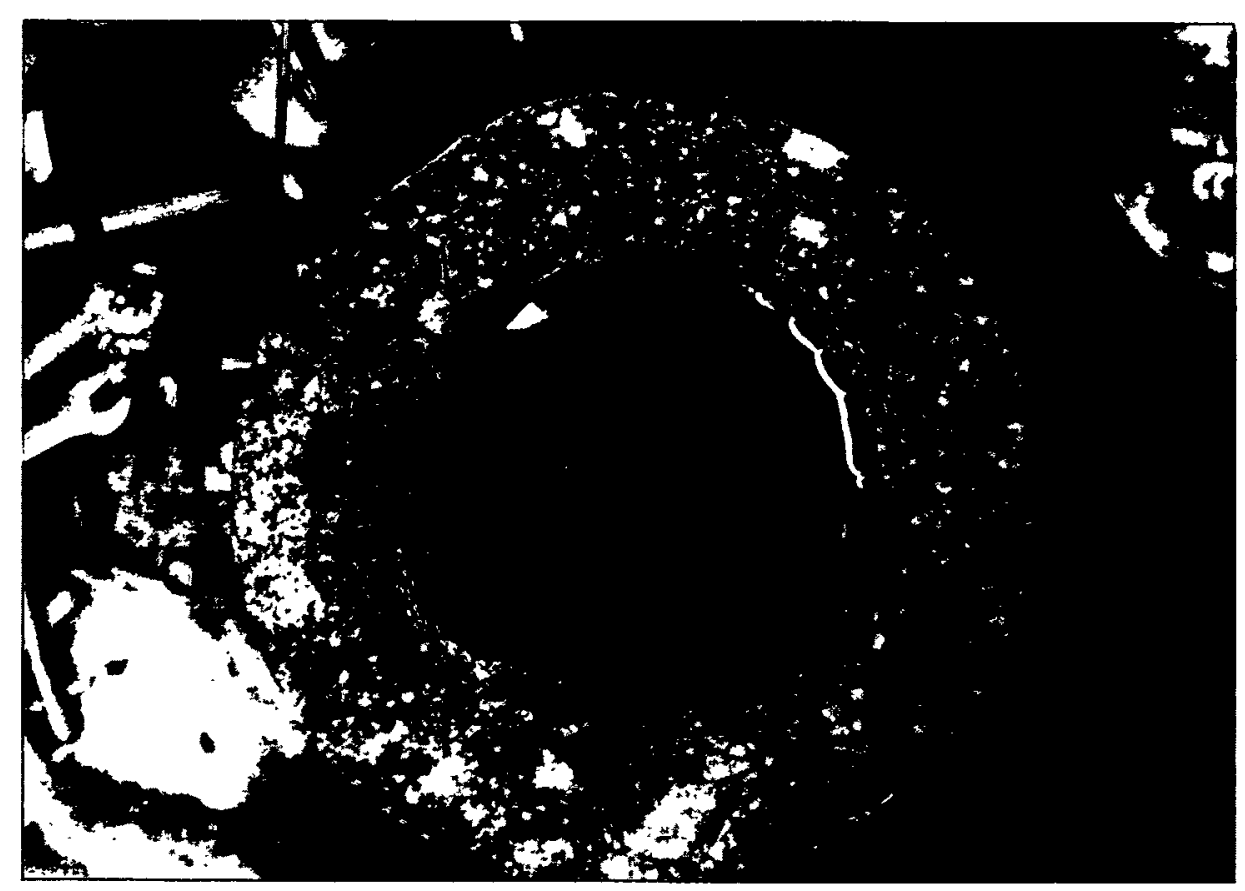

Figure 3.101 Piping channels that developed along the rock-plug interface of tuff cylinder RC-2. The channel location is indicated by the arrow. 
caused the sample to desiccate (Figure 3.102). The dessication cracks appear to extend to the bottom of the sample. This postulation is confirmed by observing the uniform, steady rise of inflow water in the peripheral crack as well as in the central crack. The cracks are healed (Figure 3.103) after the plug has been submerged for 3 days. The piping channel, however, remains as a preferential flowpath. The rehydration continued for 72 hours more before a push-out test was performed. Assuming uniform distribution of shear stress along the plug-rock interface, the bond strength is estimated to be $47.9 \mathrm{kPa}$. The average radial permeability of this Apache Leap tuff cylinder, which contains hairline cracks, ranges from 1.61 to $2.72 \times 10^{-8} \mathrm{~cm} / \mathrm{s}$. The pushed out plug is shown in Figure 3.104. The upper photo in Figure 3.104 depicts the vertical, peripheral piping channel located in the center of the photograph, where the granular texture is most conspicuous. The back side of the plug is much more homogeneous, as shown in the bottom photo of Figure 3.104. The horizontal detachment occurred during the push-out test.

\subsubsection{Mixed Sample RC-3}

The 5-cm diameter sample in RC-3 has the same composition as the plug in RC-2, i.e. $25 \%$ bentonite and 758 well-graded type A crushed tuff. This sample has been subjected to the initial saturation process for about 30 days and then to the double-pipette flow testing for an additional 50 days, with hydraulic gradients less than 10 . No positive outflow was recorded during the 80 days. In subsequent flow testing, a constanthead flow system aided by compressed air has been employed to impose higher hydraulic gradients. Positive outflow measurements have been collected at an injection pressure of $34.5 \mathrm{kPa}$ ( $5 \mathrm{psi}$ ) for 17 days with gradients ranging from 66.6 to 75.2 . The total outflow collected accounts for 9.678 of the inflow. The injection pressure has been increased to $69,103.4,137.9$ and $206.8 \mathrm{kPa}$ for a total of 33 days. The outflow collected under each injection pressure amounts to $11.3,52,58$ and 67.38 of the inflow, respectively (Table 3.16). At the injection pressure of $276 \mathrm{kPa}$, the bottom packer partially slipped out of the center hole and the flow test was terminated.

The permeability of the mixture sealant is evaluated on the assumption that the outflow collected is attributable only to the one-dimensional vertical flow through the plug. The results are summarized in Table 3.16. For the injection pressures of $103.5,138$ and $207 \mathrm{kPa}$, the mean permeabilities of this $5-\mathrm{cm}$ diameter plug are approximately two times lower than the permeabilities of the similar plug installed in a 101.6 $\mathrm{cm}$ diameter PVC permeameter as shown in Figures 3.33 through 3.35. The mean permeabilities are closely comparable to those of Sample B/AL-C-8$25 / \mathrm{A}-\mathrm{S}(20.3 \mathrm{~cm}$ in diameter) emplaced in a stainless steel permeameter (Figure 3.75 for the hydraulic gradient range 200-400). The onedimensional vertical flow assumption seems to be appropriate as the permeability values vary only slightly and all fall in the same order of $10^{-9} \mathrm{~cm} / \mathrm{s}$. Differences in plug size, compaction effort, and confining material, however, are noted.

Push-out testing was performed on the sample to determine the interface shear strength. The displacement of the plug requires a force of $890 \mathrm{~N}$. 


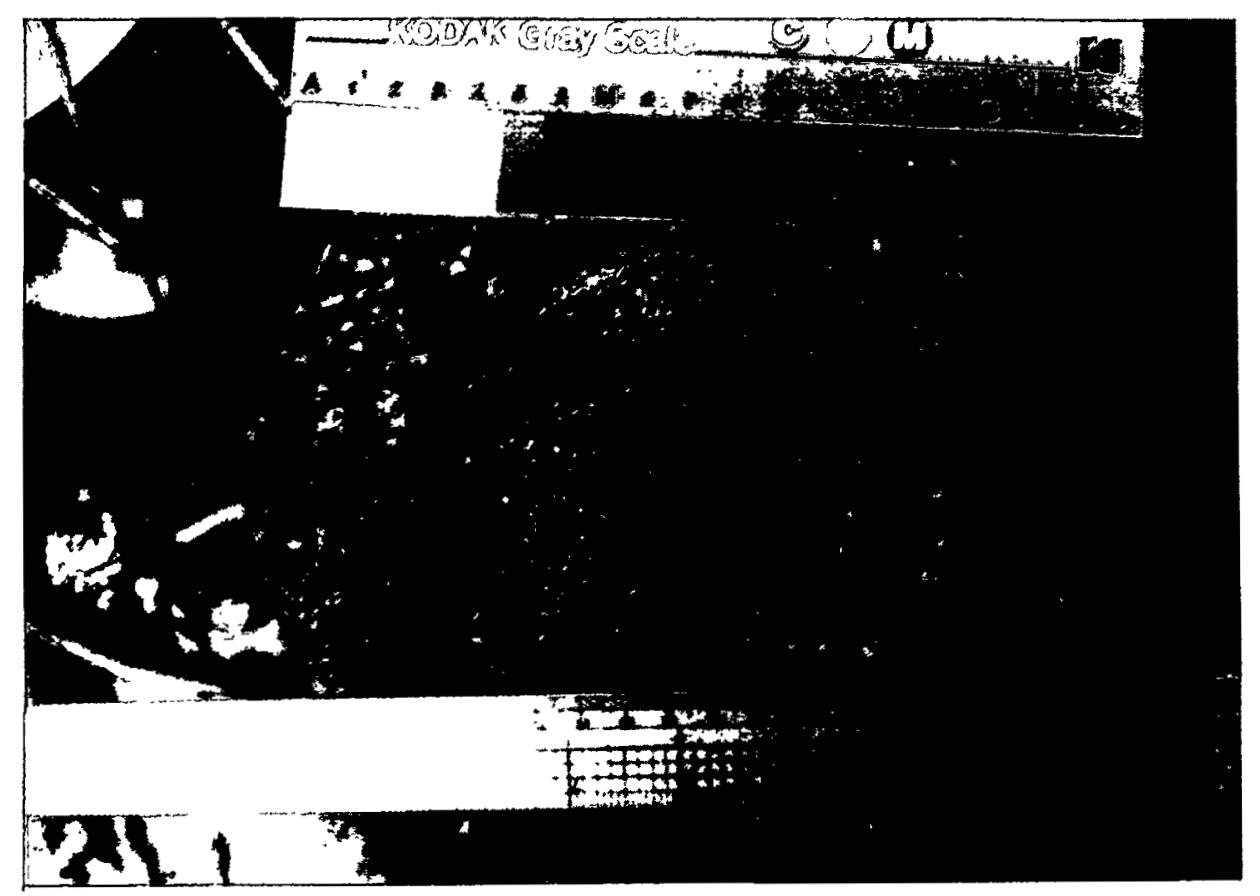

Figure 3.102 Shrinkage and desiccation cracks of the bentonite/crushed tuff plug in rock cylinder RC-2 after the top of the sample had been exposed to the atmosphere for about 14 days.

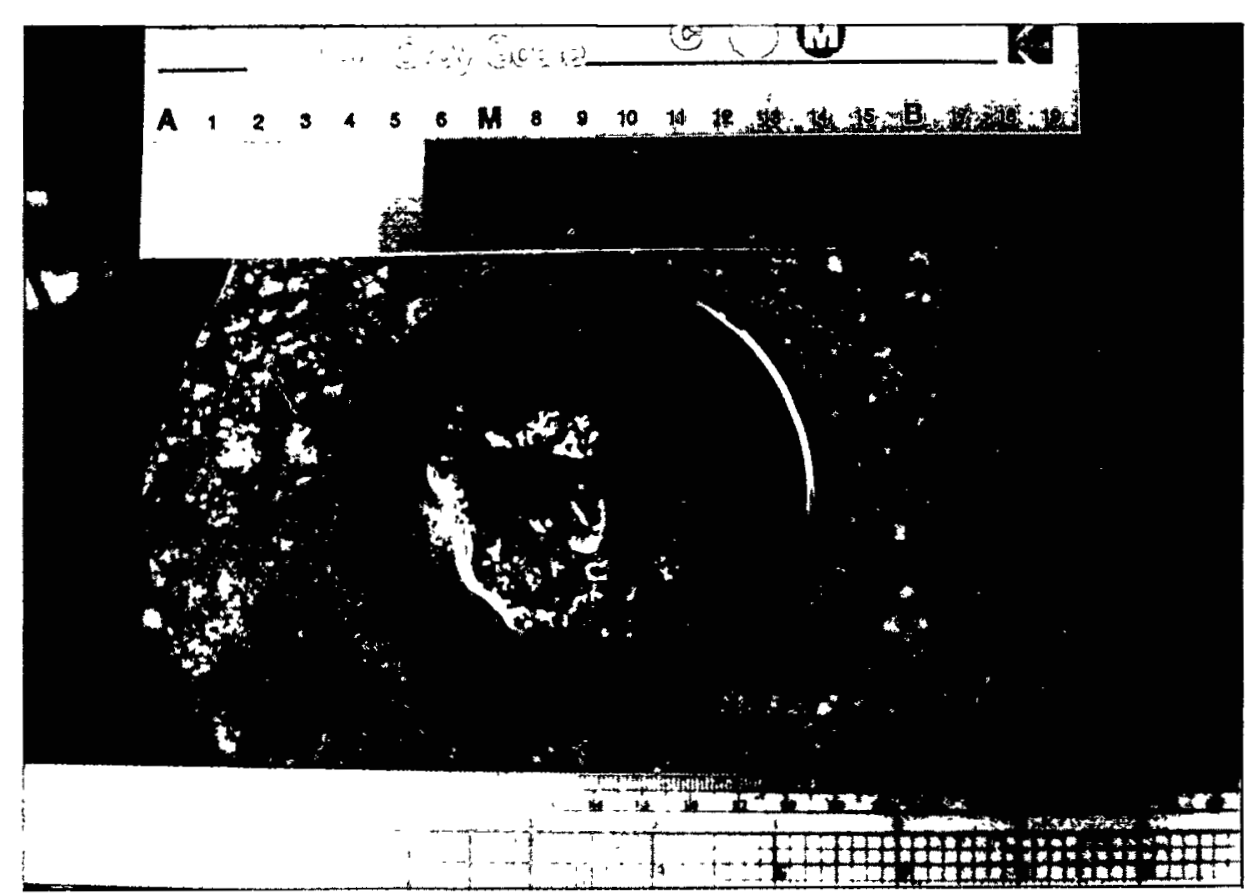

Figure 3.103 The healing of shrinkage and desiccation cracks of the bentonite/crushed tuff seal in rock cylinder RC-2 upon rehydration. The piping channel remains as a preferential flowpath. 

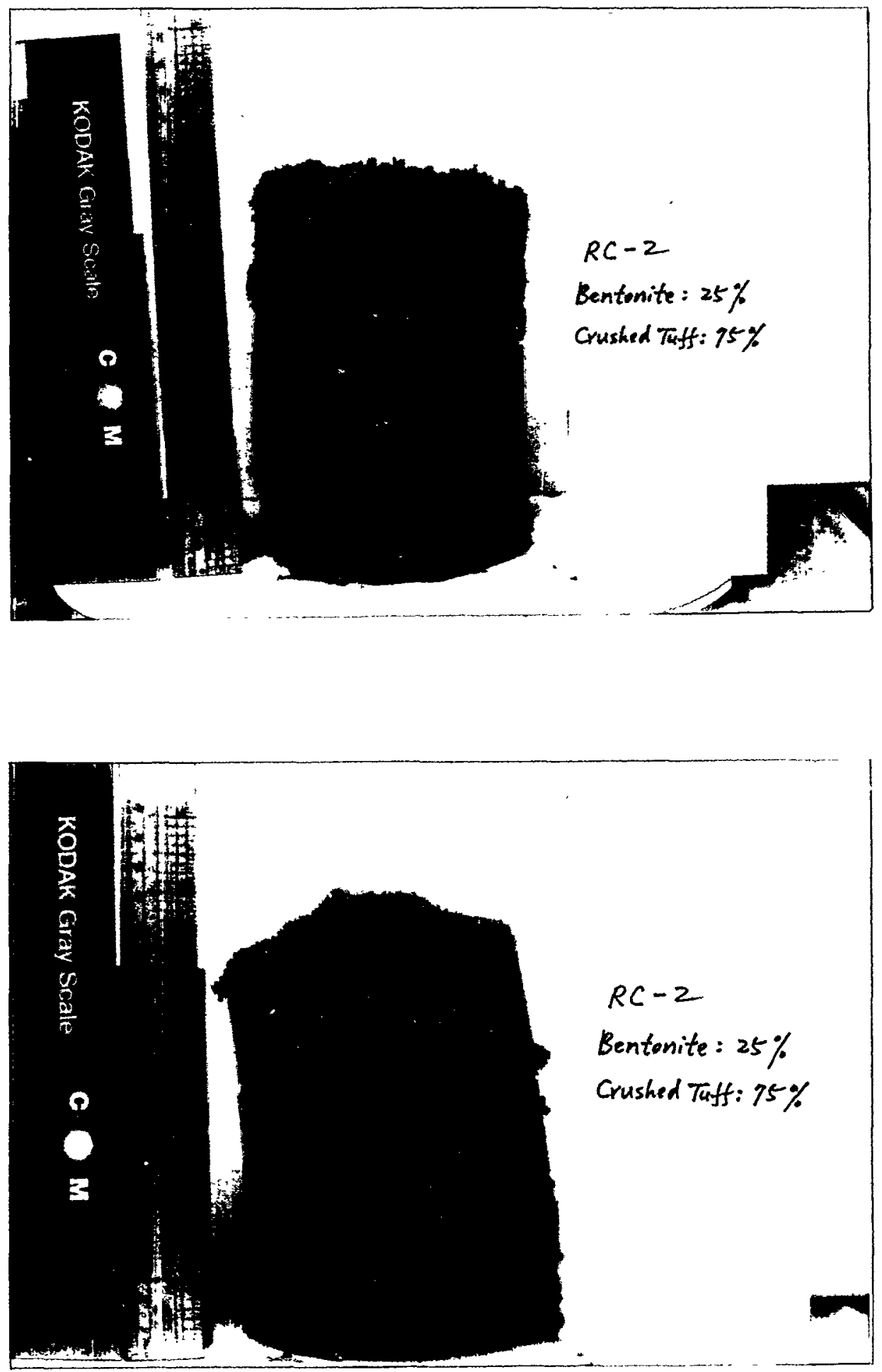

Figure 3.104 Bentonite/crushed tuff cylinder pushed out of rock cylinder RC-2. The top and bottom photographs show the front side and the back side, respectively, of the rock. Note the vertical, peripheral piping channel located in the center of the upper photograph. 
The interface shear strength is $103 \mathrm{kPa}$, assuming a uniform shear stress.

\subsubsection{Mixed Sample in RC-4}

The plug installed in tuff cylinder RC-4 consists or 358 bentonite and 658 type A crushed tuff. This $5-\mathrm{cm}$ diameter sample has been subjected to a $1.2 \mathrm{~m}$ hydraulic head and various injection pressures up to $276 \mathrm{kPa}$ (40 psi) for about 170 days. No outflow was observed during that period. The injection pressure was then raised to $345 \mathrm{kPa}$; outflow was first detected approximately 8 days later. The flow testing continued for an additional 24 days. The total outflow $(10.8 \mathrm{cc})$ accounts for 188 of the inflow $(60.4 \mathrm{cc})$. Based on the assumption of $1-D$ vertical flow through the mixture, this plug is estimated to have a mean permeability of $3.83 \times 10^{-10} \mathrm{~cm} / \mathrm{s}$ and a standard deviation of $1.42 \times 10^{-10^{2}} \mathrm{~cm} / \mathrm{s}$ (Table $3.16)$.

\section{$\underline{3.5 \text { Analysis }}$}

\subsubsection{Bentonite Permeability as a Function of Void Ratio}

Permeabilities of eleven bentonite (C/S granular) plugs are summarized in Table 3.17. The results are obtained from the flow testing under low hydraulic gradients. Also shown in the table are bentonite (MX-80) permeability measurements reported by Borgesson et al. (1988). Figure 3.105 shows bentonite permeability as a function of void ratio for the eleven samples along with a dashed line of the best fit. Figure 3.106 depicts a similar plot including the data from Borgesson et al. (1988). The regressed equations are Eqs. 3.5 and 3.6, respectively.

$$
\begin{aligned}
& K(\mathrm{~cm} / \mathrm{s})=3.72 * 10^{-11}(e)^{2.83996} \\
& K(\mathrm{~cm} / \mathrm{s})=6.68 * 10^{-11}(\mathrm{e})^{3.15926}
\end{aligned}
$$

where $e=$ void ratio. As shown in Figure $3.106, \mathrm{Eq} .(3.6)$ gives a better fit and therefore is more suitable for estimating the permeability of bentonite.

\subsubsection{Sealing Performance of Bentonite/Crushed Tuff Plugs}

Permeabilities of bentonite/crushed tuff plugs obtained from the downward flow testing under low hydraulic gradients $(<35)$ are shown in Figure 3.107 as a function of bentonite weight percent. The permeability decreases with increasing bentonite content. The permeabilities of the plugs containing 25 or 358 are close to the permeability of plugs constructed of bentonite only. Figure 3.107 also demonstrates the effect of crushed tuff gradation on the sealing performance: the greater the uniformity coefficient $\left(\mathrm{d}_{60} / \mathrm{d}_{10}\right)$ of crushed tuff $(\mathrm{e} . \mathrm{g}$. types $\mathrm{FA}$ and 
Table 3.17. Measured Permeabilities of Bentonites (C/S granular and $\mathrm{MX}-80$ )

\begin{tabular}{lcccc}
$\begin{array}{c}\text { Sample } \\
\text { Number }\end{array}$ & $\begin{array}{c}\text { Porosity } \\
(\mathrm{n})\end{array}$ & $\begin{array}{c}\text { Water } \\
\text { Content }\end{array}$ & $\begin{array}{c}\text { Hydr. } \\
\text { Grad. }\end{array}$ & $\begin{array}{l}\text { Measured } \\
\mathrm{K}, \mathrm{cm} / \mathrm{s})\end{array}$ \\
\hline (C/S granular, from this study) & & \\
B-S-1-A & 0.933 & 475.8 & $<10$ & $7.0 * 10^{-8}$ \\
B-C-1-A & 0.879 & 249.7 & $<12$ & $1.2 * 10^{-8}$ \\
B-C-1-B & 0.880 & 251.5 & $<12$ & $1.3 * 10^{-8}$ \\
B-C-2-A & 0.831 & 168.1 & $<12$ & $2.5 * 10^{-9}$ \\
B-C-2-B & 0.836 & 175.0 & $<12$ & $3.7 * 10^{-9}$ \\
B-C-4-A & 0.846 & 188.5 & $<12$ & $3.3 * 10^{-9}$ \\
B-C-4-B & 0.848 & 191.7 & $<12$ & $3.5 * 10^{-9}$ \\
B-C-1-A-S & 0.625 & 57.1 & $<57$ & $9.0 * 10^{-11}$ \\
B-C-1 3/8-A-S & 0.721 & 88.3 & $<77$ & $2.2 * 10^{-9}$ \\
B-C-2 3/8-A-S & 0.638 & 60.3 & $<50$ & $1.1 * 10^{-10}$ \\
B-C-4-A-S & 0.644 & 62.1 & $<57$ & $3.0 * 10^{-10}$ \\
\hline
\end{tabular}

\begin{tabular}{lccc}
$\begin{array}{l}\text { Saturated } \\
\begin{array}{l}\text { Density } \\
(\mathrm{g} / \mathrm{cc})\end{array}\end{array}$ & $\begin{array}{c}\text { Porosity } \\
(\mathrm{n})\end{array}$ & $\begin{array}{c}\text { Water } \\
\text { Content }\end{array}$ & $\begin{array}{l}\text { Measured } \\
\mathrm{K}(\mathrm{cm} / \mathrm{s})\end{array}$ \\
\hline$(\mathrm{MX}-80$, from Borgesson et al., 1988$)$ \\
2.1 & 0.421 & 25.08 & $3.0 \times 10^{-12}$ \\
1.9 & 0.526 & 38.31 & $1.9 * 10^{-11}$ \\
1.7 & 0.631 & 59.10 & $2.0 * 10^{-10}$ \\
1.57 & 0.7 & 80.45 & $6.0 * 10^{-10}$ \\
1.295 & 0.845 & 187.62 & $3.8 * 10^{-9}$ \\
\hline
\end{tabular}




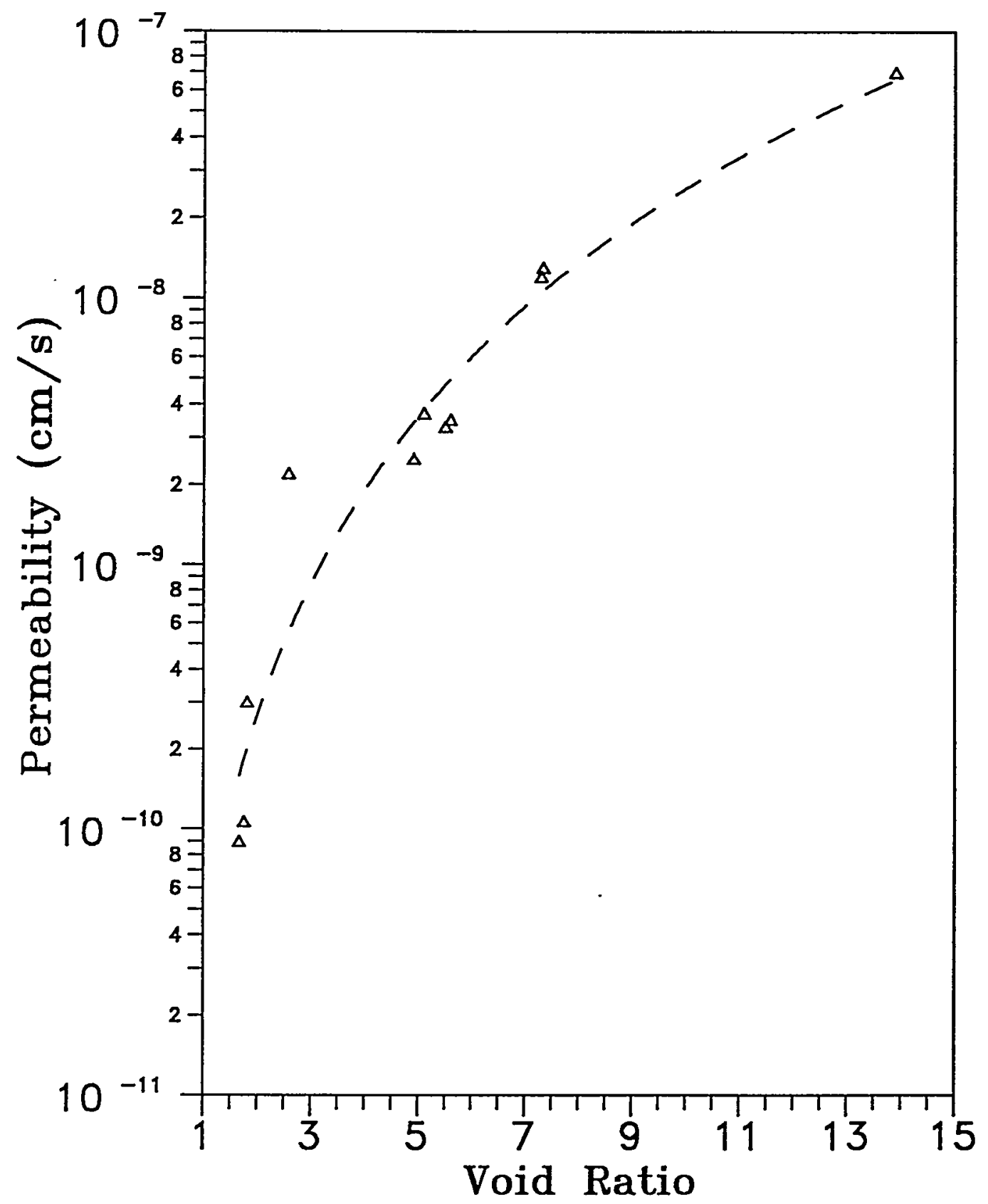

Figure 3.105 Permeability of the $\mathrm{C} / \mathrm{S}$ granular bentonite as a function of void ratio. 


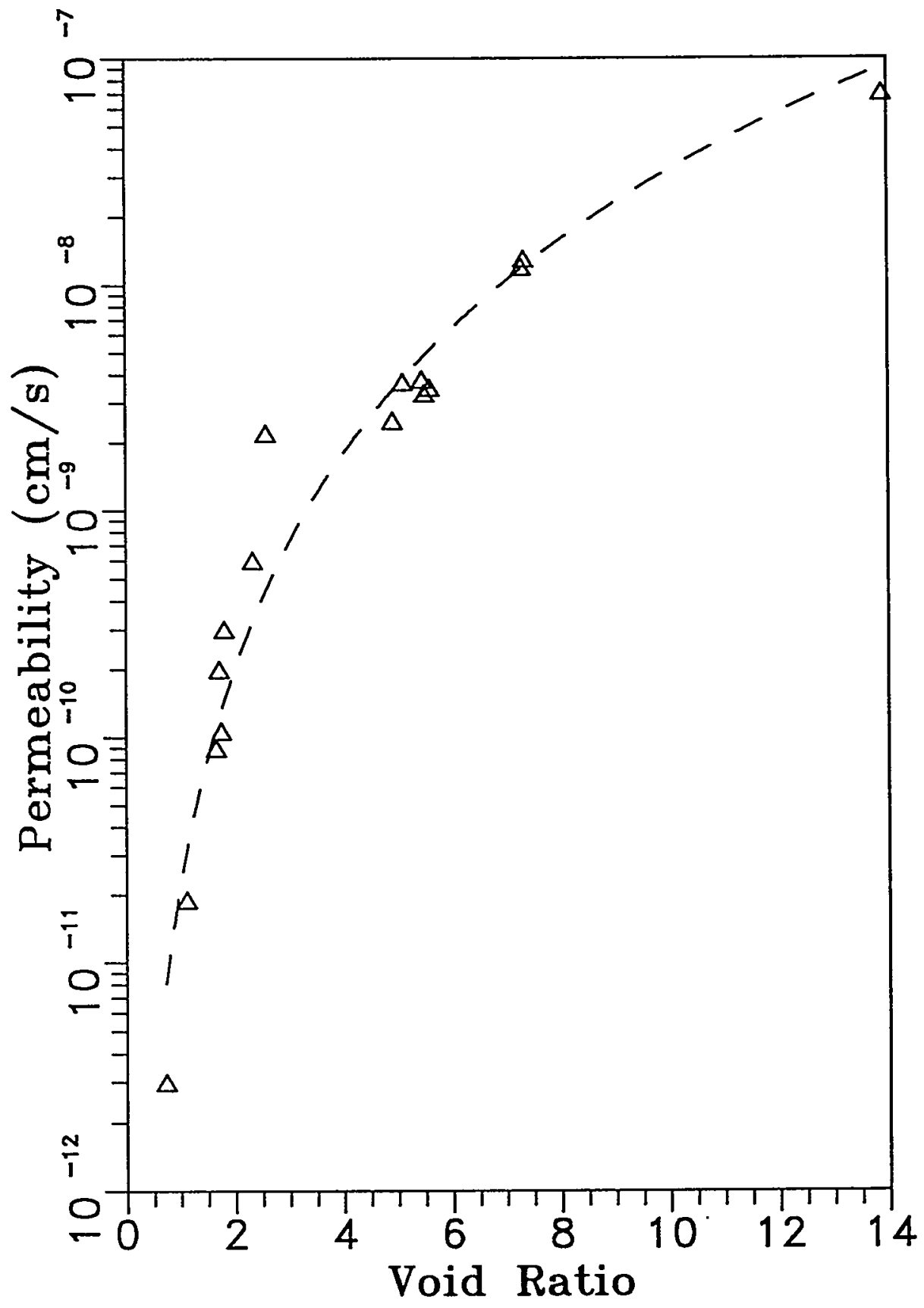

Figure 3.106 Permeability of Wyoming sodium bentonite as a function of void ratio (including measurements of eleven C/S granular samples from this study and of five $M X-80$ samples from Borgesson et al., 1988). 


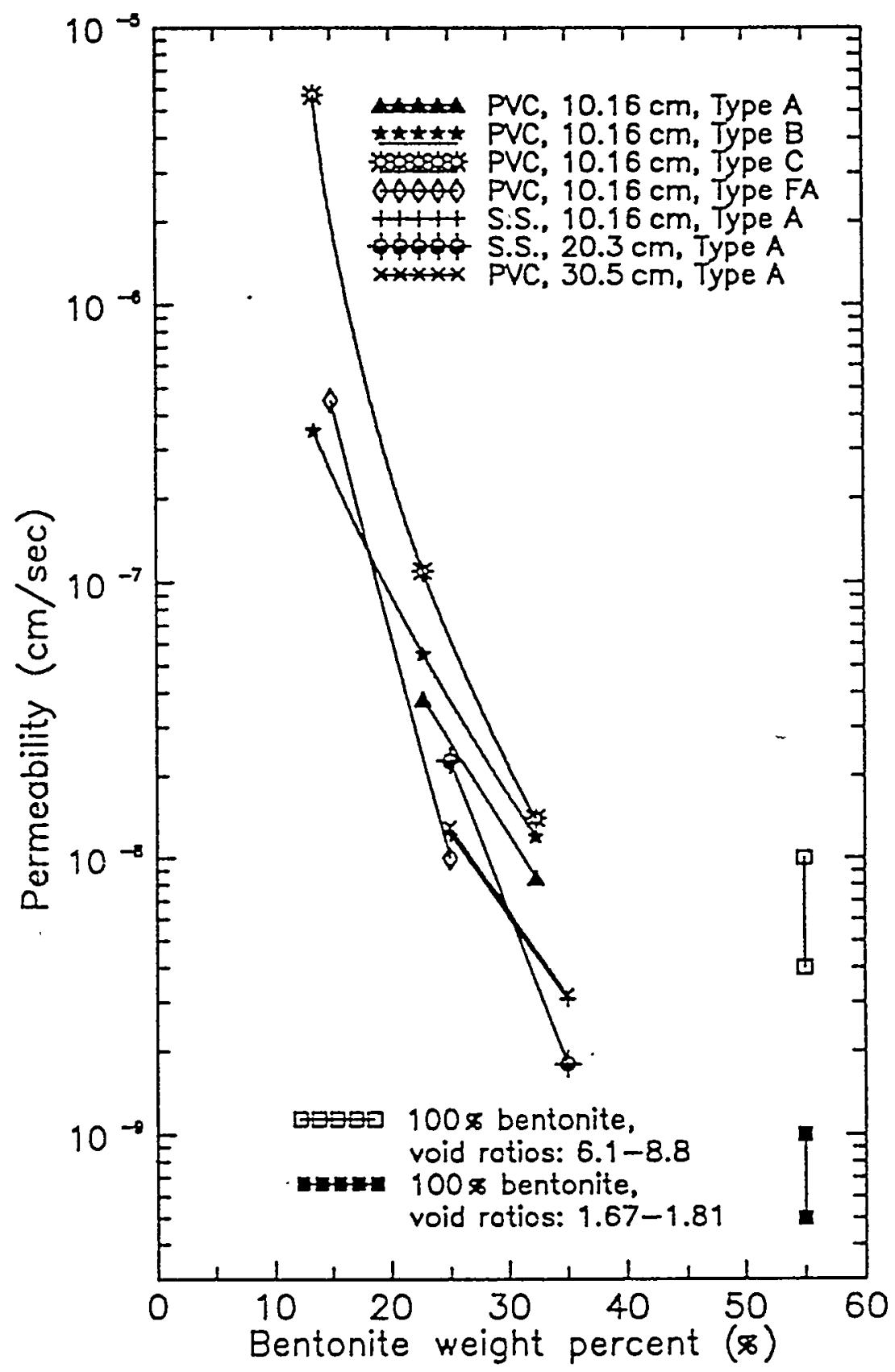

Figure 3.107 Permeability of bentonite/crushed tuff mixtures as a function of bentonite content. Hydraulic gradients are lower than 35 . 
A), the lower the permeability. The results suggest that an appropriate composition for the mixture plugs would contain at least $25 \%$ bentonite by weight mixed with well-graded crushed rock.

The effect of sample size on sealing performance is not clear. The inconsistency in the permeabilities measured for different plug sizes may be due more to variations in the stiffness of the permeameters, compaction, and the ratio of grain size to permeameter diameter (Ouyang and Daemen, 1989, 1990). For all the normally consolidated mixture samples, the upward permeability is about three times higher than the downward permeability.

According to the high injection pressure flow test results of Samples B/AL-C-8-25/A-S and B/AL-C-8-35/A-S (Figures 3.76 and 3.78 ), the potential for piping damage to the sealing performance is small if the maximum hydraulic gradient does not exceed approximately 120 and 280 , respectively. The piping test results of Sample B/AL-C-4-25/A-P-B (Section 3.4.4.1) tend to support this deduction. For a loosely or ineffectively compacted mixture containing 258 bentonite (Sample B/AL-C$8-25 / F C-S)$, the sealing performance can be damaged by dynamic disturbances. The influence of such disturbances is greatly reduced when more bentonite is added (e.g. Sample B/AL-C-8-35/FC-S).

Polyaxial flow test results indicate that a difference of up to one or two orders of magnitude may be expected between the vertical and horizontal permeabilities. The high horizontal permeability results from the uneven bentonite distribution in the pores between crushed rock grains due to particle segregation resulting from the actions of sample installation and compaction. The segregation of particles can be seen in Figure 3.108. The permeability difference may be reduced by introducing a thin layer of bentonite on top of each compacted layer, an approach which deserves further investigation.

Temperature seems to have no significant negative effects on the sealing performance within the test range from room temperature to $60^{\circ} \mathrm{C}$. The specific permeability reaches a maximum at $35^{\circ} \mathrm{C}$ and decreases slightly with increasing temperature.

Flow testing of bentonite/crushed tuff sealants installed in rock cylinders provides additional information for assessing the performance of the mixture plugs when they are emplaced in boreholes or shafts. The flow test results show a much higher permeability $\left(3.08\right.$ to $2.26 \times 10^{-6}$ $\mathrm{cm} / \mathrm{s}$ ) for the rock cylinder RC-1 (NTS tuff cylinder) than for the mixture plug ( 358 bentonite and 658 type A crushed tuff). Therefore, most of the water travels through the rock. The mixture plug in RC-2 (Apache Leap tuff cylinder) consists of $25 \%$ bentonite mixed with $75 \%$ type A crushed tuff. This $10.28 \mathrm{~cm}$ diameter sample failed as a result of piping under a hydraulic gradient of approximately 37 . The piping channel has developed vertically along the rock-plug interface. Its lateral extent, however, is limited. Localized compositional defects (e.g. uneven bentonite distribution in the pores between crushed tuff particles) must exist in the sample, which invite the development of channeling. Having the sample composition as the plug in RC-2, the $5.14-\mathrm{cm}$ diameter sample installed in $\mathrm{RC}-3$ gives a rather satisfactory 


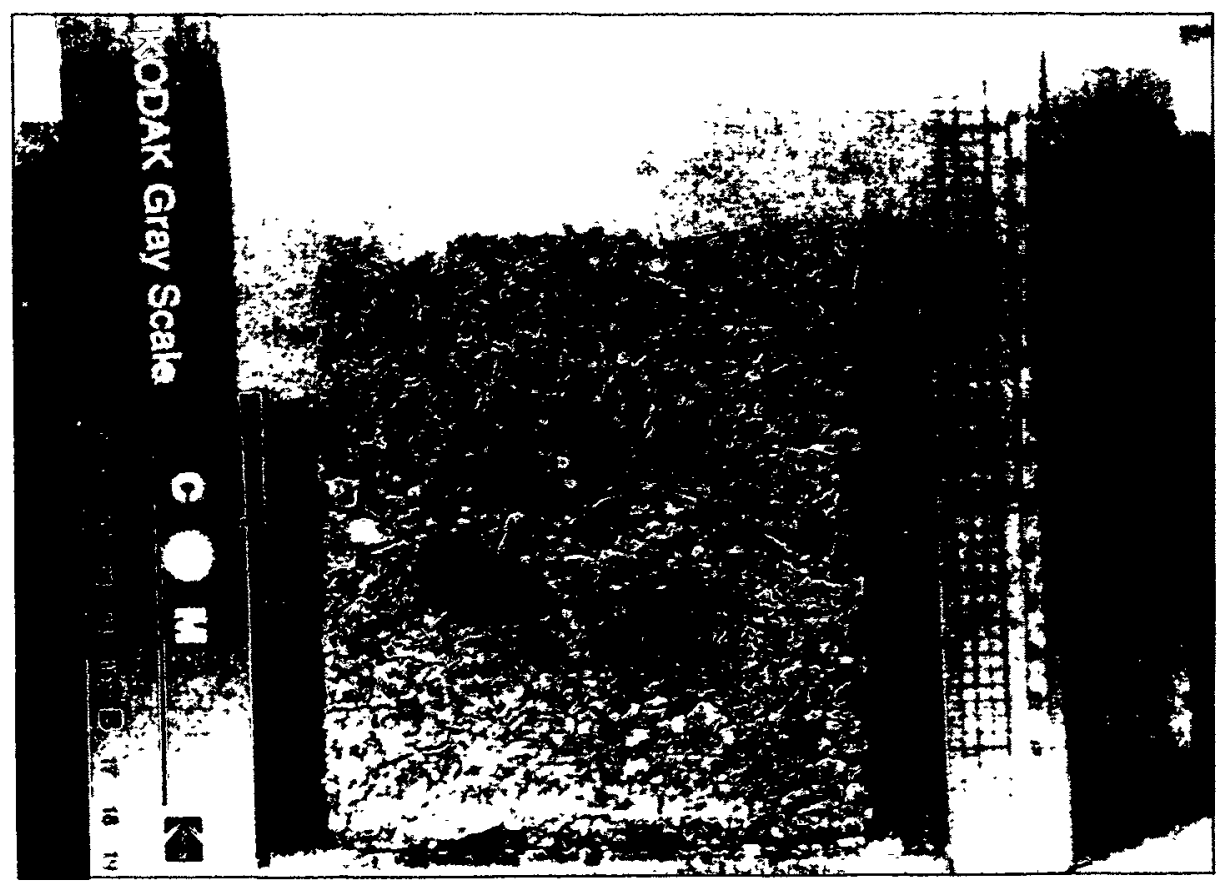

Figure 3.108 Appearance of particle segregation highlighted by desiccation cracks (sample composition: $25 \%$ bentonite and $75 \%$ type $A$ crushed tuff, air-dried after the polyaxial flow testing). 
sealing performance throughout the imposed injection pressures up to 207 $\mathrm{kPa}$. The permeability of the plug in RC-3 falls in the lower range of $10^{-9} \mathrm{~cm} / \mathrm{s}$, which is slightly smaller, but quite conformable, to the earlier results of similar plugs installed in PVC and stainless steel permeameters. The mixture plug in RC-4 contains $35 \%$ bentonite by weight and yields a mean permeability of $3.83 \times 10^{-10} \mathrm{~cm} / \mathrm{s}$ for hydraulic gradients from 668 to 658 over a 24 day period.

The flow test results of the mixture sealants installed in tuff cylinders parallel those of similar plug emplaced in permeameters. A higher degree of compaction seems to reduce the permeability of the mixtures, but is unable to totally remove compositional defects in the samples that originate during sample installation. The occurrence of such defects, manifested through piping, diminishes when more bentonite (e.g. 358 by weight) is added.

\subsubsection{Influence of Gradation of Crushed Tuff}

Figure 3.107 shows a probable trend that, for bentonite/crushed tuff plugs, the greater the uniformity coefficient $\left(C_{u}, d_{60} / d_{10}\right)$ of crushed tuff (e.g. types FA and A), the lower the permeability.

The flow test results of Samples B/AL-C-4-25/A and B/AL-C-4-25/A-S (Type $A$ crushed tuff gradation, $C_{u}=16.5,258$ bentonite by weight), B/AL-C4-25/FA-A and B/AL-C-4-25/FA-B (Type FA crushed tuff gradation, a theoretical $C_{u}=36,258$ bentonite by weight), show that their permeabilities basically fall in the lower $10^{-8} \mathrm{~cm} / \mathrm{s}$ range for the low hydraulic gradients applied. Type FA contains fewer large particles than type $A$, and more small particles, including 8.86 weight percent of particles smaller than $0.074 \mathrm{~mm}$ (U.S. mesh \#200). Nevertheless, the permeability decrease with increasing hydraulic gradient appears to be less and slower for samples constructed with crushed tuff of the FA gradation (Figures 3.85 and 3.89 ). If the decrease in permeability with increasing hydraulic gradient is primarily due to pore clogging by particle migration, then the samples with FA crushed tuff are expected to have a denser structure, smaller average pore size, and less susceptibility to piping than samples constructed with type A gradation.

Particle size distributions of nine crushed tuff gradations are given in Table 3.18. They include gradation types Coarse, A, B, C, and five ideal gradations obtained from the Fuller-Thompson grading equation (Eq. 2.2). The equation has been used in formulating backfill material for a nuclear waste disposal vault (Pusch and Alstermark, 1985, in which the exponent $\mathrm{n}$ is not reported; Yong et al., 1986, $\mathrm{n}=0.25)$. In the following subsections, the bulk porosity and permeability of some of the systems of crushed tuff are studied.

\subsubsection{Bulk Porosity of Systems of Crushed Tuff Particles}

The bulk porosity of an assembly of crushed tuff particles is primarily controlled by particle gradation and emplacement method. Porosities of crushed tuff of gradation types A, FA $(n=0.5)$, FC $(n=0.25)$, FC $(n=$ $0.45)$, and FC $(n=0.5)$ are evaluated for the pouring and scooping emplacement methods. Porosities obtained by pouring followed by shaking 
Table 3.18 Particle Size Distribution of Nine Crushed Tuff Gradations

\begin{tabular}{|c|c|c|c|c|c|c|c|c|c|}
\hline \multirow{2}{*}{$\begin{array}{l}\text { Sieve } \\
\text { Size } \\
(\mathrm{mm}) \\
\end{array}$} & \multicolumn{9}{|c|}{ Crushed Tuff Gradation Type } \\
\hline & $\mathrm{A}$ & B & C & $\begin{array}{c}F A \\
(n-0.5)\end{array}$ & $\begin{array}{c}F A \\
(n=0.45)\end{array}$ & Coarse & $\begin{array}{c}\text { FC } \\
(n-0.25)\end{array}$ & $\begin{array}{c}\text { FC } \\
(n-0.45)\end{array}$ & $\begin{array}{c}F C \\
(n-0.5)\end{array}$ \\
\hline \multicolumn{10}{|c|}{ Weight percent retained: } \\
\hline $19.05(3 / 4 \mathrm{ln})$ & & & & & & i & & & \\
\hline $12.7(1 / 2 \mathrm{in})$ & & & & & & 35.8 & 9.64 & 16.68 & 18.35 \\
\hline $9.42(10.37 \mathrm{in})$ & & & & & & 18.08 & 6.50 & 10.47 & 11.32 \\
\hline $16.68(0.26 \mathrm{In})$ & 26.98 & & & 15.8 & 14.34 & 12.4 & 6.91 & 10.45 & 11.11 \\
\hline 4.75 (\#4) & $1: 6.94$ & .26 & & 13.2 & 12.19 & 7.75 & 6.29 & 8.88 & 9.29 \\
\hline $2.0(\# 10)$ & 23.69 & 29.5 & 45 & 24.93 & 23.69 & 11.01 & 13.74 & 17.26 & 17.53 \\
\hline $0.84(\# 20)$ & 12.71 & 19.5 & 23 & 16.20 & 16.07 & 5.40 & 11.08 & 11.71 & 11.39 \\
\hline $0.42(\# 40)$ & 7.09 & 7.5 & 10 & 8.78 & 9.07 & 3.23 & 7.33 & 6.61 & 6.18 \\
\hline $0.25(\# 60)$ & 4.22 & 6.0 & 8.0 & 4.84 & 5.14 & 1.84 & 4.7 & 3.75 & 3.4 \\
\hline $0.15 \quad(\# 100)$ & 3.80 & 5.5 & 6.0 & 3.63 & 3.98 & 1.78 & 4.02 & 2.90 & 2.56 \\
\hline $0.074(\# 200)$ & 4.575 & 6.0 & 8.0 & 3.76 & 4.23 & 1.74 & 4.825 & 3.08 & 2.64 \\
\hline \multicolumn{10}{|c|}{ Weight percent passing: } \\
\hline $0.074(\# 200)$ & 0.0 & 0.0 & 0.0 & 8.86 & 11.29 & 0.97 & 24.97 & 8.23 & 6.23 \\
\hline
\end{tabular}

NOTES:

1. Crushed tuff gradation type 'coarse' is obtained by crushing chunks of Apache Leap tuff using a jaw crusher and a roller crusher in sequence.

2. The prefix ' $F$ ' in gradation type stands for a Fuller-Thompson grading curve, which is described by $P_{W}=100(d / D)^{n}$, where $P_{W}$ - weight percent passing sieve aperture $d, D-$ maximum particle size, and $\mathrm{n}=$ exponent. 
are also determined to evaluate the possible range of porosity.

Particles smaller than $0.074 \mathrm{~mm}$ are removed from the crushed tuff before the determination of the porosity. The crushed tuff aggregates emplaced by scooping are compacted using the standard Proctor method. Impact compaction is likely to be used for compacting backfill in a borehole or a shaft (Martin, 1975, p. 18).

From the sample weight and dimensions, both porosities of packings of crushed tuff particles are calculated in two ways, based on the solid density $\left(2.61 \mathrm{~g} / \mathrm{cm}^{3}\right)$ and based on the dry bulk density $\left(2.37 \mathrm{~g} / \mathrm{cm}^{3}\right)$. The results are summarized in Table 3.19 .

Shaking gives the lowest porosity, pouring the highest. Shaking also induces significant particle segregation, leaving fine particles on the bottom and large particles on top. The particle segregation is reversed for the same crushed sample emplaced by pouring because large particles fall faster than small particles. The crushed tuff aggregates installed by scooping appear to be most homogeneous. The difference between a poured and a scooped sample can be seen in Figure 3.109.

The porosities in Table 3.19 are based on at least three measurements for each emplacement method, except for the compacted samples (one measurement per sample). At least two samples have been prepared for each gradation type. The maximum particle size is $9.43 \mathrm{~mm}$ for crushed tuff gradation types $A$ and FA, $19.05 \mathrm{~mm}$ for the three FC types (i.e. $\mathrm{n}=0.25,0.45$ and 0.5$)$. For the former two gradation types, the porosities obtained for the scooped samples are similar. The porosity ranges overlap. Porosity measured after compaction is lower for type FA than for type A. For the three FC types, porosity obtained from scooping tends to increase with $\mathrm{n}$ values in both the uncompacted and the compacted cases. Among the five gradations, crushed tuff aggregates of the type FC with $\mathrm{n}=0.25$ give the lowest porosity.

\subsubsection{Permeability of Systems of Crushed Tuff Particles}

Permeabilities of the five crushed tuff gradations have been determined using the constant head method. The crushed tuff assemblies tested contain no particles smaller than $0.074 \mathrm{~mm}$. Each flow test includes at least six measurements. The results are summarized in Table 3.20. The porosities are slightly lower than those in Table 3.19. This is the result of knocking on the permeameter wall, in an attempt to remove air bubbles during the saturation process.

For the uncompacted samples, permeability decreases with increasing amount of fine particles in the aggregate, with the lowest permeability of $2.9 \times 10^{-3} \mathrm{~cm} / \mathrm{s}$ measured for type FC $(n=0.25)$. This observation parallels the results reported by Kenney et al. (1984), who state that permeability of granular materials is primarily controlled by the small particles and is practically independent of the shape of the gradation.

Compaction reduces the permeability of the mixtures for gradation types FA $(n=0.5)$, FC $(n=0.45)$, and FC $(n=0.5)$, while increasing the permeability for types $A$ and FC $(n=0.25)$. The lowest permeabilities are obtained for the compacted FC aggregates $(n=0.45$ and 0.5$)$. This 
Table 3.19 Bulk Porosities of Packings of Crushed Tuff Particles (as a function of gradation and of emplacement method)

\begin{tabular}{|c|c|c|c|c|c|c|}
\hline \multirow{3}{*}{$\begin{array}{l}\text { Crushed } \\
\text { Tuff Grada- } \\
\text { tion Type }\end{array}$} & & & & & & \\
\hline & \multicolumn{3}{|c|}{ Porosity, $n_{1}$} & \multicolumn{3}{|c|}{ Porosity, $n_{2}$} \\
\hline & Pouring, & Scooping & Shaking & Pouring & Scooping & shaking \\
\hline$\Lambda-1$ & $\begin{array}{c}0.517-0.522 \\
(0.519 \pm 0.002)\end{array}$ & $\begin{array}{c}0.483-0.503 \\
(0.492 \pm 0.009)\end{array}$ & $\begin{array}{c}0.466-0.485 \\
(0.476 \neq 0.008)\end{array}$ & $\begin{array}{c}0.168-0.474 \\
(0.470 \pm 0.002)\end{array}$ & $\begin{array}{c}0.431-0.453 \\
(0.441 * 0.010) \\
\end{array}$ & $\begin{array}{c}0.412-0.433 \\
(0.424 \pm 0.009)\end{array}$ \\
\hline $\begin{array}{c}\Lambda-1 \\
\text { (compacted) }\end{array}$ & & 0.388 & & & 0.326 & \\
\hline$\Lambda-2$ & $\begin{array}{c}0.522-0.537 \\
(0.528 \pm 0.007)\end{array}$ & $\begin{array}{c}0.478-0.496 \\
(0.488 \pm 0.008)\end{array}$ & $\begin{array}{c}0.475-0.478 \\
(0.476 \pm 0.002)\end{array}$ & $\begin{array}{c}0.473-0.491 \\
(0.479 \pm 0.008)\end{array}$ & $\begin{array}{c}0.425-0.445 \\
(0.436 * 0.008)\end{array}$ & $\begin{array}{c}0.422-0.425 \\
(0.423 \pm 0.002)\end{array}$ \\
\hline$\Lambda-3$ & $\begin{array}{c}0.524-0.533 \\
(0.527 \cdot 0.004)\end{array}$ & $\begin{array}{c}0.483-0.493 \\
(0.489 \neq 0.004)\end{array}$ & $\begin{array}{c}0.455-0.461 \\
(0.457 \pm 0.003)\end{array}$ & $\begin{array}{c}0.476-0.485 \\
(0.479 \pm 0.004)\end{array}$ & $\begin{array}{c}0.431-0.441 \\
(0.437 \pm 0.004)\end{array}$ & $\begin{array}{c}0.399-0.407 \\
(0.402 \pm 0.00(4)\end{array}$ \\
\hline $\begin{array}{c}A-4 \\
\text { (compacted) }\end{array}$ & & 0.379 & & & 0.316 & \\
\hline$\Lambda-5$ & & 0.490 & & & 0.438 & \\
\hline $\begin{array}{c}\Lambda-5 \\
\text { (compacted) }\end{array}$ & & 0.378 & & & 0.315 & \\
\hline FA-.5-1 & $\begin{array}{c}0.493 \cdot 0.528 \\
(0.516 \pm 0.014)\end{array}$ & $\begin{array}{c}0.491-0.498 \\
(0.496 \pm 0.003)\end{array}$ & $\begin{array}{c}0.486-0.492 \\
(0.489 \pm 0.003)\end{array}$ & $\begin{array}{c}0.442-0.481 \\
(0.468 \pm 0.015)\end{array}$ & $\begin{array}{c}0.439-0.448 \\
(0.444 \div 0.004)\end{array}$ & $\begin{array}{c}0.434 \cdot 0.1412 \\
(0.438 * 0.0014)\end{array}$ \\
\hline $\begin{array}{c}F \Lambda-.5-1 \\
\text { (compactied) }\end{array}$ & & 0.365 & & & 0.301 & \\
\hline FA-.5-2 & & 0.470 & & & 0.417 & \\
\hline $\begin{array}{c}1: A-.5-2 \\
\text { (conpacted) }\end{array}$ & & 0.364 & & & 0.300 & \\
\hline FC-.25-1 & $\begin{array}{c}0.483-0.494 \\
(0.484 \pm 0.007)\end{array}$ & $\begin{array}{c}0.427-0.458 \\
(0.1415 \pm 0.013)\end{array}$ & $\begin{array}{c}0.11 .5-0.1171 \\
(0.139 \pm 0.025)\end{array}$ & $\begin{array}{c}0.425-0.143 \\
(0.432 \pm 0.008)\end{array}$ & $\begin{array}{c}0.369-0.103 \\
(0.389 \pm 0.014)\end{array}$ & $\begin{array}{c}0.356 \cdot 0.118 \\
(0.383 \pm 0.027)\end{array}$ \\
\hline
\end{tabular}


Table 3.19 Bulk Porosities of Packings of Crushed Tuff Particles - -

\begin{tabular}{|c|c|c|c|c|c|c|}
\hline \multirow{3}{*}{$\begin{array}{r}\text { Crushed } \\
\text { Tuff Grada- } \\
\text { tLon Type }\end{array}$} & & & & & & \\
\hline & \multicolumn{3}{|c|}{ Porosity, $n_{1}$} & \multicolumn{3}{|c|}{ Porosity, $n_{2}$} \\
\hline & Pouring & Scooping & Shaking & Pouring & Scooping & Shaking, \\
\hline $\begin{array}{c}\text { FC }-.25-1 \\
\text { (compacted) }\end{array}$ & & 0.353 & & & 0.287 & \\
\hline FC-.25-2 & & 0.455 & & & 0.399 & \\
\hline $\begin{array}{c}\text { FC-.25-2 } \\
\text { (compacted) }\end{array}$ & & 0.348 & & & 0.282 & \\
\hline FC- $-.45-1$ & $\begin{array}{c}0.495-0.503 \\
(0.500 \pm 0.004)\end{array}$ & $\begin{array}{c}0.484-0.492 \\
(0.488 \pm 0.003)\end{array}$ & $\begin{array}{c}0.466-0.501 \\
(0.486 \pm 0.016)\end{array}$ & $\begin{array}{c}0.444-0.453 \\
(0.450 \pm 0.004)\end{array}$ & $\begin{array}{c}0.432-0.441 \\
(0.436 \pm 0.004)\end{array}$ & $\begin{array}{c}0.412-0.450 \\
(0.434 \pm 0.018)\end{array}$ \\
\hline $\begin{array}{c}\text { FC }-.45-1 \\
\text { (compacted) }\end{array}$ & & 0.377 & & & 0.314 & \\
\hline FC-. $1.5-2$ & & 0.472 & & & 0.418 & \\
\hline FC $-.5-1$ & $\begin{array}{c}0.507-0.527 \\
(0.516 * 0.010)\end{array}$ & $\begin{array}{c}0.478-0.493 \\
(0.484 \pm 0.006)\end{array}$ & $\begin{array}{c}0.469-0.517 \\
(0.492 \neq 0.026)\end{array}$ & $\begin{array}{c}0.457 \times 0.479 \\
(0.468 \pm 0.010)\end{array}$ & $\begin{array}{c}0.426-0.441 \\
(0.432 \pm 0.006)\end{array}$ & $\begin{array}{c}0.415-0.468 \\
(0.440 \pm 0.029)\end{array}$ \\
\hline $\begin{array}{c}\mathrm{FC}-.5-1 \\
\text { (compacted) }\end{array}$ & & 0.382 & & & 0.320 & \\
\hline FC. $.5-2$ & & 0.476 & & & 0.1423 & \\
\hline
\end{tabular}

\section{NOTES :}

1. Porosity $n_{1}$ is calculated based on the sample weight and sample dimensions using tuff solid density of $2.61 \mathrm{~g} / \mathrm{cm}^{3}$. Dry bulk density of $2.37 \mathrm{~g} / \mathrm{cm}^{3}$ is used in calculating porosity $\mathrm{n}_{2}$.

2. A-1 stands for sample 1 of crushed tuff gradation A. The maximum particle size is $9.42 \mathrm{~mm}$. FA-.5 stands for the Fuller-Thompson gradation with $D_{\max }=9.42 \mathrm{~mm}$ (type A) and $\mathrm{n}=0.5$. FC-.25-1 stands for the Fuller-Thompson gradation with coarse $D_{\max }=19.05 \mathrm{~mm}$ and $\mathrm{n}=0.25$.

3. The Standard Proctor compaction method is used in compacting the samples. No particles smaller than $0.074 \mathrm{~mm}$ were included in the sample preparation. 


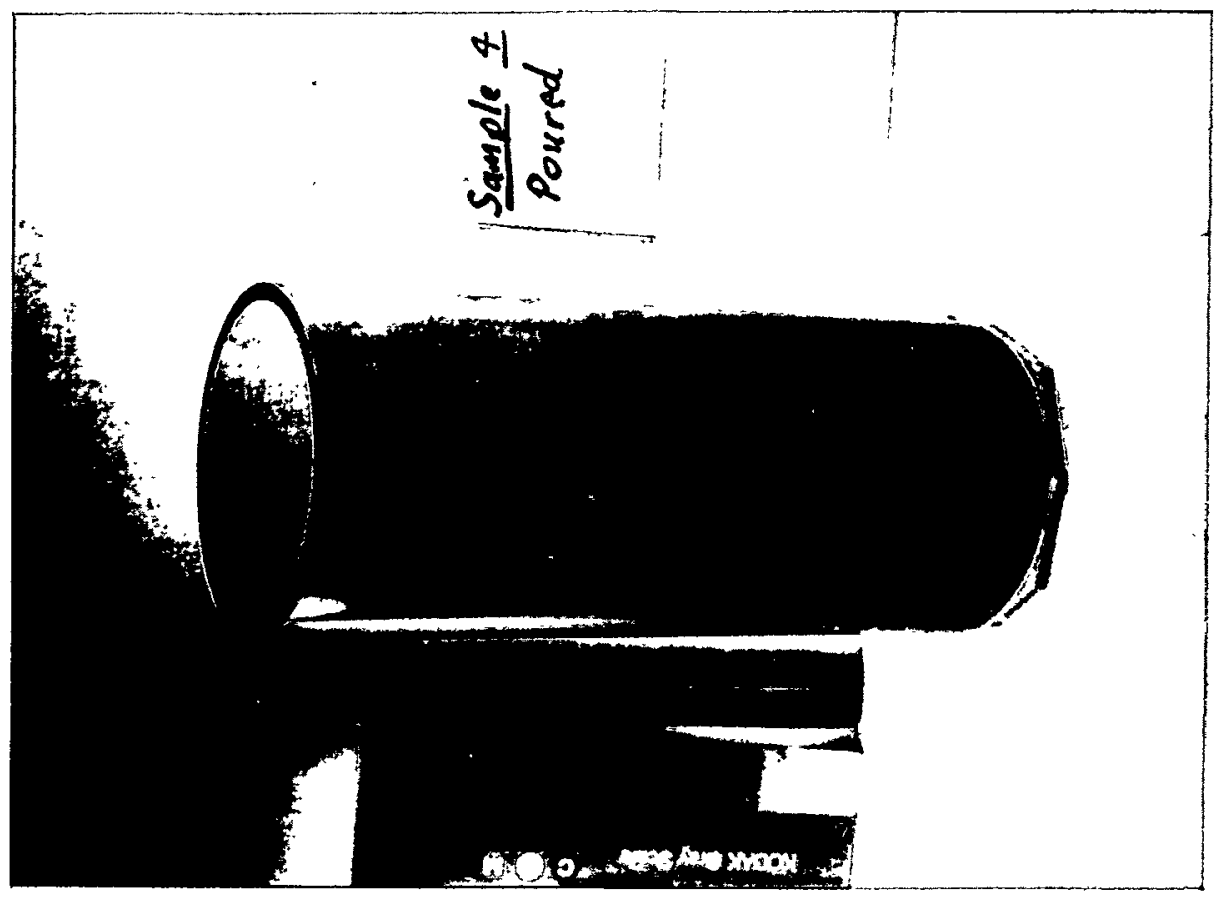

$\stackrel{\substack{4 \\ 0}}{4}$

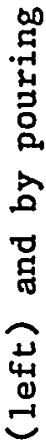

告

오

7

品

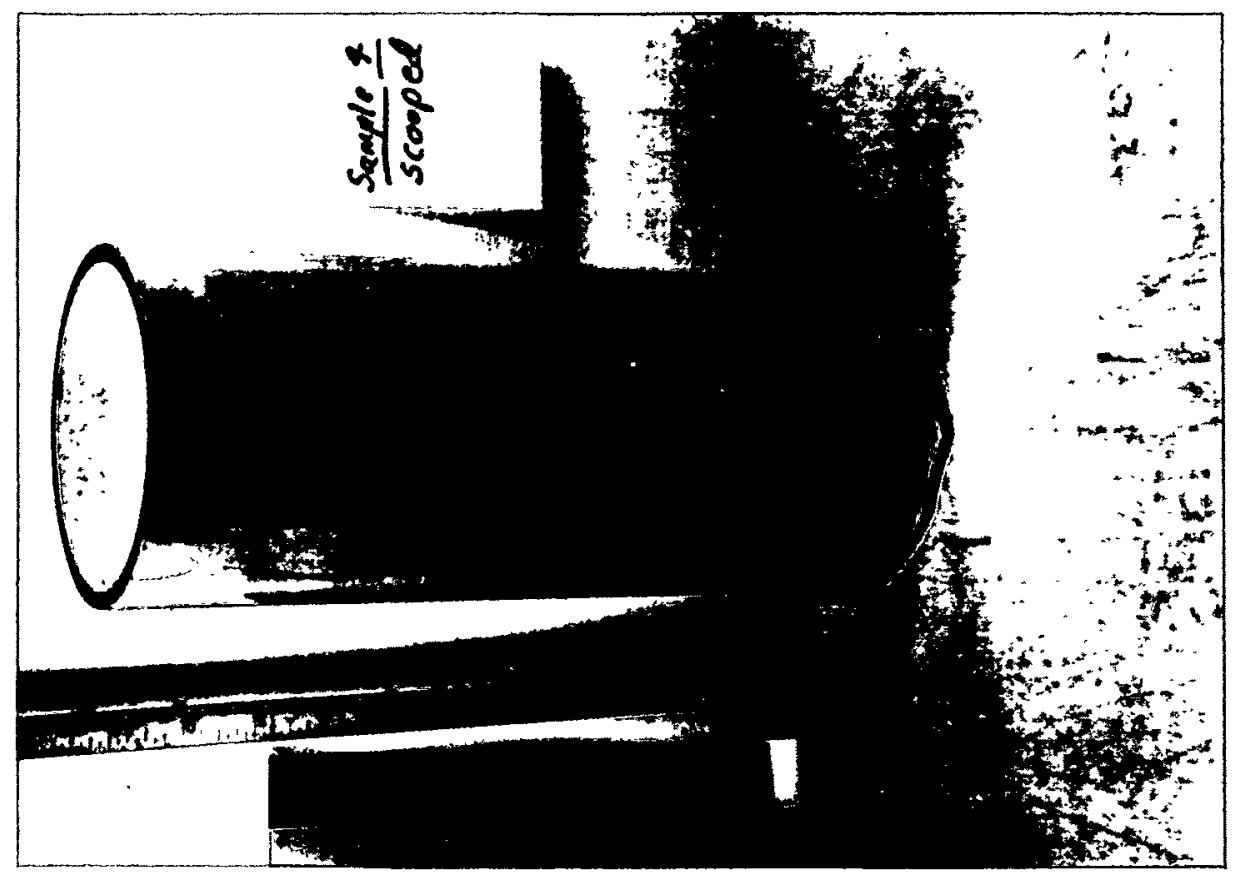

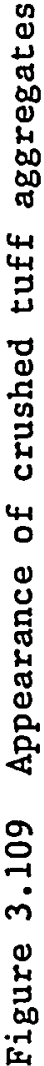


Table 3.20 Flow Test Results of Crushed Tuff Aggregates

\begin{tabular}{|c|c|c|c|}
\hline $\begin{array}{l}\text { Sample } \\
\text { Number }\end{array}$ & Porosity & $\begin{array}{l}\text { Permeability } \\
\left(\mathrm{cm} / \mathrm{s} \times 10^{-3}\right)\end{array}$ & $\begin{array}{l}\text { Hydraulic } \\
\text { Gradient }\end{array}$ \\
\hline A-4 (compacted) & 0.316 & $3.537 \pm 0.179$ & $1.71-1.37$ \\
\hline$A-5$ & 0.369 & $6.977 \pm 0.249$ & $1.66-1.05$ \\
\hline \multirow[t]{2}{*}{ A-5 (compacted) } & 0,299 & $7.965 \pm 0.197$ & $1.86-1.07$ \\
\hline & & $9.887 \pm 0.291$ & $0.37-0.13$ \\
\hline$F A-.5-2$ & 0.336 & $5.148 \pm 0.291$ & $1.68-1.15$ \\
\hline $\begin{array}{c}\text { FA-.5-2 (com- } \\
\text { pacted) }\end{array}$ & 0.300 & $3.774 \pm 0.158$ & $2.28-1.05$ \\
\hline FC- $.25-2$ & 0,347 & $2.926 \pm 0.065$ & $1.93-1.06$ \\
\hline \multirow[t]{2}{*}{$\begin{array}{c}\text { FC- } .25-2(\mathrm{com}- \\
\text { pacted) }\end{array}$} & 0,282 & $4.674 \pm 0.147$ & $2.11-1.19$ \\
\hline & & $3.253 \pm 0.329$ & $1.83-1.40$ \\
\hline $\begin{array}{c}\text { FC- } .45-1 \text { (com- } \\
\text { pacted) }\end{array}$ & 0.314 & $2.358 \pm 0.195$ & $1.44-1.05$ \\
\hline FC- $-.45-2$ & 0.362 & $4.994 \pm 0.323$ & $2.03-1.08$ \\
\hline $\begin{array}{c}\text { FC-.5-1 (com- } \\
\text { pacted) }\end{array}$ & 0.32 & $1.644 \pm 0.077$ & $1.40-1.06$ \\
\hline FC- $.5-2$ & 0.339 & $6.471=0.348$ & $2.08-1.10$ \\
\hline
\end{tabular}

NOTES :

1. Porosity is calculated using the dry bulk density of $2.37 \mathrm{~g} / \mathrm{cm}^{3}$ for tuff.

2. Knocking on the side wall of the permeameters to help remove air bubbles entrapped in the samples during the sample saturation process while a vacuum of $103.5 \mathrm{kPa}$ was applied from the top resulted in a further reduetion of porosity. 
may indicate that the crushed tuff aggregates of the Fuller-Thompson gradations ( $n=0.45$ or 0.5$)$, after compaction, can reach a denser packing state than crushed tuff of other gradation types, possibly due to the particle migration or the generation of more fine particles from compaction. The amount of fine particles produced by compaction has not been determined. In this sense, the results may suggest a permeability dependence of granular materials on particle gradation when the effect of compaction is taken into account.

\subsubsection{Bulk Porosity of Systems of Crushed Tuff Particles in the Presence of Bentonite}

The bulk porosity of the system of crushed tuff particles is different when mixed with bentonite. The effectiveness of compaction in reducing porosity is hindered by the soft bentonite buffer. The porosity of the crushed tuff component can be calculated if the sample dimensions, weight and density of the crushed tuff are known. The dry bulk density of tuff $\left(2.37 \mathrm{~g} / \mathrm{cm}^{3}\right)$ is used in computing porosity. This porosity of the crushed tuff component has been computed for each of the compacted mixture samples tested. The results are shown in Table 3.21.

For the compacted samples containing $15 \%$ bentonite, the bulk porosity lies in between those of the uncompacted and compacted counterparts in the absence of bentonite. The porosity increases with the bentonite content (Table 3.21). The samples with 158 bentonite failed to yield permeabilities lower than $10^{-8} \mathrm{~cm} / \mathrm{s}$. Piping was observed during flow testing of Samples B/AL-C-4-15/A and B/AL-C-4-15/C (Type $A$ and Type $C$ crushed tuff gradations, respectively), with permeabilities in the order of $10^{-4} \mathrm{~cm} / \mathrm{s}$. Samples B/AL-C-4-15/FA-A and B/AL-C-4-15/FA-B, with crushed tuff of type FA gradation, exhibit a permeability of $10^{-7} \mathrm{~cm} / \mathrm{s}$. These results indicate that the effect of the crushed tuff gradation on sealing performance is significant for mixtures having 158 bentonite. They also indicate that more bentonite is needed to secure lower permeabilities. The effect of crushed tuff gradation becomes insignificant when more bentonite is added, as a result of the increase of the bulk porosity with increasing bentonite content (Table 3.21). The long-term sealing performance is then heavily dependent on the resistance of bentonite to erosion or flow.

The bulk porosities of systems of crushed tuff in the compacted mixture samples containing 258 bentonite by weight (Table 3.21) are similar to those of crushed tuff only emplaced by scooping (Table 3.19). The similarity suggests that a higher compaction energy than that of the applied standard Proctor compaction is needed if a denser packing state is desired.

\subsubsection{Discussion}

Based on the results presented in Sections 3.5.3.1 and 3.5.3.2, it appears that a denser particle arrangement can be achieved if crushed tuff of gradation types FA ( $D_{\max }=9.42 \mathrm{~mm}, \mathrm{n}=0.5$ ) or, in particular, FC $\left(D_{\max }=19.05 \mathrm{~mm}, \mathrm{n}=0.25\right)^{\text {max }}$ is mixed with bentonite. The latter gradation has been recommended by Yong et al. (1986). Both gradations are quite different from the "coarse" gradation obtained by crushing 
Table 3.21 Calculated Bentonite Occupancy Percentage $\left(P / P_{W 3}\right)$ and Its Water Content at Saturation

\begin{tabular}{|c|c|c|c|c|c|c|c|c|c|c|}
\hline Sample Number & $\begin{array}{c}\text { Sample } \\
\text { Welght (g) } \\
\text { Tuff/ } \\
\text { Bentonite/ } \\
\text { Water Added }\end{array}$ & $\begin{array}{l}\text { Sample } \\
\text { Dlam- } \\
\text { eter } \\
(\mathrm{cm})\end{array}$ & $\begin{array}{l}\text { Sanple } \\
\text { Length } \\
(\mathrm{cm})\end{array}$ & $\begin{array}{c}\text { Bulk Den- } \\
\text { s } 1 \mathrm{ty} \\
\left(\mathrm{g} / \mathrm{cm}^{3}\right)\end{array}$ & $\begin{array}{l}\text { Poro- } \\
\text { sity } \\
n_{t^{a}}\end{array}$ & $\begin{array}{c}\text { Bentonit } \\
\mathrm{p}^{\mathrm{b}}\end{array}$ & $\begin{array}{l}\text { e Welght } \\
(8) \\
\mathrm{P}_{\mathrm{w} 2}\end{array}$ & $\begin{array}{c}\text { Percent } \\
P_{w 3}\end{array}$ & $\begin{array}{c}\mathrm{P} / \mathrm{P}_{w 3^{C}} \\
(8)\end{array}$ & $\begin{array}{l}\text { Saturated } \\
\text { Water } \\
\text { Content } \\
\text { of Benton- } \\
\text { lte }(8)\end{array}$ \\
\hline$B / A L-C-4-15 / F A-A$ & $1190 / 210 / 32$ & 10.16 & 10.27 & 1.719 & 0.406 & 15 & 35.19 & 27.51 & 54.52 & 143.55 \\
\hline$B / A L-C-4-15 / F A-B$ & $1190 / 210 / 32$ & 10.10 & 10.22 & 1.748 & 0.396 & 15 & 34.31 & 26.67 & 56.24 & 136.11 \\
\hline$B / A L-C-4-25 / F A-A$ & $1050 / 350 / 48$ & 10.12 & 9.92 & 1.813 & 0.453 & 25 & 39.72 & 31.54 & 79.27 & 79.99 \\
\hline$B / A L-C-4-25 / F \Lambda-B$ & $1050 / 350 / 48$ & 10.12 & 1.0 .58 & 1.701 & 0.487 & 25 & 43.02 & 34.55 & 72.36 & 96.65 \\
\hline$B / A L-C-4-15 / A^{*}$ & $1062 / 188 / 5$ & 10.16 & 9.5 & 1.631 & 0.126 & 1.4 & 37.10 & 29.19 & 46.31 & 194.55 \\
\hline$B / A L-C-4-25 / A *$ & $938 / 312 / 5$ & 10.09 & 9.5 & 1.651 & 0.487 & 23 & 42.99 & 34.52 & 66.03 & 120.61 \\
\hline$B / A L-C-4-25 / A-S$ & $1050 / 350 / 53$ & 10.24 & 10.8 & 1.633 & 0.509 & 25 & 45.19 & 36.56 & 68.38 & 108.68 \\
\hline & & 10.24 & 11.05 & 1.596 & 0.520 & 25 & 46.29 & 37.60 & 66.49 & 115.18 \\
\hline$B / A L-C-4-25 / A-P-A$ & $938 / 312 / 49$ & 10.16 & 9.31 & 1.720 & 0.484 & 25 & 42.67 & 35.71 & 70.02 & 103.48 \\
\hline$B / A L-C-4-25 / A-P-B$ & $1088 / 362 / 45$ & 10.19 & 11.27 & 1.626 & 0.508 & 25 & 45.08 & 36.46 & 68.57 & 108.07 \\
\hline$B / A L-C-8-25 / A-S$ & $4200 / 1400 / 229$ & 20.65 & 11.0 & 1.582 & 0.526 & 25 & 46.85 & 38.13 & 65.57 & 118.57 \\
\hline & & 20.65 & 10.9 & 1.597 & 0.522 & 25 & 46.41 & 37.72 & 66.28 & 115.93 \\
\hline$B / A L-C-12-25 / A$ & $9917 / 3306 / 475$ & 30.15 & 10.5 & 1.827 & 0.450 & 25 & 39.39 & 31.24 & 80.03 & 78.42 \\
\hline$B / A L-C-4-35 / A *$ & $812 / 438 / 4$ & 10.07 & 9.0 & 1.749 & 0.529 & 32 & 47.13 & 38.39 & 84.10 & 79.10 \\
\hline$B / A L-C-4-35 / A-S$ & $910 / 490 / 64$ & 10.25 & 10.8 & 1.614 & 0.575 & 35 & 51.81 & 42.91 & 81.56 & 81.16 \\
\hline & & 10.25 & 11.25 & 1.579 & 0.592 & 35 & 53.55 & 44.64 & 78.41 & 89.52 \\
\hline $\mathrm{B} / \mathrm{AL}-\mathrm{C}-4-35 / \mathrm{A}-\mathrm{P}-\mathrm{A}$ & $812 / 438 / 63$ & 10.24 & 9.42 & 1.693 & 0.564 & 35 & 50.71 & 41.84 & 83.65 & 76.19 \\
\hline
\end{tabular}


Table 3.21 Calculated Bentonite Occupancy Percentage $\left(P / P_{w 3}\right)$ and Its Water Content at Saturation - - Continued

\begin{tabular}{|c|c|c|c|c|c|c|c|c|c|c|}
\hline Sample Number & $\begin{array}{c}\text { Sample } \\
\text { Weight (E) } \\
\text { Tuff/ } \\
\text { Bentonite/ } \\
\text { Water Added } \\
\end{array}$ & $\begin{array}{l}\text { Sample } \\
\text { Dlam- } \\
\text { eter } \\
(\mathrm{cm}) \\
\end{array}$ & $\begin{array}{c}\text { Sample } \\
\text { Length } \\
(\mathrm{cm})\end{array}$ & $\begin{array}{c}\text { Bulk Den- } \\
\text { sicy } \\
\left(\mathrm{g} / \mathrm{cm}^{3}\right)\end{array}$ & $\begin{array}{l}\text { Poro- } \\
\text { slcy } \\
n_{t}^{a}\end{array}$ & $\begin{array}{c}\text { Bentonite } \\
\mathrm{pb} \\
\end{array}$ & $\begin{array}{c}\text { Weight } \\
\text { (8) } \\
\mathrm{P}_{\mathrm{w} 2} \\
\end{array}$ & $\begin{array}{c}\text { Percent } \\
\mathrm{P}_{\mathrm{w} 3} \\
\end{array}$ & $\begin{array}{c}\mathrm{P} / \mathrm{P}_{\mathrm{w} 3^{\mathrm{C}}} \\
(\mathrm{8})^{-}\end{array}$ & $\begin{array}{c}\text { Saturated } \\
\text { Hater } \\
\text { Content } \\
\text { of Benton: } \\
\text { lte (B) }\end{array}$ \\
\hline$B / A L-C-4-35 / A-P-B$ & $812 / 438 / 61$ & 10.19 & 9.45 & 1.701 & 0.562 & 35 & 50.46 & 41.60 & 84.14 & 75.09 \\
\hline$B / A L-C-8-35 / A-S$ & $3640 / 1960 / 258$ & 20.65 & 10.05 & 1.740 & 0.550 & 35 & 49.29 & 40.47 & 86.49 & 70.10 \\
\hline & & & 10.65 & 1.642 & 0.576 & 35 & 51.87 & 42.97 & 81.45 & 81.42 \\
\hline$B / A L-C-12 \cdot 35 / A$ & $9810 / 3270 / 615$ & 30.15 & 12.32 & 1.556 & 0.598 & 35 & 54.16 & 4.5 .23 & 77.38 & 92.54 \\
\hline$B / A L-C-4-15 / B *$ & $1062 / 188 / 5$ & 10.09 & 9.5 & 1.651 & 0.419 & 14 & 36.41 & 28.59 & 47.29 & 187.91 \\
\hline$B / A L-C-4-25 / B *$ & $938 / 312 / 5$ & 10.12 & 9.5 & 1.642 & 0.490 & 23 & 43.26 & 34.77 & 65.54 & 122.38 \\
\hline$B / A L-C-4-35 / B *$ & $812 / 438 / 4$ & 10.11 & 9.5 & 1.643 & 0.557 & 32 & 50.01 & 41.15 & 78.46 & 92.94 \\
\hline$B / A L-C-4-15 / C *$ & $1062 / 188 / 5$ & 10.17 & 9.6 & 1.610 & 0.434 & 14 & 37.80 & 29.82 & 45.33 & 201.59 \\
\hline$B / A L-C-4-25 / C *$ & $938 / 312 / 5$ & 10.13 & 9.8 & 1.588 & 0.507 & 23 & 44.93 & 36.32 & 62.75 & 133.33 \\
\hline$B / A L-C-4-35 / C *$ & $812 / 438 / 4$ & 10.14 & 10.0 & 1.551 & 0.582 & 32 & 52.53 & 43.61 & 74.04 & 106.43 \\
\hline$B / A L-C-8-15 / F G-S * *$ & $4760 / 840 / 144$ & 20.45 & 11.01 & 1.589 & 0.453 & 15 & 39.63 & 31.46 & 47.67 & 180.8 \\
\hline$B / A L-C-8-25 / F C-S * *$ & $4200 / 1400 / 192$ & 20.45 & 11.08 & 1.591 & 0.520 & 25 & 46.27 & 37.58 & 66.52 & 115.09 \\
\hline$B / A L-C-8-35 / F C-S * *$ & $3640 / 1960 / 258$ & 20.45 & 11.84 & 1.506 & 0.611 & 35 & 55.50 & 46.39 & 75.15 & 99.60 \\
\hline
\end{tabular}

*Bentonite used in mixing with air-dried crushed tuff contained 23.58 water by weight.

**These samples received one quarter of the Standard Proctor compactive effort.

aporosity of crushed tuff system (neglecting the internal pores in the tuff particles).

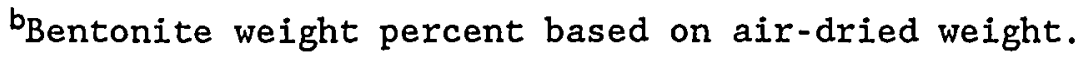

${ }^{c}$ Amount of bentonite added/amount of bentonite needed to obtain the maximum dry density of bentonite (ASTM D-698) in a mixture. 
rock chunks using a conventional $\mathrm{j}$ aw crusher and an adjustable roller crusher (set at $1.3 \mathrm{~cm}$ opening) in sequence. To form crushed tuff constituting the two gradations requires sieving and blending. Type A gradation, nevertheless, is derived from the "coarse" gradation by simply removing particles larger than $9.42 \mathrm{~mm}$. Such a production procedure for generating crushed tuff of type A gradation can be implemented easily in the field.

As shown in Tables 3.19 and 3.21 , the bulk porosity of the system of crushed tuff particles is different when mixed with bentonite. The effectiveness of compaction in reducing porosity is hindered by the soft bentonite buffer. To reduce the bulk porosities of the mixture samples containing 258 or more bentonite by weight, a compaction energy higher than that of the standard Proctor compaction is necessary.

\subsubsection{Bentonite Occupancy Percentage and Water Content at Saturation}

Whether the clay or the crushed-tuff phase will dominate the sealing performance can be anticipated to some extent in terms of the amount of bentonite in the mixture. Equation (3.7) provides a theoretical calculation for the amount of bentonite required to fill all the voids of the crushed-tuff phase and to prevent direct contact between the crushed tuff particles for any water content. This equation is derived based on the phase diagram relation (Mitche11, 1976, p. 172).

$$
\left(\frac{n_{t}}{1-n_{t}}\right) \frac{1}{\gamma_{t}}=\frac{P_{w I}}{100}\left[\frac{1}{G_{s b} \gamma_{w}}+\frac{w_{b s}}{100 \gamma_{w}}+\left(\frac{n_{t}}{1-n_{t}}\right) \frac{1}{\gamma_{t}}\right]
$$

where $P_{W 1}=$ bentonite weight percent based on dry weight

$$
\begin{aligned}
& \mathrm{n}_{\mathrm{t}}= \text { bulk porosity of crushed-tuff phase (neglecting the } \\
& \text { internal pores) } \\
& \gamma_{\mathrm{t}}=\text { dry weight of tuff particles }=2.37 \mathrm{~g} / \mathrm{cm}^{3} \\
& \mathrm{G}_{\mathrm{sb}}=\text { specific gravity of bentonite }=2.92 \\
& \gamma_{w}=\text { unit weight of water }=1 \mathrm{~g} / \mathrm{cm}^{3} \\
& \mathrm{w}_{\mathrm{bs}}=\text { water content of bentonite at saturated conditions. }
\end{aligned}
$$

Substituting the numerical values for the variables, Eq. (3.7) becomes:

$$
\left(\frac{n_{t}}{1-n_{t}}\right) \frac{1}{2.37}=\frac{P_{w 1}}{100}\left[\frac{1}{2.92}+\frac{w_{b s}}{100 \gamma_{w}}+\left(\frac{n_{t}}{1-n_{t}}\right) \frac{1}{2.37}\right]
$$

Bentonite weight percent based on the air-dried weight $\left(P_{w 2}\right)$ can be obtained using the following transformation formula: 


$$
P_{w 2}=\frac{100 P_{w 1}\left(100-w_{I t}\right)}{100\left(100-w_{r b}\right)-P_{w 1}\left(w_{r t}-w_{x b}\right)}
$$

where $P_{w 2}$ - bentonite weight percent (based on air-dried weight

$w_{r t}$ - water content of air-dried crushed tuff (1.4588)

$w_{r b}=$ water content of air-dried bentonite $(9.438)$.

Given the optimal water content of bentonite (23.558) for $w_{b s}$, the clay weight percent, $P_{K 2}$, has been calculated for each of the mixture samples using Eqs. (3.8) and (3.9). The results are shown in Table 3.21.

The initial water content of the bentonite can be controlled. For bentonite of a given initial water content and corresponding bulk density, the bentonite weight percent required to fill the pores between crushed tuff particles $\left(P_{w 3}\right.$ in Table 3.21 ) can be calculated using Eqs. (3.10) and (3.11).

$$
\begin{gathered}
W_{r b}-V_{v} \times \gamma_{b} \times \frac{1}{\left(100+W_{b}\right)} \times\left(100+W_{r b}\right) \\
P_{w 3}=\frac{100 W_{I b}}{W_{x b}+W_{I t}} \\
W_{r b}=\text { air-dried weight of bentonite } \\
V_{v}=\text { volume of voids (discounting bentonite phase and internal }
\end{gathered}
$$

where $W_{r b}=$ air-dried weight of bentonite pores in tuff aggregates)

$\mathrm{w}_{\mathrm{b}}$ = initial water content of bentonite

$\gamma_{b}=$ unit weight of bentonite at water content of $w_{b}$

$W_{r t}=$ air-dried weight of crushed tuff.

The water content of bentonite at saturation $\left(w_{b s}\right)$ can be calculated from:

$$
w_{b s}-\left(\frac{100 V_{v} \gamma_{w}}{w_{x b}\left(100-w_{x b}\right)}-\frac{1}{G_{g b}}\right) \times 100
$$

The values of $P_{w 3}$ in Table 3.21 are abtained using $w_{b}=23.55 \%, r_{b}=$ $1.508 \mathrm{~g} / \mathrm{cm}^{3}$. It can be seen from Table 3.21 that for the samples containing 15 weight percent of bentonite, the clay accounts for less than 45 to 568 of the weight required to fill the pore space (based on $P_{w 3}$ ), depending on the gradation of the crushed tuff. The expected water content of bentonite at saturation ranges from $130 \%$ to $200 \%$. As discussed in Section 3.4.1.7, this type of sample does not yield a very low permeability and its probability of failure is high. The occupancy percentage, defined as the clay added as a percentage of the clay amount 
required to completely fill all voids in between the tuff particles $\left(\mathrm{P} / \mathrm{P}_{\mathrm{H}}\right.$ in Table 3.21$)$, improves as more bentonite is introduced. This percentage amounts to 65 to 808 for the samples having 25 weight percent of bentonite, and 75 to 86.58 for the mixtures having 35 weight percent of bentonite. Moreover, the water content of bentonite at saturation is reduced and the resistance to flow and piping/erosion is enhanced by adding more clay.

Gradation of crushed tuff aggregates influences the occupancy percentage and the saturated water content of bentonite. For the same bentonite weight percent, the mixtures with crushed tuff of type FA gradation generally yield the highest occupancy percentage and the lowest water content of bentonite at saturation.

\subsubsection{Mechanical Factors Affecting the Deterioration of Sealing Performance}

Bentonite and bentonite/crushed tuff plugs, when properly engineered, can yield very low permeabilities. To preserve such sealing ability for a long time, the seals must be designed such that the loss of bentonite and fine tuff particles to the surrounding environment is minimized or prevented. The efficiency of such seals is a function of their ability to resist piping and erosion by flowing groundwater (Pusch et al., 1987).

Piping, which generally precedes erosion, refers to local, fast penetration of water, creating continuous passages through a soil (Pusch et al., 1987). It thus depends on pore size and pore distribution, and water pressure imposed. In the case of bentonite/crushed tuff mixtures, the grading of the crushed tuff is a decisive factor for the water pressure conditions at which piping takes place. If the grading is not proper or the mixture not thoroughly homogenized, or if the amount of bentonite is not sufficient to substantially fill the ballast pores, soft parts of the bentonite gel can be displaced. Torn-off fragments are transported through channels formed at a relatively low water overpressure (Pusch et al., 1987).

In addition to piping/erosion, the flow of bentonite as observed for Samples B/AL-C-8-25/A-S and B/AL-C-8-35/A-S (Figures 3.79 and 3.80) should also be taken into account in the evaluation of long-term sealing performance. The consequence of bentonite flow is that portions of a mixture plug may eventually become shortcuts for transportation of contaminated groundwater or air. As indicated by the flow testing of B/AL-C-8-25/A-S (Section 3.4.1.8.3), the pressure gradient required for bentonite to flow may be much less than that for piping to take place. It is therefore necessary to determine the yield strengths of bentonite at various water contents so that critical hydraulic gradients can be defined.

The bond strength between crushed tuff/bentonite plugs and the host rock is low (not more than $110 \mathrm{kPa}$ (16 psi)), based on the small number of push-out tests performed. While further investigations are recommended in order to determine the bond strength, and especially its sensitivity to the numerous variables that might influence it (e.g. bentonite 
content, crushed rock size and size distribution, emplacement method, water content, including its change as a function of flow), it appears probable that crushed tuff/bentonite seals, as installed here, need to be confined axially, or can be subjected only to relatively small axial loads. 
CHAPTER FOUR

\author{
PIPING, EROSION AND FLOW OF BENTONITE
}

\title{
4.1 Introduction
}

Piping, seepage erosion, and channelling have been responsible for the failures of earth dams, embankments, and natural slopes (Wolski, 1965, Rosewe11, 1977; Sherard et al., 1972, 1977). Such effects can also be detrimental to the performance of seals installed against fractured rocks (Aisenstein et al., 1961, Goodman and Sundaram, 1980). To retain sealing capacity of bentonite and bentonite/crushed rock seals, piping and erosion of bentonite should be minimized or prevented.

The laws governing piping in cohesionless soils do not apply to cohesive soils due to their neglect of cohesion between particles (Zaslavsky and Kassiff, 1965; Graf, 1971; Raudkivi, 1976, pp. 2, 16-18). Studies on surface erosion of cohesive soils for irrigation and soil conservation relate the erodibility to maximum permissible velocity (e.g. Fortier and Scobey, 1926) or to critical tractive force (Smerdon and Beasley, 1961). The concept of maximum permissible velocity does not adequately take the soil properties into consideration. Critical tractive forces of nonplastic to low plastic soils, determined by flume erosion tests, have been correlated to soil properties such as plasticity index, vane shear strength, cation exchange capacity, calcium-sodium ratio, and percent clay (Lyle and Smerdon, 1965). Although the boundary conditions of surface erosion and seepage erosion are different, their results suggest that critical tractive force may be an indication of resistance to erosion. (The term "tractive force" used in agricultural engineering is synonymous with "shear strength" commonly adopted in geotechnical engineering.)

Zaslavsky and Kassiff (1965) include the tensile strength in their simple model of piping in cohesive soils. Its application is limited because the parameters required for input are localized and thus are difficult to obtain. The model provides valuable insight into piping mechanisms and may serve for a rough estimation of the critical hydraulic gradient needed to initiate piping. The Zaslavsky-Kassiff model is described in Section 4.3.

In flow testing of bentonite/crushed tuff sealants, several samples have been subjected to high injection pressures to evaluate the sealing performance under large hydraulic gradients. A breakdown of the linear relationship between flow rate and hydraulic gradient has been observed in all high injection pressure flow tests. The abrupt departure from the initial linear relationship between flow rate and hydraulic gradient can not be explained by small strain deformation. The sudden change is believed to indicate the onset of plastic flow of bentonite in the pores between crushed tuff particles. This type of bentonite transport may damage the sealing performance locally and may eventually jeopardize the 
whole sealing ability, especially if the seals are in contact with fractures or open joints.

The pressure required to initiate bentonite flow likely relates to the yield stress of the bentonite, which, in turn, depends primarily upon its water content if the type of adsorbed cations and the $\mathrm{Ph}$ of the pore water remain the same. If the yield stress can be established as a function of water content, the critical hydraulic gradient at which flow of bentonite takes place can be calculated, provided the water content of bentonite and the mean pore size of the crushed tuff skeleton are known. The long-term sealing performance should not be impaired by bentonite loss if the maximum possible hydraulic gradient expected in the field will not exceed the critical gradient. Conversely, given a maximum hydraulic gradient, knowledge of the relation between yield stress and water content of bentonite can facilitate the design of the clay/crushed rock plugs to assure that no bentonite flow will occur.

A bentonite flow model is proposed, based on the theory of plastic flow in capillaries. The model has been examined against nine flow test results. The predicted gradients are about 1.1 to 2.1 times higher than the ones obtained experimentally. Factors that may contribute to the deviation are discussed.

\subsection{Effect of Dispersion Erosion}

Sodium bentonite is highly dispersive in water of low ion concentration and therefore is susceptible to dispersion erosion during percolation of such a permeant (Sherard et a1., 1972; Landau and Altschaeff1, 1977; Shaikh et al., 1988). Dispersion erosion is defined herein as the loss of fine particles suspended in the effluent while the flow rate remains small and relatively constant.

The amount of solids carried in the outflows has been measured when flow testing six bentonite/crushed tuff plugs. The imposed hydraulic

gradients ranged from 310 to 351 . The solid concentrations in grams per $100 \mathrm{cc}$ are given in Table 4.1. Based on the concentrations and average flow rates measured, the time required for the loss of 10 weight percent bentonite is calculated, given continuous permeation at a constant flow rate. As shown in Table 4.1, it will take a few to several tens of years to lose 10 percent bentonite for the mixture plugs initially containing $25 \%$ bentonite by weight. For the mixture plugs having an original bentonite content of 358 , it will take more than 100 years for dispersion erosion to carry away 108 of the bentonite. Assuming that the flow rates are linearly associated with hydraulic gradient and that the solid concentrations in the effluents remain constant, a reduction in hydraulic gradient by 30 times will result in an increase of the time by 30 . In other words, to lose 10 percent bentonite by dispersion erosion under a hydraulic gradient of approximately 10 may take several hundred and several thousand years for the mixture sealants containing 258 and 358 bentonite, respectively. The time span may increase further if the solid concentration reduces with decreasing hydraulic gradient. On the other hand, the calculations do not account for the fact that erosion may accelerate as bentonite is progressively removed from the system. 
Table 4.1 Dispersive Erosion Rates of Mixed Samples Installed with 258 and $35 \%$ Bentonite by Weight.

\begin{tabular}{|c|c|c|c|c|c|}
\hline $\begin{array}{l}\text { Sample } \\
\text { Number }\end{array}$ & $\begin{array}{l}\text { Hydrau- } \\
\quad \text { lic } \\
\text { Gradient }\end{array}$ & $\begin{array}{l}\text { Flow Rate } \\
\left(x 10^{-4} \mathrm{cc} / \mathrm{s}\right) \\
\text { Mean } \pm \text { S.D.* }\end{array}$ & $\begin{array}{c}\text { Solids } \\
\text { in } \\
\text { Outflow } \\
\text { (g per } 100 \\
\text { cc) }\end{array}$ & $\begin{array}{c}\text { Estim } \\
\text { for } \\
\text { Bent } \\
15 \%\end{array}$ & $\begin{array}{l}\text { Time } \\
\text { s of } \\
\text { te to } \\
258\end{array}$ \\
\hline $\begin{array}{l}\text { B/AL-C- } \\
4-25 / A\end{array}$ & $343-347$ & $0.818 \pm 0.102$ & $\begin{array}{l}0.09 \\
0.08 \\
0.075 \\
0.036\end{array}$ & $\begin{array}{r}57.3 \\
64.5 \\
68.8 \\
143.3\end{array}$ & \\
\hline \multirow[t]{2}{*}{$\begin{array}{l}\text { B/AL-C- } \\
4-25 / B\end{array}$} & $209-211$ & $1.445 \pm 0.142$ & $\begin{array}{l}0.26 \\
0.30 \\
0.12 \\
0.24\end{array}$ & $\begin{array}{r}11.2 \\
9.8 \\
24.4 \\
12.1\end{array}$ & \\
\hline & $349-351$ & $2.245 \pm 0.723$ & $\begin{array}{c}0.24 \\
0.17 \\
0.18 \\
0.15 \\
0.08 \\
0.082 \\
0.075 \\
0.088 \\
0.12\end{array}$ & $\begin{array}{r}7.9 \\
11.1 \\
10.5 \\
12.5 \\
23.5 \\
22.9 \\
25.1 \\
21.3 \\
15.6\end{array}$ & \\
\hline $\begin{array}{l}B / A L / C- \\
4-25 / C\end{array}$ & $310-314$ & $0.977 \pm 0.167$ & $\begin{array}{l}0.076 \\
0.083 \\
0.083 \\
0.081\end{array}$ & $\begin{array}{l}56.8 \\
52.0 \\
52.0 \\
53.3\end{array}$ & \\
\hline $\begin{array}{l}\text { B/AL-C- } \\
4-35 / A\end{array}$ & $333-337$ & $0.401 \pm 0.122$ & $\begin{array}{c}0.087 \\
0.08\end{array}$ & & $\begin{array}{l}137.1 \\
149.1\end{array}$ \\
\hline $\begin{array}{l}B / A L-C- \\
4-35 / B\end{array}$ & $321-325$ & $0.508 \neq 0.087$ & $\begin{array}{l}0.091 \\
0.082\end{array}$ & & $\begin{array}{l}103.5 \\
114.8\end{array}$ \\
\hline $\begin{array}{l}B / A L-C- \\
4-35 / C\end{array}$ & $342-347$ & $0.404 \pm 0.133$ & $\begin{array}{l}0.086 \\
0.081\end{array}$ & & $\begin{array}{l}137.6 \\
146.1\end{array}$ \\
\hline
\end{tabular}

${ }^{*}$ S.D. = Standard Deviation

1) The first three samples consist of $312.5 \mathrm{~g}$ (air-dried) bentonite and crushed tuff. The latter three samples consist of $437.5 \mathrm{~g}$ bentonite and $812.5 \mathrm{~g}$ crushed tuff. The last letter shown in the sample number designation is the type of crushed tuff gradation used for mixing.

2) The air-dried bentonite has a moisture content of 9.58 .

3) The calculation of time required for loss of bentonite to the next lower bentonite percentage (i.e. $15 \%$ or $25 \%$ ) is based on the assumption that flow rate and rate of bentonite removal remain constant. 
The dispersion exosion is likely to have a minimal effect on the deterioration of sealing performance of the mixture plugs containing 358 bentonite by weight, if the field hydraulic gradient is close to 1 . It is important to take into consideration, when evaluating the implications of these calculations, that the effective hydraulic gradient during bentonite flow is not necessarily the gradient over the entire seal length. Situations can readily be envisioned in which the gradient between the loaded seal end and a fracture in the host rock intersecting the sealed hole determines whether or not bentonite will flow into the fracture.

\subsection{Zaslavsky-Kassiff Mechanical Model of Piping}

A theoretical formulation of the piping mechanism has been developed by Zaslavsky and Kassiff (1965). They consider the balance of the forces acting on a soil element, namely, the gravitational force, the drag force, and the cohesive force. The basic model consists of a layer of fine cohesive soil subjected to flow of water and supported by another material, through which particles of the fine soil can leave the interface between the two materials. The model is analogous to the situations where cohesive sealants are installed against coarse filters or fractured rocks. Assuming the resultant of the gravitational force and the drag force work against the cohesive force, their model can be expressed in the form:

$$
f_{s}=\frac{a \sigma_{t}}{d_{m} \gamma_{w}\left[a_{1} j+(G-1)(1-n) \cos (\alpha)\right]}
$$

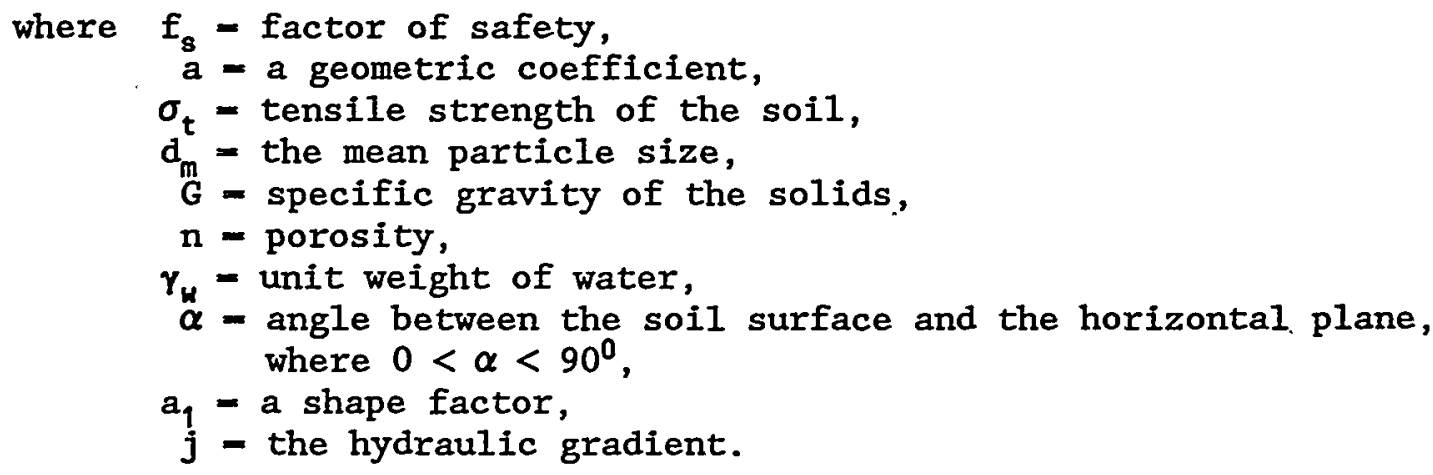

For highly cohesive soils, $\left(a \sigma_{t}\right)$ is very large compared with the submerged unit weight. Eq. (4.1) may then be approximated as: 


$$
f_{s}=\frac{b \sigma_{t}}{d_{m} \gamma_{w} j}
$$

where $b=a / a_{1}$. For compacted clays, $b$ may take a value of 1 (Kassiff et al., 1965).

Considering soil on a plate with a hole of radius $r$, the hydraulic gradient may be expressed as:

$$
j=\frac{Q}{2 \pi r^{2} K}
$$

where $Q=$ discharge through the hole,

$\mathrm{K}=$ the permeability of the soil in the region near the exit.

Introducing Eq. (4.3) into Eq. (4.2) results in:

$$
f_{s}=\frac{b \sigma_{t}}{d_{m} \gamma_{w}\left(Q / 2 \pi r^{2} K\right)}
$$

For failure conditions $f_{s}=1$, and Eq. (4.4) becomes:

$$
\ell_{c r}=\frac{2 \pi b \sigma_{t} K r^{2}}{d_{m} \gamma_{w}}
$$

where $Q_{c r}=$ critical discharge through the hole for failure conditions. Similarly, for failure conditions, Eq. (4.3) becomes:

$$
j_{c r}=\frac{Q_{C r}}{2 \pi r^{2} K}
$$

Equation (4.5) can be used to calculate $Q_{c r}$ if the tensile strength $\sigma_{t}$, the mean particle size $d_{m}$, the permeability $k$, the ratio $b$ and the radius $r$ are known. The critical hydraulic gradient then can be computed according to Eq. (4.6).

The application of the Zaslavsky-Kassiff piping model is difficult primarily for two reasons: (1) the representative tensile strength and mean particle size of the soil are difficult to obtain because of the soil-water interactions, (2) the hydraulic parameters $K$ and $j$ in the above equations are local properties in the vicinity of the outlet and may be quite different from the average macroscopic values over the whole soil layer. 
The piping test results of Sample B/AL-C-4-25/A-P-B are adopted to estimate the critical hydraulic gradient for failure, using Eq. (4.6). The discharge of $1.651 \times 10^{-4} \mathrm{cc} / \mathrm{s}$ and the permeability of $3.7 \times 10^{-6}$ $\mathrm{cm} / \mathrm{s}$, measured prior to failure, are used for $Q_{c r}$ and $K$. The diameter of the circular outlet is $0.225 \mathrm{~mm}$. The computation yields a critical gradient of 561, about 5 times higher than the experimental average critical gradient of 116 .

\subsection{Model of Bentonite Elow}

\subsubsection{Theory of Flow through Capillaries}

The rate of laminar flow of a viscous fluid through a pipe or capillary is directly proportional to the pressure gradient and can be described by the Poiseuille equation:

$$
Q=\frac{\pi R^{4} \Delta P}{8 \eta \Delta L}
$$

where $Q=$ flow rate,

$$
\begin{aligned}
& \Delta P=\text { pressure difference over a length } \mathrm{L}, \\
& \mathrm{R}=\text { radius of capillary, } \\
& \eta=\text { viscosity of the fluid. }
\end{aligned}
$$

A clay slurry or paste is not a true liquid. Its flow properties lie between those applicable to liquids and solids (Scott Blair and Crowther, 1929; Keen and Scott Blair, 1929). Bingham (1916) introduced the concept of an ideal material which does not flow until a certain shear stress, the yield stress $\tau_{f}$, is reached and thereafter flows at a rate proportional to the excess shear stress, $\tau-\tau_{f}$. The rate of shear strain, $d e_{\text {s }} / d t$ at any point in the material is given by:

$$
\frac{d e_{s}}{d t}=\frac{1}{\eta_{p I}}(\tau-\tau)
$$

where $\eta_{\mathrm{pl}}=$ plastic viscosity of the materlal.

The application of Eq. (4.8) to flow in a circular capillary was worked out by Buckingham (1921), who gave an equation without detailed derivation in the form: 


$$
\frac{V}{t}=\frac{\pi R^{4} \mu}{81}\left(P-\frac{4}{3} p_{0}+\frac{p_{o}^{4}}{3 p^{3}}\right)+\pi R^{2} V_{R}
$$

where $V=$ volume of discharge,

$t=$ elapsed time for discharge $V$,

$\mathrm{R}$ - radius of capillary,

$\mu$ - mobility of material, equal to $1 / \eta_{p l}$,

$P=$ pressure difference over a length 1 of the capillary,

$p_{0}=21 \mathrm{f} / \mathrm{R}$ ( $\mathrm{f}:$ yield stress of material),

$V_{R}=$ slip velocity at the wall of capillary.

The derivation of Eq. (4.9) is given in Appendix G.

Assuming that the slip effect at the wall is negligible, Marsland and Loudon (1963) obtained the following equation:

$$
Q=\frac{\pi R^{4}}{8 \eta_{p I}} \frac{\Delta p}{\Delta I}\left[1-\frac{4}{3}\left(\frac{2 \tau_{f}}{R} \frac{\Delta I}{\Delta p}\right)+\frac{1}{3}\left(\frac{2 \tau_{f}}{R} \frac{\Delta I}{\Delta p}\right)^{4}\right]
$$

where $Q$ - volume rate of discharge, the same as V/t in Eq. 4.9,

$\tau_{f}=$ yield stress of material (same as $f$ in $P_{0}=21 f / R$ below Eq. 4.9),

$\Delta p=$ pressure difference over a length $\Delta I$ of the capillary.

Rearranging Eq. (4.10) leads to:

$$
Q=\frac{\pi R^{3}}{4 \eta_{p 1}} \frac{R}{2} \frac{\Delta p}{\Delta I}\left[1-\frac{4}{3} \frac{\tau_{f}}{\tau_{R}}+\frac{1}{3}\left(\frac{\tau_{f}}{\tau_{R}}\right)^{4}\right]
$$

where $\tau_{R}=(\mathrm{R} / 2)(\Delta p / \Delta I)$.

Expressed in terms of the shear stress, and the rate of shear strain, $\left(\mathrm{d} \epsilon_{s} / \mathrm{dt}\right)_{R}$, Eq. $(4.11)$ becomes:

$$
\left\langle\frac{d \epsilon_{s}}{d t}\right\rangle_{R}=\frac{4 Q}{\pi R^{3}}=\frac{1}{\eta_{p I}}\left[\tau_{R}-\frac{4}{3} \tau_{f}+\frac{1}{3} \frac{\tau_{f}^{4}}{\tau_{R}^{3}}\right]
$$


Eq. (4.12) gives a bilinear flow curve. No flow occurs in region $I$, where $\tau<\tau_{f}$. The material flows according to equation (4.12) in region II. When $\tau_{R} \gg \tau_{f}$, the slope of the flow curve becomes $1 / \eta_{p l}$. Actual clay slurries or pastes do not follow this idealized law exactly but have a curved transition region (region III) in between regions II and IV (Marsland and Loudon, 1963). When the shear stress, $T_{R}$, reaches $\tau_{f}$, shear failure occurs near the wall of the capillary and the slurry moves forward as a solid plug (region II). As the pressure gradient increases, the diameter of the solid plug becomes progressively smaller (region III) until the whole of the material in the capillary flows in a streamline manner like a viscous liquid. The rate of flow then increases Iinearly with the pressure gradient (region IV).

The flow of clay within a matrix of sand or crushed rock particles is analogous to the flow of clay paste in capillaries. A mixture of bentonite and crushed rock should maintain its sealing ability if the clay remains in place. The maximum pressure gradient that such a plug can sustain without initiation of bentonite flow depends upon the yield stress of the clay. The yield stress is a function of many variables. A brief discussion of this matter is given in the following section.

\subsubsection{Factors Influencing the Yield Stress of Bentonite}

The behavior of a clay is closely related to its structure, as well as its fabric. The fabric is influenced by interactions between soil particles, adsorbed cations, and water because of unbalanced force fields at the interfaces between the constituents (Mitche11, 1976, p. 112). Clay particles, due to their small size and large surface area, are particularly susceptible to such effects.

The fabric of clay, as a result of such interactions, depends upon the thickness of the diffuse double layer, and so does the yield stress. In addition to temperature, electrolyte concentration, and type of cations adsorbed, factors such as ion size, $\mathrm{pH}$, and anion adsorption also influence the development of the diffuse double layer (Mitche11, 1976, pp. 126-127). Brandenburg and Lagaly (1988) report that the rheological behavior of montmorillonite dispersions in water also depends strongly on $\mathrm{pH}$ and salt type. For $\mathrm{NaCl}$ solutions and $\mathrm{pH}$ values between 7.5 and 9, their results show that the shear stress of Wyoming bentonite is insensitive to the salt concentration (from water to $0.1 \mathrm{M} \mathrm{NaCl}$ ). They also observe a viscosity increase with decreasing particle size.

If the pore water contains divalent cations (e.g. $\mathrm{Ca}^{++}$) exchangeable for the adsorbed sodium ions, the double layer is suppressed, as predicted by the Gouy-Chapman theory. The clay structure becomes flocculated and the shear strength increases (Lambe, 1958). For bentonite dispersed in distilled water, the yield stress depends primarily upon its water content (Marsland and Loudon, 1963), and is expected to assume a minimum value. In this study, yield stresses of bentonite pastes mixed with distilled water are used to evaluate the critical hydraulic gradient required to initiate the flow of bentonite. 


\subsubsection{Determination of Yield Stress of Bentonite Mixed with Distilled Water}

The bentonite used in this study is American Colloid $\mathrm{C} / \mathrm{S}$ granular having a liquid limit of $433 \%$ and a plastic limit of $50 \%$. Bentonite pastes having nominal water contents (using distilled water) of 75, 100, 200, and 5008, have been prepared and allowed to cure for 72 hours in air-tight containers prior to testing. The pastes were driven through glass capillaries by compressed gas(helium). The experimental design is based on the following logic: (1) once a clay paste occupies a capillary, a fixed shearing surface is established; (2) by varying the driving pressure and measuring the discharge of the clay paste, a flow curve can be obtained which is similar to the one shown in Marsland and Loudon (1963, Fig. 1c) and discussed on the previous page.

Experience from several pre-trials has indicated that a constant shearing surface condition is difficult to achieve due to the slow flow rates of the thick bentonite pastes. To prolong the test duration solves the problem but brings about another complication, i.e., a significant change in water content of the clay paste, resulting from the migration of water. Shortening the length of the capillaries was found undesirable because the clay slurry near the outlet dried out due to evaporation and therefore slowed down the advance of the paste. Since the yield stress of bentonite as a function of water content is the ultimate interest in determining the critical hydraulic gradient for bentonite flow, the experiments of bentonite flow in glass capillaries are aimed at obtaining such a relationship. The yield stress of a bentonite paste is determined by narrowing the driving pressures down to a small range within which a slight change of driving pressure results in either the flow or the no-flow condition. The yield stress is computed based on the no-flow condition. Sample preparation and experimental procedures are described in Appendix $H$.

Results of bentonite flow tests in glass capillaries of $3.6 \mathrm{~mm}$ in diameter are summarized in Table 4.2. Fig. 4.1 shows the yield stress of bentonite pastes vs. bentonite weight percent. The data points (open squares) shown in Fig. 4.1 represent the shear stress immediately after bentonite flowed into the capillaries. The open circles indicate the shear stress at which no advance of the clay can be detected with a measuring tape of $0.5 \mathrm{~mm}$ resolution. Time elapsed before the no flow conclusion was made varies from 30 minutes to more than 24 hours, depending upon water content of the samples (Section H.4, Appendix H). The selection of the time spans is arbitrary. The rate of shear strain calculated accordingly is lower than $2.1 \times 10^{-4} / \mathrm{s}$. The shear stress computed for the "no flow" condition is assumed to be the yield stress of bentonite. Also included in Fig. 4.1 are yield stresses of Wyoming bentonite grouts (triangles) determined by Marsland and Loudon (1963).

It is not always easy to find the no-flow condition quickly. When it takes a long time to identify the condition, the water content of bentonite in the capillary can be quite different from its initial value due to the migration of water. What water content the computed yield stress actually corresponds to requires a careful examination of experimental records. For the open circles in Fig. 4.1, the averaged 
Table 4.2 Results of Bentonite Flow Tests through a $3.6 \mathrm{~mm}$ Diameter Glass Capillary

(a) Water Content of Bentonite:

\begin{tabular}{|c|c|c|c|c|}
\hline \multicolumn{5}{|l|}{ Before test: } \\
\hline Nominal & 500 & 200 & 100 & 75 \\
\hline Range & $508.6-514.4$ & $217.3-222.6$ & $107.1-111.2$ & $66.1-68.8$ \\
\hline Average & 511.5 & 219.9 & 108.2 & 67.5 \\
\hline \multicolumn{5}{|l|}{ After test: } \\
\hline Top & - & 213.1 & 107.1 & 67.5 \\
\hline Bottom & - & - & 111.2 & 70.0 \\
\hline $\begin{array}{l}\text { Near the glass } \\
\text { tube }\end{array}$ & - & 212.7 & $1 \overline{1} 2.2$ & 69.3 \\
\hline Inside the tube & - & 335.3 & 194.7 & 125.0 \\
\hline \multicolumn{3}{|c|}{ (b) Bentonite Weight Percent } & \multicolumn{2}{|l|}{ ' } \\
\hline $\begin{array}{l}\text { Near the glass } \\
\text { tube }\end{array}$ & $16.35 \%$ & $32.0^{3}$ & 47.1 & $59.05^{4}$ \\
\hline Inside the tube & $16.35^{1}$ & $23.0^{2}$ & 33.9 & 44.4 \\
\hline \multicolumn{5}{|l|}{ (c) Shear Stress } \\
\hline $\begin{array}{l}\text { Range of shear } \\
\text { stress }(\mathrm{kPa})\end{array}$ & $0.705-0.134$ & $4.149-0.465$ & $16.786-2.754$ & $25.3-11.856$ \\
\hline $\begin{array}{l}\text { Rate of shear } \\
\text { strain }\left(s^{-1}\right)\end{array}$ & - & $2.1 \times 10^{-4}$ & $1.11 \times 10^{-3}$ & $1.65 \times 10^{-5}$ \\
\hline $\begin{array}{l}\text { Shear stress } \\
(\mathrm{kPa})\end{array}$ & - & $1.076^{3}$ & 13.562 & 21.069 \\
\hline $\begin{array}{l}\text { Time recor- } \\
\text { ded/total time } \\
\text { (days) }\end{array}$ & - & $1 / 6.15$ & $0.04 / 8.65$ & $6.19 / 27.2$ \\
\hline $\begin{array}{l}\text { Rate of shear } \\
\text { strain }\left(s^{-1}\right)\end{array}$ & $\mathrm{ND}^{*}$ & ND & ND & ND \\
\hline $\begin{array}{l}\text { Shear stress } \\
(\mathrm{kPa})\end{array}$ & $0.232^{1}$ & $0.465^{2}$ & 2.862 & $14.32^{4}$ \\
\hline $\begin{array}{l}\text { Time recor- } \\
\text { ded/total time } \\
\text { (days) }\end{array}$ & $0.21 / 2.92$ & $4.25 / 6.15$ & $4.58 / 8.65$ & $10.17 / 27.2$ \\
\hline \multicolumn{5}{|c|}{$\begin{array}{l}*: \quad \mathrm{ND}=\text { not detectable } \\
* *: \text { computed assuming an average water content of } 511.5 \% \\
1,2,3,4: \text { indicate the pair number of circular data points } \\
\text { Fig. } 4.1\end{array}$} \\
\hline
\end{tabular}




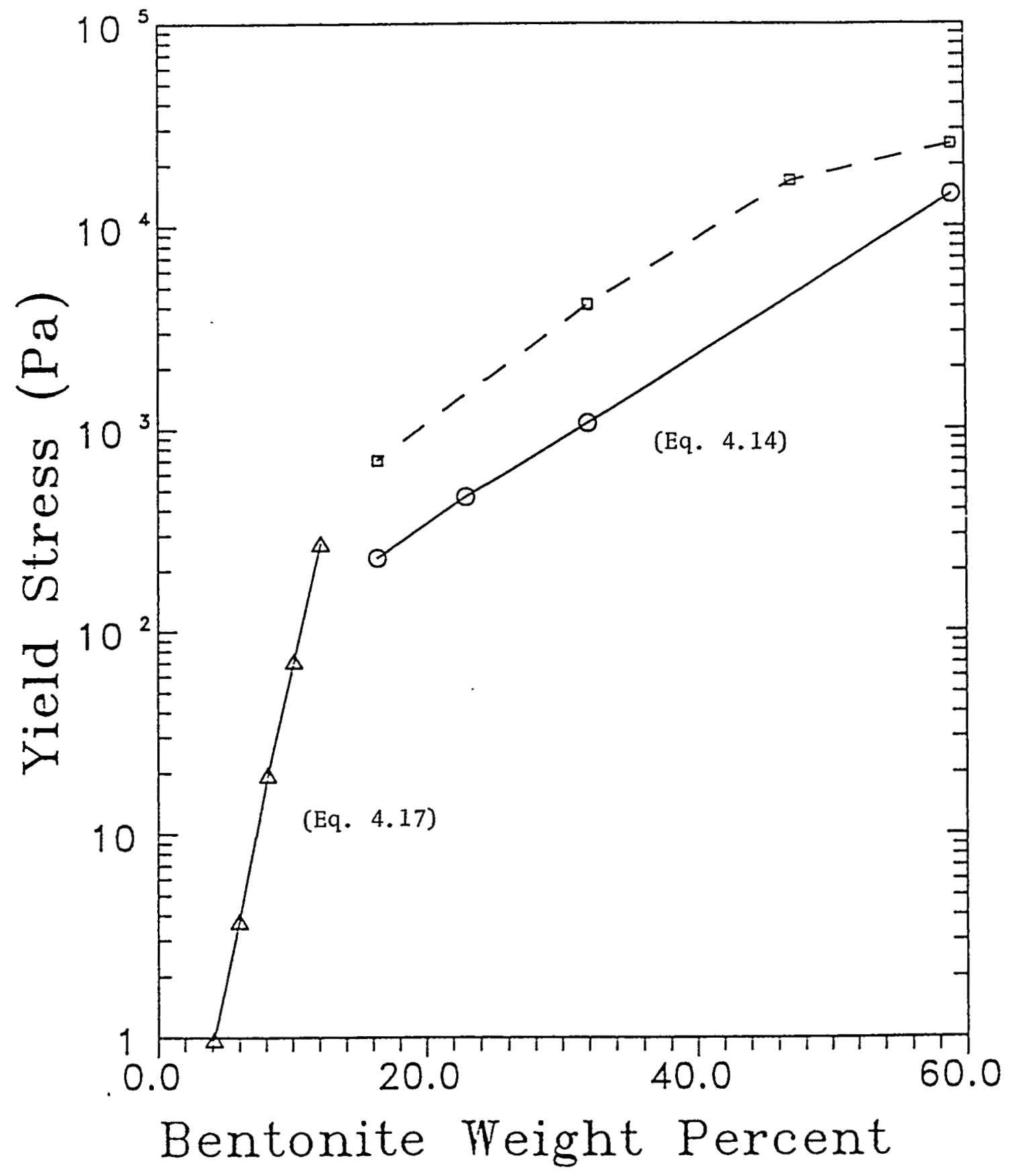

Figure 4.1 Yield stress of bentonite pastes vs. bentonite weight percent. Values of the triangle points are obtained from the work by Marsland and Loudon (1963). 
initial water content is related to the yield stress based on the early no flow condition. Water content of bentonite paste inside the capillary, determined immediately after testing, is used for the no flow condition established in the later part of a test.

\subsubsection{Relation Between Water Content and Yield Stress of Bentonite}

Statistical analysis has been performed on the bentonite flow test results. The equations obtained from curve fitting are given below:

A. Thick bentonite pastes (for $708<w<5108$ )

$$
\log \left(\tau_{f}\right)-13.728 w^{-0.2818}
$$

where $\tau_{f}=$ yield stress of bentonite $(\mathrm{Pa})$,

$w=$ water content in percent.

$$
\log \left(\tau_{f}\right)-1.6964+0.0417 x
$$

where $\mathrm{x}$ - bentonite weight percent.

B. Thin bentonite pastes (for $7208<w<23008$, based on the data from Marsland and Loudon, 1963)

$$
\log \left(\tau_{f}\right)-8.552 \operatorname{EXP}(-0.001715 \mathrm{~W})
$$

or

$$
\log \left(\tau_{f}\right)-3.841-0.00214 W
$$

$$
\log \left(\tau_{f}\right)=-1.209+0.3017 x
$$

where $\mathrm{w}$ and $\mathrm{x}$ are defined earlier.

Regression has also been performed on the pooled results of bentonite flow tests (both thick and thin clay pastes). The equations of the best fit are: 
and

$$
\log \left(\tau_{f}\right)=-1.57+1.4 \ln (x)
$$

Excluding the outlier yield stress point for $x=12.58$ ( $w=7238$ ) shown in Fig. 4.2, the regression gives:

$$
\log \left(\tau_{f}\right)-8.81-1.07 \ln (w)
$$

and

$$
\log \left(\tau_{f}\right)=-1.76+1.44 \ln (x)
$$

Table 4.3 gives correlation coefficients $\left(R^{2}\right)$ for Eqs. (4.13) through (4.21). These equations can be used to predict the yield stress of bentonite mixed with distilled water.

\subsubsection{Reliability of Prediction of Bentonite Yield Stress}

The yield stress of bentonite is the shear strength to resist the flow. Casagrande (1958) has first suggested a possible unique value of the shear strength $\left(2.65 \mathrm{kN} / \mathrm{m}^{2}\right)$ for most natural fine-grained soils at the liquid limit. Several researchers also report a narrow range of shear strengths of different soils, when measured at the liquid limit: 0.7$1.75 \mathrm{kN} / \mathrm{m}^{2}$ (Skempton and Northey, 1953), 0.8-1.6 and $1.1-2.3 \mathrm{kN} / \mathrm{m}^{2}$ (Norman, 1958), $1.3-2.4 \mathrm{kN} / \mathrm{m}^{2}$ (Youssef et al., 1965), $1-3 \mathrm{kN} / \mathrm{m}^{2}$ (Skopek and Ter-Stepanian, 1975). Although the ranges are small, the shear strength tends to decrease with increasing value of the liquid limit (Youssef et al., 1965). We speculate that the C/S granular bentonite at the liquid limit of 4338 should have a shear strength either similar to or lower than the ranges indicated above. For the given liquid limit, the shear stress computed from Eq. $(4.13)$ is $0.3 \mathrm{kN} / \mathrm{m}^{2}$, as expected.

The reliability of Eq. (4.13) has also been examined against the results from axial shear testing of annulus bentonite seals (Ogden and Ruff, 1989) listed in Table 4.4. The annulus seals were installed between an outer plexiglass pipe and an inner steel casing. Recognizing the differences in the aggregate size and supplier, some differences between the predicted shear strengths from the equation and the experimental results are considered small.

\subsubsection{Prediction of Critical Gradient Using the Relation Between Yield Stress and Water Content}

The relation between yield stress and water content has been incorporated in a model for predicting the critical pressure gradient for 


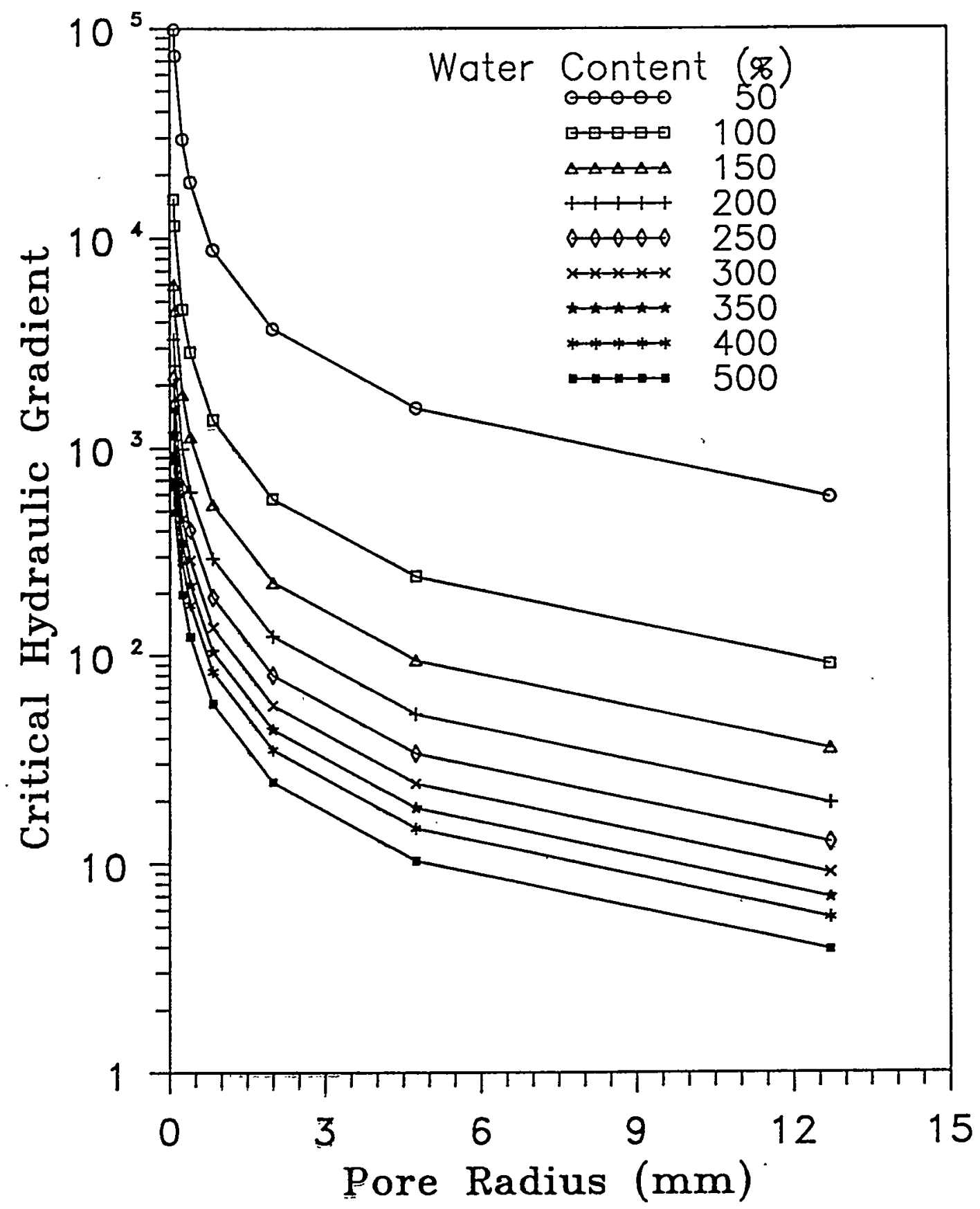

Figure 4.2 Relations between the critical hydraulic gradient and pore radius for various water contents of bentonite. 
Table 4.3 Correlation Coefficients $\left(R^{2}\right)$ of Yield Stress-Water Content

A. For $708<\mathrm{w}<5108$ or $17.48<\mathrm{x}<5958$
$\log \left(\tau_{f}\right)=13.728 \times \mathrm{w}^{-0.2818}$
0.9999
Eq. (4.13)
$\log \left(\tau_{f}\right)=1.6964+0.0417 x$
0.9998
Eq. (4.14)

B. For $7208<w<23008$ or $4.28<x<12.28$
$\log \left(\tau_{f}\right)=8.552 \times \operatorname{EXP}(-0.001715 \mathrm{w})$
0.9979
Eq. (4.15)
$\log \left(\tau_{f}\right)=3.841+0.00214 \mathrm{w}$
0.9711
Eq. (4.16)
$\log \left(\tau_{f}\right)=-1.209+0.3017 x$
0.9984
Eq. (4.17)

C. Pooled data
$\log \left(\tau_{f}\right)=4.47 \times \operatorname{EXP}(-0.0018 \mathrm{w})$
0.934
Eq. (4.18)
$\log \left(\tau_{f}\right)=-1.57+1.40 \ln (x)$
0.938
Eq. (4.19)

D. Pooled data except the yield stress for $x=12.5 \%$
$\log \left(\tau_{f}\right)=8.81-1.07 \ln (w)$
0.938
Eq. (4.20)
$\log \left(\tau_{f}\right)=-1.76+1.44 \ln (x)$
0.976
Eq. (4.21)

NOTES :

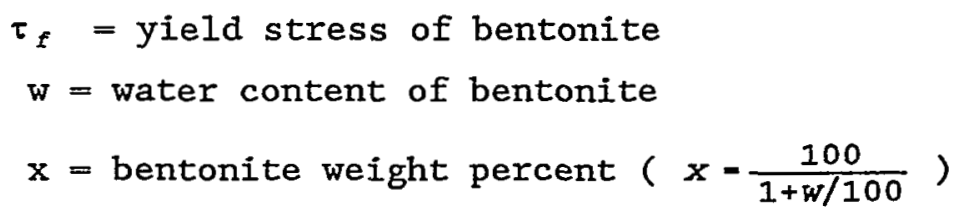


Table 4.4 Predicted Yield Stresses and Experimental Axial Shear Strengths for Different Bentonite Products

\begin{tabular}{|c|c|c|c|}
\hline Clay Type & $\begin{array}{l}\text { Water } \\
\text { Content }\end{array}$ & $\begin{array}{c}\text { Experimental } \\
\text { Shear Strength } \\
\left(\mathrm{kN} / \mathrm{m}^{2}\right)\end{array}$ & $\begin{array}{l}\text { Predicted } \\
\text { Yield Stress } \\
\left(\mathrm{kN} / \mathrm{m}^{2}\right) \\
\end{array}$ \\
\hline $\begin{array}{l}\text { American Colloid } \\
\text { Volclay chip }\end{array}$ & 80.47 & 15.45 & 9.70 \\
\hline $\begin{array}{l}\text { American Colloid } \\
\text { Volclay } 3 / 8 " \\
\text { tablets }\end{array}$ & 77.05 & 18.57 & 10.86 \\
\hline $\begin{array}{l}\text { Wyo-Ben Enviroplug } \\
\text { medium }\left(1 / 4-3 / 8^{\prime \prime}\right)\end{array}$ & 95.33 & 6.03 & 6.32 \\
\hline $\begin{array}{l}\text { NL Baroid Holeplug } \\
(3 / 4 " \text { chip) }\end{array}$ & 83.33 & 26.2 & 8.9 \\
\hline
\end{tabular}

"Ogden and Ruff (1989, Table 5-3). Shear strengths measured after the samples set for 72 hours.

${ }^{\star *}$ Based on Eq. (4.13) 
bentonite/crushed rock plugs. The model requires two additional parameters, the water content of the bentonite and the representative pore size of the crushed rock skeleton. For the saturated condition, the water content of the bentonite can be estimated using Eq. (3.11). The water content thus determined can be used to calculate the yield stress $\left(\tau_{f}\right)$, using Eq. $(4.8),(4.10)$, or $(4.11)$. The critical pressure gradient $\left(i_{c, p}\right)$ is then computed from:

$$
i_{c, p}=\frac{2 \tau_{f}}{R_{m}}
$$

where $R_{m}=$ representative pore radius of the crushed rock skeleton.

In this study, $d_{50}$ (sieve aperture at 508 passing) instead of $d_{5}$ or $d_{10}$ commonly used for predicting permeability of granular materials (Kenney et al., 1984; Hazen, 1892), is assumed to represent $R_{m}$. This selection is based on the consideration that, according to Eq. (4.13), the bentonite will first start flowing in the large pores between crushed rock particles for a given yield stress. For a crushed rock mixture of gradation type $A, d_{50}$ is $3.9 \mathrm{~mm}$. This implies a pore diameter of $8 \mathrm{~mm}$, close to the maximum particle size of $9.42 \mathrm{~mm}$ for crushed rock of type A. Such an estimation for the representative size of large pores appears to be reasonable, considering the separation of rock particles due to the bentonite filler.

The predicted critical gradients are compared with the experimental ones for nine bentonite/crushed tuff samples in Table 4.5. An example of such a comparison is given below.

Sample: B/AL-C-8-25/A-S

Composition: 25\% bentonite and $75 \%$ type A crushed tuff

$R_{m}: d_{50}=3.9 \mathrm{~mm}$

Sample length: $10.9 \mathrm{~cm}$

Estimated water content of bentonite at saturation: 115.938

(from Eq. 3.11)

Experimental critical hydraulic gradient: $i_{c, e}=120$

(from the flow rate-hydraulic gradient curve)

Computations:

$$
\begin{aligned}
& \log \left(\tau_{f}\right)=13.728 *(115.93)^{-0.2818} \text { gives } \tau_{f}=3952 \mathrm{~Pa} \\
& i_{c, p}=2 \tau_{f} / R_{m}=2026.7 \mathrm{~Pa} / \mathrm{mm}
\end{aligned}
$$

when expressed in terms of hydraulic gradient (10.2 $\mathrm{m}$ of water $=100 \mathrm{kPa}$ ): 
Table 4.5 Predicted and Experimental Critical Pressure Gradients for Bentonite Flow

\begin{tabular}{|c|c|c|c|c|c|c|}
\hline $\begin{array}{l}\text { Sample } \\
\text { Number }\end{array}$ & $\begin{array}{c}\text { Satu- } \\
\text { rated } \\
\text { Water } \\
\text { Content of } \\
\text { Bentonite } \\
(8)\end{array}$ & $\begin{array}{r}\text { Sample } \\
\text { Length } \\
(\mathrm{cm})\end{array}$ & $\begin{array}{r}\text { Cri } \\
\text { Hyd } \\
\text { Gra } \\
\text { Pre- } \\
\text { dicted } \\
\end{array}$ & $\begin{array}{l}\text { ical } \\
\text { aulic } \\
\text { ient } \\
\text { Experi- } \\
\text { mental } \\
\end{array}$ & $\begin{array}{c}\text { Gradient } \\
\text { Ratio } \\
\text { Pred./ } \\
\text { Exper } \\
\end{array}$ & $\begin{array}{c}\text { Pore } \\
\text { Radius } \\
\mathrm{R}_{\mathrm{m}} \\
(\mathrm{mm})\end{array}$ \\
\hline $\begin{array}{l}\text { B/AL-C-4- } \\
25 / A-P-B\end{array}$ & 108.07 & 10.27 & 244 & 115 & 2.12 & 3.9 \\
\hline $\begin{array}{l}\text { B/AL-C-8 - } \\
25 / A-S\end{array}$ & 115.93 & 10,9 & 207 & 120 & 1.72 & 3.9 \\
\hline $\begin{array}{l}\text { B/AL-C-8 - } \\
35 / \mathrm{A}-\mathrm{S}\end{array}$ & 83.42 & 10.65 & 492 & 280 & 1.76 & 3.9 \\
\hline $\begin{array}{l}B / A L-C-4 \\
25 / A\end{array}$ & 120.61 & 9.5 & 189 & 170 & 1.11 & 3.9 \\
\hline $\begin{array}{l}\text { B/AL-C-4- } \\
25 / B\end{array}$ & 122.38 & 9,5 & 290 & 250 & 1.16 & 2.45 \\
\hline $\begin{array}{l}\text { B/AL-C-4- } \\
25 / C\end{array}$ & 133.33 & 9.8 & 339 & 273 & 1.24 & 1.725 \\
\hline $\begin{array}{l}\text { B/AL-C-4- } \\
35 / A\end{array}$ & 79.1 & 9.0 & 530 & 424 & 1.25 & 3.9 \\
\hline $\begin{array}{l}\text { B/AL-C-4- } \\
35 / B\end{array}$ & 92.94 & 9.5 & 560 & 352 & 1.59 & 2.45 \\
\hline $\begin{array}{l}B / A L-C-4- \\
35 / C\end{array}$ & 106.43 & 10,0 & 572 & 360 & 1.59 & 1.725 \\
\hline
\end{tabular}




$$
\begin{aligned}
& i_{c, p}= 2026.7 \mathrm{~Pa} / \mathrm{mm} * 109 \mathrm{~mm} *(1 \mathrm{kPa} / 1000 \mathrm{~Pa}) * \\
&\left(10.2 \mathrm{~m} \mathrm{H}_{2} 0 / 100 \mathrm{kPa}\right) *(1 / 0.109 \mathrm{~m}) \\
&= 206.7 \\
& i_{c, p} / i_{c, e}=206.7 / 120=1.72
\end{aligned}
$$

As shown in Table 4.5, the proposed model overestimates the critical hydraulic gradient by a factor ranging from 1.11 to 2.12 . The discrepancy may be due to the following: (1) The neglect of slip at the wall of the capillary results in the overestimation of $T_{f}$. (2) In driving the clay paste through a capillary, part of the energy must have been consumed by the accompanying migration of moisture; the actual force effective for the advance of clay paste is less than the product of the driving pressure multiplied by the whole cross-sectional area of the capillary. (3) The saturated water content of bentonite obtained from Eq. (3.11) is an average one. In reality, the water content of bentonite changes as the pore pressure varies during flow testing. The variation in pore pressure causes consolidation near the outflow end and swelling near the inflow end. This time-dependent process cannot be eliminated and will necessarily create a non-uniform distribution of water content. Because of the swelling, the water content of bentonite near the inflow end is expected to be higher than the water content obtained from Eq. (3.11). The flow of bentonite therefore should first occur at the inflow end. The critical gradient extracted from a flow rate-hydraulic gradient curve most likely corresponds to the critical gradient for bentonite flow at a higher water content. For the example given above, the experimentally determined hydraulic gradient of 120 was used to back calculate the water content according to Eq. (4.14). The calculation gives a water content of 158 , about 368 higher than the 116 obtained from Eq. (3.11). (4) As described in Section 4.4.3, the yield stress of bentonite for a given water content is computed based on the no flow condition. Such a condition is established on the basis of observations and consequently limited by the resolution of the measuring tape. If the condition identified actually resided in a flow region (e.g. region II), the yield stress thus computed would be overestimated.

For practical purposes, the model can be used to give a first approximation of the critical hydraulic gradient at which bentonite starts to flow in the crushed rock matrix. A conservative estimation can be made if a factor of $1 / 2$ is introduced into Eq. (4.22).

\subsubsection{Filter Design}

The concept of yield stress and critical pressure gradient can be applied to the design of filters for bentonite seals. Given a water content of the bentonite (between 70 to $510 \%$ ) and a maximum possible field hydraulic gradient, the maximum allowable radius $\left(R_{m}\right)$ of pores in fine particles of the filter material may be computed using Eqs. (4.13) and (4.19). Similarly, for the known $R_{m}$ and hydraulic gradient, $a$ maximum water content of the seal can be specified. Such a maximum water content is useful for seal design (e.g. bentonite content needed) and for compaction specifications. 
Combining Eqs. (4.13) and (4.19), the relations among water content, critical hydraulic gradient, and pore radius $\left(R_{m}\right)$ are shown in Figs. 4.2 and 4.3. The graphs for the water content of $50 \%$ may not be justified since this water content lies outside the specified range for Eq. (4.13).

As shown in Fig. 4.2, the critical hydraulic gradient at which bentonite flow takes place increases rapidly with decreasing pore radius. A hydraulic gradient higher than 120 is required for bentonite of water contents ranging from 50 to 5008 to flow in pores of $0.4 \mathrm{~mm}$ radius. Sherard et al. (1984) suggest that successful filters for silts and clays should be able to sustain a relatively high gradient, such as 1000. To meet the requirement, filters of an effective pore radius of not more than $0.25 \mathrm{~mm}$ are necessary to prevent piping, erosion and flow of bentonite, for water contents from 50 to 3008 (Fig. 4.3). This pore diameter is in accordance with the general criteria adopted for the filter design for dam cores of fine-grained materials; i.e., filters having $d_{15}$ of $0.5 \mathrm{~mm}$ or less (U.S. Bureau of Reclamation, 1987, p. 220) or having $d_{15}$ of $0.4 \mathrm{~mm}$ or less (U.S. Army Corps of Engineers, 1955, as cited by Vaughan and Soares, 1982) are suitable for fine silts and clays. The criterion $d_{15}=0.5 \mathrm{~mm}$ may be conservative for general dam and embankment applications (Sherard et al., 1984; Sherard and Dunnigan, 1989). It appears to be necessary for filters for the highly dispersive bentonite, in light of its flow characteristics.

Crushed rocks of gradation types $A, B$, and $C\left(d_{15}=0.54,0.35\right.$, and 0.26 $\mathrm{mm}$, respectively) can be used as filters to prevent the migration of fine particles. The filtering ability of graded filters, however, is sensitive to the grading below the 158 size (Khor and Woo, 1989). They suggest that sufficient noncohesive fines of at least 58 by weight passing the $0.15 \mathrm{~mm}$ sieve are necessary for well-graded filters. Abiding by the requirement of $\mathrm{d}_{15}=0.5 \mathrm{~mm}$, uniform filters may be more suitable than graded filters (Vaughan and Soares, 1982).

Lateral migration of fine particles is possible if the sealants are in contact with open joints and/or fractures. Based on the results in Figs. 4.2 and 4.3, discontinuities having apertures larger than $0.5 \mathrm{~mm}$ must be grouted in order to preclude such migrations. 


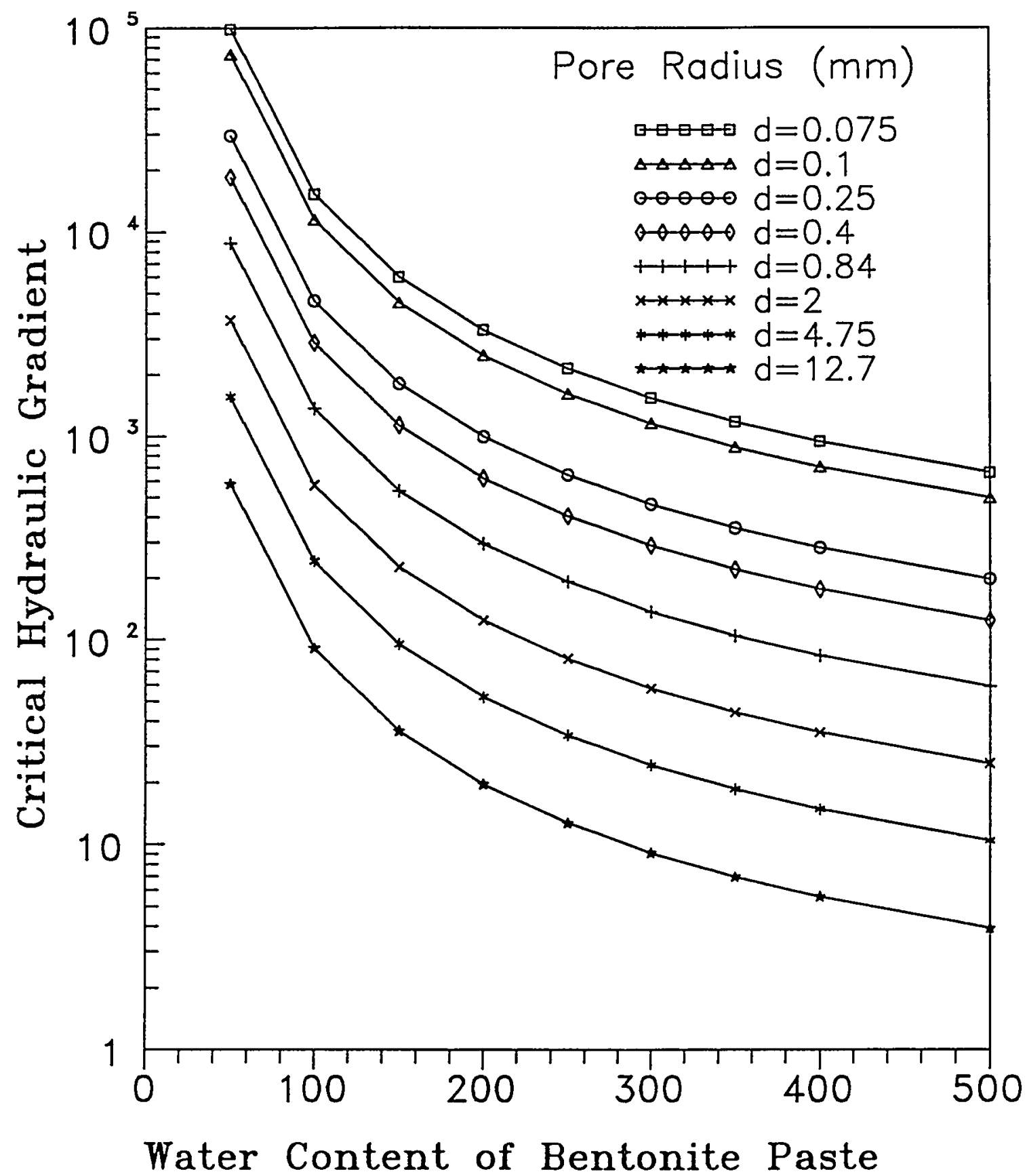

Figure 4.3 Relations between the critical hydraulic gradient and bentonite water content for different values of pore radius. 
CHAPTER FIVE

\section{PREDICTION OF PERMEABILITY AND SWELLING PRESSURE OF BENTONITE}

\subsection{Prediction of Permeability}

\subsubsection{Introduction}

The importance of evaluating the permeability of a material has long been recognized. The frequent necessity of obtaining the parameter has led to the development of theoretical as well as empirical models for the prediction of permeability.

Some empirical permeability formulae for sand and for clay are reviewed by Loudon (1952) and by Tavenas et al. (1983), respectively. Their investigations indicate that the use of the formulae is limited to the type of material and/or the range of void ratios studied. Theoretical permeability models can be grouped, according to Lagerwerff et al. (1969), into two types: grain models and pore models. In this usage, "grain" does not necessarily mean the "soil grain" only and may include the immovable water layers attached to the solid surface. The models developed by Carman (1939), Schmid (1957), and Lagerwerff et al. (1969) are of the first type. Pore models include those developed by Childs and Collis-George (1950), Marshall (1958), Millington and Quirk (1959), and Paterson (1983). Both types of theoretical models are based on the Hagen-Poiseuille equation for laminar flow in a circular pipe.

The pore models are handicapped for application to bentonite by the fact that they do not allow for swelling. The pore size distribution is usually derived from a determination of the soil-moisture characteristic, which may be subject to the effects of swelling (Lagerwerff et al., 1969). This complicates the application of the pore models to swelling materials (e.g. bentonite). The grain models suggested by Schmid (1957) and Lagerwerff et al. (1969) require several flow tests to pre-determine some key parameters before the prediction of permeability is possible. The applicability of these two grain models is thereby greatly reduced.

The Kozeny-Carman equation (Carman, 1937) gives good estimates for the permeability of clean sand (Taylor, 1948; Loudon, 1952), for quartz powder and for spherical glass particles (Garman, 1939). The equation fails to predict the permeability of clays (Michaels and Lin, 1954; Lambe, 1955). The modified Kozeny-Carman equation (Carman, 1939), incorporating the concept of stationary water films held at the surface of clay particles, appears to yield better results, but only to a certain extent.

The modified equation has been tested with the measured permeabilities of Wyoming bentonite obtained from this study and from the literature. The discrepancy between the predicted and the experimental results is small for samples of low porosity, but becomes large when porosity 
increases. Possible causes for the discrepancy are critically reviewed and a refined Kozeny-Carman equation is proposed.

\subsubsection{Kozeny-Carman Equation}

The Kozeny-Carman equation can be expressed in the form:

$$
k=\frac{n^{3}}{m t^{2} S_{0}^{2}(1-n)^{2}}
$$

where $\mathbf{k}=$ intrinsic permeability $\left(\mathrm{cm}^{2}\right)$

$\mathrm{n}=$ porosity

$m$ = shape factor of conducting pores

$t$ - tortuosity

$\mathrm{S}_{0}=$ specific surface of the particles $\left(\mathrm{cm}^{2} / \mathrm{cm}^{3}\right)$.

According to Carman (1937), $m=2.5$ and $t^{2}-2$ suit most materials.

The modified Kozeny-Carman equation is given by:

$$
k=\frac{n_{e}{ }^{3}}{m t^{2} S_{0}^{2}(1-n)^{2}}
$$

where $n_{e}=$ effective porosity, and other parameters are as defined above.

The derivations of Eqs. (5.1) and (5.2) are based upon the HagenPoiseuille equation and Darcy's law, and can be found in Carman (1939) and Yong and Warkentin (1975, pp. 144-146), and Carman (1939), respectively. The assumptions involved in the derivation are: uniform and equidimensional pores, and laminar fluid flow (0lsen, 1962).

According to Michaels and Lin (1955), discrepancies between measured and computed (from Eq. 5.1) permeabilities in clays are likely due to two factors: (1) interfacial phenomena (the influence of electrical forces at the liquid-solid interfaces that act on the permeating fluid) and, (2) particle packing characteristics (degree of particle dispersion and particle orientation). Their studies on kaolinite indicate that the effects of particle packing characteristics primarily are responsible for the discrepancies. The effects of interfacial phenomena are minor. They further conclude that the latter effects could be attributed primarily to counter electro-osmosis and consequently that the thickness of immobilized liquid films on the surface of solids must be extremely small, less than 48 of the diameter of the pores. The conclusion of the limited thickness of immobilized liquid films may be supported by the results obtained by Rosenquist (1955) and Aylmore and Quirk (1960). An important deduction from Eq. (5.2) is that clays may have zero permeability at quite considerable porosities (where the effective porosities become zero), e.g. at $n=0.207$ for a clay soil and $n=0.355$ for a plastic clay (Carman, 1939). 
Olsen (1962) investigated the relative importance of several factors on the failure of Eq. (5.1) to predict permeability in saturated clays: (1) possible violation of Darcy's law, (2) electrokinetic coupling, (3) high viscosity, (4) tortuous flow paths, and (5) unequal pore sizes. His results show that: (a) the possible violations of Darcy's law and electrokinetic coupling are insignificant, (b) high viscosity and/or tortuous flow paths fail to account completely for the discrepancies, and (c) unequal pore sizes can explain all the discrepancies.

Discrepancies between measured and predicted flow rates for kaolinite, illite, and Boston blue clay, obtained from consolidation permeation tests by 01sen (1962) are shown in Fig. 5.1. A cluster model proposed by 0lsen provides a possible explanation for the discrepancies. The model (Fig. 5.2) consists of clusters that are equidimensional, uniform in size, and porous. Three parameters define the model pore geometry: (1) $\mathrm{N}$, the number of particles per cluster; (2) $e_{c}$, the intra-cluster void ratio; and (3) $e_{p}$, the inter-cluster void ratio, which equals the total void ratio minus the intra-cluster void ratio, $e_{T}-e_{c}$. Since flow rates are proportional to the fourth power of pore radii, the contribution of the flow component through the cluster pores is assumed to be negligible.

Based on an assumed relationship between the total, intra- and intercluster void ratios, 0lsen was able to produce discrepancies for systems of clusters (Fig. 5.3) similar to those shown in Fig. 5.1. At high total vold ratios, or porosities, the compressibility of the individual clusters is considered negligible compared to that of the cluster skeleton. When, during compression, the clusters approach a density corresponding to the densest possible packing of spheres, the clusters themselves begin to compress as the total void ratio decreases. At this stage, the inter-cluster pores and consequently the flow rates likely change little. The predicted flow rates are smaller due to the reducing total void ratios. This behavior may explain why at porosities less than about 0.4 (Fig. 5.1), measured flow rates decrease less rapidly with decreasing porosity than predicted.

The concept of particle clusters is analogous to those of domains (Aylmore and Quirk, 1960), quasi-crystals (Aylmore and Quirk, 1967), tactoids (Blackmore and Miller, 1962), packets (Shainberg and Caiserman, 1971). The concept is also supported by the studies on fabric of clays (Pusch, 1973; Collins and McGown, 1974). The cluster model provides reasonable explanations for the discrepancies between measured and predicted permeabilities in clays. Refinement of the Kozeny-Carman equation is complicated by the difficulties in determining the three parameters needed to describe the pore geometry.

\subsubsection{Refined Kozeny-Garman Equation For Clays}

From the discussions in Section 5.2, it is clear that the Kozeny-Carman equation needs to be modified for the prediction of permeabilities in clays. Such an improvement requires incorporating the effects of the complex pore geometry of clays. The modified Kozeny-Carman equation (Eq. 5.2) only accounts for the effects on the pore size of the immobilized liquid films at the solid surface. Considering the influences of 


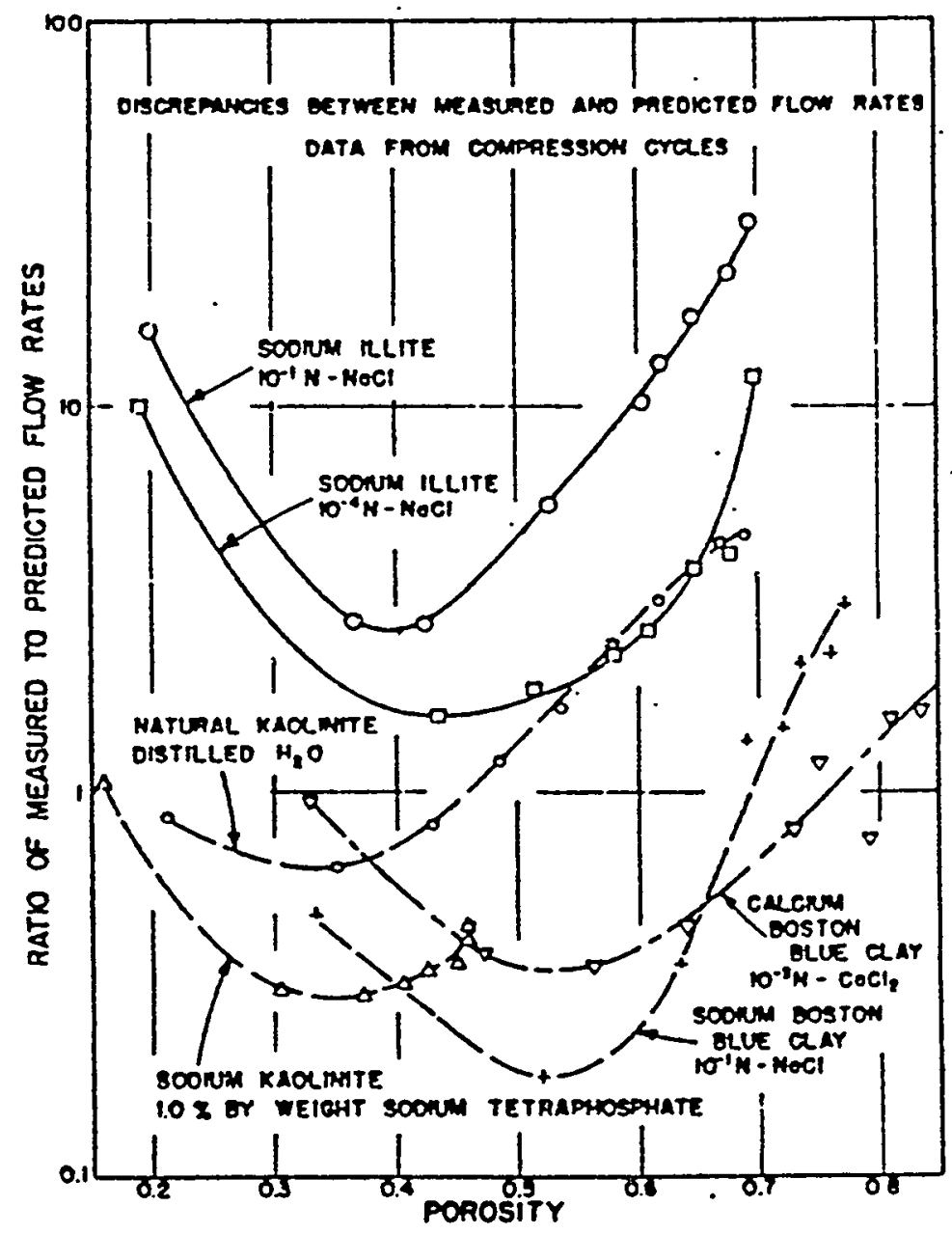

Figure 5.1 Discrepancies between measured flow rates and predicted flow rates from the Kozeny-Carman equation.

Reproduced with permission from H.W. 01sen, "Hydraulic Flow Through Saturated Clays," in Proceedings of the 9th Conference on Clays and Clay Minerals, Figure 5, p. 139. Copyright 1962 by Pergamon Press, New York. 


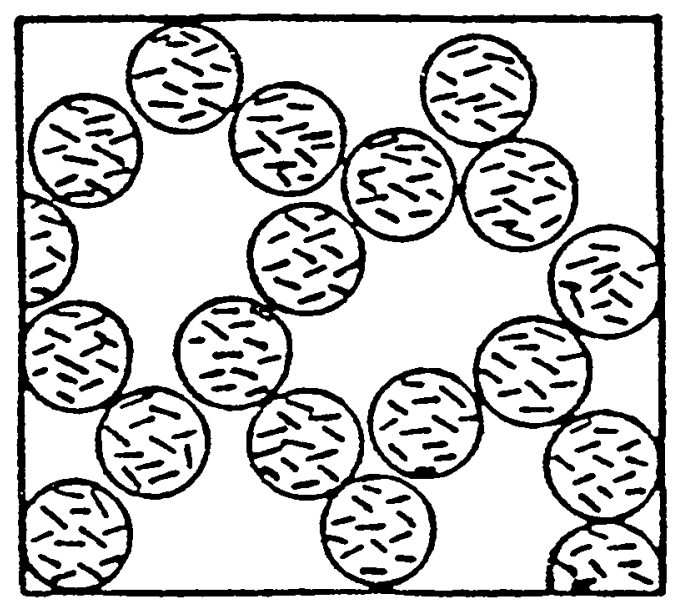

Figure 5.2 Cluster model for clays. Each circle represents a cluster, with a number of clay particles per cluster. The total void volume equals the sum of the void volumes between and within clusters.

Reproduced with permission from H.W. Olsen, "Hydraulic Flow Through Saturated Clays," in Proceedings of the 9th Conference on Clays and Clay Minerals, Figure 12, p. 151. Copyright 1962 by Pergamon Press, New York. 


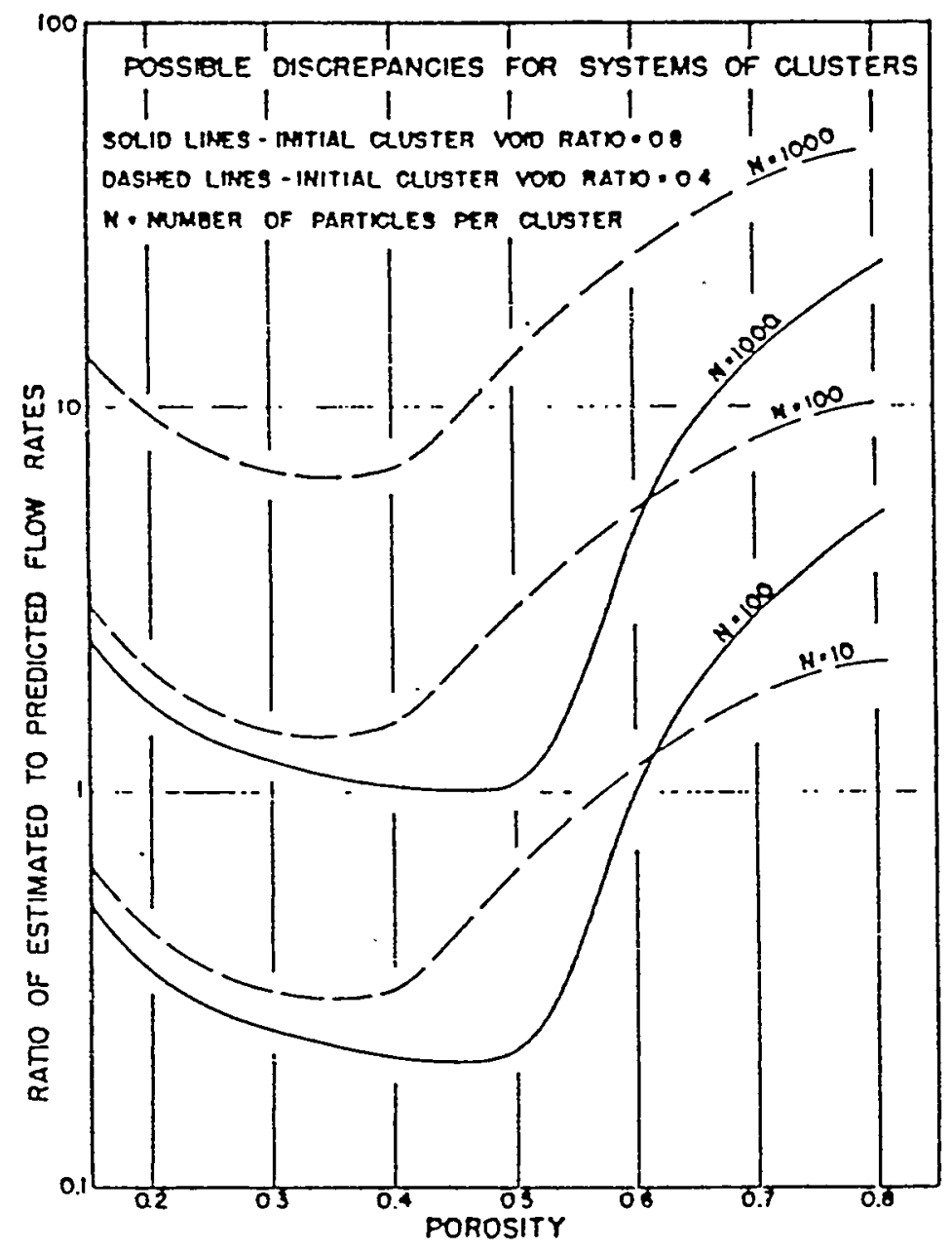

Figure 5.3 Possible discrepancies for systems of clusters.

Reproduced with permission from H.W. Olsen, "Hydraulic Flow Through Saturated Clays," in Proceedings of the 9 th Conference on Clays and Clay Minerals, Figure 14, p. 153. Copyright 1962 by Pergamon Press, New York. 
complex water-solid-electrolyte interactions on the soil structure, a complete mathematical description is not attempted. Instead, a possible collective factor (a water content ratio) is examined to account for the changes in pore geometry.

Atterberg limits are water contents where the soil behavior changes (Holtz and Kovacs, 1981, p. 36). Depending on its water content, a fine-grained soil can exist in any one of four states: solid, semisolid, plastic, or liquid. The water content corresponding to the transition between adjacent states is termed shrinkage; plastic, and liquid limit, respectively (Lambe and Whitman, 1979, p. 33). At the plastic limit, the particles or units of particles slide past one another upon application of force but there is still sufficient cohesion to allow them to retain a shape (Yong and Warkentin, p. 66-67). At the liquid limit, the cohesion becomes too small to retain a definite shape and the material acts as a liquid.

The engineering properties of a soil reflect directly the influences of structure which, in turn, depends upon the soil type, the ions adsorbed, the salt concentration, and the stress history (Mitchell, 1976, p. 222; Holtz and Kovacs, 1981, p. 40). Water contents such as plastic and liquid limits and related other indices have been found to correlate with some engineering properties of soils such as the undrained shear strength, compression index, compression ratio (e.g. Skempton, 1944; Youssef et al. 1965; Wroth and Wood, 1978; Sridharan and Jayadeva, 1982; Nagaraj and Srinivasa Murthy, 1983, 1986; Srinivasa Murthy and Nagaraj, 1988; Pandian and Nagaraj, 1990). The correlations lead to a postulation that water content of a fine-grained soil describes collectively a possible equilibrium state of the soil's structure, and therefore a corresponding state of pore structure.

The vertex of the parabola-like curves shown in Fig. 5.1 should indicate a unique state of pore structure. According to Olsen's cluster model, this unique state may correspond to the densest possible packing of clay clusters. Further reduction in total void ratio will be due primarily to the compression of the clusters themselves. The water content related to the unique pore structure of a soil should be very close to its plastic limit, at which particles or units of particles slide past one another upon application of force. The plastic limit shall be used to represent (indirectly) the unique state of pore structure.

The Kozeny-Carman equation may then be refined as follows:

$$
k=\left(\frac{w}{w_{p}}\right) \frac{n^{3}}{m t^{2} S_{o}^{2}(1-n)^{2}}
$$

$$
\begin{aligned}
& \text { for } w>w_{p}, \\
& \text { where } \quad w=\text { water content, } \\
& w_{p}=\text { the plastic limit. }
\end{aligned}
$$

and 


$$
k=\left(\frac{w_{p}}{w}\right) \frac{n^{3}}{m t^{2} S_{o}^{2}(1-n)^{2}}
$$

for $w<w_{p}$ (i.e. highly compacted clays).

Conceptually, saturated fine-grained soils having a water content less than the shrinkage limit, should be considered as solids which have zero permeability. The lower bound of water content, w, for Eq. (5.4) may therefore be set at the shrinkage limit. This lower bound can also be a water content at which all the water is held firmly by the solids, based on the concept of immobilized liquid films.

The water content of a soil sample at saturation can be expressed as:

$$
w=\frac{1}{\rho_{s}} \frac{n}{(1-n)}
$$

where $p_{s}=$ density of solids.

Using this relation, Eqs. (5.3) and (5.4) can be reduced to:

$$
k=\left(\frac{1}{\rho_{s}}\right)\left(\frac{1}{w_{p}}\right) \frac{n^{4}}{m t^{2} S_{0}^{2}(1-n)^{3}}
$$

and

$$
k=w_{p} \rho_{s} \frac{n^{2}}{m t^{2} S_{o}^{2}(1-n)}
$$

\subsubsection{Validation of the Refined Kozeny-Carman Equation}

The refined Kozeny-Carman equation has been examined to see if the discrepancies between measured and predicted (from Eq. 5.1) permeabilities in clays can be accounted for, e.g. as by the cluster model proposed by Olsen (1962). The permeabilities obtained from Eq. (5.3) and (5.4) are assumed to represent the measured permeabilities, and are compared with the ones predicted from Eq. (5.1). The assumption should be valid if a similar trend as shown in Fig. 5.1 can be obtained. The basic parameters used are $w_{p}=328, S_{0}=10 \mathrm{~m}^{2} / \mathrm{g}$ for kaolinite (Lambe and Whitman, 1979, Tables 3.4 and 5.2), and an assumed specific gravity of 2.7. Table 5.1 and Fig. 5.4 indicate that the refined equation is capable to incorporate the effects of unequal pore sizes and the changing pore geometry on the prediction of permeabilities in clays.

Approximately $90 \%$ of American Colloid C/S granular and MX-80 bentonite is montmorillonite (American Colloid Company, Data No. 202). Montmorillonite has an estimated specific surface of 760 to $800 \mathrm{~m}^{2} / \mathrm{g}$ (Quirk, 
Table 5.1 Differences between predicted permeabilities from the original and refined Kozeny-Carman equations

\begin{tabular}{ccccc} 
Porosity & $\begin{array}{c}\text { Water Content } \\
(\%)\end{array}$ & $\begin{array}{c}\text { Predicted Permeability } \\
(1)^{\mathrm{b}}\end{array}$ & $\begin{array}{c}(\mathrm{cm} / \mathrm{s}) \\
(2)^{\mathrm{b}}\end{array}$ & $\begin{array}{c}\text { Ratio } \\
(2) /(1)\end{array}$ \\
\hline 0.2 & 9.26 & $3.360 * 10^{-9}$ & $1.161 * 10^{-8}$ & 3.45 \\
0.3 & 15.87 & $1.482 * 10^{-8}$ & $2.988 * 10^{-8}$ & 2.02 \\
0.4 & 24.69 & $4.779 * 10^{-8}$ & $6.194 * 10^{-8}$ & 1.30 \\
0.5 & 37.04 & $1.344 * 10^{-7}$ & $1.556 * 10^{-7}$ & 1.16 \\
0.6 & 55.55 & $3.630 * 10^{-7}$ & $6.300 * 10^{-7}$ & 1.74 \\
0.7 & 86.42 & $1.025 * 10^{-6}$ & $2.768 * 10^{-6}$ & 2.70 \\
\hline
\end{tabular}

a: Water content $(w)$ is computed using the relation $\mathrm{Se}=\mathrm{Gw}$, where $\mathbf{S}=$ degree of saturation ( $100 \%$ in this calculation), $e=$ void ratio, $G=$ specific gravity of the clay.

b: For kaolinite with $S_{\circ}=10 \mathrm{~m}^{2} / \mathrm{g}, \mathrm{G}=2.7$ (from Lambe and Whitman, 1979). Values in columns (1) and (2) are obtained from Eqs. (5.1), and $(5.3)$ or $(5.4)$, respectively. 


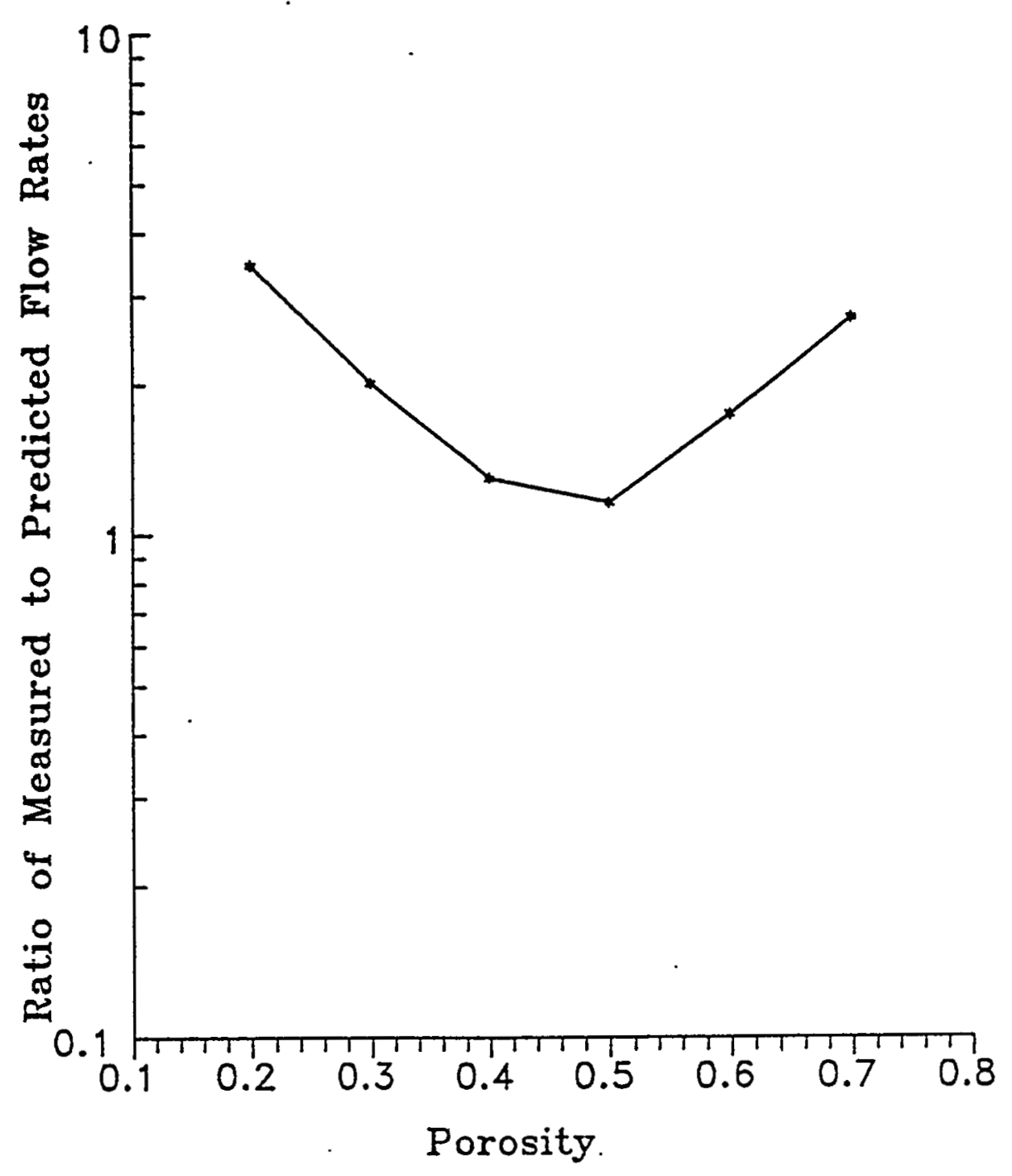

Figure 5.4 Discrepancies between predicted flow rates from the KozenyCarman equation and the refined equation. 
1968; Shainberg et al., 1971; Mitchel1, 1976, p. 45; Yong and Warkentin, 1975, p. 46). Using the specific surface value of $800 \mathrm{~m}^{2} / \mathrm{g}$ and assuming the other $10 \%$ of the materials have a negligible effect on the permeability, the refined Kozeny-Carman equation has been used to check measured permeabilities (C/S granular bentonite) reported in Chapter 3 , as well as those of MX-80 bentonite reported by Borgesson et al. (1988). The plastic limit and specific gravity are $50 \%$ and 2.92 for the $\mathrm{C} / \mathrm{S}$ granular, $70 \%$ and 2.9 for the MX-80. The results are shown in Tables 5.2 and 5.3 , respectively.

For eleven $\mathrm{C} / \mathrm{S}$ granular bentonite samples, the ratios between measured and predicted permeabilities vary from 1.02 to 3.66 . For five MX-80 samples, the ratios differ from 0.8 to 3.75 . Such narrow deviations substantiate the usefulness of the refined Kozeny-Carman equation proposed for the prediction of permeabilities in clays. The credibility of the equation is further enhanced by the wide range of porosities ( 0.42 to 0.93 ) covered by the model validations. Moreover, according to Eq. (5.6), permeability should be linearly related to the porosity function $\mathrm{n}^{4} /(1-n)^{3}$ for clay samples having water content greater than $w_{p}$ (plastic limit). Such a relationship is demonstrated in Fig. 5.5.

\subsubsection{Discussion}

The refined Kozeny-Carman equation can predict permeabilities of Wyoming sodium bentonite mixed and permeated with distilled water. The equation is believed to be able to handle other fine-grained soils and situations of different pore water chemistry. This deduction is based on three reasons: (1) the refined equation can account for the common parabolalike discrepancies observed between measured and predicted (from the Kozeny-Carman Equation) permeabilities in different clays (Figs. 5.1 and 5.4), (2) the specific surface and the plastic limit change with materials, therefore implicitly account for the material type, (3) changes in pore water chemistry should result in different values of the plastic limit, as they do for the liquid limit (Borgesson et al., 1988); the suggested water content ratios may still be able to account for the effects of changes in the pore geometry.

The same liquid as used for the permeation should be employed in determining the liquid limit. Further investigations are recommended.

\subsection{Prediction of Swelling Pressure}

\subsubsection{Introduction}

Montmorillonite (the predominant mineral in bentonites) has an unbalanced electronic charge in its crystal structure (Mitchel1, 1976, pp. $33,38)$. The unbalanced charge can be satisfied by cations, hydrated cations and polar fluids (e.g. water). As water is adsorbed between successive sheets of montmorillonite crystals, the crystal lattices separate from one another with a resulting expansion or swelling. If swelling is restrained, pressure will build up.

The swelling pressure generated by bentonites (as hydraulic barriers) should prove useful since it can provide good clay-rock contacts. More- 
Table 5.2 Measured and Predicted Permeabilities of Bentonites (C/S granular)

\begin{tabular}{|c|c|c|c|c|c|c|}
\hline $\begin{array}{ll}\text { Sample Por } & \text { Pomber ty } \\
\text { Numb }\end{array}$ & $\begin{array}{l}\text { orosi- } \\
y(n)\end{array}$ & $\begin{array}{l}\text { Water } \\
\text { Content }\end{array}$ & $\begin{array}{l}\text { Hydr. } \\
\text { Grad. }\end{array}$ & $\begin{array}{l}\text { Measured } \\
\mathrm{k}_{1}(\mathrm{~cm} / \mathrm{s})\end{array}$ & $\begin{array}{l}\text { Predicted } \\
\mathrm{K}_{2}(\mathrm{~cm} / \mathrm{s})\end{array}$ & $\begin{array}{r}\text { Ratio } \\
\left(\mathrm{K}_{1} / \mathrm{K}_{2}\right)\end{array}$ \\
\hline$B-S-1-A$ & 0.933 & 475.8 & $<10$ & $7.0 * 10^{-8}$ & $6.15 * 10^{-8}$ & 1.14 \\
\hline$B-C-1-A$ & 0.879 & 249.7 & $<12$ & $1.2 * 10^{-8}$ & $8.39 \div 10^{-9}$ & 1.43 \\
\hline$B-C-1-B$ & 0.880 & 251.5 & $<12$ & $1.3 * 10^{-8}$ & $8.57 * 10^{-9}$ & 1.52 \\
\hline$B-C-2-A$ & 0.831 & 168.1 & $<12$ & $2.5 * 10^{-9}$ & $2.44 \div 10^{-9}$ & 1.02 \\
\hline$B-C-2-B$ & 0.836 & 175.0 & $<12$ & $3.7 \div 10^{-9}$ & $2.74 \div 10^{-9}$ & 1.35 \\
\hline$B-C-4-A$ & 0.846 & 188.5 & $<12$ & $3.3 * 10^{-9}$ & $9.21 \div 10^{-10}$ & 3.58 \\
\hline$B-C-4-B$ & 0.848 & 191.7 & $<12$ & $3.5 * 10^{-9}$ & $9.57 * 10^{-10}$ & 3.66 \\
\hline$B-C-1-A-S$ & 0.625 & 57.1 & $<57$ & $9.0 \times 10^{-11}$ & $6.23 \div 10^{-11}$ & 1.44 \\
\hline $\begin{array}{l}B-C-1 \quad 3 / 8 \\
-A-S\end{array}$ & 0.721 & 88.3 & $<77$ & $2.2 * 10^{-9}$ & $1.73 * 10^{-9}$ & 1.27 \\
\hline $\begin{array}{l}B-C-2 \quad 3 / 8 \\
-A-S\end{array}$ & 0.638 & 60.3 & $<50$ & $1.1 \div 10^{-10}$ & $8.58 * 10^{-11}$ & 1.25 \\
\hline$B-C-4-A-S$ & 0.644 & 62.1 & $<57$ & $3.0 \times 10^{-10}$ & $9.39 * 10^{-11}$ & 3.19 \\
\hline
\end{tabular}

Note: Predicted permeabilities were obtained from Eq. (5.3), using $G=2.92, w_{p}=50, S_{o}=800 \mathrm{~m}^{2} / g$. 
Table 5.3 Measured and Predicted Permeabilities of Bentonite (MX-80)

\begin{tabular}{llllll}
$\begin{array}{l}\text { Saturated } \\
\text { Density } \\
(\mathrm{g} / \mathrm{cc})\end{array}$ & $\begin{array}{l}\text { Porosi- } \\
\text { ty }(\mathrm{n})\end{array}$ & $\begin{array}{c}\text { Water } \\
\text { Content }\end{array}$ & $\begin{array}{l}\text { Measured } \\
\mathrm{K}_{1}(\mathrm{~cm} / \mathrm{s})\end{array}$ & $\begin{array}{c}\text { Predicted }^{2} \\
\mathrm{~K}_{2}(\mathrm{~cm} / \mathrm{s})\end{array}$ & $\begin{array}{c}\text { Ratio } \\
\left(\mathrm{K}_{1} / \mathrm{K}_{2}\right)\end{array}$ \\
\hline 2.1 & 0.421 & 25.08 & $3.0 * 10^{-12}$ & $2.90 * 10^{-12}$ & 1.03 \\
1.9 & 0.526 & 38.31 & $1.9 * 10^{-11}$ & $2.36 * 10^{-11}$ & 0.80 \\
1.7 & 0.631 & 59.10 & $2.0 * 10^{-10}$ & $6.75 \div 10^{-11}$ & 2.96 \\
1.57 & 0.7 & 80.45 & $6.0 * 10^{-10}$ & $1.60 * 10^{-10}$ & 3.75 \\
1.295 & 0.845 & 187.62 & $3.8 * 10^{-9}$ & $2.90 * 10^{-9}$ & 1.56 \\
\hline
\end{tabular}

1. From SKB Report 88-30, Fig. 4.2; $G=2.9, w_{p}=70$ (Borgesson et al., 1988).

2. Calculated from Eqs. (5.3) and (5.4) with $S_{0}=800 \mathrm{~m}^{2} / g$.

Reproduced with permission from L. Borgesson, et al., "Rheological Properties of Sodium Smectite Clay," SKG Technical Report 88-30, Swedish Nuclear Fuel and Waste Management Co., Stockholm, Sweden, Figure 4.2, p. 21, Dec. 1988. 


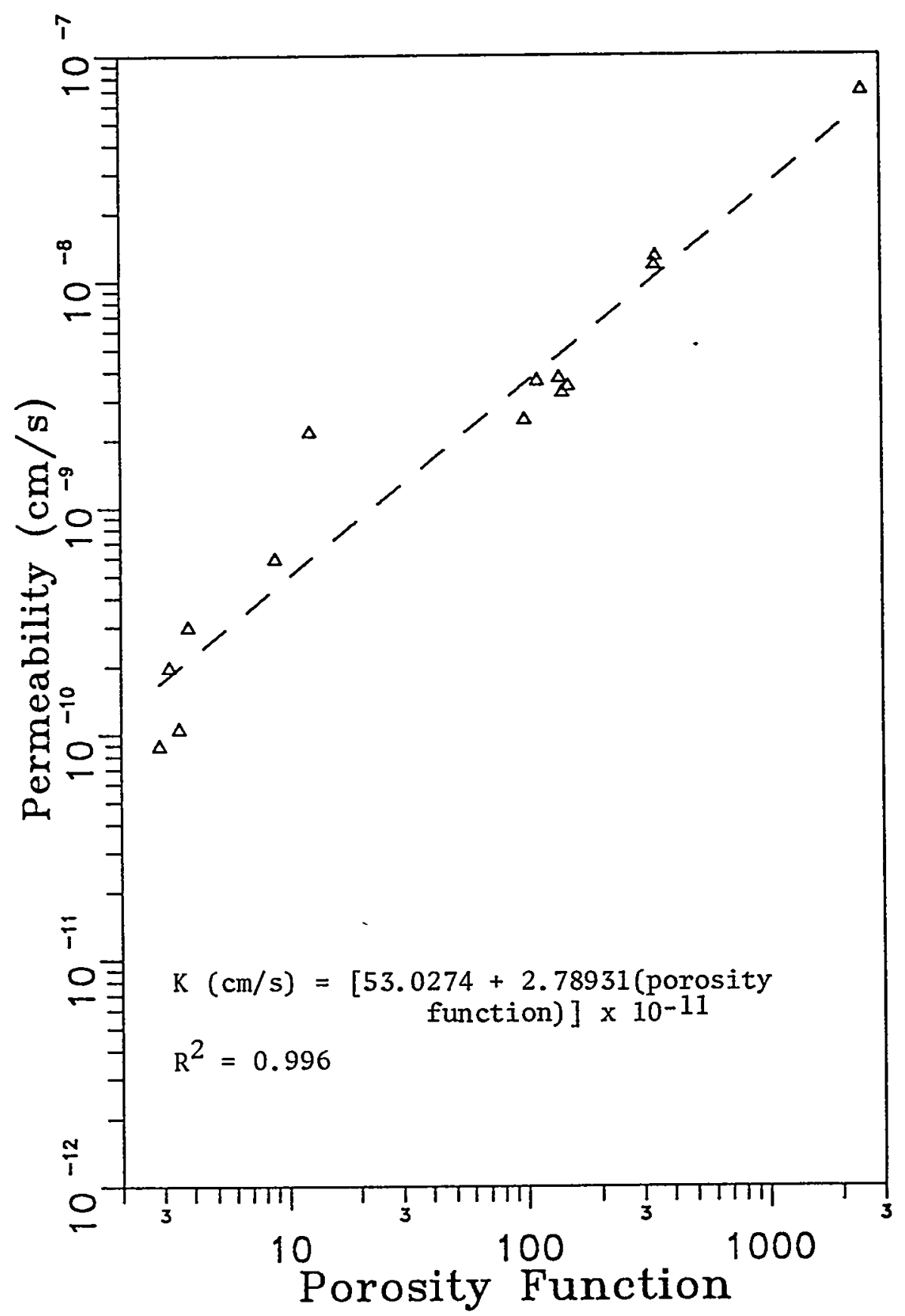

Figure 5.5 Linear relationship between the permeability of bentonite and the porosity function $n^{4} /(1-n)^{3}$. 
over, air voids in the barriers and rock fractures in the host rock could be filled by the expanding bentonites (Pusch, 1978, 1982). Conversely, if the swelling pressure is too high, unfavorably oriented fractures and joints may be propagated, which may have a deleterious effect on the sealing performance and even on the stability of the host formation (e.g. Fyfe et al., 1984), and on radionuclide isolation (Neretnieks, 1987), at least locally. This would be particularly true in locations with either original or induced highly anisotropic stressfields (Sawyer and Daemen, 1987, Section 6.3.1; Daemen et al., 1983). The swelling pressure therefore, is important to the evaluation of sealing performance, and to the design of waste isolation facilities.

The swelling of bentonites can be explained best by the Gouy-Chapman diffuse double layer theory (Bolt, 1956; Warkentin et al., 1957;

Warkentin and Schofield, 1962). Discussed below are models available for the prediction of swelling pressure. Predicted swelling pressures from some of these models have been compared with measured ones for bentonite samples mixed and permeated with distilled water.

\subsubsection{Bolt's Model}

Based on the double layer theory and the Van 't Hoff equation, Bolt (1956) proposed a method to calculate the swelling pressure. The calculation consists of three steps (Eqs. 5.8 to 5.10):

$$
\begin{gathered}
\left(B C_{0}\right)^{1 / 2}\left(X_{0}+d\right)=2\left(\frac{C_{0}}{C_{c}}\right)^{1 / 2^{\pi / 2}} \int_{\phi-0} \frac{d \phi}{\left[1-\left(\frac{C_{0}}{C_{c}}\right)^{2} \sin ^{2} \phi\right]^{1 / 2}} \\
P_{s}-R T C_{0}\left(\frac{C_{c}}{C_{0}}+\frac{C_{0}}{C_{c}}-2\right)
\end{gathered}
$$

where $P_{s}=$ swelling pressure in atmosphere (approximately $0.1 \mathrm{MPa}$ for 1 atm.)

$\mathrm{R}$ - gas constant, $8.314 \times 10^{7}$ ergs $/ \mathrm{mole}^{\circ} \mathrm{K}$

$\mathrm{T}$ - absolute temperature $\left({ }^{0} \mathrm{~K}\right)$

$c_{0}$ - concentration of the bulk liquid (mmole $/ \mathrm{cm}^{3}$ )

$C_{c}=$ concentration of cations midway between two clay plates (mole $/ \mathrm{cm}^{3}$ )

$\mathrm{V}$ - valence of the exchangeable cation

$\mathrm{B}=8 \pi \mathrm{F} / 1000 \mathrm{DRT}$, approximately $1 \times 10^{15} \mathrm{~cm} / \mathrm{mmole}$ at $20^{\circ} \mathrm{C}, \mathrm{F}$ is Faraday's constant and $D$ the dielectric constant of the pore fluid

$\mathrm{X}_{0}-4 / \mathrm{vBI}$ in $\mathrm{cm}$, where $\mathrm{I}=$ surface charge density of the clay; generally $0.1 / \mathrm{v} \mathrm{nm}$ for illite, $0.2 / \mathrm{v} \mathrm{nm}$ for kaolinite and $0.4 / \mathrm{v} \mathrm{nm}$ for montmorillonite 
$\mathrm{d}=$ half-distance between two clay plates (angstroms)

$\phi=a$ variable related to $\mathrm{C}_{c}$.

For saturated clays and assuming the clay plates are parallel, $d$ can be approximated using the equation:

$$
e=G_{c} \gamma_{w} S_{0} d
$$

where $e=$ void ratio

$\mathrm{S}_{0}=$ specific surface of the clay $\left(\mathrm{cm}^{2} / \mathrm{g}\right)$

$\mathrm{G}_{c}=$ specific gravity of the clay

$\boldsymbol{\gamma}_{\mathrm{w}}=$ density of water.

Substituting $x_{0}=4 \times 10^{-8} \mathrm{~cm}$ ( 4 angstroms) and $s_{0}=8 \times 10^{6} \mathrm{~cm}^{2} / \mathrm{g}$, Bolt calculated theoretical swelling pressures for Wyoming bentonite and compared them with experimental results. Although the calculated swelling pressures deviate from the measured values, a parallel trend can be observed. Bolt attributed the deviations to structural effects such as a "dead volume" resulting from the terraced nature of the clay surface and to the possible retention of salt by the cellophane membrane used in the experiments. Use of the approximate value of $\mathrm{x}_{0}$ may also lead to deviations, as suggested by the work of Sridharan and Jayadeva (1982).

Bolt's work clearly indicates the potential of using double layer theory and the Van't Hoff equation for the prediction of the swelling pressures. The application of this model is limited because of the difficulties in evaluating Eq. (5.8).

\subsubsection{Yong and Warkentin's Model}

Yong and Warkentin (1975) have proposed a simpler model for the prediction of swelling pressures. For monovalent ions, the swelling pressure in $\mathrm{kg} / \mathrm{cm}^{2}\left(1 \mathrm{~kg} / \mathrm{cm}^{2}=98.07 \mathrm{kPa}\right)$ is:

$$
P_{s}=R T\left(C_{c}-2 C_{0}\right)
$$

and

$$
C_{c}=\frac{T^{2}}{V^{2} B\left(d+X_{o}\right)^{2} 10^{-16}}
$$

where $C_{c}=$ concentration of cations midway between two clay plates (moles/liter)

$C_{0}=$ concentration of the bulk liquid (moles/liter)

$\mathrm{X}_{0}=4 / \mathrm{vBI}$ in angstroms, where $\mathrm{I}$ - surface charge density of the clay; generally $1 / \mathrm{v}$ A for illite, $2 / \mathrm{v}$ A for kaolinite and $4 / \mathrm{v}$ A for montmorillonite

$\mathrm{d}=$ half-distance between two clay plates (angstroms) 
$\mathrm{B}-8 \pi \mathrm{F} / 1000 \mathrm{DRT}$, approximately $1 \times 10^{15} \mathrm{~cm} / \mathrm{mmole}$ at $20^{\circ} \mathrm{C}$, $F$ is Faraday's constant and $D$ the dielectric constant of pore fluid

$\mathrm{R}$ - gas constant, $0.0848 \mathrm{~kg} \times 1$ iter $/ \mathrm{cm}^{2}{ }_{\mathrm{K}}$.

The other terms are as defined before.

The value of $d$ (in angstroms) can be estimated using the relation:

$$
w=0.01 s_{0} d
$$

for water content $(w)$ expressed in percent and specific surface $\left(S_{0}\right)$ in $\mathrm{m}^{2} / \mathrm{g}$.

According to Warkentin and Schofield (1962), these calculations adequately predict measured swelling pressures for the high-swelling sodium montmorillonite at low salt concentrations. At higher salt concentrations the measured pressures exceed calculated values. They note that the deviations may be due to the errors in using concentrations rather than activities of the exchangeable cations, and to neglecting the tactoid structure of the clay.

In the presence of deionized water, Eq. (5.11) can be simplified as:

$$
P_{s}=R T C_{C}
$$

For saturated clays, water content can be expressed in terms of dry clay density $\left(\gamma_{c}\right)$ and specific gravity $\left(G_{c}\right)$ :

$$
w=100\left(\frac{1}{\gamma_{c}}-\frac{1}{G_{c}}\right)
$$

For $T=293^{\circ} \mathrm{K}\left(20^{\circ} \mathrm{C}\right), v=1, B=10^{15} \mathrm{~cm} / \mathrm{mmole}, G_{c}=2.75$, and $X_{0}=4 \mathrm{~A}$, Gray et al. (1985) obtain the following relation:

$$
P_{s}=\frac{241}{\left[\frac{10^{4}}{S_{0}}\left(\frac{1}{\gamma_{c}}-0.364\right)\right]^{2}}
$$

for $P_{z}$ in $\mathrm{Mpa}, S_{0}$ in $\mathrm{m}^{2} / \mathrm{g}$, and $\gamma_{c}$ in $\mathrm{g} / \mathrm{cc}$. Compared with the experimental results, they suggest a correction factor of $1 / 3$ for Eq. (5.16) for an effective clay dry density up to $1.7 \mathrm{~g} / \mathrm{cm}^{3}$.

Following the same approach and taking the correction factor into account, a more general form of Eq. (5.16) is proposed in this study: $\mathrm{x}_{0}=4 \mathrm{~A}$ for montmorillonite. 


$$
P_{s}=\frac{80.13}{\left[\frac{10^{4}}{S_{0}}\left(\frac{1}{\gamma_{c}}-\frac{1}{G_{c}}\right)+X_{0}\right]^{2}}
$$

\subsubsection{Sridharan-Jayadeva's Model}

Sridharan and Jayadeva (1982) carried out a detailed study on the relation between double layer theory and compressibility of clays. Their work suggests that $\mathrm{X}_{0}$ depends not only on clay and fluid properties but also on the midplane potential, which is a function of $d$ (halfdistance between two clay plates) or e (void ratio). The use of an approximate value of $\mathrm{x}_{0}$ in calculating swelling pressure may lead to large errors. They further indicate that the $e-\log \left(P_{s}\right)$ relationship is influenced significantly by clay type, while the $d-\log \left(P_{s}\right)$ relationship is essentially unaffected by clay type. They propose the following equation for predicting swelling pressure:

$$
\log (d)-f-g * \log \left(P_{s}\right)
$$

where $g=0.5263$

$$
f=-2.7286+0.0263 * \log \left(C_{0}\right)+0.5263 * \log \left[\mathrm{T}(\mathrm{DT})^{0.95}\right]-\log (v)
$$

for $P_{\mathrm{g}}$ in $\mathrm{kg} / \mathrm{cm}^{2}, C_{o}$ in molarity, and $\mathrm{d}$ in $\mathrm{nm}$. Other terms are as defined earlier. The half-distance, $d$, can be obtained from Eq. (5.13) with known water content and specific surface of the clay.

For $C_{0}=10^{-4} \mathrm{M}, T=293 \mathrm{~K}, \mathrm{D}=80.36$, and $\mathrm{v}=1$, the value of $\mathrm{f}$ is 0.65 . Therefore, for low concentrations, Eq. (5.18) becomes:

$$
\log (d)=0.65-0.5236 * \log \left(P_{s}\right)
$$

\subsubsection{Validation}

The swelling pressures of four compacted bentonite samples (C/S granular) have been measured using the Soil Test FHA Volume Change meter. This device is essentially a frame with a displacement-reading dial gage attached to a proving ring. A bentonite sample is compacted in a circular, stainless steel ring and loaded into the swell meter. Porous stones are placed on the top and bottom of the compacted bentonite. The proving ring is placed in contact with the bentonite through an aluminum seat atop the upper porous stone. Distilled water is poured into a plastic container surrounding the bentonite/steel ring. Water is adsorbed into bentonite through holes in the steel ring and the porous stones. Table 5.4 summarizes the results. 
Table 5.4 Summary of Swelling Test Results

Sample Number

\begin{tabular}{cccc}
\multicolumn{4}{c}{ Sample Number } \\
\hline 非 1 & 非 2 & 非 &
\end{tabular}

\begin{tabular}{lllll}
$\begin{array}{l}\text { Initial water } \\
\text { content (\%) }\end{array}$ & 30.52 & 17.82 & 40.5 & 19.26 \\
$\begin{array}{l}\text { Initial dry } \\
\text { density (g/cc) }\end{array}$ & 1.153 & 1.210 & 1.031 & 1.362 \\
$\begin{array}{l}\text { Calc. Final dry } \\
\text { density (g/cc) }\end{array}$ & 1.145 & 1.204 & 1.026 & 1.346 \\
$\begin{array}{l}\text { Expected water } \\
\text { content (\%) } \\
\text { at saturation }\end{array}$ & 53.10 & 48.84 & 63.21 & 40.00 \\
$\begin{array}{l}\text { Calc. final } \\
\text { porosity }\end{array}$ & 0.608 & 0.588 & 0.649 & 0.539 \\
$\begin{array}{l}\text { Max. swel1ing } \\
\text { pressure (MPa) }\end{array}$ & 0.71 & 0.62 & 0.52 & 1.17 \\
$\begin{array}{l}\text { Water content } \\
\text { (top 1/3 sample) }\end{array}$ & 43.31 & 50.00 & 57.74 & 39.34 \\
$\begin{array}{l}\text { Water content } \\
\text { (middle 1/3) }\end{array}$ & 40.96 & 45.02 & 46.88 & 37.68 \\
$\begin{array}{l}\text { Water content } \\
\text { (lower 1/3) } \\
\text { - }\end{array}$ & 41.87 & 45.60 & 47.96 & 38.83 \\
\hline
\end{tabular}


As shown in Table 5.4, the bentonite samples might not be saturated when the maximum swelling pressures are recorded. However, the middle portion of the samples should be very close to saturation, due to the compression resulting from the swelling of the upper and lower portions. The water contents measured from the middle 1/3 of the samples have been used to calculate d (using Eq. 5.13). Predicted swelling pressures are obtained from Eq. (5.19). The final dry density (Table 5.4) is used in Eq. (5.17). The specific gravity and specific surface of the bentonite are 2.92 and $800 \mathrm{~m}^{2} / \mathrm{g}$, respectively. Measured and predicted swelling pressures are shown in Table 5.5.

Swelling pressures calculated from Eq. (5.17) agree well with measured ones. Note that a correction factor of $1 / 3$ has been included in the derivation of Eq. (5.17). This correction supports the work by Gray et a1. (1985). Predicted swelling pressures from Eq. (5.19) exceed the measured ones by 7 to 9 times. Considering the effects of the cluster or tactoid clay structure and assuming an average of 3 clay sheets per cluster, the effective specific surface becomes: $800 / 3=266.67 \mathrm{~m}^{2} / \mathrm{g}$. The reduced surface leads to higher $d$ values than the previous d values by a factor of 3 . This adjustment results in a good agreement between measured and predicted swelling pressures.

\section{2 .6 Discussion}

The swelling behavior of clays can be accounted for reasonably well by the electric diffuse double layer theory. The assumption of parallel clay plates in the theory, however, appears to deviate from what exists in natural fine-grained soils. The influence of clay structure probably is responsible for the discrepancies between measured and predicted swelling pressures.

For pore water of low salt concentrations $(<0.0001 \mathrm{M})$, Eq. (5.17), from Yong and Warkentin (1975) and Gray et al. (1985), can be used for the prediction of swelling pressure of clays for dry clay densities up to $1.7 \mathrm{~g} / \mathrm{cm}^{3}$. Eq. (5.19) also yields good predictions but requires finding an appropriate value of the effective specific surface.

According to the results presented by Gray et al. (1985), the swelling pressures of clays having dry densities larger than $1.7 \mathrm{~g} / \mathrm{cm}^{3}$ may be predicted from Eq. (5.16), or from Eq. (5.17) without correction. It is speculated that Eq. (5.19) can also be used for the same purpose, as the cluster structure may have been destroyed at the high density conditions. 
Table 5.5 Measured and Predicted Swelling Pressures of Bentonite

\begin{tabular}{|c|c|c|c|c|}
\hline \multirow[b]{2}{*}{$\begin{array}{l}\text { Sample } \\
\text { number }\end{array}$} & \multirow{2}{*}{$\begin{array}{l}\text { Measured } \\
\text { swelling pressure } \\
\text { (MPa) }\end{array}$} & \multicolumn{3}{|c|}{ Predicted swelling pressure } \\
\hline & & $\begin{array}{c}\mathrm{Eq} \cdot(5.17) \\
(\mathrm{MPa})\end{array}$ & $\begin{array}{c}\mathrm{Eq} \cdot(5.19) \\
\text { (MPa) }\end{array}$ & $\begin{array}{c}\mathrm{Eq} \cdot(5.19)^{*} \\
(\mathrm{MPa})\end{array}$ \\
\hline & , & & & \\
\hline 非 1 & 0.71 & 0.71 & 5.27 & 0.74 \\
\hline 非 2 & 0.62 & 0.78 & 5.02 & 0.62 \\
\hline 非 3 & 0.52 & 0.56 & 4.65 & 0.58 \\
\hline 非 4 & 1.17 & 0.99 & 7.04 & 0.87 \\
\hline
\end{tabular}

* Based on the adjusted specific surface of $266.67 \mathrm{~m}^{2} / \mathrm{g}$, assuming three clay sheets per cluster. 
CHAPTER SIX

\section{SUMMARY, CONCLUSIONS, AND RECOMMENDATIONS}

Bentonite is an excellent sealant material due to its swelling and selfhealing characteristics, low permeability, sorptive qualities, and longevity in nature. The use of bentonite in constructing hydraulic barriers has greatly increased in recent years, particularly for liquid and solid waste containment. Bentonite and bentonite/crushed rock plugs are being proposed for sealing underground nuclear waste repositories. The sealing performance of such plugs under diverse conditions needs to be studied to allow for overall repository performance assessments.

American Colloid C/S granular bentonite and Apache Leap tuff have been used to prepare samples for flow testing. Bentonite content and crushed tuff gradation are the major variables in sample composition. Material characterization and properties of the bentonite and tuff are described in Chapter 2. The sealing performance assessments include high injection pressure flow tests, polyaxial flow tests, high temperature flow tests, and piping tests. Analytical work includes the introduction of bentonite occupancy percentage and water content at saturation as two primary parameters for plug design. A piping model is developed to evaluate the susceptibility of the seals to piping as a result of bentonite flow. The permeability model proposed allows for the prediction of permeability in clays.

\subsection{Summary of Results}

\subsubsection{Bentonite Plugs}

Flow test results of the sedimented bentonite plugs $(2.54 \mathrm{~cm}$ in diameter) indicate the dependence of permeability on the molding water and permeant. The plug deposited in and tested with 28 sodium pyrophosphate solution (a dispersing agent) yields a permeability of $1.4 \times 10^{-8} \mathrm{~cm} / \mathrm{s}$, compared to $6.9 \times 10^{-8} \mathrm{~cm} / \mathrm{s}$ for the plug sedimented in and permeated with deaired distilled water. After having been flushed with the dispersing solution, the permeability of the latter plug reduces to 2 to $3 \times 10^{-8} \mathrm{~cm} / \mathrm{s}$. The same bentonite, when dropped in a 28 calcium hydroxide suspension (a flocculent agent), and later tested with distilled water, gives a permeability of $10^{-5} \mathrm{~cm} / \mathrm{s}$. Subsequently, this sample has been flushed with a 48 sodium pyrophosphate solution for about two and a half months, and the permeability decreases to $3.4 \times 10^{-7} \mathrm{~cm} / \mathrm{s}$. The sample sedimented in the water previously boiled in the presence of tuff aggregates has a permeability of $7.7 \times 10^{-8} \mathrm{~cm} / \mathrm{s}$ when permeated with the synthetic water and of $2 \times 10^{-8} \mathrm{~cm} / \mathrm{s}$ with a 28 dispersing solution.

Permeabilities of compacted bentonite plugs with diameters from 2.54 to $10.16 \mathrm{~cm}$ appear invariant with size. For samples of the same diameter and similar bulk density, permeability values vary by no more than a factor of three. Water contents of bentonite Sample B-C-4-B have been 
determined at different depths along the sample, and indicate a nonuniform distribution. The water content is high at the inflow end and low at the outflow end. High injection pressure flow tests of four compacted bentonite samples installed in stainless steel permeameters give permeabilities from $2 \times 10^{-9}$ to $5 \times 10^{-11} \mathrm{~cm} / \mathrm{s}$. The saturated bulk densities of the four samples vary from 1.54 to $1.72 \mathrm{~g} / \mathrm{cm}^{3}$. The permeabilities decrease with increasing hydraulic gradient.

The permeability to air of compacted $2.54 \mathrm{~cm}$ diameter bentonite samples ranges from $10^{-11}$ to $10^{-17} \mathrm{~m}^{2}$ (10 darcy to 0.01 milidarcy), depending upon water content and dry density. The water content has a large influence on the permeability to air. The permeability may increase due to pore enlargement resulting from loss of moisture. To minimize the migration of gaseous radionuclides, highly compacted bentonite plugs at low water content are recommended.

The modification made to the Kozeny-Carman equation includes a correction factor to account for the microstructural changes in clays, corresponding to the changes in water content. The selection of water content ratios $w$ relative to the plastic limit $w_{p}\left(w / w_{p}\right.$ or $\left.w_{p} / w\right)$ as the correction factor is based on referenced phenomenological studies on microstructures of clays. The influence of water content on engineering properties of clays, reported in the literature, is used as supplemental support for the selection. The correction factor serves effectively as a collective parameter to represent complex interactions of an electrolyte-clay system. Predicted bentonite permeabilities from the refined Kozeny-Carman equation agree to within from 2 to 738 with the experimental results over a wide range of void ratios (Tables 5.2 and 5.3 ).

Swelling pressures of bentonite measured with a volume change meter are used to check several published swelling pressure models. A generalized form of the Yong and Warkentin (1975) model has been derived for low salt concentrations with a correction factor of $1 / 3$ as suggested by Gray et al. (1985). The generalized equation (5.17) gives predictions to within 0 to 268 from the measured swelling pressures. Swelling pressures calculated from the Sridharan-Jayadeva model (Eq. 5.19) are approximately 8 times higher than the experimental results. Assuming a microstructure of three clay sheets per cluster and consequently reducing the specific surface of montmorillonite $\left(800 \mathrm{~m}^{2} / \mathrm{g}\right)$ three fold, Eq. (5.19) yields predictions comparable to the experimental results. The difference between predicted and measured swelling pressures also varies from 0 to 268 .

\subsubsection{Bentonite/Crushed Tuff Plugs}

The permeability of bentonite/crushed tuff plugs decreases with increasing bentonite content. The sealing performance of the samples containing 158 bentonite by weight is erratic. Piping, erosion, and channeling have been observed for Samples B/AL-C-4-15/A and B/AL-C-4-15/C during the falling head flow testing. The samples containing $15 \%$ bentonite mixed with type $B$ or type FA crushed tuff yield permeabilities in the upper $10^{-8}$ and in the middle $10^{-7} \mathrm{~cm} / \mathrm{s}$ range, respectively. 
The permeabilities of the plugs containing 25 or 358 bentonite are close to the permeability of plugs constructed of bentonite only. Samples $B / A L-C-4-25 / A, B / A L-C-4-35 / A, B / A L-C-4-35 / B$, and B/AL-C-4-35/C have been tested for more than 9 months, and subjected to various injection pressures up to $1 \mathrm{MPa}$ ( $145 \mathrm{psi}, 102 \mathrm{~m}$ water head; induced hydraulic gradients: 900 to 1000). Although depositions of dispersed or eroded bentonite have been observed in the outflow tubing, no deterioration of sealing ability has been detected. Piping has developed in Sample B/ALC-4-25/B under hydraulic gradients over 400 .

The effect of crushed tuff gradation on the sealing performance shows that the greater the uniformity coefficient $\left(d_{60} / d_{10}\right)(e . g$. types $F A$ and A), the lower the permeability. Samples containing 258 bentonite and mixed with type $A\left(C_{u}=16.5\right)$ or type FA (a theoretical $C_{u}=36$ ) crushed tuff, however, give similar permeabilities for low hydraulic gradients. Type FA contains fewer large particles and more small particles than type A, including 8.86 weight percent of particles smaller than $0.074 \mathrm{~mm}$ (U.S. mesh \#200). Nevertheless, the permeability decrease with increasing gradient is less and slower for samples constructed with crushed tuff of the FA gradation. In general, an appropriate composition for the mixture plugs to yield permeabilities lower than $5 \times 10^{-8} \mathrm{~cm} / \mathrm{s}$ would contain at least $25 \%$ bentonite by weight mixed with well-graded crushed rock.

The effect of sample size on the sealing performance is not clear. The inconsistency in the permeabilities measured for different plug sizes appears to be due more to variations in the stiffness of the permeameters, compaction, and the ratio of grain size to permeameter diameter. For the normally consolidated mixture samples, the upward permeability is about three times higher than the downward permeability, suggesting an effect of the upward seepage forces on the sealing performance. Such an effect is insignificant for the overconsolidated samples.

Bentonite content and compaction are important in constructing good seals. Samples B/AL-C-4-15/A and B/AL-C-4-15/C, in which piping occurs, have an occupancy percentage of bentonite lower than $50 z$ and a water content of bentonite at saturation in the vicinity of $200 \%$. In these two cases, piping likely has occurred in preferential passageways that originally exist in the samples. For mixtures consisting of $25 \%$ and $35 \%$ bentonite, the occupancy percentage of bentonite improves to 65 to 808 and to 75 to 86.58 , respectively. No piping has been observed in these samples except for Sample B/AL-C-4-25/B. For a loosely or ineffectively compacted mixture containing 258 bentonite (Sample B/AL-C-8-25/FC-S), the sealing performance can be damaged by dynamic disturbances. The influence of such disturbances is greatly reduced when more bentonite is added (e.g. Sample B/AL-C-8-35/FC-S).

Based on the high injection pressure flow test results, the potential for piping damage to the sealing performance is small if the maximum hydraulic gradient does not exceed approximately 120 and 280 for mixture samples containing 25 and 358 bentonite by weight, respectively. The piping test results of Sample B/AL-C-4-25/A-P-B (Section 3.4.4.1) tend to support this conclusion. Bentonite is found to flow between crushed tuff particles when higher hydraulic gradients are imposed. The piping 
model developed in this study, based on plastic flow theory, provides a means to evaluate the critical hydraulic gradient at which bentonite starts to flow. Input parameters for this model are the representative pore diameter of a crushed rock system and the yield stress of bentonite (depending upon its water content). This model has been validated with results from the high injection pressure flow tests and from the piping tests. The ratio between predicted and experimental critical hydraulic gradients varies from 1.11 to 2.12 .

The concept of yield stress and critical pressure gradient has been extended to filter design. Filters with an effective pore diameter of $0.5 \mathrm{~mm}$ are necessary to prevent piping, erosion and flow of bentonite, for water contents from 50 to 3008 and hydraulic gradients no more than 1000. To prevent lateral migration of fine particles, which is possible if the sealants are in contact with open joints and/or fractures, discontinuities having apertures larger than $0.5 \mathrm{~mm}$ must be grouted.

Polyaxial flow test results indicate that a difference of up to one or two orders of magnitude may be expected between the vertical and horizontal permeabilities. The high horizontal permeability results from the uneven bentonite distribution in the pores between crushed rock grains due to particle segregation during sample installation and compaction. The segregation can be seen in Figure 3.108. Increasing the bentonite content from 25 to 358 reduces the vertical permeability by almost one order of magnitude (from $1.7 \times 10^{-8}$ to $2.5 \times 10^{-9} \mathrm{~cm} / \mathrm{s}$ ), but changes the relatively high horizontal permeability only slightly $\left(1.4\right.$ vs. $1.9 \times 10^{-7} \mathrm{~cm} / \mathrm{s}$ ). This observation indicates that increasing the bentonite content is likely to be an ineffective means to resolve the problem of particle segregation.

\section{$\underline{6.2}$ Conclusions}

Flow test results on the sedimented bentonite plugs indicate the dependence of permeability on the molding water and permeant. The bentonite sample deposited in and permeated with the synthetic groundwater gives a permeability $\left(7 \times 10^{-8} \mathrm{~cm} / \mathrm{s}\right)$ very similar to that of the sample prepared and tested with deaired distilled water. Permeability of bentonite can be reduced by molding or percolating with a dispersing solution (e.g. 28 sodium pyrophosphate solution). In view of the nonuniform water content distribution developed in bentonite, permeability calculated based on the assumption that the sample is uniform should be treated as some equivalent measure (for a system of layers) of a sample's ability to transmit water. To effectively minimize the migration of gaseous radionuclides, highly compacted bentonite plugs at low water content are recommended.

Mixtures of bentonite and crushed densely welded Apache Leap tuff can be engineered to yield a low permeability, close to that of bentonite itself. An appropriate composition for this purpose would contain at least 258 bentonite by weight mixed with well-graded crushed rock. A mixture containing 258 bentonite and 758 crushed tuff of type A (maximum particle size of $9.42 \mathrm{~mm}$ ) gradation appears to be a promising seal material. Limited flow test results suggest that crushed tuff of FA or FC gradations (Fuller-Thompson gradations, $n=0.5$ and $D_{\max }=9.42 \mathrm{~mm}$ 
and $19.05 \mathrm{~mm}$, respectively) may also be good candidates for mixing with bentonite. The sealing performance of mixture plugs is enhanced by increasing the amount of bentonite to 358. The increase in bentonite content improves the bentonite occupancy percentage and reduces the water content of bentonite at saturation, giving better resistance to piping, erosion and flow. Similar effects have been observed when crushed rock constituting a Fuller-Thompson grading curve (e.g. type FA with $\mathrm{n}=0.5$ ) is used.

Compaction and the amount of bentonite are decisive factors in producing good mixture seals. The effectiveness of compaction in reducing porosity is hindered by the soft bentonite buffer. To reduce the bulk porosities of the mixture plugs containing 258 or more bentonite by weight, a compaction energy higher than that of the standard Proctor compaction is necessary. For a loosely or ineffectively compacted mixture containing $25 \%$ bentonite or less, the sealing performance can be damaged by dynamic disturbances. The influence of such disturbances is greatly reduced when more bentonite is added.

Bentonite/crushed tuff mixtures tested in this study exhibit heterogeneity and anisotropy. A difference of up to one or two orders of magnitude can be expected between the vertical and horizontal permeabilities. The high horizontal permeability results from the uneven bentonite distribution in the pores between crushed rock particles due to particle segregation during installation and compaction. Moreover, the contact between adjacent compacted layers may serve as a preferential flow path. Increasing the bentonite content from 25 to $35 \%$ reduces the vertical permeability by nearly one order of magnitude but results in little change in the horizontal permeability. Adequate sealing ability of mixture plugs in the transverse direction may be necessary to minimize the possibility of flow of groundwater or gases laterally into a connected fracture system in a host rock formation. Compromising the sealing ability in the transverse direction ultimately may jeopardize the entire sealing performance if piping occurs laterally. This consideration can be significant if seals are installed at locations intercepted by joints and/or fractures.

Temperature has no negative effects on the sealing performance of bentonite/crushed tuff plugs over the test range from room temperature to $60^{\circ} \mathrm{C}$. The specific permeability reaches a maximum at $35^{\circ} \mathrm{C}$ and decreases with increasing temperature, indicating the effect of temperature on the structure of the samples. The decreases in the specific permeability are likely due to the thermal expansion of crushed tuff particles and the expansion of the diffuse double layer of bentonite. The structural change is reversible over the temperature range tested.

The possibility for piping to occur in passageways that may be created by the radial expansion of pores due to an increasing injection pressure is small, except for the mixture plugs with a low bentonite content (e.g. $15 \%$ by weight). The effect of pore expansion is believed to be counteracted by pore clogging resulting from the migration of fine particles. The fine particle migration is evidenced by the observation of bentonite flow between crushed tuff aggregates. The migration argument may be further supported by the breakdown of the linear 
relation between flow rate and hydraulic gradient observed in all high injection pressure flow tests. The breakdown is believed to indicate the onset of bentonite flow in the mixtures. For mixtures of type A crushed tuff with $25 \%$ or $35 \%$ bentonite, piping damage is small if the maximum hydraulic gradient does not exceed approximately 120 or 280 , respectively.

Piping can occur if bentonite is lost externally. The piping model developed in this study combines yield stress characteristics of bentonite and the flow of bentonite through capillaries. The model provides an analytical means to determine the critical pressure gradient at which bentonite of a given water content may start to flow. The concept of yield stress and critical pressure gradient has been extended to filter design. For bentonite with water content from 50 to 3008 and subjected to hydraulic gradients of no more than 1000 , filters of an effective pore diameter of no more than $0.5 \mathrm{~mm}$ are necessary to prevent piping, erosion and flow of bentonite. If the sealants are in contact with open joints and/or fractures, discontinuities having apertures larger than $0.5 \mathrm{~mm}$ must be grouted to minimize the risk of lateral migration of fine particles. The relation between yield stress of bentonite and its water content can also be used in the design of bentonite grouting.

The Kozeny-Carman equation has been reevaluated to improve the predictability of the saturated permeability in clays. The modification made to the Kozeny-Carman equation includes a correction factor to account for the microstructural changes in clays, responding to changes in water content. Permeability measurements of eleven bentonite samples obtained in this study, along with five measurements reported in the literature, are used to examine the validity of the model proposed. Predicted bentonite permeabilities from the refined Kozeny-Carman equation agree to within a factor of 0.8 to 3.75 with the experimental ones over a wide range of void ratios. The prediction is within 348 of the measured permeability for eleven out of sixteen samples.

Swelling pressures of bentonite can be predicted using the modified Yong and Warkentin model as well as the Sridharan-Jayadeva model for low salt concentrations. The former model gives predictions close to the measured swelling pressures, with variations ranging from 0 to 25.88 . Predicted swelling pressures from the Sridharan-Jayadeva's model are approximately 8 times higher than the experimental measurements. Assuming a microstructure of three clay sheets per cluster and consequently reducing the specific surface of montmorillonite $\left(800 \mathrm{~m}^{2} / \mathrm{g}\right)$ by a factor of three, the Sridharan-Jayadeva's model yields predictions comparable to the experimental results. The difference between predicted and measured swelling pressures varies from 0 to 25.68 .

\subsection{Recommendations}

Several recommendations for future studies can be drawn from this investigation: 
(1) High injection pressure flow tests and piping tests in the transverse direction are recommended to evaluate the consequences of the permeability anisotropy.

(2) If more homogeneous and isotropic bentonite/crushed tuff plugs are deemed desirable, methods are needed to minimize particle segregation and to assure $a$ uniform distribution of the bentonite. The permeability anisotropy may be reduced by introducing a layer of bentonite on top of each compacted layer. The crushed rock, during subsequent compaction, should carve into the bentonite layers above and below to tie together adjacent lifts. This method deserves further investigation.

(3) In this study, precautions have been taken to reduce particle segregation during sample installation, e.g. thoroughly mixed material is emplaced by scooping. The differences observed between the vertical and horizontal permeabilities therefore may only be minimal. Problems caused by particle segregation and uneven distribution of bentonite are expected to be more severe when the mixtures are used to seal small diameter and/or long boreholes. A sealing performance evaluation of small-diameter, long bentonite/crushed tuff plugs is warranted, particularly with regard to the influence of installation methods.

(4) The proposed permeability model adequately predicts permeabilities of Wyoming sodium bentonite mixed and permeated with distilled water. The model is believed to be applicable for other fine-grained clays and situations of different pore water chemistry. Different material type and pore water chemistry likely result in changes only in the specific surface and plastic limit. Further studies are recommended to verify the validity of these postulates.

(5) For bentonite molded with distilled water, the yield stress is expected to assume a minimum value due to the development of a dispersive microstructure. When the pore water chemistry changes, bentonite can have a flocculated structure and thus a higher yield stress. Studies of the influence of pore water chemistry on the yield stress of bentonite are recommended.

(6) The effect of bentonite loss into fractures on the sealing performance deserves further investigation. This effect may be evaluated by conducting flow tests on seals installed in permeameters with rectangular slits of carefully controlled dimensions. Such a test configuration is more representative of in-situ conditions than a circular opening in the wall of a permeameter. While experiments on samples or boreholes in rock containing fractures ultimately may be desirable to confirm any results and conclusions from simulations, experiments in real rock are likely to be complicated greatly by the complex geometry, aperture, and flow path distribution experienced during investigationts of fracture grouting (Sharpe and Daemen, 1991).

(7) Dynamic effects on the sealing performance of bentonite-based plugs should be studied, in view of the possible disturbances caused by earthquakes. For loosely emplaced mixture plugs containing 258 or less bentonite by weight, dynamic disturbances can lead to failure in the sealing ability of the plugs. 
(8) Throughout this report, the calculations assume that the seals are homogeneous. Frequent observations, particularly with regard to water content, indicate that the actual systems, after a longer or shorter test period, tend to become highly nonuniform. The changes result from water flow (and resulting water content redistribution), drying, or water injection, as well as from bentonite flow. From the mechanisms causing the changes and from the (small) number of measurements and observations reported here, it appears highly likely that changes in seal composition, and hence in associated properties, occur in very systematic patterns. It should be possible, therefore, to incorporate such variations within more realistic theoretical models, e.g. of bentonite flow or of water flow. The development of such models, more realistic descriptions of the physical behavior of the bentonite-water system than presently used averaged properties, would seem very desirable. A correct accounting of non-uniform behavior would seem particularly desirable for predictions of performance of seal systems over very long periods of time, i.e. periods over which even the very low flowrates that can be expected in bentonite may be sufficient to cause substantial differences in behavior and properties of different sections of seals.

(9) The (relatively small number of measurements of) bond strengths of seals constructed of crushed rock and bentonite and emplaced in boreholes in rock indicate that the bond strength of such seals is likely to be small (probably less than $100 \mathrm{kPa}$, or $10 \mathrm{psi}$ ). If seals of the type investigated here are to be subjected to significant axial loads, they probably should be confined axially (e.g. by filling the entire hole, or by supporting them with cementitious plugs). Further investigations are needed to determine long-term bond strengths, particularly as they are affected by water and by bentonite flow.

(10) Flow of bentonite in capillaries deserves further investigation. The macroscopic analysis presented here may oversimplify the flow patterns and mechanics. A detailed observation and description of bentonite flow in capillaries should assist. in identifying any major shortcomings in presently available flow models.. This, in turn, will allow the development of more complete models that account for all major aspects of bentonite flow through capillaries. Such models would be very desirable to predict long-term flow of bentonite, e.g. through pore spaces in crushed rock matrices, and through fractures in the host rock. 
. 


\section{REFERENCES}

Aisenstein, B., E. Diamant, and I. Saidoff, 1961, "Fat Clay as a Blanketing Material for Leaky Reservoirs," Proceedings of the 5th International Conference on Soil Mechanics and Foundation Engineering, Paris, Vol. II, Division 3B-7, pp. 523-529.

American Colloid Company, Data No. 202, Arlington Heights, I11inois.

Anderson, L.A., 1981, "Rock Property Analysis of Core Samples from the Yucca Mountain VE25a-1 Borehole, Nevada Test Site, Nevada," OpenFile Report 81-1338, prepared by the U.S. Geological Survey, for Nevada Operation Office, U.S. Department of Energy.

ASTM D698-78, "Standard Test Methods for Moisture-Density Relations of Soils and Soil-Aggregate Mixtures Using 5.5-lb (2.49-kg) Rammer and 12-in (305-mm) Drop," Annual Book of ASTM Standards, Section 4, Construction, Vol. 04.08, Soil and Rock; Building Stones, American Society for Testing and Materials, Philadelphia.

ASTM D854-83, "Standard Test Method for Specific Gravity of Soils," Annual Book of ASTM Standards, Section 4, Vol. 04.08, Soil and Rock; Building Stones, American Society for Testing and Materials, Philadelphia.

ASTM D2216-80, "Standard Method for Laboratory Determination of Water (Moisture) Content of Soil, Rock and Soil-Aggregate Mixtures," Annual Book of ASTM Standards, Section 4, Vol. 04.08, Soil and Rock; Building Stones, American Society for Testing and Materials, Philadelphia.

ASTM D2434-68, "Standard Test Method for Permeability of Granular Soils (Constant Head)," Annual Book of ASTM Standards, Section 4, Vol. 04.08, Soil and Rock; Building Stones, American Society for Testing and Materials, Philadelphia.

ASTM D4318-84, "Standard Test Method for Liquid Limit, Plastic Limit, and Plasticity Index of Soils," Annual Book of ASTM Standards, Section 4, Vol. 04.08, Soil and Rock; Building Stones, American Society for Testing and Materials, Philadelphia.

ASTM D4525-85, "Standard Test Method for Permeability of Rocks by Flowing Air," Annual Book of ASTM Standards, Section 4, Construction, Vol. 04.08, Soil and Rock; Building Stones, American Society for Testing and Materials, Philadelphia.

Aylmore, L.A.G. and J.P. Quirk, 1960, "Domain or Turbostratic Structure of Clays, " Nature, Vol. 187, p. 1046. 
Aylmore, L.A.G. and J.P. Quirk, 1967, "The Micropore Size Distributions of Clay Mineral Systems," Journal of Soil Science, Vol. 18, No. 1, pp. $1-17$.

Bingham, E.C., 1916, "An Investigation of the Laws of Plastic Flow," Scientific Paper No. 278, U.S. Bureau of Standards.

Binnal1, E.P., S.M. Benson, L. Tsao, H.A. Wollenberg, T.K. Tokunaga, and E.M. Didwa11, 1987, "Critical Parameters for a High-Level Waste Repository, Volume 2: Tuff," NUREG/CR-4161, U.S. Regulatory Commission, Washington, DC.

Bish, D.L., A.E. Ogard, D.T. Vaniman and L. Benson, 1984, "MineralogyPetrology and Groundwater Geochemistry of Yucca Mountain Tuffs," pp. 283-291, Scientific Basis for Nuclear Waste Management VII, Materials Research Society Symposia Proceedings, Vol. 26, G.L. McVay, ed., North-Holland, New York.

Blackmore, A.V. and R.D. Miller, 1962, "Tactoid Size and Osmotic Swelling in Calcium Montmorillonite," Proceedings, Soil Science Society of America, Vol. 25, pp. 169-173.

Bolt, G.H., 1956, "Physico-Chemical Analysis of the Compressibility of Pure Clays," Geotechnique, Vol. 6, pp. 86-93.

Bonne, A., J. Black, G. Gera, P. Gonze, E. Tassoni, and J.F. Thimus, 1985, "Characterization and Behaviour of Argillaceous Rocks," Radioactive Waste Management and Disposal, Proceedings of the Second European Community Conference, Luxembourg, April 22-26, R. Simon, ed., Commission of the European Communities, Directorate-General Science, Research and Development, Brussels, pp. 487-504.

Borgesson, L., H. Hokmark, and 0. Karnland, 1988, "Rheological Properties of Sodium Smectite Clay," SKB Technical Report 88-30, Swedish Nuclear Fuel and Waste Management Co., Stockholm, 65 pp.

Boyes, R.G.H., 1986, "Bentonite for Seepage Contro1," Civil Engineering (London), May, pp. 30-33.

Brace, W.F., J.B. Walsh, and W.T. Frangos, 1968, "Permeability of Granite Under High Pressure," Journal of Geophysical Research, Vol. 73, No. 6, pp. 2225-2236.

Brandenburg, U. and G. Lagaly, 1988, "Rheological Properties of Sodium Montmorillonite Dispersions," Applied Clay Science, Vol. 3, No. 3, pp. 263-279.

Buckingham, E., 1921, "On Plastic Flow Through Capillary Tubes," Proceedings of The American Society for Testing Materials, Vol. 24, 24th Annual Meeting, pp. 1154-1161.

Carman, P.C., 1937, "Fluid Flow Through Granular Beds," Transactions, Institute of Chemical Engineers, London, Vol. 15, pp. 150-166. 
Carman, P.C., 1939, "Permeability of Saturated Sands, Soils and Clays," Journal of Agricultural Science, Vol. XXIX, Part 2, pp. 262-273.

Casagrande, A., 1958, "Notes. on the Design of the Liquid Limit Device," Geotechnique, Vol. 8, No. 2, pp. 84-91.

Chapman, D.L., 1913, "A Contribution to the Theory of Electrocapillarity," Philosophical Magazine, Sixth Series, Vol. 25, No. CXLVIII, Pp. 475-481.

Childs, E.C. and N. Collis-George, 1950, "The Permeability of Porous Material," Proceedings of Royal Society, London, Vol. 201A, pp. $392-405$.

Collins, K. and A. McGown, 1974, "The Form and Function of Microfabric Features in a Variety of Natural Soils," Geotechnique, Vol. 24, No.2, pp. 223-254.

CRC Handbook of Chemistry and Physics, 1982, R.C. Weast, ed., CRC Press, Inc., Boca Raton, FL.

Daemen, J.J.K., J.C. Stormont, N.I. Colburn, D.L. South, S.A. Dischler, K. Fuenkajorn, W.B. Greer, G.S. Adisoma, D.E. Miles, B. Kousari, and J. Bertuca, 1983, "Rock Mass Sealing - Experimental Assessment of Borehole Plug Performance, Annual Report, June 1982 - May 1983," NUREG/CR-3473, prepared for U.S. Nuclear Regulatory Commission, by Department of Mining and Geological Engineering, University of Arizona, Tucson.

Daniel, D.E., 1984, "Predicting Hydraulic Conductivity of Clay Liners," Journal of Geotechnical Engineering, ASCE, Vol. 110, No. 2, pp. 285-300.

Day, S.R. and D.E. Danie1, 1985, "Hydraulic Conductivity of Two Prototype Clay Liners," Journal of Geotechnical Engineering, ASCE, Vol. 111, No. 8, pp. 957-970.

Dixon, D.A., M.N. Gray, and A.W. Thomas, 1985, "A Study of the Compaction Properties of Potential Clay-Sand Buffer Mixtures for Use in Nuclear Fuel Waste Disposal," Engineering Geology, Vol. 21, pp. 247-255.

Dunn, R.J., 1985, "Laboratory Measurement of Fine-Grained Soil Fluid Conductivity," Engineering Geology, Vol. 21, No. 3/4, pp. 215-223.

Dunn, R.J., 1986, "Clay Liners and Barriers - Considerations of Compacted Clay Structure," Proceedings of the International Symposium on Environmental Geotechnology, Allentown, PA, April 21-23, 1986, ed. by H.Y. Fang, Vol. 1, pp. 293-302.

Ende11, K., W. Loos, H. Meischeider, and V. Berg, 1938, "Ueber Zusammenhaenge zwischen Wasserhaushalt der Tonminerale und Bodenphysikalischen Eigenschaften Bindiger Boeden," (On the relations between water management by clay minerals and the soil 
physical properties of cohesive soils), Veroeff. Dtsch. Forsch. Bodenmech. 5 .

Fernandez, J.A., P.C. Kelsall, J.B. Case, and D. Meyer, 1987, "Technical Basis for Performance Goals, Design Requirements, and Material Recommendations for the NNWSI Repository Sealing Program," SAND84-1895, prepared for the U.S. Department of Energy, by Sandia National Laboratories, Albuquerque, NM, and Livermore, CA.

Fortier, S. and F.C. Scobey, 1926, "Permissible Canal Velocities," Transactions of A.S.C.E., Vol. 89, pp. 940-984.

Freeze, R.A. and J.A. Cherry, 1979, Groundwater, Prentice-Ha11, Inc., Englewood Cliffs, NJ.

Fyfe, W.F., V. Babuska, N.J. Price, E. Schmid, C.F. Tsang, S. Uyeda, and B. Velde, 1984, "The Geology of Nuclear Waste Disposal," Nature, Vol. 310, pp. 537-540, August 16.

Gaudette, M.V. and J.J.K. Daemen, 1988, "Bentonite Borehole Plug Flow Testing with Five Water Types," NUREG/CR-5130, prepared for U.S. Nuclear Regulatory Commission, by the Department of Mining and Geological Engineering, University of Arizona, Tucson.

Gibson, R.E., G.L. England, and M.J.L. Hussey, 1967, "The Theory of OneDimensional Consolidation of Saturated Clays, 1, Finite Non-Linear Consolidation of Thin Homogeneous Layers," Geotechnique, Vol. 17, No. 3, pp. 261-273.

Goodman, R.E. and P.N. Sundaram, 1980, "Permeability and Piping in Fractured Rocks," ASCE Journal of Geotechnical Engineering, Vol. 106, No. GT5, pp. 485-498.

Gouy, G., 1910, "Sur la Constitution de la Charge Electrique a la Surface d'un Electrolyte (On the formation of an electrical charge on the surface of an electrolyte)," Annales Physique (Paris), Serie 4, Vol. 9, pp. 457-468.

Graf, W.H., 1971, Hydraulics of Sediment Transport, McGraw-Hill Book Company, New York, 513 pp.

Gray, M.N., S.C.H. Cheung, and D.A. Dixon, 1985, "Swelling Pressures of Compacted Bentonite/Sand Mixtures," in Scientific Basis for Nuclear Waste Management VIII, Materials Research Society Symposia Proceedings, Vol. 44, C.M. Jantzen, J.A. Stone, and R.C. Ewing, Editors, November 26-29, 1984, Boston, Ma., Materials Research Society, Pittsburgh, $\mathrm{Pa} ., \mathrm{pp}$. 523-530.

Grim, R.E., 1953, Glay Mineralogy, McGraw-Hil1 Book Co., Inc., New York, $384 \mathrm{pp}$.

Grim, R.E., 1968, Glay Mineralogy, Second Edition, McGraw-Hill Book Co., Inc., New York, 596 pp. 
Grim, R.E. and N. Guven, 1978, Bentonites - Geology, Mineralogy, Properties and Uses, Developments in Sedimentology 24, Elsevier Scientific Publishing Company, Amsterdam.

Gupta, D.C., M. Nataraja, and J.J.K. Daemen, 1989, "Regulatory Questions and Concerns about Sealing a HLW Repository in an Unsaturated Environment," pp. 201-212, Proceedings, Workshop on Sealing of Radioactive Waste Repositories, organized by OECD Nuclear Energy Agency and Commission of the European Communities in cooperation with Gesellschaft fur Strahlen-Und Umweltforschung MBH Munchen, Institut fur Tieflagerung, Braunschweig, Federal Republic of Germany, 22-25 May, OECD, Paris.

Hazen, A., 1892, "Some Physical Properties of Sand and Gravel, with Special Reference to Their Use in Filtration," 24th Annual Report, Massachussets State Board of Health, Boston, pp. 539-566.

Head, K.H., 1980, Manual of Soil Laboratory Testing, Volume 1: Soil Classification and Compaction Tests, Engineering Laboratory Equipment Limited, 339 pp.

Holopainen, P., 1985, "Crushed Aggregate-Bentonite Mixtures as Backfill Material for Repositories of Low- and Intermediate-Level Radioactive Wastes," Engineering Geology, Vol. 21, pp. 239-245.

Holtz, R.D. and W.D. Kovacs, 1981, An Introduction to Geotechnical Engineering, Prentice-Hall, Inc., Englewood Cliffs, New Jersey, $733 \mathrm{p}$.

Hsieh, M.D., 1988, "The Permeability of Cohesive Soils in ThreeDimensions," a Report by Dept. of Civil Engineering, State University of New York at Buffalo, 35 p.

Hsieh, P.A., J.V. Tracy, C.E. Neuzil, J.D. Bredehoeft, and S.E. Silliman, 1981, "A Transient Laboratory Method for Determining the Hydraulic Properties of 'Tight' Rocks, I, Theory," International Journal of Rock Mechanics and Mining Sciences, Vol. 18, No. 3, pp. 245-252.

International Atomic Energy Agency, 1984, "Effects of Heat from High-Level Waste on Performance of Deep Geological Repository Components," IAEA-TECDOC-319, Vienna, 81 pp.

Jepsen, C.P., 1984, "Sodium Bentonite: Still a Viable Solution for Hazardous Waste Containment," Pollution Engineering, Vol. 16, No. 4, pp. 50,52,53, April.

Jepsen, C.P. and M. Place, 1985, "Evaluation of Two Methods for Constructing Vertical Cutoff Walls at Waste Containment Sites," Hydraulic Barriers in Soil and Rock, ASTM STP 874, edited by Johnson, A.I., R.K. Frobel, N.J. Cavalli, and C.B. Pettersson, American Society for Testing and Materials, Philadelphia, pp. 45-63. 
Jones, G.K., 1963, "Chemistry and Flow Properties of Bentonite Grouts," Grouts and Drilling Muds in Engineering Practice, Butterworth, London, pp. 22-28.

Jones, J.A.A., 1981, The Nature of Soil Piping: A Review of Research, Geo Books, Norwich, England, 301 pp.

Kassiff, G., D. Zaslavsky, and J.G. Zeitlen, 1965, "Analys is of Filter Requirements for Compacted Clays," Proceedings of the 6 th International Conference on Soil Mechanics and Foundation Engineering, Montreal, Vol. 2, pp. 495-499.

Keen, B.A. and G.W. Scott Blair, 1929, "Plastometric Studies of Soil and Clay Pastes," Journal of Agriculture Science, Vo1. 19, part IV, pp. 684-700.

Kenney, T.C., D. Lau, and G.I. Ofoegbu, 1984, "Permeability of Compacted Granular Materials," Canadian Geotechnical Journal, Vol. 21, pp. 726-729.

Kharaka, Y.K. and W.C. Smalley, 1976, "Flow of Water and Solutes through Compacted Clays," American Association of Petroleum Geology, Bulletin, Vol. 60, No. 6, pp. 973-980.

Khor, C.H. and H.K. Woo, 1989, "Investigation of Crushed Rock Filters for Dam Embankment," ASCE Journal of Geotechnical Engineering, Vol. 115, No. 3, pp. 399-412.

Lagerwerff, J.V., F.S. Nakayama, and M.H. Frere, 1969, "Hydraulic Conductivity Related to Porosity and Swelling of Soil,"

Proceedings of Soil Science Society of America, Vol. 33, pp. 3-11.

Lambe, T.W., 1951, Soil Testing for Engineers, John Wiley and Sons, New York, $165 \mathrm{p}$.

Lambe, T.W., 1955, "The Permeability of Fine-Grained Soils," ASTM, STP 163, American Society for Testing and Materials, Philadelphia, Pa, pp. 56-67.

Lambe, T.W., 1958, "The Structure of Compacted Clay," ASCE Journal of the Soil Mechanics and Foundations Division, Vol. 84., No. SM 2, May, pp. 1654-1 to 1654-33.

Lambe, T.W. and R.V. Whitman, 1979, Soil Mechanics, SI Version, John Wiley and Sons, New York, 553 pp.

Landau, H.G. and A.G. Altschaeff1, 1977, "Conditions Causing Piping in Compacted Clay," in Dispersive Clays, Related Piping, and Erosion in Geotechnical Projects, ASTM STP 623, Sherard, J.L. and R.S. Decker, Eds., American Society for Testing and Materials, Philadephia, PA, PP. 240-259.

Libicki, J.S., 1989, "Use of Abandoned Coal/Lignite Open Pits for Waste Disposal in Selected European Countries," EPA/600/9-89/072, 
Proceedings of Third International Conference on New Frontiers for Hazardous Waste Management, September 10-13, Pittsburgh, pp. 7683.

Loudon, A.G., 1952, "The Computation of Permeability from Simple Soil Tests," Geotechnique, Vol. III, No. 4, pp. 165-183.

Lyle, W.M. and E.T. Smerdon, 1965, "Relation of Compaction and Other Soil Properties to Erosion Resistance of Soils," Transactions of The American Society of Agricultural Engineering, Vol. 8, No. 3, pp. 419-422.

Marsha11, T.J., 1958, "A Relation Between Permeability and Size Distribution of Pores," Journal of Soil Science, Vol. 9, pp. 1-8.

Marsland, A. and A.G. Loudon, 1963, "The Flow Properties and Yield Gradients of Bentonite Grouts in Sands and Capillaries," Grouts and Drilling Muds in Engineering Practice, Butterworth, London, pp. 15-21.

Martin, R.T., 1975, "Feasibility of Sealing Boreholes with Compacted Natural Earthen Material, Vol. I," MIT Research Report 75-28, 121 pp.

Mesri, G. and R.E. Olson, 1971, "Mechanisms Controlling the Permeability of Clays," Clays and Clay Minerals, Vol. 19, pp. 151-158.

Meyer, D. and J.J. Howard, 1983, "Evaluation of Clays and Clay Minerals for Application to Repository Sealing," ONWI-486, Office of Nuclear Waste Isolation, Battelle Memorial Institute, Columbus, $\mathrm{OH}$.

Michaels, A.S. and C.S. Lin, 1954, "Permeability of Kaolinite," Industrial and Engineering Chemistry, Vol. 46, No. 6, pp. 12391246 .

Michaels, A.S. and C.S. Lin, 1955, "Effects of Counterelectro-osmosis and Sodium Ion Exchange on Permeability of Kaolinite," Industrial and Engineering Chemistry, Vol. 47, No. 6, pp. 1249-1253.

Millington, R.J. and J.P. Quirk, 1959, "Permeability of Porous Media," Nature, Vol. 183, pp. 387-388.

Mitchell, J.K., 1976, Fundamentals of Soil Behavior, John Wiley \& Sons, New York, 422 pp.

Mitchell, J.K. and J.S. Younger, 1967, "Abnormalities in Hydraulic Flow Through Fine-Grained Soils," ASTM STP 417, American Society for Testing and Materials, Philadelphia, PA, pp. 106-139.

Nagaraj, T.S. and B.R. Srinivasa Murthy, 1983, "Rationalization of Skempton's Compressibility Equation," Geotechnique, Vol. 33, No. 4, pp. 433-443. 
Nagaraj, T.S. and B.R. Srinivasa Murthy, 1986, "A Critical Reappraisal of Compression Index Equations," Geotechnique, Vo1. 36, No. 1, pp. 27-32.

Neretnieks, I., 1987, "Some Coupled Processes Which May be Important for a Nuclear Waste Repository," Ch. 55, pp. 759-763, Coupled Processes Associated with Nuclear Waste Repositories, C.F. Tsang, ed., Academic Press, Inc., Orlando.

Neuzil, C.E., 1986, "Groundwater Flow in Low-Permeability Environments," Water Resources Research, Vol. 22, No. 8, pp. 1163-1195.

Neuzil, C.E., C. Cooley, S.E. Silliman, J.D. Bredehoeft, and P.A. Hsieh, 1981, "A Transient Laboratory Method for Determining the Hydraulic Properties of 'Tight' Rocks, II, Application," International Journal of Rock Mechanics and Mining Sciences, Vol. 18, No. 3, pp. 245-252.

Nilsson, J., 1985, "Field Compaction of Bentonite-Based Backfilling," Engineering Geology, Vo1. 21, No. 3/4, pp. 367-376.

Norman, L.E.J., 1958, "A Comparison of Values of Liquid Limit Determined with Apparatus Having Bases of Different Hardness," Geotechnique, Vol. 8, No. 2, pp. 79-83.

Ogden, F.L. and J.F. Ruff, 1989, "Axial Shear Strength Testing of Bentonite Water Well Annulus Seals," a Report by Dept. of Civil Engineering, Colorado State University, Fort Collins, 98 p.

Olsen, H.W., 1962, "Hydraulic Flow Through Saturated Clays," Proceedings, 9th National Conference on Clays and Clay Minerals, Clays and Clay Minerals, Vol. 9, Pergamon Press, New York, pp. 131-161.

Olsen, H.W., 1966, "Darcy's Law in Saturated Clays," Water Resources Research, Vol. 2, No. 2, pp. 287-295.

Olsen, H.W., R.W. Nichols, and T. L. Rice, 1985, "Low-Gradient Permeability Measurements in a Triaxial System," Geotechnique, Vol. 35, No. 2, pp. 145-157.

Olson, R.E. and D.E. Daniel, 1981, "Measurement of the Hydraulic Conductivity of Fine-Grained Soils," Permeability and Groundwater Contaminant Transport, ASTM STP 746, Am. Soc. for Testing and Materials, Philadelphia, PA, pp. 18-64.

Organization for Economic Cooperation and Development, Nuclear Energy Agency, 1982, "Geological Disposal of Radioactive Waste Research in the OECD Area," OECD, Paris, May.

Ouyang, S. and J.J.K. Daemen, 1990, "Performance of Bentonite/Crushed Tuff Seals for Nuclear Waste Repositories," Waste Management '90, Feb. 25 - March 1, Tucson, Arizona, Vol. 2, pp. 605-611. 
Ouyang, S. and J.J.K. Daemen, 1989, "Crushed Salt Consolidation,", NUREG/CR-5402, prepared for U.S. Nuclear Regulatory Commission, Office of Nuclear Regulatory Research, Division of Engineering, by the Department of Mining and Geological Engineering, University of Arizona, Tucson.

Pandian, N.S. and T.S. Nagaraj, 1990, "Critical Reappraisal of Colloidal Activity of Clays," ASCE, Journal of Geotechnical Engineering, Vo1. 116, No. 2, pp. 285-296.

Paterson, M.S., 1983, "The Equivalent Channel Model for Permeability and Resistivity in Fluid-Saturated Rock - a Re-Appraisal," Mechanics of Materials, Vol. 2, pp. 345-352.

Perry, E., 1975, "Piping in Earth Dams Constructed of Dispersive Clay: Literature Review and Design of Laboratory Testing," U.S. Corps of Engineers, Technical Report 5-75-15, U.S. Army Engineer Waterways Experiment Station, Vicksburg, MS.

Price, R.H. and S.U. Bauer, 1985, "Analysis of the Elastic and Strength Properties of Yucca Mountain Tuff, Nevada," Proceedings of the 26th U.S. Symposium on Rock Mechanics, Rapid City, SD, June 26-29, pp. 89-96.

Pusch, R., 1973, "Influence of Salinity and Organic Matter on the Formation of Clay Microstructure," Proceedings of the

International Symposium on Soil Structure, Gothenburg, Sweden, Pp. 161-173.

Pusch, R., 1978, "Highly Compacted Na Bentonite as a Buffer Substance," KBS 74, Karnbranslesakerhet, Stockholmn Sweden.

Pusch, R., 1978, "Self Injection of Highly Compacted Bentonite into Rock Joints," KBS 73, Karnbranslesakerhet, Stockholm, Sweden.

Pusch, R., 1983, "Borehole Sealing for Underground Waste Storage," ASCE, Journal of Geotechnical Engineering, Vol. 109, No. 1, pp. 113-119.

Pusch, R. and G. Alstermark, 1985, "Experience from Preparation and Application of Ti11/Bentonite Mixtures," Engineering Geology, Vol. 21 , pp. 377-382.

Pusch, R., T. Eriksen, and A. Jacobsson, 1982, "Ion/Water Migration Phenomena in Dense Bentonites," in Scientific Basis for Nuclear Waste Management $V$, W. Lutze, editor, Proceedings of the Symposium, June 7-10, 1982, Berlin, Germany, Elsevier Science Publishing Co., Inc, New York, pp. 649-658.

Pusch, R., M. Eristrom, and L. Borgensson, 1987, "Piping and Erosion Phenomena in Soft Clay Gels," SKB Technical Report 87-09, for Stripa Project, prepared for Div. of Research and Development, Swedish Nuclear Fuel and Waste Mangement Co., by the Swedish Geological Co., Stockholm, $40 \mathrm{pp}$. 
Pusch, R., O. Karnland, and A. Muurinen, 1989, "Transport and Microstructural Phenomena in Bentonite Clay with Respect to the Behavior and Influence of $\mathrm{Na}, \mathrm{Cu}$, and $\mathrm{U}, "$ SKB Technical Report 8934, Swedish Nuclear Fuel and Waste Management Co., Stockholm.

Pusch, R., A. Jacobsson, and A. Bergstrom, 1980, "Bentonite-Based Buffer Substances for Isolating Radioactive Waste Products at Great Depths in Rock," in Underground Disposal of Radioactive Wastes, Vol. 1, Proceedings of a Symposium, Otaniemi, July 2-6, 1979, International Atomic Energy Agency, Vienna.

Quirk, J.P., 1968, "Particle Interactions and Soil Swelling," Israel Journal of Chemistry, Vol. 6, pp. 213-234.

Raudkivi, A.J., 1976, Loose Boundary Hydraulics, 2nd Edition, Pergamon Press, Oxford, $397 \mathrm{pp}$.

Rosenquist, I.T., 1955, "Investigations in the Clay-Electrolyte-Water System, "Publication No. 9, Norwegian Geotechnical Institute, Os lo, pp. 92-100.

Rosewe11, C.J., 1977, "Identification of Susceptible Soils and Control of Tunnelling Failure in Small Earth Dams," in Dispersive Clays, Related Piping, and Erosion in Geotechnical Projects, ASTM STP 623, Sherard, J.L. and R.S. Decker, Eds., American Society for Testing and Materials, Philadephia, PA, pp. 362-369.

Ross, C.S. and S.B. Hendricks, 1945, "Minerals of the Montmorillonite Group," U.S. Geological Survey Professional Paper, 205-B, pp. 2377.

Rouse, H., 1946, Elementary Mechanics of Fluids, Dover Publications, Inc., New York, 376 pp.

Samuels, S.G., 1950, "The Effect of Base Exchange on the Engineering Properties of Soils," Build. Res. Stn. G.B., Note C 176.

Sawyer, W.D., II, and J.J.K. Daemen, 1987, "Experimental Assessment of the Sealing Performance of Bentonite Borehole Plugs," NUREG/CR-4995, prepared for Division of Engineering, Office of Nuclear Regulatory Research, U.S. Nuclear Regulatory Commission, by Department of Mining and Geological Engineering, University of Arizona, Tucson.

Schmid, W.E., 1957, "The Permeability of Soils and the Concept of a Stationary Boundary-Layer," Proceedings of American Society for Testing and Materials, Vol. 57, pp. 1195-1218.

Schoenberger, R. J., 1988, "Design of Future Hazardous Waste Landfill What are the Objectives?", Land Disposal of Hazardous Waste: Engineering and Environmental Issues, Ellis Horwood Limited, Chichester, England, 311 pp. 
Scott Blair, G.W. and E.M. Crowther, 1929, "The Flow of Clay Pastes Through Narrow Tubes," Journal of Physical Chemistry, Vol 33, pp. $321-330$.

Scully, L.W., 1984, "Design Considerations to Minimize the Impact of a Repository on a Host Rock," in Scientific Basis for Nuclear Waste Management VII, Materials Research Society Symposia Proceedings, Boston, MA, Nov. 14-17, 1983, Vo1. 26, G.L. McVay, ed., NorthHolland, New York, pp. 293-300.

Seaber, P.R., and J. Vecchioli, 1966, "Use of Soil-Consolidation Test Data to Determine Permeability of Clays," U.S. Geological Survey Water Supply Paper 1822, pp. 105-112.

Shaikh, A., J.F. Ruff, and S.R. Abt, 1988, "Erosion Rate of Compacted Na-Montmorillonite Soils," ASCE Journal of Geotechnical Engineering, Vol. 114, No. 3, pp. 296-305.

Shainberg, I., E. Bresler, and Y. Klausner, 1971, "Studies on $\mathrm{Na} / \mathrm{Ca}$ Montmorillonite Systems: 1. The Swelling Pressure," Soil Science, Vol. 111, No. 4, pp. 214-219.

Shainberg, I. and A. Caiserman, 1971, "Studies on $\mathrm{Na} / \mathrm{Ca}$ Montmorillonite Systems: 2. The Hydraulic Conductivity," Soil Science, Vol. 111, No. 5 , pp. 276-281.

Sharpe, C. and J.J.K. Daemen, 1991, "Laboratory Testing of Cement Grouting of Fractures in Welded Tuff," NUREG/CR-5683, Technical Report prepared for the U.S. Nuclear Regulatory Commission, by the Department of Mining and Geological Engineering, University of Arizona, Tucson.

Sherard, J.L., R.S. Decker, and N.L. Ryker, 1972, "Piping in Earth Dams of Dispersive Clay," Proceedings of ASCE Special Conference on Performance of Earth and Earth-Supported Structures, Vol. 1, pp. 589-626.

Sherard, J.L. and L.P. Dunnigan, 1989, "Critical Filters for Impervious Soils," ASCE Journal of Geotechnical Engineering, Vol. 115, No. 7, pp. 927-947.

Sherard, J.L., L.P. Dunnigan, and R.S. Decker, 1977, "Some Engineering Problems with Dispersive Clays," in Dispersive Clays, Related Piping, and Erosion in Geotechnical Projects, ASTM STP 623, Sherard, J.L. and R.S. Decker, Eds., American Society for Testing and Materials, Philadephia, PA, pp. 3-12.

Sherard, J.L., L.P. Dunnigan, and J.R. Talbot, 1984, "Filters for Silts and Clays," ASCE Journal of Geotechnical Engineering, Vol. 110, No. 6, pp. 701-718.

Singh, S.K., 1982, "Chemical, Physical, and Engineering Characterization of Candidate Backfill Clays and Clay Admixtures for a Nuclear Waste Repository - Part I," in Scientific Basis for Nuclear Waste 
Management, Materials Research Society Symposia Proceedings, Annual Meeting, Nov. 1981, Boston, MA, Vol. 6, S.V. Topp, editor, pp. 413-433.

Skempton, A.W., 1944, "Notes on the Compressibility of Clays," Quarterly Journal of the Geological Society of London, Vol. 100C, pp. 119135 .

Skempton, A.W. and R.D. Northey, 1953, "The Sensitivity of Clays," Geotechnique, Vol. 3, No. 1, pp. 30-53.

Skopek, J. and G. Ter-Stepanian, 1975, "Comparison of Liquid Limit Values Determined According to Casagrande and Vasilev," Geotechnique, Vol. 25, No. 1, pp. 135-136.

Smerdon, E.T. and R.P. Beasley, 1961, "Critical Tractive Forces in Cohesive Soils," Agricultural Engineering, Vo1. 46, pp. 26-29.

Smiles, D.E., 1969, "Steady Flow Experiments in Saturated Clays," Australia Journal of Soil Research, Vol. 7, pp. 91-98.

Smiles, D.E. and M.J. Rosenthal, 1968, "The Movement of Water in Swelling Materials," Australia Journal of Soil Research, Vol. 6, pp. 237-248.

Smith, M.J., G.J. Anttonen, G.S. Barney, W.E. Coons, F.N. Hodges, R.G. Johnston, J.D. Haser, R.M. Manabe, S.C. McCarel, E.L. Moore, A.F. Noonan, J.E. O'Rourke, W.W. Schulz, C.L. Taylor, B.J. Wood, and M.I. Wood, 1980, "Engineered Barrier Development for a Nuclear Waste Repository in Basalt: An Integration of Current Knowledge," RHO-BWI-ST-7, Rockwell Hanford Operations, Richland, Washington, May.

South, D.L. and J.J.K. Daemen, 1986, "Permeameter Studies of Water Flow Through Cement and Clay Borehole Seals in Granite, Basalt and Tuff," NUREG/CR-4748, prepared for U.S. Nuclear Regulatory Commission, by the Department of Mining and Geological Engineering, University of Arizona, Tucson.

Southwestern Pay Dirt, 1990, March, Bisbee, Arizona, 24 pp.

Sridharan, A. and M. S. Jayadeva, 1982, "Double Layer Theory and Compressibility of Clays," Geotechnique, Vol. 32, No. 2, pp. 133144 .

Srinivasa Murthy, B.R., A. Vatsala, and T.S. Nagaraj, 1988, "Can CamClay Model Be Generalized ?," ASCE, Journal of Geotechnical Engineering, Vol. 114, No. 5, pp. 601-613.

Statton, C.T. and J.K. Mitchel1, 1977, "Influence of Eroding Solution Composition on Dispersive Behavior of a Compacted Clay Shale," in Dispersive Clays, Related Piping, and Erosion in Geotechnical Projects, ASTM STP 623, J.L. Sherard and R.S. Decker, eds., American Society for Testing and Materials, Philadephia, PA. 
Stegmann, R., 1988, "Design of Hazardous Waste Landfill in the Future in West Germany", Land Disposal of Hazardous Waste: Engineering and Environmental Issues, Ellis Horwood Limited, Chichester, England, 311 .

Streeter, V.L. and E.B. Wylie, 1979, Fluid Mechanics, 7th Edition, McGraw-Hill Book Co., New York, 562 pp.

Tavenas, F., P. Jean, P. Leblond, and S. Leroueil, 1983, "The Permeability of Natural Soft Clays. Part II: Permeability Characteristics," Canadian Geotechnical Journal, Vo1. 20, pp. 645660.

Tavenas, F., P. Leblond, P. Jean, and S. Leroueil, 1983, "The Permeability of Natural Soft Clays, I, Methods of Laboratory Measurement," Canadian Geotechnical Journal, Vol. 20, No. 4, pp. 629-644.

Taylor, D.W., 1948, Fundamentals of Soil Mechanics, John Wiley and Sons Inc., New York, $700 \mathrm{pp}$.

Taylor, C.L., G.J. Anttonen, J.E. O'Rourke, and D. Allirot, 1980, "Preliminary Geochemical and Physical Testing of Materials for Plugging of Man-Made Accesses to a Repository in Basalt," RHO-BWIC-66. Prepared for the U.S. Department of Energy, Contract DEAC06-77RL01030, Rockwell International, Rockwell Hanford Operations, Energy System Group, Richland, WA, by Woodward-Clyde Consultants, San Francisco, CA, April.

Terzaghi, K., 1927, "Principles of Final Soil Classification," Public Roads, Vol. 8, No. 3, pp. 41-5̦3.

Thompson, H.P., 1988, "Review and Comment on the U.S. Department of Energy Site Characterization Plan Conceptual Design Report," NWPOTR-009-88, prepared by Engineering Company, Inc., in conjunction with Sea Inc., Dunn Geoscience Corp., and W.F. Guyton Associated, Inc., for Nuclear Waste Project Office, Agency for Nuclear Projects, Nevada.

U.S. Army Corps of Engineers, 1955, "Drainage and Erosion ControlSurface Drainage Facilities for Airfields," Part 13, Chapter 2, Engineering Manual, Military Construction, Washington, DC.

U.S.B.P.R., 1962, "Aggregate Gradation for Highways," Bureau of Public Roads, Washington, DC.

U.S. Bureau of Reclamation, 1987, Design of Smal1 Dams, 3rd Edition, U.S. Government Printing Office, Denver, 860 pp.

U.S. Department of Energy, 1988, "Site Characterization Plan: Overview," DOE/RW-0198, U.S. Department of Energy, Office of Civilian Radioactive Waste Management, Washington, DC 
van 01phen, H., 1963, An Introduction to Clay Colloid Chemistry, Interscience Publishers, a Division of John Wiley and Sons, New York.

Vaughan, P.R. and H.F. Soares, 1982, "Design of Filters for Clay Cores of Dams," ASCE Journal of Geotechnical Engineering, Vol. 108, No. GT1, pp. 17-31.

Warkentin, B.P., G.H. Bolt, and R.D. Miller, 1957, "Swelling Pressure of Montmorillonite," Proceedings of Soil Science of America, Vol. 21, No. 5, pp. 495-497.

Warkentin, B.P. and R.K. Schofield, 1962, "Swelling Pressure of NaMontmorillonite in NaCl Solutions," Journal of Soil Science, Vol. 13, No. 1, pp. 98-105.

Williams, J.R. and J.J.K. Daemen, 1987, "The Sealing Performance of Bentonite/Crushed Basalt Borehole Plugs," NUREG/CR-4983, Technical Report prepared for U.S. Nuclear Regulatory Commission, by Department of Mining and Geological Engineering, University of Arizona, Tucson.

Winterkorn, H.F., 1975, "Soil Stabilization," in Foundation Engineering Handbook, H.F. Winterkorn and H.Y. Fang, eds., Van Nostrand Reinhold Co., New York, pp. 312-336.

Wolski, W., 1965, "Model Tests on the Seepage Erosion in the Silty Clay Core of an Earth Dam," Proceedings of the 6th International Conference on Soil Mechanics and Foundation Engineering, Montreal, Vol. 2, pp. 583-587.

Wroth, C.P. and D.M. Wood, 1978, "The Correlation of Index Properties with Some Basic Engineering Properties of Soils," Canadian Geotechnical Journal, Vol. 15, No. 2, PP. 137-145.

Wu, T.H., 1976, Soil Mechanics, Second Edition, Allyn and Bacon, Inc., Boston, $440 \mathrm{pp}$.

Yong, R.N., P. Boonsinuk, and G. Wong, 1986, "Formulation of Backfill Material for a Nuclear Fuel Waste Disposal Vault," Canadian Geotechnical Journal, Vol. 23, pp. 216-228.

Yong, R.N. and B.P. Warkentin, 1975, Soil Properties and Behaviour, Elsevier Scientific Publishing Co., Amsterdam, $449 \mathrm{p}$.

Youssef, M.S., A.H. E1 Ramli, and M. El Demery, 1965, "Relationships Between Shear Strength, Consolidation, Liquid Limit, and Plastic Limit for Remoulded Clays," Proceedings, 6th International Conference on Soil Mechanics and Foundation Engineering, Montreal, Vol. 1, pp. 126-129.

Zaslavsky, D. and G. Kassiff, 1965, "Theoretical Formulation of Piping Mechanism in Cohesive Soils," Geotechnique, Vol. XV, No. 2, pp. 305-316. 
Zimmerman, R.M., F.B. Nimick, and M.P. Board, 1985, "Geoengineering Characterization of Welded Tuffs from Laboratory and Field Investigations," in Scientific Bas is for Nuclear Waste Management VIII, Vol. 44, Materials Research Society Symposia Proceedings, Pittsburgh, pp. 547-554. 



\section{APPENDIX A}

SAMPLE PREPARATION AND INSTALIATION PROCEDURE FOR PERMEABILITY TESTING OF BENTONITE AND BENTONITE/CRUSHED TUFF PLUGS

\section{Objective}

The objective of this procedure is to prepare bentonite and bentonite/crushed tuff samples for permeability testing. This procedure consists of two parts: sample preparation and sample installation.

\section{Apparatus}

(1) Balance (readable to $0.1 \mathrm{~g}$ )

(2) Plastic mixing pan

(3) Small plastic shovel or scoop

(4) Distilled water supply

(5) Sprayer

(6) Plastic jar with air-tight cap

(7) Standard proctor compactor (ASTM D698-78, 3.2.1)

(8) A fabricated hammer compactor (1.16 1b, $0.53 \mathrm{~kg}$ hammer weight; circular specimen contact of 1 in $(25.4 \mathrm{~mm})$ diameter)

(9) Funnel with special extended cylindrical spout 1 in $(25.4 \mathrm{~mm}$ ) in diameter. The length of the spout should be close to the full length of the permeameter.

\section{$\underline{3 . \text { Procedure }}$}

(I) Sample preparation

a. Bentonite samples

1. Weigh out an appropriate amount (W) of air-dried bentonite (with predetermined water content $w_{i}$ ) to the nearest $1 \mathrm{~g}$. The bentonite should have an excess $100-200 \mathrm{~g}$ over the weight needed for the sample to be prepared.

2. Determine the weight of water to be added to the bentonite to render a desired water content $\left(w_{f}\right)$. This weight can be calculated using the following formula:

$$
W_{a d d}=W \times\left(\frac{100-W_{i}}{100}\right) \times\left(\frac{W_{E}-W_{i}}{100}\right)
$$

3. Add a small amount of distilled water to the bentonite and mix thoroughly. Repeat this process several times until the water added reaches the prescribed amount. Use a plant sprayer to distribute the distilled water evenly across the sample. 
4. Transfer the sample into a plastic jar with an air-tight cap. Let the sample cure for at least 72 hours before installing it in the permeameter.

b. Bentonite/crushed tuff samples

1. Select the weight ratio of air-dried bentonite vs. air-dried crushed tuff.

2. Select the desired grain size distribution curve for crushed tuff.

3. Weigh out appropriate amounts of air-dried bentonite and of crushed tuff. The combined weight of the bentonite and crushed tuff should have an excess of 100 to $200 \mathrm{~g}$, in appropriate proportions, over the weight needed for sample construction.

4. Prepare the bentonite to the desired water content following steps 1 to 3 in part a.

5. Add the crushed tuff to the bentonite and mix thoroughly. (This is designated as dry crushed tuff/wet bentonite mixing.)

6. Transfer the sample into a plastic jar with air-tight cap. Let the sample cure for at least 72 hours before emplacing it in a permeameter.

7. Alternatively, the crushed tuff can be added to the bentonite at the end of step 3, followed by the addition of distilled water, which is parallel to step 3 as described in part a. (This is designated as wet crushed tuff/wet bentonite mixing.)

(II) Sample installation

1. Place a porous stone at the bottom of a permeameter. A layer of clean sand of a desired thickness, with grain size between $0.841 \mathrm{~mm}$ (U.S standard \#20) and $0.259 \mathrm{~mm}$ (U.S. standard \#60), may be emplaced before the placement of the porous stone such that the sample length can either be reduced or measured from outside (when a transparent PVC permeameter is used).

2. Use a small shovel or scoop (if it can be lowered to placement position) to transfer the sample to the permeameter by tilting it at a small angle and sliding it back gradually toward the center of the permeameter so that the sample is placed as a stripe from the perimeter to the center. Turn 90 degrees for the next placement. Repeat this process two more times. Spread the sample gently and evenly over the area of the permeameter. For small diameter permeameters, place the sample by pouring through funnels with spouts long enough to reach the placement location.

3. Repeat step 2 until the layer thickness is suitable for compaction. ASTM D2434, 6.4 requires that the layer be approximately equal in thickness, after compaction, to the maximum size of the particles, but not less than about $15 \mathrm{~mm}(0.60 \mathrm{in})$. 
4. Compact each layer thoroughly by tamping uniformly using a sliding weight Rammer compactor. For sample diameters greater than $50.8 \mathrm{~mm}$ (2 in), a manual rammer as specified by ASTM D698-78 (Standard Proctor Test), D3.2.1, shall be used to compact the sample. A fabricated hammer compactor with hammer weight of $1.16 \mathrm{lb}(0.53 \mathrm{~kg})$ and circular specimen contact of 1 in $(25.4 \mathrm{~mm})$ in diameter, shall be used for compacting samples of smaller diameters. For either case, the drop height and the number of drops per layer shall be adjusted to provide the same energy input as for the Standard Proctor Test $\left(593 \mathrm{~kJ} / \mathrm{m}^{3}\right.$; $\left.12375 \mathrm{ft}-\mathrm{lb} / \mathrm{ft}^{3}\right)$.

5. Repeat steps 2 through 4 until the desired sample length is reached.

6. Place a porous stone and/or a sand layer on top of the sample. The sample may be further confined axially with a piston if a stainless steel permeameter is used. This will hold the placement density and volume of sample without significant change during the saturation of the specimen. [NOTE: Any consolidation influences during permeability testing (change of void ratio or sample volume) will be monitored by the displacement of the piston rod.] This step may be omitted for samples installed in PVC permeameters to allow bentonite to swell during the saturation of the specimen such that the swelling pressure can be reduced and will not damage the PVC pipe. [NOTE: Measure, e.g. with a tape, changes of the sample length during saturation and permeability testing.] 


\section{APPENDIX B \\ EXPERTIENTAL PROGEDURE FOR PERLEABIIITT TESTITG \\ OF BENTONITE AND BENTONITE/GRUSHED TUKF PLUGS}

\section{Objective}

This procedure covers the determination of the coefficient of permeability for bentonite and bentonite/crushed tuff plugs. The procedure includes three methods: constant head, standard falling head, and double-pipette tests.

\section{Apparatus}

(A) Constant head method

1. Permeameters (PVC or stainless steel) (Figure B.1)

2. Constant-head reservoir

3. Outlet reservoir with overflow to maintain a constant water level.

4. Measuring cylinders

5. Stop clock

6. Inflow and outflow tubing

7. Dial gage (for monitoring the displacement of piston rod of stainless steel permeameter)

8. Precision tape or ruler (for measuring the sample length)

9. Helium tank (for high-pressure tests)

10. Gas-over-water pump (for high-pressure tests)

(B) Falling head method

1. Permeameters (PVC or stainless steel)

2. Precision pipettes and caps

3. Inflow and outflow tubing (stainless steel or high-pressure rubber or plastic)

4. Precision tape

5. Stop clock

6. Outlet reservoir with overflow to maintain a constant water level

\section{Testing Procedure}

Sample preparation and installation shall follow the procedures described in Appendix A. ASTM D2434, 6.6.4, shall be followed for sample saturation prior to permeability testing.

(A) Constant head method (Fig. B.2.a)

1. Connect the inlet of the permeameter with the constant head reservoir. Remove any air trapped in the connecting tubing. 


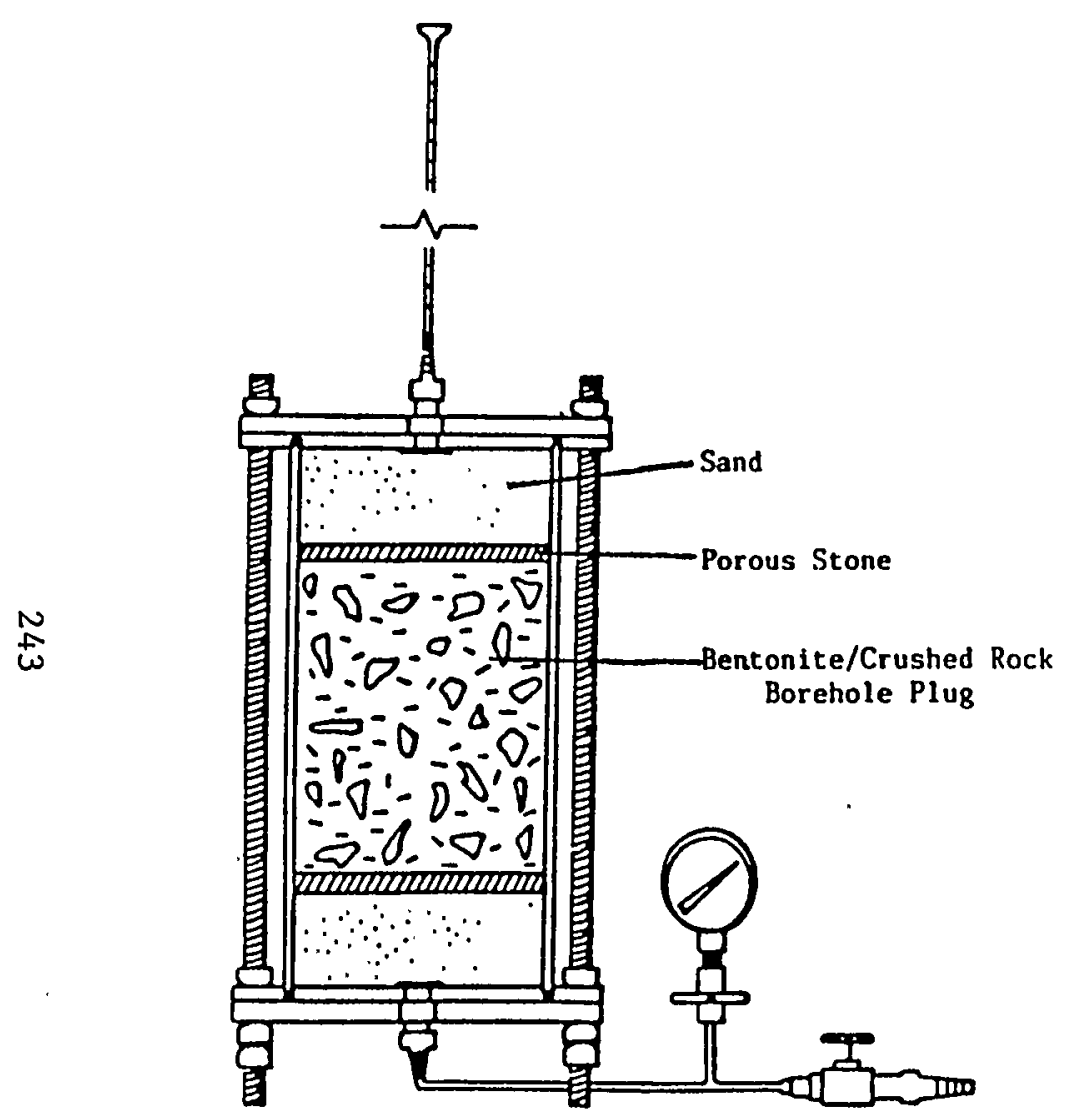

(a)

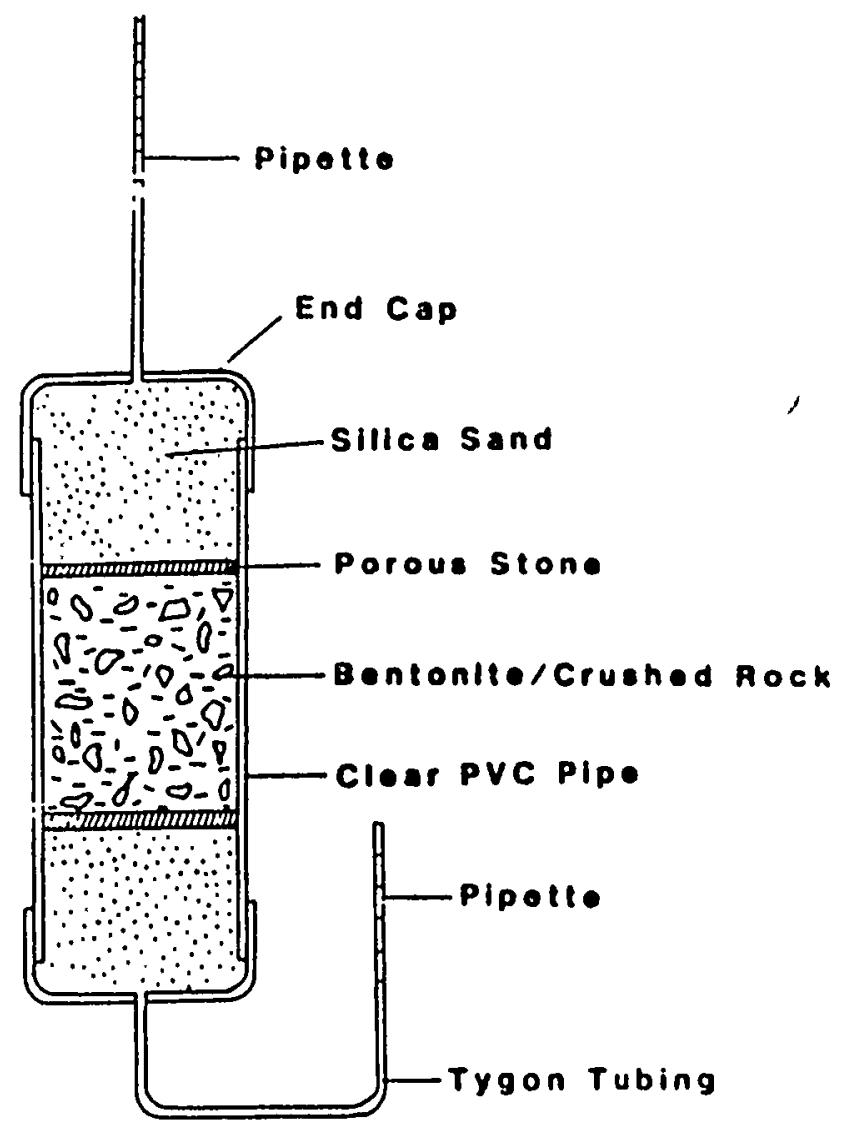

(b)

Fig'. B.1. Schematic of pipe flow permeameters: (a) stainless steel permeameter, (b) Pvc permeameter. 


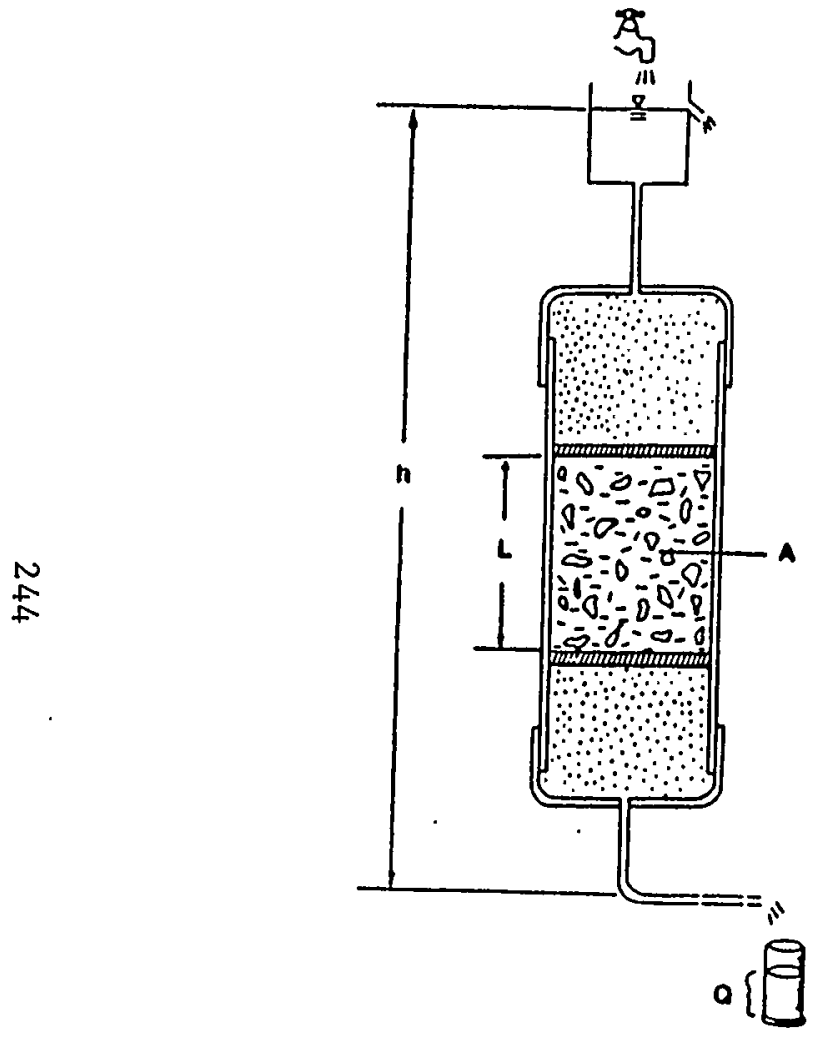

(a)

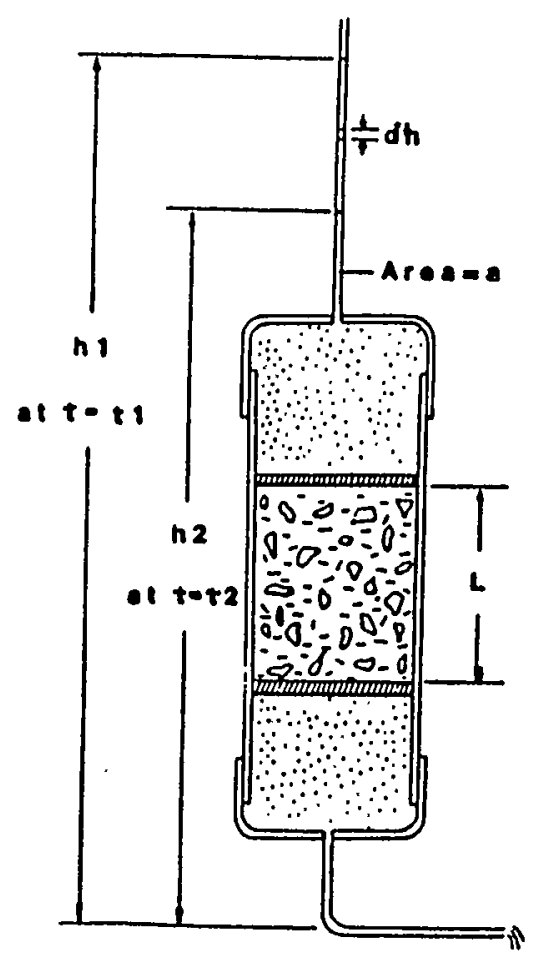

(b)

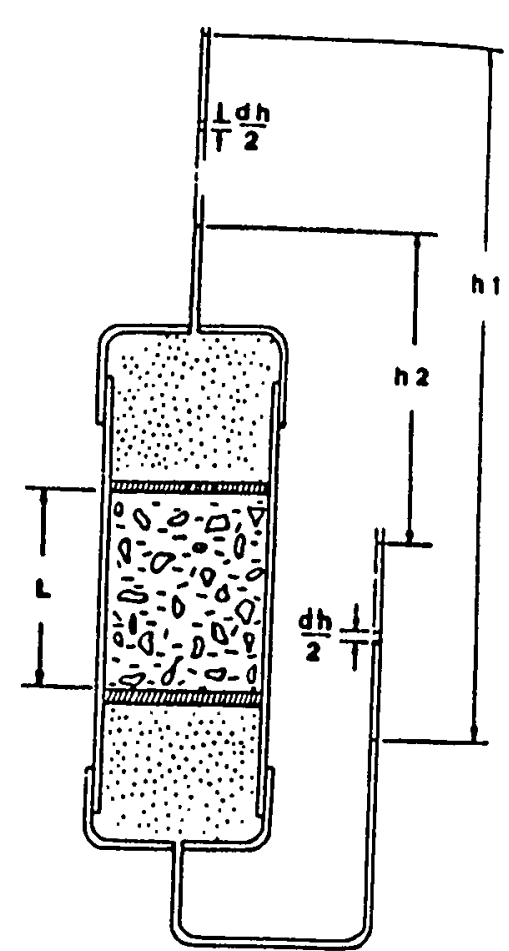

(c)

Fig. B.2. Schematic of flow test set-ups: (a) standard constant head test, (b) standard falling head test, (c) modified falling head test. 
2. Connect the outlet of the permeameter with the outlet reservoir. Pinch or tap the connecting tubing to remove any trapped air.

3. Place a graduated cylinder to catch the overflow of the outlet reservoir.

4. Cover the outlet reservoir and the graduated cylinder with plastic sheet or aluminum foil to prevent evaporation.

5. Calculate the cross-sectional area of the sample (A). Measure the difference in the head between the water columns above and below the sample $(h)$. Record the time $(t)$, amount of outflow (Q), and sample length (L). The head difference (h) should be adjusted if the sample length changes. Monitor and record the test room temperature.

6. Calculate the coefficient of permeability, $K$, as follows:

$$
K=\frac{Q L}{A t h}
$$

$$
\text { where } \begin{aligned}
K & =\text { coefficient of permeability }(\mathrm{cm} / \mathrm{s}) \\
Q & =\text { quantity of water discharged over } t(\mathrm{cc}) \\
L & =\text { sample length }(\mathrm{cm}) \\
A & =\text { cross-sectional area of specimen }\left(\mathrm{cm}^{2}\right) \\
t & =\text { elapsed time of discharge }(\mathrm{s}) \\
h & =\text { head difference across the specimen }(\mathrm{cm}) .
\end{aligned}
$$

7. To increase the rate of flow for samples of low permeability, a gas pressure (e.g. compressed helium) can be applied to the surface of the water supply (Lambe, 1951, p. 58). The surface of the water supply should be covered with a membrane to reduce the amount of gas going into solution. Alternatively, a gas-overwater pump can be used for this purpose. The head difference is then $h$ plus the applied pressure changed to units of water head.

8. The outflow can be collected under gravity fall in a graduated cylinder. The water head at the bottom of the sample is equal to the atmospheric pressure, and the surface tension is assumed negligible.

(B) Falling head method

1. Standard falling head method (Fig. B.2.b)

(a) Measure and record the inside diameter and calculate the cross-sectional area of the pipette $\left(\mathrm{a}, \mathrm{cm}^{2}\right)$ and permeameter $\left(A, \mathrm{~cm}^{2}\right)$ and sample length $(\mathrm{L}, \mathrm{cm})$.

(b) Measure the length of the entire graduation. Calculate the length per unit volume $\left(l, \mathrm{~cm}^{-2}\right)$. Record the total volume for the graduation of the pipette $\left(V_{t}, \mathrm{~cm}^{3}\right)$. 
(c) Connect the necessary tubing and pipette according to Fig. B.2.b. Especially if low pressure tubing is used between the pipette and the permeameter, keep the length of tubing to a minimum. This will minimize the effect of volume changes due to the decrease of tubing diameter as the hydrostatic pressure decreases.

(d) Measure the height between the level of outflow and the level of the lowest graduate mark on the pipette. This will be the reference height $(\mathrm{H}, \mathrm{cm})$.

(e) Fill the pipette with deaired distilled water and let the water level drop.

(f) When the water level drops within the graduation, record the time $\left(t_{1}\right)$ and the graduation reading $\left(v_{1}\right)$. Calculate $h_{1}$, using the equation:

$$
h_{1}=H+\left(V_{t}-V_{1}\right) \times \ell
$$

(g) At the time $t_{2}$ record the graduation reading $\left(v_{2}\right)$. Calculate $h_{2}$ from:

$$
\mathrm{h}_{2}=\mathrm{H}+\left(\mathrm{V}_{\mathrm{t}}-\mathrm{V}_{2}\right) \times \ell
$$

(h) The coefficient of permeability $K$ can be computed from:

$$
K=\frac{a L}{A\left(t_{2}-t_{1}\right)} \ln \frac{h_{1}}{h_{2}}
$$

in which $K=$ coefficient of permeability $(\mathrm{cm} / \mathrm{s})$

$a=$ cross-sectional area of the pipette $\left(\mathrm{cm}^{2}\right)$

$A=$ cross-sectional area of the permeameter $\left(\mathrm{cm}^{2}\right)$

$L=$ sample length $(\mathrm{cm})$

$t_{1}=$ time when water in the pipette is at $h_{1}$

$t_{2}=$ time when water in the pipette is at $h_{2}$

$h_{1}=$ height of water level at $t_{1}$

$h_{2}=$ height of water level at $t_{2}$

$t_{2}-t_{1}=$ duration of the permeability measurement(s).

(i) The outflow may be allowed to drain vertically under gravity fall without using the outlet reservoir. The reference height (H) should then be measured from the lowest graduate mark to the bottom of the sample. It is advisable to cover the open end of the pipette to minimize evaporation.

2. Double-pipette falling head method (Fig. B.2.c)

(a) Measure and record the inside diameter of the pipettes and of the permeameter, and the sample length (L). Calculate the crosssectional area of the pipettes $\left(a, \mathrm{~cm}^{2}\right)$ and permeameter $\left(A, \mathrm{~cm}^{2}\right)$. 
Make sure that the inflow pipette and the outflow pipette are of the same diameter and graduated length.

(b) Measure the length of the pipette graduation $\left(L_{p}\right)$. Record the total graduation volume $\left(V_{t}, c c\right)$. Calculate the length per unit volume $\left(l, \mathrm{~cm}^{-2}\right)$, i.e. $\mathrm{I}_{\mathrm{p}} / \mathrm{V}_{\mathrm{t}}$.

(c) Connect tubing and pipettes. Keep the tubing length as short as possible.

(d) Measure the height between the lowest graduation mark of the outflow pipette and that of the inflow pipette. This will be the reference height $(\mathrm{H}, \mathrm{cm})$.

(e) Record the time $t_{1}$, and read the water level in terms of the graduation marks for the inflow pipette $\left(V_{\text {in }}\right)$ and the outflow pipette $\left(V_{\text {out }}\right)$.

(f) Calculate $h_{1}$ as follows:

$$
\mathrm{h}_{1}=\mathrm{H}+\left(\mathrm{V}_{\text {out }}-\mathrm{V}_{\text {in }}\right) \ell
$$

(g) Record the time $t_{2}$ and read the water levels in both pipettes as described in step e.

(h) Calculate $h_{2}$.

$$
h_{2}=H+\left(V_{\text {out }}-V_{\text {in }}\right) \ell
$$

where $V_{\text {out }}$ and $V_{\text {in }}$ are the readings measured at time $t_{2}$.

(i) Calculate the coefficient of permeability $\mathrm{K}$ :

$$
K=\frac{a L}{2 A\left(t_{2}-t_{1}\right)} \ln \frac{h_{1}}{h_{2}}
$$

where $\quad K=$ coefficient of permeability $(\mathrm{cm} / \mathrm{s})$

$a=$ cross-sectional area of the pipette $\left(\mathrm{cm}^{2}\right)$

$A=$ cross-sectional area of the permeameter $\left(\mathrm{cm}^{2}\right)$

$L$ = sample length $(\mathrm{cm})$

$t_{1}=$ time when water in the pipette is at $h_{1}$

$t_{2}=$ time when water in the pipette is at $h_{2}$

$h_{1}=$ height of water level at $t_{1}$

$h_{2}=$ height of water level at $t_{2}$ 


\section{APPERDIX G}

FLOT TEST RESULTS OF COMPAGTED BENTONITE PLUGS

Table C.I First Test Sequence

Table C.2 Second Test Sequence 
Appendix C.I Results of Compacted Bentonite Plug Flow Testing

\begin{tabular}{|c|c|c|c|c|c|c|c|c|c|}
\hline $\begin{array}{c}\text { Elapsed } \\
\text { Time } \\
\text { (min) }\end{array}$ & $\begin{array}{l}\text { Inf low } \\
(c c)\end{array}$ & $\begin{array}{l}\text { Outflow } \\
\text { (cc) }\end{array}$ & $\begin{array}{c}\text { Permeability } \\
(\mathrm{cm} / \mathrm{s})\end{array}$ & $\begin{array}{l}\text { Inflow } \\
\text { (cc) }\end{array}$ & $\begin{array}{l}\text { Outf low } \\
(c c)\end{array}$ & $\begin{array}{c}\text { Permeability } \\
(\mathrm{cm} / \mathrm{s})\end{array}$ & $\begin{array}{l}\text { Inflow } \\
(c c)\end{array}$ & $\begin{array}{c}\text { Oute low } \\
\text { (cc) }\end{array}$ & $\begin{array}{c}\text { Permeability } \\
(\mathrm{cm} / \mathrm{s})\end{array}$ \\
\hline & \multicolumn{3}{|c|}{$B-C-1-\Lambda$} & \multicolumn{3}{|c|}{$B-C-1-B$} & \multicolumn{3}{|c|}{$B-C-2-\Lambda$} \\
\hline 1635 & 0.1150 & -0.0175 & $0.602 E-08$ & 0.0950 & 0.0275 & $0.752 E-08$ & 0.100 & 0.025 & $0.161 \mathrm{E}-08$ \\
\hline 1355 & 0.0450 & -0.0075 & $0.280 \mathrm{E}-08$ & 0.0575 & 0.0050 & $0.465 E-08$ & 0.125 & 0.075 & $0.310 E-08$ \\
\hline 1525 & 0.0550 & -0.002 .5 & $0.350 E-08$ & 0.0650 & 0.0050 & $0.465 E-08$ & 0.125 & 0.025 & $0.207 E-08$ \\
\hline 1420 & 0.0600 & -0.0050 & $0.395 \mathrm{E}-08$ & 0.0700 & 0.0100 & $0.573 \mathrm{E}-08$ & 0.100 & 0.000 & $0.148 \mathrm{E}-08$ \\
\hline 3845 & 0.1700 & 0.0050 & $0.467 \mathrm{E}-08$ & 0.2050 & 0.0300 & $0.627 \mathrm{E}-08$ & 0.250 & 0.100 & $0.192 \mathrm{E}-08$ \\
\hline 1930 & 0.0800 & 0.0025 & $0.442 \mathrm{E}-08$ & 0.0975 & 0.0225 & $0.645 E-08$ & 0.125 & 0.100 & $0.247 \mathrm{E}-08$ \\
\hline 1445 & 0.0625 & 0.0075 & $0.503 E-08$ & 0.0700 & 0.0175 & $0.632 E-08$ & 0.125 & 0.075 & $0.293 \mathrm{E}-08$ \\
\hline 1470 & 0.0750 & 0.0025 & $0.550 \mathrm{E}-08$ & 0.0850 & 0.0125 & $0.697 \mathrm{E}-08$ & 0.125 & 0.025 & $0.217 \mathrm{E}-08$ \\
\hline 1395 & 0.0725 & 0.0025 & $0.563 \mathrm{~F}-08$ & 0.0875 & 0.0150 & $0.775 E-08$ & 0.125 & 0.050 & $0.267 E-08$ \\
\hline 2900 & 0.1575 & 0.0100 & $0.610 \mathrm{E}-08$ & 0.1800 & 0.0325 & $0.782 \mathrm{E}-08$ & 0.200 & 0.050 & $0.183 \mathrm{E}-08$ \\
\hline 1410 & 0.0750 & 0.0075 & $0.622 E-08$ & 0.0825 & 0.0150 & $0.743 \mathrm{E}-08$ & 0.100 & 0.100 & $0.302 . \mathrm{E}-08$ \\
\hline 1495 & 0.0825 & 0.0000 & $0.590 \mathrm{E}-08$ & 0.0950 & 0.0200 & $0.833 E-08$ & 0.150 & 0.050 & $0.285 \mathrm{E}-08$ \\
\hline 1400 & 0.0700 & 0.0100 & $0.613 \mathrm{E}-08$ & 0.0825 & 0.0150 & $0.758 \mathrm{E}-08$ & 0.100 & 0.050 & $0.228 \mathrm{E}-08$ \\
\hline 1470 & 0.0725 & 0.0100 & $0.605 E-08$ & 0.0800 & 0.0225 & $0.765 \mathrm{E}-08$ & 0.125 & 0.000 & $0.182 \mathrm{E}-08$ \\
\hline 1405 & 0.0725 & 0.0075 & $0.617 \mathrm{E}-08$ & 0.0875 & 0.0225 & $0.865 \mathrm{E}-08$ & 0.100 & 0.050 & $0.228 \mathrm{E}-08$ \\
\hline 1385 & 0.0700 & 0.0125 & $0.648 \mathrm{E}-08$ & 0.0775 & 0.0225 & $0.802 E-08$ & 0.125 & 0.050 & $0.270 \mathrm{E}-08$ \\
\hline 1205 & 0.0625 & $\underline{0.0025}$ & $0.590 \mathrm{E}-08$ & $\underline{0.0750}$ & 0.0175 & $0.858 \mathrm{E}-08$ & $\underline{0.100}$ & $\underline{0.025}$ & $0.222 E-08$ \\
\hline $\begin{array}{l}\text { Sub- } \\
\text { total: }\end{array}$ & 1.3975 & 0.0475 & & 1.5925 & 0.3125 & & 2.200 & 0.850 & \\
\hline 1655 & 0.1625 & 0.0250 & $0.177 \mathrm{E}-07$ & 0.0300 & -0.0350 & $-0.467 \mathrm{E}-09 *$ & 0.150 & 0.075 & $0.387 \mathrm{E}-08$ \\
\hline 1585 & 0.0825 & 0.0300 & $0.112 \mathrm{E}-07$ & 0.0275 & -0.0100 & $0.170 \mathrm{E}-08$ & 0.100 & 0.100 & $0.358 \mathrm{E}-08$ \\
\hline 1245 & 0.0500 & 0.0200 & $0.893 \mathrm{E}-08$ & 0.0225 & -0.0150 & $0.930 \mathrm{E}-09$ & 0.050 & 0.000 & $0.114 \mathrm{E}-08$ \\
\hline 1500 & 0.0700 & 0.0200 & $0.960 E-08$ & 0.0225 & -0.0300 & $-0.773 E-09 *$ & 0.100 & 0.000 & $0.190 \mathrm{E}-08$ \\
\hline 2830 & 0.1150 & 0.0475 & $0.928 \mathrm{E}-08$ & 0.0500 & -0.0150 & $0.192 \mathrm{E}-08$ & 0.150 & 0.075 & $0.227 \mathrm{E}-08$ \\
\hline 1465 & 0.0675 & 0.0225 & $0.100 E-07$ & 0.0425 & -0.0000 & $0.450 E-08$ & 0.100 & 0.025 & $0.243 \mathrm{E}-08$ \\
\hline
\end{tabular}


Appendix C.I Results of Compacted Bentonite Plug Flow Testing-.-Continued

\begin{tabular}{|c|c|c|c|c|c|c|c|c|c|}
\hline $\begin{array}{l}\text { Elapsed } \\
\text { Time } \\
\text { (min) }\end{array}$ & $\begin{array}{l}\text { Inf low } \\
\text { (cc) }\end{array}$ & $\begin{array}{l}\text { Oute flow } \\
\text { (cc) }\end{array}$ & $\begin{array}{c}\text { Permeability } \\
(\mathrm{cm} / \mathrm{s})\end{array}$ & $\begin{array}{c}\text { Inflow } \\
(c c)\end{array}$ & $\begin{array}{l}\text { Outf low } \\
\text { (cc) }\end{array}$ & $\begin{array}{c}\text { Permeability } \\
(\mathrm{cm} / \mathrm{s})\end{array}$ & $\begin{array}{l}\text { Inf low } \\
(c c)\end{array}$ & $\begin{array}{l}\text { Outf low } \\
\text { (cc) }\end{array}$ & $\begin{array}{c}\text { Permeability } \\
(\mathrm{cm} / \mathrm{s})\end{array}$ \\
\hline 1805 & 0.0850 & 0.0250 & $0.100 \mathrm{E}-07$ & 0.0475 & -0.0100 & $0.323 \mathrm{E}-08$ & 0.100 & 0.075 & $0.277 \mathrm{E}-08$ \\
\hline 1055 & $\underline{0.0475}$ & 0.0175 & $0.102 E-07$ & 0.0300 & -0.0050 & $0.370 \mathrm{E}-08$ & $\underline{0.075}$ & $\underline{0.025}$ & $0.272 \mathrm{E}-08$ \\
\hline $\begin{array}{l}\text { Sub- } \\
\text { total }\end{array}$ & 0.6800 & 0.2075 & & 0.2725 & -0.1200 & & 0.825 & 0.375 & \\
\hline 1455 & 0.0675 & 0.0175 & $0.100 \mathrm{E}-07$ & 0.0450 & 0.0100 & $0.602 E-08$ & 0.075 & 0.050 & $0.248 E-08$ \\
\hline 1440 & 0.0750 & 0.0150 & $0.108 \mathrm{E}-07$ & 0.0550 & -0.0100 & $0.500 \mathrm{E}-08$ & 0.075 & 0.050 & $0.252 \mathrm{E}-08$ \\
\hline 1430 & 0.0600 & 0.0200 & $0.977 E-08$ & 0.0375 & 0.0000 & $0.420 \mathrm{E}-08$ & 0.075 & 0.000 & $0.152 \mathrm{E}-08$ \\
\hline 1460 & 0.0625 & 0.0225 & $0.102 \mathrm{E}-07$ & 0.0375 & 0.0000 & $0.413 \mathrm{D}-08$ & 0.100 & 0.000 & $0.198 \mathrm{E}-08$ \\
\hline 1470 & 0.0800 & 0.0150 & $0.115 \mathrm{E}-07$ & 0.0575 & 0.0000 & $0.632 \mathrm{E}-08$ & 0.100 & 0.050 & $0.297 \mathrm{E}-08$ \\
\hline 1420 & 0.0700 & 0.0175 & $0.110 \mathrm{E}-07$ & 0.0550 & 0.0100 & $0.743 \mathrm{E}-08$ & 0.100 & 0.050 & $0.308 E-08$ \\
\hline 1420 & 0.0725 & 0.0175 & $0.114 \mathrm{E}-07$ & 0.0550 & 0.0000 & $0.632 \mathrm{E}-08$ & 0.100 & 0.050 & $0.308 \mathrm{E}-08$ \\
\hline 1600 & 0.0825 & 0.0150 & $0.111 \mathrm{E}-07$ & 0.0650 & 0.0000 & $0.667 \mathrm{E}-08$ & 0.100 & 0.100 & $0.365 E-08$ \\
\hline 1270 & 0.0700 & 0.0150 & $0.123 \mathrm{E}-07$ & 0.0550 & 0.0000 & $0.713 E-08$ & 0.100 & -0.050 & $0.115 E-08$ \\
\hline 3020 & 0.1450 & 0.0325 & $0.109 \mathrm{E}-07$ & 0.1175 & 0.0100 & $0.702 \mathrm{E}=08$ & 0.200 & 0.100 & $0.292 \mathrm{E}-08$ \\
\hline 1285 & 0.0750 & 0.0100 & $0.124 \mathrm{E}-07$ & 0.0625 & 0.0000 & $0.815 E-08$ & 0.075 & 0.000 & $0.172 \mathrm{E}-08$ \\
\hline 1480 & 0.0775 & 0.0150 & $0.118 \mathrm{E}-07$ & 0.0650 & 0.0000 & $0.740 \mathrm{E}-08$ & 0.100 & 0.050 & $0.298 \mathrm{E}-08$ \\
\hline 1375 & 0.0675 & 0.0100 & $0.108 \mathrm{E}-07$ & 0.0600 & 0.0100 & $0.863 E-08$ & 0.125 & 0.050 & $0.375 E-08$ \\
\hline 1470 & 0.0750 & 0.0125 & $0.115 \mathrm{E}-07$ & 0.0650 & 0.0100 & $0.870 E-08$ & 0.075 & 0.000 & $0.150 \mathrm{E}-08$ \\
\hline 1480 & 0.0850 & 0.0100 & $0.125 \mathrm{E}-07$ & 0.0725 & 0.0000 & $0.840 E-08$ & 0.100 & 0.025 & $0.248 \mathrm{E}-08$ \\
\hline 1540 & 0.0825 & 0.0100 & $0.118 \mathrm{E}-07$ & 0.0750 & 0.0100 & $0.953 \mathrm{E}-08$ & 0.125 & 0.000 & $0.240 \mathrm{E}-08$ \\
\hline 1100 & 0.0575 & $\underline{0.0100}$ & $0.121 \mathrm{E}-07$ & $\underline{0.0500}$ & 0.0050 & $0.870 E-08$ & 0.050 & 0.050 & $0.268 \mathrm{E}-08$ \\
\hline $\begin{array}{l}\text { Sub- } \\
\text { total: }\end{array}$ & 1.3050 & 0.265 & & 1.0300 & 0.0550 & & 1.675 & 0.575 & \\
\hline 1595 & 0.0850 & 0.0125 & $0.123 \mathrm{E}-07$ & 0.0750 & 0.0100 & $0.940 \mathrm{E}-08$ & 0.150 & 0.050 & $0.370 \mathrm{E}-08$ \\
\hline 1510 & 0.0950 & 0.0050 & $0.134 \mathrm{E}-07$ & 0.0875 & 0.0050 & $0.109 E-07$ & 0.100 & -0.025 & $0.147 \mathrm{E}-08$ \\
\hline 1410 & 0.0800 & 0.0075 & $0.127 \mathrm{E}-07$ & 0.0725 & 0.0000 & $0.922 \mathrm{E}-08$ & 0.100 & 0.000 & $0.210 \mathrm{E}-08$ \\
\hline 1415 & 0.0725 & 0.0125 & $0.124 \mathrm{E}-07$ & 0.0675 & 0.0000 & $0.860 \mathrm{E}-08$ & 0.100 & 0.100 & $0.418 \mathrm{E}-08$ \\
\hline
\end{tabular}


Appendix C.1 Results of Compacted Bentonite Plug Flow Testing--Continued

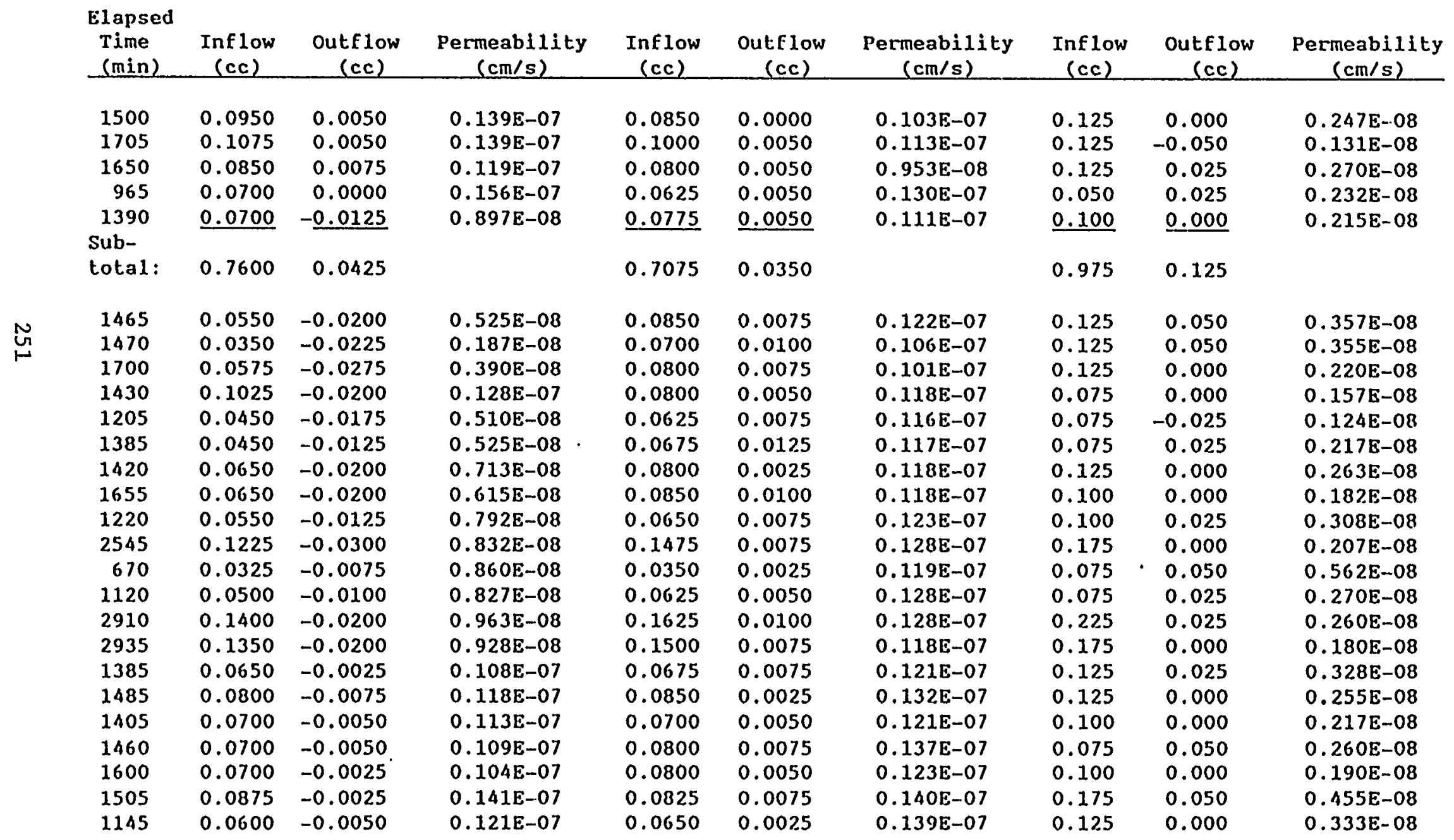


Appendix C.I Results of Compacted Dentonite Plug Flow Testing-- Continued

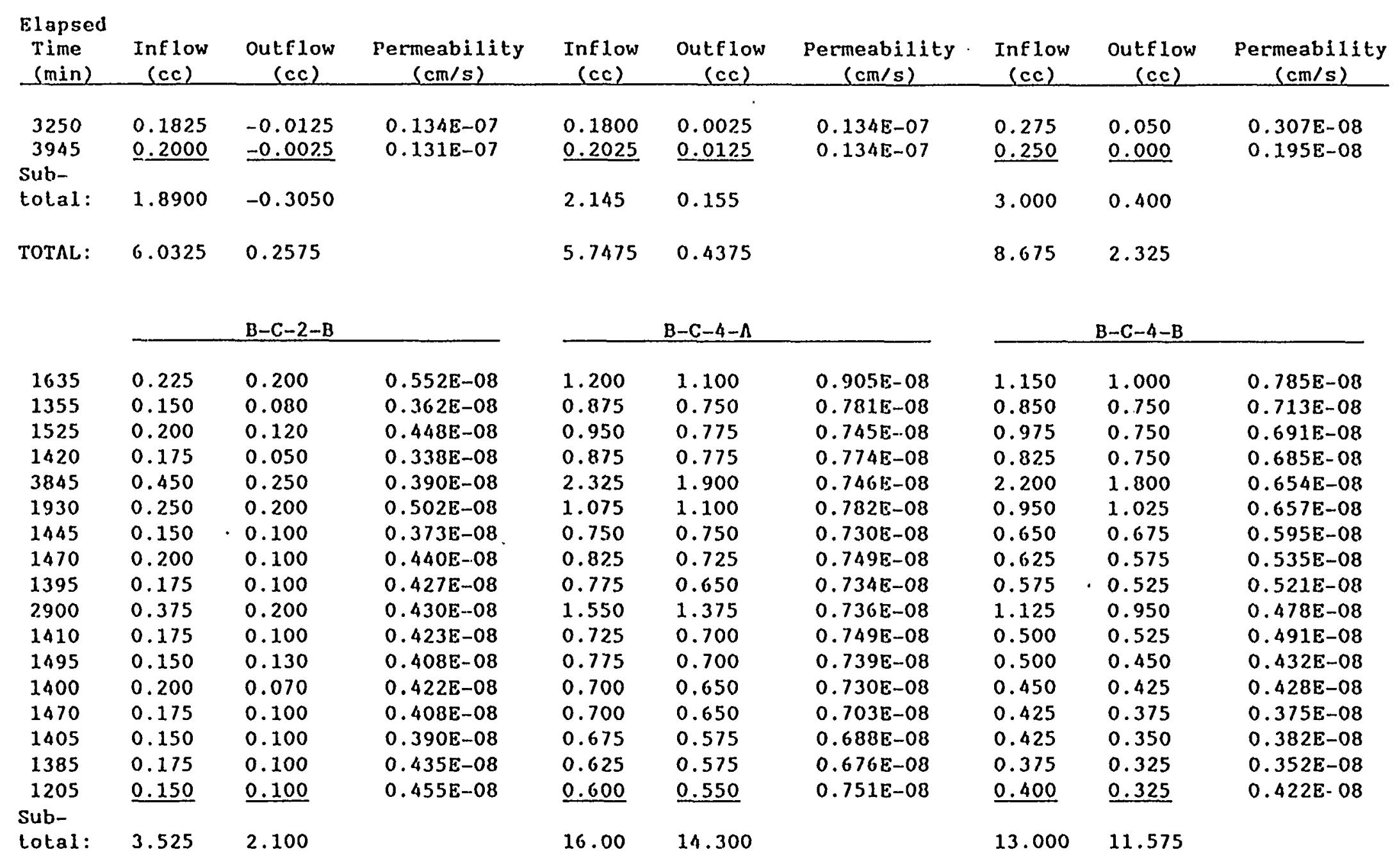


Appendix C.1 Results of Compacted Bentonite plug Flow Testing-- Continued

\begin{tabular}{|c|c|c|c|c|c|c|c|c|c|}
\hline $\begin{array}{l}\text { Elapsed } \\
\text { Time } \\
\text { (min) }\end{array}$ & $\begin{array}{l}\operatorname{In} f \text { low } \\
(c c)\end{array}$ & $\begin{array}{l}\text { Outflow } \\
\text { (cc) }\end{array}$ & $\begin{array}{c}\text { Permeability } \\
(\mathrm{cm} / \mathrm{s})\end{array}$ & $\begin{array}{l}\text { Inf low } \\
\text { (cc) }\end{array}$ & $\begin{array}{l}\text { Outf low } \\
\text { (cc) }\end{array}$ & $\begin{array}{c}\text { Permeability } \\
(\mathrm{cm} / \mathrm{s})\end{array}$ & $\begin{array}{c}\text { Inflow } \\
\text { (cc) }\end{array}$ & $\begin{array}{l}\text { Outf low } \\
\text { (cc) }\end{array}$ & $\begin{array}{c}\text { Permeability } \\
(\mathrm{cm} / \mathrm{s})\end{array}$ \\
\hline $\begin{array}{l}1655 \\
1585\end{array}$ & $\begin{array}{l}0.150 \\
0.150\end{array}$ & $\begin{array}{l}0.150 \\
0.150\end{array}$ & $\begin{array}{l}0.517 E-08 \\
0.540 E-08\end{array}$ & $\begin{array}{l}0.950 \\
0.750\end{array}$ & $\begin{array}{l}0.900 \\
0.800\end{array}$ & $\begin{array}{l}0.931 \mathrm{E}-08 \\
0.826 \mathrm{E}-08\end{array}$ & $\begin{array}{l}0.500 \\
0.400\end{array}$ & $\begin{array}{l}0.500 \\
0.400\end{array}$ & $\begin{array}{l}0.466 E-08 \\
0.392 E-08\end{array}$ \\
\hline 1245 & 0.100 & 0.000 & $0.230 E-08$ & 0.600 & 0.475 & $0.738 \mathrm{E}-08$ & 0.275 & 0.200 & $0.298 E-08$ \\
\hline 1500 & 0.150 & 0.050 & $0.382 E-08$ & 0.750 & 0.500 & $0.719 E-08$ & 0.325 & 0.175 & $0.261 \mathrm{E}-08$ \\
\hline 2830 & 0.225 & 0.150 & $0.380 E-08$ & 1.200 & 1.100 & $0.712 \mathrm{E}-08$ & 0.625 & 0.450 & $0.300 E-08$ \\
\hline 1465 & 0.125 & 0.100 & $0.442 E-08$ & 0.625 & 0.575 & $0.729 E-08$ & 0.350 & 0.300 & $0.353 \mathrm{E}-08$ \\
\hline 1805 & 0.175 & 0.100 & $0.440 E-08$ & 0.725 & 0.650 & $0.686 \mathrm{E}-08$ & 0.425 & 0.375 & $0.355 E-08$ \\
\hline 1055 & $\underline{0.100}$ & $\underline{0.080}$ & $0.493 E-08$ & 0.425 & 0.500 & $0.797 \mathrm{E}-08$ & 0.200 & 0.200 & $0.305 E-08$ \\
\hline $\begin{array}{l}\text { Sut- } \\
\text { total: }\end{array}$ & 1.175 & 0.780 & & 6.025 & 5.500 & & 3.100 & 2.600 & \\
\hline 1455 & 0.125 & 0.050 & $0.360 \mathrm{E}-08$ & 0.575 & 0.475 & $0.669 \mathrm{E}-08$ & 0.325 & 0.000 & $0.180 \mathrm{E}-08$ \\
\hline 1440 & 0.100 & 0.070 & $0.353 E-08$ & 0.525 & 0.500 & $0.666 \mathrm{E}-08$ & 0.325 & 0.100 & $0.239 E-08$ \\
\hline 1430 & 0.150 & 0.050 & $0.420 \mathrm{E}-08$ & 0.475 & 0.425 & $0.594 \mathrm{E}-08$ & 0.300 & 0.100 & $0.227 \mathrm{E}-08$ \\
\hline 1460 & 0.150 & 0.050 & $0.412 \mathrm{E}-08$ & 0.500 & 0.100 & $0.587 \mathrm{E}-08$ & 0.300 & 0.100 & $0.223 \mathrm{E}-08$ \\
\hline 1470 & 0.100 & 0.100 & $0.408 E-08$ & 0.500 & 0.450 & $0.62 .1 \mathrm{E}-08$ & 0.350 & 0.200 & $0.306 E-08$ \\
\hline 1420 & 0.150 & 0.080 & $0.488 E-08$ & 0.400 & 0.450 & $0.580 \mathrm{E}-08$ & 0.300 & 0.250 & $0.319 E-08$ \\
\hline 1420 & 0.150 & 0.070 & $0.468 E-08$ & 0.400 & 0.350 & $0.516 \mathrm{E}-08$ & 0.300 & 0.250 & $0.320 E-08$ \\
\hline 1600 & 0.150 & 0.050 & $0.378 E-08$ & 0.400 & 0.275 & $0.415 \mathrm{E}-08$ & 0.325 & 0.100 & $0.221 E-08$ \\
\hline 1270 & 0.125 & 0.080 & $0.488 \mathrm{E}-08$ & 0.325 & 0.275 & $0.467 \mathrm{E}-08$ & 0.275 & 0.200 & $0.312 \mathrm{E}-08$ \\
\hline 3020 & 0.275 & 0.120 & $0.397 \mathrm{E}-08$ & 0.725 & 0.725 & $0.480 \mathrm{E}-08$ & 0.625 & 0.500 & $0.313 E-08$ \\
\hline 1285 & 0.150 & 0.100 & $0.592 \mathrm{E}-08$ & 0.300 & 0.275 & $0.451 E-08$ & 0.275 & 0.250 & $0.346 \mathrm{E}-08$ \\
\hline 1480 & 0.100 & 0.100 & $0.412 \mathrm{E}-08$ & 0.350 & 0.350 & $0.180 \mathrm{E}-08$ & 0.325 & 0.250 & $0.330 \mathrm{E}-08$ \\
\hline 1375 & 0.050 & 0.050 & $0.222 \mathrm{E}-08$ & 0.300 & 0.250 & $0.409 \mathrm{E}-08$ & 0.275 & 0.175 & $0.279 E-08$ \\
\hline 1470 & 0.250 & 0.050 & $0.625 E-08$ & 0.300 & 0.300 & $0.4198-08$ & 0.300 & 0.225 & $0.306 E-08$ \\
\hline 1480 & 0.150 & 0.100 & $0.518 \mathrm{E}-08$ & 0.325 & 0.300 & $0.437 \mathrm{E}-08$ & - & - & - \\
\hline 1540 & 0.150 & 0.000 & $0.300 E-08$ & 0.325 & 0.175 & $0.338 E-08$ & - & - & - \\
\hline 1100 & $\underline{0.100}$ & $\underline{0.050}$ & $0.420 \mathrm{E}-08$ & 0.250 & 0.125 & $0.356 E-08$ & - & $=$ & - \\
\hline $\begin{array}{l}\text { Sub- } \\
\text { total }\end{array}$ & 2.425 & 1.170 & & 6.975 & 6.100 & & 4.600 & 2.700 & \\
\hline
\end{tabular}


Appendix C.1 Results of Compacted Bentonite Plug Flow Testing--..Continued

\begin{tabular}{|c|c|c|c|c|c|c|c|c|c|}
\hline $\begin{array}{c}\text { Elapsed } \\
\text { Time } \\
\text { (min) } \\
\end{array}$ & $\begin{array}{c}\text { Inf low } \\
(\mathrm{cc})\end{array}$ & $\begin{array}{l}\text { Outflow } \\
\text { (cc) }\end{array}$ & $\begin{array}{c}\text { Permeability } \\
(\mathrm{cm} / \mathrm{s})\end{array}$ & $\begin{array}{l}\text { Inflow } \\
(\mathrm{cc})\end{array}$ & $\begin{array}{c}\text { Outf low } \\
\text { (cc) }\end{array}$ & $\begin{array}{c}\text { Permeability } \\
(\mathrm{cm} / \mathrm{s})\end{array}$ & $\begin{array}{l}\text { Inflow } \\
(\mathrm{cc})\end{array}$ & $\begin{array}{l}\text { Outf low } \\
\text { (cc) }\end{array}$ & $\begin{array}{c}\text { Permeability } \\
(\mathrm{cm} / \mathrm{s})\end{array}$ \\
\hline 1595 & 0.200 & 0.130 & $0.632 \mathrm{E}-08$ & 0.375 & 0.450 & $0.538 \mathrm{E}-08$ & - & - & - \\
\hline 1510 & 0.125 & 0.020 & $0.293 \mathrm{E}-08$ & 0.325 & 0.100 & $0.295 E-08$ & 0.950 & - & $0.850 \mathrm{E}-08$ \\
\hline 1410 & 0.150 & 0.050 & $0.435 E-08$ & 0.275 & 0.200 & $0.355 \mathrm{E}-08$ & 0.850 & - & $0.820 \mathrm{E}-08$ \\
\hline 1415 & 0.150 & 0.100 & $0.542 \mathrm{E}-08$ & 0.300 & 0.350 & $0.486 \mathrm{E}-08$ & 0.800 & - & $0.773 \mathrm{E}-08$ \\
\hline 1500 & 0.175 & 0.050 & $0.462 \mathrm{E}-08$ & 0.300 & 0.150 & $0.320 \mathrm{E}-08$ & 0.850 & - & $0.780 E-08$ \\
\hline 1705 & 0.175 & 0.000 & $0.317 E-08$ & 0.350 & 0.150 & $0.314 \mathrm{E}-08$ & 1.000 & - & $0.813 \mathrm{E}-08$ \\
\hline 1650 & 0.150 & 0.080 & $0.430 E-08$ & 0.325 & 0.175 & $0.326 \mathrm{E}-08$ & 0.900 & - & $0.761 \mathrm{E}-08$ \\
\hline 965 & 0.100 & 0.020 & $0.383 \mathrm{E}-08$ & 0.225 & 0.200 & $0.476 \mathrm{E}-08$ & 0.550 & - & $0.800 \mathrm{E}-08$ \\
\hline 1390 & 0.175 & 0.100 & $0.612 \mathrm{E}-08$ & 0.275 & 0.200 & $0.371 \mathrm{E}-08$ & $\underline{0.775}$ & $=$ & $0.786 \mathrm{E}-08$ \\
\hline $\begin{array}{l}\text { Sub- } \\
\text { total: }\end{array}$ & 1.300 & 0.550 & & 2.750 & 1.975 & & 6.675 & & \\
\hline 1465 & 0.150 & 0.080 & $0.487 E-08$ & 0.325 & 0.275 & $0.452 \mathrm{E}-08$ & 0.750 & - & $0.741 E-08$ \\
\hline 1470 & 0.150 & 0.050 & $0.423 \mathrm{E}-08$ & 0.250 & 0.200 & $0.340 \mathrm{E}-08$ & 0.775 & - & $0.767 \mathrm{E}-08$ \\
\hline 1700 & 0.150 & 0.070 & $0.403 \mathrm{E}-08$ & 0.325 & 0.150 & $0.312 \mathrm{E}-08$ & 0.900 & - & $0.775 \mathrm{E}-08$ \\
\hline 1430 & 0.150 & 0.000 & $0.327 \mathrm{E}-08$ & 0.250 & 0.100 & $0.274 E-08$ & 0.750 & - & $0.773 \mathrm{E}-08$ \\
\hline 1205 & 0.100 & 0.030 & $0.337 \mathrm{E}-08$ & 0.250 & 0.100 & $0.327 \mathrm{E}-08$ & 0.650 & - & $0.799 \mathrm{E}-08$ \\
\hline 1385 & 0.125 & 0.100 & $0.508 \mathrm{E}-08$ & 0.250 & 0.275 & $0.429 \mathrm{E}-08$ & 0.675 & - & $0.726 \mathrm{E}-08$ \\
\hline 1420 & 0.175 & 0.070 & $0.540 \mathrm{E}-08$ & 0.300 & 0.075 & $0.300 \mathrm{E}-08$ & 0.725 & - & $0.765 \mathrm{E}-08$ \\
\hline 1655 & 0.150 & 0.030 & $0.342 \mathrm{E}-08$ & 0.275 & 0.250 & $0.362 \mathrm{E}-08$ & 0.825 & - & $0.751 \mathrm{E}-08$ \\
\hline 1220 & 0.125 & 0.070 & $0.503 \mathrm{E}-08$ & 0.225 & 0.100 & $0.306 \mathrm{E}-08$ & 0.650 & - & $0.807 \mathrm{E}-08$ \\
\hline 2545 & 0.250 & 0.080 & $0.408 \mathrm{E}-08$ & 0.500 & 0.300 & $0.363 \mathrm{E}-08$ & 1.300 & - & $0.780 \mathrm{E}-08$ \\
\hline 670 & 0.075 & 0.050 & $0.590 \mathrm{E}-08$ & 0.100 & 0.125 & $0.390 \mathrm{E}-08$ & 0.300 & - & $0.688 \mathrm{E}-08$ \\
\hline 1120 & 0.100 & 0.020 & $0.338 \mathrm{E}-08$ & 0.200 & 0.125 & $0.338 \mathrm{E}-08$ & 0.600 & - & $0.826 \mathrm{E}-08$ \\
\hline 2910 & 0.300 & 0.150 & $0.490 \mathrm{E}-08$ & 0.600 & 0.550 & $0.465 E-08$ & 1.450 & - & $0.775 \mathrm{E}-08$ \\
\hline 2935 & 0.300 & 0.100 & $0.433 \mathrm{E}-08$ & 0.475 & 0.225 & $0.283 \mathrm{E}-08$ & 1.475 & - & $0.791 \mathrm{E}-08$ \\
\hline 1385 & 0.125 & 0.030 & $0.357 \mathrm{E}-08$ & 0.225 & 0.125 & $0.302 \mathrm{E}-08$ & 0.675 & - & $0.774 E-08$ \\
\hline 1485 & 0.175 & 0.070 & $0.527 \mathrm{E}-08$ & 0.300 & 0.100 & $0.324 \mathrm{E}-08$ & 0.750 & - & $0.807 \mathrm{E}-08$ \\
\hline 1405 & 0.125 & 0.030 & $0.353 E-08$ & 0.200 & 0.075 & $0.236 \mathrm{E}-08$ & 0.675 & - & $0.772 \mathrm{E}-08$ \\
\hline
\end{tabular}


Appendix C.1 Results of Compacted Bentonite Plug Flow Testing--Continued

\begin{tabular}{|c|c|c|c|c|c|c|c|c|c|}
\hline $\begin{array}{l}\text { Elapsed } \\
\text { Time } \\
\text { _(min) }\end{array}$ & $\begin{array}{l}\text { Inf low } \\
\text { (cc) }\end{array}$ & $\begin{array}{c}\text { Outf low } \\
\text { (cc) }\end{array}$ & $\begin{array}{c}\text { Permeability } \\
(\mathrm{cm} / \mathrm{s})\end{array}$ & $\begin{array}{c}\text { Inflow } \\
(\mathrm{cc})\end{array}$ & $\begin{array}{l}\text { Outf low } \\
\text { (cc) }\end{array}$ & $\begin{array}{c}\text { Permeability } \\
(\mathrm{cm} / \mathrm{s})\end{array}$ & $\begin{array}{l}\text { Inf low } \\
(c c)\end{array}$ & $\begin{array}{l}\text { Oute low } \\
\text { (cc) }\end{array}$ & $\begin{array}{c}\text { Permeability } \\
(\mathrm{cm} / \mathrm{s})\end{array}$ \\
\hline 1460 & 0.150 & 0.070 & $0.483 E-08$ & 0.300 & 0.225 & $0.436 \mathrm{E}-08$ & 0.700 & - & $0.775 \mathrm{E}-08$ \\
\hline 1505 & 0.150 & 0.050 & $0.428 \mathrm{E}-08$ & 0.250 & 0.125 & $0.306 \mathrm{E}-08$ & 0.775 & - & $0.843 E-08$ \\
\hline 1145 & 0.125 & 0.030 & $0.437 \mathrm{E}-08$ & 0.200 & 0.075 & $0.296 \mathrm{E}-08$ & 0.575 & - & $0.826 \mathrm{E}-08$ \\
\hline 3250 & 0.325 & 0.020 & $0.343 \mathrm{E}-08$ & 0.600 & 0.325 & $0.353 \mathrm{E}-08$ & 1.625 & - & $0.831 \mathrm{E}-08$ \\
\hline 3945 & 0.375 & 0.130 & $0.415 \mathrm{E}-08$ & 0.600 & 0.275 & $0.278 \mathrm{E}-08$ & 2.050 & $=$ & $0.877 \mathrm{E}-08$ \\
\hline TOTAL: & 12.425 & 6.030 & & 39.000 & 32.375 & & 47.750 & - & \\
\hline
\end{tabular}

$*_{\Lambda}$ negative permeability value has no physical meaning. 
Table C.2.1 Flow Test Record for Sample B-C-1-A.

\begin{tabular}{|c|c|c|c|c|}
\hline$\stackrel{\Delta T}{(M i n)}$ & $\begin{array}{l}\text { Cumu- } \\
\text { lative } \\
\text { Time } \\
\text { (Days) }\end{array}$ & $\begin{array}{c}\text { Inflow } \\
(c c)\end{array}$ & $\begin{array}{l}\text { Outflow } \\
\text { (cc) }\end{array}$ & $\begin{array}{c}\mathrm{K} \\
\left(\times 10^{-7}\right. \\
\mathrm{cm} / \mathrm{s})\end{array}$ \\
\hline $\begin{array}{l}1600 \\
1470\end{array}$ & $\begin{array}{l}1.111 \\
2.132\end{array}$ & $\begin{array}{l}0.23 \\
0.14\end{array}$ & $\begin{array}{l}0.05 \\
0.05\end{array}$ & $\begin{array}{l}0.3020 \\
0.2268\end{array}$ \\
\hline 1440 & 3.132 & 0.16 & 0.05 & 0.2537 \\
\hline 1390 & 4.097 & 0.13 & 0.05 & 0.2287 \\
\hline 1660 & 5.250 & 0.14 & 0.05 & 0.2112 \\
\hline 1270 & 6.132 & 0.11 & 0.04 & 0.2148 \\
\hline 1555 & 7.212 & 0.12 & 0.05 & 0.1991 \\
\hline 1325 & 8.132 & 0.13 & 0.04 & 0.2446 \\
\hline 1410 & 9.111 & 0.11 & 0.04 & 0.2025 \\
\hline 1250 & 9.979 & 0.10 & 0.03 & 0.2080 \\
\hline 1670 & 11.139 & 0.14 & 0.04 & 0.2118 \\
\hline 2485 & 12.865 & 0.32 & 0.06 & 0.3134 \\
\hline 1800 & 14.115 & 0.00 & 0.04 & 0.0465 \\
\hline 1445 & 15.118 & 0.11 & 0.03 & 0.2048 \\
\hline 1435 & 16.115 & 0.11 & 0.03 & 0.2094 \\
\hline 1440 & 17.115 & 0.10 & 0.03 & 0.1966 \\
\hline 1396 & 18.084 & 0.10 & 0.02 & 0.1937 \\
\hline 1405 & 19.060 & 0.08 & 0.03 & 0.1751 \\
\hline 1395 & 20.028 & 0.09 & 0.03 & 0.1908 \\
\hline 1610 & 21.147 & 0.12 & 0.03 & 0.2107 \\
\hline 1440 & 22.147 & 0.10 & 0.02 . & 0.1987 \\
\hline 1355 & 23.088 & 0.09 & $0.03^{\circ}$ & 0.2098 \\
\hline 1480 & 24.115 & 0.10 & 0.02 & 0.1908 \\
\hline 2900 & 26.129 & 0.18 & 0.05 & 0.1904 \\
\hline 2865 & 28.119 & 0.18 & 0.04 & 0.1937 \\
\hline 1365 & 29.067 & 0.09 & 0.02 & 0.2028 \\
\hline 1505 & 30.112 & 0.10 & 0.02 & 0.2040 \\
\hline 1445 & 31.115 & 0.10 & 0.02 & 0.2065 \\
\hline 1705 & 32.299 & 0.11 & 0.02 & 0.2095 \\
\hline 2580 & 34.091 & 0.14 & 0.04 & 0.1868 \\
\hline 1480 & 35.119 & 0.10 & 0.02 & 0.2279 \\
\hline 1450 & 36.126 & 0.09 & 0.01 & 0.2068 \\
\hline 1465 & 37.143 & 0.10 & 0.01 & 0.2078 \\
\hline 1475 & 38.167 & 0.09 & 0.02 & 0.2196 \\
\hline 2845 & 40.143 & 0.16 & 0.03 & 0.2011 \\
\hline 1648 & 41.287 & 0.11 & 0.01 & 0.2292 \\
\hline 2637 & 43.119 & $\underline{0.20}$ & $\underline{0.00}$ & 0.2429 \\
\hline \multicolumn{2}{|c|}{ Totals: } & 4.59 & 1.14 & \\
\hline
\end{tabular}


Table C.2.2 Flow Test Record for Sample $\mathrm{B}-\mathrm{C}-1-\mathrm{B}$.

\begin{tabular}{|c|c|c|c|c|}
\hline $\begin{array}{c}\Delta T \\
(\mathrm{Min})\end{array}$ & $\begin{array}{l}\text { Cumu- } \\
\text { lative } \\
\text { Time } \\
\text { (Days) }\end{array}$ & $\begin{array}{l}\text { Inflow } \\
\text { (cc). }\end{array}$ & $\begin{array}{l}\text { Outflow } \\
\text { (cc) }\end{array}$ & $\begin{array}{c}K \\
\left(\times 10^{-7}\right. \\
\mathrm{cm} / \mathrm{s})\end{array}$ \\
\hline 1600 & 1.111 & 0.23 & 0.05 & 0.2914 \\
\hline 1470 & 2.132 & 0.15 & 0.05 & 0.2263 \\
\hline 1440 & 3.132 & 0.12 & 0.04 & 0.1930 \\
\hline 1390 & 4.097 & 0.13 & 0.04 & 0.2216 \\
\hline 1660 & 5.250 & 0.13 & 0.05 & 0.1912 \\
\hline 1270 & 6.132 & 0.10 & 0.03 & 0.1971 \\
\hline 1555 & 7.212 & 0.13 & 0.05 & 0.1983 \\
\hline 1325 & 8.132 & 0.11 & 0.04 & 0.2153 \\
\hline 1410 & 9.111 & 0.11 & 0.03 & 0.1853 \\
\hline 1250 & 9.979 & 0.10 & 0.02 & 0.1776 \\
\hline 1670 & 11.139 & 0.13 & 0.04 & 0.1921 \\
\hline 2485 & 12.865 & 0.17 & 0.01 & 0.1470 \\
\hline 1800 & 14.115 & 0.13 & 0.08 & 0.2318 \\
\hline 1445 & 15.118 & 0.11 & 0.03 & 0.1889 \\
\hline 1435 & 16.115 & 0.10 & 0.03 & 0.1784 \\
\hline 1440 & 17.115 & 0.09 & 0.02 & 0.1656 \\
\hline 1396 & 18.084 & 0.09 & 0.02 & 0.1729 \\
\hline 1405 & 19.060 & 0.08 & 0.03 & 0.1625 \\
\hline 1395 & 20.028 & 0.09 & 0.03 & 0.1772 \\
\hline 1610 & 21.147 & 0.11 & 0.03 & 0.1827 \\
\hline 1440 & 22.147 & 0.10 & 0.02 & 0.1880 \\
\hline 1355 & 23.088 & 0.08 & 0.02 & 0.1693 \\
\hline 1480 & 24.115 & 0.10 & 0.02 & 0.1761 \\
\hline 2900 & 26.129 & 0.17 & 0.05 & 0.1752 \\
\hline 2865 & 28.119 & 0.17 & 0.03 & 0.1695 \\
\hline 1365 & 29.067 & 0.08 & 0.02 & 0.1678 \\
\hline 1505 & 30.112 & 0.09 & 0.01 & 0.1744 \\
\hline 1445 & 31.115 & 0.09 & 0.02 & 0.1926 \\
\hline 1705 & 32.299 & 0.11 & 0.02 & 0.1767 \\
\hline 2580 & 34.091 & 0.13 & 0.04 & 0.1635 \\
\hline 1480 & 35.119 & 0.09 & 0.01 & 0.1890 \\
\hline 1450 & 36.126 & 0.09 & 0.01 & 0.1819 \\
\hline 1465 & 37.143 & 0.09 & 0.01 & 0.1825 \\
\hline 1475 & 38.167 & 0.08 & 0.01 & 0.1745 \\
\hline 2845 & 40.143 & 0.16 & 0.03 & 0.1796 \\
\hline 1648 & 41.287 & 0.11 & 0.01 & 0.1925 \\
\hline 2637 & 43.119 & 0.18 & -0.01 & 0.1938 \\
\hline \multicolumn{2}{|c|}{ Totals: } & 4.34 & 1.04 & \\
\hline
\end{tabular}


Table C.2.3 Flow Test Record for Sample B-C-2-A.

\begin{tabular}{|c|c|c|c|c|}
\hline$\stackrel{\Delta T}{(\operatorname{Min})}$ & $\begin{array}{l}\text { Cumu- } \\
\text { lative } \\
\text { Time } \\
\text { (Days) }\end{array}$ & $\begin{array}{l}\text { Inflow } \\
(c c)\end{array}$ & $\begin{array}{l}\text { Outflow } \\
\text { (cc) }\end{array}$ & $\begin{array}{c}K \\
\left(\times 10^{-7}\right. \\
\mathrm{cm} / \mathrm{s})\end{array}$ \\
\hline 1600 & 1.111 & 0.22 & 0.10 & 0.0592 \\
\hline 1470 & 2.132 & 0.15 & 0.10 & 0.0497 \\
\hline 1440 & 3.132 & 0.15 & 0.08 & 0.0457 \\
\hline 1390 & 4.097 & 0.15 & 0.05 & 0.0422 \\
\hline 1660 & 5.250 & 0.15 & 0.05 & 0.0354 \\
\hline 1270 & 6.132 & 0.15 & 0.05 & 0.0463 \\
\hline 1555 & 7.212 & 0.15 & 0.08 & 0.0426 \\
\hline 1325 & 8.132 & 0.10 & 0.07 & 0.0390 \\
\hline 1410 & 9.111 & 0.13 & 0.00 & 0.0262 \\
\hline 1250 & 9.979 & 0.15 & 0.08 & 0.0533 \\
\hline 1670 & 11.139 & 0.13 & 0.05 & 0.0311 \\
\hline 2485 & 12.865 & 0.22 & 0.08 & 0.0359 \\
\hline 1800 & 14.115 & 0.15 & 0.10 & 0.0414 \\
\hline 1445 & 15.118 & 0.13 & 0.05 & 0.0361 \\
\hline 1435 & 16.115 & 0.10 & 0.02 & 0.0260 \\
\hline 1440 & 17.115 & 0.13 & 0.00 & 0.0260 \\
\hline 1396 & 18.084 & 0.13 & 0.03 & 0.0322 \\
\hline 1405 & 19.060 & 0.10 & 0.00 & 0.0213 \\
\hline 1395 & 20.028 & 0.13 & 0.03 & 0.0323 \\
\hline 1610 & 21.147 & 0.13 & 0.05 & 0.0327 \\
\hline 1440 & 22.147 & 0.13 & 0.05 & 0.0366 \\
\hline 1355 & 23.088 & 0.10 & 0.08 & 0.0389 \\
\hline 1480 & 24.115 & 0.10 & 0.00 & 0.0204 \\
\hline 2900 & 26.129 & 0.25 & 0.08 & 0.0339 \\
\hline 2865 & 28.119 & 0.22 & 0.02 & 0.0265 \\
\hline 1365 & 29.067 & 0.10 & 0.00 & 0.0222 \\
\hline 1505 & 30.112 & 0.10 & 0.00 & 0.0202 \\
\hline 1445 & 31.115 & 0.10 & 0.03 & 0.0263 \\
\hline 1705 & 32.299 & 0.15 & 0.05 & 0.0357 \\
\hline 2580 & 34.091 & 0.18 & 0.05 & 0.0266 \\
\hline 1480 & 35.119 & 0.13 & 0.05 & 0.0361 \\
\hline 1450 & 36.126 & 0.13 & 0.03 & 0.0317 \\
\hline 1465 & 37.143 & 0.10 & 0.00 & 0.0209 \\
\hline 1475 & 38.167 & 0.13 & 0.00 & 0.0260 \\
\hline 2845 & 40.143 & 0.20 & 0.10 & 0.0324 \\
\hline 1648 & 41.287 & 0.13 & 0.10 & 0.0421 \\
\hline 2637 & 43.119 & 0.28 & 0.00 & 0.0322 \\
\hline \multicolumn{2}{|c|}{ Totals: } & 5.33 & 1.67 & \\
\hline
\end{tabular}


Table C.2.4 Flow Test Record for Sample $\mathrm{B}-\mathrm{C}-2-\mathrm{B}$.

\begin{tabular}{|c|c|c|c|c|}
\hline 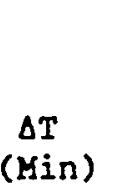 & $\begin{array}{l}\text { Cumu- } \\
\text { lative } \\
\text { Time } \\
\text { (Days) }\end{array}$ & $\begin{array}{l}\text { Inflow } \\
\text { (cc) }\end{array}$ & $\begin{array}{l}\text { Outf low } \\
\text { (cc) }\end{array}$ & $\begin{array}{c}\mathrm{K} \\
\left(\times 10^{-7}\right. \\
\mathrm{cm} / \mathrm{s})\end{array}$ \\
\hline 1600 & 1.111 & 0.10 & 0.05 & 0.0279 \\
\hline 1470 & 2.132 & 0.15 & 0.10 & 0.0506 \\
\hline 1440 & 3.132 & 0.17 & 0.05 & 0.0466 \\
\hline 1390 & 4.097 & 0.15 & 0.05 & 0.0430 \\
\hline 1660 & 5.250 & 0.17 & 0.05 & 0.0406 \\
\hline 1270 & 6.132 & 0.15 & 0.05 & 0.0472 \\
\hline 1555 & 7.212 & 0.15 & 0.07 & 0.0435 \\
\hline 1325 & 8.132 & 0.15 & 0.05 & 0.0454 \\
\hline 1410 & 9.111 & 0.13 & 0.05 & 0.0374 \\
\hline 1250 & 9.979 & 0.15 & 0.08 & 0.0543 \\
\hline 1670 & 11.139 & 0.15 & 0.05 & 0.0362 \\
\hline 2485 & 12.865 & 0.28 & 0.08 & 0.0427 \\
\hline 1800 & 14.115 & 0.20 & 0.13 & 0.0549 \\
\hline 1445 & 15.118 & 0.13 & 0.07 & 0.0422 \\
\hline 1435 & 16.115 & 0.15 & 0.03 & 0.0372 \\
\hline 1440 & 17.115 & 0.15 & 0.05 & 0.0425 \\
\hline 1396 & 18.084 & 0.13 & 0.03 & 0.0329 \\
\hline 1405 & 19.060 & 0.15 & 0.05 & 0.0436 \\
\hline 1395 & 20.028 & 0.13 & 0.05 & 0.0385 \\
\hline 1610 & 21.147 & 0.15 & 0.10 & 0.0478 \\
\hline 1440 & 22.147 & 0.15 & 0.02 & 0.0375 \\
\hline 1355 & 23.088 & 0.13 & 0.08 & 0.0467 \\
\hline 1480 & 24.115 & 0.14 & 0.05 & 0.0408 \\
\hline 2900 & 26.129 & 0.27 & $0.10^{\circ}$ & 0.0401 \\
\hline 2865 & 28.119 & 0.28 & 0.07 & 0.0380 \\
\hline 1365 & 29.067 & 0.15 & 0.03 & 0.0400 \\
\hline 1505 & 30.112 & 0.13 & 0.05 & 0.0363 \\
\hline 1445 & 31.115 & 0.15 & 0.08 & 0.0487 \\
\hline 1705 & 32.299 & 0.17 & 0.05 & 0.0414 \\
\hline 2580 & 34.091 & 0.25 & 0.10 & 0.0426 \\
\hline 1480 & 35.119 & 0.13 & 0.07 & 0.0426 \\
\hline 1450 & 36.126 & 0.15 & 0.03 & 0.0381 \\
\hline 1465 & 37.143 & 0.15 & 0.05 & 0.0432 \\
\hline 1475 & 38.167 & 0.13 & 0.03 & 0.0322 \\
\hline 2845 & 40.143 & 0.28 & 0.17 & 0.0502 \\
\hline 1648 & 41.287 & 0.17 & 0.13 & 0.0580 \\
\hline 2637 & 43.119 & $\underline{0.28}$ & $\underline{0.03}$ & 0.0363 \\
\hline \multicolumn{2}{|c|}{ Totals: } & 6.18 & 2.35 & \\
\hline
\end{tabular}


Table C.2.5 Flow Test Record for Sample $B-C-4-A$.

\begin{tabular}{|c|c|c|c|c|}
\hline$\underset{(\operatorname{Min})}{\Delta \mathrm{T}}$ & $\begin{array}{l}\text { Cumu- } \\
\text { lative } \\
\text { Time } \\
\text { (Days) }\end{array}$ & $\begin{array}{l}\text { Inflow } \\
\text { (cc) }\end{array}$ & $\begin{array}{l}\text { Outf low } \\
\text { (cc) }\end{array}$ & $\begin{array}{c}K \\
\left(\times 10^{-7}\right. \\
\mathrm{cm} / \mathrm{s})\end{array}$ \\
\hline 1600 & 1.111 & 0.40 & 0.25 & 0.0395 \\
\hline 1470 & 2.132 & 0.33 & 0.20 & 0.0349 \\
\hline 1440 & 3.132 & 0.30 & 0.13 & 0.0290 \\
\hline 1390 & 4.097 & 0.33 & 0.18 & 0.0355 \\
\hline 1660 & 5.250 & 0.30 & 0.10 & 0.0239 \\
\hline 1270 & 6.132 & 0.25 & 0.10 & 0.0274 \\
\hline 1555 & 7.212 & 0.33 & 0.23 & 0.0354 \\
\hline 1325 & 8.132 & 0.28 & 0.22 & 0.0379 \\
\hline 1410 & 9.111 & 0.30 & 0.20 & 0.0358 \\
\hline 1250 & 9.979 & 0.22 & 0.10 & 0.0260 \\
\hline 1650 & 11.125 & 0.35 & 0.10 & 0.0268 \\
\hline 2485 & 12.851 & 0.47 & 0.15 & 0.0251 \\
\hline 1800 & 14.101 & 0.35 & 0.38 & 0.0405 \\
\hline 1445 & 15.104 & 0.27 & 0.25 & 0.0368 \\
\hline 1435 & 16.101 & 0.28 & 0.10 & 0.0266 \\
\hline 1440 & 17.101 & 0.22 & 0.13 & 0.0248 \\
\hline 1396 & 18.070 & 0.28 & 0.05 & 0.0238 \\
\hline 1405 & 19.046 & 0.22 & 0.15 & 0.0274 \\
\hline 1395 & 20.015 & 0.25 & 0.10 & 0.0259 \\
\hline 1610 & 21.133 & 0.30 & 0.22 & 0.0338 \\
\hline 1440 & 22.133 & 0.25 & 0.28 & 0.0380 \\
\hline 1355 & 23.074 & 0.27 & 0.20 & 0.0363 \\
\hline 1480 & 24.101 & 0.13 & 0.10 & 0.0163 \\
\hline 2900 & 26.115 & 0.50 & 0.33 & 0.0301 \\
\hline 2865 & 28.105 & 0.50 & 0.20 & 0.0260 \\
\hline 1365 & 29.053 & 0.23 & 0.02 & 0.0196 \\
\hline 1505 & 30.098 & 0.27 & 0.10 & 0.0268 \\
\hline 1445 & 31.101 & 0.25 & 0.23 & 0.0355 \\
\hline 1705 & 32.285 & 0.30 & 0.23 & 0.0334 \\
\hline 2580 & 34.077 & 0.40 & 0.30 & 0.0296 \\
\hline 1480 & 35.105 & 0.25 & 0.20 & 0.0334 \\
\hline 1450 & 36.112 & 0.22 & 0.10 & 0.0247 \\
\hline 1465 & 37.129 & 0.28 & 0.05 & 0.0246 \\
\hline 1475 & 38.153 & 0.22 & 0.15 & 0.0283 \\
\hline 2845 & 40.129 & 0.48 & 0.50 & 0.0384 \\
\hline 1648 & 41.274 & 0.30 & 0.40 & 0.0480 \\
\hline 2637 & 43.105 & 0.40 & 0.10 & 0.0216 \\
\hline \multicolumn{2}{|c|}{ Totals: } & 11.27 & 6.80 & \\
\hline
\end{tabular}


APPENDIX D

FLON TEST RESULTS OF THE 10.16 CY DIAKETER

YIXTURE SAKTIES INSTALI.ED IN PVC PERKRAKITTERS

(First Test Sequence) 


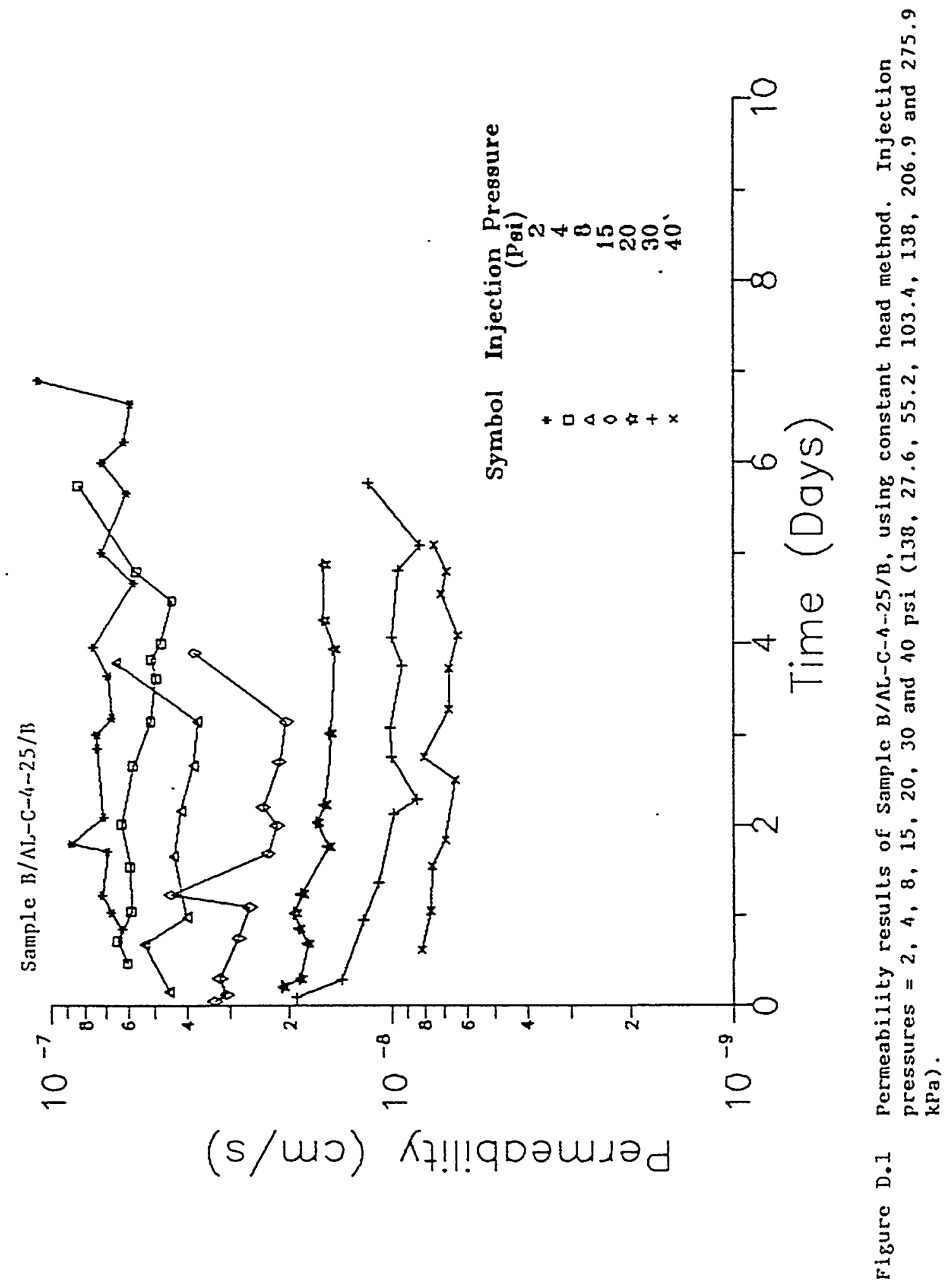




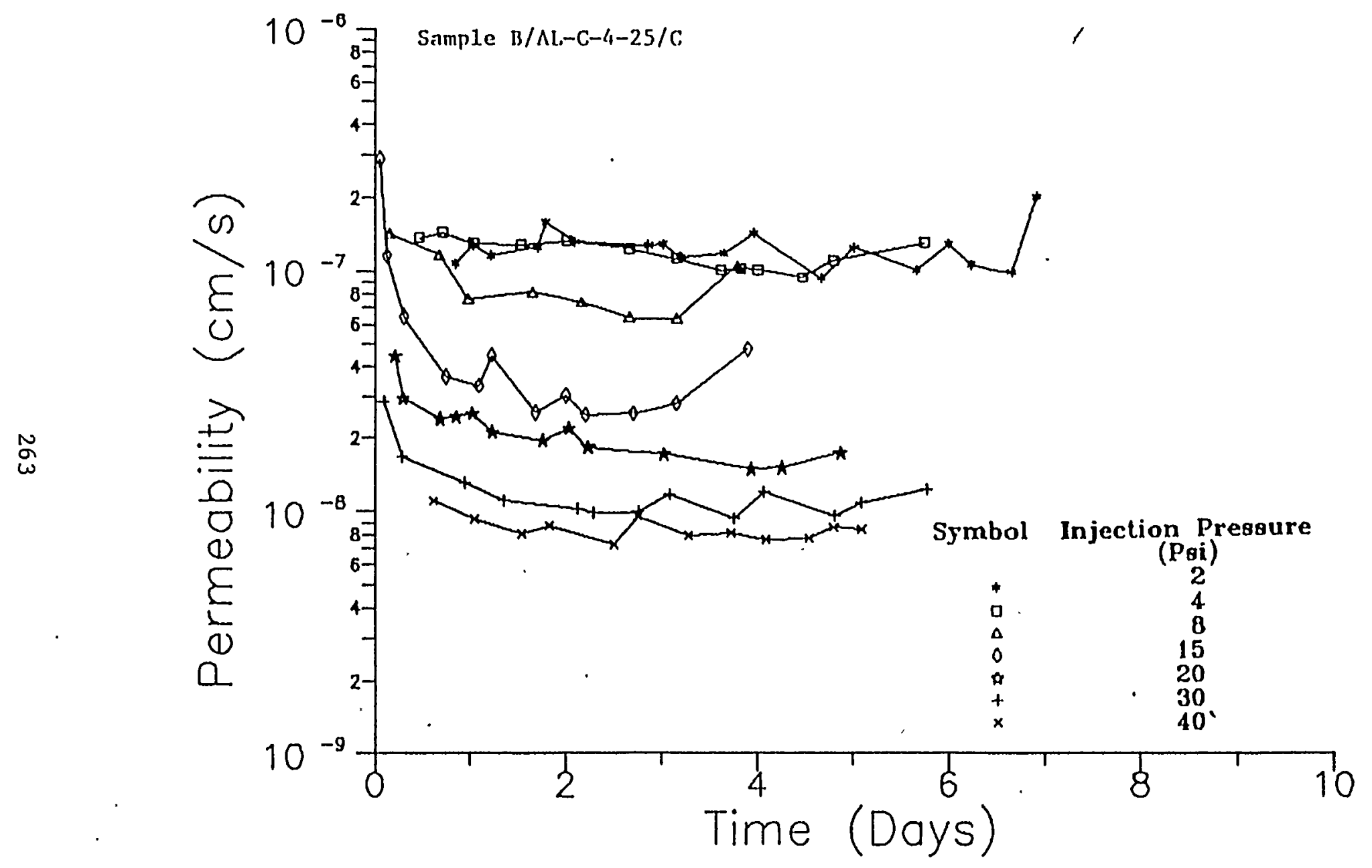

Figure D.2 Permeability results of Sample B/AL-C-1-25/C, using constant head method. Injection pressures $=2,4,8,15,20,30$ and $40 \mathrm{psi}(138,27.6,55.2,103.4,138,206.9$ and 275.9 $\mathrm{kPa}$ ). 


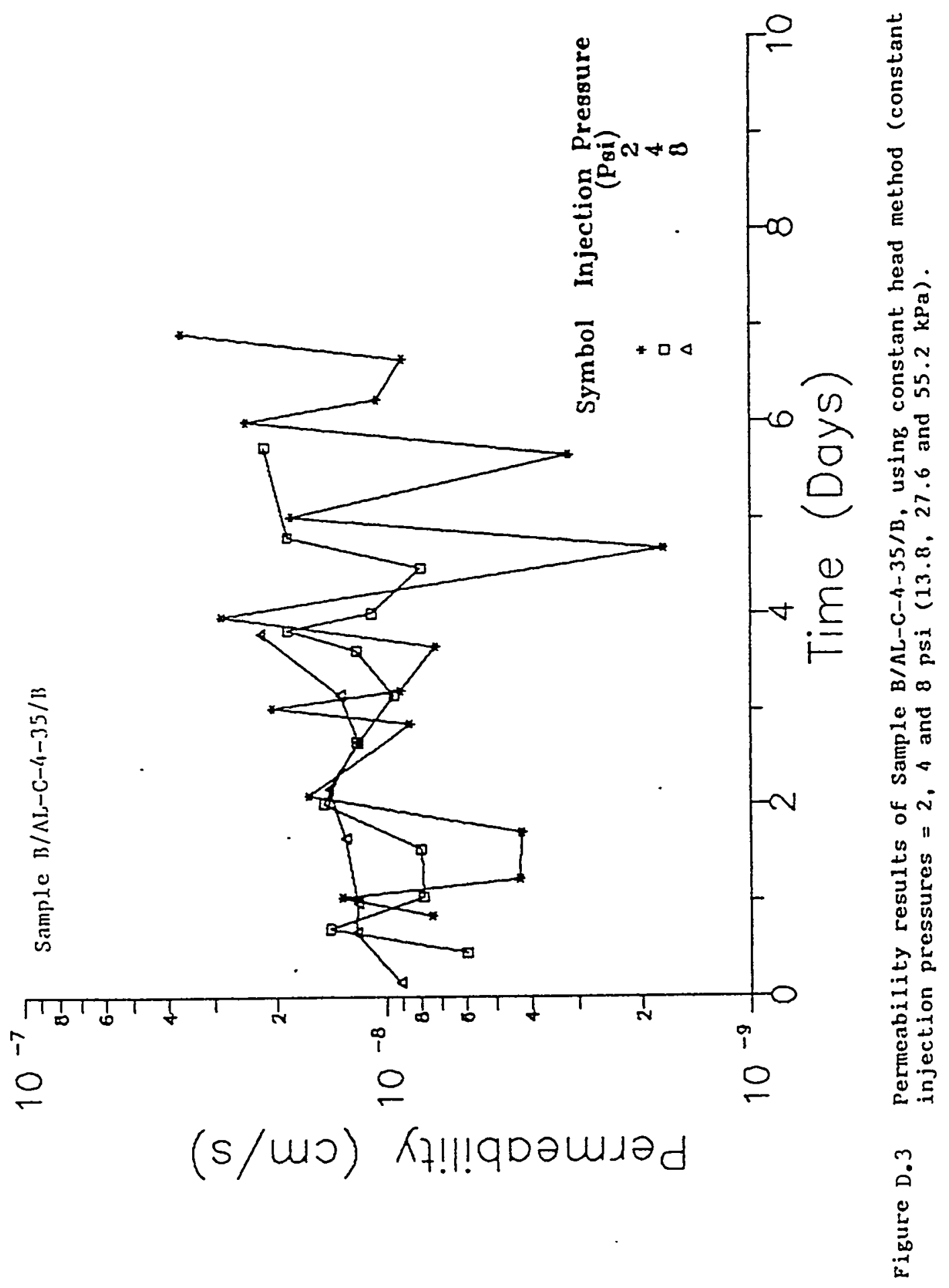




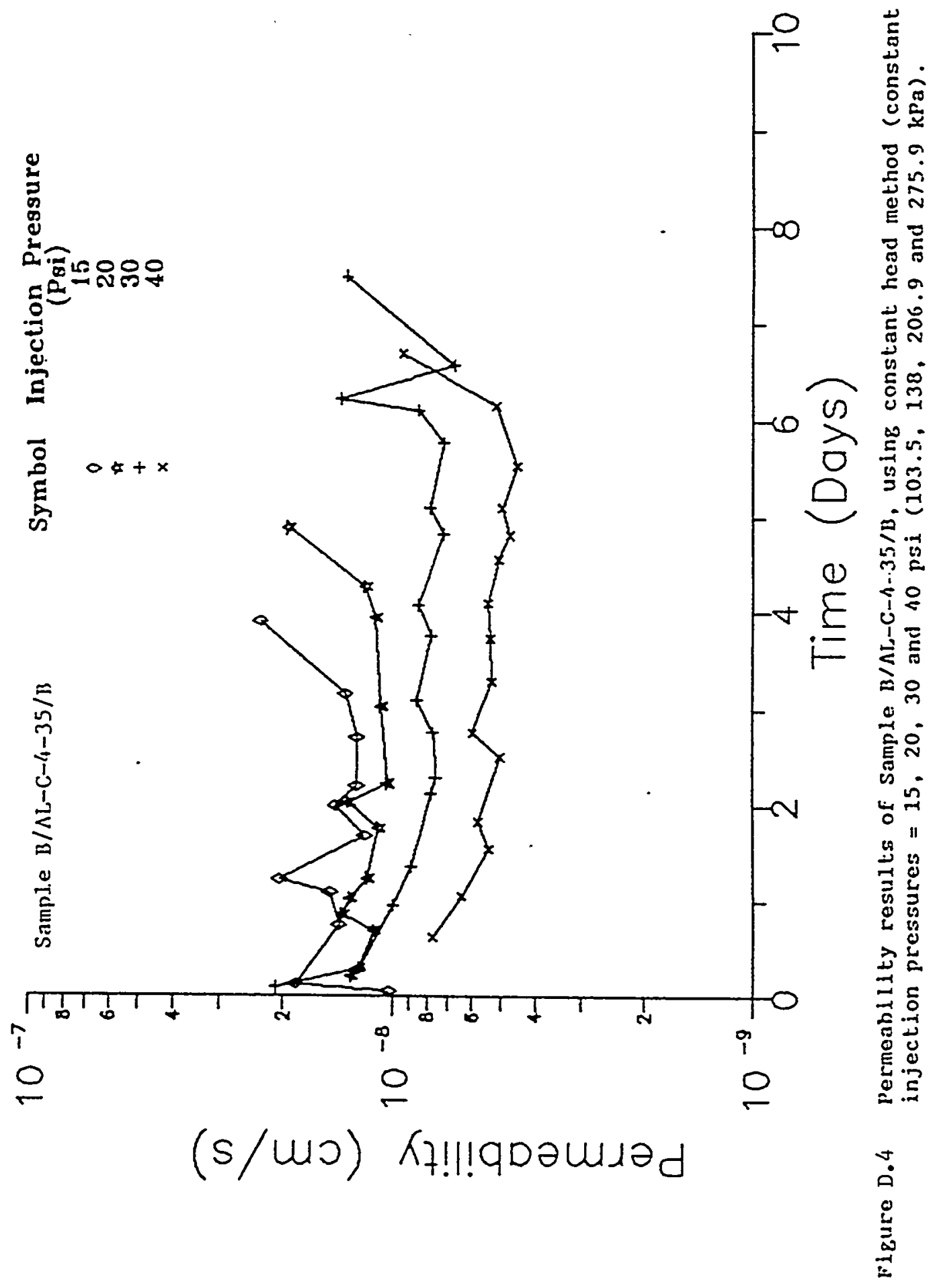



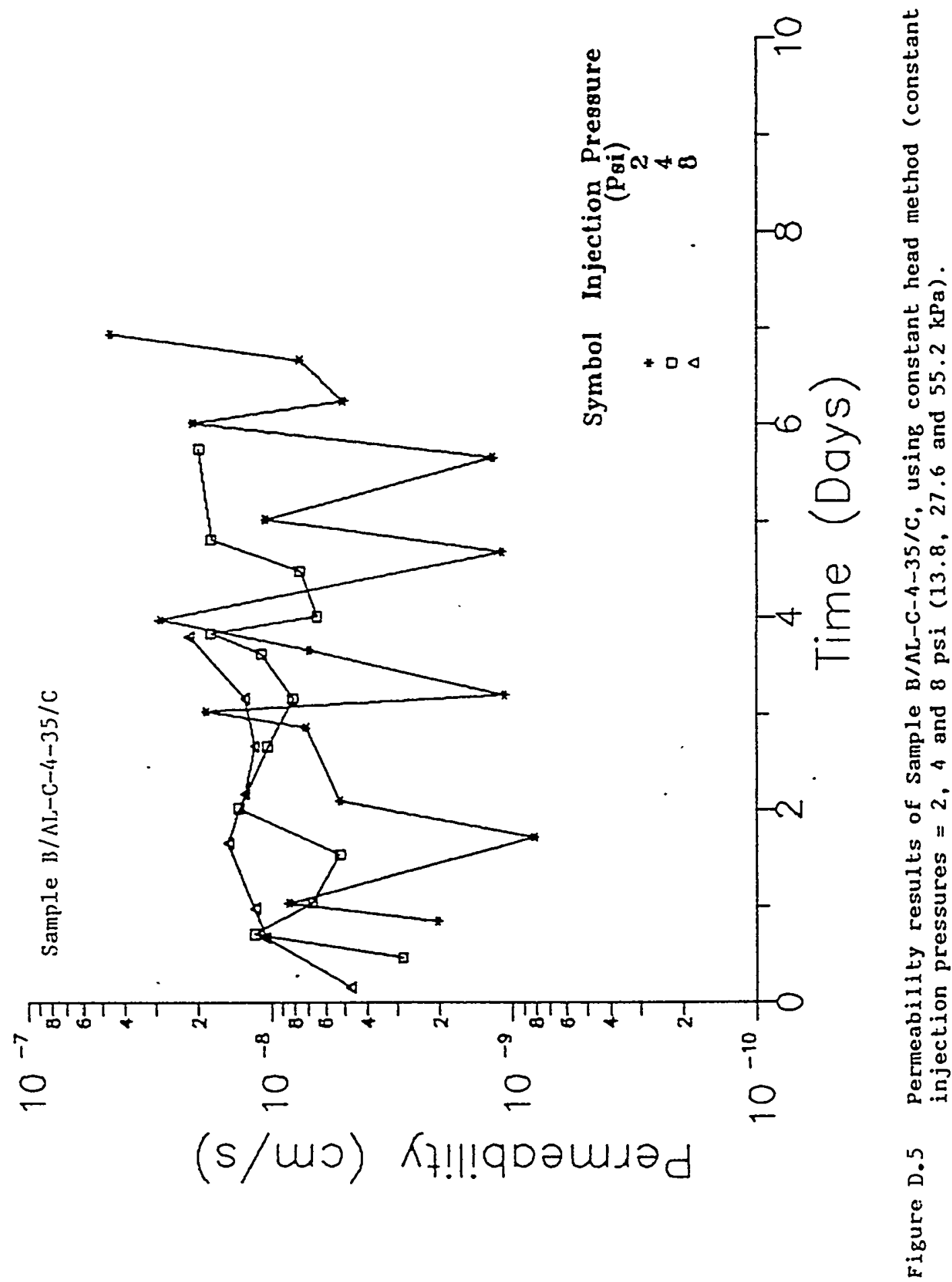


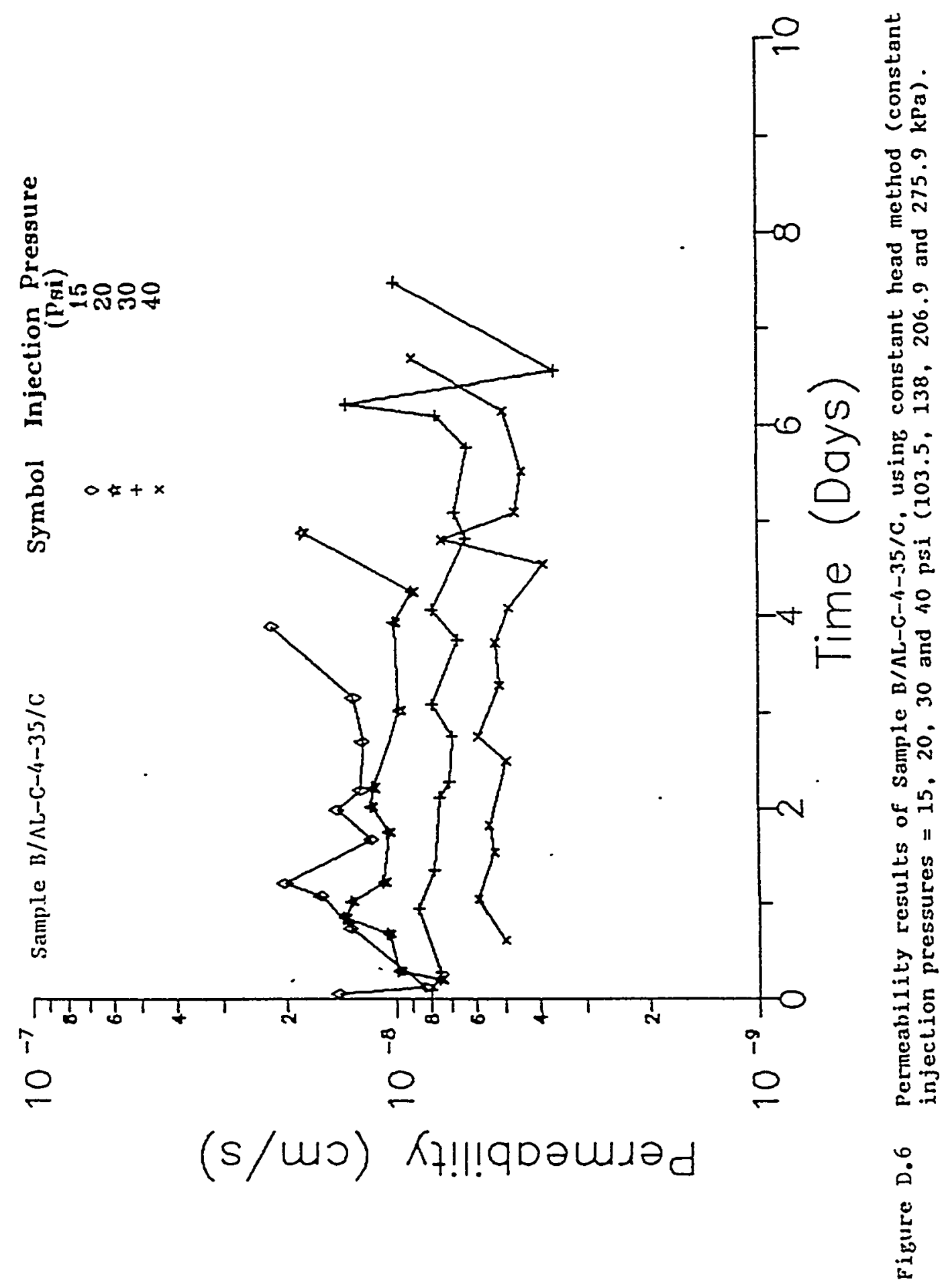




\section{APPERDIX E}

FLOV TEST RESULTS OF THE 10.16 GE DTAKETER

YIXITURE SAYTILS INSTALIED IN PVG PERKTRAYRTERS

(Second and Third Test Sequences) 


\section{Sample B/AL-C-4-25/C}

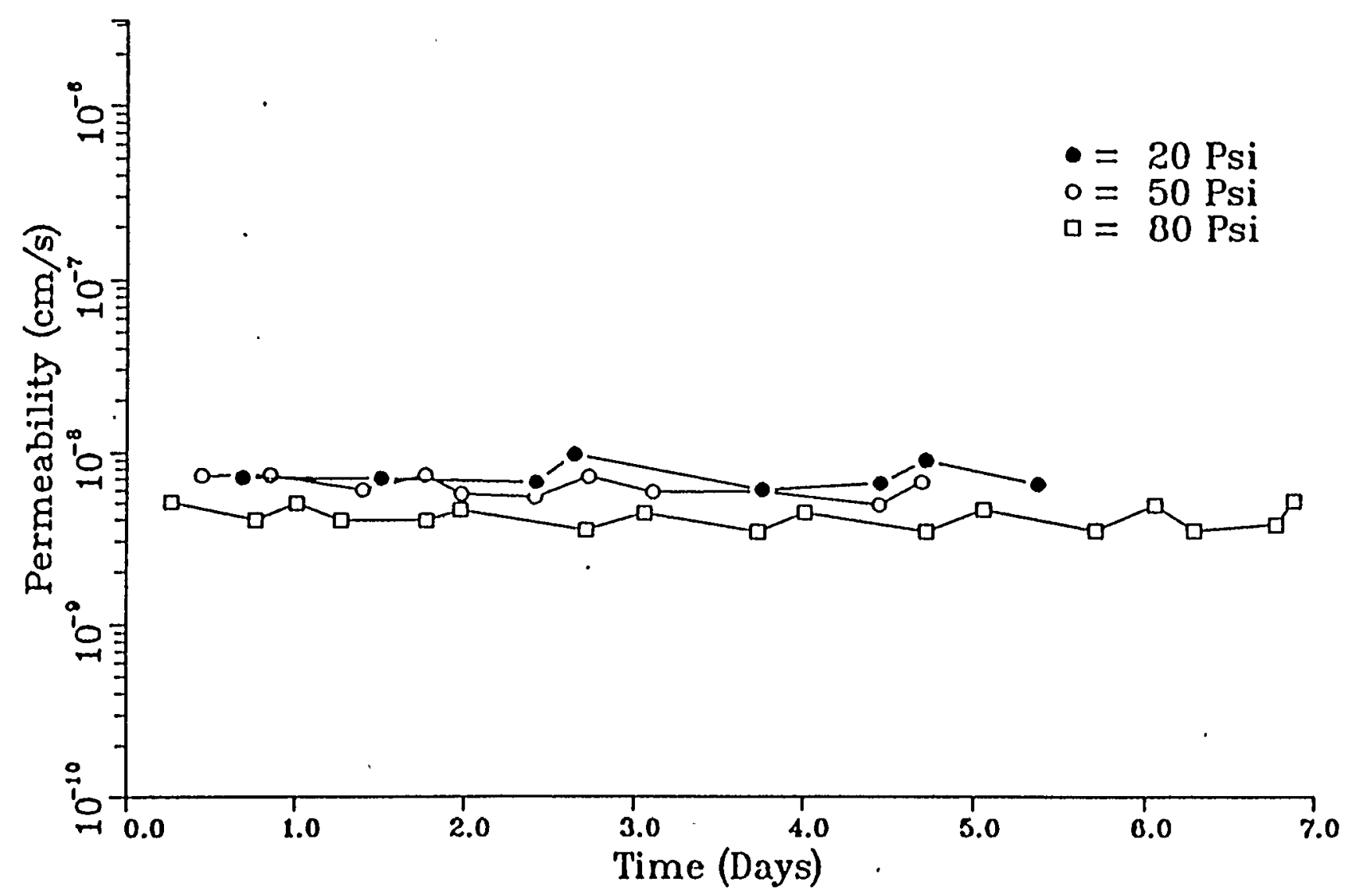

Figure E.1 Permeability results of sample B/AL-C-4-25/C at injection pressures $=138$, 345 and $552 \mathrm{kPa}(20,50$ and $80 \mathrm{psi})$. 


\section{Sample B/AL-C-4-25/C}

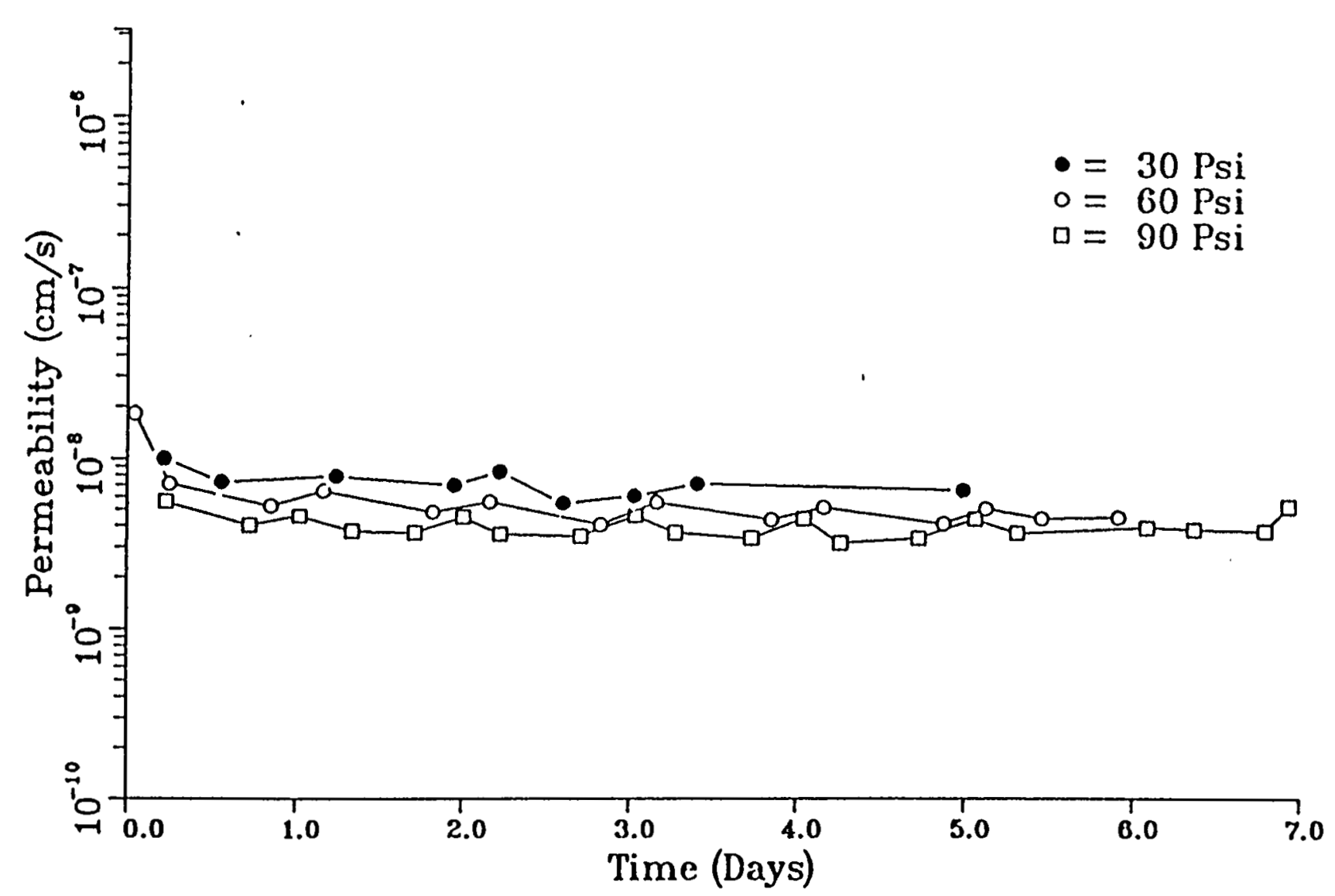

Figure E.2 Permeability results of Sample B/AL-C-4-25/C at injection pressures $=207$, 414 and $621 \mathrm{kPa}(30,60$ and $90 \mathrm{psi})$. 


\section{Sample B/AL-C-4-25/C}

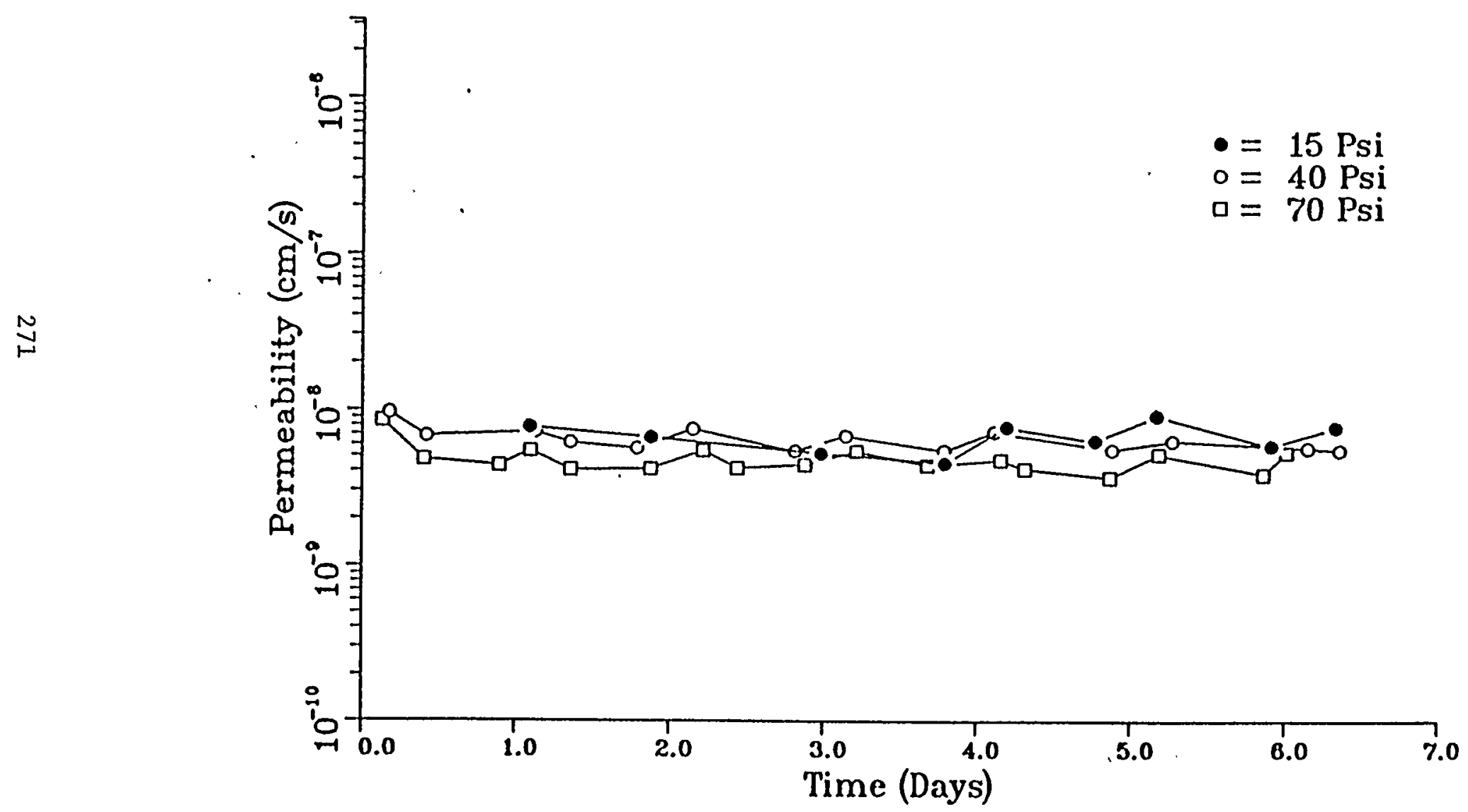

Figure E.3 Permeability results of Sample B/AL-C-4-25/C at injection pressures $=104$, 276 and $483 \mathrm{kPa}(15,40$ and $70 \mathrm{psi})$. 


\section{Sample B/AL-C-4-35/B}

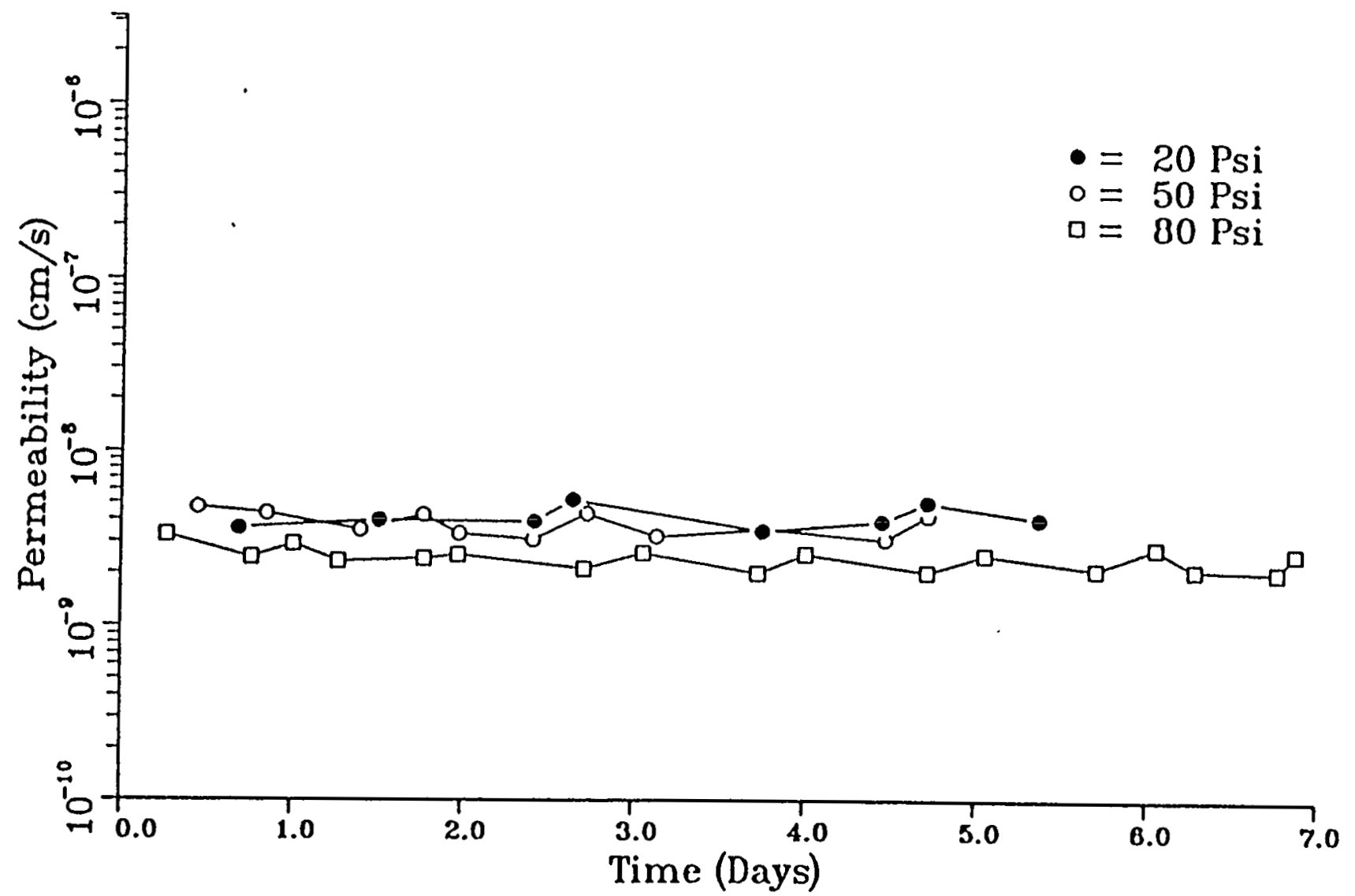

Figure E.4 Permeability results of Sample B/AL-C-4-35/B at injection pressures $=138$, 345 and $552 \mathrm{kPa}(20,50$ and $80 \mathrm{psi})$. 


\section{Sample B/AL-C-4-35/B}

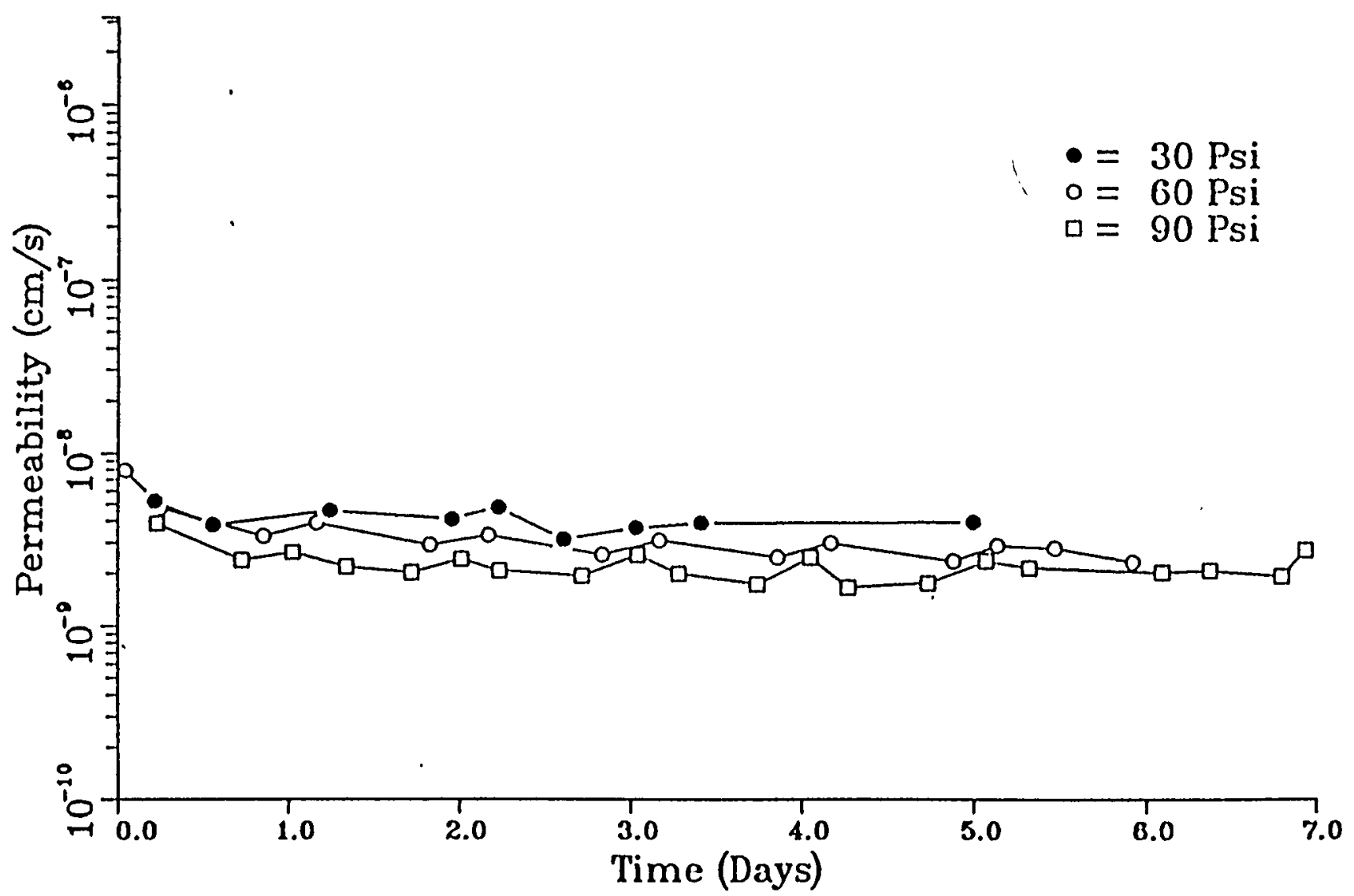

Figure E.5 Permeability results of Sample B/AL-C-4-35/B at injection pressures $=207$, 414 and $621 \mathrm{kPa}(30,60$ and $90 \mathrm{psi})$. 


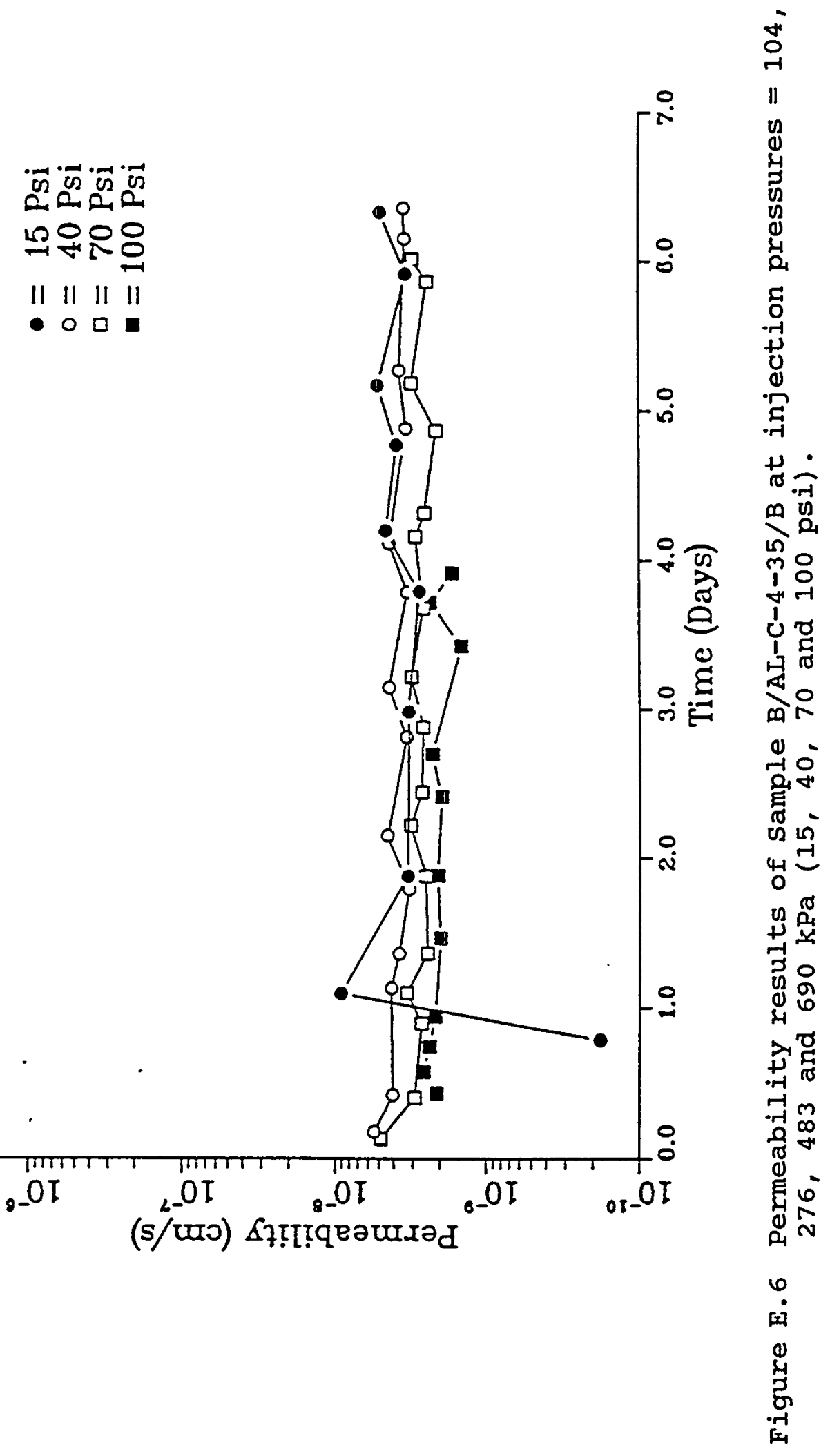




\section{Sample B/AL-C-4-35/C}

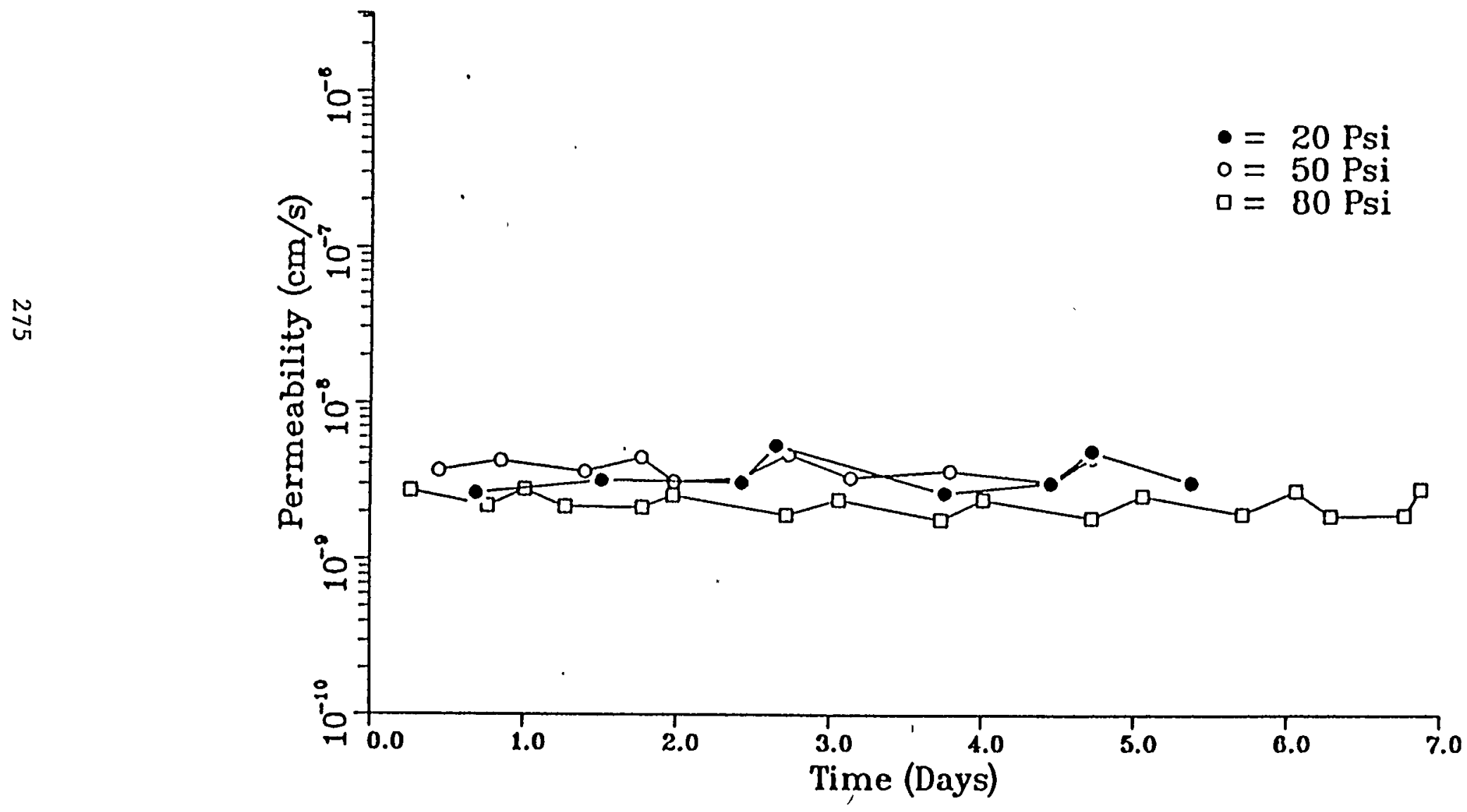

Figure E.7 Permeability results of Sample B/AL-C-4-35/C at injection pressures $=138$, 345 , and $552 \mathrm{kPa}(20,50$, and $80 \mathrm{psi})$. 


\section{Sample B/AL-C-4-35/C}

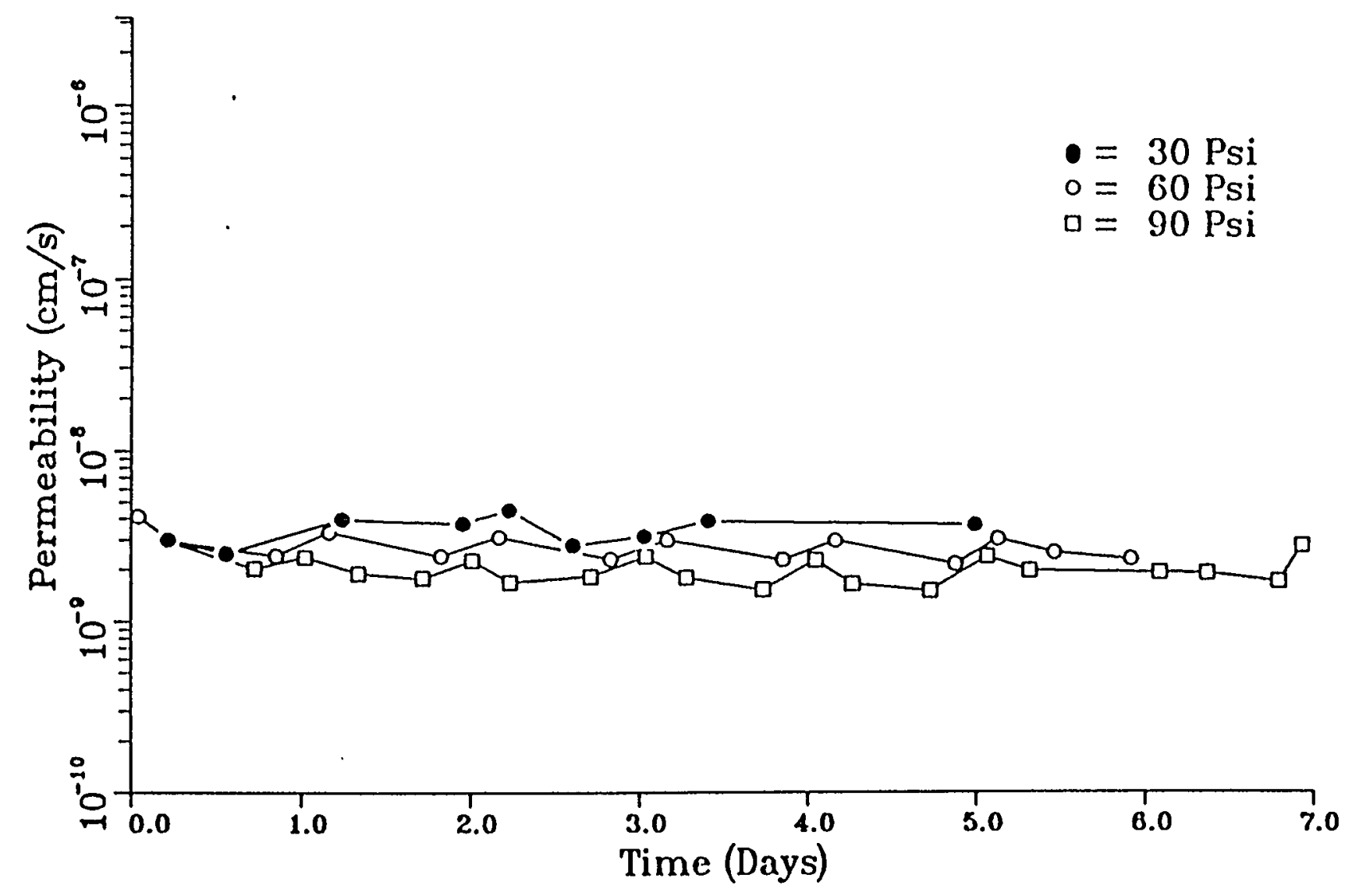

Figure E.8 Permeability results of Sample B/AL-C-4-35/C at injection pressures $=207$, 414 , and $621 \mathrm{kPa}(30,60$, and $90 \mathrm{psi})$. 


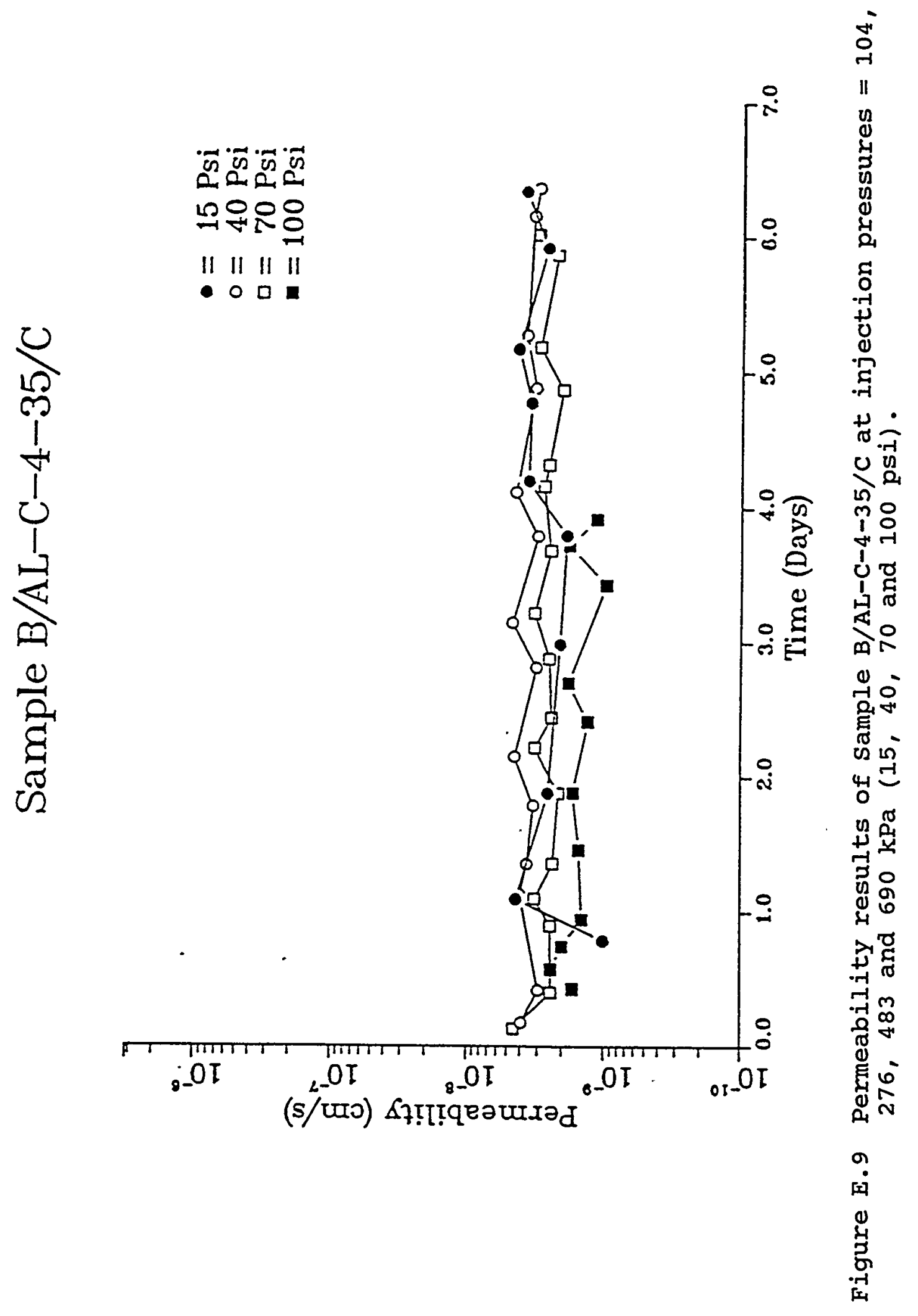


Sample B/AL-C-4-25/C

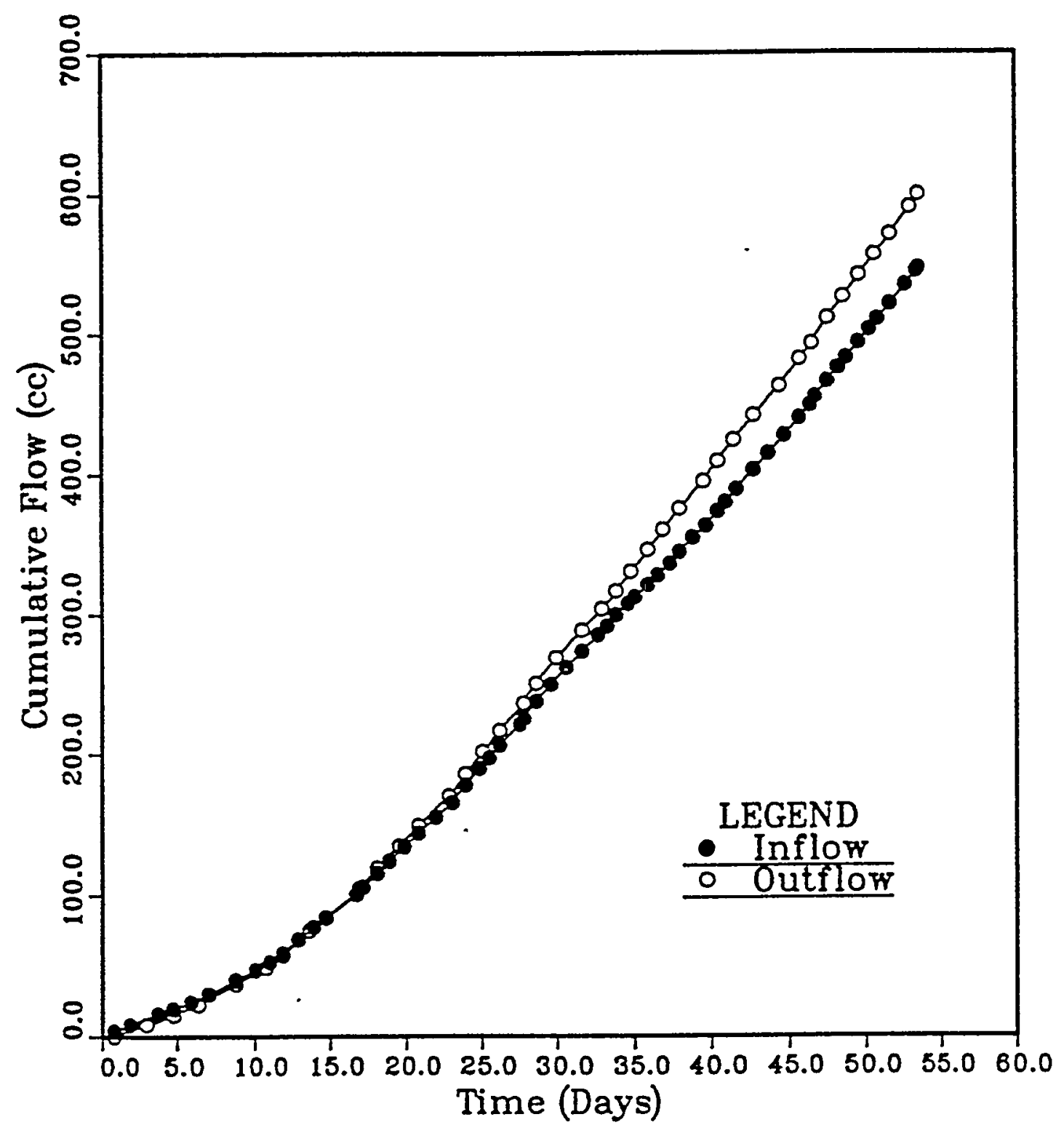

Figure E.10 Cumulative inflow and outflow vs. time for Sample B/AL-C-4-25/C. 
Sample B/AL-C-4-35/B

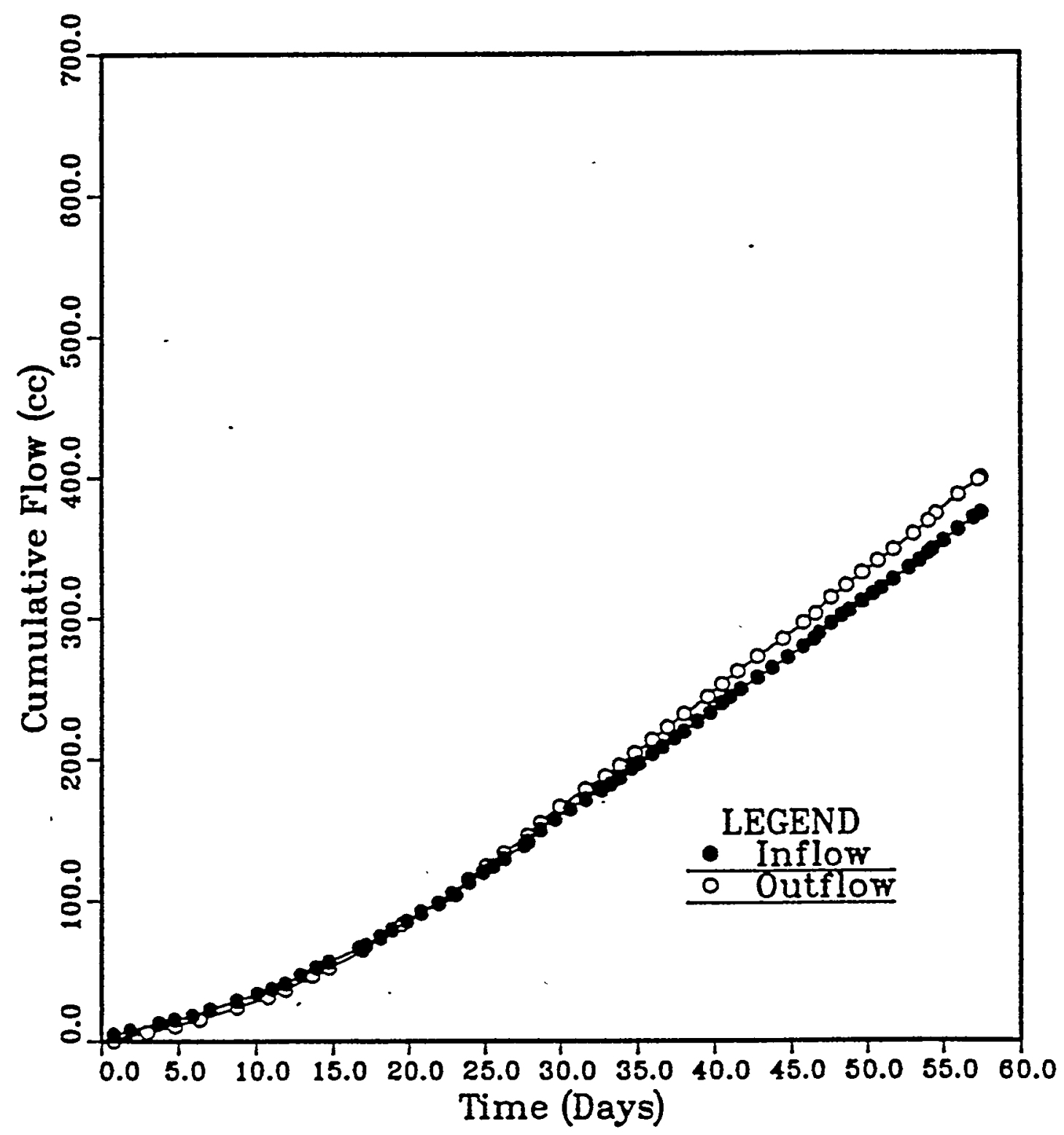

Figure E.11 Cumulative inflow and outflow vs. time for Sample B/AL-C-4-35/B. 
Sample B/AL-C-4-35/C

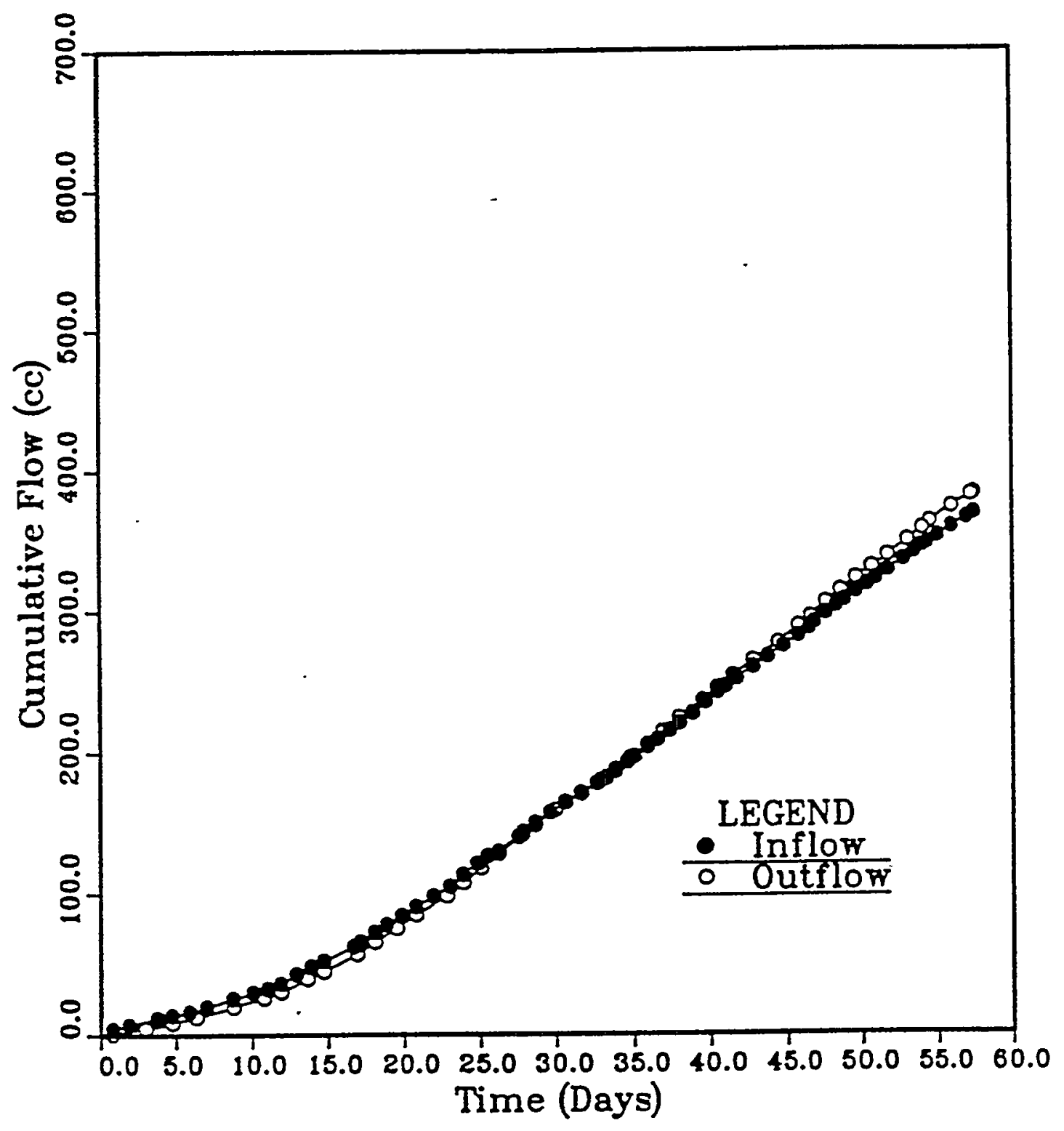

Figure E.12 Cumulative inflow and outflow vs. time for Sample B/AL-C-4-35/C. 

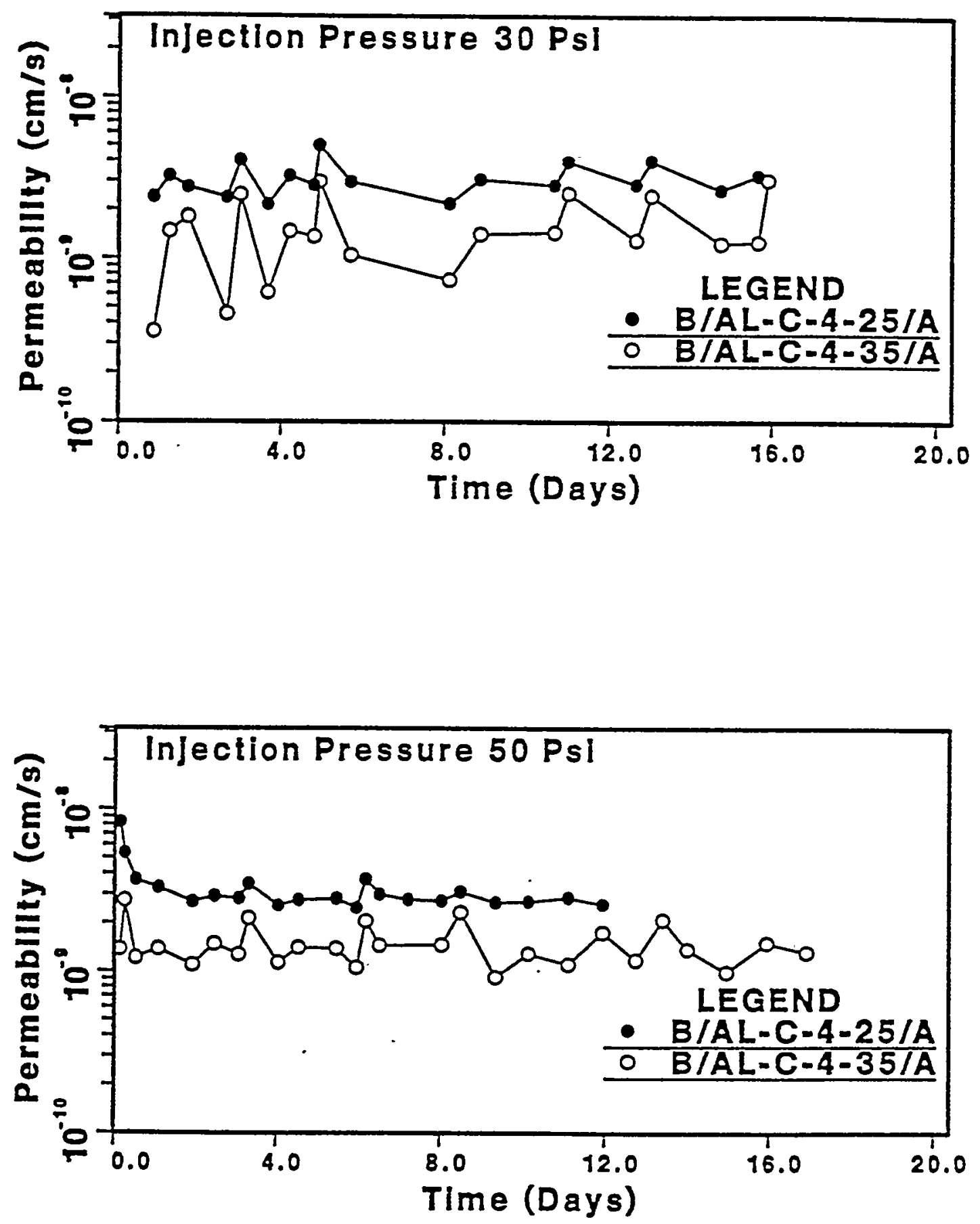

Figure E.13 Permeability results of samples B/AL-C-4-25/A and B/AL-C-435/A at injection pressures of 207 and $345 \mathrm{kPa}$ ( 30 and 50 psi). Hydraulic gradient: 200 to 208 for the former injection pressure, 333 to 347 -for the latter. 

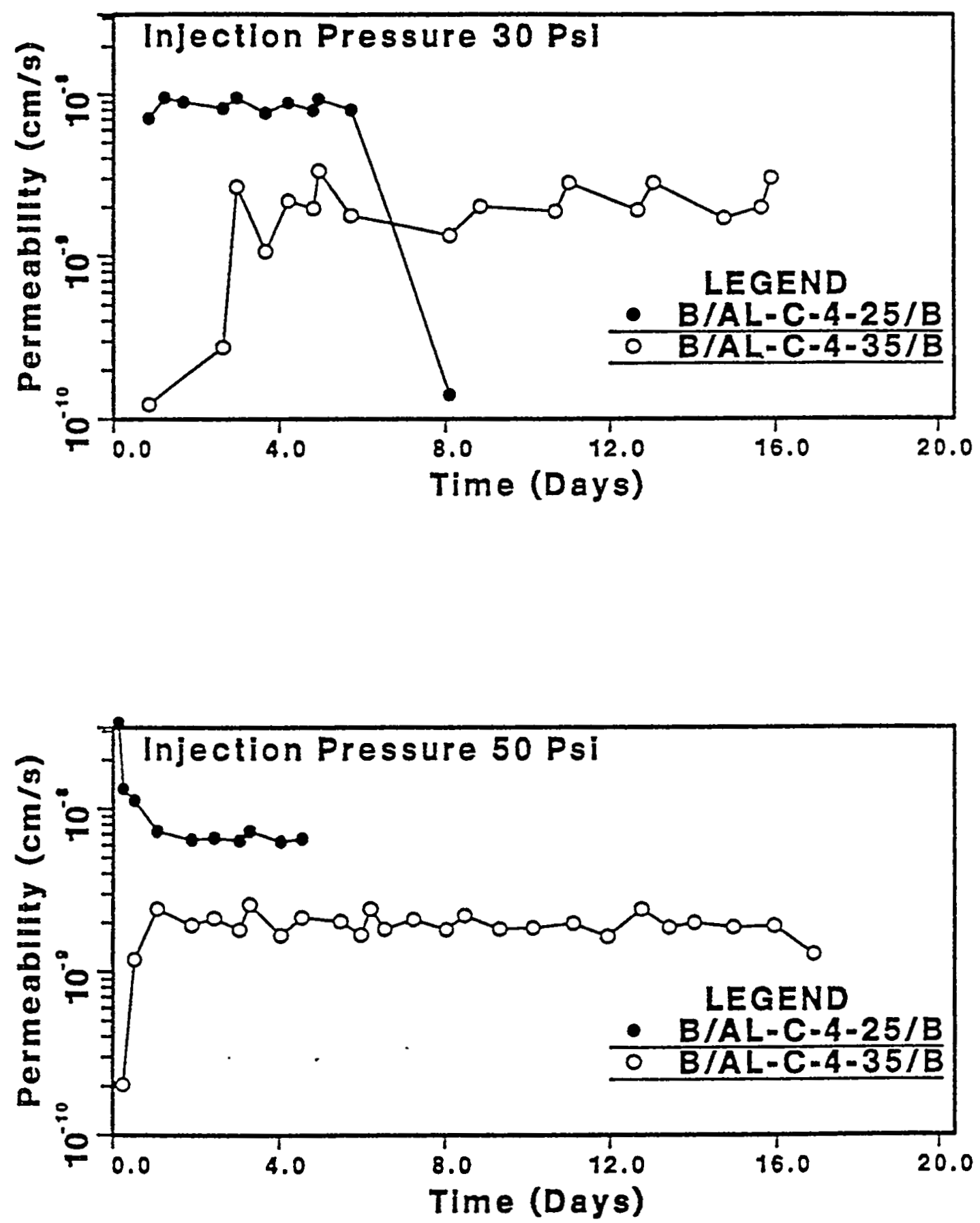

Figure E.14 Permeability results of samples B/AL-C-4-25/B and B/AL-C-4$35 / B$ at injection pressures of 207 and $345 \mathrm{kPa}$ (30 and 50 psi). Hydraulic gradient: 193 to 211 for the former injection pressure, 321 to 351 for the latter. 

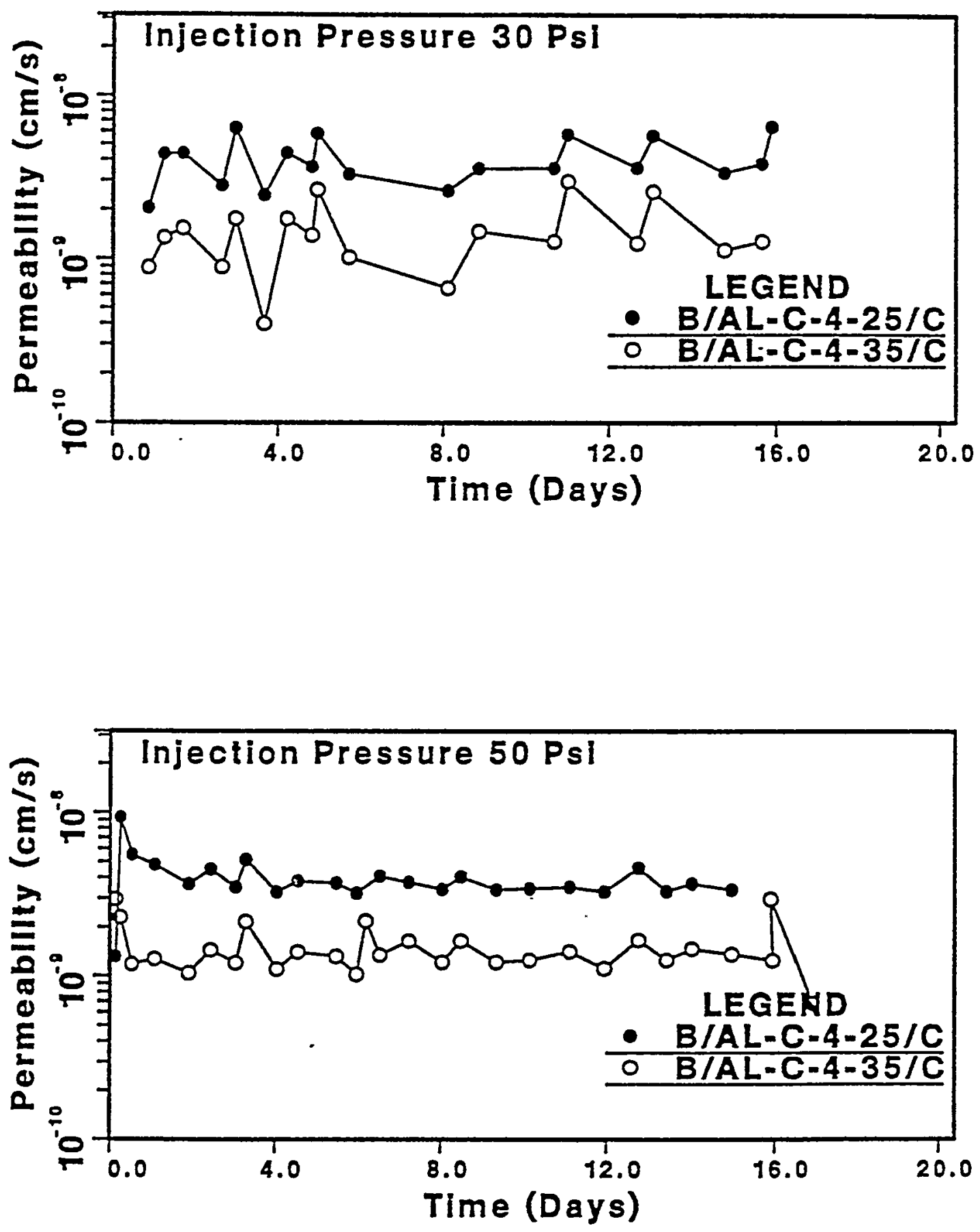

Figure E.15 Permeability results of samples B/AL-C-4-25/C and B/AL-C-4$35 / \mathrm{C}$ at injection pressures of 207 and $345 \mathrm{kPa}$ ( 30 and 50 psi). Hydraulic gradient: 186 to 208 for the former injection pressure, 310 to 347 for the latter. 

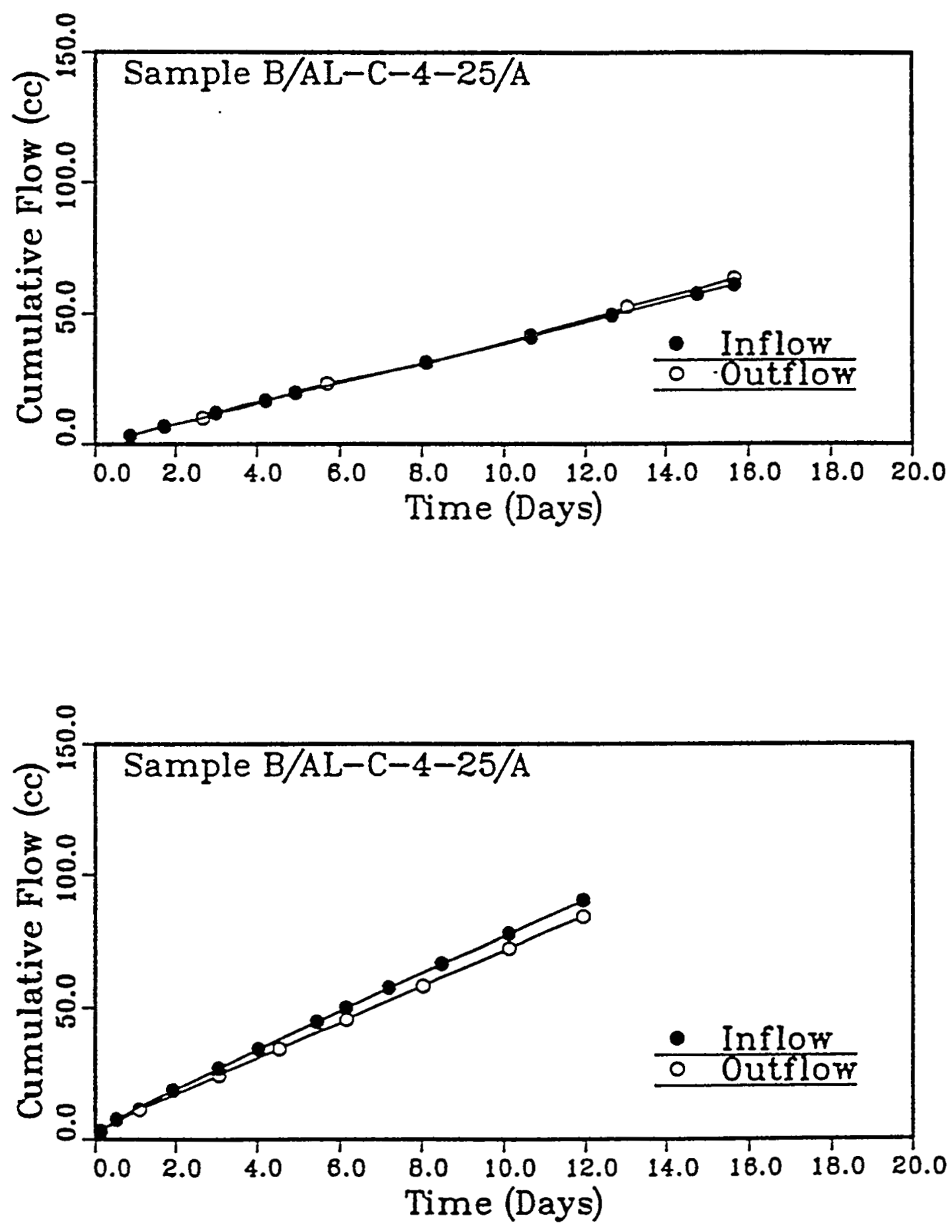

Figure E.16 Cumulative inflow and outflow vs. time for sample B/AL-C-425/A (top: injection pressure $=207 \mathrm{kPa}$; bottom: injection pressure $=345 \mathrm{kPa})$. 

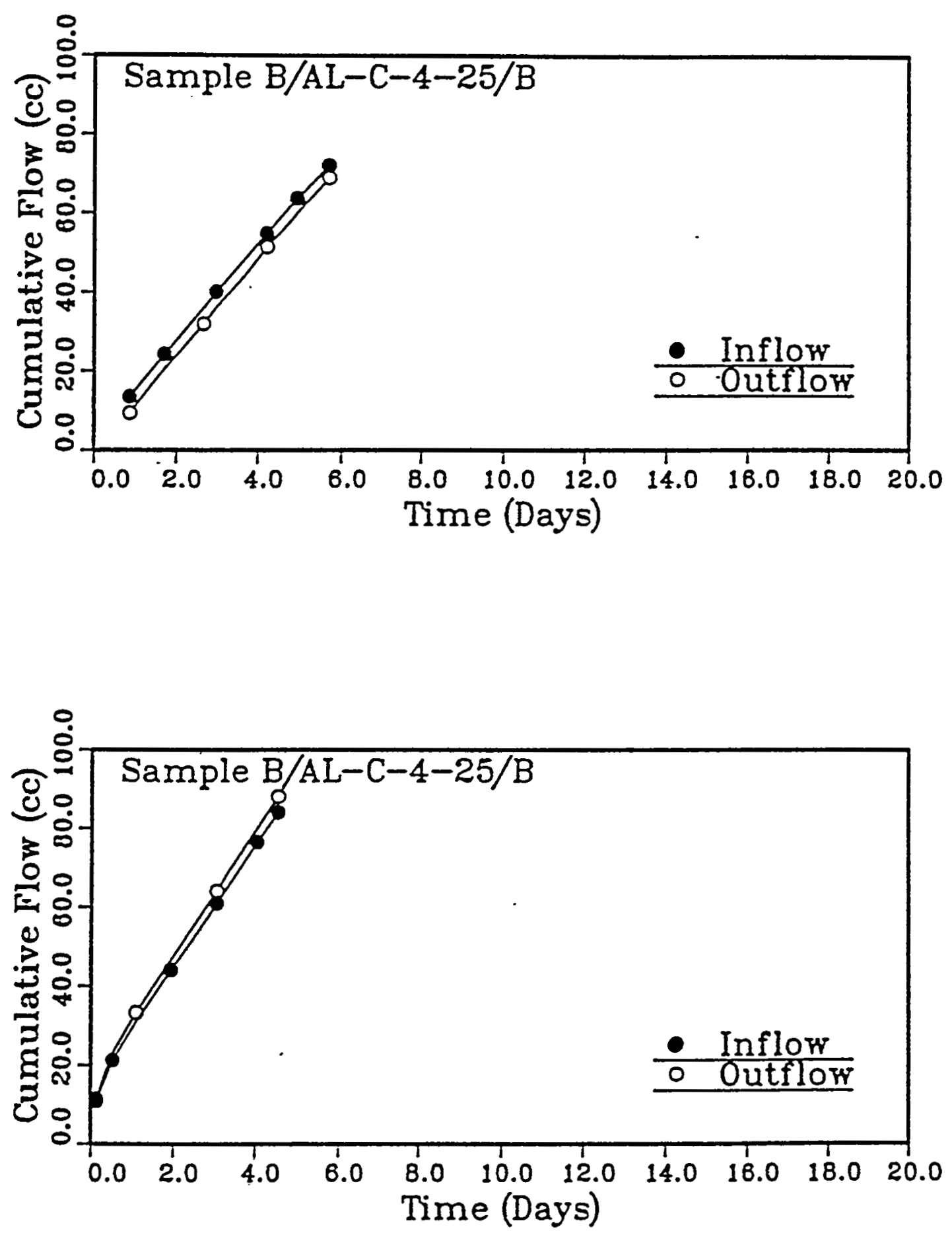

Figure E.17 Cumulative inflow and outflow vs. time for sample B/AL-C-425/B (top: injection pressure $=207 \mathrm{kPa}$; bottom: injection pressure $=345 \mathrm{kPa}$ ). 

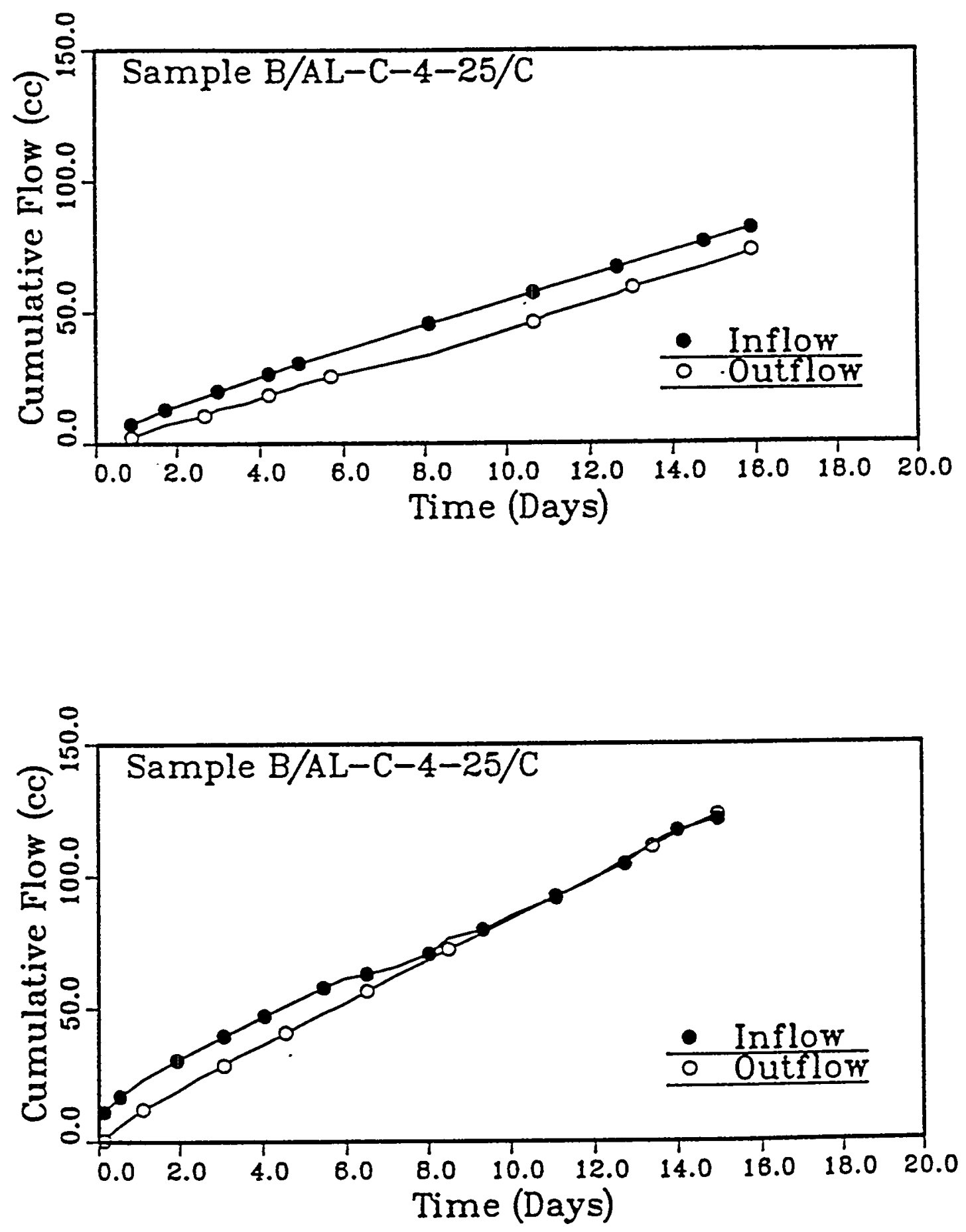

Figure E.18 Cumulative inflow and outflow vs. time for sample B/AL-C-4$25 / \mathrm{C}$ (top: injection pressure $=207 \mathrm{kPa}$; bottom: injection pressure $=345 \mathrm{kPa}$ ). 

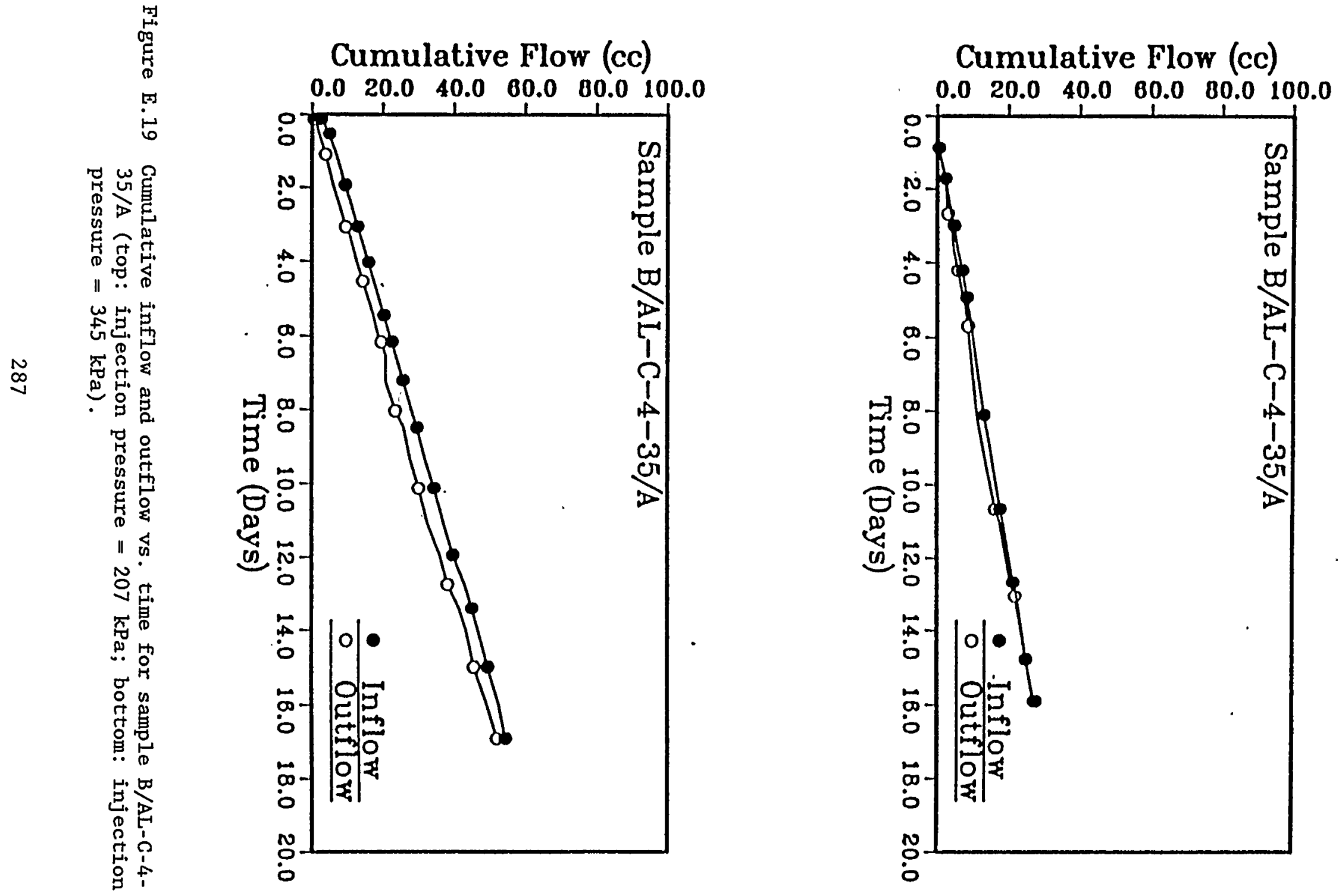

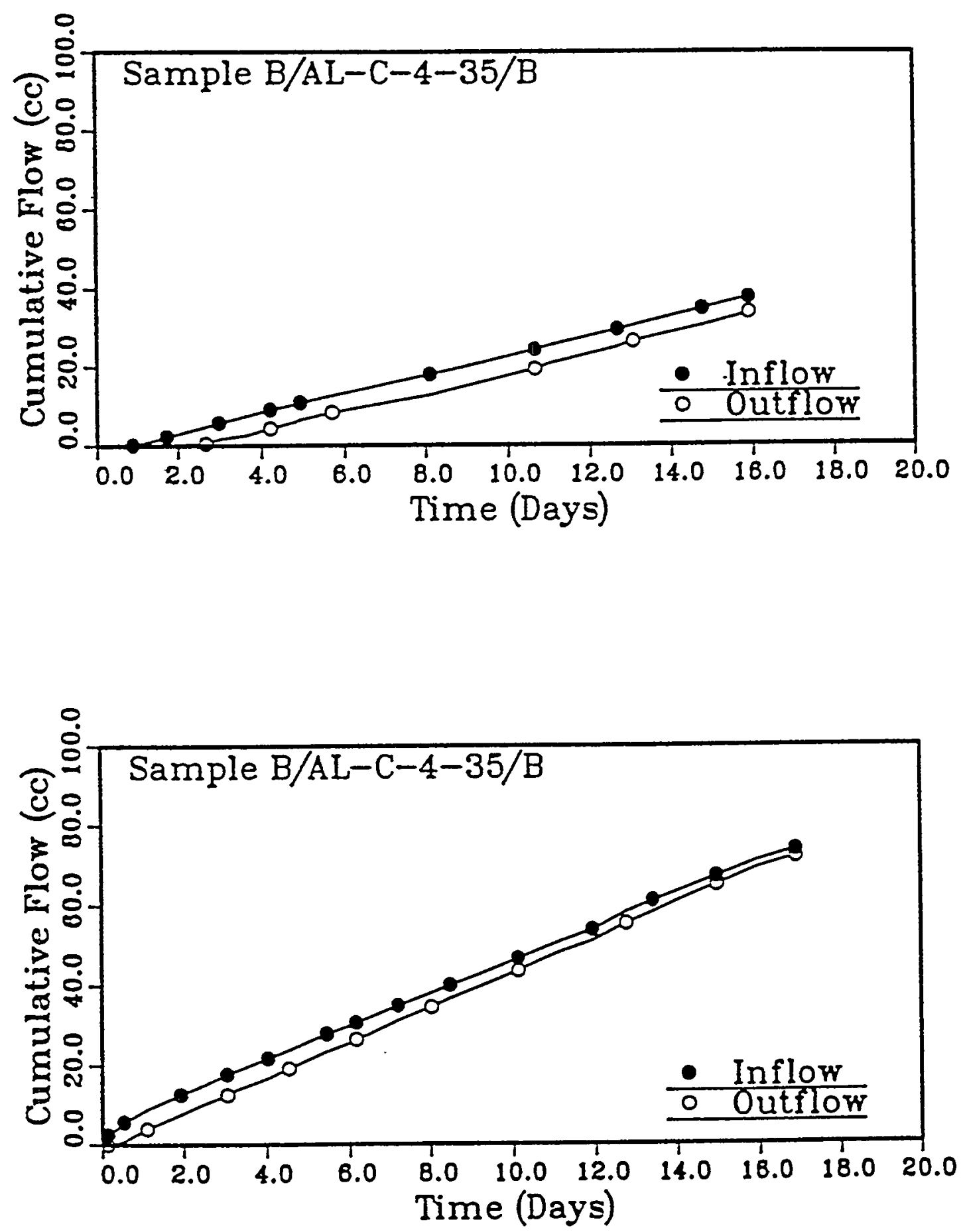

Figure E.20 Cumulative inflow and outflow vs. time for sample B/AL-C-435/B (top: injection pressure $=207 \mathrm{kPa}$; bottom: injection pressure $=345 \mathrm{kPa}$ ). 

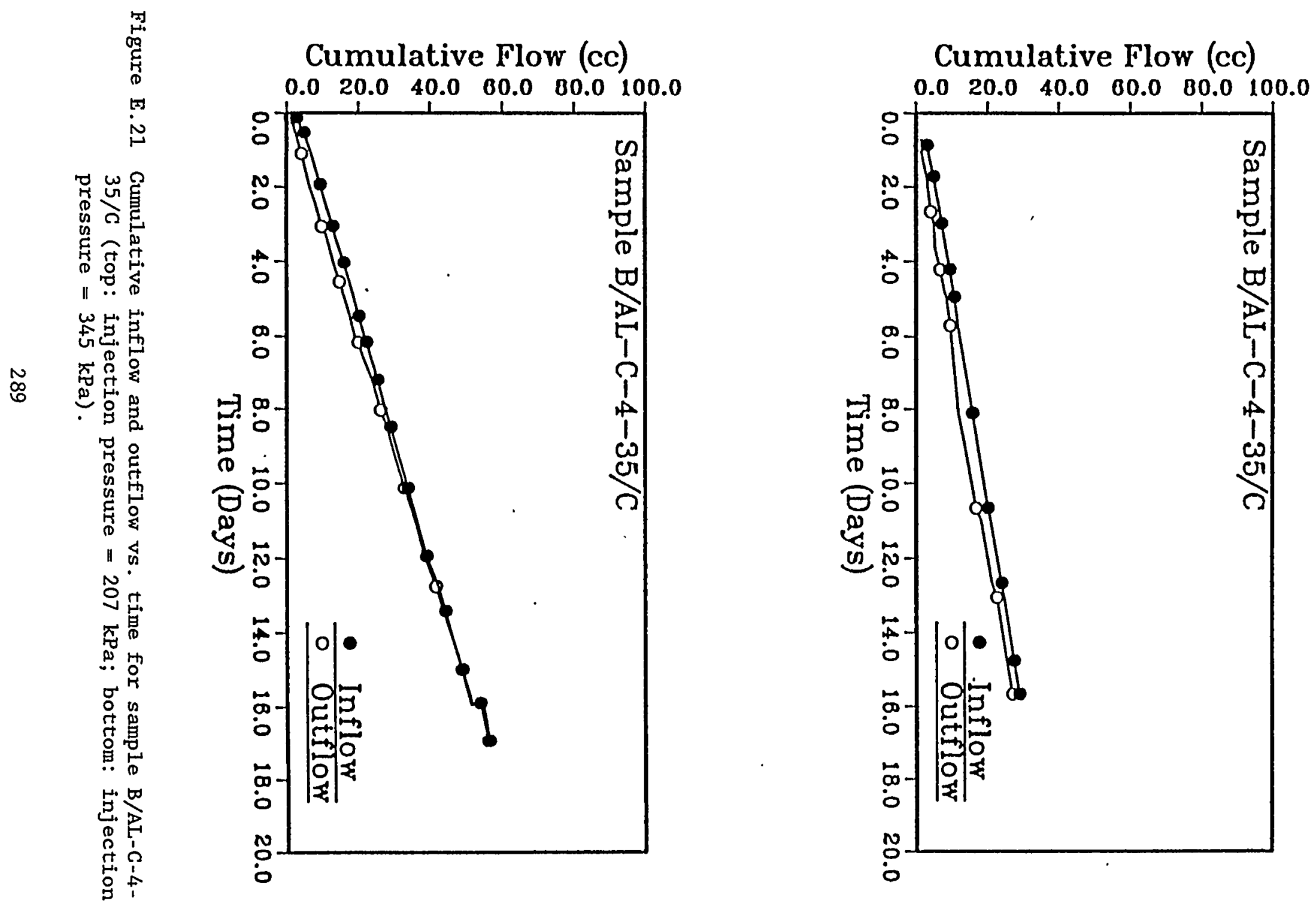

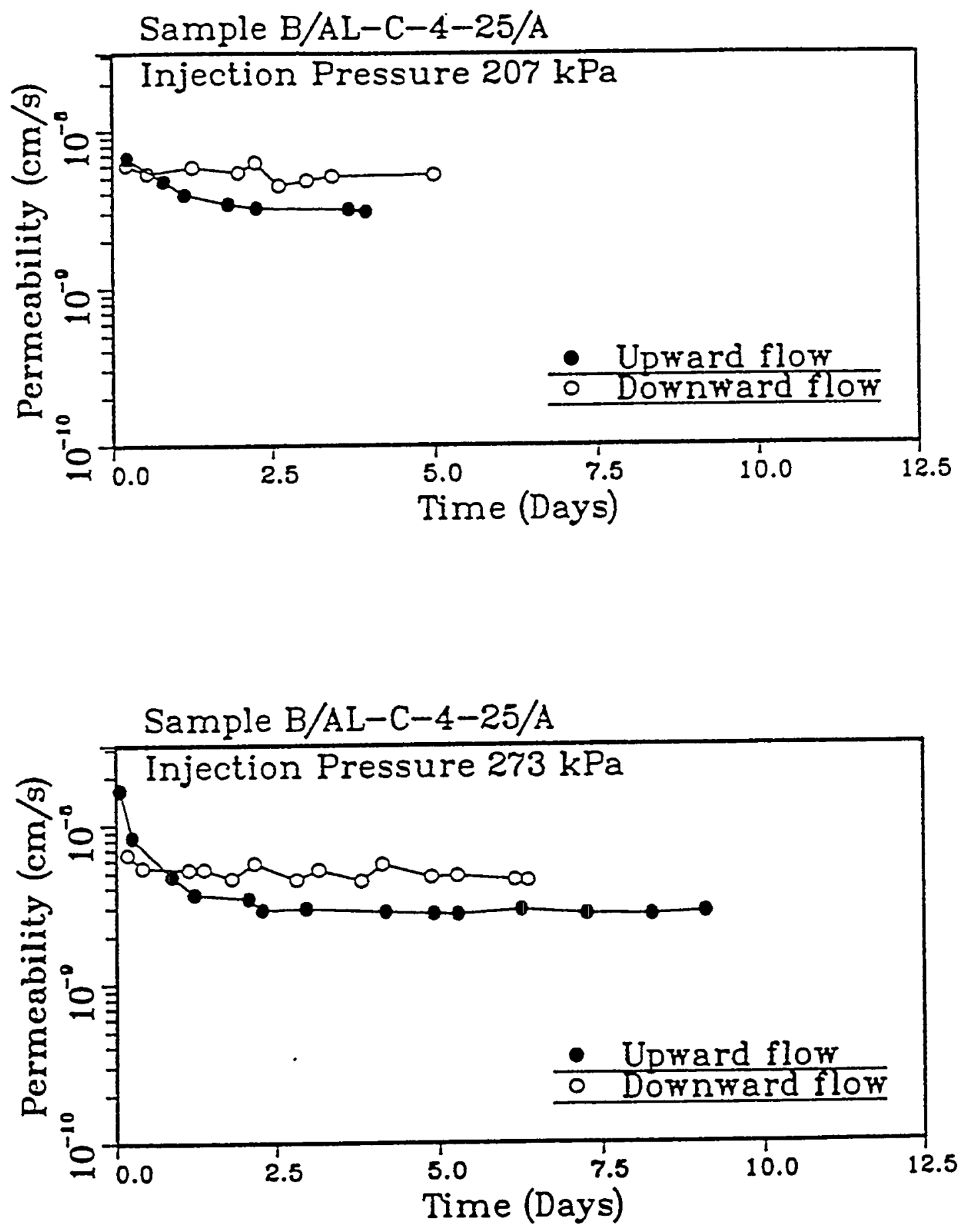

Figure E.22 Upward and downward permeability of sample B/AL-C-4-25/A under injection pressures of $207 \mathrm{kPa}$ ( $30 \mathrm{psi}$ ) and $273 \mathrm{kPa}$ (40 psi). Hydraulic gradient: 208.4-210.3 (top), 273.8-278.6 (bottom). 

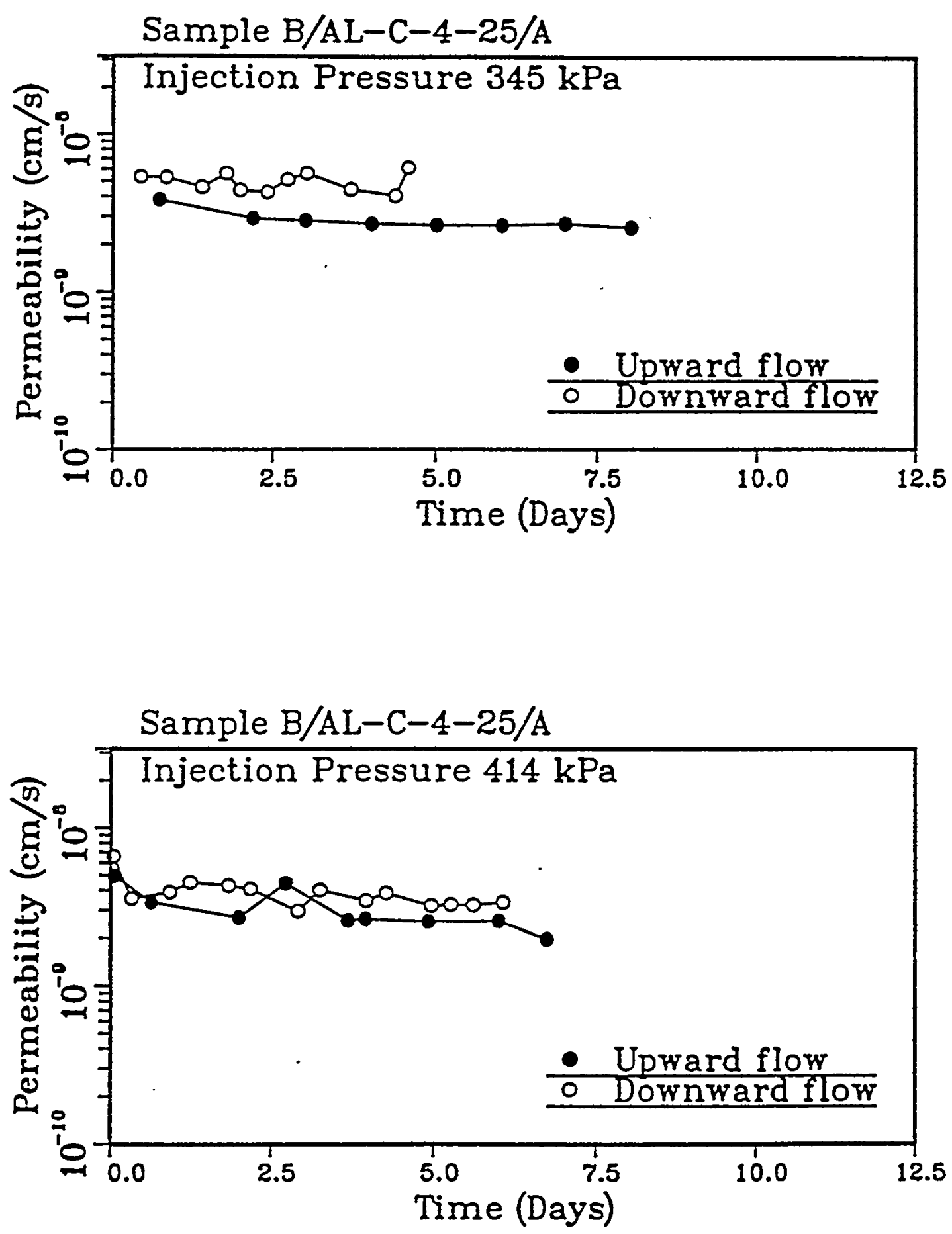

Figure E.23 Upward and downward permeability of sample B/AL-C-4-25/A under injection pressures of $345 \mathrm{kPa}(50 \mathrm{psi}$ ) and $414 \mathrm{kPa}$ (60 psi). Hydraulic gradient: $342.9-347.9$ (top), 409.6-415.4 (bottom). 

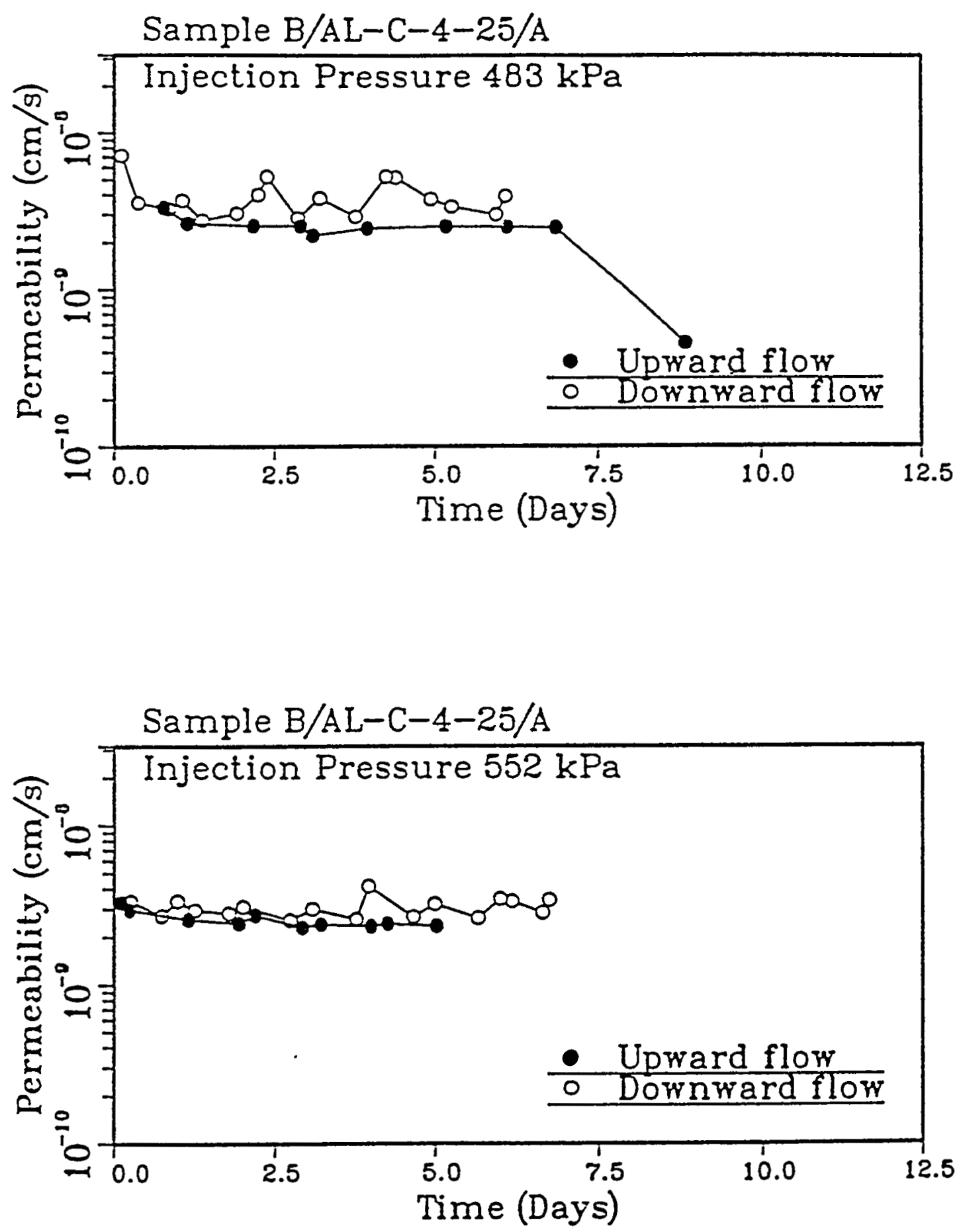

Figure E.24 Upward and downward permeability of sample B/AL-C-4-25/A under injection pressures of $483 \mathrm{kPa}(70 \mathrm{psi})$ and $552 \mathrm{kPa}$ (80 psi). Hydraulic gradient: 479-485.4 (top), 545.4-552.8 (bottom). 

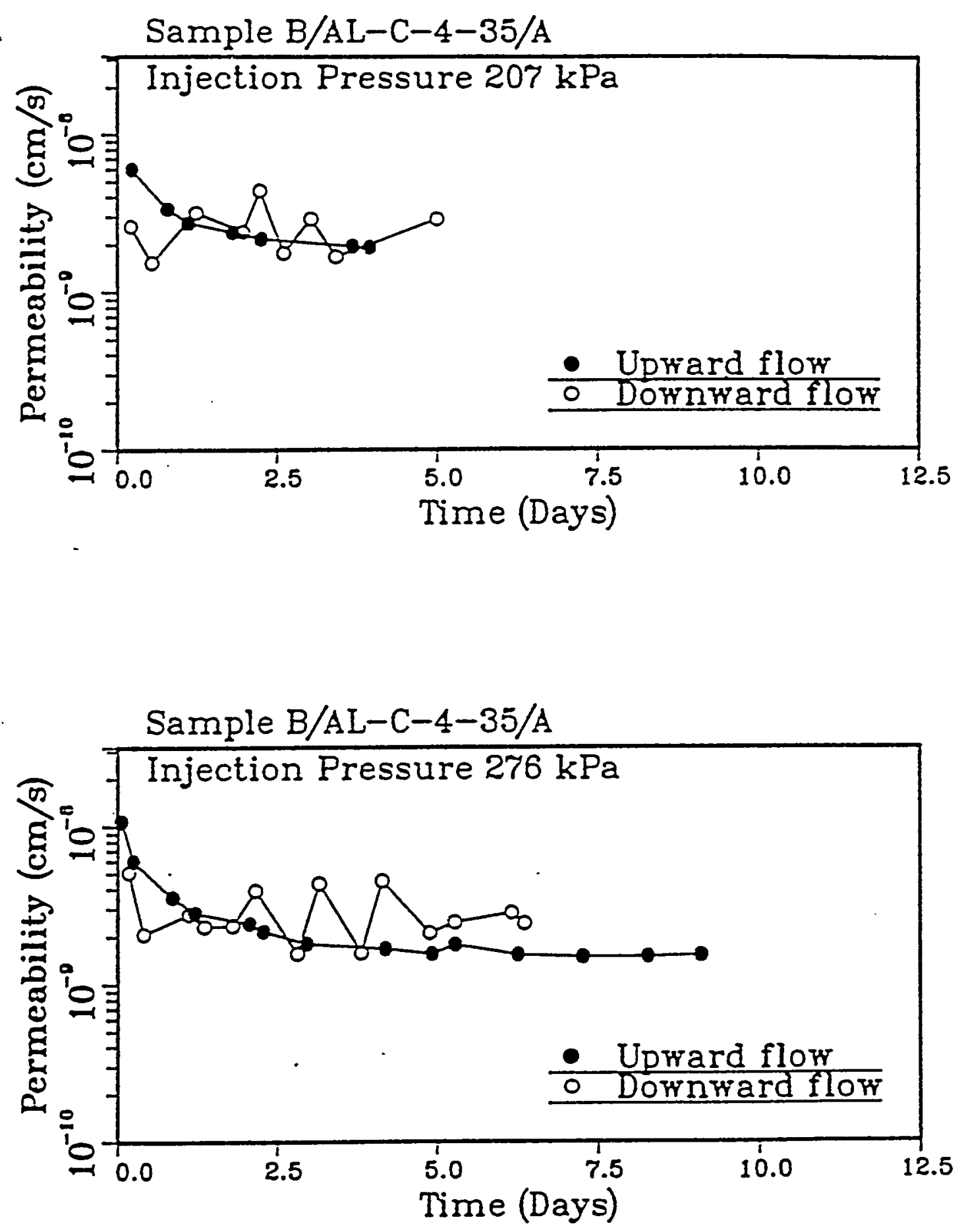

Figure E.25 Upward and downward permeability of sample B/AL-C-4-35/A under injection pressures of $207 \mathrm{kPa}$ ( $30 \mathrm{psi}$ ) and $276 \mathrm{kPa}$ (40 psi). Hydraulic gradient: 203.9-204.8 (top), 266.7-269.9 (bottom). 

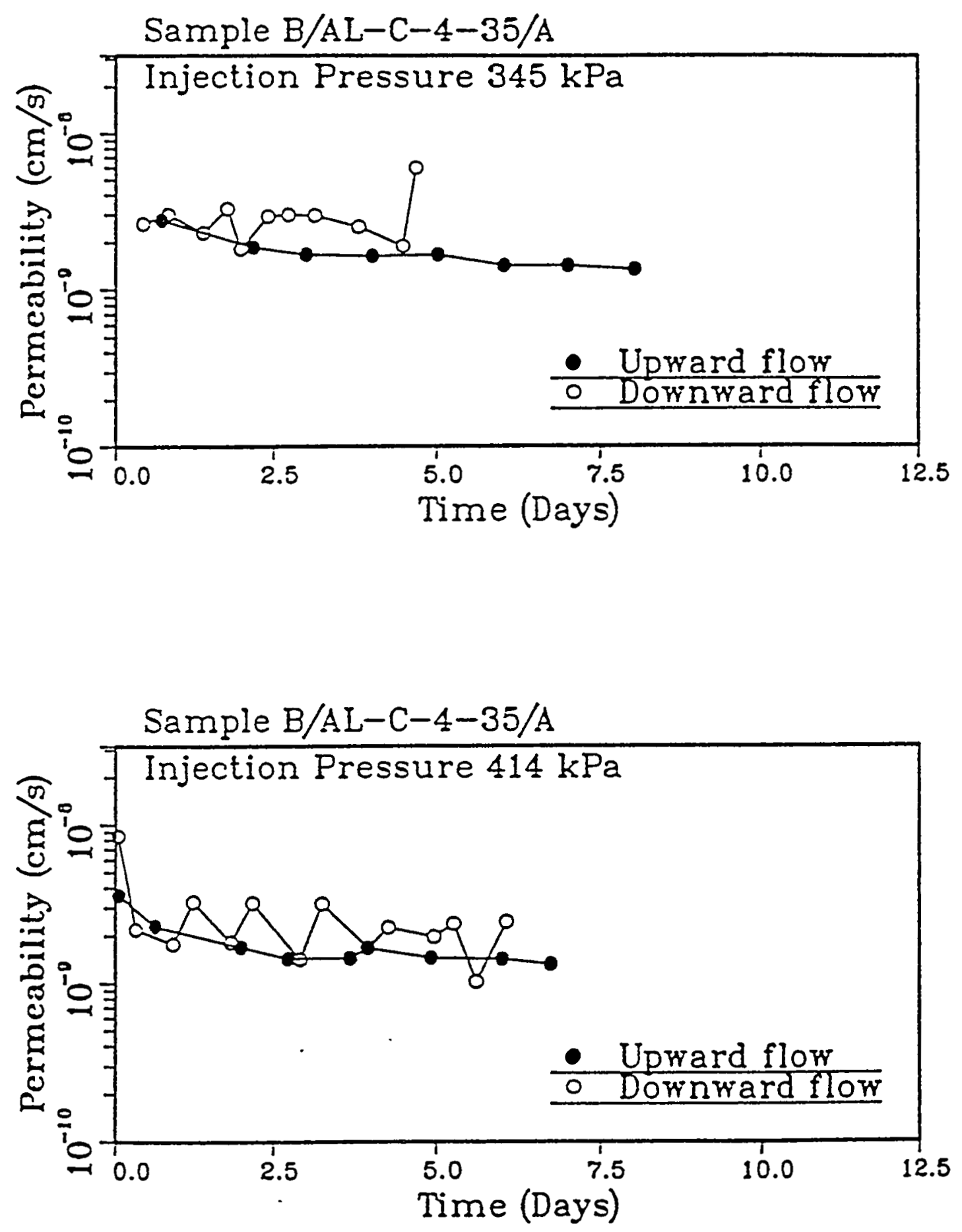

Figure E.26 Upward and downward permeability of sample B/AL-C-4-35/A under injection pressures of $345 \mathrm{kPa}$ ( $50 \mathrm{psi}$ ) and $414 \mathrm{kPa}$ (60 psi). Hydraulic gradient: $333.2-338$ (top), 398.1-403.7 (bottom). 

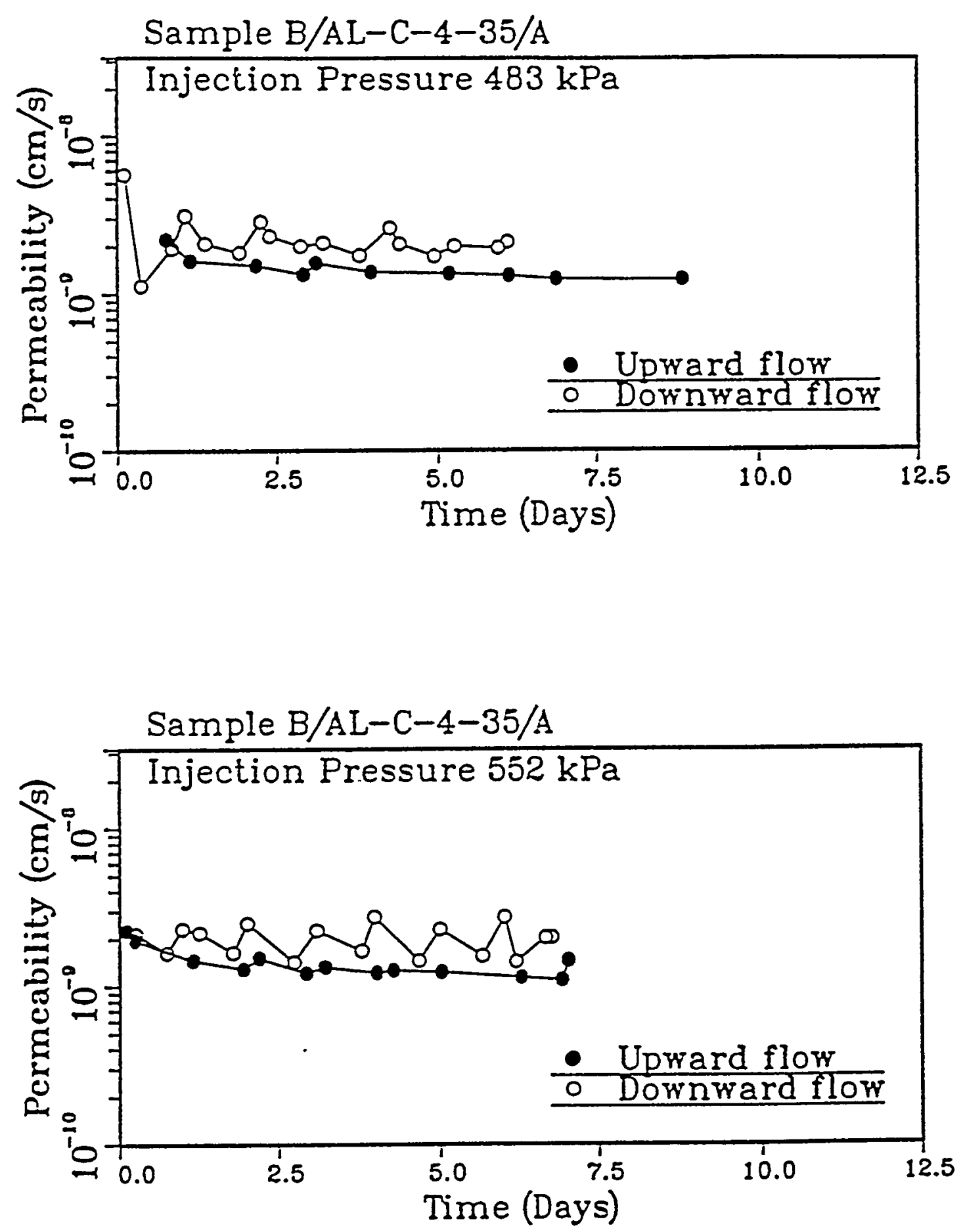

Figure E.27 Upward and downward permeability of sample B/AL-C-4-35/A under injection pressures of $483 \mathrm{kPa}(70 \mathrm{psi}$ ) and $552 \mathrm{kPa}$ (80 psi). Hydraulic gradient: $465.2-471.1$ (top), 531.1-537.8 (bottom). 


\section{APPENDIX F}

EXPERIMENTAL RECORDS OF PIPING TESTS 
Table F.1 Flow Test Results of Sample B/AL-C-4-25/A-P-B

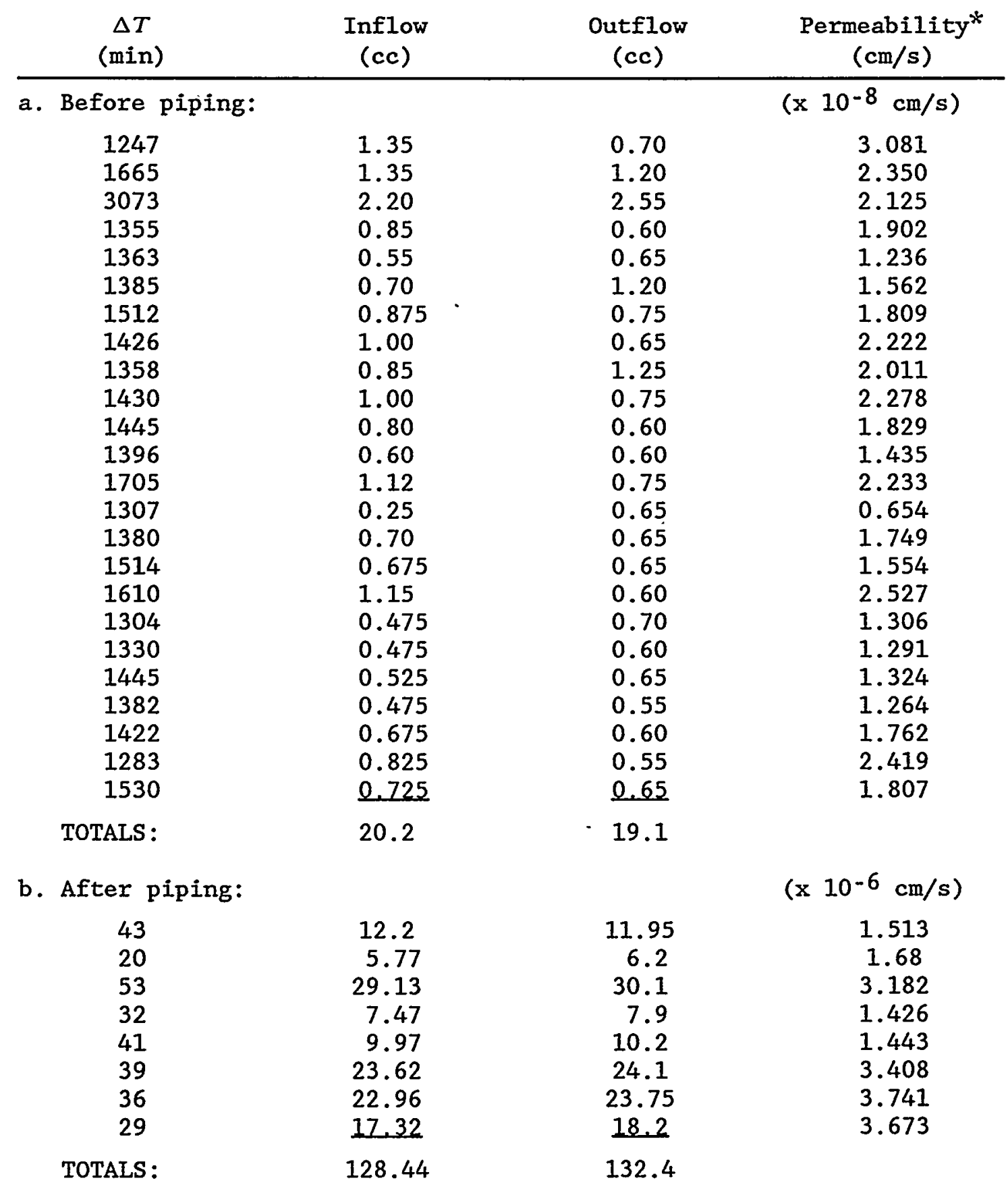

${ }^{*}$ Based on outflow. 
Table F.2 Flow Test Results of Sample B/AL-C-4-25/A-P-A

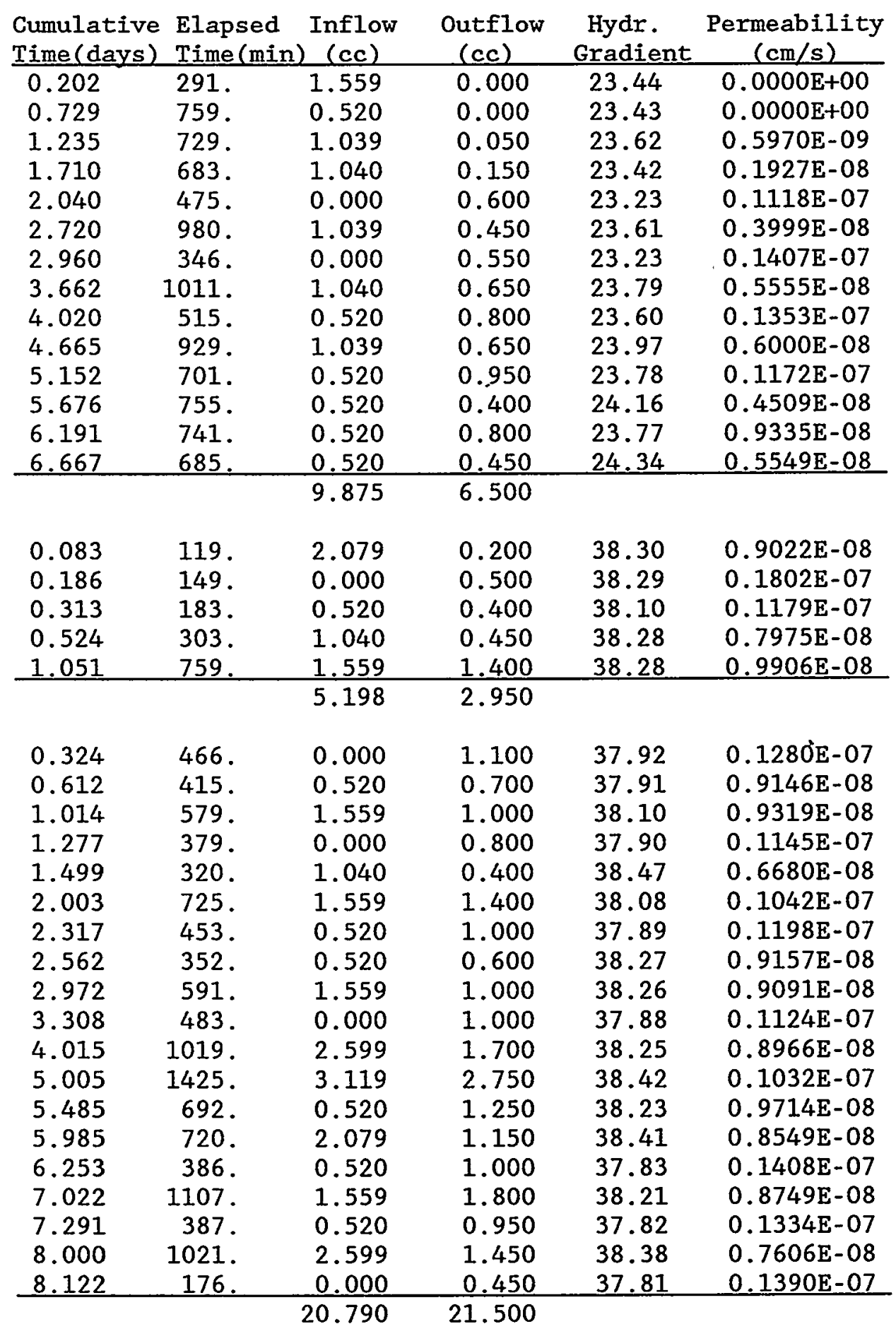




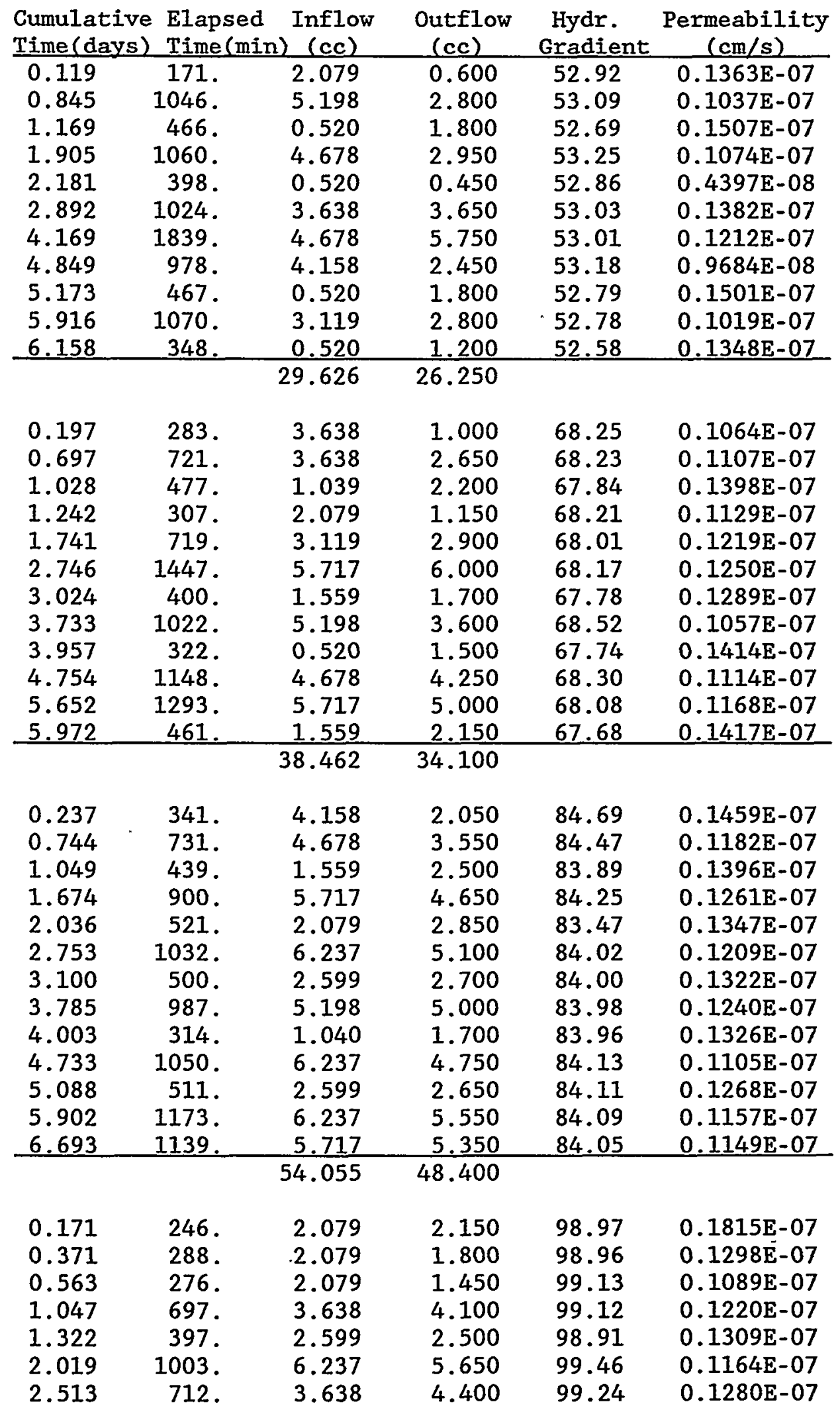




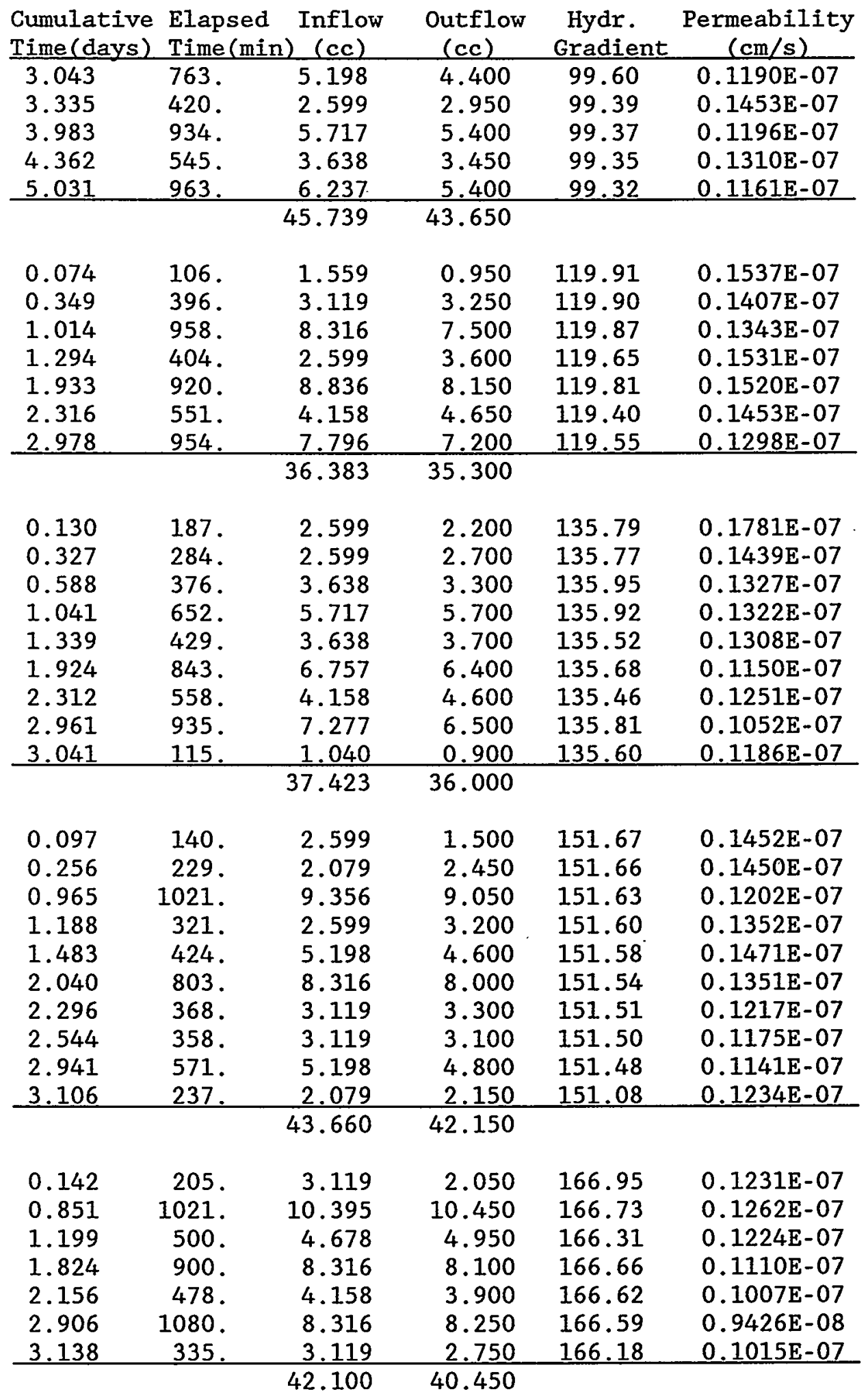




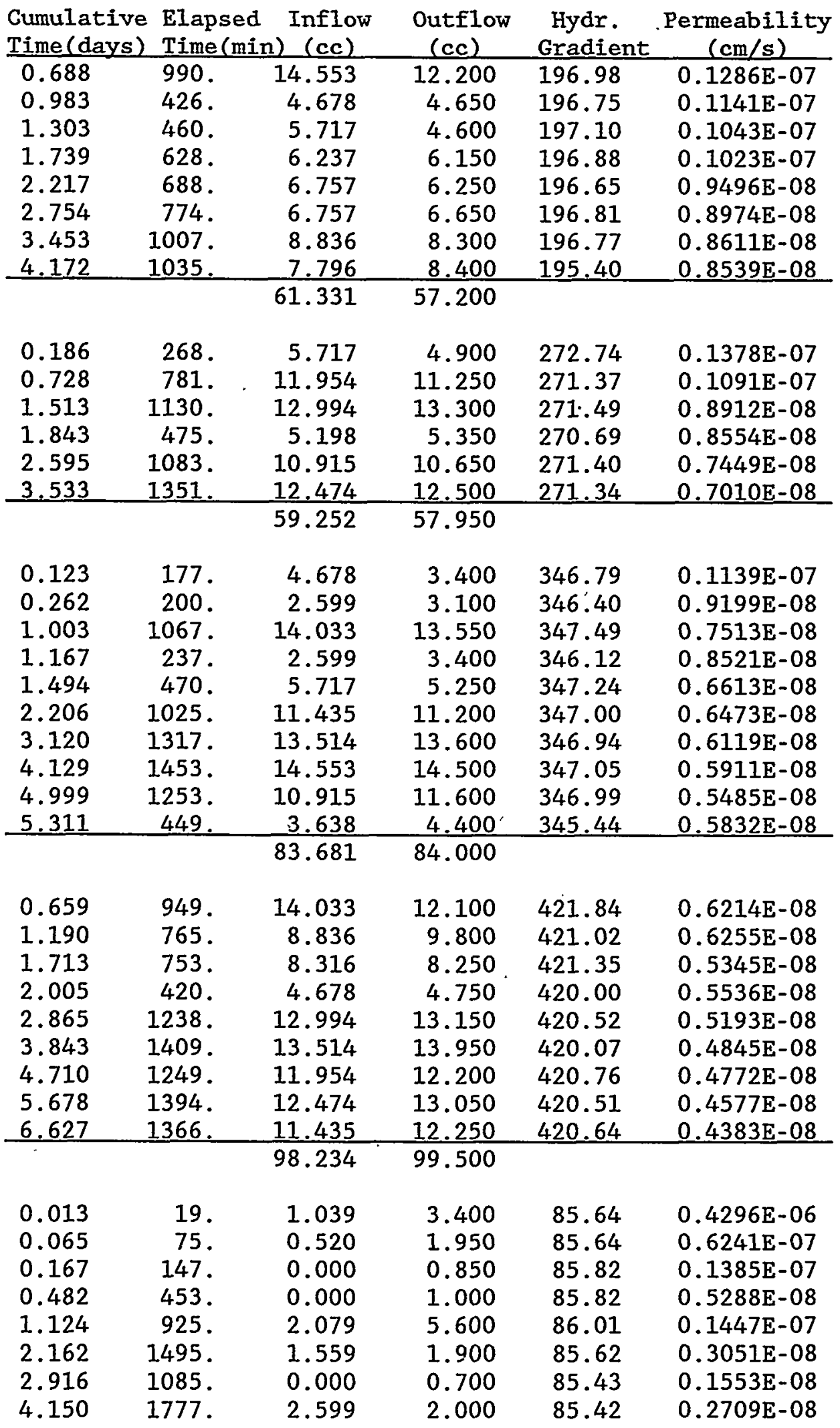




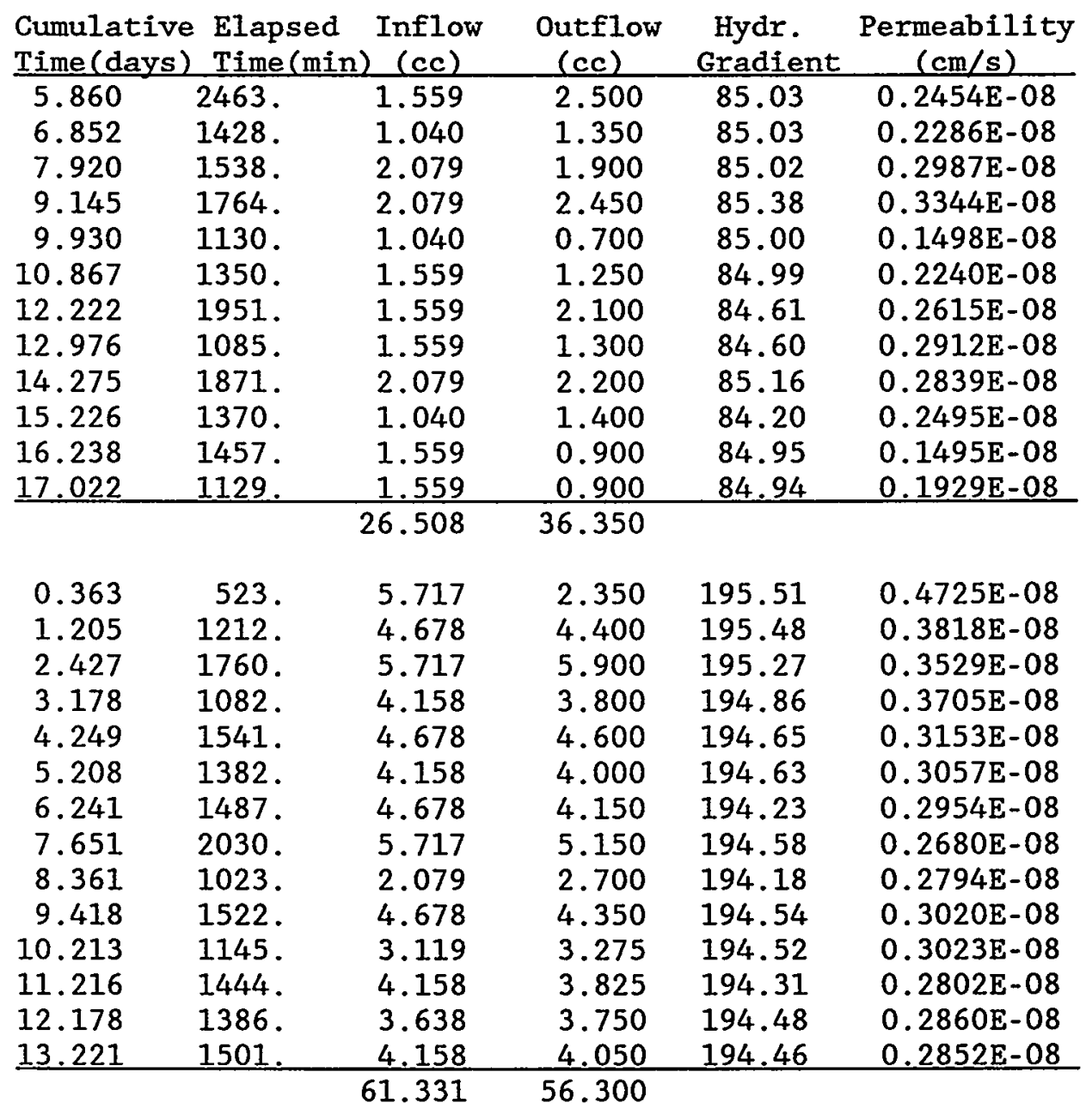


Table F.3 Flow Test Results of Sample B/AL-C-4-35/A-P-A

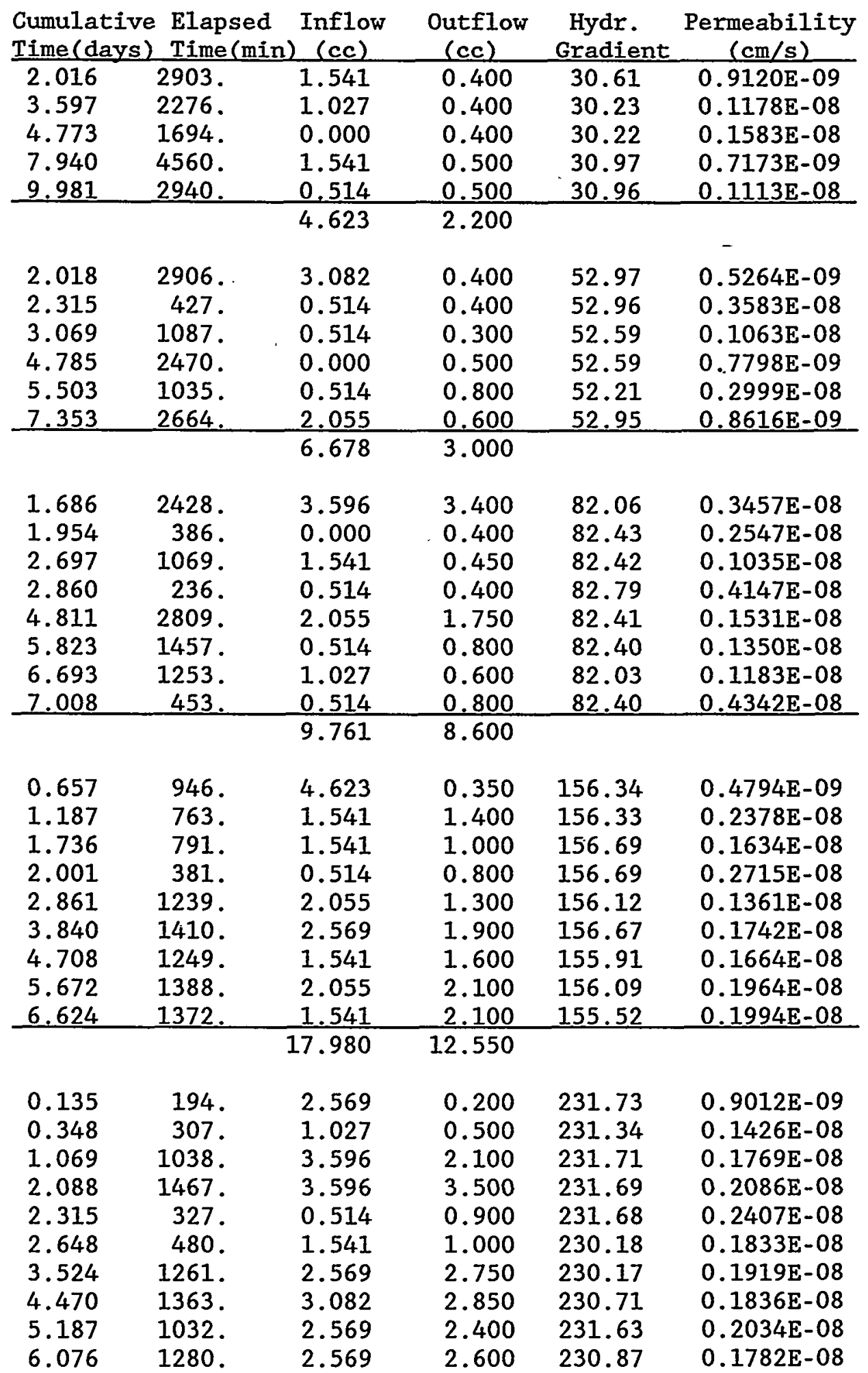




\begin{tabular}{|c|c|c|c|c|c|}
\hline $\begin{array}{l}\text { Cumulative } \\
\text { Time (days) }\end{array}$ & $\begin{array}{l}\text { Elapsed } \\
\text { Time (min) }\end{array}$ & $\begin{array}{l}\text { Inflow } \\
\text { (cc) }\end{array}$ & $\begin{array}{l}\text { Outflow } \\
\text { (cc) }\end{array}$ & $\begin{array}{l}\text { Hydr. } \\
\text { Gradient }\end{array}$ & $\begin{array}{l}\text { Permeability } \\
(\mathrm{cm} / \mathrm{s})\end{array}$ \\
\hline 6.371 & 425 & 1.027 & 1.400 & 231.61 & $0.2881 \mathrm{E}-08$ \\
\hline 7.308 & 1349. & 3.082 & 2.850 & 231.60 & $0.1848 \mathrm{E}-08$ \\
\hline 8.065 & 1091. & 2.055 & 2.150 & 229.72 & $0.1738 \mathrm{E}-08$ \\
\hline 8.339 & 394. & 1.027 & 1.000 & 230.83 & $0.2227 \mathrm{E}-08$ \\
\hline 9.093 & 1086. & 2.569 & 2.400 & 231.19 & $0.1936 \mathrm{E}-08$ \\
\hline & & 33.391 & 28.600 & & \\
\hline 0.055 & 79 . & 0.514 & 3.250 & 81.92 & $0.1017 \mathrm{E}-06$ \\
\hline 0.157 & 147. & 0.000 & 1.200 & 81.92 & $0.2019 E-07$ \\
\hline 0.473 & 455. & 0.000 & 1.400 & 81.92 & $0.7609 \mathrm{E}-08$ \\
\hline 1.114 & 923. & 0.000 & 1.350 & 81.92 & $0.3617 \mathrm{E}-08$ \\
\hline 2.152 & 1495. & 0.000 & 1.200 & 81.92 & $0.1985 \mathrm{E}-08$ \\
\hline 2.906 & 1085. & 0.000 & 0.200 & 81.92 & $0.4558 \mathrm{E}-09$ \\
\hline 4.140 & 1777. & 0.000 & 0.800 & 81.92 & $0.1113 \mathrm{E}-08$ \\
\hline 5.850 & 2463 . & 0.000 & 1.000 & 81.92 & $0.1004 \mathrm{E}-08$ \\
\hline 6.842 & 1428. & 0.000 & 0.500 & 81.17 & $0.8738 \mathrm{E}-09$ \\
\hline 7.911 & 1540. & 0.000 & 1.100 & 81.92 & $0.1766 \mathrm{E}-08$ \\
\hline 9.135 & 1762 . & 0.000 & 0.800 & 81.92 & $0.1123 E-08$ \\
\hline 9.919 & 1130. & 1.027 & 0.200 & 81.54 & $0.4397 \mathrm{E}-09$ \\
\hline 10.857 & 1350. & 0.514 & 0.400 & 81.91 & $0.7328 \mathrm{E}-09$ \\
\hline 12.212 & 1951. & 1.027 & 1.000 & 81.91 & $0.1268 \mathrm{E}-08$ \\
\hline 12.965 & 1085. & 0.000 & 0.800 & 81.90 & $0.1824 \mathrm{E}-08$ \\
\hline 14.181 & 1751. & 0.514 & 1.000 & 81.90 & $0.1413 E-08$ \\
\hline 15.133 & 1371. & 0.514 & 0.600 & 81.90 & $0.1082 E-08$ \\
\hline 16.144 & 1456. & 1.027 & 0.200 & 81.90 & $0.3398 E-09$ \\
\hline 16.933 & 1135. & 0.514 & 0.200 & 81.89 & $0.4359 \mathrm{E}-09$ \\
\hline & & 5.651 & 17.200 & & \\
\hline 0.359 & 517. & 4.110 & 0.600 & 230.38 & $0.1020 \mathrm{E}-08$ \\
\hline 1.201 & 1213. & 2.569 & 1.200 & 230.37 & 0.8699E-09 \\
\hline 2.424 & 1760. & 3.082 & 2.400 & 230.54 & $0.1198 E-08$ \\
\hline 3.174 & 1081. & 2.055 & 1.800 & 230.34 & $0.1464 \mathrm{E}-08$ \\
\hline 4.203 & 1481. & 2.055 & 2.150 & 230.51 & $0.1276 \mathrm{E}-08$ \\
\hline 5.204 & 1442 . & 2.569 & 2.050 & 230.50 & $0.1249 E-08$ \\
\hline 6.238 & 1488. & 2.569 & 2.250 & 229.74 & $0.1333 E-08$ \\
\hline 7.647 & 2029 . & 2.569 & 2.750 & 229.73 & $0.1195 \mathrm{E}-08$ \\
\hline 8.358 & 1024. & 1.027 & 1.600 & 230.28 & $0.1375 E-08$ \\
\hline 9.414 & 1521. & 2.569 & 2.050 & 229.34 & $0.1191 E-08$ \\
\hline 10.209 & 1145. & 1.541 & 1.950 & 230.45 & $0.1497 \mathrm{E}-08$ \\
\hline 11.212 & 1444. & 2.055 & 2.200 & 229.32 & $0.1346 \mathrm{E}-08$ \\
\hline 12.174 & 1386. & 2.569 & 1.800 & 230.43 & $0.1142 \mathrm{E}-08$ \\
\hline 13.217 & 1502. & 2.055 & 2.400 & 230.41 & $0.1405 E-08$ \\
\hline & & 33.391 & 27.200 & & \\
\hline
\end{tabular}


Table F.4 Flow Test Results of Sample B/AL-C-4-35/A-P-B

\begin{tabular}{|c|c|c|c|c|c|}
\hline $\begin{array}{l}\text { Cumulativ } \\
\text { Time (days }\end{array}$ & $\begin{array}{l}\text { e Elapsed } \\
2 \text { Time (min) }\end{array}$ & $\begin{array}{l}\text { Inflow } \\
\text { (cc) }\end{array}$ & $\begin{array}{l}\text { Outflow } \\
\text { (cc) }\end{array}$ & $\begin{array}{l}\text { Hydr. } \\
\text { Gradient }\end{array}$ & $\begin{array}{c}\text { Permeability } \\
(\mathrm{cm} / \mathrm{s})\end{array}$ \\
\hline 2.016 & 2903. & 1.033 & 6.350 & 30.45 & $0.1468 \mathrm{E}-07$ \\
\hline 3.597 & 2276 . & 3.100 & 0.650 & 30.07 & $0.1940 \mathrm{E}-08$ \\
\hline 4.773 & 1694. & 0.517 & 0.450 & 30.06 & $0.1805 E-08$ \\
\hline 7.940 & 4560. & 2.584 & 0.550 & 30.80 & $0.8001 E-09$ \\
\hline \multirow[t]{2}{*}{10.228} & 3295. & 1.033 & 1.050 & 30.79 & $0.2115 \mathrm{E}-08$ \\
\hline & & 8.268 & 9.050 & & \\
\hline 2.018 & 2906 . & 2.584 & 0.900 & 52.72 & $0.1200 \mathrm{E}-08$ \\
\hline 2.315 & 427. & 0.517 & 0.400 & 52.71 & $0.3631 E-08$ \\
\hline 3.069 & 1087. & 0.517 & 0.500 & 52.34 & $0.1796 \mathrm{E}-08$ \\
\hline 4.785 & 2470 . & 2.067 & 0.900 & 52.33 & $0.1423 E-08$ \\
\hline 5.503 & 1035. & 0.517 & 0.750 & 51.95 & $0.2850 \mathrm{E}-08$ \\
\hline \multirow[t]{2}{*}{7.353} & 2664. & 3.100 & 0.500 & 52.69 & $0.7278 \mathrm{E}-09$ \\
\hline & & 9.301 & 3.950 & & \\
\hline 1.686 & 2428 . & 4.651 & 2.600 & 81.70 & $0.2678 \mathrm{E}-08$ \\
\hline 2.697 & 1455. & 2.067 & 0.550 & 82.06 & $0.9412 \mathrm{E}-09$ \\
\hline 2.860 & 236 & 0.517 & 0.500 & 82.43 & $0.5252 \mathrm{E}-08$ \\
\hline 4.811 & 2809 & 2.584 & 2.100 & 82.04 & $0.1862 \mathrm{E}-08$ \\
\hline 5.823 & 1457. & 1.550 & 0.650 & 82.03 & $0.1111 E-08$ \\
\hline 6.693 & 1253. & 1.550 & 0.600 & 81.65 & $0.1198 \mathrm{E}-08$ \\
\hline \multirow{2}{*}{7.008} & 453. & 0.517 & 0.550 & 82.02 & $0.3024 E-08$ \\
\hline & & 13.435 & 7.550 & & \\
\hline 0.657 & 946. & 6.718 & 3.350 & 155.74 & $0.4646 \mathrm{E}-08$ \\
\hline 1.187 & 763. & 2.067 & 1.550 & 155.71 & $0.2666 \mathrm{E}-08$ \\
\hline 1.736 & 791. & 2.584 & 1.650 & 156.07 & $0.2731 E-08$ \\
\hline 2.001 & 381. & 1.033 & 0.550 & 156.07 & $0.1890 \mathrm{E}-08$ \\
\hline 2.861 & 1239. & 3.100 & 1.550 & 155.50 & $0.1644 \mathrm{E}-08$ \\
\hline 3.840 & 1410. & 3.617 & 1.500 & 156.04 & $0.1393 E-08$ \\
\hline 4.708 & 1249. & 2.067 & 1.600 & 155.28 & $0.1686 E-08$ \\
\hline 5.672 & 1388. & 2.584 & 1.650 & 155.45 & $0.1562 E-08$ \\
\hline \multirow[t]{2}{*}{6.624} & 1372. & 2.584 & 1.600 & 154.88 & $0.1538 \mathrm{E}-08$ \\
\hline & & 26.353 & 15.000 & & \\
\hline 0.135 & 194. & 3.617 & 1.550 & 230.85 & $0.7071 E-08$ \\
\hline 0.348 & 307. & 2.584 & 1.800 & 230.47 & $0.5198 \mathrm{E}-08$ \\
\hline 1.069 & 1038. & 5.684 & 3.600 & 230.82 & $0.3070 \mathrm{E}-08$ \\
\hline 2.088 & 1467. & 6.201 & 3.200 & 230.79 & $0.1931 \mathrm{E}-08$ \\
\hline 2.315 & 327. & 0.517 & 0.600 & 230.77 & $0.1625 \mathrm{E}-08$ \\
\hline 2.648 & 480 . & 1.550 & 0.800 & 229.27 & $0.1485 E-08$ \\
\hline 3.524 & 1261. & 4.134 & 2.200 & 229.26 & $0.1555 \mathrm{E}-08$ \\
\hline 4.470 & 1363. & 4.134 & 1.800 & 229.80 & $0.1174 \mathrm{E}-08$ \\
\hline 5.187 & 1032 . & 3.617 & 1.700 & 230.71 & $0.1459 \mathrm{E}-08$ \\
\hline 6.076 & 1280 . & 3.617 & 2.050 & 229.95 & $0.1423 E-08$ \\
\hline 6.371 & 425. & 1.550 & 0.650 & 230.68 & $0.1355 E-08$ \\
\hline
\end{tabular}




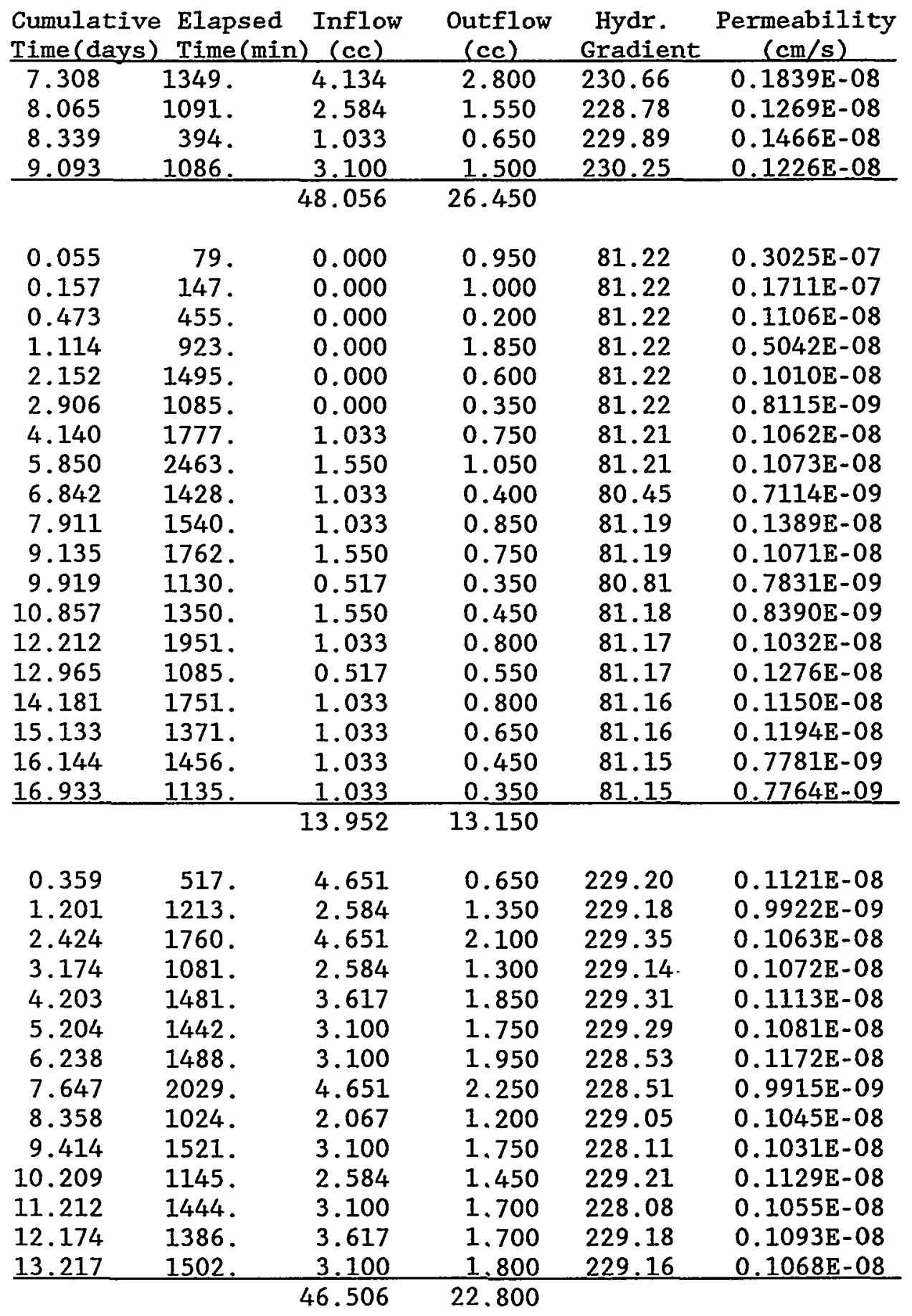




\section{APPENDIX G}

DERIVATION OF THE BUCRINGHAM EQUATION FOR PLASTIC FLOW THROUGH PIPES

According to Bingham's plastic flow concept, the rate of shear in a plastic material is proportional to the excess of the shearing stress $F$ over a certain constant yield stress $f$, below which the material behaves as a solid. If $v$ is the speed at any point and $y$ denotes the distance perpendicular to $v$, the hypothesis can be described by the equation:

$$
\frac{d v}{d y}=\mu(F-f) \quad \text { for } F \geq f
$$

where $\mu=$ mobility of the material,

$F=$ shearing stress

$f=$ yield stress of the material.

This equation does not fit the observations well. Buckingham (1921) gave a refined formula in the following form:

$$
\frac{V}{t}=\frac{\pi R^{4} \mu}{8 l}\left(P-\frac{4}{3} p_{o}+\frac{p_{o}^{4}}{3 P^{3}}\right)+\pi R^{2} v_{R}
$$

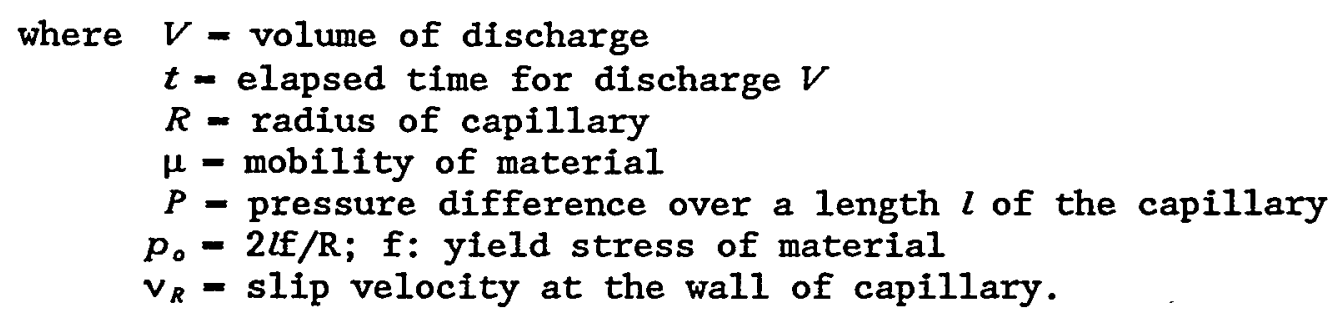

The equation was given without detailed derivations. The theory of plastic flow through capillaries has been applied to the determination of the yield stress of bentonite flow in glass tubes. The derivation of Buckingham's equation is given below.

Consider the steady state flow of a plastic material under a pressure $P$, through a circular tube of length $l$ and radius $R$. Assume that the end and kinetic energy corrections are negligible (i.e. slow steady flow). The longitudinal force, due to pressure $P$, on the material inside a cylindrical surface of radius $r$, coaxial with the tube, is $\pi r^{2} P$, and the resulting shearing stress at radius $r$ is:

$$
F=\pi r^{2} P / 2 \pi r l=P r / 2 l
$$

Substituting Eq. G.3 in Eq. G.1, and changing $y$ to $-r$, because the speed decreases as $r$ increases, results in: 


$$
-\frac{d v}{d r}=\mu\left(\frac{P r}{2 l}-f\right)
$$

For $\operatorname{Pr} / 2 l<f$, there is no shear; hence, the material inside the cylinder of radius $r_{0}=2 l f / P$ moves as a solid plug. Between $r_{0}$ and $R$, the material yields. Let $v_{R}$ be the slip at the wall and $v_{r}$ the speed at radius r. Thus:

$$
v_{r}-v_{R}=-\mu \int_{R}^{r}\left(\frac{P r}{2 l}-f\right) d r=\mu\left[\frac{P}{4 l}\left(R^{2}-r^{2}\right)-f(R-r)\right]
$$

Setting $r=r_{0}=2 l f / P$ in Eq. 5, the speed of the solid plug is given by:

$$
v_{0}-v_{R}=\mu\left[\frac{P R^{2}}{4 l}+\frac{l f^{2}}{P}-f R\right]
$$

Thus, the speed distribution curve is not a complete parabola, but has the vertex cut off. The volume rate of discharge can then be described by:

$$
\frac{V}{t}=\int_{0}^{R} 2 \pi r d r v=\pi r_{0}^{2} v_{0}+2 \pi \int_{r_{0}}^{R} v_{r} r d r
$$

Substituting the following expressions:

$$
\begin{aligned}
& r_{0}=2 l f / P \\
& v_{0}=\mu\left[\frac{P R^{2}}{4 l}+\frac{l f^{2}}{P}-f R\right]+v_{R} \\
& v_{r}=\mu\left[\frac{P}{4 l}\left(R^{2}-r^{2}\right)-f(R-r)\right]+v_{R}
\end{aligned}
$$


Eq. G.7 becomes:

$$
\begin{aligned}
\frac{V}{t}= & \pi \frac{4 l^{2} f^{2}}{P^{2}}\left\{\mu\left[\frac{P R^{2}}{4 l}+\frac{l f^{2}}{P}-f R\right]+v_{R}\right\} \\
& +2 \pi \int_{r_{0}}^{R}\left\{\mu\left[\frac{P}{4 l}\left(R^{2}-r^{2}\right)-f(R-r)\right]+v_{R}\right\} r_{i} d r
\end{aligned}
$$

Expand the second term on the right hand side:

$$
\begin{aligned}
& 2 \pi \int_{r_{0}}^{R}\left\{\mu\left[\frac{P}{4 l}\left(R^{2}-r^{2}\right)-f(R-r)\right]+v_{R}\right\} r d r \\
& =2 \pi\left[\int_{r_{0}}^{R} \mu \frac{P}{4 l}\left(R^{2} r-r^{3}\right) d r-\int_{r_{0}}^{R} \mu f\left(R r-r^{2}\right) d r+\int_{r_{0}}^{R} v_{R} r d r\right] \\
& =2 \pi\left[\left.\mu \frac{P}{4 l}\left(\frac{1}{2} R^{2} r^{2}-\frac{1}{4} r^{4}\right)\right|_{r_{0}} ^{R}-\left.\mu f\left(\frac{1}{2} R r^{2}-\frac{1}{3} r^{3}\right)\right|_{r_{0}} ^{R}+\left.v_{R} \frac{1}{2} r^{2}\right|_{r_{0}} ^{R}\right] \\
& =2 \pi\left\{\frac{\mu P}{4 l}\left[\left(\frac{1}{2} R^{4}-\frac{1}{4} R^{4}\right)-\left(\frac{1}{2} R^{2} r_{0}^{2}-\frac{1}{4} r_{0}^{4}\right)\right]-\mu f\left[\left(\frac{1}{2} R^{3}-\frac{1}{3} R^{3}\right)-\right.\right. \\
& \left.\left.\left(\frac{1}{2} R r_{0}^{2}-\frac{1}{3} r_{0}^{3}\right)\right]+v_{R}\left[\frac{1}{2} R^{2}-\frac{1}{2} r_{0}^{2}\right]\right\} \\
& =2 \pi\left\{\frac{\mu P}{4 l}\left[\frac{R^{4}}{4}-\frac{R^{2} r_{0}^{2}}{2}+\frac{r_{0}^{4}}{4}\right]-\mu f\left[\frac{R^{3}}{6}-\frac{R r_{0}^{2}}{2}+\frac{r_{0}^{3}}{3}\right]+v_{R}\left[\frac{R^{2}}{2}-\frac{r_{0}^{2}}{2}\right]\right\} \\
& =\frac{\pi \mu P}{2 l}\left[\frac{R^{4}}{4}-\frac{2 l^{2} f^{2} R^{2}}{P^{2}}+\frac{4 l^{4} f^{4}}{P^{4}}\right]-2 \pi \mu f\left[\frac{R^{3}}{6}-\frac{2 l^{2} f^{2} R}{P^{2}}+\frac{8 l^{3} f^{3}}{3 P^{3}}\right] \\
& +2 \pi v_{R}\left[\frac{R^{2}}{2}-\frac{2 l^{2} f^{2}}{P^{2}}\right] \quad \text { (since } \quad r_{0}=2 l f / P \text { ) }
\end{aligned}
$$


Therefore, Eq. G.8 can be written as:

$$
\begin{aligned}
\frac{V}{t}= & \frac{\pi \mu l f^{2} R^{2}}{P}+\frac{4 \pi \mu l^{3} f^{4}}{P^{3}}-\frac{4 \pi \mu l^{2} f^{3} R}{P^{2}}+\frac{4 \pi l^{2} f^{2} v_{R}}{P^{2}} \\
& +\frac{\pi \mu P R^{4}}{8 l}-\frac{\pi \mu l f^{2} R^{2}}{P}+\frac{2 \pi \mu l^{3} f^{4}}{P^{3}}-\frac{\pi \mu f R^{3}}{3} \\
& +\frac{4 \pi \mu l^{2} f^{3} R}{P^{2}}-\frac{16 \pi \mu l^{3} f^{4}}{3 P^{3}}+\pi v_{R} R^{2}-\frac{4 \pi l^{2} f^{2} v_{R}}{P^{2}} \\
& =\frac{\pi \mu R^{4} P}{8 l}-\frac{\pi \mu f R^{3}}{3}+\pi R^{2} v_{R}+\frac{2 \pi \mu l^{3} f^{4}}{3 P^{3}} \\
& =\frac{\pi R^{4} \mu}{8 l}\left[P-\frac{8 l f}{3 R}+\frac{16 l^{4} f^{4}}{3 P^{3} R^{4}}\right]+\pi R^{2} v_{R}
\end{aligned}
$$

Introducing $p_{0}=2 l f / R$, then:

$$
\frac{V}{t}=\frac{\pi R^{4} \mu}{8 l}\left[P-\frac{4}{3} p_{\circ}+\frac{p_{o}^{4}}{3 P^{3}}\right]+\pi R^{2} v_{R}
$$




\section{APPENDIX H \\ PROCEDURE FOR THE DETERMINATION OF YIELD STRESS \\ OF BENTONITE FLOWING IN GLASS TUBES}

\section{H.1 Objective}

This procedure describes a method for the determination of the yield stress of bentonite at various water contents. The method is based on the theory of plastic flow through capillaries (Bingham, 1916; Buckingham, 1921) and closely follows the work by Marsland and Loudon (1963).

\section{H.2 Apparatus}

- a PVC pipe reservoir (ID: $10.16 \mathrm{~cm}, \mathrm{~L}: 18 \mathrm{~cm}$, wall thickness: $6.15 \mathrm{~mm}$ )

- glass capillaries (3.6, 2.4, and $1.65 \mathrm{~mm}$ diameter and $28 \mathrm{~cm}$ long)

- compressed helium tank

- pressure regulator

- pressure gauge (resolution of 0.2 psi $=1379 \mathrm{~Pa}$ )

- measuring tape (readable to $0.5 \mathrm{~mm}$ )

- cap plates

- assembling bolts and nuts

Figure H.1 shows the test setup.

\section{H. 3 Sample Preparation and Installation}

- Weigh an appropriate amount of bentonite and mix it with distilled water to yield a desired water content.

- Knead to achieve a uniform distribution of the water content, especially for relatively dry samples.

- Store the sample in an airtight plastic container and allow the sample to cure for 72 hours.

- Transfer a portion of the cured sample into the PVC pipe reservoir until the top of the installed sample lies approximately $5 \mathrm{~cm}$ above the horizontal capillaries. The amount of bentonite emplaced should be enough for one test.

- Remove air voids entrapped in the sample as much as possible by hand kneading and compressing to prevent air flow during the testing.

- Level the sample's surface. Seal the reservoir with the top cap plate. Connect the pressure line to the pressure gauge and the compressed helium tank. 


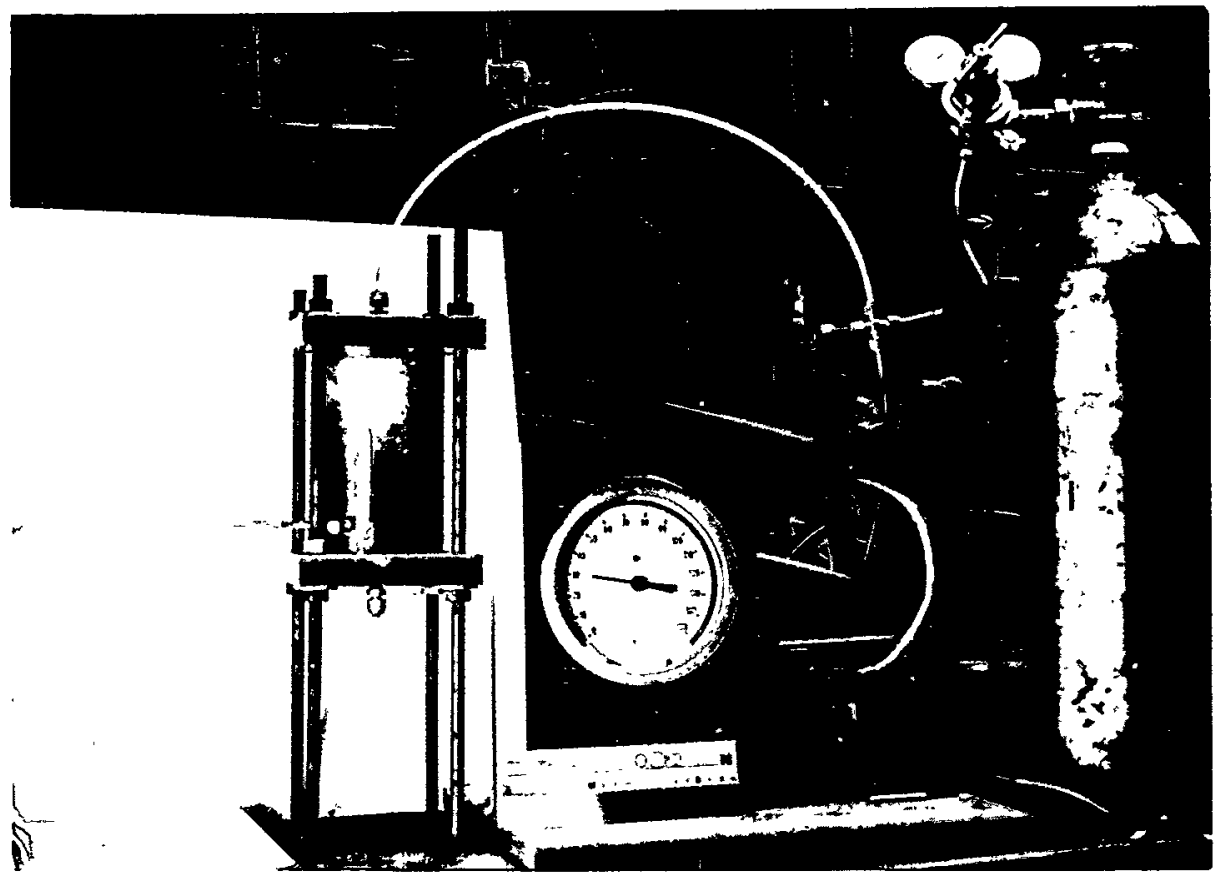

Figure H.I Setup for testing of bentonite flowing through glass capillaries. 
- Cover the ends of the glass tubes with aluminum foil to reduce evaporation.

- Take two or three samples from the remaining bentonite paste and determine their water content.

- Obtain three density measurements using the remaining sample.

H.4 Experimental Procedure

(1) Open the valve of the compressed helium tank. Adjust the regulator until the advance of bentonite in the capillaries can be observed.

(2) Record the gauge pressure $\left(P_{g}\right)$, the height (L) between the sample top and the tubes, and the length (1) of the bentonite paste in the capillaries with time.

(3) If the clay paste continues to advance for 30 minutes, reduce the pressure and repeat Step 2 until no flow can be detected. If the bentonite has a high water content (e.g. 500\%), stop the test and go to Step 5. This stage is assumed to indicate the "no flow" condi-. tion. The shear stress at the wall of the tube is designated as the yield stress for the given water content. Otherwise, go to Step 4.

(4) For drier samples, times elapsed before reaching the "no flow" conclusion are 4, 12, and 24 hours for nominal water contents of 200, 100 , and $75 \%$, respectively. If flow is observed, reduce the pressure further until the "no flow" condition is obtained.

(5) After the test, determine the water content of the bentonite near the capillary tubes, as well as the water content of bentonite in the tubes.

\section{H.5 Calculation of Yield Stress}

The total driving pressure at the entrance of a glass tube $\left(P_{t}\right)$ is the sum of the gauge pressure $\left(P_{g}\right)$ and the lithostatic pressure $\left(P_{S}\right)$, assuming that the shear between bentonite and the inner wall of the PVC pipe is negligible and the coefficient of lateral earth pressure $\left(\mathrm{K}_{0}\right)$ equals 1. That is :

$$
\begin{aligned}
& P_{t}=P_{g}+P_{s} \\
& P_{g}(\text { in Pascal })=P_{g}(\text { in psi) } \times 6894.7572 \text { Pascal } / p s i \\
& P_{S}(\text { in Pascal })=L(m) \times \text { material density }\left(\mathrm{kg} / \mathrm{m}^{3}\right) \times 9.80665\left(\mathrm{~m} / \mathrm{s}^{2}\right)
\end{aligned}
$$

Let $R$ be the radius of the capillary (in $\mathrm{cm}$ ). From the force balance, we obtain: 


$$
\mathrm{P}_{\mathrm{t}} \times \mathrm{R}^{2}=\tau_{f} \times 2 \mathrm{R} \times 1_{0}
$$

and

$$
\tau_{f}=P_{t} \times R / 21_{0}
$$

where $\tau_{f}=$ yield stress in $\mathrm{Pa}$

$1_{0}=$ length of clay paste in the capillary (in $\mathrm{cm}$ ) at the "no flow" condition.

Several determinations of water content are required in the procedure because the actual water content of bentonite in a capillary can be quite different from its initial water content due to the migration of water. To what water content a computed yield stress corresponds needs a careful examination of experimental records. In principle, the calculated yield stress is related to the averaged initial water content if the "no flow" condition is established early in the test. For the case where the condition is arrived at in the later part of the test, the water content of bentonite paste in the capillary is selected. 


\begin{tabular}{|c|c|}
\hline $\begin{array}{l}\text { U.S. NUCLEAR REGULATORY COMMISSION } \\
\text { BIBLIOGRAPHIC DATA SHEET } \\
\text { (See instructions on the reverse) }\end{array}$ & 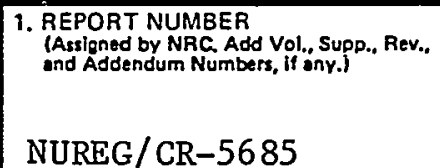 \\
\hline \multirow{4}{*}{$\begin{array}{l}\text { 2. TITLE AND SUBTITLE } \\
\text { Sealing Performance of Bentonite and Bentonite/Crushed Rock } \\
\text { Borehole Plugs }\end{array}$} & \multirow{2}{*}{\begin{tabular}{|l} 
NUREG/CR-5685 \\
3. DATE REPORT PUBLISHED
\end{tabular}} \\
\hline & \\
\hline & \begin{tabular}{|c|r|} 
MONTH & YEAR \\
July & 1992 \\
\end{tabular} \\
\hline & $\begin{array}{l}\text { 4. FIN OR GRANT NUMBER } \\
\text { L1476 }\end{array}$ \\
\hline $\begin{array}{l}\text { 5. AUTHOR(S) } \\
\text { S. Ouyang, J. J. K. Daemen }\end{array}$ & $\begin{array}{l}\text { 6. TYPE OF REPORT } \\
\text { Technical }\end{array}$ \\
\hline & $\begin{array}{l}\text { 7. PERIOD COVERED (Inclusive Dates) } \\
1988-1990\end{array}$ \\
\hline
\end{tabular}

8. PERFORMING ORGANIZATION - NAME AND ADDRESS IIN NRC, provide Division, Office or Region, U.S. Nuclear Regulatory Commission, and mailing address; if contractor, provide name and mailing address.)

Department of Mining and Geological Engineering

University of Arizona

Tucson, AZ 85721

9. SPONSORING ORGANIZATION - NAME AND ADDRESS (II NRC, type "Same as above"; if contractor, provide NRC Division, Office or Region, U.S. Nuclear Regulatory Commission. and mailing address.l

Division of Regulatory Applications

Office of Nuclear Regulatory Research

U.S. Nuclear Regulatory Commission

Washington, DC 20555

10. SUPPLEMENTARY NOTES

11. ABSTRACT (200 words or less) This study includes a systematic investigation of the sealing performance of bentonite and bentonite/crushed rock plugs. American Colloid C/S granular bentonite and crushed Apache Leap tuff have been mixed to prepare samples. Bentonite weight percent and crushed tuff gradation are the major variables studied. High injection pressure flow tests, polyaxial flow tests, high temperature flow tests, and piping tests have been performed. A composition to yield a permeability lower than $5 \times 10^{-8} \mathrm{~cm} / \mathrm{s}$ would have at least $25 \%$ bentonite by weight mixed with well-graded crushed rock. Hydraulic properties of the mixture plugs may be highly anisotropic if significant particle segregation occurs during sample installation and compaction. Temperature has no significant effect on sealing performance from room temperature to $60^{\circ} \mathrm{C}$. Piping damage is small if the hydraulic gradient does not exceed 120 and 280 for samples with a bentonite content of 25 and $35 \%$, respectively. The hydraulic gradients above which flow of bentonite may take place are deemed critical.

Bentonite occupancy percentage and water content at saturation are two major parameters for plug design. A model is developed for predicting the permeability in clays. A piping model, based on plastic flow theory, permits estimating the critical hydraulic gradients at which flow of bentonite takes place. The model can also be used to define the maximum allowable pore diameter of a protective filter layer.

12. KEY WORDS/DESCR!PTORS (List words or phrases that will assist researchers in locating the report.)

permeability

hydraulic conductivity

clay

piping

erosion

flow testing

tuff

plastic flow

longevity dispersion

bentonite content

grading

anisotropy

plug design

filter design
13. AVAILABILITY STATEMENT

Unlimited

14. SECURITY CLASSIFICATION

(This Pagel

Unclassified

This Report

Unclassified

15. NUMBER OF PAGES 